Prepared for the U.S. Department of Energy

under Contract DE-AC05-76RL01830

\title{
Equilibrium Speciation of Select Lanthanides in the Presence of Acidic Ligands in Homo- and Heterogeneous Solutions
}

\section{T Robinson}

August 2011

Pacific Northwest

NATIONAL LABORATORY

Proudly Operated by Battelle Since 1965 


\title{
DISCLAIMER
}

This report was prepared as an account of work sponsored by an agency of the United States Government. Neither the United States Government nor any agency thereof, nor Battelle Memorial Institute, nor any of their employees, makes any warranty, express or implied, or assumes any legal liability or responsibility for the accuracy, completeness, or usefulness of any information, apparatus, product, or process disclosed, or represents that its use would not infringe privately owned rights. Reference herein to any specific commercial product, process, or service by trade name, trademark, manufacturer, or otherwise does not necessarily constitute or imply its endorsement, recommendation, or favoring by the United States Government or any agency thereof, or Battelle Memorial Institute. The views and opinions of authors expressed herein do not necessarily state or reflect those of the United States Government or any agency thereof.

\author{
PACIFIC NORTHWEST NATIONAL LABORATORY \\ operated by \\ BATTELLE \\ for the \\ UNITED STATES DEPARTMENT OF ENERGY \\ under Contract DE-AC05-76RL01830
}

Printed in the United States of America
Available to DOE and DOE contractors from the Office of Scientific and Technical Information,
P.O. Box 62, Oak Ridge, TN 37831-0062;
ph: (865) 576-8401
fax: $(865)$ 576-5728
email: reports@adonis.osti.gov

\begin{abstract}
Available to the public from the National Technical Information Service, U.S. Department of Commerce, 5285 Port Royal Rd., Springfield, VA 22161 ph: (800) 553-6847 fax: $(703) 605-6900$ email: orders@ntis.fedworld.gov online ordering: http://www.ntis.gov/ordering.htm
\end{abstract}

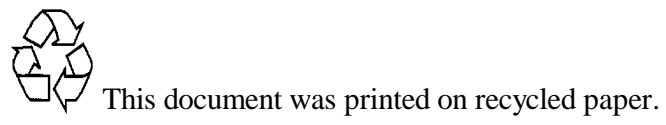


EQUILIBRIUM SPECIATION OF SELECT LANTHANIDES IN THE PRESENCE OF ACIDIC

LIGANDS IN HOMO- AND HETEROGENEOUS SOLUTIONS

By

Troy Robinson

Bachelor of Science

Brigham Young University

2005

A dissertation submitted in partial fulfillment of the requirements for the

Doctor of Philosophy in Radiochemistry

Department of Chemistry

College of Science

Graduate College

University of Nevada, Las Vegas

August 2011 
Copyright by Troy A Robinson 2011

All Rights Reserved 


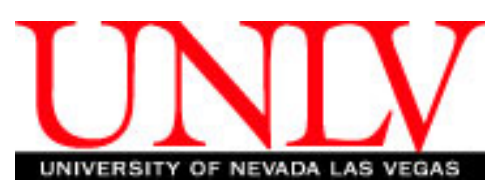

THE GRADUATE COLLEGE

We recommend the dissertation prepared under our supervision by

\section{Troy Robinson}

entitled

\section{Equilibrium Speciation of Select Lanthanides in the Presence of Acidic Ligands in Homo- and Heterogeneous Solutions}

be accepted in partial fulfillment of the requirements for the degree of

\section{Doctorate of Philosophy in Radiochemistry}

Department of Chemistry

Ken Czerwinski, Committee Chair

Sam Bryan, Committee Member

Patricia Paviet-Hartman, Committee Member

Tatiana Leviskaia, Committee Member

Ralf Sudowe, Graduate College Representative

Ronald Smith, Ph. D., Vice President for Research and Graduate Studies and Dean of the Graduate College

August 2011 
ABSTRACT

\title{
Equilibrium Speciation of Select Lanthanides in the Presence of Acidic Ligands in Homo- and Heterogeneous Solutions
}

\author{
by \\ Troy Robinson \\ Dr. Kenneth R. Czerwinski, Examination Committee Chair \\ Professor of Chemistry \\ Chair of the Department of Radiochemistry \\ University of Nevada, Las Vegas
}

This dissertation explores lanthanide speciation in liquid solution systems related to separation schemes involving the acidic ligands: bis(2-ethylhexyl) phosphoric acid (HDEHP), lactate, and 8-hydroxyquinoline. Equilibrium speciation of neodymium $\left(\mathrm{Nd}^{3+}\right)$, sodium $\left(\mathrm{Na}^{+}\right)$, HDEHP, water, and lactate in the TALSPEAK liquid-liquid extraction system was explored under varied $\mathrm{Nd}^{3+}$ loading of HDEHP in the organic phase and through extraction from aqueous $\mathrm{HCl}$ and lactate media. System speciation was probed through vapor pressure osmometry, visible and Fourier Transform Infrared (FTIR) spectroscopy, ${ }^{22} \mathrm{Na}$ and ${ }^{13} \mathrm{C}$ labeled lactate radiotracer distribution measurements, Karl Fischer titrations, and equilibrium $\mathrm{pH}$ measurements. Distribution of $\mathrm{Nd}^{3+}, \mathrm{Na}^{+}$, lactate, and equilibrium $\mathrm{pH}$ were modeled using the SXLSQI software to obtain $\log K_{\mathrm{Nd}}$ and $\log K_{\mathrm{Na}}$ extraction constants under selected conditions. Results showed that high $\mathrm{Nd}^{3+}$ loading of the HDEHP led to $\mathrm{Nd}^{3+}$ speciation that departs from the ion exchange mechanism and includes formation of highly aggregated, polynuclear [NdLactate(DEHP $\left.)_{2}\right]_{x}$; (with $\mathrm{x}>1$ ). By substituting lanthanum $\left(\mathrm{La}^{3+}\right)$ for $\mathrm{Nd}^{3+}$ in this system, NMR scoping experiments using ${ }^{23} \mathrm{Na},{ }^{31} \mathrm{P}$ nuclei and ${ }^{13} \mathrm{C}$ labeled lactate were performed. Results indicated that this technique is sensitive to changes in system speciation, and that further experiments are warranted. In a homogeneous system representing the TALSPEAK aqueous phase, Lactate protonation behavior at various temperatures was characterized using a combination of 
potentiometric titration and modeling with the Hyperquad computer program. The temperature dependent deprotonation behavior of lactate showed little change with temperature at $2.0 \mathrm{M}$ $\mathrm{NaCl}$ ionic strength.

Cloud point extraction is a non-traditional separation technique that starts with a homogeneous phase that becomes heterogeneous by the micellization of surfactants through the increase of temperature. To better understand the behavior of europium $\left(\mathrm{Eu}^{3+}\right)$ and 8hydroxyquinoline under cloud point extraction conditions, potentiometric and spectrophotometric titrations coupled with modeling with Hyperquad and SQUAD computer programs were performed to assess europium $\left(\mathrm{Eu}^{3+}\right)$ and 8-hydroxyquinoline speciation. Experiments in both water and a $1 \mathrm{wt} \%$ Triton X-114/water mixed solvent were compared to understand the effect of Triton X-114 on the system speciation. Results indicated that increased solvation of 8-hydroxyquinoline by the mixed solvent lead to more stable complexes involving 8hydroxyquinoline than in water, whereas competition between hydroxide and Triton X-114 for $\mathrm{Eu}^{3+}$ led to lower stability hydrolysis complexes in the mixed solvent than in water.

Lanthanide speciation is challenging due to the trivalent oxidation state that leads to multiple ligand complexes, including some mixed complexes. The complexity of the system demands well-designed and precise experiments that capture the nuances of the chemistry. This work increased the understanding of lanthanide speciation in the explored systems, but more work is required to produce a comprehensive understanding of the speciation involved. 


\section{ACKNOWLEDGEMENTS}

I would like to take this opportunity to recognize those who have helped me to this milestone in my development. I would like to thank my wife Linda has been patient and supportive of my long work hours and travel that has taken me away from her and our four children on numerous occasions. I would like to thank the members of my thesis committee: Ken Czerwinski, Tatiana Levitskaia, Sam Bryan and Patricia Paviet-Hartmann for their guidance while writing this dissertation. I would like to thank my thesis advisor, Ken Czerwinski, specifically for all he taught about a career in academia through his example of hard work and dedication. I would also like to thank Tatiana Levitskaia for the individual attention she provided to help improve my analytical chemistry, and attention to details in technical communication. Thanks to Brady Hanson and Sam Bryan who made it possible for me to come to Pacific Northwest National Laboratory and to gain experience performing research in the Radiochemical Processing Laboratory. Thanks to Dean Peterman, Scott Herbst and Terry Todd who gave me the chance to experience Idaho National Laboratory, and who provided valuable experience by entrusting me to work with ${ }^{14} \mathrm{C}$ in their laboratories. 


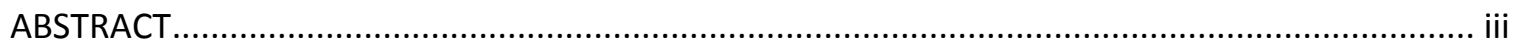

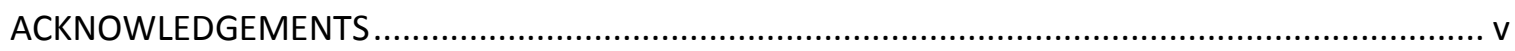

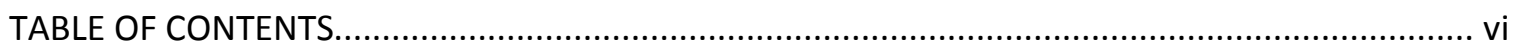

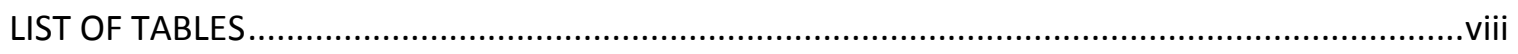

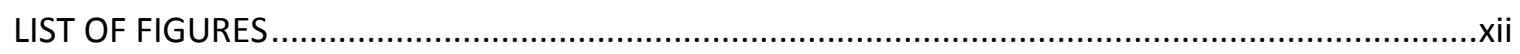

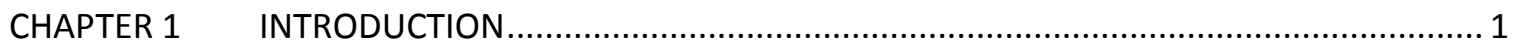

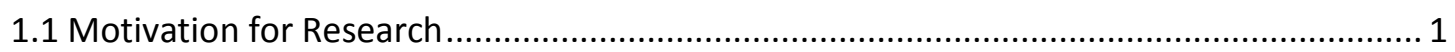

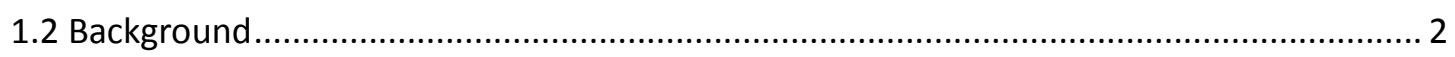

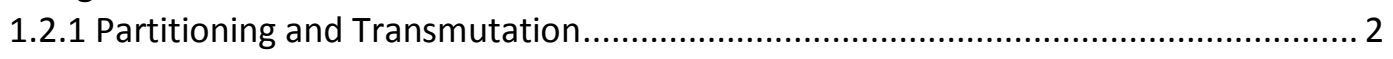

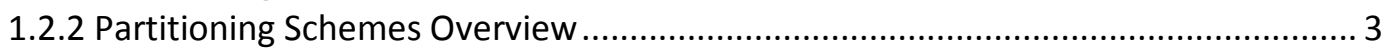

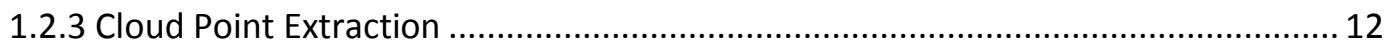

1.2.4 Trivalent Actinide/Lanthanide Separation Principles ........................................... 13

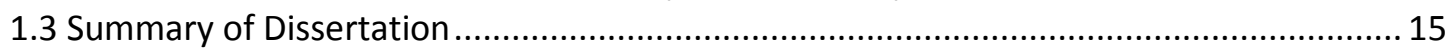

CHAPTER 2 TALSPEAK ORGANIC PHASE SPECIATION UNDER VARIABLE LOADING CONDITIONS: HCL AND LACTATE AQUEOUS MEDIUM STUDIES ........................16

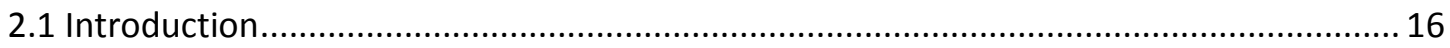

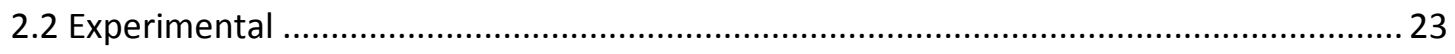

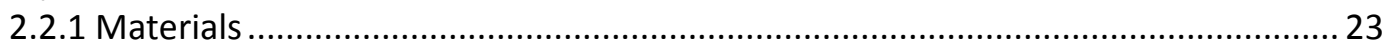

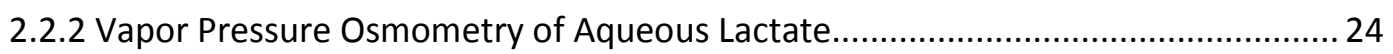

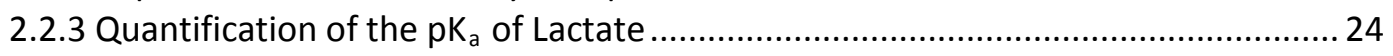

2.2.4 Liquid-Liquid Distribution Measurements Methodology ...................................... 26

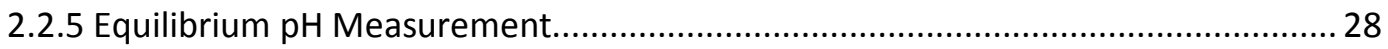

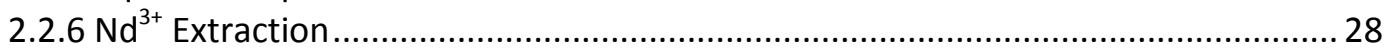

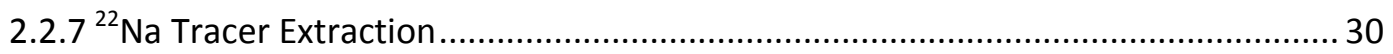

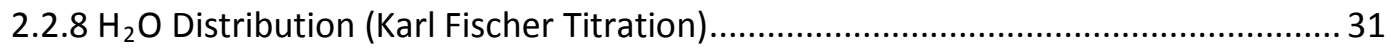

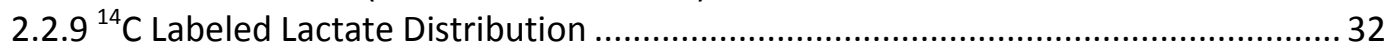

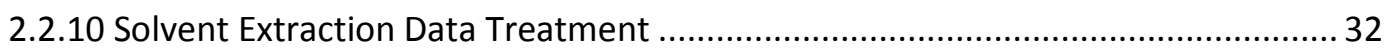

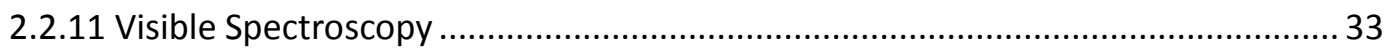

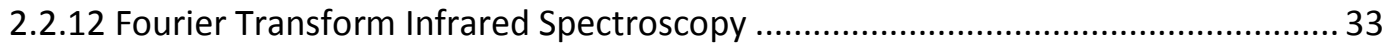

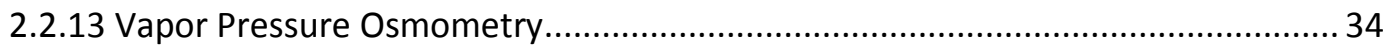

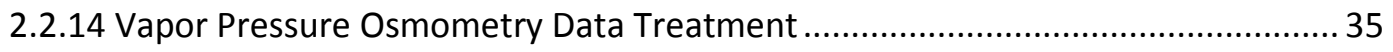

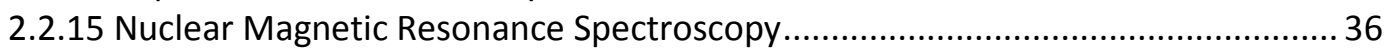

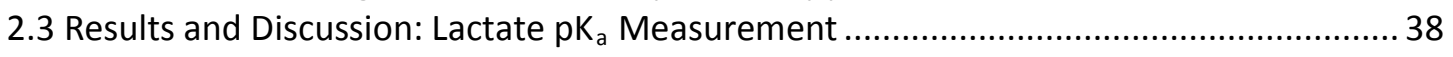

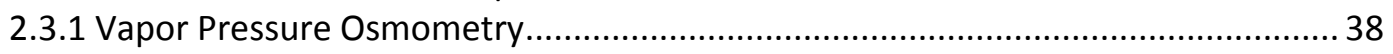

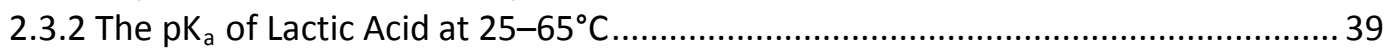

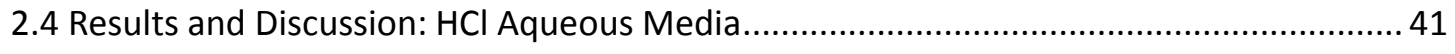

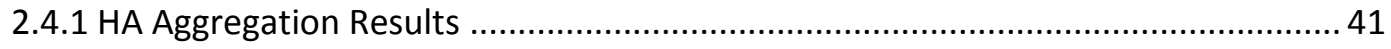

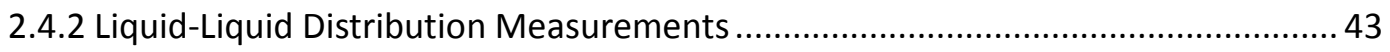

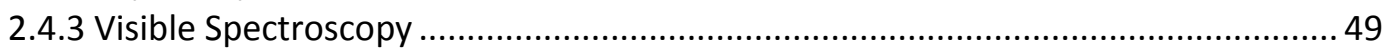


2.4.4 Fourier Transform Infrared Spectroscopy of the Organic Phase ........................... 53

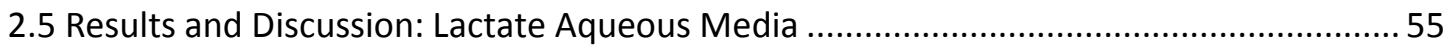

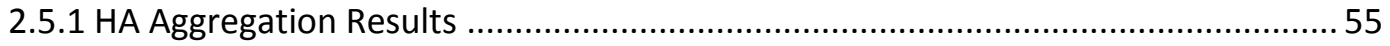

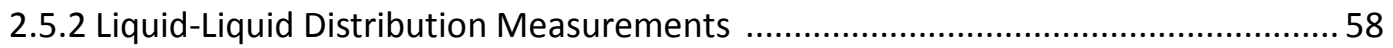

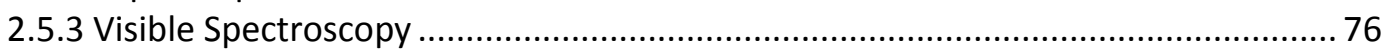

2.5.4 Fourier Transform Infrared Spectroscopy of the Organic Phase ............................ 78

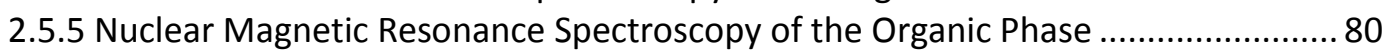

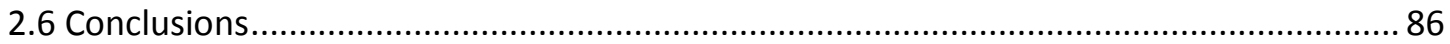

CHAPTER 3 THE DETERMINATION OF BINDING CONSTANTS OF 8-HYDROXYQUINOLINE IN THEWATER/TRITON X-114 MIXED SOLVENT SYSTEM .......................................90

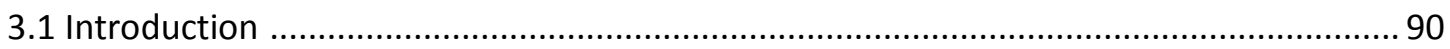

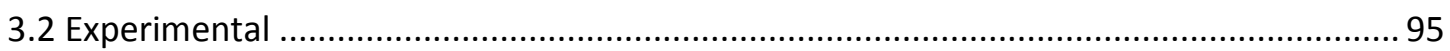

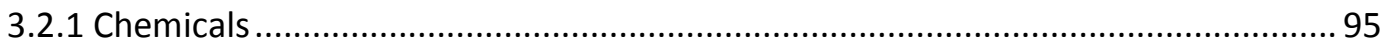

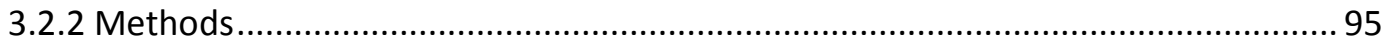

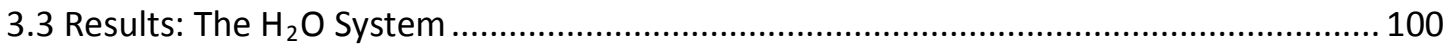

3.3.1 Determination of the $\mathrm{pK}_{\mathrm{a}}$ 's of 8-Hydroxyquinoline by Potentiometric

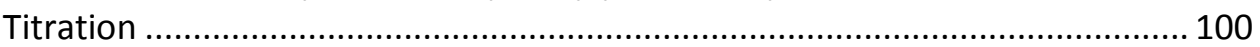

3.3.2 Determination of the $\mathrm{pK}_{\mathrm{a}}{ }^{\text {'s }}$ of 8-Hydroxyquinoline by Spectrophotometric

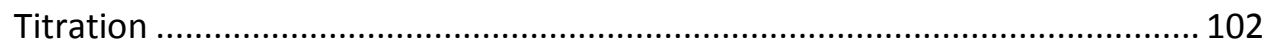

3.3.3 Determination of the Europium Hydrolysis Constants by Potentiometric

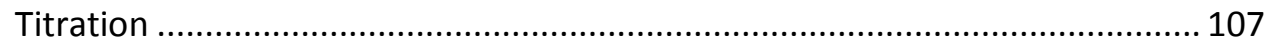

3.3.4 Determination of the Europium 8-Hydroxyquinoline Complex Stability Constants by Potentiometric Titration ........................................................... 109

3.3.5 Determination of the Europium 8-Hydroxyquinoline Complex Stability Constants by Spectrophotometric Titration ................................................... 116

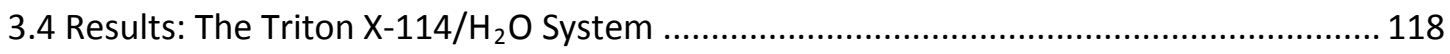

3.4.1 Characterization of Protolytic Behavior of Triton X-114 by Potentiometric

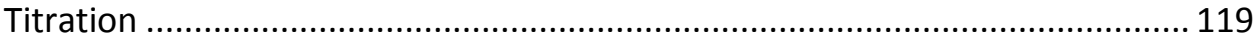

3.4.2 Determination of the $\mathrm{pK}_{\mathrm{a}}{ }^{\prime} \mathrm{s}$ of 8-Hydroxyquinoline by Potentiometric Titration 121

3.4.3 Determination of the $\mathrm{pK}_{\mathrm{a}}$ 's of 8-Hydroxyquinoline by Spectrophotometric

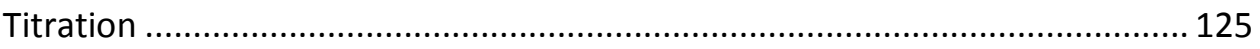

3.4.4 Determination of the Europium Hydrolysis Constants by Potentiometric Titration 129

3.4.5 Determination of the Europium 8-Hydroxyquinoline Complex Stability Constants by Potentiometric Titration ....................................................... 131

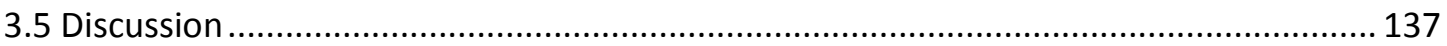

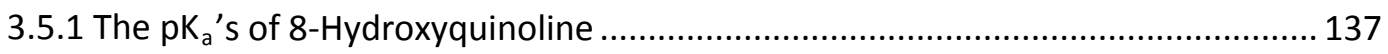

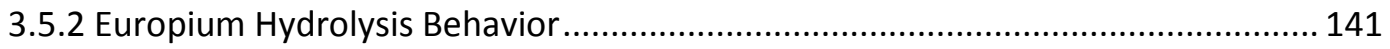

3.5.3 The Europium 8-Hydroxyquinoline Stability Constants ...................................... 145

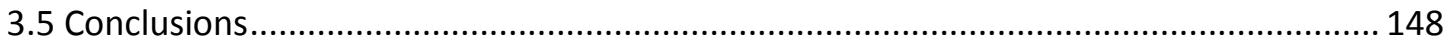

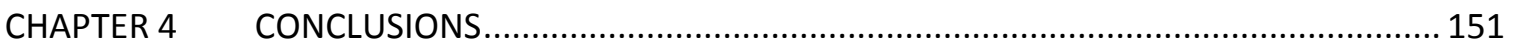

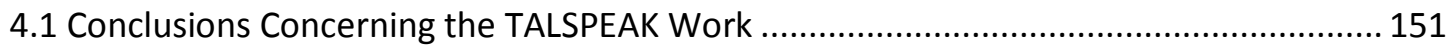

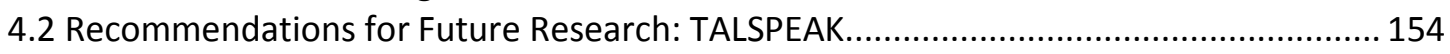

4.3 Conclusions Concerning the Europium 8-Hydroxyquinoline Work ................................. 157

4.4 Recommendations for Future Research: Europium 8-Hydroxyquinoline ....................... 157 
APPENDIX $1 \quad$ INSTRUCTIONS AND DESCRIPTION OF THE USE OF SQUAD …......................... 159

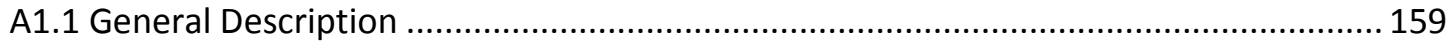

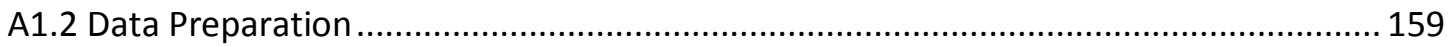

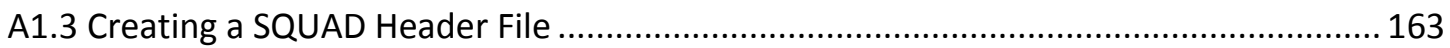

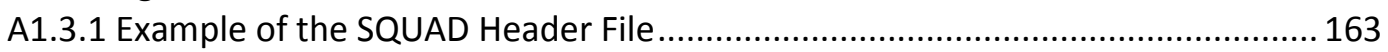

A1.3.2 Description of the SQUAD Header File Line-by-line ......................................... 165

A1.4 Creating the SQUAD Input File using GWBASIC.EXE while running the SQUAD-IN.BAS Instruction File. 171

A1.4.1 Step-by-step Instructions for using GWBASIC.EXE and SQUAD-IN.BAS to Create a

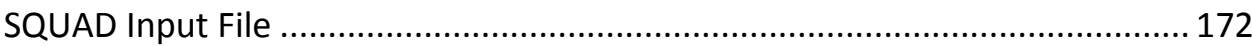

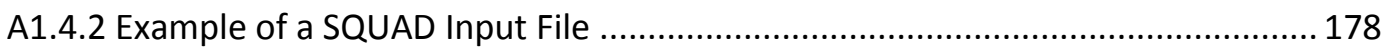

A1.4.3 Line-by-line Description of the SQUAD Input File .......................................... 179

A1.5 Using SQUAD to Read-in Data from the Input File and Create an Output File .............. 181

A1.5.1 Step-by-step Instructions for using SQUAD to Create an Output File.................. 181

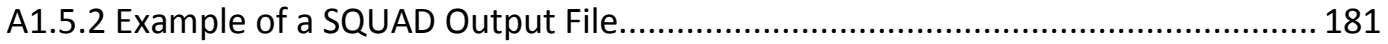

A1.5.3 Line-by-line Description of the SQUAD Output File .......................................... 190

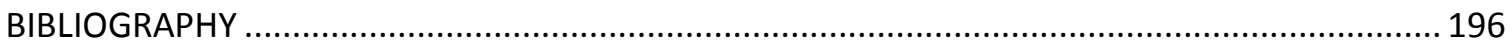

VITA 


\section{LIST OF TABLES}

Table 1.1

Table 1.2

Table 1.3

Table 1.4

Table 2.1

Table 2.2

Table 2.3

Table 2.4

Table 2.5

Table 2.6

Table 2.7

Table 2.8

Table 2.9

Table 2.10

Table 2.11

Table 2.12

Table 2.13

Table 2.14

Table 2.15

Table 2.16

Table 2.17

Table 2.18

Table 2.19

Table 2.20

Table 2.21

Thermal neutron capture cross section ${ }^{[16]}$ (barns) of the fission product lanthanides. Isotopes with fission yields of zero were omitted. Values in bold font are for isotopes with $T_{1 / 2}>20$ years that are more likely to be present after the post-irradiation cooling period.

The top 15 elements in irradiated fuel sorted by mass percent. Category is either Actinides (Act.) or Fission Product (F.P).

\section{5}

The top 15 elements in irradiated fuel sorted by Percent of total activity. Category is either Actinides (Act.) or Fission Product (F.P.) ................................ 6 Ionic radius of Trivalent lanthanide and actinide ions. ${ }^{[48]}$..................................... 14 IUPAC recommended values for the conditional $\mathrm{pK}_{\mathrm{a}}$ of Lactic Acid. ${ }^{[55]}$.............. 17 IUPAC recommended data for the conditional $\mathrm{pK}_{\mathrm{a}}$ of Lactic acid in $\mathrm{NaCl}$ media. ${ }^{[55]}$

Aqueous phase solution concentrations for the $\mathrm{HCl}$ medium experiments. ...... 27 Aqueous phase solution concentrations for the lactate medium experiments. . 26 Constants used to model ionic strength of aqueous phase for phase contacts.. 26 Molar absorptivities observed for $\mathrm{Nd}^{3+}$ in the aqueous phase...........................28 Summary of the molar absorptivity data, and calibration techniques used in the quantification of $\mathrm{Nd}^{3+}$ in the aqueous phase solutions. .........................................28 The detection limits for the $\mathrm{Na}^{+}$extraction experiments by system.................. 30 Aqueous solutions used for the NMR scoping experiments................................36 Standard enthalpy of reaction obtained for lactic acid potentiometric titrations at $25-65^{\circ} \mathrm{C}$ at ionic strength of $2.0 \mathrm{M} \mathrm{NaCl}$.

Equilibrium $\mathrm{pH},\left[\mathrm{Na}^{+}\right]_{\mathrm{org}}$, and $\left[\mathrm{Nd}^{3+}\right]_{\text {org }}$ results for the $\mathrm{pH} 1+(0,30) \mathrm{mM} \mathrm{Nd}+\mathrm{HCl}$ systems. Estimated error for these values is: $\mathrm{pH}_{\text {eq }} \pm 0.01$, $\left[\mathrm{Nd}^{3+}\right]_{\text {org }} \pm 2 \% \ldots \ldots \ldots . . .44$ Equilibrium $\mathrm{pH},\left[\mathrm{Na}^{+}\right]_{\text {org }}$, and $\left[\mathrm{Nd}^{3+}\right]_{\text {org }}$ results for the $\mathrm{pH} 4+(0,30) \mathrm{mM} \mathrm{Nd}+\mathrm{HCl}$ systems. Estimated error for these values is: $\mathrm{pH}_{\mathrm{eq}} \pm 0.01,\left[\mathrm{Nd}^{3+}\right]_{\mathrm{org}} \pm 2 \% \ldots \ldots \ldots . . .44$ Organic phase water concentration for the $\mathrm{pH}(1,4)+(0,30) \mathrm{mM} \mathrm{Nd}+\mathrm{HCl}$ systems.

Prominent bands of the molar absorptivity of $\mathrm{Nd}^{3+}$ in the aqueous and organic phase of the $\mathrm{HCl}$ systems. Bands that found in the shoulder of other bands without a prominent maximum are shown in parentheses. Equilibrium $\mathrm{pH},\left[\mathrm{Na}^{+}\right]_{\mathrm{org}},\left[\mathrm{Nd}^{3+}\right]_{\mathrm{org}}$, and $[\mathrm{Lac}]_{\mathrm{org}}$ for the $\mathrm{pH} 1+(0,30) \mathrm{mM} \mathrm{Nd}+$ Lac systems. Error for the values shown is: \pm 0.01 for $\mathrm{pH}_{\text {eq }}, 2 \%$ for $\left[\mathrm{Nd}^{3+}\right]_{\text {org,eq }} .58$ Equilibrium $\mathrm{pH},\left[\mathrm{Na}^{+}\right]_{\text {org }},\left[\mathrm{Nd}^{3+}\right]_{\text {org }}$, and $[\mathrm{Lac}]_{\text {org }}$ for the $\mathrm{pH} 4+(0,30,60) \mathrm{mM} \mathrm{Nd}$ + Lac systems. Error for the values shown is: \pm 0.01 for $\mathrm{pH}_{\text {eq }}, 2 \%$ for $\left[\mathrm{Nd}^{3+}\right]_{\text {org,eq }} .60$ Organic phase water concentration for the $\mathrm{pH} 1+(0,30) \mathrm{mM} \mathrm{Nd}+$ Lac systems.

Organic phase water concentration for the $\mathrm{pH} 4+(0,30,60) \mathrm{mM} \mathrm{Nd}+\mathrm{Lac}$ systems. 71 Molar absorptivities observed for $\mathrm{Nd}^{3+}$ in the aqueous phase. $\mathrm{R}^{2}$ ranged from 0.997-0.9999 for the listed molar absoptivities shown in this table. .75 Chemical shift for selected functional groups that can be found in this work's system. Values are from Bruice. ${ }^{[106]}$

Summary of species identified in the systems studied with the conditions where they were observed. 88 
Table 3.1

Table 3.2

Table 3.3

Table 3.4

Table 3.5

Table 3.6

Table 3.7

Table 3.8

Table 3.9

Table 3.10

Table 3.11

Table 3.12

Table 3.13

Table 3.14

Table 3.15

Table 3.16

Table 3.17

Table 3.18

Table 3.19

Table 3.20

Table 3.21

Table 3.22

Table 3.23

Table 3.24

Table 3.25

Table 3.26
Solution constituent concentrations for the $\mathrm{H}_{2} \mathrm{O}$ system potentiometric

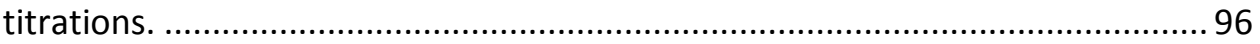

Solution constituent concentrations for the $\mathrm{H}_{2} \mathrm{O} /$ Triton X-114 system

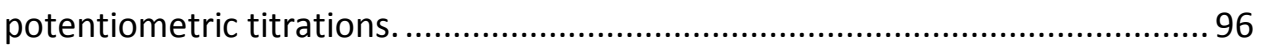

Solution constituent concentrations for the $\mathrm{H}_{2} \mathrm{O}$ and $\mathrm{H}_{2} \mathrm{O}$ /Triton X-114 system

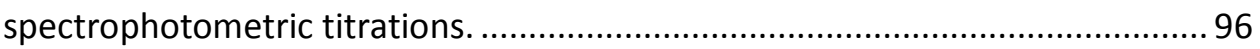

Hyperquad Fit parameters for 8-hydroxyquinoline deprotonation constants.. 100 The observed spectral maxima in the titration spectrum of 8-hydroxyquinoline. The wavelength of the peak in parenthesis is estimated, but does not have a

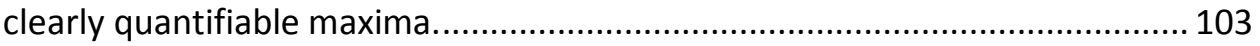

SQUAD modeled parameters using varied spectral windows.......................... 105 Hyperquad fit parameters for the europium hydrolysis constants................... 108 Hyperquad fit parameters for the 1:1 ligand to metal ratio solutions.............. 109 Hyperquad fit Parameters for the 2:1 ligand to metal solution...................... 111 Parameters obtained from the Hyperquad fit of the 3:1 Ligand to metal solution titration.

Fit parameters for the deprotonation of the protolytic impurity. 120 Concentration of unknown protolytic species obtained from Hyperquad simulation for the $1 \mathrm{wt} \%$ Triton solution titration. 120 Hyperquad parameters obtained for the protonation of 8-hydroxyquinoline in the $\mathrm{H}_{2} \mathrm{O} /$ Triton $\mathrm{X}-114$ system when the $\mathrm{pK}_{\mathrm{xx}}$ parameter is excluded from the model. 122 Hyperquad parameters obtained for the protonation of 8-hydroxyquinoline in the $\mathrm{H}_{2} \mathrm{O}$ /Triton X-114 system when the $\mathrm{pK}_{\mathrm{xx}}$ parameter is included in the model.

SQUAD modeling parameters for the $\mathrm{pK}_{\mathrm{a}}$ 's of 8-hydroxyquinoline in 1 wt\% Triton, $\mathrm{T}=0^{\circ} \mathrm{C}$. 125 Hyperquad fit parameters for the titration data from the $\mathrm{Eu}^{3+}$ in 1 wt\% Triton.

130

Table 3.17- Hyperquad fit parameters for the 1:1 ligand to metal solution titration in $1 \mathrm{wt} \%$ Triton. 130 Hyperquad fit parameters for the 2:1 ligand to metal solution titration in $1 \mathrm{wt} \%$ Triton...... 133 Parameters from the four models used to fit the 3:1 ligand to metal ratio solution titration. 134 Hyperquad fit parameters for the 3:1 ligand to metal solution titration in 1 wt\%

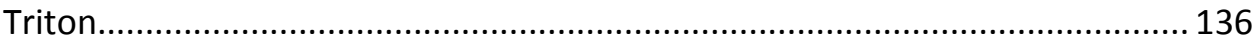
Calculated Standard Enthalpy of reaction for the deprotonation of 8hydroxyquinoline. Data utilized for this calculation are shown in Table 3.22... 138 Data used in the Van't Hoff Analysis of the $\mathrm{pK}_{\mathrm{a}}$ 's of 8-hydroxyquinoline in the water system. 138 Summary of $\mathrm{pK}_{\mathrm{a}}$ results from potentiometric (Potent.) and spectrophotometric (Spect.) titration methods. The results from these methods were averaged for a final result from this study. 138 Values used for the inverse ionic radius of the trivalent lanthanides. ${ }^{[14]}$.......... 141 Comparison of the $\mathrm{H}_{2} \mathrm{O}$ /Triton $\mathrm{X}-114$ vs. $\mathrm{H}_{2} \mathrm{O}$ system europium hydrolysis constants. 143 Comparison of the mixed ligand hydrolysis species parameters. 144 
Table 3.27 A comparison of the stability constants obtained from the $\mathrm{H}_{2} \mathrm{O}$ and the $\mathrm{H}_{2} \mathrm{O} /$ Triton X-114 systems...................................................................... 147

Table 3.28 Summary of parameters measured in this work. All parameters were measured at $0.1 \mathrm{M} \mathrm{NaCl}$ and $\mathrm{T}=1^{\circ} \mathrm{C}$. The $\mathrm{H}_{2} \mathrm{O}$ /Triton $\mathrm{X}-114$ system also contained $1 \mathrm{wt} \%$ Triton X-114. 150

Table 4.1 Summary of species identified in the systems studied with the conditions where they were observed. 153 


\section{LIST OF FIGURES}

Figure 1.1 A schematic representations showing the basic material flow for the PUREX, COEX, UREX and NPEX processes. The separation of neptunium in the PUREX was not historically performed (see Irish et al. ${ }^{[19]}$ ), but has been added in more modern applications of this process, and is thus shown in parentheses .............. 6

Figure 1.2 Extractants developed for Uranium, Neptunium and Plutonium extraction: A) Tributyl phosphate; B) N',N' dialkyl amide functional group. The R groups in ' $\mathrm{B}$ ' are varied between $\mathrm{n}$-alkyl and branched alkyl groups (see Musikas et al. ${ }^{[20]}$ ).... 7

Figure 1.3 Extractants used in cesium and strontium extraction: A) Chlorinated Cobalt Dicarbollide; B) Polyethylene Glycol; C) Calix[4]arene-bis(4-tert-octylbenzocrown-6); D) bis [4,4'(5')-(tert-butyl)cyclohexano]-18-crown-6.

\section{7}

Figure 1.4 A schematic representation of the material flow in the Cs and Sr extraction. These processes have been developed for use with dissolved irradiated fuel or other $\mathrm{Cs}$ and $\mathrm{Sr}$ bearing wastes. The parentheses enclosing $\mathrm{U}, \mathrm{Np}, \mathrm{Pu}, \mathrm{Am}$, and $\mathrm{Cm}$ are to indicate that these processes are effective with or without these elements present.

Figure 1.5 Examples of extractants developed for the trivalent actinide/trivalent lanthanide co-extraction: A) octyl (phenyl)-N,N-diisobutylcarbamoyl-methylphosphine oxide; B) generic form of the diamide extractant.

Figure 1.6 A schematic representation of the material flow in the TRUEX or DIAMEX systems. 9

Figure 1.7 Selective complexants developed for the trivalent actinide/trivalent lanthanide separation: A) diethlyenetriaminepentaacetic acid; B) bis(2,4,4trimethylpentyl)dithiophosphinic acid; C) bis-triazinyl-pyridine; D) terpyridine. 11

Figure 1.8

Figure 1.9

Figure 2.1

Figure 2.2

Figure 2.3

Figure 2.4

Figure 2.5

Figure 2.6

Figure 2.7

Figure 2.8

Figure 2.9

Figure 2.10 .. 11

A schematic representations showing the basic material flow for the UNEX and TRUSPEAK systems. 12 An example of the instrument response curve obtained from the bibenzyl standard and unwashed HA solutions. 34 VPO Instrument response data from the aqueous lactate samples................... 37 The conditional equilibrium constants obtained from the potentiometric titrations of lactic acid at $25-65^{\circ} \mathrm{C}$ at ionic strength of $2.0 \mathrm{M} \mathrm{NaCl}$.................... 38 Van't Hoff plot of the acid dissociation constant results for lactic acid titrations at $25-65^{\circ} \mathrm{C}$ at ionic strength of $2.0 \mathrm{M} \mathrm{NaCl}$.................................................. 39 Instrument response curve for the $\mathrm{pH} 4+0 \mathrm{mM} \mathrm{Nd}+\mathrm{HCl}$ system. At low $[\mathrm{HA}]_{\text {initial }}$ there is a small portion of the data that shows a different slope, indicating increased aggregation.

Calculated by SXLSQI (line) and observed (symbols) organic $\mathrm{Nd}^{3+}$ concentration as a function of initial HA molarity.

Log-slope analysis of $\mathrm{Na}^{+}$Extraction in the $\mathrm{pH} 4+0 \mathrm{mM} \mathrm{Nd}+\mathrm{HCl}$ system......... 46 Log-slope analysis of water in the washed solvent and the $\mathrm{pH} 4+0 \mathrm{mM} \mathrm{Nd}+\mathrm{HCl}$ system. The matching slopes indicate that no water was co-extracted with $\mathrm{Na}^{+}$ in the $\mathrm{pH} 4+0 \mathrm{mM} \mathrm{Nd}+\mathrm{HCl}$ system.

A comparison of the absorbance spectra of the $\mathrm{Nd}^{3+}$ ion in the aqueous and organic phases. The HA concentration for the organic solution shown is $0.2 \mathrm{M} .48$ Organic phase molar absorptivity from the $\mathrm{pH} 4+30 \mathrm{mM} \mathrm{Nd}+\mathrm{HCl}$ system. Plot (B) shows a more detailed view of Plot (A) from 560-615 nm 51 
Figure 2.11

Figure 2.12

Figure 2.13

Figure 2.14

Figure 2.15

Figure 2.16

Figure 2.17

Figure 2.18

Figure 2.19

Figure 2.20

Figure 2.21

Figure 2.22

Figure 2.23

Figure 2.24

Figure 2.25

Figure 2.26

Figure 2.27

Figure 2.28

Figure 2.29

Figure 2.30
Trend in the $570 \mathrm{~nm}$ absorbance band as a function of $[\mathrm{HA}]_{\text {initial }}$ from the $\mathrm{pH}$ $(1,4)+30 \mathrm{mM} \mathrm{Nd}+\mathrm{HCl}$ systems. Also included in this plot is the percent theoretical loading of the solvent based on the assumption of HA dimer stoichiometric coefficient $(\mathrm{n})$ of 2 52 Infrared spectra normalized by $[\mathrm{HA}]_{\text {org }}$. The isooctane peaks have been removed from the spectrum 53 Aggregation results for the $\mathrm{pH} 4+(30,60) \mathrm{mM} \mathrm{Nd}+$ Lac systems ..................... 56 Instrument response curve used to calculate the aggregation results for the $\mathrm{pH} 4$ $+(30,60) \mathrm{mM} \mathrm{Nd}+$ Lac systems. 56 $\mathrm{Nd}^{3+}$ extraction results. Observed data are depicted as points, SXLSQI modeling results are depicted as the solid curve.

The transition between saturation and quantitative extraction supports the 1:2 $\mathrm{Nd}^{3+}:$ HA stoichiometry of the $\mathrm{NdLacA}_{2}$ complex. Log-slope analysis of Lac extraction vs. $[\mathrm{HA}]_{\text {org }}$ for the $\mathrm{pH} 1+(0,30) \mathrm{mM} \mathrm{Nd}+$ Lac systems. 63 Log-slope plot for the $\mathrm{Nd}^{3+}$ vs. Lac dependence in the $\mathrm{pH} 1+30 \mathrm{mM} \mathrm{Nd}+\mathrm{Lac}$ system. 64 Dependence of organic $\mathrm{Nd}^{3+}$ and Lac concentrations on HA molarity in the $\mathrm{pH} 4$ $+30 \mathrm{mM} \mathrm{Nd}+$ Lac systems. 66 Correlation between organic phase $\mathrm{Na}^{+}$and lactate. This slope is not indicative of the stoichiometry between $\mathrm{Na}^{+}$and lactate in the organic phase, but indicates that $\mathrm{Na}^{+}$must exist as both a lactate and $\mathrm{HA}$ salt..... 68 Linear scale dependence of the extraction of Lac and $\mathrm{Nd}^{3+}$ in the $\mathrm{pH} 4+(30,60)$ $\mathrm{mM} \mathrm{Nd} \mathrm{+} \mathrm{Lac} \mathrm{systems.} \mathrm{This} \mathrm{data} \mathrm{is} \mathrm{limited} \mathrm{to} \mathrm{the} \mathrm{data} \mathrm{points} \mathrm{where} \mathrm{the}$ $\mathrm{Nd}(\mathrm{LaC})(\mathrm{HA})_{2}$ species was shown to be present. 68 The extraction of water in excess of the HA hydration of the washed solvent for the $\mathrm{pH} 4+(0,30,60) \mathrm{mM} \mathrm{Nd}+$ Lac systems. 72 Dependence of organic $\mathrm{H}_{2} \mathrm{O}$ concentration on the organic Lactate molarity. These data were selected from the entire $\mathrm{pH} 4+(0,30) \mathrm{mM} \mathrm{Nd}+$ Lac systems under conditions of NaLac extraction 73 Linear scale correlation between organic phase $\mathrm{Nd}^{3+}$ and water concentration in the $\mathrm{pH} 4+(30,60) \mathrm{mM} \mathrm{Nd}+$ Lac systems. This data was selected from the $\mathrm{pH} 4$ $+(30,60) \mathrm{mM} \mathrm{Nd}+$ Lac systems where $\mathrm{Nd}(\mathrm{Lac})(\mathrm{HA})_{2}$ was shown to exist in the organic phase. Data points used were restricted to the $[\mathrm{HA}]_{\text {org }}$ of $0-0.075$ and $0-$ $0.125 \mathrm{M} \mathrm{HA}$ respectively. 73 Linear scale correlation of organic phase water and $\mathrm{Na}^{+}$concentrations from the $\mathrm{pH} 4+(0,30,60) \mathrm{mM} \mathrm{Nd}+$ Lac systems using only data where $\mathrm{Na}^{+}$extraction was observed. $[\mathrm{HA}]_{\text {org }}$ utilized in this plot are: $0.025-1,0.2-1$ and $0.3-1 \mathrm{M} \mathrm{HA}$ respectively. 74 Comparison of the molar absorptivities that are representative of those observed for $\mathrm{pH}(1,4)+(30,60) \mathrm{mM} \mathrm{Nd}+$ Lac systems.................................... 76 A comparison of the molar absorptivities for $\mathrm{Nd}^{3+}$ in the loaded organic phase solutions in the $\mathrm{HCl}$ and Lac systems at $\mathrm{pH} 4+30 \mathrm{mM} \mathrm{Nd}$ systems................... 77 A comparison of the FTIR spectra for the $\mathrm{pH}(1,4)+(0,30) \mathrm{mM} \mathrm{Nd}+$ Lac systems and the washed solvent. 79 Aggregation results for the $\mathrm{pH} 4+(30,60) \mathrm{mM} \mathrm{La}+$ Lac systems. ..................... 80 ${ }^{23} \mathrm{Na}$ NMR scan for pH $4+30 \mathrm{mM} \mathrm{La}+\operatorname{Lac}(1.0 \mathrm{M} \mathrm{HA})$ sample. 81 
Figure 2.31

Figure 2.32

Figure 3.1

Figure 3.2

Figure 3.3

Figure 3.4

Figure 3.5

Figure 3.6

Figure 3.7

Figure 3.8

Figure 3.9

Figure 3.10

Figure 3.11

Figure 3.12

Figure 3.13

Figure 3.14

Figure 3.15

Figure 3.16

Figure 3.17
${ }^{31} \mathrm{P}$ NMR spectra obtained from the $\mathrm{pH} 4+60 \mathrm{mM} \mathrm{La}+\mathrm{Lac}(0.125 \mathrm{M} \mathrm{HA})$ and $\mathrm{pH}$ $4+30 \mathrm{mM} \mathrm{La}+\operatorname{Lac}(1.0 \mathrm{M} \mathrm{HA})$ samples.

${ }^{13} \mathrm{C}$ NMR scan spectra obtained from the $\mathrm{pH} 4+60 \mathrm{mM} \mathrm{La}+\mathrm{Lac}(0.125 \mathrm{M} \mathrm{HA})$ and $\mathrm{pH} 4+30 \mathrm{mM}$ La + Lac (1.0 M HA) samples.

Chemical Structure of Triton $\mathrm{X}-114$. This chemical is typically sold as a mixture with $n=7-8$ with the total mixture having an average molecular weight of 537 gm/mol. ${ }^{[108]}$.....

The protonation states of 8-hydroxyquinoline. 91 The aqueous solubility and speciation of 8-hydroxyquinoline as a function of $\mathrm{pH}$.

92

Titration of $[\mathrm{HQ}]=3 \times 10^{-4} \mathrm{M},[\mathrm{NaCl}]=0.1 \mathrm{M},[\mathrm{HCl}]=0.001 \mathrm{M}$ by $[\mathrm{NaOH}]$

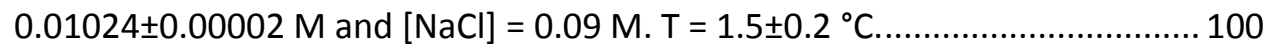
Hyperquad modeling results for the potentiometric titration of 8hydroxyquinoline at $\mathrm{T}=1.5 \pm 0.2^{\circ} \mathrm{C}$. 101 Spectral changes for $[\mathrm{HQ}]=10^{-5} \mathrm{M},[\mathrm{NaCl}]=0.1 \mathrm{M},[\mathrm{HCl}]=0.001 \mathrm{M}$ titrated by $\mathrm{NaOH}$ of varying concentration and volume at $1.8 \pm 0.2{ }^{\circ} \mathrm{C}$. The solution $\mathrm{pH}$ for each spectrum is listed in the figure legend. Arrows indicate the direction of the spectral change as $\mathrm{pH}$ increases. These spectral changes represent the transition from 8-hydroxyquinoline protonation state $A$ to $B$ in Figure 3.2. 102 Spectral changes for $[\mathrm{HQ}]=10^{-5} \mathrm{M},[\mathrm{NaCl}]=0.1 \mathrm{M},[\mathrm{HCl}]=0.001 \mathrm{M}$ titrated by $\mathrm{NaOH}$ of varying concentration and volume at $1.8 \pm 0.2{ }^{\circ} \mathrm{C}$. The solution $\mathrm{pH}$ for each spectrum is listed in the figure legend. Arrows indicate the direction of the spectral change as $\mathrm{pH}$ increases. These spectral changes represent the transition from 8-hydroxyquinoline protonation state $B$ to $C$ in Figure 3.2 .................. 103 Molar absorptivity Spectra obtained from SQUAD modeling using the 280-430 $\mathrm{nm}$ spectral window called window $B$. 104 Calculated speciation curve obtained from SQUAD modeling using the 280-430 $\mathrm{nm}$ spectral window also called window $B$. 104 Titration of $\left[\mathrm{Eu}^{3+}\right]=3 \times 10^{-4} \mathrm{M},[\mathrm{NaCl}]=0.1 \mathrm{M},[\mathrm{HCl}]=0.001 \mathrm{M}$ by $[\mathrm{NaOH}]$ $0.01024 \pm 0.00002 \mathrm{M}$ and $[\mathrm{NaCl}]=0.09 \mathrm{M} . \mathrm{T}=1.7 \pm 0.2^{\circ} \mathrm{C}$. 107 Hyperquad modeling results for the titration of $\left[\mathrm{Eu}^{3+}\right]=3 \times 10^{-4} \mathrm{M},[\mathrm{NaCl}]=0.1 \mathrm{M}$, $[\mathrm{HCl}]=0.001 \mathrm{M}$ by $[\mathrm{NaOH}] 0.01024 \pm 0.00002 \mathrm{M}$ and $[\mathrm{NaCl}]=0.09 \mathrm{M} . \mathrm{T}=1.7 \pm 0.2$ ${ }^{\circ} \mathrm{C}$. 107

Titration of $[\mathrm{HQ}]=3 \times 10^{-4} \mathrm{M},\left[\mathrm{Eu}^{3+}\right]=3 \times 10^{-4} \mathrm{M},[\mathrm{NaCl}]=0.1 \mathrm{M},[\mathrm{HCl}]=0.001 \mathrm{M}$ by $[\mathrm{NaOH}]=0.01024 \pm 0.00002 \mathrm{M}$ and $[\mathrm{NaCl}]=0.09 \mathrm{M} . \mathrm{T}=1.7 \pm 0.2^{\circ} \mathrm{C}$. 110 Hyperquad fit results for titration of $[\mathrm{HQ}]=3 \times 10^{-4} \mathrm{M},\left[\mathrm{Eu}^{3+}\right]=3 \times 10^{-4} \mathrm{M},[\mathrm{NaCl}]=$ $0.1 \mathrm{M},[\mathrm{HCl}]=0.001 \mathrm{M}$ by $[\mathrm{NaOH}]=0.01024 \pm 0.00002 \mathrm{M}$ and $[\mathrm{NaCl}]=0.09 \mathrm{M}$. $\mathrm{T}=1.7 \pm 0.2^{\circ} \mathrm{C}$. 110

Titration of $[\mathrm{HQ}]=3 \times 10^{-4} \mathrm{M},\left[\mathrm{Eu}^{3+}\right]=1.5 \times 10^{-4} \mathrm{M},[\mathrm{NaCl}]=0.1 \mathrm{M},[\mathrm{HCl}]=0.001 \mathrm{M}$ by $[\mathrm{NaOH}]=0.01024 \pm 0.00002 \mathrm{M}$ and $[\mathrm{NaCl}]=0.09 \mathrm{M} . \mathrm{T}=1.9 \pm 0.2^{\circ} \mathrm{C}$. Hyperquad fit results for titration of $[\mathrm{HQ}]=3 \times 10^{-4} \mathrm{M},\left[\mathrm{Eu}^{3+}\right]=1.5 \times 10^{-4} \mathrm{M},[\mathrm{NaCl}]$ $=0.1 \mathrm{M},[\mathrm{HCl}]=0.001 \mathrm{M}$ by $[\mathrm{NaOH}]=0.01024 \pm 0.00002 \mathrm{M}$ and $[\mathrm{NaCl}]=0.09 \mathrm{M}$. $\mathrm{T}=1.7 \pm 0.2{ }^{\circ} \mathrm{C}$. 112

Titration of $[\mathrm{HQ}]=3 \times 10^{-4} \mathrm{M},\left[\mathrm{Eu}^{3+}\right]=1 \times 10^{-4} \mathrm{M},[\mathrm{NaCl}]=0.1 \mathrm{M},[\mathrm{HCl}]=0.001 \mathrm{M}$ by $[\mathrm{NaOH}]=0.01024 \pm 0.00002 \mathrm{M}$ and $[\mathrm{NaCl}]=0.09 \mathrm{M} . \mathrm{T}=2.0 \pm 0.2^{\circ} \mathrm{C} \ldots \ldots \ldots \ldots . .114$ Hyperquad fit results for titration of $[\mathrm{HQ}]=3 \times 10^{-4} \mathrm{M},\left[\mathrm{Eu}^{3+}\right]=1 \times 10^{-4} \mathrm{M},[\mathrm{NaCl}]=$ $0.1 \mathrm{M},[\mathrm{HCl}]=0.001 \mathrm{M}$ by $[\mathrm{NaOH}]=0.01024 \pm 0.00002 \mathrm{M}$ and $[\mathrm{NaCl}]=0.09 \mathrm{M}$. $\mathrm{T}=2.0 \pm 0.2^{\circ} \mathrm{C}$. 114 
Figure 3.18

Figure 3.19

Figure 3.20

Figure 3.21

Figure 3.22

Figure 3.23

Figure 3.24

Figure 3.25

Figure 3.26

Figure 3.27

Figure 3.28

Figure 3.29

Figure 3.30

Figure 3.31

Figure 3.32

Figure 3.33
Spectral changes for $[\mathrm{HQ}]=10^{-5} \mathrm{M},\left[\mathrm{Eu}^{3+}\right]=2 \times 10^{-6} \mathrm{M},[\mathrm{NaCl}]=0.1 \mathrm{M},[\mathrm{HCl}]=$ $0.001 \mathrm{M}$ titrated by $\mathrm{NaOH}$ of varying concentration and volume at $\mathrm{T}=1.8 \pm 0.2$ ${ }^{\circ} \mathrm{C}$. The solution $\mathrm{pH}$ for each spectrum is listed in the figure legend. Arrows indicate the direction of the spectral change as $\mathrm{pH}$ increases. 116 Spectral changes for $[\mathrm{HQ}]=10^{-5} \mathrm{M},\left[\mathrm{Eu}^{3+}\right]=2 \times 10^{-6} \mathrm{M},[\mathrm{NaCl}]=0.1 \mathrm{M},[\mathrm{HCl}]=$ $0.001 \mathrm{M}$ titrated by $\mathrm{NaOH}$ of varying concentration and volume at $\mathrm{T}=1.8 \pm 0.2$ ${ }^{\circ} \mathrm{C}$. The solution $\mathrm{pH}$ for each spectrum is listed in the figure legend. Arrows indicate the direction of the spectral change as $\mathrm{pH}$ increases. 116 Spectral changes for $[\mathrm{HQ}]=10^{-5} \mathrm{M},\left[\mathrm{Eu}^{3+}\right]=2 \times 10^{-6} \mathrm{M},[\mathrm{NaCl}]=0.1 \mathrm{M},[\mathrm{HCl}]=$ $0.001 \mathrm{M}$ titrated by $\mathrm{NaOH}$ of varying concentration and volume at $\mathrm{T}=1.8 \pm 0.2$ ${ }^{\circ} \mathrm{C}$. The solution $\mathrm{pH}$ for each spectrum is listed in the figure legend. Arrows indicate the direction of the spectral change as $\mathrm{pH}$ increases. 117 Titration of $[\mathrm{HCl}]=0.001 \mathrm{M}$, $[$ Triton $\mathrm{x}-114=1 \mathrm{wt} \%(\sim 0.02 \mathrm{~mol} / \mathrm{L})$ by $[\mathrm{NaOH}]=$ $0.01024 \pm 0.00002 \mathrm{M}$ and $[\mathrm{NaCl}]=0.09 \mathrm{M} . \mathrm{T}=1.9 \pm 0.2^{\circ} \mathrm{C}$. Hyperquad fit for the titration of $[\mathrm{HCl}]=0.001 \mathrm{M}$, [Triton X-114 $=1 \mathrm{wt} \%(\sim 0.02$ $\mathrm{mol} / \mathrm{L})$ by $[\mathrm{NaOH}]=0.01024 \pm 0.00002 \mathrm{M}$ and $[\mathrm{NaCl}]=0.09 \mathrm{M} . \mathrm{T}=1.9 \pm 0.2{ }^{\circ} \mathrm{C} .121$ Titration of $[\mathrm{HQ}]=3 \times 10^{-4} \mathrm{M},[\mathrm{NaCl}]=0.1 \mathrm{M},[\mathrm{HCl}]=0.001 \mathrm{M},[$ Triton X-114] $=1$ wt\% $(\sim 0.02 \mathrm{M})$ by $[\mathrm{NaOH}]=0.01024 \pm 0.00002 \mathrm{M}$ and $[\mathrm{NaCl}]=0.09 \mathrm{M}$. $\mathrm{T}=1.8 \pm 0.2^{\circ} \mathrm{C}$. 121 Hyperquad fit for the titration of $[\mathrm{HQ}]=3 \times 10^{-4} \mathrm{M},[\mathrm{NaCl}]=0.1 \mathrm{M},[\mathrm{HCl}]=0.001$ $\mathrm{M}$, [Triton X-114] $=1 \mathrm{wt} \%(\sim 0.02 \mathrm{M})$ by $[\mathrm{NaOH}]=0.01024 \pm 0.00002 \mathrm{M}$ and $[\mathrm{NaCl}]=0.09 \mathrm{M} . \mathrm{T}=1.8 \pm 0.2^{\circ} \mathrm{C}$. 122 Hyperquad fit for the Hyperquad fit for the titration of $[\mathrm{HQ}]=3 \times 10^{-4} \mathrm{M},[\mathrm{NaCl}]=$ $0.1 \mathrm{M},[\mathrm{HCl}]=0.001 \mathrm{M},[$ Triton X-114] $=1 \mathrm{wt} \%(\sim 0.02 \mathrm{M})$, by $[\mathrm{NaOH}]=0.01024 \pm 0.00002 \mathrm{M}$ and $[\mathrm{NaCl}]=0.09 \mathrm{M} . \mathrm{T}=1.8 \pm 0.2^{\circ} \mathrm{C} \ldots \ldots \ldots \ldots . . .123$ Spectra obtained while titrating a solution containing: $[\mathrm{HQ}]=0.0001 \mathrm{M},[\mathrm{NaCl}]=$ $0.1 \mathrm{M}$, [Triton X-114] = $1 \mathrm{wt} \%$. Spectra shown are of $\mathrm{pH} 3.19-6.95$. The arrows indicate the spectral change as $\mathrm{pH}$ increases. 125 A select window of the spectra obtained while titrating a solution containing: $[\mathrm{HQ}]=0.0001 \mathrm{M},[\mathrm{NaCl}]=0.1 \mathrm{M},[$ Triton X-114] $=1 \mathrm{wt} \%$. Spectra shown are of $\mathrm{pH}$ 3.19-6.95. The arrows indicate the spectral change as $\mathrm{pH}$ increases. ........ 126 Spectra obtained while titrating a solution containing: $[\mathrm{HQ}]=0.0001 \mathrm{M},[\mathrm{NaCl}]=$ $0.1 \mathrm{M}$, [Triton X-114] = $1 \mathrm{wt} \%$. Spectra shown are of $\mathrm{pH}$ 6.95-9.96. The arrows indicate the spectral change as $\mathrm{pH}$ increases. 126 A select window of the spectra obtained while titrating a solution containing: $[\mathrm{HQ}]=0.0001 \mathrm{M},[\mathrm{NaCl}]=0.1 \mathrm{M},[$ Triton X-114] $=1 \mathrm{wt} \%$. Spectra shown are of $\mathrm{pH}$ 6.95-9.96. The arrows indicate the spectral change as $\mathrm{pH}$ increases. ........ 127 Molar absorptivity Spectra obtained from SQUAD modeling using the 300-412 nm spectral window. 127 Calculated speciation curves obtained from SQUAD modeling using the 300-412 $\mathrm{nm}$ window. 128 Titration of $\left[\mathrm{Eu}^{3+}\right]=3 \times 10^{-4} \mathrm{M},[\mathrm{NaCl}]=0.1 \mathrm{M},[\mathrm{HCl}]=0.001 \mathrm{M},[$ Triton X-114 $]=1$ wt\% $(\sim 0.02 \mathrm{M})$, by $[\mathrm{NaOH}]=0.01024 \pm 0.00002 \mathrm{M}$ and $[\mathrm{NaCl}]=0.09 \mathrm{M}$. $\mathrm{T}=1.8 \pm 0.2^{\circ} \mathrm{C}$. 129 Hyperquad fit for the titration of $\left[\mathrm{Eu}^{3+}\right]=3 \times 10^{-4} \mathrm{M},[\mathrm{NaCl}]=0.1 \mathrm{M},[\mathrm{HCl}]=0.001$ $\mathrm{M}$, [Triton X-114] $=1 \mathrm{wt} \%(\sim 0.02 \mathrm{M})$, by $[\mathrm{NaOH}]=0.01024 \pm 0.00002 \mathrm{M}$ and $[\mathrm{NaCl}]=0.09 \mathrm{M} . \mathrm{T}=1.8 \pm 0.2^{\circ} \mathrm{C}$. 129 
Figure 3.34

Figure 3.35

Figure 3.36

Figure 3.37

Figure 3.38

Figure 3.39

Figure 3.40

Figure 3.41

Figure 3.42

Figure 3.43

Figure 3.44

Figure 3.45

Figure 3.46

Figure 3.47

Figure A1.1

Figure A1.2

Figure A1.3

Figure A1.4

Figure A1.5

Figure A1.6

Figure A1.7
Titration of $[\mathrm{HQ}]=3 \times 10^{-4} \mathrm{M},\left[\mathrm{Eu}^{3+}\right]=3 \times 10^{-4} \mathrm{M},[\mathrm{NaCl}]=0.1 \mathrm{M},[\mathrm{HCl}]=0.001 \mathrm{M}$, $[$ Triton $X-114]=1 \mathrm{wt} \% \quad(\sim 0.02 \mathrm{M})$, by $[\mathrm{NaOH}]=0.01024 \pm 0.00002 \mathrm{M}$ and $[\mathrm{NaCl}]=0.09 \mathrm{M} . \mathrm{T}=1.8 \pm 0.2^{\circ} \mathrm{C}$. 131 Hyperquad fit for the titration of $[\mathrm{HQ}]=3 \times 10^{-4} \mathrm{M},\left[\mathrm{Eu}^{3+}\right]=3 \times 10^{-4} \mathrm{M},[\mathrm{NaCl}]=0.1$ $\mathrm{M},[\mathrm{HCl}]=0.001 \mathrm{M},[$ Triton X-114] $=1 \mathrm{wt} \%(\sim 0.02 \mathrm{M})$, by $[\mathrm{NaOH}]=$ $0.01024 \pm 0.00002 \mathrm{M}$ and $[\mathrm{NaCl}]=0.09 \mathrm{M} . \mathrm{T}=1.8 \pm 0.2^{\circ} \mathrm{C}$.

Titration of $[\mathrm{HQ}]=3 \times 10^{-4} \mathrm{M},\left[\mathrm{Eu}^{3+}\right]=1.5 \times 10^{-4} \mathrm{M},[\mathrm{NaCl}]=0.1 \mathrm{M},[\mathrm{HCl}]=0.001 \mathrm{M}$, $[$ Triton $X-114]=1 \mathrm{wt} \% \quad(\sim 0.02 \mathrm{M})$, by $[\mathrm{NaOH}]=0.01024 \pm 0.00002 \mathrm{M}$ and $[\mathrm{NaCl}]=0.09 \mathrm{M} . \mathrm{T}=1.9 \pm 0.1^{\circ} \mathrm{C}$. 132 Hyperquad fit for the titration of $[\mathrm{HQ}]=3 \times 10^{-4} \mathrm{M},\left[\mathrm{Eu}^{3+}\right]=1.5 \times 10^{-4} \mathrm{M},[\mathrm{NaCl}]=$ $0.1 \mathrm{M},[\mathrm{HCl}]=0.001 \mathrm{M}$, [Triton $\mathrm{X}-114]=1 \mathrm{wt} \%(\sim 0.02 \mathrm{M})$, by $[\mathrm{NaOH}]=$ $0.01024 \pm 0.00002 \mathrm{M}$ and $[\mathrm{NaCl}]=0.09 \mathrm{M} . \mathrm{T}=1.9 \pm 0.1^{\circ} \mathrm{C}$. Titration of $[\mathrm{HQ}]=3 \times 10^{-4} \mathrm{M},\left[\mathrm{Eu}^{3+}\right]=1 \times 10^{-4} \mathrm{M},[\mathrm{NaCl}]=0.1 \mathrm{M},[\mathrm{HCl}]=0.001 \mathrm{M}$, [Triton $X-114]=1 \mathrm{wt} \% \quad(\sim 0.02 \mathrm{M})$, by $[\mathrm{NaOH}]=0.01024 \pm 0.00002 \mathrm{M}$ and $[\mathrm{NaCl}]=0.09 \mathrm{M} . \mathrm{T}=1.9 \pm 0.2^{\circ} \mathrm{C}$. 134 Results from four attempts at fitting the 3:1 ligand to metal titration curve. .. 135 Hyperquad fit using Model $\mathrm{C}$ for the titration of $[\mathrm{HQ}]=3 \times 10^{-4} \mathrm{M},\left[\mathrm{Eu}^{3+}\right]=1 \times 10^{-4}$ $\mathrm{M},[\mathrm{NaCl}]=0.1 \mathrm{M},[\mathrm{HCl}]=0.001 \mathrm{M},[$ Triton X-114] $=1 \mathrm{wt} \%(\sim 0.02 \mathrm{M})$, by $[\mathrm{NaOH}]=0.01024 \pm 0.00002 \mathrm{M}$ and $[\mathrm{NaCl}]=0.09 \mathrm{M} . \mathrm{T}=1.9 \pm 0.1{ }^{\circ} \mathrm{C}$. 135 Comparison of measured $\mathrm{pK}_{\mathrm{a}}$ values for 8-hydroxyquinoline with literature data from Lobanov et al. ${ }^{[151-152]}$ utilizing the Van't Hoff relation. Data used in this plot are shown in Table 3.22 . 139 Comparison of the $\mathrm{pK}_{\mathrm{a}}$ of 8-hydroxyquinoline in Triton X-100/water with the $\mathrm{pK}_{\mathrm{a}}$ of 8-hydroxyquinoline in Triton X-114/water measured in this work. The literature values are from Hoshino et al. ${ }^{[134]}$ 139

Comparison of the first hydrolysis constant $\left(\log \beta_{1-10}\right)$ obtained in this work at $1^{\circ} \mathrm{C}$ with literature data at $25^{\circ} \mathrm{C}$. 141 Comparison of observed and literature data values for the first hydrolysis constant as a function of inverse ionic radius. 142 A comparison of the formation constants for $[\mathrm{Eu}(\mathrm{Q})]^{2+}\left(\log \beta_{101}\right)$ in $0.1 \mathrm{M}$ lonic media at various temperatures, to various lanthanides' $[\operatorname{Ln}(Q)]^{2+}$ formation constants represented as a function of ionic radius....................................... 145 Comparison of Literature data for the formation of $[\mathrm{Eu}(\mathrm{Q})]^{2+}$ in $50 \%$ dioxane (at $\mathrm{T}=30{ }^{\circ} \mathrm{C}$ ), and $0 \%$ dioxane (at $\mathrm{T}=20^{\circ} \mathrm{C}$ ) with the observed values at $1 \mathrm{wt} \%$ Triton $\mathrm{X}-114\left(\right.$ at $1{ }^{\circ} \mathrm{C}$ ) and $0 \mathrm{wt} \%$ Triton $\mathrm{X}-114\left(\right.$ at $1{ }^{\circ} \mathrm{C}$ ). 146 Comparison of Literature data for the formation of $\left[\mathrm{Eu}(\mathrm{Q})_{2}\right]^{+}$in $50 \%$ dioxane (at $\mathrm{T}=30^{\circ} \mathrm{C}$ ), and $0 \%$ dioxane $\left(\right.$ at $\mathrm{T}=25^{\circ} \mathrm{C}$ ) with the observed values at $1 \mathrm{wt} \%$ Triton $\mathrm{X}-114\left(\right.$ at $1{ }^{\circ} \mathrm{C}$ ) and 0 wt\% Triton $\mathrm{X}-114\left(\right.$ at $1{ }^{\circ} \mathrm{C}$ ). 147 $\mathrm{pH}$ titration of $\mathrm{HQ}$ from $\mathrm{pH} 3.18$ to 6.87 at $\mathrm{T}=0^{\circ} \mathrm{C}$........................................ 160

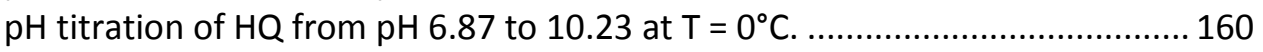
Spectrum preparation spreadsheet screenshot............................................ 161 Format manipulation by "GWBASIC.EXE" on each Spectum file....................... 162 Screen shot showing the start-up of the "GWBASIC.EXE" program.................. 172 Screen shot showing the first user prompts upon starting the "SQUAD-IN.BAS" program. 173 Screen shot showing the error result from entering a header file name that does not exist in the folder that contains the "SQUAD-IN.BAS" program that is running. 174 
Figure A1.8 Screen shot showing the result of entering an input file name that does not exist in the folder that contains the "SQUAD-IN.BAS" program that is running........ 175

Figure A1.9 Screen shot showing the user prompt displayed upon supplying the "SQUADIN.BAS" program with the correct information for 1 spectrum file. ................. 176

Figure A1.10 Screen shot showing the error that results following the input of the 26th consecutive spectrum file....................................................................... 177 


\section{CHAPTER 1}

\section{INTRODUCTION}

\subsection{Motivation for Research}

The "Trivalent Actinide Lanthanide Separation by Phosphorus reagent Extraction from Aqueous Komplexes" (TALSPEAK) process ${ }^{[1]}$ has been demonstrated to successfully separate trivalent actinides from the lanthanides on the laboratory scale. ${ }^{[2]}$ Despite this success, there are aspects of the fundamental chemistry that are not completely understood. In a recent review of the process, Nilsson and Nash ${ }^{[3]}$ have shown that modeling the TALSPEAK system leads to predictions that are not observed in the experimental data. The authors suggest that the differences may be caused by the formation of aqueous mixed complexes that are not included in the model or that the thermodynamic parameters are significantly changed at $\mathrm{pH}>3.6$ due to the increase in ionic strength and free lactate concentration. Leggett et al. ${ }^{[4]}$ studied mixed complexes in the TALSPEAK aqueous phase and reported that they form in such small quantities that they can't account for the previously noted modeling inconsistencies.

Prior to implementing the TALSPEAK process on the industrial scale it is necessary for the fundamental chemistry to be understood so the process can be satisfactorily modeled. Otherwise, a premature application may lead to unsatisfactory separation results, process delays or failures. This work was undertaken to increase understanding of the TALSPEAK process that will lead to accurate modeling of the process in the future. This work studied the extraction of $\mathrm{Nd}^{3+}$ over a range of loading regimes, characterized the speciation of $\mathrm{Nd}^{3+}$ in the organic phase, and modeled the extraction behavior where aqueous thermodynamic parameters are available. For conditions where aqueous thermodynamic data is not available, the speciation will still be characterized so that when this data is made available, the system can be accurately modeled 
Due to the narrow operating envelope required by the TALSPEAK system, other separation systems may prove to be more convenient for the trivalent lanthanide/trivalent actinide separation. Recent work with cloud point extraction has shown that lanthanum was selectively removed from gadolinium using 8-hydroxyquinoline. ${ }^{[5-7]}$ This same separation system is under exploration as a possible candidate for trivalent lanthanide/trivalent actinide separation. The cloud point extraction technique was developed as an alternative to liquid-liquid extraction that enables the extraction of metal ion complexes. ${ }^{[8-9]}$ This work explores the complexation behavior of 8-hydroxyquinoline with europium in the cloud point medium of Triton $\mathrm{X}-114 / \mathrm{H}_{2} \mathrm{O}$ and also the $\mathrm{H}_{2} \mathrm{O}$ system behavior for comparison at $0^{\circ} \mathrm{C}$.

\subsection{Background}

\subsubsection{Partitioning and Transmutation}

The fundamental premise of partitioning and transmutation (P\&T) is separating long-lived radioisotopes from irradiated nuclear fuel and re-irradiating to convert them to shorter-lived or stable radioisotopes. In irradiated nuclear fuel, with a few exceptions, fission products are shortlived isotopes and transuranium elements are longer lived. Past simulation of dose from irradiated nuclear fuel shows that between 1,000 and 10,000 years after irradiation, the major contributors are plutonium and the minor actinides (neptunium, americium and curium). ${ }^{[10]}$ Thus, plutonium and the minor actinides are prime targets of P\&T research.

Due to the focus on recovery of plutonium for nuclear weapons purposes during the cold war, its chemistry and separation is well understood. ${ }^{[11]}$ Neptunium separation has been a more recent achievement, ${ }^{[12-13]}$ but it appears to be feasible within current nuclear reprocessing facilities. Americium and curium represent a more difficult problem in P\&T research. During nuclear fuel irradiation, these trivalent actinides and the lighter lanthanides, $\mathrm{La}^{3+}$ through $\mathrm{Gd}^{3+}$, are introduced into the fuel through neutron activation and fission respectively. When the fuel 
is dissolved in acidic solution these actinides and lanthanides all exist as trivalent ions with no other easily accessible or stable oxidation states available. In addition, these trivalent ions are also very similar in size. ${ }^{[14]}$ Due to the similar charge density and affinity to complexing agents between the trivalent actinides and the lanthanides, the separation has been described as “...the most challenging aspect of advanced nuclear fuel partitioning schemes." ${ }^{[15]}$ It is necessary to separate the trivalent actinides from the trivalent lanthanides in a P\&T scheme because the neutron absorption cross-section of the lanthanides. ${ }^{[15]}$ Because the lanthanides have such large cross-sections (Table 1.1), they would act as a neutron poison and hinder the utility of a nuclear fuel containing these elements.

Table 1.1- Thermal neutron capture cross section ${ }^{[16]}$ (barns) of the fission product lanthanides. Isotopes with fission yields of zero were omitted. Values in bold font are for isotopes with $T_{1 / 2}>$ 20 years that are more likely to be present after the post-irradiation cooling period.

\begin{tabular}{|c|c|c|c|c|c|c|c|c|}
\hline $\begin{array}{c}\text { Element } \\
\left(1^{\mathrm{st}} \text { mass }\right)\end{array}$ & $\begin{array}{c}\text { La } \\
\text { (139) }\end{array}$ & $\begin{array}{c}\mathrm{Ce} \\
(140)\end{array}$ & $\begin{array}{c}\mathrm{Pr} \\
(141)\end{array}$ & $\begin{array}{c}\mathrm{Nd} \\
(143)\end{array}$ & $\begin{array}{c}\text { Pm } \\
(147)\end{array}$ & $\begin{array}{c}\text { Sm } \\
(147)\end{array}$ & $\begin{array}{c}\text { Eu } \\
(151)\end{array}$ & $\begin{array}{c}\text { Gd } \\
(155)\end{array}$ \\
\hline $1^{\text {st }}$ mass +0 & 9.0 & 0.58 & $11.4^{*}$ & 330 & $182^{*}$ & 57 & $\sim 9200^{*}$ & 61,000 \\
\hline+1 & 2.7 & 29 & & 3.6 & & 3 & & 2 \\
\hline+2 & & 0.97 & 90 & 45 & 140 & 40,000 & 350 & 255,000 \\
\hline+3 & & 6 & & 1.4 & & 103 & 1,400 & 2.4 \\
\hline+4 & & 1.0 & & 400 & 200 & 15,100 & 3,900 & \\
\hline+5 & & & & 2.5 & & 210 & & 1 \\
\hline+6 & & & & & & 400 & & 20,000 \\
\hline+7 & & & & 1.0 & & 7 & & \\
\hline
\end{tabular}

*These values were listed as a sum of two or more cross-sections (i.e. $x+x+\ldots$ ), but are listed here as the single value of the sum of the separately listed values.

\subsubsection{Partitioning Schemes Overview}

Partitioning schemes are developed both to target species in the nuclear fuel and to achieve various purposes. These purposes include the reduction of overall bulk or mass, limitation of 
dose, recycling of fissile and fertile fuel materials, or the transmutation of long-lived radiotoxic constituents. For purposes of the discussion in this section, irradiated nuclear fuel will be assumed to be $30 \mathrm{MWd} / \mathrm{kg} \mathrm{M}$ burnup fuel with 10 year decay period and a $2.9 \%$ initial ${ }^{235} \mathrm{U}$ enrichment with elemental constituent breakdown obtained from ORIGEN2 ${ }^{[17]}$ simulation shown in the appendix of Guenther et al. ${ }^{[18]}$ The major constituents of irradiated fuel are ranked by their bulk (mass) and activity in Table 1.2 and Table 1.3. The data is shown grouped in an elemental rather than isotopic break-down because partitioning schemes discussed in this work target elements.

In the PUREX reprocessing scheme ${ }^{[11,19]}$ the fertile and fissile isotopes of uranium and plutonium are targeted for reuse in nuclear power reactors, or for production of nuclear weapons. Because the PUREX process effectively removes the uranium from the remaining irradiated fuel constituents and uranium makes up the vast bulk of irradiated fuel $(98.4 \mathrm{wt} \%$, see Table 1.2) the PUREX process in its original or modified form is used as a head-end process for subsequent nuclear separations. Different modifications of PUREX enable the co-recovery of neptunium, ${ }^{[12]}$ or prevent the creation of separated plutonium as in the COEX, and UREX + NPEX ${ }^{[2]}$ processes (see Figure 1.1). An alternative to the PUREX process was developed using completely incinerable extractants, specifically $\mathrm{N}, \mathrm{N}$-dialkylamide type extractants, that recovered uranium and plutonium using different conditions and chemistry, but providing a similar separation as PUREX. ${ }^{[20]}$ The use of $\mathrm{N}, \mathrm{N}$-dialkylamide for the recovery of uranium and plutonium has been abandoned due to problems with third phase formation causing problems for the separation. ${ }^{[20]}$ Structure of the PUREX process extractant, tributyl phosphate, and the $\mathrm{N}, \mathrm{N}$-dialkylamide alternative are shown in Figure 1.2.

A large reduction of radioactive dose from the used fuel materials is a goal that is achieved by targeting cesium and strontium for separation from the irradiated fuel mixture. As shown in 
Table 1.3, cesium and strontium and their daughter products barium and yttrium account for $71 \%$ of the total activity, but only $0.16 \%$ of the mass. Separation schemes using the chlorinated cobalt dicarbollide (CCD) with Polyethylene Glycol (PEG), ${ }^{[21-22]}$ or calixarene type ${ }^{[23-24]}$ molecules enable the selective extraction of cesium and strontium. The structures of the molecules used in cesium and strontium extraction are shown in Figure 1.3. A schematic representation of the cesium and Strontium extraction is shown in Figure 1.4.

Table 1.2- The top 15 elements in irradiated fuel sorted by mass percent. Category is either Actinides (Act.) or Fission Product (F.P).

\begin{tabular}{|c|c|c|c|c|}
\hline Rank & Category & Element & Mass\% & Activity\% \\
\hline 1 & Act. & $\mathrm{U}$ & $\mathbf{9 8 . 4 3} \%$ & $0.00 \%$ \\
\hline 2 & Act. & $\mathrm{Pu}$ & $\mathbf{0 . 8 5} \%$ & $22.37 \%$ \\
\hline 3 & F.P. & $\mathrm{Nd}$ & $\mathbf{0 . 1 3} \%$ & $0.00 \%$ \\
\hline 4 & F.P. & $\mathrm{Cs}$ & $\mathbf{0 . 1 3} \%$ & $22.19 \%$ \\
\hline 5 & F.P. & $\mathrm{Ce}$ & $\mathbf{0 . 1 0} \%$ & $0.01 \%$ \\
\hline 6 & F.P. & $\mathrm{Tc}$ & $\mathbf{0 . 0 7} \%$ & $0.00 \%$ \\
\hline 7 & F.P. & $\mathrm{Zr}$ & $\mathbf{0 . 0 7} \%$ & $0.00 \%$ \\
\hline 8 & Act. & $\mathrm{Am}$ & $\mathbf{0 . 0 6} \%$ & $0.59 \%$ \\
\hline 9 & Act. & $\mathrm{Np}$ & $\mathbf{0 . 0 4 \%}$ & $0.00 \%$ \\
\hline 10 & F.P. & $\mathrm{Sr}$ & $\mathbf{0 . 0 4} \%$ & $14.77 \%$ \\
\hline 11 & F.P. & $\mathrm{Rb}$ & $\mathbf{0 . 0 2} \%$ & $0.00 \%$ \\
\hline 12 & F.P. & $\mathrm{Sm}$ & $\mathbf{0 . 0 2 \%}$ & $0.09 \%$ \\
\hline 13 & F.P. & $\mathrm{I}$ & $\mathbf{0 . 0 2} \%$ & $0.00 \%$ \\
\hline 14 & Act. & $\mathrm{Cm}$ & $\mathbf{0 . 0 1 \%}$ & $0.33 \%$ \\
\hline 15 & F.P. & $\mathrm{Sn}$ & $\mathbf{0 . 0 0 \%}$ & $0.00 \%$ \\
\hline
\end{tabular}


Table 1.3- The top 15 elements in irradiated fuel sorted by Percent of total activity. Category is either Actinides (Act.) or Fission Product (F.P.).

\begin{tabular}{|c|c|c|c|c|}
\hline Rank & Category & Element & Mass\% & Activity\% \\
\hline 1 & Act. & $\mathrm{Pu}$ & $0.85 \%$ & $\mathbf{2 2 . 3 7 \%}$ \\
\hline 2 & F.P. & $\mathrm{Cs}$ & $0.13 \%$ & $\mathbf{2 2 . 1 9 \%}$ \\
\hline 3 & F.P. & $\mathrm{Ba}$ & $0.00 \%$ & $\mathbf{2 0 . 2 3 \%}$ \\
\hline 4 & F.P. & $\mathrm{Y}$ & $0.00 \%$ & $\mathbf{1 4 . 7 7 \%}$ \\
\hline 5 & F.P. & $\mathrm{Sr}$ & $0.04 \%$ & $\mathbf{1 4 . 7 7 \%}$ \\
\hline 6 & F.P. & $\mathrm{Pm}$ & $0.00 \%$ & $\mathbf{1 . 6 3 \%}$ \\
\hline 7 & F.P. & $\mathrm{Eu}$ & $0.00 \%$ & $\mathbf{1 . 5 1 \%}$ \\
\hline 8 & F.P. & $\mathrm{Kr}$ & $0.00 \%$ & $\mathbf{1 . 0 9 \%}$ \\
\hline 1 & Act. & $\mathrm{Am}$ & $0.06 \%$ & $\mathbf{0 . 5 9 \%}$ \\
\hline 11 & Act. & $\mathrm{Cm}$ & $0.01 \%$ & $\mathbf{0 . 3 3 \%}$ \\
\hline 12 & F.P. & $\mathrm{Sb}$ & $0.00 \%$ & $\mathbf{0 . 1 6 \%}$ \\
\hline 13 & F.P. & $\mathrm{Sm}$ & $0.02 \%$ & $\mathbf{0 . 0 9 \%}$ \\
\hline 14 & F.P. & $\mathrm{H}$ & $0.00 \%$ & $\mathbf{0 . 0 7 \%}$ \\
\hline 15 & F.P. & $\mathrm{Rh}$ & $0.00 \%$ & $\mathbf{0 . 0 6 \%}$ \\
\hline
\end{tabular}
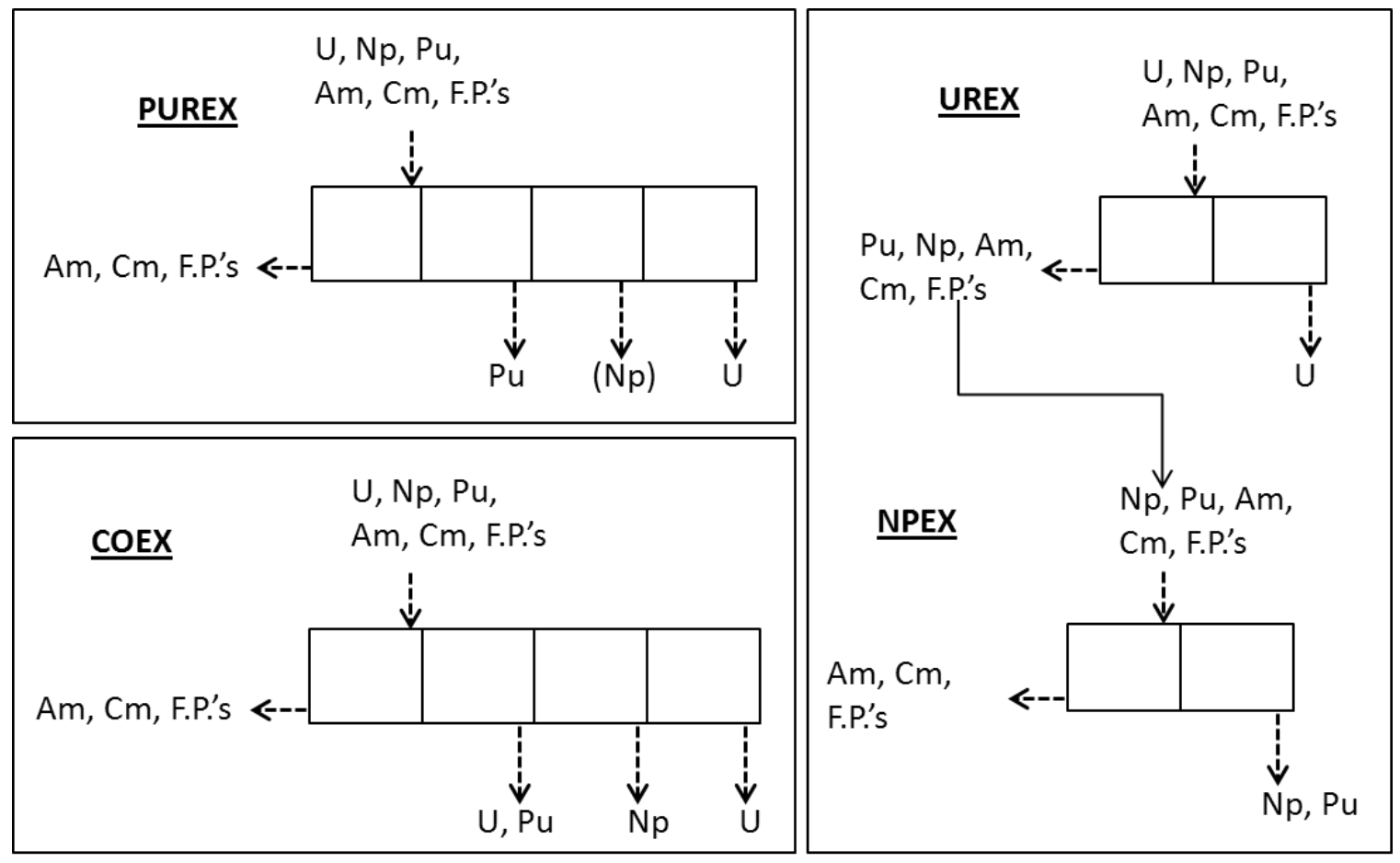

Figure 1.1- A schematic representations showing the basic material flow for the PUREX, COEX, UREX and NPEX processes. The separation of neptunium in the PUREX was not historically performed (see Irish et al. ${ }^{[19]}$ ), but has been added in more modern applications of this process, and is thus shown in parentheses. 

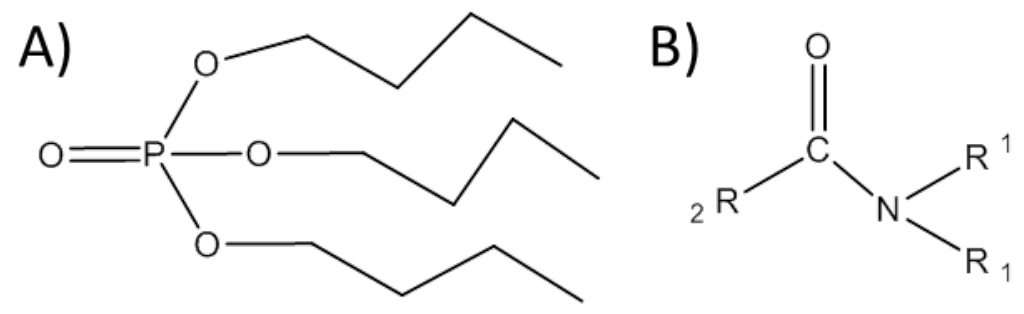

Figure 1.2- Extractants developed for Uranium, Neptunium and Plutonium extraction: A) Tri-nbutyl phosphate; B) N',N' dialkyl amide functional group. The R groups in 'B' are varied between n-alkyl and branched alkyl groups (see Musikas et al. ${ }^{[20]}$ ).

A)
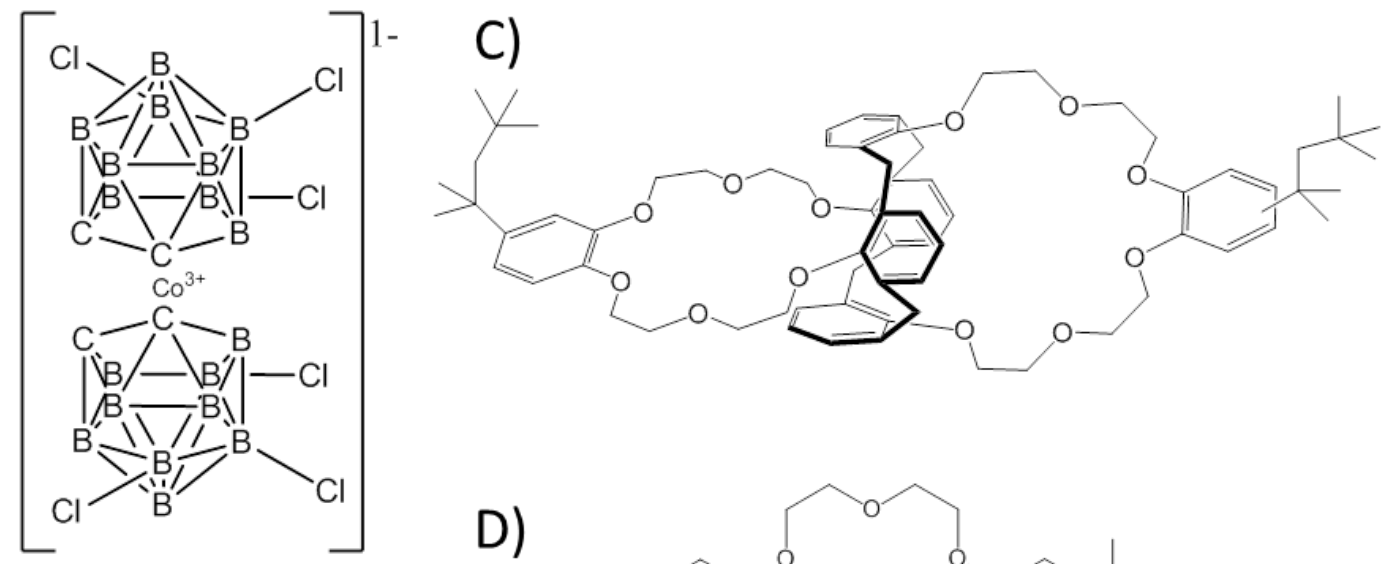

B)

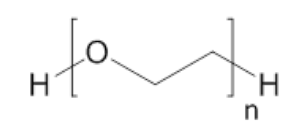

D)

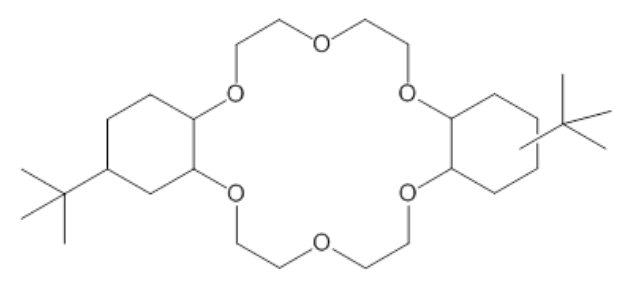

Figure 1.3- Extractants used in cesium and strontium extraction: A) Chlorinated Cobalt Dicarbollide; B) Polyethylene Glycol; C) Calix[4]arene-bis(4-tert-octylbenzo-crown-6); D) bis $\left[4,4^{\prime}\left(5^{\prime}\right)\right.$-(tert-butyl)cyclohexano]-18-crown-6. 


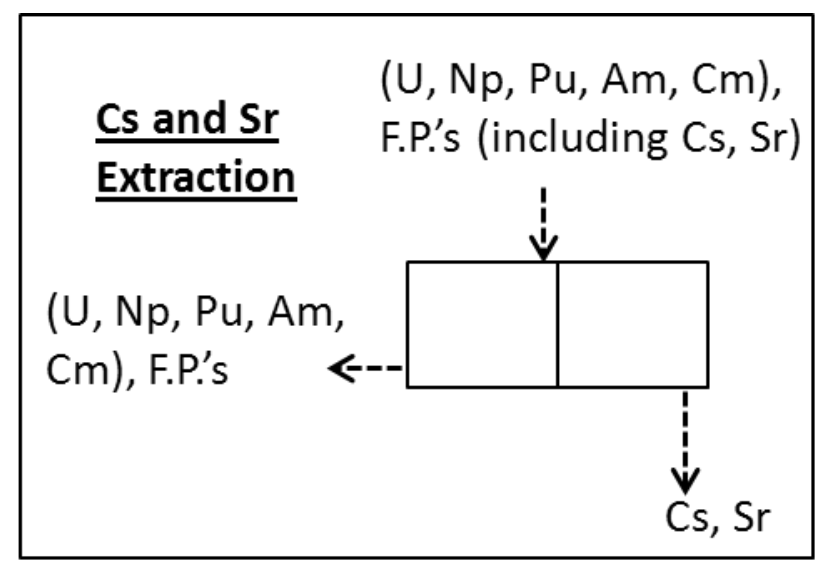

Figure 1.4- A schematic representation of the material flow in the Cs and Sr extraction. These processes have been developed for use with dissolved irradiated fuel or other Cs and Sr bearing wastes. The parentheses enclosing $\mathrm{U}, \mathrm{Np}, \mathrm{Pu}, \mathrm{Am}$, and $\mathrm{Cm}$ are to indicate that these processes are effective with or without these elements present.

The transmutation of the long-lived radiotoxic minor actinides is a goal that requires the separation of these elements from the other fission products (see section 1.2.1). Because neptunium extraction is feasible through a modified PUREX process, ${ }^{[12]}$ the separation of americium and curium from the fission product mixture has been developed as a separate process. A preliminary extraction of all trivalent actinides and trivalent lanthanides from the other fission products precedes the separation of the trivalent actinides from the lanthanides. ${ }^{[25]}$ This separation has been performed using the phosphate based ligand octyl (phenyl)- $\mathrm{N}, \mathrm{N}$ diisobutylcarbamoyl-methylphosphine oxide (CMPO) in a process named the Transuranic Extraction (TRUEX) process, ${ }^{[25]}$ or bifunctional malonamides in a process named the Diamide Extraction (DIAMEX) process. ${ }^{[20]}$ The structures of these extractants are shown in Figure 1.5. Once the other fission products are removed from the trivalent actinide / trivalent lanthanide mixture, the separation of the trivalent actinides can be achieved. The trivalent actinide / trivalent lanthanide separation was first developed as a fractional precipitation ${ }^{[26]}$ and later using ion exchange. ${ }^{[26]}$ Solvent extraction processes are under continual development for 
deployment at the industrial scale in advanced recycling schemes that utilizes thiophosphinic acid extractants, ${ }^{[27]}$ aromatic nitrogen ligands such as triazines and terpyridines, ${ }^{[28]}$ the TALSPEAK Process ${ }^{[1]}$ that utilizes a combination of organophosphoric acid and aminopolycarboxylic acid ligands.

A)

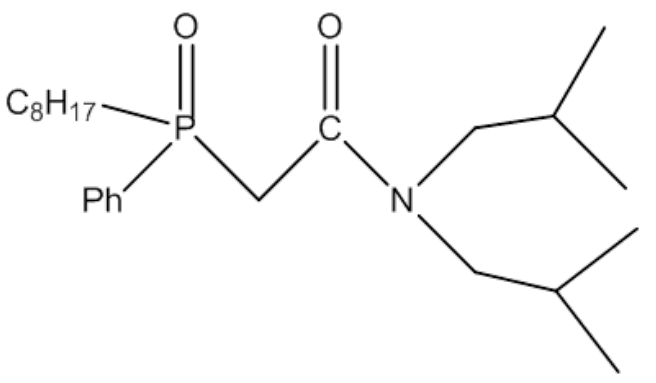

B)<smiles>[R]C(C(=O)N([R])[R])C(=O)N([R])[R]</smiles>

Figure 1.5- Examples of extractants developed for the trivalent actinide/trivalent lanthanide coextraction: A) octyl (phenyl)-N,N-diisobutylcarbamoyl-methylphosphine oxide; B) generic form of the diamide extractant.

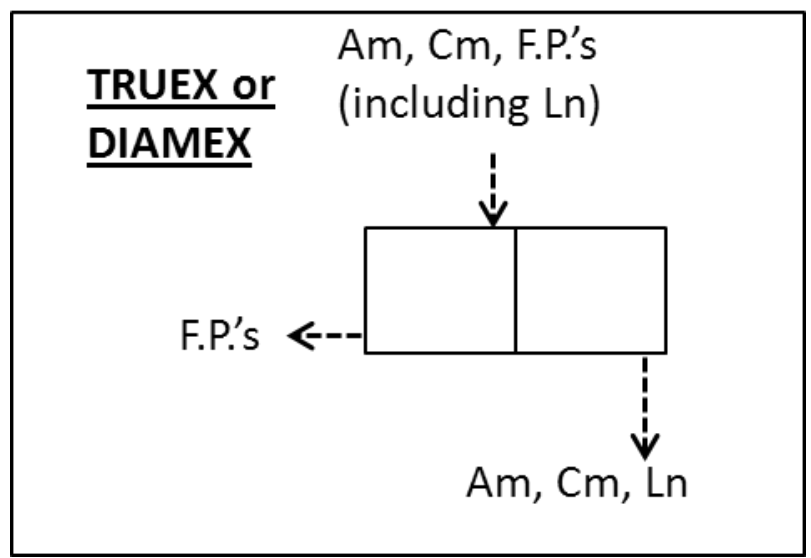

Figure 1.6- A schematic representation of the material flow in the TRUEX or DIAMEX systems. 
Extraction processes can be used in sequence in an overall waste partitioning scheme. The UREX+ set of processes was designed and demonstrated at the lab-scale. ${ }^{[29-30]}$ This reported series of extractions consisted of 1) Modified PUREX for uranium only extraction (named 'UREX'), 2) CCD-PEG for cesium and strontium recovery, 3) a $2^{\text {nd }}$ modified PUREX for neptunium and plutonium co-extraction (named 'NPEX'), 4) TRUEX for trivalent actinide / trivalent lanthanide co-extraction, and 5) Cyanex 301 (the trade name for bis(2,4,4trimethylpentyl)dithiophosphinic acid) for trivalent actinide separation from trivalent lanthanides.

Some efforts have been made to simplify the overall fuel recycling flowsheets into fewer processes by combining processes. The Universal Solvent Extraction (UNEX) process was developed more as a decontamination rather than recycling process that targets the highest activity species for removal from an acidic nuclear waste stream. The UNEX process solvent was a combination of the TRUEX and the CCD/PEG processes that achieved extraction of all actinides, lanthanides and cesium and strontium. ${ }^{[31-32]}$ The TRUSPEAK process ${ }^{[33-34]}$ is currently under development and seeks to combine the TRUEX and TALSPEAK processes to enable extraction of both trivalent actinides and trivalent lanthanides, but achieve the separation through selective stripping. 
<smiles>CC1CCP(=S)(S)CCC(C)CC(C)(C)CC(C)(C)CC(C)(C)C1</smiles><smiles>Clc1cccc(-c2nccnn2)n1</smiles><smiles>c1ccc(-c2cccc(-c3ccccn3)n2)nc1</smiles>

Figure 1.7- Selective complexants developed for the trivalent actinide/trivalent lanthanide separation: A) diethlyenetriaminepentaacetic acid; B) bis(2,4,4-trimethylpentyl)dithiophosphinic acid; C) bis-triazinyl-pyridine; D) terpyridine.

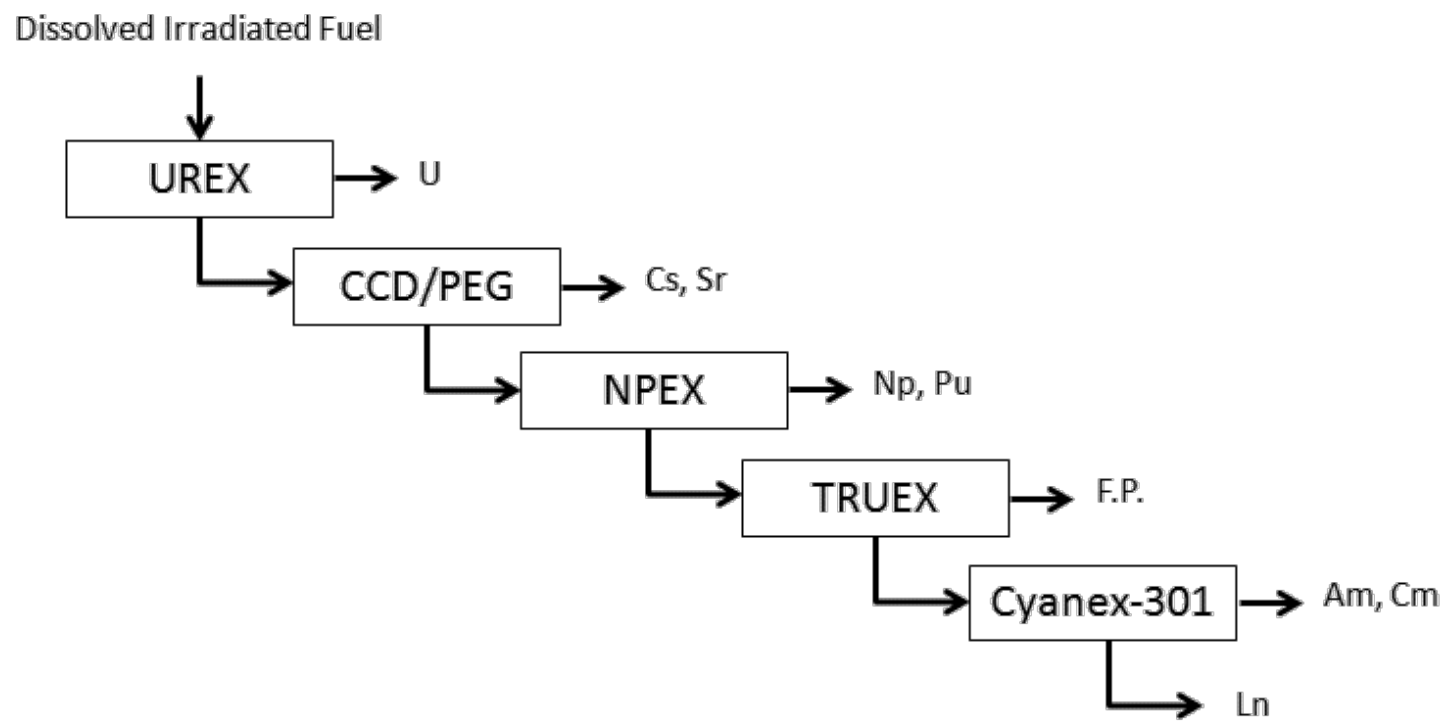

Figure 1.8- A schematic representation of the UREX+ separation scheme. 


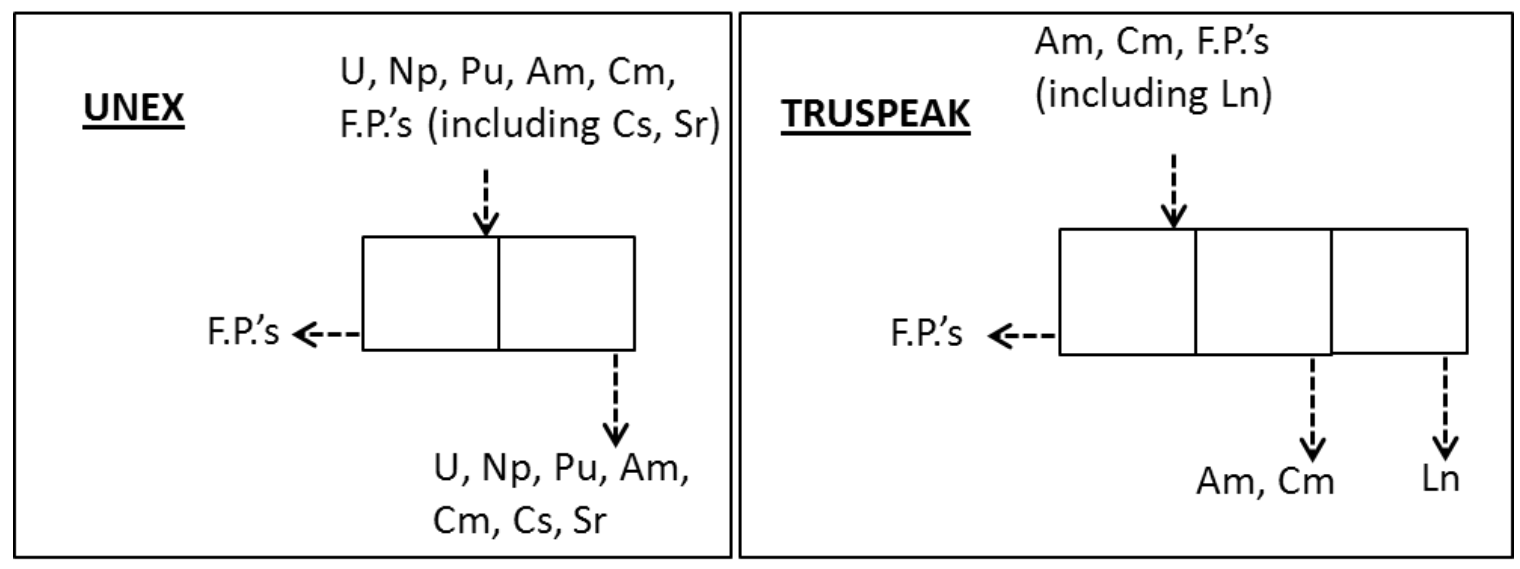

Figure 1.9- A schematic representations showing the basic material flow for the UNEX and TRUSPEAK systems.

\subsubsection{Cloud Point Extraction}

Cloud point extraction is a method utilizing surfactants (surface active agents) as the phase separating reagents. Cloud point extraction is similar to liquid-liquid extraction because it utilizes two phases with selective distribution of hydrophobic species but it differs in that the system begins as a single phase. The term "Cloud Point" refers to the temperature of transition where the surfactants change from solutes in the bulk phase to self-aggregate micelles. ${ }^{[35]}$ Because temperature is the driving force in non-ionic surfactant micellization, it is believed to be an entropic process ${ }^{[36-37]}$ that can be described by the Gibbs free energy equation shown in equation (1.1).

$$
\Delta G_{\text {micelle }}=\Delta H_{\text {micelle }}-T \Delta S_{\text {micelle }}
$$

As the temperature is increased the magnitude of the T $\Delta \mathrm{S}$ term in the equation will exceed that of the $\Delta \mathrm{H}$ term, causing spontaneous micellization where the solution becomes a cloudy, twophase system. The reaction is reversible if the temperature is decreased. ${ }^{[36]}$ 
Cloud point extraction was introduced as an alternative separation technique to solvent extraction ${ }^{[36]}$ by Watanabe and co-workers. ${ }^{[8]}$ Since then most of the work utilizing this technique has been separation and pre-concentration of trace metals, ${ }^{[35,37-38]}$ and protein isolation. ${ }^{[38-39]}$ Although reviews concerned with cloud point extraction deal mostly with laboratory applications, ${ }^{[35-37,40]}$ there are some efforts to apply this method to industrial scale waste treatment using the continuous disk rotating contactor. ${ }^{[41-42]}$

\subsubsection{Trivalent Actinide/Lanthanide Separation Principles}

Trivalent actinides and lanthanides must be separated by exploiting their chemical and physical differences. The difference in ionic radius is one basis for separating these elements. A separation designed purely on the basis of size will be difficult for the actinide/lanthanide separation because the ionic radius of americium and curium are approximately the same size as promethium and samarium respectively (Table 1.4). Some success has been achieved through ion exchange that leads to the elution of progressively larger trivalent ions. ${ }^{[43]}$ Differences in ionic radius and thus charge density lead to variations in affinity for the ion exchange resin, and complexants in the mobile phase such as a-hydroxy isobutyrate, ${ }^{[43-44]}$ citrate $_{,}^{[43]}$ lactate, ${ }^{[43]}$ or ethylenediaminetetraacetic acid (EDTA). ${ }^{[43,45-46]}$ The ionic radius differences are small, but when a sufficiently large column is used, a successful separation is achieved.

The "Hard-Soft Acid-Base" character of the actinides and lanthanides is another difference that can be exploited to achieve separation. The Hard-Soft Acid Base theory ${ }^{[47]}$ suggests that certain electron pair acceptor acids will form stronger complexes with certain electron pair donor bases when the acid-base pair share either Hard or Soft character. Hard or Soft character is determined by the polarizability of the acid or base and the degree of covalency in the resulting complex's bond. Soft acid/ base complexes have polarizable valence electrons and

form bonds with higher covalent character than hard acid/base complexes. ${ }^{[47]}$ 
Table 1.4- lonic radius of Trivalent lanthanide and actinide ions. ${ }^{[48]}$

\begin{tabular}{|c|c|c|c|}
\hline \multicolumn{2}{|c|}{ Lanthanides } & \multicolumn{2}{|c|}{ Actinides } \\
\hline$\left(\mathrm{M}^{+3}\right)$ & $\begin{array}{c}\text { Ionic Radius } \\
(\mathrm{pm})\end{array}$ & $\left(\mathrm{M}^{+3}\right)$ & $\begin{array}{c}\text { Ionic Radius } \\
(\mathrm{pm})\end{array}$ \\
\hline $\mathrm{La}$ & 103.2 & $\mathrm{Ac}$ & 112 \\
\hline $\mathrm{Ce}$ & 101 & $\mathrm{Th}$ & \\
\hline $\mathrm{Pr}$ & 99 & $\mathrm{~Pa}$ & \\
\hline $\mathrm{Nd}$ & 98.3 & $\mathrm{U}$ & 102.5 \\
\hline $\mathrm{Pm}$ & 97 & $\mathrm{~Np}$ & 101 \\
\hline $\mathrm{Sm}$ & 95.8 & $\mathrm{Pu}$ & 100 \\
\hline $\mathrm{Eu}$ & 94.7 & $\mathrm{Am}$ & 97.5 \\
\hline $\mathrm{Gd}$ & 93.8 & $\mathrm{Cm}$ & 96 \\
\hline $\mathrm{Tb}$ & 92.3 & $\mathrm{Bk}$ & 96 \\
\hline $\mathrm{Dy}$ & 91.2 & $\mathrm{Cf}$ & 95 \\
\hline $\mathrm{Ho}$ & 90.1 & $\mathrm{Es}$ & \\
\hline $\mathrm{Er}$ & 89 & $\mathrm{Fm}$ & \\
\hline $\mathrm{Tm}$ & 88 & $\mathrm{Md}$ & \\
\hline $\mathrm{Yb}$ & 86.8 & $\mathrm{No}$ & \\
\hline $\mathrm{Lu}$ & 86.1 & $\mathrm{Lr}$ & \\
\hline $\mathrm{CoOrdin}$ & & \\
\hline
\end{tabular}

* Coordination number is 6 for all ions in table.

The actinides and lanthanides are in general hard acids, ${ }^{[47,49]}$ but the actinides show a small but significant preference for soft donor ligands. ${ }^{[48,50]}$ Relativistic calculations predict that the valence $d$ - and $f$ - orbitals extend further for actinides than lanthanides. ${ }^{[49]}$ Electron spin resonance (ESR) experiments with iso-electronic uranium and neodymium fluoride compounds show some interaction between the fluorine nuclei and the electron spin of the uranium ion, while the neodymium showed no similar interaction. ${ }^{[51]}$ Both the relativistic calculations and the ESR experiments support the hypothesis of increased covalency in actinide bonding. In a review by $\mathrm{Nash}^{[48]}$ the comparison of the enthalpy of complexation for $\mathrm{Am}^{3+}, \mathrm{Cm}^{3+}$ and $\mathrm{Eu}^{3+}$ with aminocarboxylate ligands showed no clear difference between actinides and lanthanides, thus indicated no clear increased covalency in the actinide complexes. Choppin ${ }^{[49]}$ points out that, although a large difference was not manifest, a small difference that resulted in a correlation of 1.08 when the stability constants of Am vs. Nd were plotted, indicating there may be a slightly 
more covalent bonding for Am than for Nd. Choppin ${ }^{[49]}$ also mentions data from optical, Mössbauer, and nuclear magnetic resonance spectroscopy that led him to conclude concerning f-element covalent bonding:

"In summary, the data are consistent with ionicity as the predominant character of lanthanide and actinide bonding. However, there is compelling evidence for some small amount of covalency, which is stronger in the actinide bonds. The argument for this extra covalency involving the forbitals does not seem to have strong experimental support, and more likely, the s orbitals play the major role in the covalency of the bonding f-element cations."

So, although bonds through forbitals were not implicated in increased covalent character of actinide bonding, data from a variety of techniques supports a slightly increased covalent character of actinide bonds over the analogous lanthanide bonds. This slightly increased covalent character of actinide bonds appears to be the fundamental reason behind the effectiveness of soft donor ligands to selectively complex actinides over lanthanides and is the basis for much of the ligand design theory and practice in trivalent actinide / trivalent lanthanide separations.

\subsection{Summary of Dissertation}

This work explores various principles and phenomena related to the trivalent lanthanide / trivalent actinide separation. This chapter established the motivation for this research and presented background information introducing the subject area that will be explored later in this work. Chapter 2 discusses the characterization of the organic phase of the TALSPEAK solvent extraction system under variable loading conditions. Chapter 3 discusses a study of the temperature dependent behavior of lactate, which is used in the TALSPEAK solvent extraction system as a major component of the aqueous phase. Chapter 4 discusses a study of the complexation constants between europium and 8-hydroxyquinoline, a ligand that is being evaluated for selectivity in the trivalent lanthanide/trivalent actinide separations. 


\section{CHAPTER 2}

\section{TALSPEAK ORGANIC PHASE SPECIATION UNDER VARIABLE LOADING CONDITIONS: HCI AND LACTATE AQUEOUS MEDIUM STUDIES}

\subsection{Introduction}

The Trivalent Actinide-Lanthanide Separation by Phosphorus reagent Extraction from Aqueous Komplexes (TALSPEAK) Process ${ }^{[1]}$ has been demonstrated to work on various scales. ${ }^{[2]}$ Under carefully controlled conditions this process achieves one of the most difficult separations that are encountered in nuclear separations: that of trivalent actinides $\left(\mathrm{An}^{3+}\right)$ from trivalent lanthanides $\left(\mathrm{Ln}^{3+}\right)$. This separation is a necessary part of a partitioning and transmutation scheme that involves the recycle of all transuranium elements in irradiated nuclear fuel. The TALSPEAK is a well-studied process that is nearing the level of understanding required for successful industrial application, but this process still lacks a comprehensive extraction model that will account for the system behavior at all conditions. ${ }^{[52]}$

Lactate plays a vital role in the TALSPEAK system. It acts as an aqueous phase buffer that prevents the $\mathrm{pH}$ from leaving the narrow operating window required for this challenging separation. As will be discussed in this chapter, lactate also affects the system speciation in various ways, including: co-extraction with $\mathrm{M}^{3+}$ and causing the formation of highly aggregated species. Understanding the ionization behavior of lactate under various temperatures is vital to

creating a complete model of the TALSPEAK system. Lactate is the major constituent $(1 \mathrm{M})^{[53]}$ of the aqueous phase of the TALSPEAK system. The dissociation of lactic acid will significantly change the ionic strength of the aqueous phase, resulting in large activity coefficient changes. Measuring the acid/base behavior of lactate is therefore an important step to establishing a complete TALSPEAK model. 
For the chemical reaction in equation (2.1), the general form for the chemical equilibrium constants $^{[54]}$ is shown in equation (2.2)

$$
\begin{gathered}
a A+b B \longleftrightarrow c C+d D \\
K=\frac{\mathcal{A}_{C}^{c} \mathcal{A}_{D}^{d}}{\mathcal{A}_{A}^{a} \mathcal{A}_{B}^{b}}=\frac{[C]^{c} \gamma_{C}^{c}[D]^{d} \gamma_{D}^{d}}{[A]^{a} \gamma_{A}^{a}[B]^{b} \gamma_{B}^{b}}
\end{gathered}
$$

where $\mathcal{A}$ is the activity, the quantities shown in square brackets are the molar concentrations, and $\gamma$ is the activity coefficient. Analytical chemistry techniques provide a measure of the bracketed quantities in the equilibrium expression. The activity coefficients are more difficult to measure and correlate. Because activity coefficients depend on ionic strength, performing experiments at constant ionic strength is a way to keep the activity coefficients constant and lump them in with the equilibrium constant as shown in equation (2.3).

$$
K \frac{\gamma_{A}^{a} \gamma_{B}^{b}}{\gamma_{c}^{c} \gamma_{D}^{d}}=\frac{[C]^{c}[D]^{d}}{[A]^{a}[B]^{b}}=K_{\text {cond }}
$$

This new expression shows the value known as the conditional equilibrium constant. In this work the chemical equilibrium constants presented as either equilibrium constants as shown in equation (2.2) or conditional chemical equilibrium constants as shown in equation (2.3).

The ionization equation and derivation of $\mathrm{pK}_{\mathrm{a}}$ for lactic acid is:

$$
\begin{aligned}
& H A \stackrel{K_{a}}{\longleftrightarrow} H^{+}+A^{-} \\
& K_{a}=\frac{\left[H^{+} \rrbracket A^{-}\right]}{[H A]} \\
& p K_{a}=-\log _{10}\left(K_{a}\right)
\end{aligned}
$$

The current IUPAC recommended values of the $\mathrm{pK}_{\mathrm{a}}$ of lactic acid are listed in Table 2.1. 
Table 2.1- IUPAC recommended values for the conditional $\mathrm{pK}_{\mathrm{a}}$ of Lactic Acid. ${ }^{[55]}$

\begin{tabular}{|c|c|c|}
\hline $\begin{array}{c}\text { lonic Strength } \\
\text { (medium unspecified) }\end{array}$ & Temp $\left({ }^{\circ} \mathrm{C}\right)$ & $\mathrm{pK}_{\mathrm{a}}$ of Lactic Acid \\
\hline $0 \mathrm{M}$ & 25 & 3.86 \\
\hline $1.0 \mathrm{M}$ & 25 & 3.64 \\
\hline $2.0 \mathrm{M}$ & 25 & 3.80 \\
\hline
\end{tabular}

From the literature, it appears the medium most used was $\mathrm{NaClO}_{4} .{ }^{[55]}$ The data recommended by the IUPAC for the lactic acid $\mathrm{pK}_{\mathrm{a}}$ in $\mathrm{NaCl}$ medium are shown in Table 2.2.

Table 2.2- IUPAC recommended data for the conditional $\mathrm{pK}_{\mathrm{a}}$ of Lactic acid in $\mathrm{NaCl}$ media. ${ }^{[55]}$

\begin{tabular}{|c|c|c|}
\hline $\begin{array}{c}\text { Ionic Strength } \\
(\mathrm{NaCl})\end{array}$ & $\begin{array}{c}\text { Temp } \\
\left({ }^{\circ} \mathrm{C}\right)\end{array}$ & $\mathrm{pK}_{\mathrm{a}}$ of Lactic Acid \\
\hline $0 \mathrm{M}$ & 25 & 3.86 \\
\hline $0.15 \mathrm{M}$ & 25 & 3.51 \\
\hline $0.15 \mathrm{M}$ & 37 & 3.70 \\
\hline $0.5 \mathrm{M}$ & 25 & 3.50 \\
\hline $0.6 \mathrm{M}$ & 25 & 3.572 \\
\hline
\end{tabular}

The selection of $25^{\circ} \mathrm{C}$ for laboratory experiments is a convenient choice, but may not be relevant to industrial applications. Because TALSPEAK is being evaluated and prepared for industrial scale application, a wider temperature range will be necessary. For this purpose extending the current published data record with lactic acid $\mathrm{pK}_{\mathrm{a}}$ values for the temperature range of $25-65^{\circ} \mathrm{C}$ is desirable.

Research on a simplified TALSPEAK process has shown the mechanism of extraction of $\mathrm{Ln}^{3+}$ by bis(2-ethylhexyl) phosphoric acid (HA). By excluding lactate (Lac) and diethyltriaminepentaacetic acid (DTPA) from the aqueous phase Peppard et al. ${ }^{[56]}$ showed that 
lanthanides are extracted from $\mathrm{HCl}$ or $\mathrm{HNO}_{3}$ by $\mathrm{HA}$ according to the mechanism shown in equation (2.7).

$$
\mathrm{Ln}^{+3}+\mathrm{n} \overline{(\mathrm{HA})_{2}} \leftrightarrow 3 \mathrm{H}^{+}+\overline{\operatorname{LnA}_{3}(\mathrm{HA})_{2 \mathrm{n}-3}}
$$

Various values for the HA dimer stoichiometric coefficient $(n)$ have been reported. Peppard et al. ${ }^{[56]}$ concluded that $\mathrm{n}=3$ when extracting tracer quantities into an aromatic diluent. Lundqvist et al. ${ }^{[57]}$ later showed that $\mathrm{n}=2.5$ or 3 depending on the diluent. For aromatic diluents and aliphatic diluents with very low $[\mathrm{HA}]_{\text {org }}\left(10^{-4.5}-10^{-3.5} \mathrm{M} \mathrm{HA}\right) \mathrm{n}=3$, however, $n=2.5$ for aliphatic diluents where the $[\mathrm{HA}]_{\text {org }}$ is greater. Other authors ${ }^{[58-59]}$ have expressed $\mathrm{n}$ as a function of the number of deprotonated HA complexed to the metal (d), the number of protonated HA complexed to the metal ( $p)$, and the degree of polymerization of HA in the diluent (s) as shown in equation (2.8).

$$
n=\frac{d+p}{s}
$$

This formulation does not explicitly assume that HA is strictly a dimer as other authors have.

Dubuquoy et al. ${ }^{[58]}$ first proposed this formulation for $n$ and suggested that $\mathrm{s}$ for HA was 2 below $[\mathrm{HA}]_{\text {org }}<0.3 \mathrm{M}$ but was 3 at where $[\mathrm{HA}]_{\text {org }}>0.3 \mathrm{M}$. In contrast, Thomas and Burkhart ${ }^{[59]}$ note that other research by various authors has shown that $s$ is not strictly 2 or 3 at any given time, but that partial trimerization of HA occurs over a range of $[\mathrm{HA}]_{\text {org }}(0.1-1 \mathrm{M} \mathrm{HA})$ and increases with increasing $[\mathrm{HA}]_{\text {org }}$.

Mel'nik et al. ${ }^{[60]}$ reported that there are conditions where the HA- $\mathrm{Ln}^{3+}$ extraction behavior deviates from that shown in equation (2.7). When performing extractions with weighable quantities of $\mathrm{Ln}^{3+}$, log-slope analysis consistently produced $2.7 \pm 0.1$ instead of the previous published 3. ${ }^{[56]}$ Mel'nik et al. reasoned that when sufficient quantities of $\mathrm{Ln}^{3+}$ salts are present, 
aqueous complexes between $\mathrm{Ln}^{3+}$ and the counter ions can be extracted by ion-pair extraction by the mechanism shown in equation (2.9).

$$
\operatorname{LnB}_{b}^{3-b}+n \overline{(H A)_{2}} \leftrightarrow(3-b) H^{+}+\overline{\operatorname{LnB}_{b} A_{3-b}(H A)_{2 n-(3-b)}}
$$

This behavior occurs under two types of conditions: 1 ) where $\mathrm{pH}$ drives $\mathrm{Ln}^{3+}$ extraction beyond the ideal HA extraction stoichiometry in equation (2.1 $)^{[53]}$ and 2 ) where high concentrations of acid counter ion (B) exist to shift the extracted species to $\operatorname{LnB}_{3}(H A)_{3}$. This second behavior was observed at $\mathrm{HCl}$ concentrations greater than $5 \mathrm{M} \mathrm{HCl}$ for $\mathrm{Y}^{3+},{ }^{[61]}$ and at $\mathrm{HNO}_{3}$ concentrations greater 5-7 $\mathrm{M} \mathrm{HNO}_{3}$ for $\mathrm{La}^{3+}$ through $\mathrm{Lu}^{3+}$ respectively. ${ }^{[62-63]}$

The $\mathrm{Na}^{+}$distribution data in the literature shows that it is transferred to the organic phase of non-polar diluents when $\mathrm{HA}$ is converted to $\mathrm{NaA}$ by the mechanism ${ }^{[57]}$ shown in equation (2.10) with $n=2$.

$$
\mathrm{Na}^{+}+\mathrm{n} \overline{(\mathrm{HA})_{2}} \leftrightarrow \mathrm{H}^{+}+\overline{\mathrm{NaA}(\mathrm{HA})_{2 \mathrm{n}-1}}
$$

If more than $25 \%$ of the $\mathrm{HA}$ is converted to $\mathrm{NaA}$, the $\mathrm{HA}$ dimers are destroyed and $\mathrm{NaA}$ aggregates, forming a third phase. ${ }^{[57]}$

Average aggregation increases with the extraction of $\mathrm{Ln}^{3+}$ ions. ${ }^{[64]}$ This is a result of the speciation [equation (2.1)] that has two to three HA dimers complexing the $\mathrm{Ln}^{3+}$ metal center. High loading conditions can cause problems in the TALSPEAK process if they lead to loss of extractant solubility. Under extremely high trivalent metal loading conditions, insoluble gels will form as an interphase between the organic and aqueous phases resulting in loss of system flow properties. ${ }^{[65-66]}$ The formation of a gel interphase can occur under equilibrium conditions, or temporarily under high mass transfer conditions. ${ }^{[65]}$ A detailed physical description of the process through which interphase species form is in Kizim et al. ${ }^{[67]}$ Previous work on the 
formation of gel interphase indicate that this behavior is dependent on HA concentration, aqueous acidity, and aqueous metal concentration. ${ }^{[66]}$ Targeted synthesis to understand other high aggregation species has produced the following compounds: $\operatorname{LnA}\left(\mathrm{NO}_{3} \mathrm{HA}\right)_{2}{ }^{[68]}$ $\operatorname{LnA}_{2}(\mathrm{ClHA}){ }^{[68]} \operatorname{Ln}(\mathrm{A})_{3},{ }^{[69-70]}$ and $\operatorname{Ln}(\text { Acetate })_{b}(\mathrm{~A})_{2-\mathrm{b}}{ }^{[71]}$ Additional tests on the species $\operatorname{Ln}(\mathrm{A})_{3}$ and $\operatorname{Ln}(\text { Acetate })_{b}(A)_{2-b}$ showed that they were insoluble in excess diluent (octane ${ }^{15}$ and decane ${ }^{16}$ ), but are solvated by the addition of excess $\mathrm{HA}^{\left[{ }^{[71-72]}\right.} \mathrm{Neutralization}$ of $\mathrm{HA}$ by $\mathrm{NaOH}$ in the absence of $\mathrm{Ln}^{3+}$ can also lead to extreme aggregation of $\mathrm{HA}^{[73]}$ and formation of a gel-like third phase. ${ }^{[74]}$ This extractant loss can be prevented by adding strong electrolytes such as $\mathrm{NaCl}, \mathrm{NaNO}_{3}$, or $\mathrm{Na}_{2} \mathrm{SO}_{4}$ to the aqueous phase ${ }^{[73-74]}$ or organic phase modifiers. ${ }^{[75-76]}$ Blake et al. ${ }^{[75]}$ tested 63 possible candidates and showed that a variety of primary alcohols with 8-13 carbons, tributyl phosphate, dibutly butyl phosphonate, butyl dibutyl phosphonate, and tributyl phosphine oxide were among the most promising organic phase modifiers.

Visible spectroscopic studies of the complexes formed under TALSPEAK conditions increase the understanding of their structures. Jensen et al. ${ }^{[64]}$ showed hypersensitive bands in the $\mathrm{Nd}^{3+}$ spectrum that were indicative of rearrangement of ligands surrounding the $\mathrm{Nd}^{+3}$ metal center. The change occurring as suggested by Jensen et al. was from $\mathrm{Nd}(\mathrm{AHA})_{3}$ or $\mathrm{NdA}_{3}$ to $\mathrm{Nd}_{2}(A)_{6}$ that occurred with increasing metal loading. Comparison of the visible spectra of various $\mathrm{Nd}^{3+}$ complexes has shown that the coordination chemistry surrounding the $\mathrm{Nd}^{3+}$ metal center leads to a recognizable spectral profile. In systems where organophosphorus ligand dimers complex $\mathrm{Nd}^{3+}\left[\right.$ Cyanex $272^{[77]}, \mathrm{HA}$ in $\left.\mathrm{Ln}(\mathrm{AHA})_{3}\right]$ the $\mathrm{Nd}^{3+}$ absorbance spectrum shows bands at 570,577 , 585,590 and $605 \mathrm{~nm} \cdot{ }^{[64,78]}$ In systems where organophosphorus ligand monomers complex $\mathrm{Nd}^{3+}$ (Cyanex-302,Cyanex-301 ${ }^{[77]} ; \mathrm{HA}$ in $\mathrm{LnA}_{3}$ ) the $\mathrm{Nd}^{3+}$ absorbance spectrum shows a band at $585 \mathrm{~nm}$ with a shoulder at $577 \mathrm{~nm} .{ }^{[64,69]}$ The interpretation of these references indicates that the 
presence of the $570 \mathrm{~nm}$ band indicates the presence of dimerized HA in the coordination sphere of $\mathrm{Nd}^{3+}$.

Studies of the IR spectrum of HA complexed with $\mathrm{Ln}^{3+}$ or $\mathrm{Na}^{+}$have shown spectral changes indicative of metal complexation. The IR spectrum of $\mathrm{HA}^{[79-80]}$ and its $\mathrm{Na}^{+},{ }^{[79-81]}$ and $\mathrm{Ln}^{3+}[68,70-71]$ complexes has been presented in detail in various sources. The IR spectral features of highest interest is the asymmetric stretching of the $\mathrm{P}=\mathrm{O}$ and $\mathrm{P}-\mathrm{O}$ bonds that show bands at 1230 and $1035 \mathrm{~cm}^{-1}$ respectively. When $\mathrm{HA}$ is complexed with metals, the $1230 \mathrm{~cm}^{-1}$ band will shift depending on the metal involved in complexation $\left(\mathrm{Na}^{+}=1220, \mathrm{Ln}^{3+}=1200 \mathrm{~cm}^{-1}\right) .{ }^{[68,81]}$ Literature also has shown that large aggregate $\mathrm{Ln}^{3+}: \mathrm{HA}$ complexes contain -O-P-O- bridging bands that appear at 1090 or $1100 \mathrm{~cm}^{-1} .{ }^{[68,71]} \mathrm{IR}$ absorbance bands in $\mathrm{Ln}^{3+}: \mathrm{HA}$ complexes appearing in the $1500-1700$ region have been given various assignments. A vibration band at $1620 \mathrm{~cm}^{-1}$ in $\operatorname{LaA}\left(\mathrm{NO}_{3}\right)_{2} \cdot 2 \mathrm{H}_{2} \mathrm{O}$ complex was assigned to $\mathrm{H}_{2} \mathrm{O}$ bending. A vibration band in LnAcetate $\mathrm{A}_{2}$ complex involving $\mathrm{Ho}^{3+}, \mathrm{Er}^{3+}, \mathrm{Yb}^{3+}$ and $\mathrm{Lu}^{3+}$ at $1575-1590 \mathrm{~cm}^{-1}$ was assigned to the acetate- $\mathrm{Ln}^{3+}$ complex vibrations in bridging acetate molecules. ${ }^{[71]}$

The presence of water in non-polar diluent phases is predominantly bound in organic complexes. However, past work with HA has shown that water is absent from the inner coordination sphere of the $\mathrm{Ln}^{3+}: \mathrm{HA}$ extracted species. ${ }^{[82-83]}$ Thus, it is expected that little water will be found in the organic phase unless there exists complexes between $\mathrm{HA}$ and non- $\mathrm{Ln}^{3+}$ species in solution. Non- $\mathrm{Ln}^{3+}$ complexes that may contain water are the HA dimer, and $\mathrm{NaA}$, which has been shown to contain water in various studies. ${ }^{[73,81,84]}$

Past research has focused on isolated aspects of the chemistry, at times connecting a few related phenomena, but rarely providing a comprehensive characterization of the organic phases. The conditions studied also tend to focus on either low or high loading so that speciation is predictable and uniform. Published research has demonstrated formation of high- 
order aggregates of $\left(\mathrm{LnA}_{3}\right)_{x}{ }^{[69,72]}$ or $\left(\operatorname{LnBA}_{2}\right)_{x}{ }^{[71]}$ at high organic phase loading, but the gap in understanding of the dynamic change from the high to low HA aggregation and speciation change due to transition from high to moderate and low loading conditions still exists. The investigation reported herein enhances the knowledge of the HA speciation that exists over the dynamic range of high to low loading in the organic phase. In this investigation the organic phase was initially characterized by measuring the distribution of $\mathrm{Nd}^{3+}, \mathrm{Na}^{+}, \mathrm{H}_{2} \mathrm{O}$ and $\mathrm{H}^{+}$. Additional characterization included vapor pressure osmometry to assess the HA aggregation, and visible and IR absorbance spectroscopy to probe the coordination environment of $\mathrm{Nd}^{3+}$ and HA respectively. This work is designed to develop organic phase characterization methods that will be used in future studies of the TALSPEAK system.

\subsection{Experimental}

\subsubsection{Materials}

Bis(2-ethylhexyl) phosphoric acid (HA or HA) (97\%) was obtained from sigma-Aldrich and was diluted using 2,2,4-trimethylpentane (99\%) (isooctane) obtained from Lancaster Synthesis, Inc. to make solutions ranging from 0-1 M. HA was not purified, however, all solutions were washed once with deionized water prior to use. Bibenzyl (99\%) was obtained from Aldrich and recrystallized twice from methanol prior to dissolution in isooctane. Water used in preparing solutions was at least $15 \mathrm{M} \Omega-\mathrm{cm} . \mathrm{NdCl}_{3} \cdot 6 \mathrm{H}_{2} \mathrm{O}$ (99.9\% metals basis), concentrated DL-lactic acid $(85 \%+), 50 \mathrm{wt} \% \mathrm{NaOH}, \mathrm{NaCl}$, were obtained from Aldrich and used as received. The concentrated lactic acid was diluted with DI water, boiled for 24 hours to eliminate polymerization, and then standardized by potentiometric titration prior to use. For the radiotracer distribution measurements of lactate and sodium, ${ }^{14} \mathrm{C}$ labeled sodium lactate, and

${ }^{22} \mathrm{Na}$ stocks were obtained from Perkin Elmer. For the NMR experiments, ${ }^{13} \mathrm{C}$ labeled lactate (carbonyl C label) and d18-isooctane (98 atom\%) were obtained from Sigma-Aldrich. Isooctane 
was used as the diluent in these studies because $\mathrm{n}$-dodecane that is typically used in the TALSPEAK process did not have a vapor pressure high enough for the vapor pressure osmometer used in this study.

\subsubsection{Vapor Presssure Osmometry of Aqueous Lactate}

A series of varied lactic acid concentrations $(0.1-1.9 \mathrm{~mol} / \mathrm{L})$ dissolved in DI water was also prepared for the vapor pressure osmometry (VPO) experiments. Measurements by VPO were performed on a Wescor Vapro 5520 vapor pressure osmometer. The instrument operates at ambient temperature, $21.9 \pm 0.2^{\circ} \mathrm{C}$ for these experiments. The instrument was calibrated and operated according to the manufacturer's recommended instructions. ${ }^{[85]}$

\subsubsection{Quantification of the $\mathrm{pK}_{\mathrm{a}}$ of Lactate}

Lactic acid in the titration solution was $0.15 \mathrm{~mol} / \mathrm{L}$ in $1.925 \mathrm{~mol} / \mathrm{L} \mathrm{NaCl}$. The $\mathrm{pcH}$ (described later in this section) titration solution was $0.1 \mathrm{M} \mathrm{HCl}$ in $1.9 \mathrm{M} \mathrm{NaCl}$. The titrant for these experiments was $1 \mathrm{M} \mathrm{NaOH}$ in $1 \mathrm{M} \mathrm{NaCl}$. The $\mathrm{NaOH}$ solution was made using boiled $>15 \mathrm{M} \Omega \mathrm{cm}$ de-ionized (DI) water and stored in an Ascarite vented bottle to prevent $\mathrm{CO}_{2}$ absorption.

The automatic potentiometric titrations were performed using a Metrohm TIAMO autotitration system that was running the TIAMO version 1.2 software. Titration curves were obtained using the static titration function measuring $\mathrm{mV}$ with the fixed volume additions of 50 $\mu \mathrm{L}$. The initial solution volume for each titration was $30 \mathrm{~mL}$. lonic strength was maintained at $I_{\text {total }, \mathrm{mol} / \mathrm{L}}=2.0 \mathrm{M}$. Temperature was held constant by an aluminum heating block that was controlled by a J-Kem Model 150T Temperature controller that was self-tuned at start-up every day. Stirring was provided by a stir-plate on which the heating block was placed. Titrations were performed in Teflon beakers manufactured for use with the heating block. Temperature and $\mathrm{pH}$ measurements were recorded simultaneously and automatically by a combination $\mathrm{pH} /$ temperature electrode that was connected to the TIAMO system. 
Initial experiments were performed using $\mathrm{pH} 4,7$ and 10 buffers to calibrate the $\mathrm{pH}$ electrode prior to each potentiometric titration. This method of $\mathrm{pH}$ calibration proved to be insufficiently precise and the titration curves obtained did not yield satisfactory results. A second, more robust method of $\mathrm{pH}$ calibration proved to offer much better results. This method is known as the $\mathrm{pcH}$ method. In the $\mathrm{pcH}$ method adapted from the Gran Plot method, ${ }^{[86-88]}$ the actual concentration of $\underline{H}^{+}(\mathrm{cH})$ is correlated with the potentiometer's response in $\mathrm{mV}$. The conversion of $\mathrm{mV}$ to the concentration of $\mathrm{H}^{+}$is more convenient, because the conditional equilibrium constant is not a function of hydrogen ion activity, but rather the concentration of $\mathrm{H}^{+}$[see equation (2.3)]. In the $\mathrm{pcH}$ method, the potentiometer's response in $\mathrm{mV}$ is measured against an entire titration of a strong acid of known concentration. Because the strong acid is completely ionized, the $\mathrm{cH}$ can be calculated from the original solution concentration and the volume of titrant added using equations $(2.11)^{[88]}$ and $(2.12)^{[88]}$.

$$
\begin{gathered}
{\left[\mathrm{H}^{+}\right]=\frac{V H-v B}{V+v}=\frac{V H\left(v_{e}-v\right)}{v_{e}(V+v)}=\frac{B\left(v_{e}-v\right)}{V+v}} \\
{\left[\mathrm{H}^{+}\right]=\frac{\mathrm{K}_{\mathrm{w}}}{\left.\mathrm{OH}^{-}\right]}=\frac{\mathrm{K}_{\mathrm{w}}(\mathrm{V}+\mathrm{v})}{\mathrm{B}\left(\mathrm{v}-\mathrm{v}_{\mathrm{e}}\right)}}
\end{gathered}
$$

where $\mathrm{H}$ is the $\mathrm{H}^{+}$concentration in the initial acid solution, $\mathrm{V}$ is the initial volume of acid, $\mathrm{v}$ is the volume of titrant added, $v_{e}$ is the equivalence point volume, $B$ is the concentration of base in the titrant, and $K_{w}$ is the dissociation constant of water. Equation $(2.11)^{[88]}$ is used for the titration points before the equivalence point and equation $(2.12)^{[88]}$ is used for titration points after the equivalence point. $A$ linear regression of the $\mathrm{mV}$ response of the potentiometer and the $-\log (\mathrm{cH})$ (i.e. $\mathrm{pcH}$ ) can be obtained and used on $\mathrm{mV}$ data from titrations of weak acids to obtain pcH curves. This method can be applied to various temperatures and ionic strengths as long as a new $\mathrm{mV}$ vs. $\mathrm{pcH}$ correlation is obtained for each new experimental condition (ionic 
strength and temperature). Plotting the results in a Van't Hoff curve require changing the base of the logarithmic $\mathrm{pK}_{\mathrm{a}}$, which is in base 10 .

\subsubsection{Liquid-Liquid Distribution Measurements Methodology}

To assess effect of the extractant loading on the distribution of the aqueous phase components and speciation of the organic phase, HA concentration was varied from $0-1 \mathrm{M}$ in all extraction systems. For each extraction system, the aqueous phase composition was held constant. For the $\mathrm{HCl}$ medium experiments, the initial aqueous $\mathrm{pH}$ was adjusted to about either 1 or 4 using $\mathrm{HCl}$. For the lactate medium experiments, the $\mathrm{pH}$ of the lactic acid solutions was adjusted by adding $\mathrm{NaOH}$. Aqueous concentration of $\mathrm{Nd}^{3+}$ was 0,30 , or $60 \mathrm{mM}$. Aqueous phase ionic strength was held constant at $1.2 \mathrm{M}$ using $\mathrm{NaCl}$ as a supporting electrolyte. The initial aqueous phase concentrations are shown in Table 2.3 and Table 2.4. The initial aqueous phase concentrations were calculated based on the mass of 50 wt $\% \mathrm{NaOH}, \mathrm{NdCl}_{3} \cdot 6 \mathrm{H}_{2} \mathrm{O}$ or $\mathrm{NaCl}$ salt, or volume of standardized lactic acid or $\mathrm{HCl}$ solution added to these solutions.

Table 2.3- Aqueous phase solution concentrations for the $\mathrm{HCl}$ medium experiments.

\begin{tabular}{|c|c|c|c|c|}
\hline & $\begin{array}{c}\mathrm{pH} \mathrm{1+0} \mathbf{~ m M} \\
\mathbf{N d + H C l}\end{array}$ & $\begin{array}{c}\mathbf{p H ~ 1 + 3 0 ~} \\
\mathbf{N d}+\mathbf{H C l}\end{array}$ & $\begin{array}{c}\mathbf{p H ~ 4 + 0 ~ m M} \\
\mathbf{N d + H C l}\end{array}$ & $\begin{array}{c}\mathbf{p H ~ 4 + 3 0} \\
\mathbf{m M ~ N d + H C l}\end{array}$ \\
\hline $\mathbf{p H}_{\text {initial }}$ & 0.93 & 0.94 & 4.05 & 4.31 \\
{$\left[\mathrm{Nd}^{3+}\right] / \mathbf{M}$} & 0 & 0.03 & 0 & 0.03 \\
\hline$\left[\mathrm{Na}^{+}\right] / \mathbf{M}$ & 1.134 & 0.884 & 1.172 & 1.000 \\
\hline$\left[\mathrm{Cl}^{-}\right] / \mathbf{M}$ & 1.219 & 1.059 & 1.172 & 1.090 \\
\hline $\mathbf{I}_{\text {Calc }} / \mathbf{M}$ & 1.20 & 1.19 & 1.20 & 1.20 \\
\hline
\end{tabular}

To control the ionic strength of the aqueous phase, the aqueous phase compositions were modeled prior to mixing the solution using the Debye-Hückel approximation of the activity of 
the lactate and hydrogen ions in solution ${ }^{[89]}$ and the complexation constants for the $\mathrm{Nd}$ :lactate complexes. ${ }^{[90]}$ Complexation constants used are shown in Table 2.5.

Table 2.4- Aqueous phase solution concentrations for the lactate medium experiments.

\begin{tabular}{|c|c|c|c|c|c|}
\hline & $\begin{array}{c}\text { pH 1+0 mM } \\
\text { Nd+Lac }\end{array}$ & $\begin{array}{c}\text { pH 1+30 mM } \\
\text { Nd+Lac }\end{array}$ & $\begin{array}{c}\text { pH 4+0 mM } \\
\text { Nd+Lac }\end{array}$ & $\begin{array}{c}\mathrm{pH} \mathrm{4+30} \\
\mathrm{mM} \mathrm{Nd+Lac}\end{array}$ & $\begin{array}{c}\mathrm{pH} \mathrm{4+60} \\
\mathrm{mM} \mathrm{Nd+Lac}\end{array}$ \\
\hline $\mathrm{pH}_{\text {initial }}$ & 1.10 & 1.03 & 4.21 & 4.13 & 4.04 \\
\hline$\left[\mathrm{Nd}^{3+}\right] / \mathrm{M}$ & 0 & 0.03 & 0 & 0.03 & 0.06 \\
\hline Lactate / M & 1 & 1 & 1 & 1 & 1 \\
\hline$\left[\mathrm{Na}^{+}\right] / \mathrm{M}$ & 0.76 & 0.67 & 1.26 & 1.03 & 1.03 \\
\hline$\left[\mathrm{Cl}^{\prime}\right] / \mathrm{M}$ & 0.83 & 0.83 & 1.26 & 1.22 & 1.21 \\
\hline $\mathrm{I}_{\text {calc }} / \mathrm{M}$ & 1.20 & 1.18 & 1.21 & 1.10 & 1.17 \\
\hline
\end{tabular}

Table 2.5- Constants used to model ionic strength of aqueous phase for phase contacts.

\begin{tabular}{|c|c|c|c|c|}
\hline Nd:Lactate & $\begin{array}{c}\text { Nd:Lactate } \\
\log (\beta)\end{array}$ & Condition & $\begin{array}{c}\text { H:Lactate } \\
\log (\beta)\end{array}$ & Condition \\
\hline 1:1 & $2.45^{[90]}$ & \multirow{3}{*}{$\begin{array}{c}21^{\circ} \mathrm{C}, 2.0 \mathrm{M} \\
\mathrm{NaClO}_{4}\end{array}$} & \multirow[t]{4}{*}{$3.86^{[89]}$} & \multirow[t]{4}{*}{$25^{\circ} \mathrm{C}, \mathrm{NaCl}, 0 \mathrm{M}$} \\
\hline $1: 2$ & $4.39^{[90]}$ & & & \\
\hline $1: 3$ & $5.44^{[90]}$ & & & \\
\hline $1: 4$ & $5.82^{[91]}$ & $\begin{array}{c}20^{\circ} \mathrm{C}, 1.0 \mathrm{M}, \\
\mathrm{NaClO}_{4}\end{array}$ & & \\
\hline
\end{tabular}

In the text, each extraction system is referenced by initial aqueous $\mathrm{pH}, \mathrm{Nd}^{3+}$ concentration, and aqueous medium ( $\mathrm{HCl}$ or $\mathrm{Lac})$. Multiple systems are referenced by referring to more than one of the system variables enclosed in parentheses. An example is $\mathrm{pH} 4+(0,30) \mathrm{mM}$ Nd would refer to both $\mathrm{pH} 4+0 \mathrm{mM} \mathrm{Nd}$ and $\mathrm{pH} 4+30 \mathrm{mM} \mathrm{Nd}$. All samples were shaken overnight (15+ hours) at $20 \pm 2^{\circ} \mathrm{C}$. The organic and aqueous phase volumes were equal for phase contacts except in the ${ }^{22} \mathrm{Na}$ and ${ }^{14} \mathrm{C}$ tracer tests where the addition of tracer spike increased the aqueous 
volume to $5 \%$ greater than the organic phase. Control distribution experiments were conducted for each analyte using isooctane diluent without HA. Multiple measurements were performed for each experiment.

\subsubsection{Equilibrium pH Measurement}

The $\mathrm{pH}$ was measured for all pre- and post-contact aqueous phase samples using a Radiometer TIMS900 Auto-titration Manager in pH measurement mode with an Orion ROSS Ultra Semi-micro $\mathrm{pH}$ probe. The $\mathrm{pH}$ probe was calibrated before use using $\mathrm{pH} 4$ and 7 buffers from Fisher Scientific. Measurement error is $0.01 \mathrm{pH}$ units.

\subsection{6 $\mathrm{Nd}^{3+}$ Extraction}

The $\mathrm{Nd}^{3+}$ distribution into an organic phase was quantified using the visible spectra obtained from the aqueous phase assuming the $100 \%$ mass balance is sustained. For the $\mathrm{HCl}$ system the molar absorptivity was quantified for each system using a calibration curve with $\left[\mathrm{Nd}^{3+}\right]_{\text {aq }}$ from 0 $40 \mathrm{mM}$ at both $\mathrm{pH} 1$ and 4 . The molar absorptivity observed for $574.94 \mathrm{~nm}$ was $6.77 \pm 0.02$ for the $\mathrm{pH} 1+30 \mathrm{mM} \mathrm{Nd}+\mathrm{HCl}$ and $6.88 \pm 0.03 \mathrm{~L} \mathrm{~mol}^{-1} \mathrm{~cm}^{-1}$ for the $\mathrm{pH} 4+30 \mathrm{mM} \mathrm{Nd}+\mathrm{HCl}$ system (see all absorptivities for $\mathrm{pH} 1$ and 4 systems in Table 2.6). Only the $574 \mathrm{~nm}$ band was utilized for quantifying the aqueous $\mathrm{Nd}^{3+}$ concentration in the $\mathrm{HCl}$ systems. An example of the $\mathrm{Nd}^{3+}$ absorbance bands observed in the aqueous phase is shown in the upper spectrum of Figure 2.9.

Other molar absorptivities published for $574.94 \mathrm{~nm}$ are 6.93 (in $\mathrm{ClO}_{4}{ }^{-}$media) ${ }^{[92]}$, and estimated from a figure at 6.74 (in $\mathrm{Cl}^{-}$media). ${ }^{[93]}$ The concentration of neodymium in the aqueous post-contact phase was determined using the average of the observed absorptivies of $\mathrm{Nd}^{3+}$ at $574 \mathrm{~nm}$ in $\mathrm{HCl}$ media. This aqueous concentration was used in the mass balance to calculate equilibrium organic $\mathrm{Nd}^{3+}$ concentration. A conservative $2 \%$ error is assumed for all $\mathrm{Nd}^{3+}$ concentrations based on the $1.1 \%$ standard deviation that was observed in the average of the two molar absorptivities of $\mathrm{Nd}^{3+}$ in the $\mathrm{HCl}$ systems. 
Table 2.6- Molar absorptivities observed for $\mathrm{Nd}^{3+}$ in the aqueous phase.

\begin{tabular}{|c|c|c|c|c|}
\hline \multirow[b]{2}{*}{$\begin{array}{l}\text { Wavelength } \\
\text { (nm) }\end{array}$} & \multicolumn{2}{|c|}{$\mathrm{pH} 1+30 \mathrm{mM} \mathrm{Nd}+\mathrm{HCl}$} & \multicolumn{2}{|c|}{$\mathrm{pH} 4+30 \mathrm{mM} \mathrm{Nd}+\mathrm{HCl}$} \\
\hline & $\begin{array}{c}\varepsilon \\
\left(\mathrm{L} \mathrm{mol}^{-1} \mathrm{~cm}^{-1}\right)\end{array}$ & $\mathbf{R}^{2}$ & $\frac{\varepsilon}{\left(\mathrm{L} \mathrm{mol}^{-1} \mathrm{~cm}^{-1}\right)}$ & $\mathbf{R}^{2}$ \\
\hline 512.18 & $1.71 \pm 0.02$ & 0.9986 & $1.66 \pm 0.02$ & 0.9985 \\
\hline 521.28 & $3.92 \pm 0.02$ & 0.9996 & $3.96 \pm 0.02$ & 0.9995 \\
\hline 574.94 & $6.77 \pm 0.02$ & 0.9999 & $6.88 \pm 0.03$ & 0.9996 \\
\hline 731.66 & $3.78 \pm 0.03$ & 0.9993 & $3.81 \pm 0.02$ & 0.9995 \\
\hline 740.32 & $6.50 \pm 0.04$ & 0.9993 & $6.60 \pm 0.03$ & 0.9997 \\
\hline 794.19 & $10.59 \pm 0.05$ & 0.9997 & $10.78 \pm 0.04$ & 0.9998 \\
\hline 801.37 & $6.14 \pm 0.04$ & 0.9994 & $6.24 \pm 0.03$ & 0.9997 \\
\hline 865.68 & $3.72 \pm 0.03$ & 0.9988 & $3.79 \pm 0.02$ & 0.9996 \\
\hline
\end{tabular}

The presence of lactate in the aqueous phase lead to the formation of $\mathrm{Nd}^{3+}$ :Lactate complexes for the $\mathrm{pH} 4$ systems, changing the absorbance spectra. Thus, $\mathrm{pH} 4$ system $\mathrm{Nd}^{3+}$ concentration was measured from the observed molar absorptivity of the pre-contact aqueous solution of known concentration using the aqueous phase absorbance at $797.94 \mathrm{~nm}$. The $\mathrm{pH} 1+$ $30 \mathrm{mN} \mathrm{Nd}+$ Lac system displayed similar absorbance to the $\mathrm{pH} 1+30 \mathrm{mM} \mathrm{Nd}+\mathrm{HCl}$ system, so same method used for the $\mathrm{HCl}$ systems was used. A summary of the absorptivities used for the Nd systems is shown in Table 2.7.

Table 2.7- Summary of the molar absorptivity data, and calibration techniques used in the quantification of $\mathrm{Nd}^{3+}$ in the aqueous phase solutions.

\begin{tabular}{|c|c|c|}
\hline Systems & $\begin{array}{l}\mathrm{pH}(1,4)+30 \mathrm{mM} \mathrm{Nd}+\mathrm{HCl} \\
\mathrm{pH} 1+30 \mathrm{mM} \mathrm{Nd}+\mathrm{HCl}\end{array}$ & $\mathrm{pH} 4+(30,60) \mathrm{mM} \mathrm{Nd}+\mathrm{Lac}$ \\
\hline Calibration Data Set & $\begin{array}{c}\text { 0-40 } \mathrm{mM} \mathrm{Nd} \\
\text { calibration curve }\end{array}$ & $\begin{array}{c}\text { 30, } 60 \text { mM Nd pre-contact aqueous } \\
\text { sample (single point) }\end{array}$ \\
\hline Wavelength (nm) & 574.94 & 797.94 \\
\hline $\begin{array}{l}\text { Molar Absorptivity Used } \\
\left(\mathrm{L} \mathrm{mol}^{-1} \mathrm{~cm}^{-1}\right)\end{array}$ & $6.82 \pm 0.08$ & $10.5 \pm 0.2$ \\
\hline
\end{tabular}


The calculation of the theoretical loading was performed under the assumption that extracted $\mathrm{Nd}^{3+}$ exists as $\mathrm{NdA}_{3}(\mathrm{HA})$ in the organic phase under low loading conditions and $\mathrm{n}=2$ in equation (2.1). This assumption is consistent with the aggregation data presented herein. The equation defining theoretical loading is shown in equation (2.13)

$$
\text { Loading }_{\text {theoretical }}=\frac{[\mathrm{Nd}]_{\text {org }}}{[\mathrm{HA}]_{\text {org }} / 2 \mathrm{n}}
$$

where $2 \mathrm{n}$ is the ratio of $\mathrm{HA}$-to- $\mathrm{Nd}^{3+}$ in the organic phase when equation (2.7) is the mechanism that describes $\mathrm{Nd}^{3+}$ extraction.

\subsection{7 ${ }^{22} \mathrm{Na}^{+}$Tracer Extraction}

For ${ }^{22} \mathrm{Na}^{+}$experiments, equal volumes $(0.4 \mathrm{~mL})$ of organic and aqueous solutions were aliquoted into cryogenic centrifuge tubes. A spike of $20 \mu \mathrm{L}$ of a ${ }^{22} \mathrm{Na}^{+}$tracer was added to each two phase sample. These samples were contacted as described in section 2.2.4. Following the contacts, $200 \mu \mathrm{L}$ of each phase were transferred to separate test-tubes for counting. Gamma counting was performed using a Perkin Elmer Cobra II Auto-Gamma. The distribution ratios for were measured by calculating the ratio of ${ }^{22} \mathrm{Na}^{+}$back-ground subtracted activity in the organic vs. aqueous phase. The equation used for the calculation of distribution ratios is shown in equation (2.14)

$$
D_{N a}=\frac{\left[\mathrm{Na}^{+}\right]_{o r g}}{\left[N a^{+}\right]_{a q}}=\frac{C P M_{o r g}}{C P M_{a q}}
$$

where CPM is counts per minute. The concentration of sodium in each phase was then calculated from the distribution ratio through an iterative calculation that guessed what fraction of the total sodium was in each phase that resulted in a distribution equal to that observed. The recovery of ${ }^{22} \mathrm{Na}^{+}$was confirmed by comparison with the mass balance samples. The detection limit for these experiments for $\left[\mathrm{Na}^{+}\right]_{\text {org }}$ was $0.6-6 \mathrm{mM}$ depending on the background count 
rates. Because these samples were counted using an automated counting instrument, and due to the penetrating nature of gamma radiation there was an elevated background for systems with higher organic $\mathrm{Na}^{+}$concentrations. The $\mathrm{HCl}$ and lactate systems were counted separate from each other, which contributed to high detection limits for the lactate systems than the $\mathrm{HCl}$ systems. Empty counting vials were counted interspersed with the samples to correct the countrates for the added background in the high count rate systems, but the increased background led to a detection limit that varied by system. A table showing the detection limits for the $\mathrm{Na}^{+}$ extraction experiments is shown in Table 2.8 .

Table 2.8- The detection limits for the $\mathrm{Na}^{+}$extraction experiments by system.

\begin{tabular}{|c|c|}
\hline System & {$[\mathrm{Na}]_{\text {org }} / \mathrm{M}$} \\
\hline $\mathrm{pH} 1+0 \mathrm{mM} \mathrm{Nd}+\mathrm{HCl}$ & 0.0013 \\
$\mathrm{pH} 1+30 \mathrm{mM} \mathrm{Nd}+\mathrm{HCl}$ & 0.0009 \\
$\mathrm{pH} 4+0 \mathrm{mM} \mathrm{Nd}+\mathrm{HCl}$ & 0.0006 \\
$\mathrm{pH} 4+30 \mathrm{mM} \mathrm{Nd}+\mathrm{HCl}$ & 0.001 \\
$\mathrm{pH} 1+0 \mathrm{mM} \mathrm{Nd}+\mathrm{Lac}$ & 0.003 \\
\hline $\mathrm{pH} 1+30 \mathrm{mM} \mathrm{Nd}+\mathrm{Lac}$ & 0.003 \\
\hline $\mathrm{pH} 4+0 \mathrm{mM} \mathrm{Nd}+\mathrm{Lac}$ & 0.006 \\
\hline $\mathrm{pH} 4+30 \mathrm{mM} \mathrm{Nd}+\mathrm{Lac}$ & 0.0016 \\
\hline $\mathrm{pH} 4+60 \mathrm{mM} \mathrm{Nd}+\mathrm{Lac}$ & 0.004 \\
\hline
\end{tabular}

\subsection{8 $\quad \mathrm{H}_{2} \mathrm{O}$ Distribution (Karl Fischer Titration)}

Water content in the organic phase was measured using a Photovolt Aquatest 2010 Coulometric Moisture Analyzer. The instrument function was verified at the start of each set of samples by titrating samples of $2 \mu \mathrm{L}$ of $\mathrm{DI} \mathrm{H}_{2} \mathrm{O}$. After instrument sensitivity was verified using water test samples, organic phase samples $(0.1-2 \mathrm{~mL})$ were introduced into the titration vessel 
using manual pipettes by opening the vessel briefly. Sample sizes were varied to keep the measurement signal below $2000 \mu \mathrm{g} \mathrm{H}_{2} \mathrm{O}$ per sample.

\subsection{9 ${ }^{14} \mathrm{C}$ labeled Lactate Distribution}

For ${ }^{14} \mathrm{C}$ lactate experiments, $0.4 \mathrm{~mL}$ of both the organic and aqueous phase were measured into polypropylene cryogenic vials. A spike of $30 \mu \mathrm{L}$ of the sodium lactate tracer was then added to the two phase sample to achieve a ${ }^{14} \mathrm{C}$ activity of $60 \mu \mathrm{Ci}$, which were contacted as previously described in section 2.2.4. Liquid scintillation counting was performed using a Packard Tricarb $2550 \mathrm{TR} / \mathrm{AB} .{ }^{14} \mathrm{C}$ labeled lactate distribution ratios $\left(\mathrm{D}_{\mathrm{Lac}}=[\mathrm{Lac}]_{\mathrm{org}} /[\mathrm{Lac}]_{\mathrm{aq}}=\mathrm{CPM}_{\mathrm{org}} / \mathrm{CPM}_{\mathrm{aq}}\right.$, where CPM is counts per minute) were measured by taking the ratio of ${ }^{14} \mathrm{C}$ activity in the organic vs. aqueous phase (both corrected for background CPM) and used to calculate equilibrium organic

${ }^{14} \mathrm{C}$ labeled lactate concentration. Mass balance calculations were performed to ensure measured count rates accounted for the total activity added. Separate mass balance samples were prepared for each extraction system using spiked aliquots of $0.4 \mathrm{~mL}$ aqueous phase from each system studied. These spiked aliquots were sampled using the same technique as the batch contact samples.

\subsubsection{Solvent extraction data treatment}

Based on literature evidence ${ }^{[57]}$ and our vapor pressure osmometry (VPO) results described herein, the adopted mechanism of $\mathrm{Nd}^{3+}$ and $\mathrm{Na}^{+}$extraction can be described by equations (2.7) and (2.10) with $n=2$ and 1 , respectively. Equilibrium constants corresponding to the equations (2.7) and (2.10) were obtained using solvent extraction modeling program SXLSQI. ${ }^{[94]}$ Equilibrium values of the $[\mathrm{Nd}]_{\text {org }}$ and aqueous $\mathrm{pH}$ were used as input values to the program. Based on the VPO results, the HA aggregation number of 2 was applied in the SXLSQI modeling. Under the conditions of the applied HA concentration $>0.025 \mathrm{M}$, the concentration of monomeric HA species was assumed to be negligible based on the literature LogK $_{\text {dimer }}=4.59$ for HA in the 
isooctane diluent. ${ }^{[95]}$ For the $\mathrm{Nd}^{3+}$ or $\mathrm{Na}^{+}$extraction, the single organic-phase product species were included in the computer model: $\mathrm{Nd}(\mathrm{A})_{3}(\mathrm{HA})_{2}$ or $\mathrm{Na}(\mathrm{A})(\mathrm{HA})$, respectively. For both, $\mathrm{Nd}^{3+}$ and $\mathrm{Na}^{+}$extraction systems, two parameters were refined in the fit, namely the corresponding $\log K$ value and the solubility parameter of the product species. Non-ideality effects were taken into account by using Pitzer ${ }^{[96]}$ parameters for aqueous ions, the Hildebrand-Scott treatment for non-ionic effects in the organic phase ${ }^{[97]}$ and the Debye-Hückel treatment for electrostatic effects in the organic phase. ${ }^{[98]}$ All constants are corrected to infinite dilution. The solubility parameters and molar volumes of the HA extractant and isooctane diluent were estimated from group contributions. In the SXLSQI modeling, experimental precision for each data point was determined to be $\pm 6 \%$ as estimated from a combination of replicate determinations, volumetric error, and counting precision.

\subsubsection{Visible Spectroscopy}

For the aqueous phase, the visible absorbance spectrum of each solution was recorded using an Oceanoptics USB2000 spectrophotometer $(500-880 \mathrm{~nm})$ with a tungsten light source and a 2.1 Optical Density glass filter. The data were collected using $1 \mathrm{~cm}$ pathlength PMMA cuvettes. The spectra recorded were averages of 50 scans of 15 milliseconds each with the boxcar averaging feature disabled. Resolution for the scans was $0.35 \mathrm{~nm}$.

For the organic phase, the same spectrophotometer and light source were employed except the 2.9 optical density glass filter was used. The data were collected using $1 \mathrm{~cm}$ pathlength quartz cuvettes. The spectra recorded were averages of 60 scans of 60 milliseconds each with the boxcar averaging feature disabled. Resolution for the scans was $0.35 \mathrm{~nm}$.

\subsubsection{Fourier Transform Infrared Spectroscopy}

A sample of the organic phase was taken following the phase contacts and analyzed using a Thermo Scientific Nicolet 6700 series spectrometer. Each solution spectrum was collected in 
absorbance mode and was an average of 64 scans collected at a resolution of $2 \mathrm{~cm}^{-1}$ over the range of $650-4000 \mathrm{~cm}^{-1}$. The instrument was continuously purged with dry air to prevent any water from ambient air to interfere with the absorbance spectrum. The spectra of the solvent absorbance bands were subtracted from the sample spectra normalized (divided) by the HA concentration prior to analysis [calculation shown in equation (2.6)].

$$
A b s_{\text {Norm }}=\frac{\mathrm{Abs}_{\text {sample }}-\mathrm{Abs}_{\text {solvent }} X_{\text {solvent }}}{[\mathrm{HA}]_{\text {sample }}}
$$

where $\mathrm{Abs}_{\text {Norm }}$ is normalized, solvent subtracted resultant spectrum; $\mathrm{Abs}_{\text {sample }}$ is original absorbance spectrum of sample; Abs $_{\text {solvent }}$ is absorbance spectrum of pure solvent; $\mathrm{X}_{\text {solvent }}$ is mole fraction of solution sample; $[\mathrm{HA}]_{\text {sample }}$ is the molar concentration of $\mathrm{HA}$ in the sample.

\subsubsection{Vapor Pressure Osmometry}

Organic phase samples were analyzed using a Wescor Vapro 5520 and was operated at room temperature. The instrument was calibrated at the start of each sample series using the manufacturer provided calibration standards: $100,290,1000 \mathrm{mmol} \mathrm{NaCl} / \mathrm{kg}$ of water. Bibenzyl dissolved in isooctane was used as a non-aggregating standard. The average aggregation for HA was calculated using the ratio of the slopes from the instrument response for the bibenzyl standards divided by the slope of the sample of interest [see Figure 2.1 for an example of instrument response curves, and equations (2.16) and (2.17)]. Each experimental datum in this work was taken as average of 3 replicate measurements. For samples whose instrument response curve displayed non-linear regions, a 3-point slope was used to obtain an approximation of the average aggregation at the central point.

$$
\text { Slope }=\frac{\Delta(\text { VPO_Signal })}{\Delta\left(\text { molality }_{\text {sample }}\right)}
$$




$$
\mathrm{N}_{\text {agg,average }}=\frac{\text { Slope }_{\text {Bibenzyı_tandard }}}{\text { Slope }_{\text {sample }}}
$$

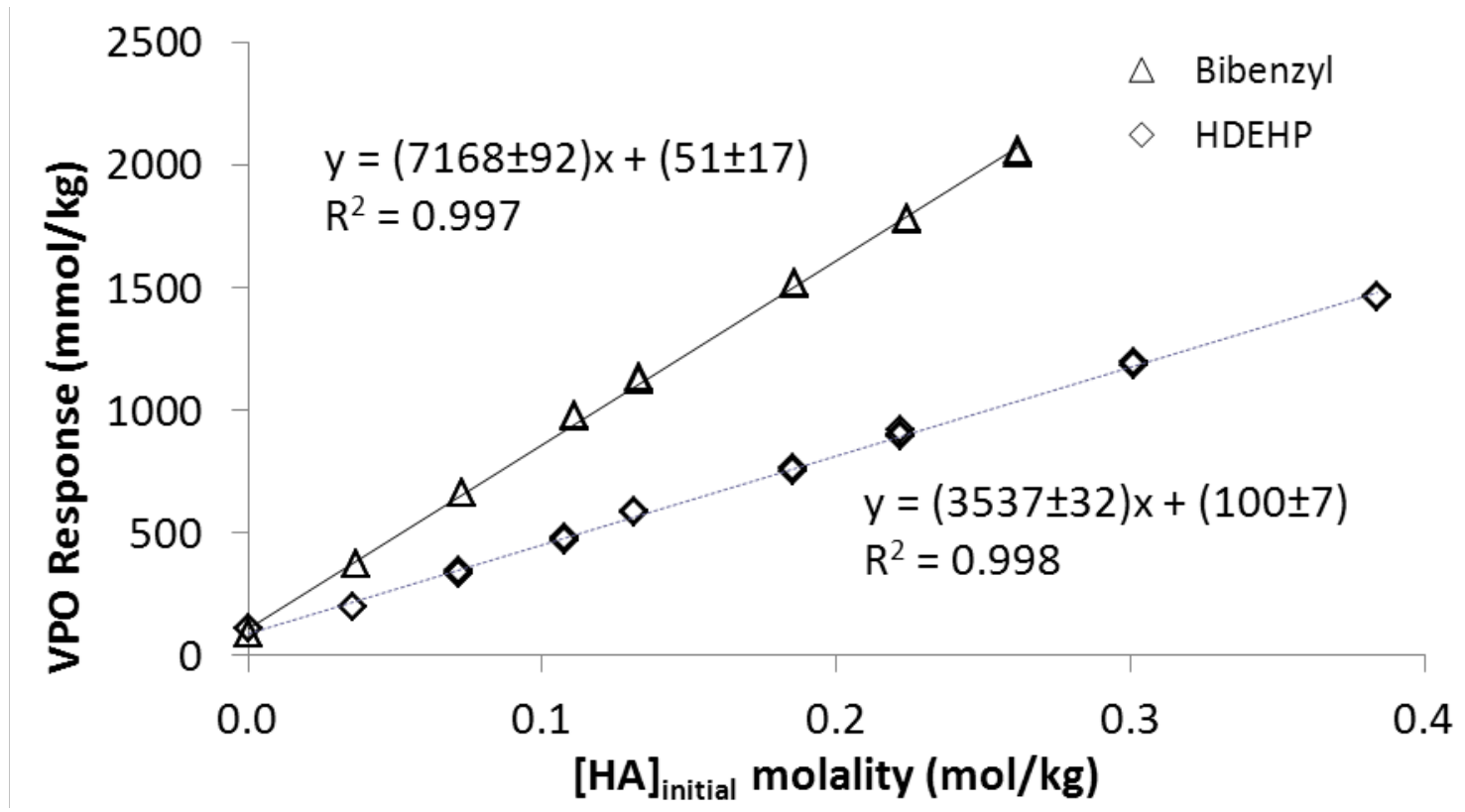

Figure 2.1- An example of the instrument response curve obtained from the bibenzyl standard and unwashed HA solutions.

\subsubsection{Vapor Pressure Osmometry Data Treatment}

The aggregation of HA was modeled based on the extraction of $\mathrm{Nd}^{3+}$ in the organic phase.

The expression for the average aggregation number of species containing " $\mathrm{A}$ " within the solution is the summation of all products of the " $\mathrm{A}$ " containing species with an aggregation value $Z_{i}$, and a species fraction value $X_{i}$. In this simple system it is assumed two species exist: the extracted $\mathrm{Nd}^{3+}$ species containing an unspecified number of " $\mathrm{A}$ " ions $\left(\mathrm{NdA}_{\mathrm{z}}\right)$, and the $\mathrm{HA} \operatorname{dimer}$ which gives the expression shown below in equation (2.18) 


$$
\mathrm{N}_{\text {agg,Average }}=\sum \mathrm{Z}_{\mathrm{i}} \mathrm{X}_{\mathrm{i}}=\mathrm{Z}_{2} \mathrm{X}_{2}+\mathrm{Z}_{\mathrm{NdA}_{\mathrm{z}}} \mathrm{X}_{\mathrm{NdA}_{\mathrm{z}}}
$$

The fraction of the total HA involved in the extracted $\mathrm{Nd}^{3+}$ complex $\left(\mathrm{X}_{\mathrm{NdA}}\right)$ is

$$
X_{\mathrm{NdA}_{z}}=\frac{Z_{\mathrm{NdA}_{z}}[\mathrm{Nd}]_{\mathrm{org}}}{[\mathrm{HA}]_{\text {total }}}
$$

The remaining fraction of the HA that resides in the dimeric form is equal to

$$
X_{2}=1-X_{\mathrm{NdA}_{2}}
$$

Since the $Z_{2}$ is known to be 2 , that can substituted into the equation. By combining equations (2.18), (2.19), and (2.20), equation (2.21) is then obtained:

$$
\mathrm{N}_{\text {agg, Average }}=2\left(1-\frac{\mathrm{Z}_{\mathrm{NdAz}}[\mathrm{Nd}]_{\mathrm{org}}}{[\mathrm{HA}]_{\text {total }}}\right)+\mathrm{Z}_{\mathrm{NdAz}}\left(\frac{\mathrm{Z}_{\mathrm{NdAz}}[\mathrm{Nd}]_{\text {org }}}{[\mathrm{HA}]_{\text {total }}}\right)
$$

which by inspection is a quadratic equation. By solving for $Z_{N d A z}$, equation (2.22) is obtained:

$$
\mathrm{Z}_{\mathrm{NdA}_{\mathrm{z}}}=\frac{2[\mathrm{Nd}]_{\mathrm{org}} \pm \sqrt{\left(-2[\mathrm{Nd}]_{\text {org }}\right)^{2}-4[\mathrm{Nd}]_{\text {org }}\left\{\left(2-\mathrm{N}_{\text {agg,Average }}\right)[\mathrm{HA}]_{\text {total }}\right\}}}{2[\mathrm{Nd}]_{\text {org }}}
$$

and was then used to calculate explicitly the value for $Z_{\mathrm{NdAz}}$ for each measured VPO data point.

\subsubsection{Nuclear Magnetic Resonance Spectroscopy}

Due to the paramagnetic shielding effects intrinsic in most lanthanides (including $\mathrm{Nd}^{3+}$ ), solutions for NMR study were made with the same conditions, except lanthanum was used as the trivalent metal. Lanthanum lacks the unpaired f-electrons that all other lanthanides except lutetium possess, and thus will not shield the sample's NMR signal. Under the assumption that $\mathrm{La}^{3+}$ extraction behavior should mimic that of $\mathrm{Nd}^{3+}$, aqueous phase samples were prepared with 20 mol\% ${ }^{13} \mathrm{C}$ labeled lactate with the sample composition shown in Table 2.9. The organic phase for these experiments was the same as that used for the regular contact experiments except $20 \%$ of the isooctane diluent was deuterated. 
The assumption that $\mathrm{La}^{3+}$ extraction behavior mimics $\mathrm{Nd}^{3+}$ behavior was confirmed by performing batch contact experiments under the same conditions as $\mathrm{Nd}^{3+}$, but with $\mathrm{La}^{3+}$. Extraction behavior of $\mathrm{La}^{3+}$ in these batch contact experiments was verified through characterization of the HA aggregation behavior by VPO. The results and discussion of these batch contact experiment are presented in section 2.5.5.

Table 2.9- Aqueous solutions used for the NMR scoping experiments.

\begin{tabular}{|c|c|c|}
\hline & $\mathbf{p H ~ 4 + 6 0 ~ m M ~ L a ~ + ~ L a c ~}$ & $\mathbf{p H ~ 4 ~ + ~ 3 0 ~ m M ~ L a ~ + ~ L a c ~}$ \\
\cline { 2 - 3 } $\mathrm{pH}_{\text {initial }}$ & 4.00 & 4.10 \\
\hline$\left[\mathrm{La}^{+3}\right] / \mathrm{M}$ & 0.060 & 0.030 \\
\hline$[\mathrm{Lac}]_{\text {total }} / \mathrm{M}$ & 1.00 & 1.00 \\
\hline${ }^{13} \mathrm{C}[\mathrm{Lac}] / \mathrm{M}$ & 0.20 & 0.20 \\
\hline$\left[\mathrm{Na}^{+}\right] / \mathrm{M}$ & 1.06 & 1.14 \\
\hline$\left[\mathrm{Cl}^{-}\right] / \mathrm{M}$ & 0.69 & 0.68 \\
\hline $\mathrm{I}_{\text {calc }} / \mathrm{M}$ & 1.19 & 1.19 \\
\hline$\left[\mathrm{HA}_{\text {org }} / \mathrm{M}\right.$ & 0.125 & 1.0 \\
\hline
\end{tabular}

The NMR samples were run using a $300 \mathrm{MHz}$ Oxford magnet with a Techmag computer console. The temperature of the samples was regulated at $15^{\circ} \mathrm{C}$. Deuterated isooctane was used as the locking solvent for all scans. The IUPAC recommended NMR standards ${ }^{[99]}$ were used for the ${ }^{23} \mathrm{Na}$ and ${ }^{31} \mathrm{P}$ experiments $\left(0.1 \mathrm{NaCl}\right.$ in $\mathrm{D}_{2} \mathrm{O}$, and $85 \% \mathrm{H}_{3} \mathrm{PO}_{4}$, respectively). For the ${ }^{13} \mathrm{C}$ experiments the signal was taken in reference to the deuterated isooctane peaks. The NMR frequency used was $75.480,79.395$, and $121.502 \mathrm{MHz}$ for ${ }^{13} \mathrm{C},{ }^{23} \mathrm{Na}$ and ${ }^{31} \mathrm{P}$ respectively. 


\subsection{Results and Discussion: Lactate $\mathrm{pK}_{\underline{a}} \underline{\text { Measurement }}$}

\subsubsection{Vapor Pressure Osmometry of Aqueous Lactic Acid}

Prior to measuring the $\mathrm{pK}_{\mathrm{a}}$ of lactic acid, it was necessary to verify that lactic acid does not form dimers or larger aggregates in solution. The degree of aggregation was measured by standard VPO technique as described below. If lactic acid was found to dimerize in the aqueous solution there would be other competing reactions in addition to the reaction shown in equation (2.4) and this would complicate the analysis of the data.

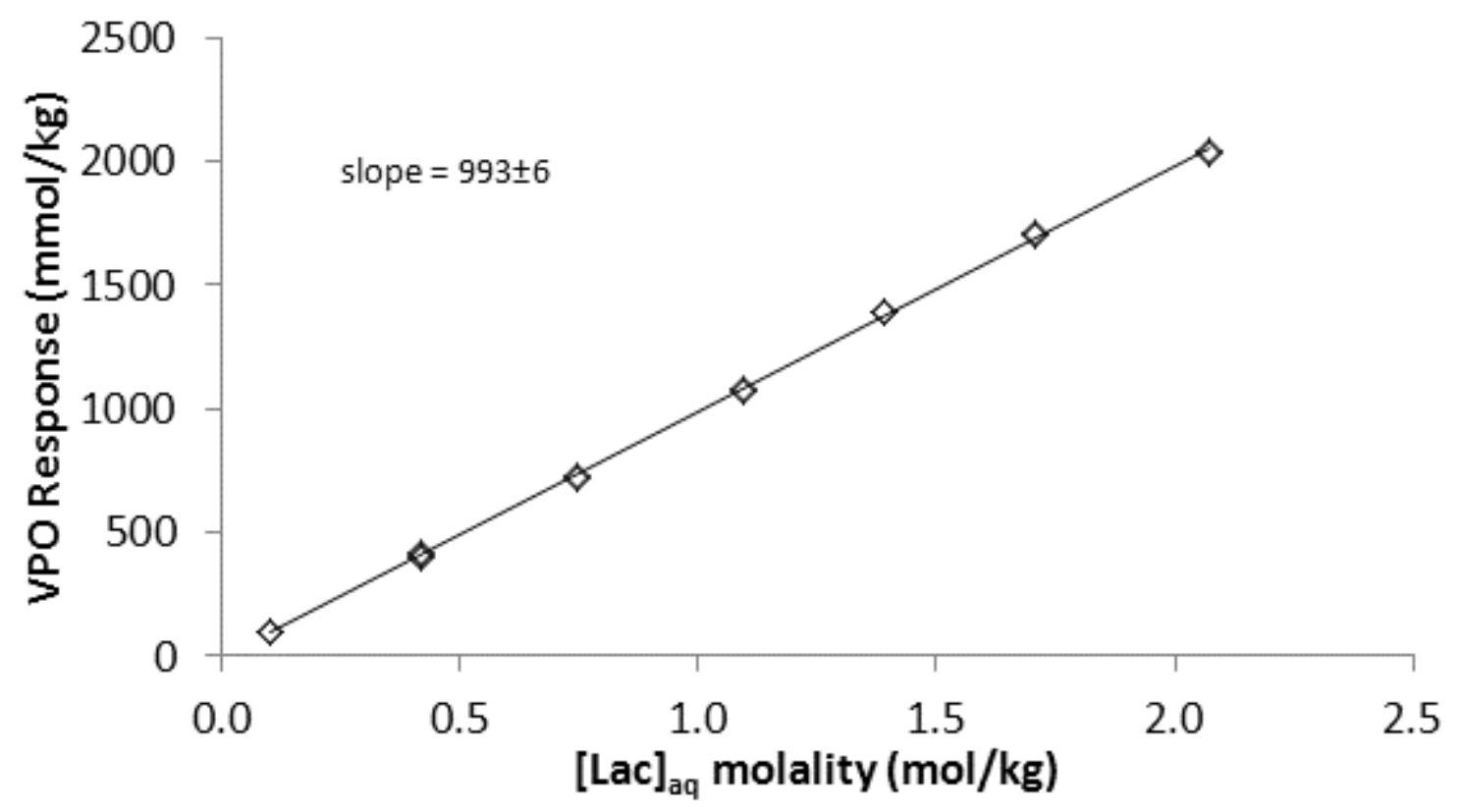

Figure 2.2- VPO Instrument response data from the aqueous lactate samples.

The VPO instrument was calibrated with aqueous $\mathrm{NaCl}$ solutions, which also serve as the non-aggregating standard for the aqueous lactic acid in this experiment. The slope from $\mathrm{NaCl}$ calibration (non-aggregating aqueous standard) is 1000 . The instrument response data obtained 
for aqueous lactate is shown in Figure 2.2. Using equation (2.17) to calculate lactate aggregation, the aggregation number for lactic acid in DI water was $0.993 \pm 0.006$. This result indicates that lactic acid remains monomeric, and thus the simple equilibrium reaction shown in equation (2.4) will be sufficient for our analysis.

\subsubsection{The $\mathrm{pK}_{\mathrm{a}}$ of Lactic acid at $25-65^{\circ} \mathrm{C}$}

The titration results gave $\mathrm{pK}_{\mathrm{a}}$ values with a linear trend when plotted $\mathrm{vs} 1 / \mathrm{T}(\mathrm{K})$. Results are shown in Figure 2.3. According to the Van't Hoff equation ${ }^{[100]}$ [see equation (2.23)], there exists a linear relationship between the natural log of the equilibrium constant and the inverse of the temperature.

$$
\frac{d(\ln K)}{d(1 / \mathrm{T})}=-\frac{\Delta H^{\circ}}{R}
$$

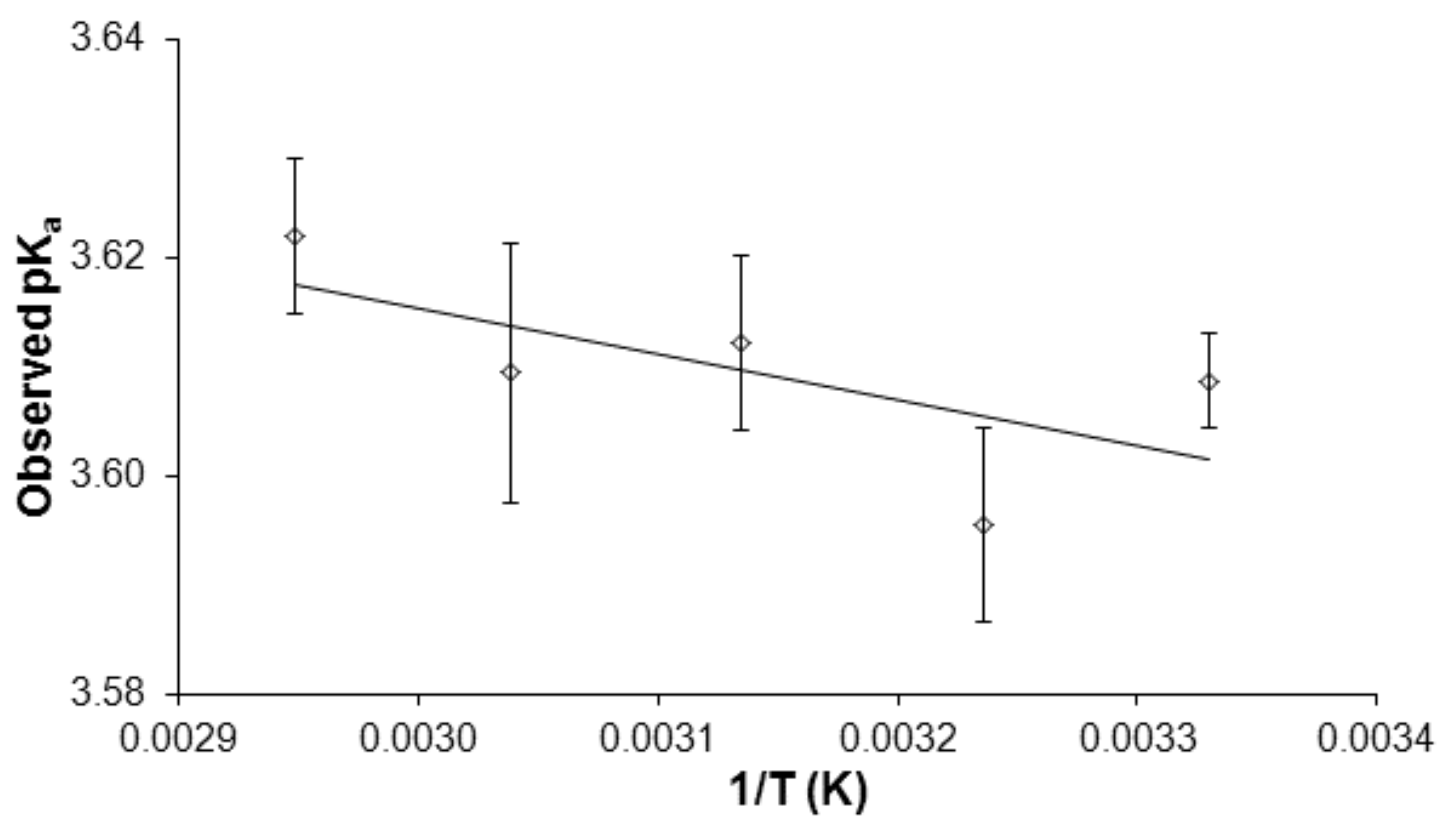

Figure 2.3- The conditional equilibrium constants obtained from the potentiometric titrations of lactic acid at $25-65^{\circ} \mathrm{C}$ at ionic strength of $2.0 \mathrm{M} \mathrm{NaCl}$. 
Therefore, if a series of equilibrium constants at various temperatures are obtained, they can be plotted as $\ln (K)$ vs $1 / T$ and the data will fall on a line whose slope is equal to $-\Delta H^{\circ} / R$. The symbol $\Delta \mathrm{H}^{\circ}$ signifies the standard enthalpy of the reaction. The Van't Hoff plot of the data obtained in the potentiometric titrations of Lactic acid are shown in Figure 2.4. Using the slope from Figure 2.4 and the Van't Hoff Equation, the standard enthalpy for this reaction was calculated with the result shown in Table 2.10.

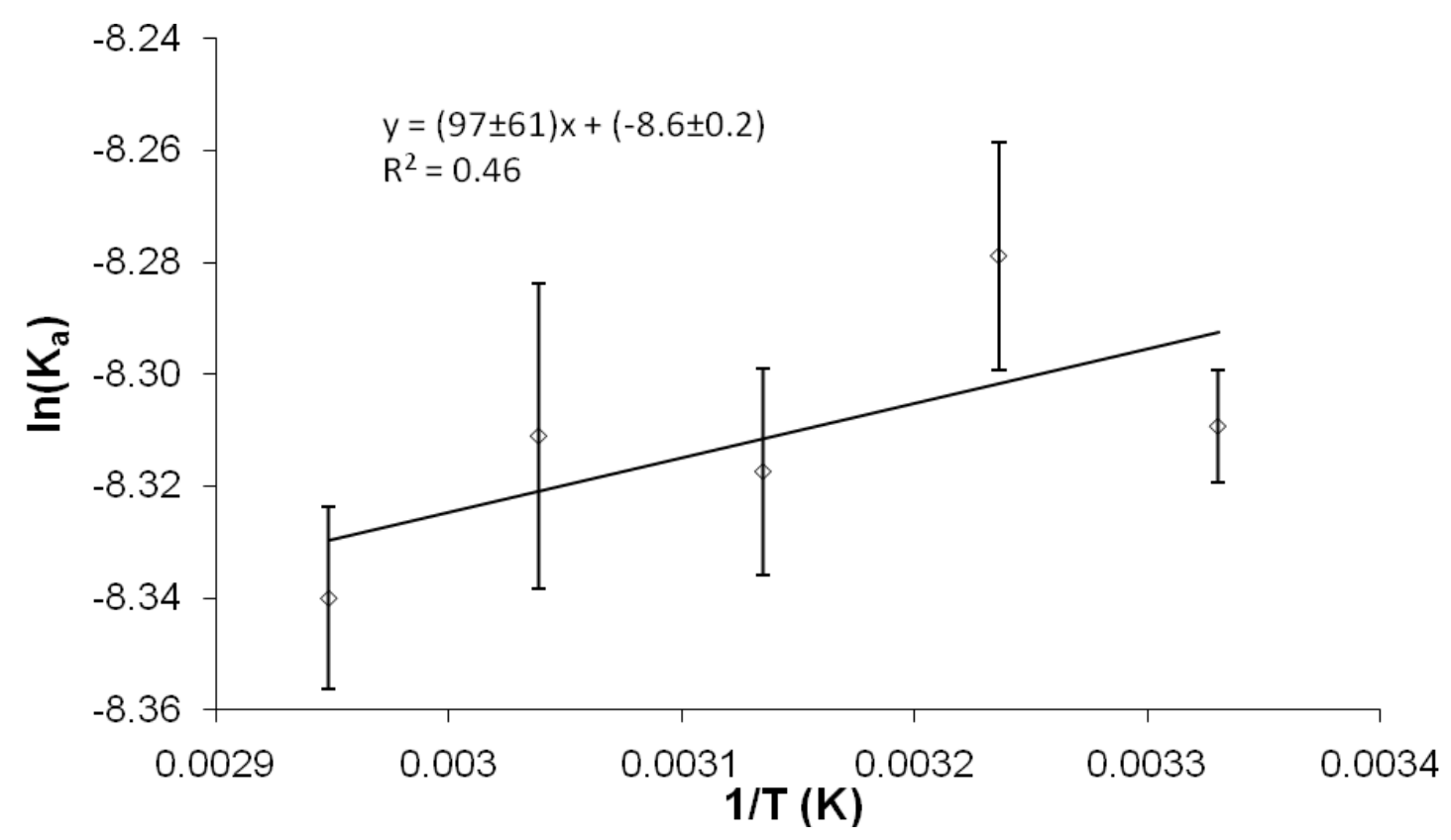

Figure 2.4- Van't Hoff plot of the acid dissociation constant results for lactic acid titrations at 25$65^{\circ} \mathrm{C}$ at ionic strength of $2.0 \mathrm{M} \mathrm{NaCl}$.

The values for the conditional $\mathrm{pK}_{\mathrm{a}}$ of lactic acid are lower than the IUPAC recommended data for $\mathrm{I}=2.0 \mathrm{M}$ and $25^{\circ} \mathrm{C}$. The 3.80 recommended by IUPAC vs. the $3.6 \pm 0.1$ measured in our experiment shows a small but significant difference. The standard enthalpy of reaction 
calculated for lactate deprotonation indicates that temperature has a small effect on the $\mathrm{pK}_{\mathrm{a}}$ at I $=2.0 \mathrm{M} \mathrm{NaCl}$.

This result is consistent with published data measured for poly-carboxylic acids by De Stefano et al. ${ }^{[101]}$ In De Stefano et al.'s work, the measured enthalpy of the first protonation of malonic, propanetricarboxylic, citric, and butanetetracarboxylic acids show that with increasing ionic strength the enthalpy of protonation decreases. ${ }^{[102]}$ De Stefano et al. reports that for malonic, propanetricarboxylic, and citric acids the reaction enthalpy changes from positive to negative enthalpy near I $=2.0 \mathrm{M} \mathrm{NaCl}$. Thus, our result showing an enthalpy near zero $(-0.8 \pm 0.1$ $\mathrm{kJ} / \mathrm{mol}$ ) at $2.0 \mathrm{M} \mathrm{NaCl}$ for the lactic acid is consistent with the behavior of other carboxylic acids of similar molecular weights and functionalities. While the data point at $2.0 \mathrm{M} \mathrm{NaCl}$ is consistent, the trend in the deprotonation enthalpy for lactic acid needs to be measured over a range of ionic strengths before it can be compared to the literature data.

Table 2.10-Standard enthalpy of reaction obtained for lactic acid potentiometric titrations at $25-65^{\circ} \mathrm{C}$ at ionic strength of $2.0 \mathrm{M} \mathrm{NaCl}$.

\begin{tabular}{|c|c|}
\hline $\begin{array}{c}\mathbf{I} \\
(\mathrm{mol} / \mathrm{L})\end{array}$ & $\begin{array}{c}\Delta \mathbf{H}^{\circ} \\
(\mathrm{KJ} / \mathrm{mol})\end{array}$ \\
\hline $2.0 \mathrm{M}(\mathrm{NaCl})$ & $-0.8 \pm 0.1$ \\
\hline
\end{tabular}

\subsection{Results and Discussion: $\mathrm{HCl}$ Aqueous Media}

\subsubsection{HA Aggregation Results}

HA exists predominantly in dimeric form in non-polar diluents, ${ }^{[103]}$ however, the aggregation will change upon complexation with a metal. Experiments performed on both unwashed and washed HA in isooctane showed that $\mathrm{HA}$ was present exclusively in its dimeric form $\left(\mathrm{N}_{\mathrm{agg}}=2.03\right.$ 
$\pm 0.03,2.02 \pm 0.03$, respectively). The $\mathrm{pH}(1,4)+0 \mathrm{mM} \mathrm{Nd}+\mathrm{HCl}$ systems also showed an average aggregation number of $2.03 \pm 0.03$ and $1.99 \pm 0.03$. The aggregation increased in the $\mathrm{pH}$ $(1,4)+30 \mathrm{mM} \mathrm{Nd}+\mathrm{HCl}$ systems with values of $2.45 \pm 0.04$ and $2.54 \pm 0.04$ respectively. The increase in aggregation for these systems is a result of the extraction mechanism shown in equation (2.7) that indicates that for every $\mathrm{Nd}^{3+}$ extracted there are two to three dimerized $\mathrm{HA}$ per metal center. There was a small portion of the $\mathrm{pH} 4+30 \mathrm{mM} \mathrm{Nd}+\mathrm{HCl}$ system at the lowest $[\mathrm{HA}]_{\text {org }}(0.025-0.05 \mathrm{M})$ that showed a non-linearity in the instrument response curve (see Figure 2.5) that was consistent with the formation of large aggregation seen in other systems with similar aggregation present (see Figure 2.14). Because of the data indicating high aggregation formation was only two to three data points, the best result that can be calculated is a lower bound of the average aggregation in this region. The lower bound of the average aggregation was approximated using a 3-point slope of the instrument response data centered on the 0.025 M HA data point showed an elevated aggregation of $4.0 \pm 0.5$. This aggregation was not unexpected due to the high loading of the organic phase at these low $[\mathrm{HA}]_{\mathrm{org}}$. This result is consistent with the formation of $\left(\mathrm{NdA}_{3}\right)_{\mathrm{x}}$ for a small fraction of the total extracted $\mathrm{Nd}^{3+}$ that was observed in the FTIR data.

When the aggregation results were modeled according to the method described in section 2.2.14, the $Z_{\mathrm{NdAz}}$ was calculated $\left(Z_{\mathrm{NdAz}}=2 \mathrm{n}\right)$. The calculation averaging both $\mathrm{pH}(1,4)+30 \mathrm{mM}$ $\mathrm{Nd}+\mathrm{HCl}$ systems showed that the observed $Z_{\mathrm{NdAz}}$ is $4.0 \pm 0.4$. This value is lower than $\mathrm{n}=2.5$ (or $Z_{\mathrm{NdAZ}}=5$ ) reported in the literature for aliphatic diluents ${ }^{[57]}$ under low loading of $\mathrm{HA}$ and indicates a 4:1 stoichiometry for $\mathrm{HA}: \mathrm{Nd}^{3+}$ complex under moderately high extractant loading. The lower stoichiometry observed through the aggregation modeling indicates that there are only $4 \mathrm{HA}$ molecules per $\mathrm{Nd}^{3+}$ in the organic phase. This can indicate that 3 deprotonated and 1 protonated HA molecules are present, or that 2 deprotonated and 2 protonated HA molecules 
are present with a co-extracted $\mathrm{Cl}^{-}$to counterbalance the charge. It is impossible to judge between these possibilities at this time, however, future plans for this research include quantification of the $\mathrm{Cl}^{-}$distribution in this system that could resolve this question.

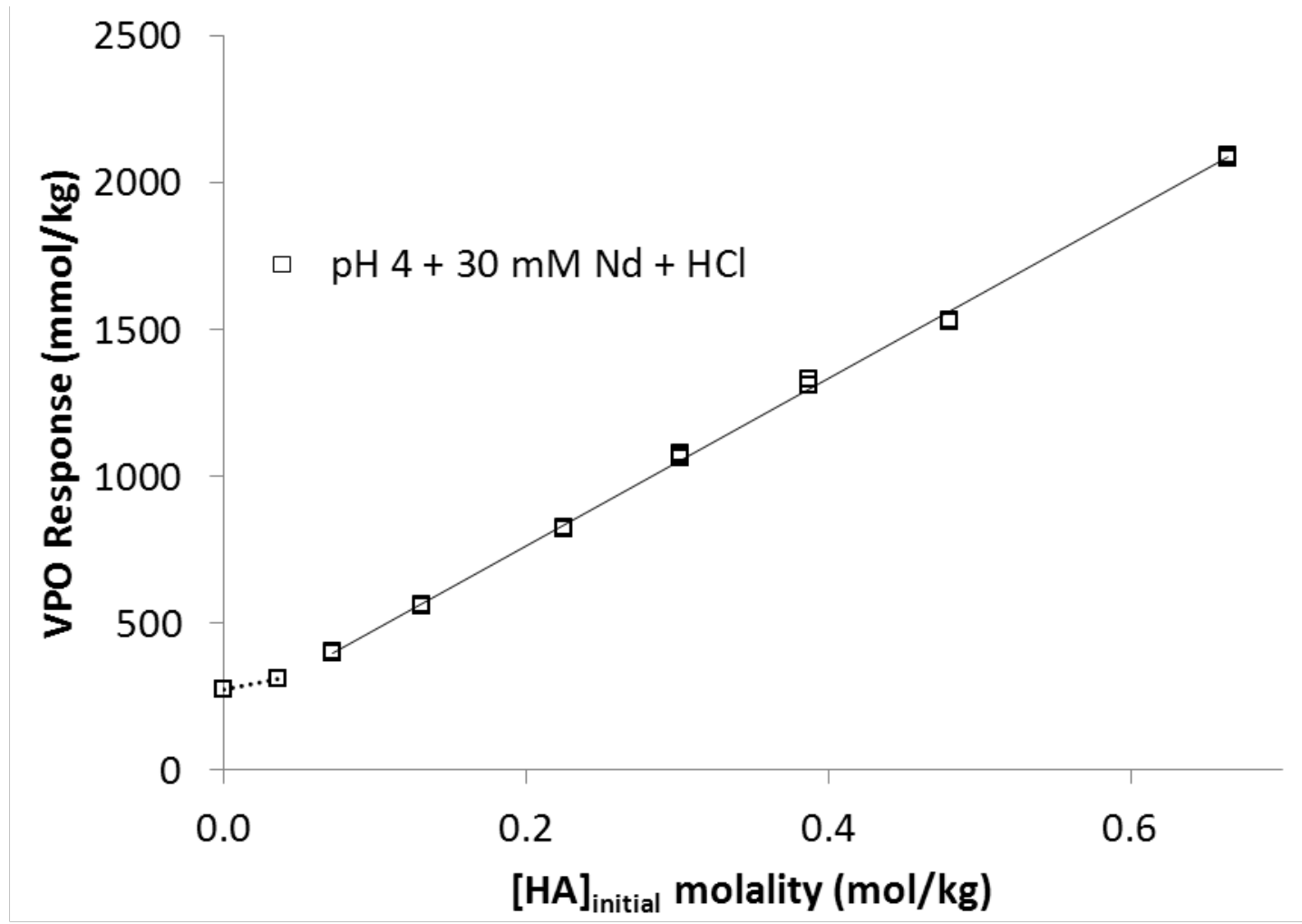

Figure 2.5- Instrument response curve for the $\mathrm{pH} 4+0 \mathrm{mM} \mathrm{Nd}+\mathrm{HCl}$ system. At low $[\mathrm{HA}]_{\text {initial }}$ there is a small portion of the data that shows a different slope, indicating increased aggregation.

\subsubsection{Liquid-Liquid Distribution Measurements}

Overall results for the liquid-liquid distribution measurements are shown in Table 2.11 and will be discussed in the upcoming sections. 


\subsubsection{1 $\underline{\mathrm{Nd}}^{3+} \underline{\text { Distribution Results }}$}

Figure 2.6 shows the effect of initial HA concentration on the organic equilibrium $\mathrm{Nd}^{3+}$ concentration. At initial $\mathrm{pH}$ of $1, \mathrm{Nd}^{3+}$ extraction gradually increased with increasing $\mathrm{HA}$ concentration. As observed from the data in Table 2.11, there is a concurrent decrease in the $\mathrm{pH}$ from the initial pre-contact to equilibrium post-contact values supports the ion exchange extraction mechanism as described by equation (2.7). Increase of initial aqueous $\mathrm{pH}$ from 1 to 4 facilitated $\mathrm{HA}$ ionization and thus $\mathrm{Nd}^{3+}$ extraction. For the $\mathrm{pH} 4+30 \mathrm{mM} \mathrm{Nd}+\mathrm{HCl}$ system, the concurrent aqueous $\mathrm{pH}$ change upon phase equilibration was significant and also consistent with the equation (2.7) $(\mathrm{pH}=4.31$ and 0.94 for pre- and post-equilibration, respectively in the 1 M HA isooctane solution. See Table 2.12).

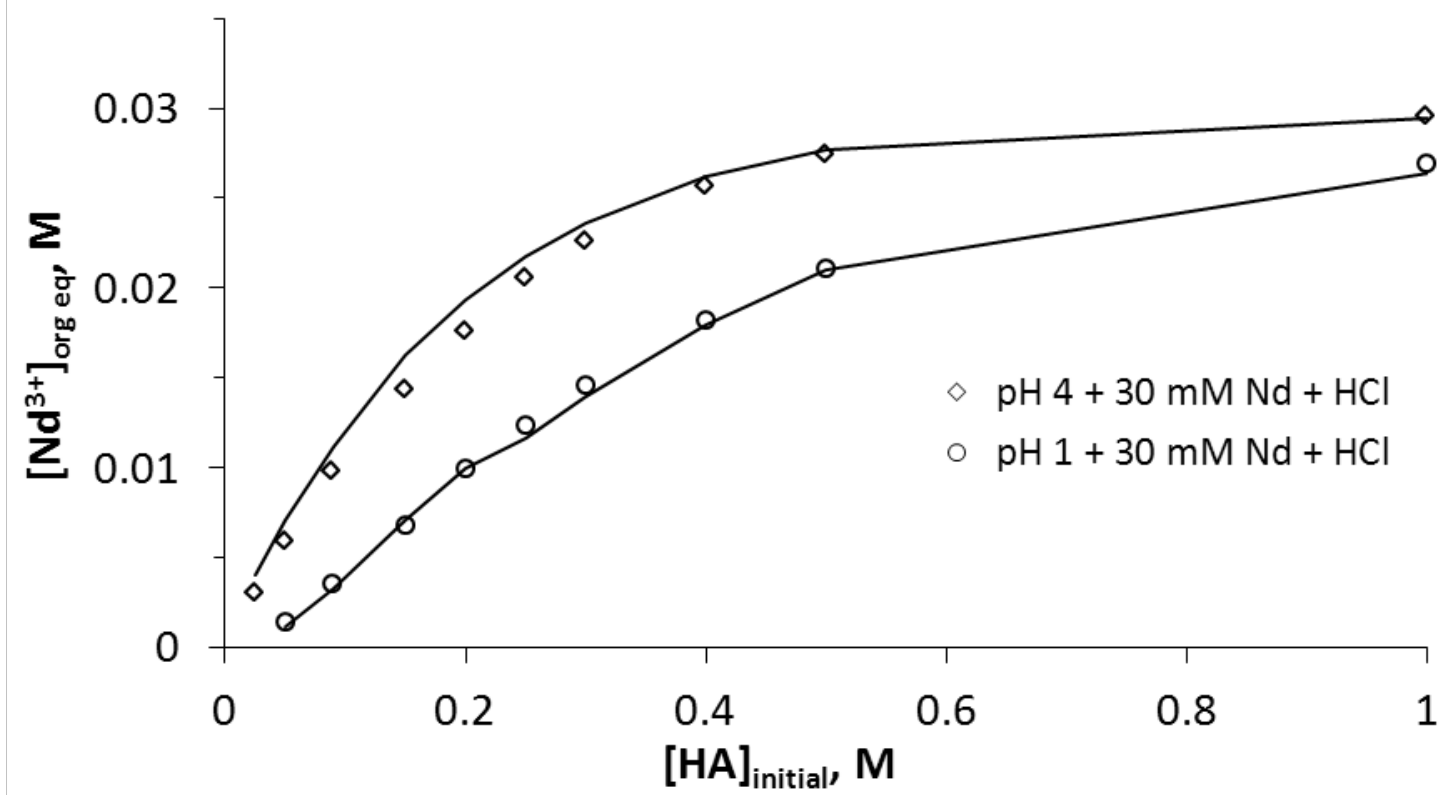

Figure 2.6- Calculated by SXLSQI (line) and observed (symbols) organic $\mathrm{Nd}^{3+}$ concentration as a function of initial HA molarity. 
Table 2.11- Equilibrium $\mathrm{pH},\left[\mathrm{Na}^{+}\right]_{\mathrm{org}}$, and $\left[\mathrm{Nd}^{3+}\right]_{\text {org }}$ results for the $\mathrm{pH} 1+(0,30) \mathrm{mM} \mathrm{Nd}+\mathrm{HCl}$ systems. Estimated error for these values is: $\mathrm{pH}_{\text {eq }} \pm 0.01$, $\left[\mathrm{Nd}^{3+}\right]_{\text {org }} \pm 2 \%$.

\begin{tabular}{|c|c|c|c|c|c|}
\hline \multirow[b]{2}{*}{ [HA] } & \multicolumn{2}{|c|}{$\begin{array}{c}\mathrm{pH} 1+0 \mathrm{mM} \mathrm{Nd}+ \\
\mathrm{HCl}\end{array}$} & \multicolumn{3}{|c|}{$\mathrm{pH} 1+30 \mathrm{mM} \mathrm{Nd}+\mathrm{HCl}$} \\
\hline & $\mathrm{pH}_{\mathrm{eq}}$ & {$[\mathrm{Na}]_{\mathrm{org}, \mathrm{eq}} / \mathrm{M}$} & $\mathrm{pH}_{\mathrm{eq}}$ & {$[\mathrm{Na}]_{\mathrm{org}, \mathrm{eq}} / \mathrm{M}$} & {$[\mathrm{Nd}]_{\mathrm{org}, \mathrm{eq}} / \mathrm{M}$} \\
\hline 0 & 0.93 & \multirow{10}{*}{$B D$} & 0.94 & \multirow{10}{*}{$B D$} & \\
\hline 0.05 & 0.93 & & 0.92 & & 0.0014 \\
\hline 0.09 & 0.93 & & 0.89 & & 0.0035 \\
\hline 0.15 & 0.93 & & 0.86 & & 0.0068 \\
\hline 0.2 & 0.93 & & 0.83 & & 0.0099 \\
\hline 0.25 & 0.93 & & 0.80 & & 0.0123 \\
\hline 0.3 & 0.93 & & 0.78 & & 0.0146 \\
\hline 0.4 & 0.93 & & 0.75 & & 0.0182 \\
\hline 0.5 & 0.93 & & 0.73 & & 0.0211 \\
\hline 1 & 0.92 & & 0.63 & & 0.0269 \\
\hline
\end{tabular}

$\mathrm{BD}=$ Below detection

Table 2.12- Equilibrium $\mathrm{pH},\left[\mathrm{Na}^{+}\right]_{\text {org }}$, and $\left[\mathrm{Nd}^{3+}\right]_{\text {org }}$ results for the $\mathrm{pH} 4+(0,30) \mathrm{mM} \mathrm{Nd}+\mathrm{HCl}$ systems. Estimated error for these values is: $\mathrm{pH}_{\text {eq }} \pm 0.01,\left[\mathrm{Nd}^{3+}\right]_{\text {org }} \pm 2 \%$.

\begin{tabular}{|c|c|c|c|c|c|}
\hline \multirow[b]{2}{*}{ [HA] } & \multicolumn{2}{|c|}{$\mathrm{pH} 4+0 \mathrm{mM} \mathrm{Nd}+\mathrm{HCl}$} & \multicolumn{3}{|c|}{$\mathrm{pH} 4+30 \mathrm{mM} \mathrm{Nd}+\mathrm{HCl}$} \\
\hline & $\mathrm{pH}_{\mathrm{eq}}$ & {$[\mathrm{Na}]_{\text {org,eq }} / \mathrm{M}$} & $\mathrm{pH}_{\mathrm{eq}}$ & {$[\mathrm{Na}]_{\text {org,eq }} / \mathrm{M}$} & {$[\mathrm{Nd}]_{\mathrm{org}, \mathrm{eq}} / \mathrm{M}$} \\
\hline 0 & 4.08 & $\mathrm{BD}$ & 4.31 & \multirow{11}{*}{ BD } & \\
\hline 0.025 & & & 1.91 & & 0.0031 \\
\hline 0.05 & 3.17 & $0.0005 \pm 0.0002$ & 1.63 & & 0.0059 \\
\hline 0.09 & 2.85 & $0.0008 \pm 0.0003$ & 1.41 & & 0.0098 \\
\hline 0.15 & 2.65 & $0.0014 \pm 0.0004$ & 1.25 & & 0.0144 \\
\hline 0.2 & 2.53 & $0.0020 \pm 0.0005$ & 1.16 & & 0.0176 \\
\hline 0.25 & 2.43 & $0.0025 \pm 0.0006$ & 1.10 & & 0.0206 \\
\hline 0.3 & 2.37 & $0.0031 \pm 0.0006$ & 1.06 & & 0.0226 \\
\hline 0.4 & 2.27 & $0.0044 \pm 0.0007$ & 1.01 & & 0.0257 \\
\hline 0.5 & 2.19 & $0.0056 \pm 0.0009$ & 0.98 & & 0.0275 \\
\hline 1 & 1.95 & $0.011 \pm 0.001$ & 0.94 & & 0.0296 \\
\hline
\end{tabular}

$\mathrm{BD}=$ Below detection 
The logarithmic value of the equilibrium constant $K_{\mathrm{Nd}}$ corresponding to equation (2.7) were determined using SXLSQI model by fitting the $\mathrm{Nd}^{3+}$ distribution results collected for the $\mathrm{pH}(1,4)$ $+30 \mathrm{mM} \mathrm{Nd}+\mathrm{HCl}$ systems simultaneously. The composition of the product species $\mathrm{Nd}(\mathrm{A})_{3}(\mathrm{HA})_{\text {(org) }}$ was deduced using the average aggregation numbers (VPO data), total HA and $\mathrm{Nd}^{3+}$ concentrations in the equilibrium organic phases for these two extraction systems. Based upon the assumption that in these systems HA exists in two forms: 1) a free dimer and 2) within a Nd complex, the analysis of these data indicated that the latter contained $4(4.0 \pm 0.4)$ extractant molecules. The equilibrium constant or $\log K_{\mathrm{Nd}}$ obtained for these systems was -0.14 \pm 0.06 .

\subsubsection{2 $\underline{\mathrm{Na}}^{ \pm} \underline{\text { Distribution Results }}$}

No $\mathrm{Na}^{+}$extraction was observed in the $\mathrm{pH} 1+(0,30) \mathrm{mM} \mathrm{Nd}+\mathrm{HCl}$ system or in the $\mathrm{pH} 4+$ $30 \mathrm{mM} \mathrm{Nd}+\mathrm{HCl}$ system (see Table 2.11). In the $\mathrm{pH} 4+0 \mathrm{mM}$ Nd system, $\mathrm{Na}^{+}$extraction was observed with approximately $1 \%$ of the HA converted to NaA. Log-slope analysis of the extraction mechanism (shown in Figure 2.7) shows a slope dependence of $0.99 \pm 0.02\left(R^{2}=\right.$ 0.997) indicating 1:1 stoichiometry between $\mathrm{Na}^{+}$and $\mathrm{HA}$ (see data in Table 2.12). The extraction of $\mathrm{Na}^{+}$in the $\mathrm{pH} 4+0 \mathrm{mM} \mathrm{Nd}+\mathrm{HCl}$ system was modeled by the SXLSQI program as described in section 2.2.10. The modeling results showed an equilibrium constant or Log $\mathrm{K}_{\mathrm{Na}}$ of $-0.989 \pm$ 0.009 for the reaction shown in equation (2.10) where $n=1$.

\subsubsection{3 $\underline{\mathrm{H}}_{2}$ O Distribution Results}

When contacted with water there is no measurable water extracted by pure isooctane. However, when HA is dissolved in isooctane, it does extract water upon contact. Karl Fischer titrations results are shown in Table 2.13. When the data obtained for the other systems [pH ( 1 , 4) $+(0,30) \mathrm{mM} \mathrm{Nd}+\mathrm{HCl}]$ is compared with that of the washed solvent it is notable that the organic phase water concentration observed under all systems is equal to that of the washed 
solvent within experimental error. This indicates that $\mathrm{Nd}^{3+}$ and $\mathrm{Na}^{+}$are extracted as dehydrated ions into the organic phase. Other lanthanides have also been shown to extract by HA as

dehydrated ions. ${ }^{[22-83]}$ Past studies with $\mathrm{Na}^{+}$extraction have shown that little water is extracted with $\mathrm{Na}^{+}$until the percent conversion of $\mathrm{HA}$ to $\mathrm{NaA}$ is above $10 \% .{ }^{[73]}$ In these experiments the conversion of $\mathrm{HA}$ to $\mathrm{NaA}$ did not exceed more than $2 \%$ (see results for $\mathrm{pH} 4+0 \mathrm{mM} \mathrm{Nd}+\mathrm{HCl}$ system in Table 2.11). A log-slope comparison of the washed solvent and the $\mathrm{pH} 4+0 \mathrm{mM} \mathrm{Nd}+$ $\mathrm{HCl}$ system is shown in Figure 2.8 shows matching slopes that is consistent with slight excess water extraction with $\mathrm{Na}^{+}$.

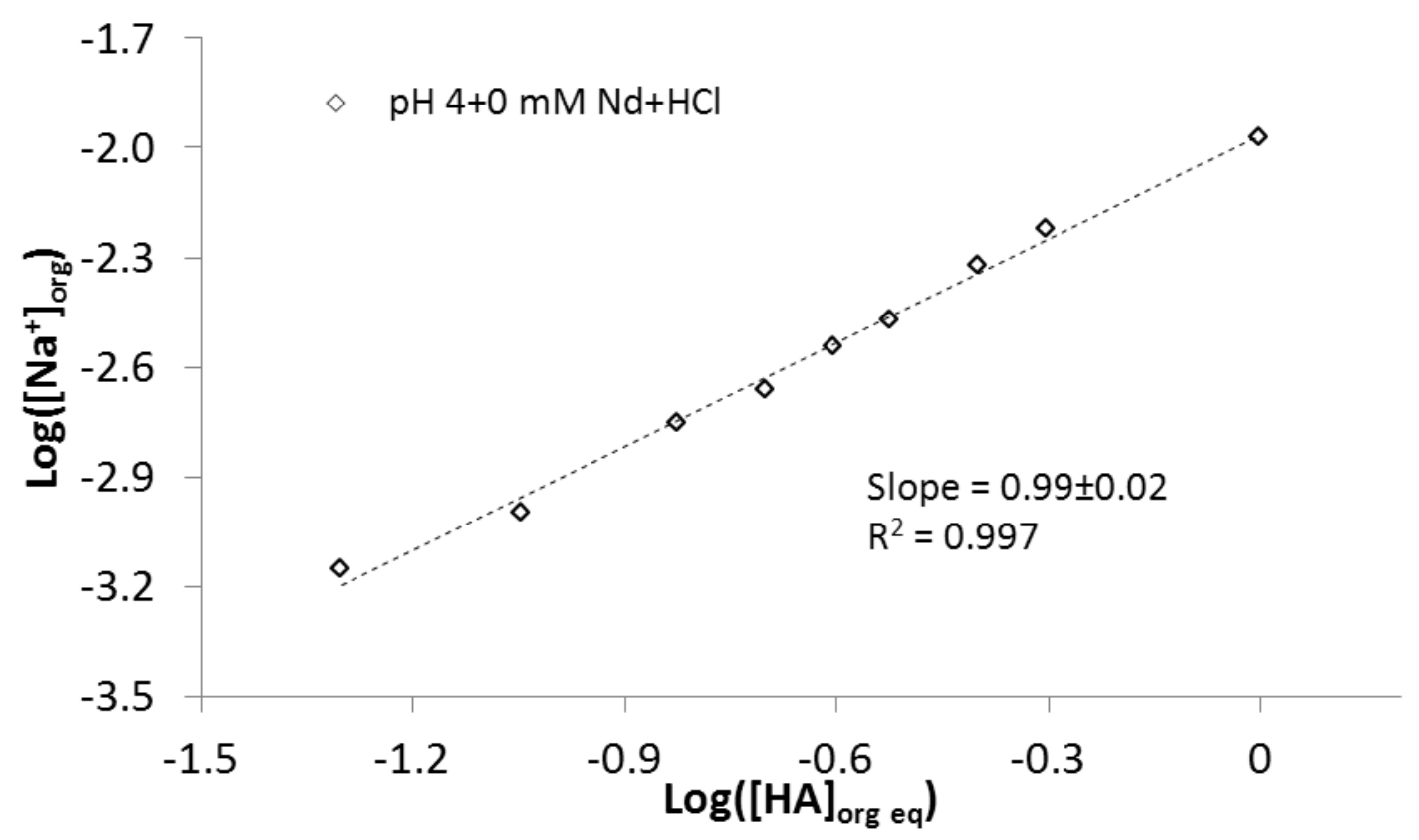

Figure 2.7- Log-slope analysis of $\mathrm{Na}^{+}$Extraction in the $\mathrm{pH} 4+0 \mathrm{mM} \mathrm{Nd}+\mathrm{HCl}$ system. 
Table 2.13- Organic phase water concentration for the $\mathrm{pH}(1,4)+(0,30) \mathrm{mM} \mathrm{Nd}+\mathrm{HCl}$ systems.

\begin{tabular}{|c|c|c|c|c|c|}
\hline \multirow[b]{3}{*}[\mathrm{HA}]{$_{\text {org }} / \mathrm{M}$} & \multirow{3}{*}{$\begin{array}{c}\text { Washed Solvent } \\
{\left[\mathrm{H}_{2} \mathrm{O}\right]_{\text {org }} / \mathrm{M}}\end{array}$} & \multicolumn{2}{|c|}{$\mathrm{pH} 1+\mathrm{HCl}$} & \multicolumn{2}{|c|}{$\mathrm{pH} 4+\mathrm{HCl}$} \\
\hline & & $0 \mathrm{mM} \mathrm{Nd}$ & $30 \mathrm{mM} \mathrm{Nd}$ & $0 \mathrm{mM} \mathrm{Nd}$ & $30 \mathrm{mM} \mathrm{Nd}$ \\
\hline & & {$\left[\mathrm{H}_{2} \mathrm{O}\right]_{\text {org }} / \mathrm{M}$} & {$\left[\mathrm{H}_{2} \mathrm{O}\right]_{\text {org }} / \mathrm{M}$} & {$\left[\mathrm{H}_{2} \mathrm{O}\right]_{\text {org }} / \mathrm{M}$} & {$\left[\mathrm{H}_{2} \mathrm{O}\right]_{\text {org }} / \mathrm{M}$} \\
\hline 0 & $0.000 \pm 0.003$ & $0.005 \pm 0.003$ & $0.003 \pm 0.003$ & $0.002 \pm 0.003$ & $0.004 \pm 0.003$ \\
\hline 0.05 & $0.003 \pm 0.003$ & $0.005 \pm 0.003$ & $0.005 \pm 0.003$ & $0.003 \pm 0.003$ & $0.004 \pm 0.003$ \\
\hline 0.09 & $0.007 \pm 0.003$ & $0.007 \pm 0.003$ & $0.006 \pm 0.003$ & $0.004 \pm 0.003$ & $0.007 \pm 0.003$ \\
\hline 0.15 & $0.010 \pm 0.003$ & $0.010 \pm 0.003$ & $0.012 \pm 0.003$ & $0.010 \pm 0.003$ & $0.009 \pm 0.003$ \\
\hline 0.2 & $0.015 \pm 0.003$ & $0.017 \pm 0.003$ & $0.017 \pm 0.003$ & $0.017 \pm 0.003$ & $0.014 \pm 0.003$ \\
\hline 0.25 & $0.023 \pm 0.003$ & $0.022 \pm 0.003$ & $0.020 \pm 0.003$ & $0.021 \pm 0.003$ & $0.020 \pm 0.003$ \\
\hline 0.3 & $0.029 \pm 0.005$ & $0.035 \pm 0.006$ & $0.027 \pm 0.005$ & $0.037 \pm 0.006$ & $0.025 \pm 0.006$ \\
\hline 0.4 & $0.053 \pm 0.007$ & $0.051 \pm 0.008$ & $0.046 \pm 0.007$ & $0.057 \pm 0.008$ & $0.049 \pm 0.006$ \\
\hline 0.5 & $0.07 \pm 0.01$ & $0.08 \pm 0.01$ & $0.07 \pm 0.01$ & $0.08 \pm 0.01$ & $0.07 \pm 0.01$ \\
\hline 1 & $0.21 \pm 0.03$ & $0.20 \pm 0.04$ & $0.20 \pm 0.03$ & $0.28 \pm 0.04$ & $0.17 \pm 0.02$ \\
\hline
\end{tabular}

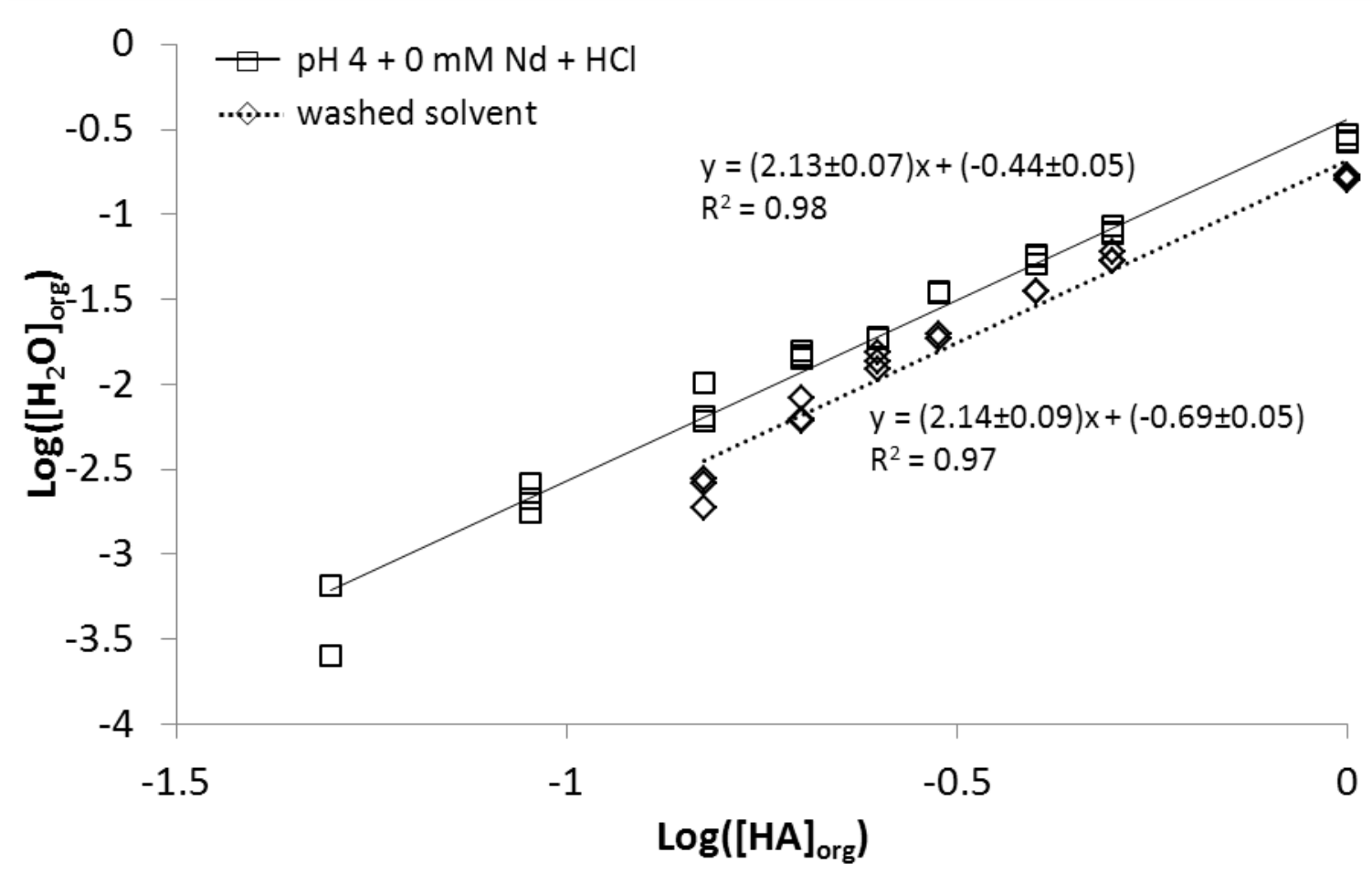

Figure 2.8- Log-slope analysis of water in the washed solvent and the $\mathrm{pH} 4+0 \mathrm{mM} \mathrm{Nd}+\mathrm{HCl}$ system. The matching slopes indicate that no water was co-extracted with $\mathrm{Na}^{+}$in the $\mathrm{pH} 4+0$ $\mathrm{mM} \mathrm{Nd}+\mathrm{HCl}$ system. 


\subsubsection{Visible Spectroscopy}

\subsubsection{Aqueous Phase}

The molar absorptivity observed for the aqueous phase showed numerous absorbance bands for the $\mathrm{Nd}^{3+}$ ion in systems studied. In the observed window $(500-881 \mathrm{~nm})$ the absorbance bands with an extinction coefficient at least $1.0 \mathrm{~L} \mathrm{~mol}^{-1} \mathrm{~cm}^{-1}$ are $511,521,575,732$, 740, 794, 801 and $865 \mathrm{~nm}$ (See trace "pH $4+30 \mathrm{mM} \mathrm{Nd}=\mathrm{HCl}(\mathrm{Aq})$ " in Figure 2.9). These bands are also listed in Table 2.14. The spectrum shown is representative of $\mathrm{pH}(1,4)+30 \mathrm{mM} \mathrm{Nd}+\mathrm{HCl}$ systems and was not changed between $\mathrm{pH} 1$ and $\mathrm{pH} 4$ systems. A comparison of the organic phase (containing $\mathrm{HA}$ ) and aqueous phase $\mathrm{Nd}^{3+}$ spectra for $\mathrm{pH} 4+30 \mathrm{mM} \mathrm{Nd}+\mathrm{HCl}$ are shown in Figure 2.9.

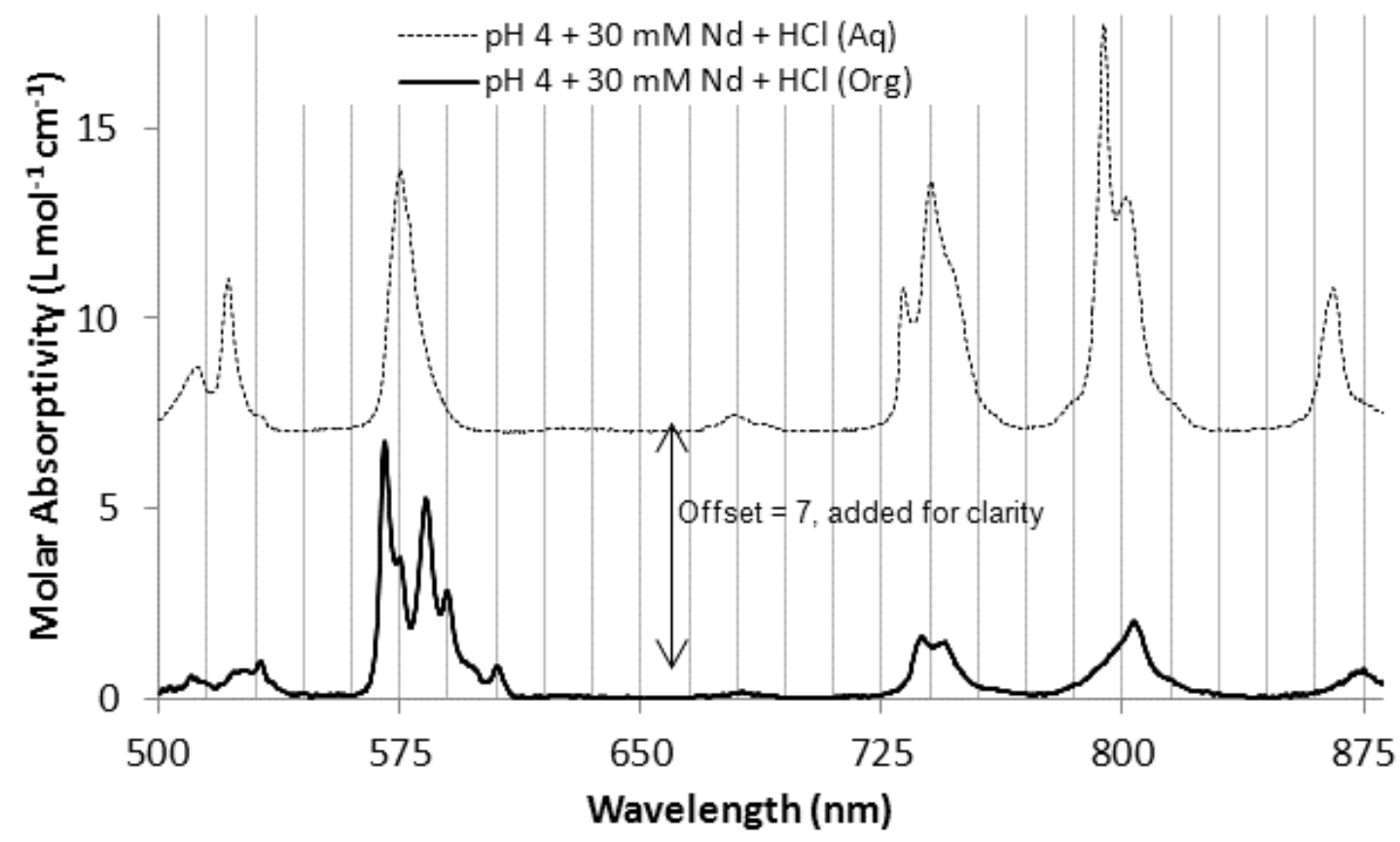

Figure 2.9- A comparison of the absorbance spectra of the $\mathrm{Nd}^{3+}$ ion in the aqueous and organic phases. The HA concentration for the organic solution shown is $0.2 \mathrm{M}$. 
Table 2.14- Prominent bands of the molar absorptivity of $\mathrm{Nd}^{3+}$ in the aqueous and organic phase of the $\mathrm{HCl}$ systems. Bands that found in the shoulder of other bands without a prominent maximum are shown in parentheses.

\begin{tabular}{|c|c|}
\hline $\begin{array}{c}\mathrm{HCl} \text { System } \\
\text { Aqueous Phase } \\
\text { (nm) }\end{array}$ & $\begin{array}{c}\mathrm{HCl} \text { System } \\
\text { Organic Phase } \\
\text { (nm) }\end{array}$ \\
\hline 511 & 512 \\
\hline 521 & 524 \\
\hline & 571 \\
\hline 575 & 575 \\
& 583 \\
& 588 \\
& 604 \\
\hline 732 & 738 \\
\hline 740 & 745 \\
\hline$(745)$ & $(794)$ \\
\hline 794 & 802 \\
\hline 801 & 874 \\
\hline 865 & \\
\hline
\end{tabular}

\subsubsection{Organic Phase}

The absorbance bands in the organic phase show band shifts and band splits due to different speciation (See Figure 2.9). The 511 and $521 \mathrm{~nm}$ aqueous absorbance bands were essentially unchanged in position, but are lower in intensity, appearing at 512 and $524 \mathrm{~nm}$ in the organic absorbance spectrum respectively. The $575 \mathrm{~nm}$ aqueous band appears to have split into 5 different bands at 571,575, 583, 588, and $604 \mathrm{~nm}$. The $732 \mathrm{~nm}$ aqueous band appears to disappear in the organic phase. The $740 \mathrm{~nm}$ band plus its shoulder ( 745 nm) in the aqueous spectrum appear to have resolved into bands at 738 and $745 \mathrm{~nm}$ in the organic absorbance spectrum, remaining relatively unchanged. The $794 \mathrm{~nm}$ aqueous band appears to decrease in organic phase spectrum until it is lost in the shoulder of the $802 \mathrm{~nm}$, which is unchanged from 
the aqueous to the organic phase spectrum. Finally, the $865 \mathrm{~nm}$ aqueous band shifted to an 874 nm organic band.

Hypersensitive bands in the $\mathrm{Nd}^{3+}$ organic phase visible spectrum changes with shifts in the $\mathrm{Nd}^{3+}$ speciation. The molar absorptivity spectra (see Figure 2.10 Plots $\mathrm{A}$ and $\mathrm{B}$ ) demonstrate that many of the bands show some little change, but the $570 \mathrm{~nm}$ band has the largest absorbance change in the ${ }^{4} \mathrm{I}_{9 / 2} \rightarrow{ }^{4} \mathrm{G}_{5 / 2},{ }^{2} \mathrm{G}_{7 / 2}$ electronic transition. ${ }^{[104]}$

The absence of the shift of the position of the spectral bands with HA concentration indicates the presence of a single $\mathrm{Nd}^{3+}$ complex species, namely $\mathrm{NdA}_{3}(\mathrm{HA})$ in the organic phase. The modulation of the molar absorptivity value that is especially pronounced for the $571 \mathrm{~nm}$ band can be attributed to the change of organic solvent properties with increased HA concentration. It has been demonstrated that the organic spectrum of $\mathrm{Nd}^{3+}$ provides information about $\mathrm{Nd}^{3+}-\mathrm{HA}$ speciation. ${ }^{[64]}$ In this past cited work, the changes in the absorbance spectrum are associated with a speciation shift of $\mathrm{Nd}^{3+}-\mathrm{HA}$ from the $\mathrm{NdA}_{3}$ or $\mathrm{NdA}_{3}(\mathrm{HA})_{2}$ that form at low loading, to $\mathrm{Nd}_{2}(A)_{6}$ that forms at high loading. The data presented here shows that the change observed in the $570 \mathrm{~nm}$ band did not correlate with the organic phase $\mathrm{Nd}^{3+}$ loading at $\mathrm{pH}$ 1, but it did at $\mathrm{pH} 4$ (see Figure 2.11). The molar absorptivity decreases with increasing $[\mathrm{HA}]_{\text {org }}$ for both $\mathrm{pH}(1,4)+30 \mathrm{mM} \mathrm{Nd}+\mathrm{HCl}$ systems. The data are consistent with the conclusion that the change in the $570 \mathrm{~nm}$ band appears to show rearrangement of ligands in the coordination sphere of $\mathrm{Nd}^{3+}$, but the changes in the $570 \mathrm{~nm}$ band correlate to the overall $[\mathrm{HA}]_{\text {org }}$ and not to theoretical $\mathrm{Nd}^{3+}$ loading. 

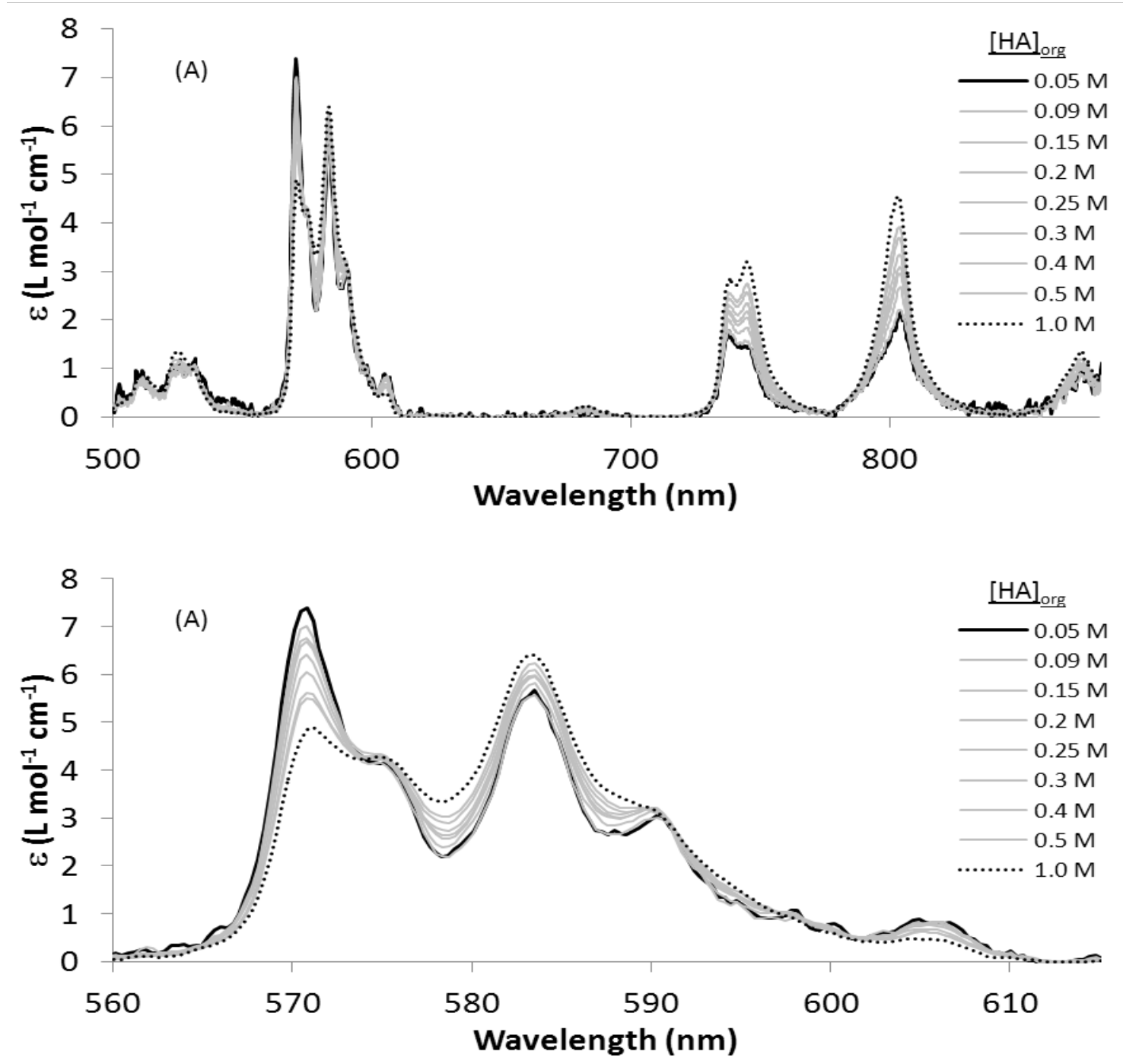

Figure 2.10- Organic phase molar absorptivity from the $\mathrm{pH} 4+30 \mathrm{mM} \mathrm{Nd}+\mathrm{HCl}$ system. Plot (B) shows a more detailed view of Plot (A) from 560-615 nm.

The spectral profile of the $\mathrm{Nd}^{3+}$ in the organic phase did offer some information regarding the coordination sphere surrounding the metal center. For the multiple band spectral profile with peaks at $570,575,583,588$, and $604 \mathrm{~nm}$ in the $560-620 \mathrm{~nm}$ window, the $\mathrm{Nd}^{3+}$ complex present in our systems showed the greatest similarity with and is practically identical to, the spectra observed for $\mathrm{Nd}(\mathrm{AHA})_{3}$ complex for both $\mathrm{Nd}-\mathrm{Cyanex} 272$ and $\mathrm{Nd}-\mathrm{HA}$ previously published. ${ }^{[64]}$ The spectra published for $\mathrm{NdA}_{3}$ complex is different with only one absorbance 
band at $585 \mathrm{~nm}$ and a shoulder band at $577 \mathrm{~nm} .{ }^{[64,69]}$ The shape of the spectrum in the $560-620$ $\mathrm{nm}$ window is consistent with the low $\mathrm{Nd}^{3+}$ loading regime where dimeric $\mathrm{HA}$ surround the $\mathrm{Nd}^{3+}$ center.

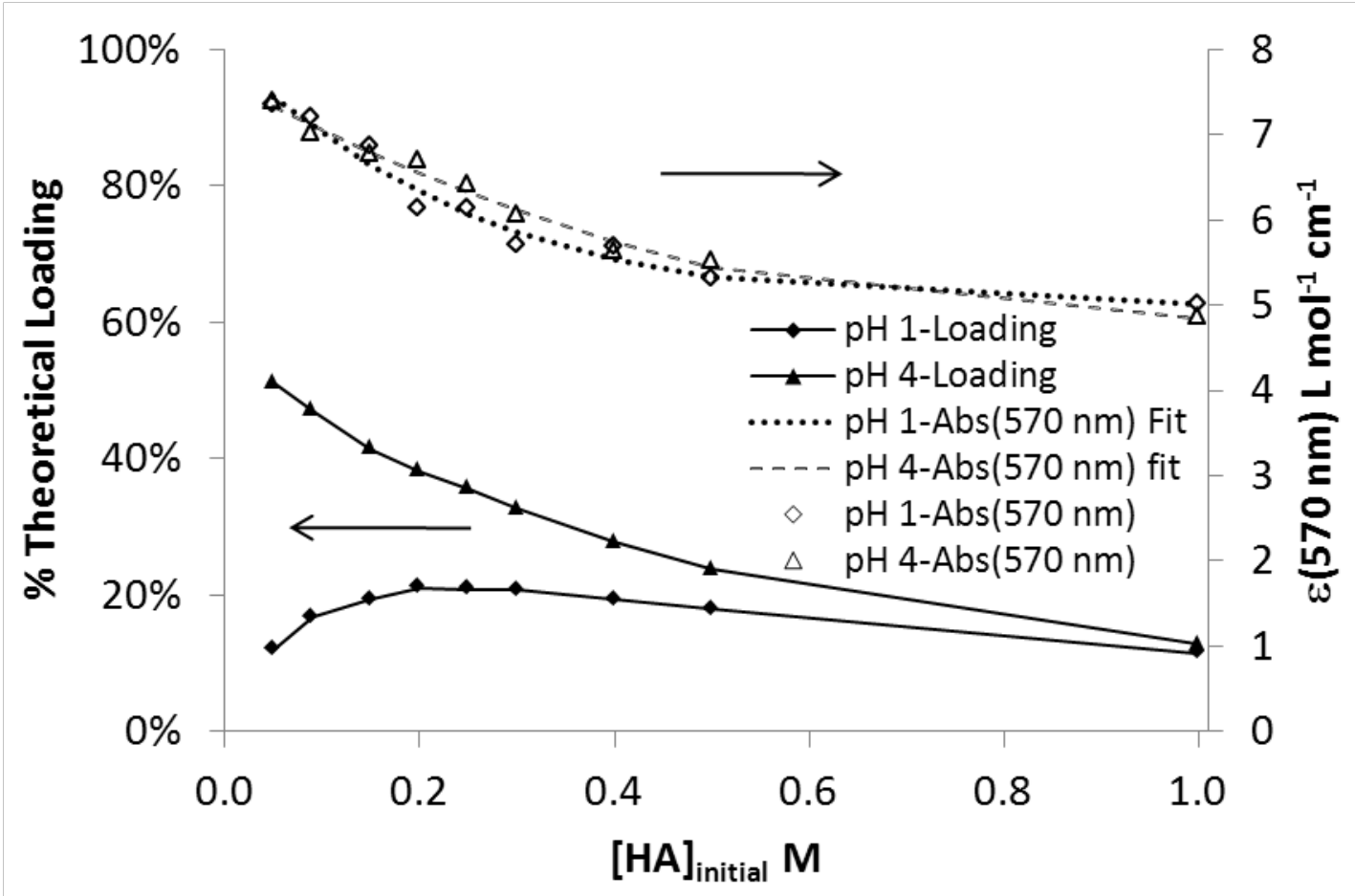

Figure 2.11- Trend in the $570 \mathrm{~nm}$ absorbance band as a function of $[\mathrm{HA}]_{\text {initial }}$ from the $\mathrm{pH}(1,4)+$ $30 \mathrm{mM} \mathrm{Nd}+\mathrm{HCl}$ systems. Also included in this plot is the percent theoretical loading of the solvent based on the assumption of HA dimer stoichiometric coefficient (n) of 2.

\subsubsection{Fourier Transform Infrared Spectroscopy of the Organic Phase}

In all systems, the two peaks of highest interest for the presented study are the 1230 and $1035 \mathrm{~cm}^{-1}$ associated with $\mathrm{P}=\mathrm{O}$ and $\mathrm{P}-\mathrm{O}$ stretching, respectively. ${ }^{[105]}$ The complexation of $\mathrm{Nd}^{3+}$ by $\mathrm{HA}$ leads to a withdrawal of electron density in the $\mathrm{P}=\mathrm{O}$ bond resulting in the decrease of the stretching mode frequency. This is supported by the shift in the 1230 band to $1200 \mathrm{~cm}^{-1}$ in pH (1, 
4) $+30 \mathrm{mM} \mathrm{Nd}+\mathrm{HCl}$ systems where $\mathrm{Nd}^{+3}$ extraction occurs (see Figure 2.12). $\mathrm{Na}^{+}$extraction in the $\mathrm{pH} 4+0 \mathrm{M} \mathrm{Nd}+\mathrm{HCl}$ system was too small to change the FTIR spectrum. Formation of highly aggregated $\left(\mathrm{NdA}_{3}\right)_{x}$ species leads to bridging -O-P-O-groups as evident from the appearance of the weak $1097 \mathrm{~cm}^{-1}$ band $^{[71]}$ for $\mathrm{pH} 4+30 \mathrm{mM} \mathrm{Nd}+\mathrm{HCl}$ systems at low $[\mathrm{HA}]_{\text {org }}\left([\mathrm{HA}]_{\text {org }}=0.05 \mathrm{M}\right.$ is shown in Figure 2.12 as the dotted spectrum).

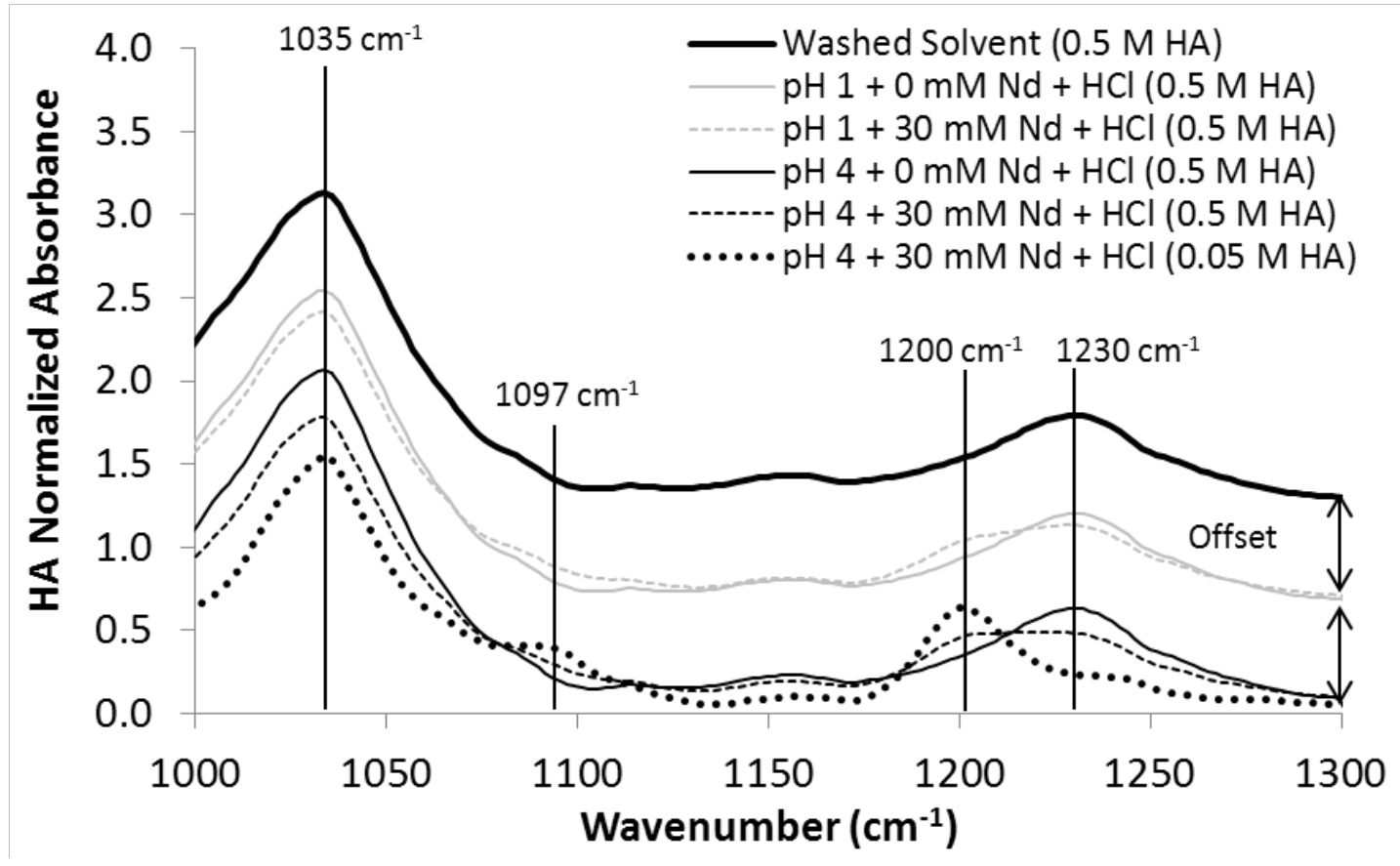

Figure 2.12- Infrared spectra normalized by $[\mathrm{HA}]_{\text {org }}$. The isooctane peaks have been removed from the spectrum. 


\subsection{Results and Discussion: Lactate Aqueous Media}

\subsubsection{HA aggregation}

In the washed solvent, an HA aggregation number of $2.02 \pm 0.03$ was observed. The same aggregation number was also observed for the $\mathrm{pH} 1+0 \mathrm{mM} \mathrm{Nd}+$ Lac system $(1.97 \pm 0.03)$. Average $\mathrm{HA}$ aggregation was observed to increase when $\mathrm{Nd}^{3+}$ extraction was significant, which occurred in the $\mathrm{pH} 1+30 \mathrm{mM} \mathrm{Nd}+$ Lac system $(2.37 \pm 0.04)$. This increase in average aggregation was due to the formation of $\mathrm{NdA}_{3}(\mathrm{HA})$. The $\mathrm{HA}$ aggregation model described in section 2.2.14 was applied to the $\mathrm{pH} 1+30 \mathrm{mM} \mathrm{Nd}+$ Lac system and revealed a $Z_{\mathrm{NdAz}}$ of $4.2 \pm$ 0.3. This value is in agreement with the results obtained for $\mathrm{pH}(1,4)+30 \mathrm{mM} \mathrm{Nd}+\mathrm{HCl}$ systems $(4.0 \pm 0.4)$. In the $\mathrm{pH} 4+0 \mathrm{mM} \mathrm{Nd}+$ Lac system a significant increase in the aggregation number was observed $\left(\mathrm{N}_{\mathrm{agg}}=3.82 \pm 0.05\right)$. The distribution measurements for this system showed that significant $\mathrm{Na}^{+}$and Lac were extracted with $2.7 \pm 0.4$ times the $\left[\mathrm{Na}^{+}\right]_{\text {org }}$ as $[\mathrm{Lac}]_{\text {org }}$. The aggregation for this system was modelled using the equations described in section 2.2.14, but it was modified as follows. Species present were $(\mathrm{HA})_{2}, \mathrm{NaA}(\mathrm{HA})$, and $\mathrm{NaLac}(\mathrm{HA})_{\mathrm{ZNaLac}(\mathrm{HA}) z}$. Species aggregation was 2 for both $(\mathrm{HA})_{2}$ and $\mathrm{NaA}(\mathrm{HA})$. The adapted model for the NaLac species was derived similar to the $\mathrm{Nd}-\mathrm{HA}$ system described in equations (2.18)-(2.22). The model for the Na-Lactate-HA system is formulated using the organic phase species: $\mathrm{NaA}, \mathrm{NaLac}(\mathrm{HA})_{2}$, and $(\mathrm{HA})_{2}$ and is derived in equations $(2.24)-(2.27)$ where $\left(N_{\text {agg, average }}\right)$ is the average $\mathrm{HA}$ aggregation number, $\left(Z_{i}\right)$ is the species HA aggregation number, $\left(X_{i}\right)$ is the species fraction, and bracketed terms are the concentration of species in molarity. The modelling results agreed with the experimental data within error.

$$
\mathrm{N}_{\text {agg,Average }}=\sum \mathrm{Z}_{\mathrm{i}} \mathrm{X}_{\mathrm{i}}=\mathrm{Z}_{2} \mathrm{X}_{2}+\mathrm{Z}_{\mathrm{NaA}} \mathrm{X}_{\mathrm{NaA}}+\mathrm{Z}_{\mathrm{NaLac}(\mathrm{HA})_{2}} \mathrm{X}_{\mathrm{NaLac}(\mathrm{HA})_{2}}
$$




$$
\begin{gathered}
X_{\mathrm{NaA}}=\frac{Z_{\mathrm{NaA}}\left([\mathrm{Na}]_{\text {org }}-[\mathrm{LaC}]_{\mathrm{org}}\right)}{[\mathrm{HA}]_{\text {total }}} \\
\mathrm{X}_{\mathrm{NaLac}(\mathrm{HA})_{2}}=\frac{Z_{\mathrm{NaLac}(\mathrm{HA})_{2}}[\mathrm{LaC}]_{\mathrm{org}}}{[\mathrm{HA}]_{\text {total }}} \\
X_{2}=1-\mathrm{X}_{\mathrm{NdA}_{2}}-\mathrm{X}_{\mathrm{Nalac}(\mathrm{HA})_{2}}
\end{gathered}
$$

Aggregation modelling results showed that the $Z_{\text {NaLac(HA)z }}$ was $5.1 \pm 0.3$ for this system.

In the $\mathrm{pH} 4+(30,60) \mathrm{mM} \mathrm{Nd}+$ Lac systems the aggregation varied as a function of both $\mathrm{HA}$ and total $\mathrm{Nd}^{3+}$ (see Figure 2.13). The average aggregation $\left(\mathrm{N}_{\text {agg }}\right)$ was measured as high as $100 \pm$ 40 at low $[\mathrm{HA}]_{\text {org }}(\sim 0.01 \mathrm{M})$ in the $\mathrm{pH} 4+60 \mathrm{mM} \mathrm{Nd}+$ Lac system, and dropped systematically to a value of $\mathrm{N}_{\mathrm{agg}} \approx 3$ at high $\mathrm{HA}(>0.2 \mathrm{M}$ ) (see Figure 2.13). The maximum average aggregation in the $\mathrm{pH} 4+30 \mathrm{mM} \mathrm{Nd}+$ Lac system also occurred at the lowest $[\mathrm{HA}]_{\text {org }}$ but was only $20 \pm 4$. The instrument response curve is shown with the aggregation data as a demonstration (see Figure 2.14). As $[\mathrm{HA}]_{\text {org }}$ was increased in these systems, the aggregation number decreased until a constant value of $(2.75 \pm 0.04,2.82 \pm 0.06$ respectively $)$ was attained. The initially high aggregation numbers results indicate that large aggregates form due to high $\mathrm{Nd}^{3+}$ loading. Literature accounts have characterized species including $\mathrm{NdA}_{3}{ }^{[69,72]}$ and $\mathrm{Nd}($ Acetate $) \mathrm{A}_{2}{ }^{[71]}$ that form large aggregates. Similar species were most likely present under the present test conditions. 


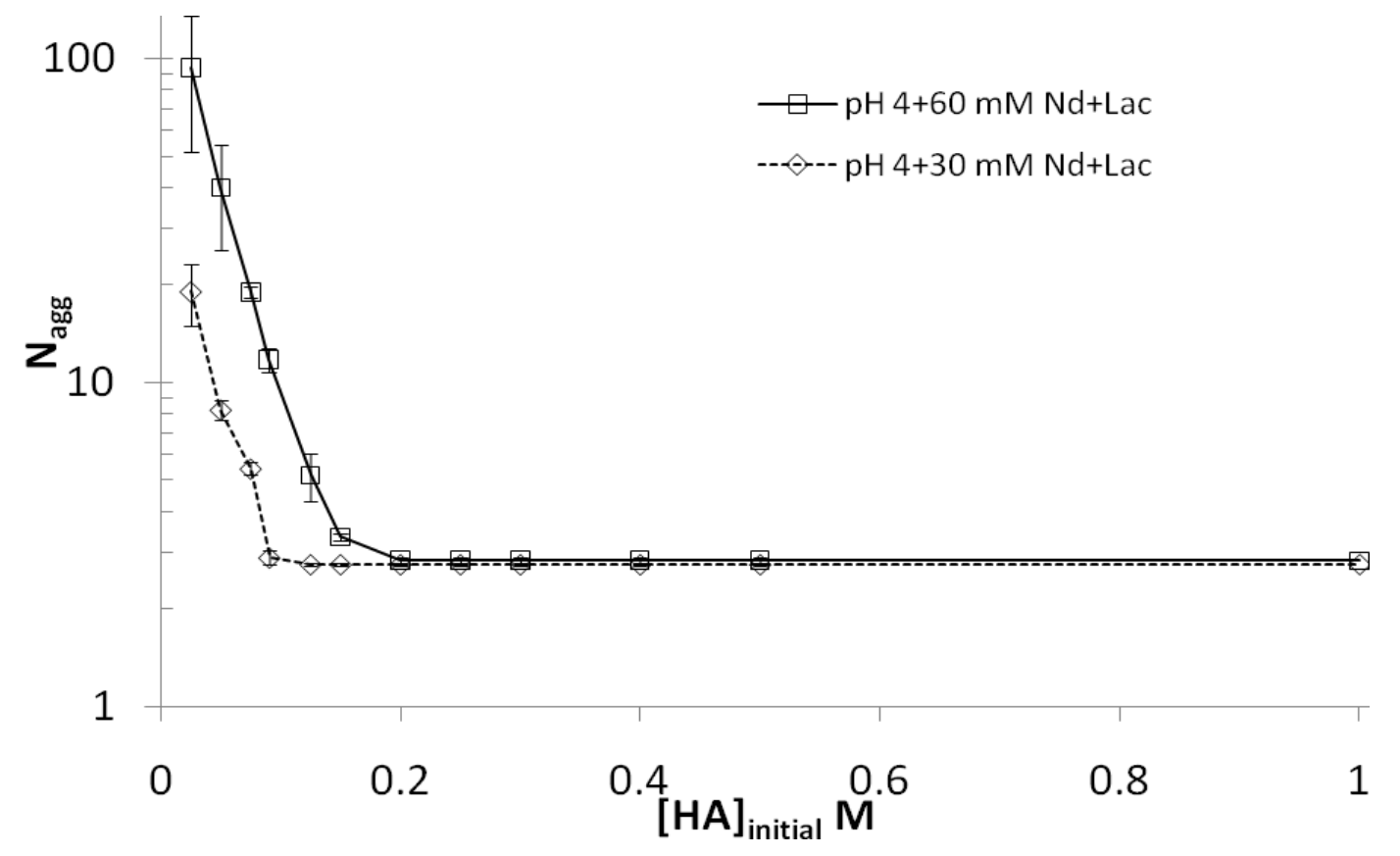

Figure 2.13- Aggregation results for the $\mathrm{pH} 4+(30,60) \mathrm{mM} \mathrm{Nd}+$ Lac systems.

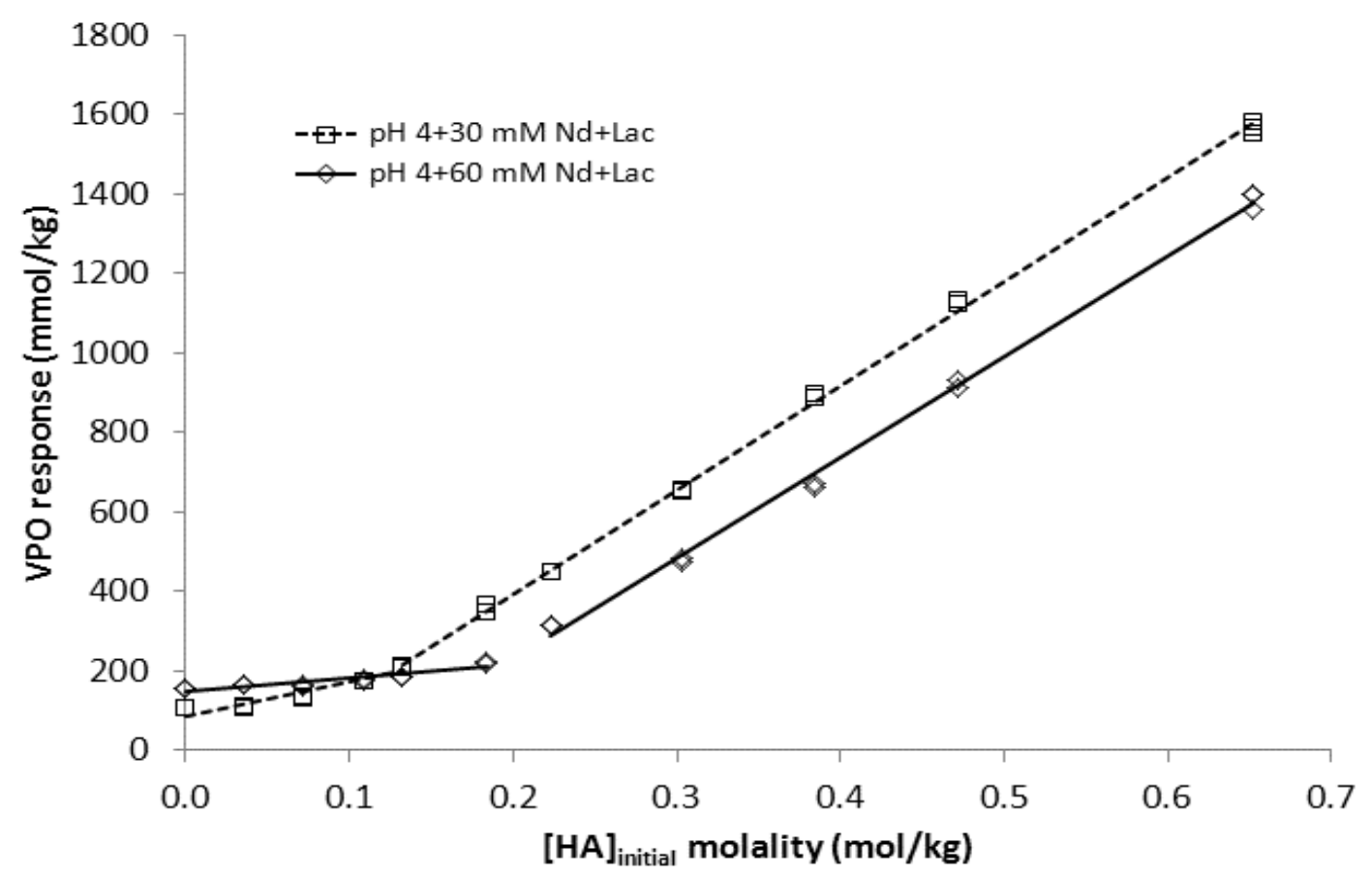

Figure 2.14- Instrument response curve used to calculate the aggregation results for the $\mathrm{pH} 4+$ $(30,60) \mathrm{mM} \mathrm{Nd}+$ Lac systems. 


\subsubsection{Liquid-Liquid Distribution Measurements}

Overall results for the extraction measurements are shown in Table 2.15 and Table 2.16 and will be discussed in sections that follow.

\subsubsection{1 $\underline{\mathrm{Nd}}^{3+} \underline{\text { Distribution Results }}$}

$\mathrm{Nd}^{3+}$ extraction showed significantly different behavior between $\mathrm{pH} 1$ and $\mathrm{pH} 4$. The extraction of $\mathrm{Nd}^{3+}$ in the $\mathrm{pH} 1+30 \mathrm{mM} \mathrm{Nd}+$ Lac system (see Table 2.15) was very similar to the $\mathrm{pH} 1+30 \mathrm{mM} \mathrm{Nd}+\mathrm{HCl}$ system (see Table 2.11). At $\mathrm{pH} 1$, the extraction of $\mathrm{Nd}^{3+}$ is governed by the ion exchange mechanism shown in equation (2.7). Comparison of the $\mathrm{pH}$ data between the $\mathrm{pH} 1+30 \mathrm{mM} \mathrm{Nd}+(\mathrm{HCl}, \mathrm{Lac})$ systems also showed that pH change was likewise the same within experimental error indicating that no lactate buffering occurred at this low pH (Table 2.11 and Table 2.15). The extraction results from the $\mathrm{pH} 1+30 \mathrm{mM} \mathrm{Nd}+$ Lac system were modeled by SXLSQI ${ }^{[94]}$ as described in section 2.2.10 and showed excellent fit with the observed data using only the $(H A)_{2}$ and $\mathrm{Nd}(\mathrm{A})_{3}(\mathrm{HA})$ species (see Figure 2.15). Use of these species was justified by the vapor pressure aggregation modeling results described in section 2.5.1.

As $\mathrm{pH}$ is elevated past the $\mathrm{pk}_{\mathrm{a}}$ of lactate $\left(\mathrm{pK}_{\mathrm{a}}=3.86^{[55]}\right)$ its buffering effects, and the aqueous complexation both impact the extraction of $\mathrm{Nd}^{3+}$. In the $\mathrm{pH} 4+(30,60) \mathrm{mM} \mathrm{Nd}+$ Lac systems, the buffering effect of lactate causes the extraction of $\mathrm{Nd}^{3+}$ at the saturation level of $\mathrm{HA}$ at low $[\mathrm{HA}]_{\text {org }}$ and quantitative extraction at higher $[\mathrm{HA}]_{\text {org }}$. The transition concentration of $[\mathrm{HA}]_{\text {org }}$ between the saturation and the quantitative extraction occurred at twice the aqueous $\mathrm{Nd}^{3+}$ concentration $(0.0619 \pm 0.0009$ and $0.123 \pm 0.001 \mathrm{M} \mathrm{HA}$ for $\mathrm{pH} 4+(30,60) \mathrm{mM} \mathrm{Nd}+\mathrm{Lac}$ systems respectively; see Figure 2.16). This result is consistent with a neodymium complex containing two deprotonated HA molecules, and one lactate molecule for charge balance as in the $\operatorname{NdX}(A)_{2}$ complex. Results presented later in this work (see section 2.5.2.3) indicate that the 
counter ion in the $\mathrm{NdX}^{2+}$ salt is lactate. Due to a lack of aqueous phase thermodynamic modeling parameters required by the SXLSQI software (Pitzer parameters for $\mathrm{Nd}^{3+}$ and lactate in lactate aqueous media), it is not possible at this time to achieve a modeling result for the extraction of $\mathrm{Nd}^{3+}$ in the $\mathrm{pH} 4+(30,60) \mathrm{mM} \mathrm{Nd}+$ Lac systems.

Table 2.15- Equilibrium $\mathrm{pH},\left[\mathrm{Na}^{+}\right]_{\mathrm{org}},\left[\mathrm{Nd}^{3+}\right]_{\text {org }}$, and $[\mathrm{Lac}]_{\text {org }}$ for the $\mathrm{pH} 1+(0,30) \mathrm{mM} \mathrm{Nd}+\mathrm{Lac}$ systems. Error for the values shown is: \pm 0.01 for $\mathrm{pH}_{\text {eq }}, 2 \%$ for $\left[\mathrm{Nd}^{3+}\right]_{\text {org,eq }}$.

\begin{tabular}{|c|c|c|c|c|c|c|}
\hline \multirow{2}{*}{ [HA] } & \multicolumn{3}{|c|}{$\mathrm{pH} 1+0 \mathrm{mM} \mathrm{Nd}+\mathrm{Lac}$} & \multicolumn{3}{|c|}{$\mathrm{pH} 1+30 \mathrm{mM} \mathrm{Nd}+\mathrm{Lac}$} \\
\hline & $\mathrm{pH}_{\mathrm{eq}}$ & {$[\mathrm{Na}]_{\text {org,eq }}$} & $\mathrm{pH}_{\mathrm{eq}}$ & {$[\mathrm{Na}]_{\text {org,eq }}$} & {$[\text { Lac }]_{\text {org,eq }}$} & {$[\text { Lac }]_{\text {org,ec }}$} \\
\hline $\mathbf{M}$ & & $\mathbf{M}$ & & $\mathbf{M}$ & M & $\mathbf{M}$ \\
\hline 0 & 1.10 & \multirow{13}{*}{ BD } & 4.21 & \multirow{2}{*}{$\mathrm{BD}$} & $\begin{array}{c}0.0007 \pm \\
0.0004\end{array}$ & $\begin{array}{c}0.0007 \pm \\
0.0003\end{array}$ \\
\hline 0.025 & 1.10 & & 4.21 & & $\begin{array}{c}0.0013 \pm \\
0.0004\end{array}$ & $\begin{array}{c}0.0011 \pm \\
0.0003\end{array}$ \\
\hline 0.05 & 1.10 & & 4.20 & $\begin{array}{c}0.010 \pm \\
0.006\end{array}$ & $\begin{array}{c}0.0031 \pm \\
0.0004\end{array}$ & $\begin{array}{c}0.0013 \pm \\
0.0003\end{array}$ \\
\hline 0.075 & 1.09 & & 4.19 & $\begin{array}{c}0.015 \pm \\
0.007\end{array}$ & $\begin{array}{c}0.0054 \pm \\
0.0005\end{array}$ & $\begin{array}{c}0.0014 \pm \\
0.0003\end{array}$ \\
\hline 0.09 & 1.09 & & 4.18 & $\begin{array}{c}0.021 \pm \\
0.009\end{array}$ & $\begin{array}{c}0.006 \pm \\
0.001\end{array}$ & $\begin{array}{c}0.0015 \pm \\
0.0003\end{array}$ \\
\hline 0.125 & 1.10 & & 4.16 & $\begin{array}{c}0.03 \pm \\
0.01\end{array}$ & $\begin{array}{c}0.012 \pm \\
0.001\end{array}$ & $\begin{array}{c}0.0017 \pm \\
0.0003\end{array}$ \\
\hline 0.15 & 1.10 & & 4.16 & $\begin{array}{c}0.04 \pm \\
0.01\end{array}$ & $\begin{array}{c}0.015 \pm \\
0.001\end{array}$ & $\begin{array}{c}0.0019 \pm \\
0.0003\end{array}$ \\
\hline 0.2 & 1.10 & & 4.14 & $\begin{array}{c}0.06 \pm \\
0.02\end{array}$ & $\begin{array}{c}0.023 \pm \\
0.002\end{array}$ & $\begin{array}{c}0.0023 \pm \\
0.0004\end{array}$ \\
\hline 0.25 & 1.09 & & 4.12 & $\begin{array}{c}0.08 \pm \\
0.02\end{array}$ & $\begin{array}{c}0.025 \pm \\
0.002\end{array}$ & $\begin{array}{c}0.0026 \pm \\
0.0004\end{array}$ \\
\hline 0.3 & 1.09 & & 4.08 & $\begin{array}{c}0.10 \pm \\
0.02\end{array}$ & $\begin{array}{c}0.039 \pm \\
0.003\end{array}$ & $\begin{array}{c}0.0029 \pm \\
0.0005\end{array}$ \\
\hline 0.4 & 1.10 & & 4.02 & $\begin{array}{c}0.13 \pm \\
0.02\end{array}$ & $\begin{array}{c}0.054 \pm \\
0.004\end{array}$ & $\begin{array}{c}0.0039 \pm \\
0.0005\end{array}$ \\
\hline 0.5 & 1.10 & & 3.99 & $\begin{array}{c}0.17 \pm \\
0.03\end{array}$ & $\begin{array}{c}0.061 \pm \\
0.004\end{array}$ & $\begin{array}{c}0.0047 \pm \\
0.0005\end{array}$ \\
\hline 1 & 1.09 & & 3.76 & $\begin{array}{c}0.31 \pm \\
0.04\end{array}$ & $\begin{array}{c}0.125 \pm \\
0.005\end{array}$ & $\begin{array}{c}0.0095 \pm \\
0.0009\end{array}$ \\
\hline
\end{tabular}

$\mathrm{BD}=$ Below detection 


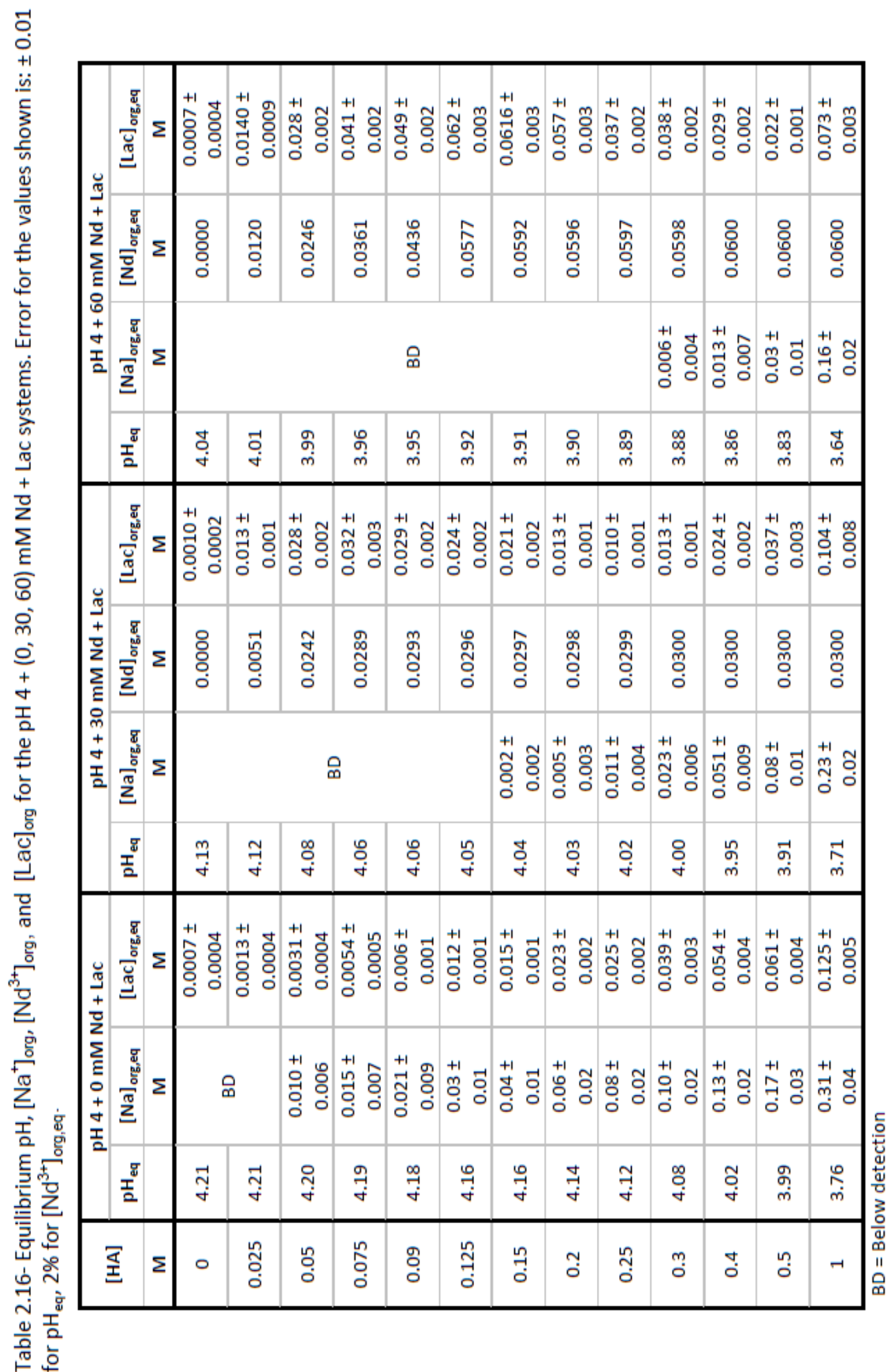




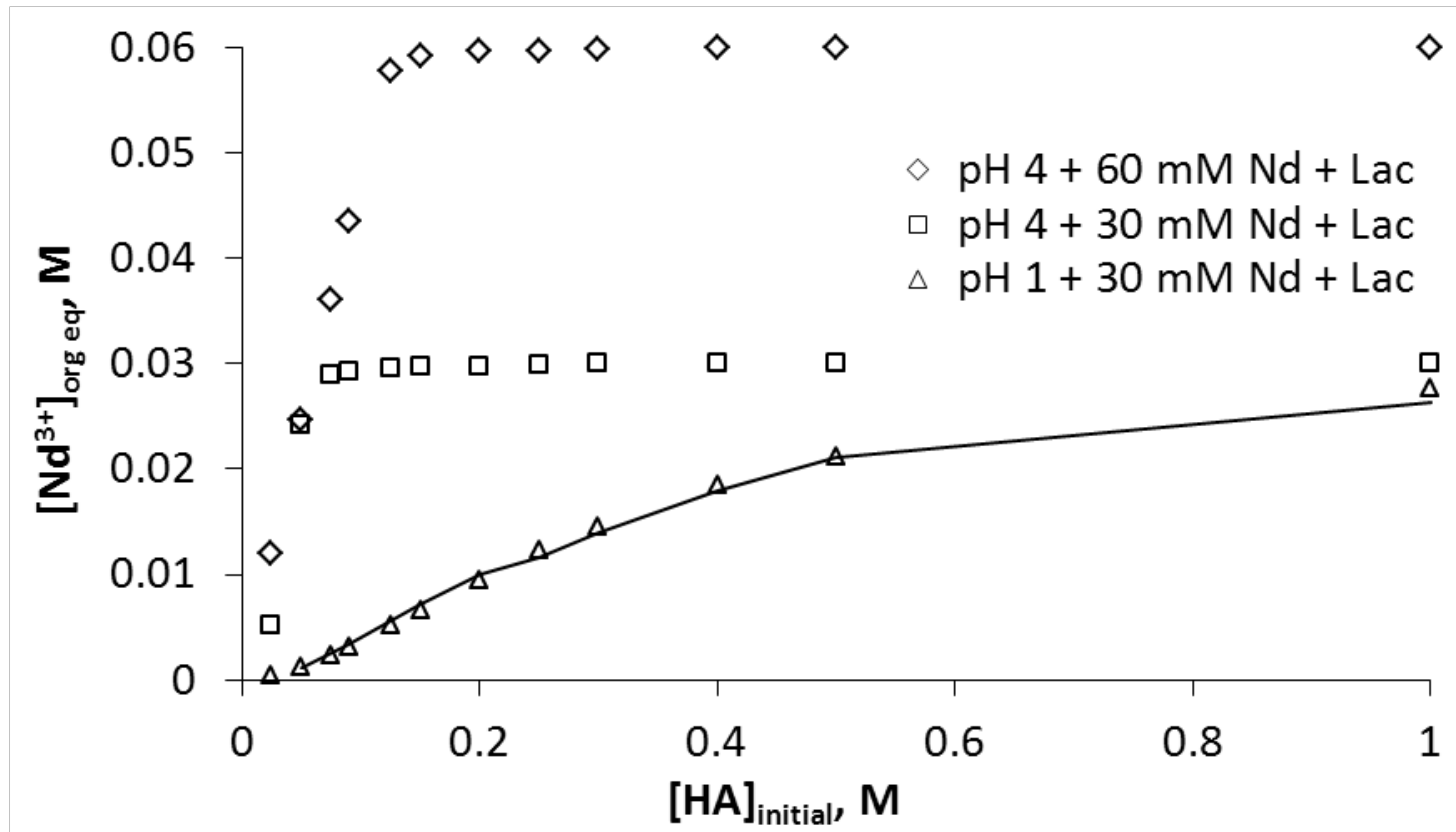

Figure 2.15- $\mathrm{Nd}^{3+}$ extraction results. Observed data are depicted as points, SXLSQI modeling results are depicted as the solid curve.

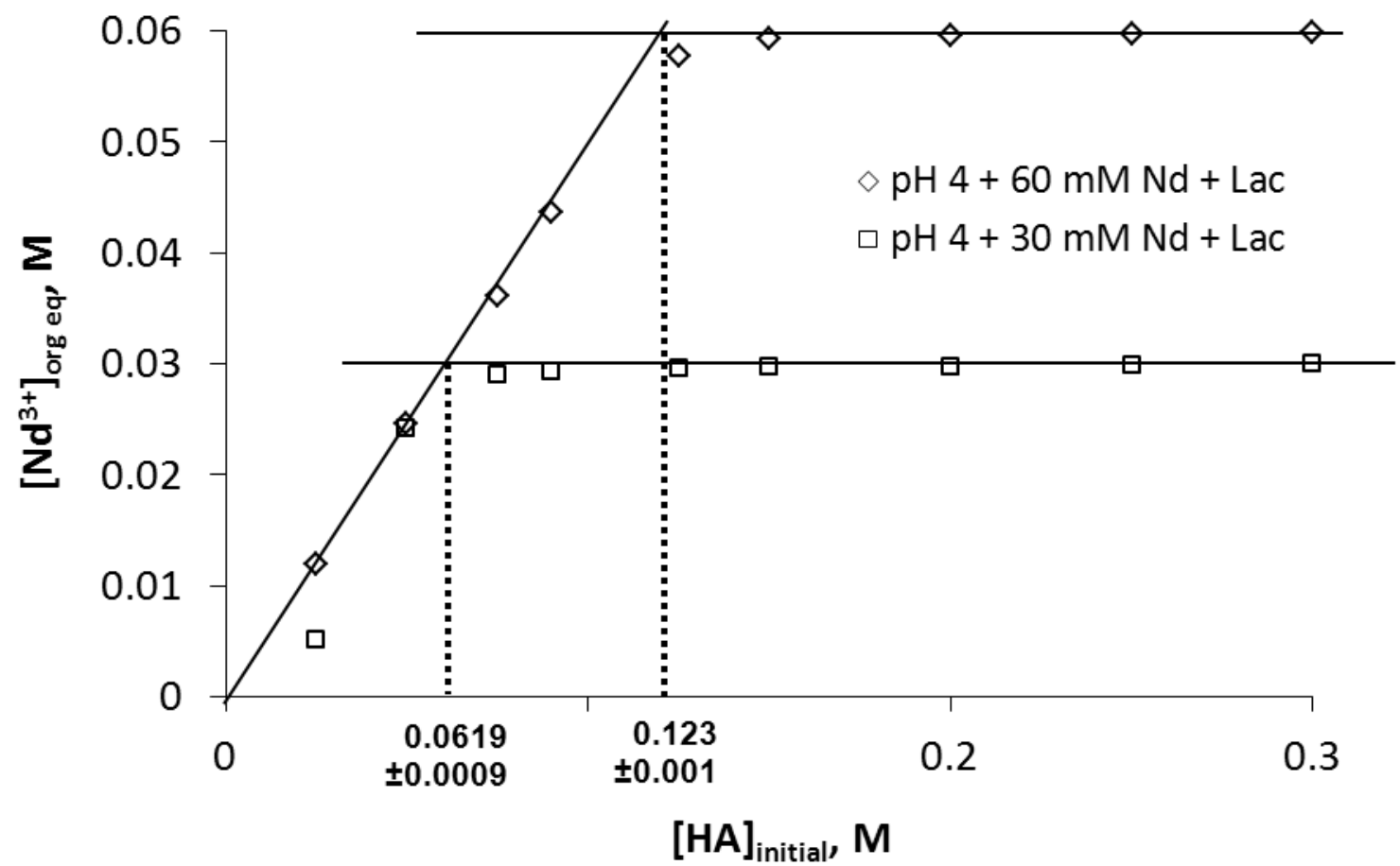

Figure 2.16- The transition between saturation and quantitative extraction supports the 1:2 $\mathrm{Nd}^{3+}$ : HA stoichiometry of the $\mathrm{NdLacA}_{2}$ complex. 
As $\mathrm{pH}$ is elevated past the $\mathrm{pk}_{\mathrm{a}}$ of lactate $\left(\mathrm{pK}_{\mathrm{a}}=3.86^{[55]}\right)$ its buffering effects, and the aqueous complexation both impact the extraction of $\mathrm{Nd}^{3+}$. In the $\mathrm{pH} 4+(30,60) \mathrm{mM} \mathrm{Nd}+$ Lac systems, the buffering effect of lactate causes the extraction of $\mathrm{Nd}^{3+}$ at the saturation level of $\mathrm{HA}$ at low $[\mathrm{HA}]_{\text {org }}$ and quantitative extraction at higher $[\mathrm{HA}]_{\text {org }}$. The transition concentration of $[\mathrm{HA}]_{\text {org }}$ between the saturation and the quantitative extraction occurred at twice the aqueous $\mathrm{Nd}^{3+}$ concentration $(0.0619 \pm 0.0009$ and $0.123 \pm 0.001 \mathrm{M} \mathrm{HA}$ for $\mathrm{pH} 4+(30,60) \mathrm{mM} \mathrm{Nd}+\mathrm{Lac}$ systems respectively; see Figure 2.16). This result is consistent with a neodymium complex containing two deprotonated HA molecules, and one lactate molecule for charge balance as in the $\operatorname{NdX}(A)_{2}$ complex. Results presented later in this work (see section 2.5.2.3) indicate that the counter ion in the $\mathrm{NdX}^{2+}$ salt is lactate. Due to a lack of aqueous phase thermodynamic modeling parameters required by the SXLSQI software (Pitzer parameters for $\mathrm{Nd}^{3+}$ and lactate in lactate aqueous media), it is not possible at this time to achieve a modeling result for the extraction of $\mathrm{Nd}^{3+}$ in the $\mathrm{pH} 4+(30,60) \mathrm{mM} \mathrm{Nd}+$ Lac systems.

\subsubsection{2 $\underline{\mathrm{Na}}^{ \pm} \underline{\text { Distribution Results }}$}

Just as with the $\mathrm{Nd}^{3+}$ systems, the $\mathrm{Na}^{+}$extraction data also shows a significantly different behavior between $\mathrm{pH} 1$ and $\mathrm{pH} 4$. Extraction of $\mathrm{Na}^{+}$was below detection in the $\mathrm{pH} 1+(0,30)$ $\mathrm{mM} \mathrm{Nd}+$ Lac systems. A linear increase in $\mathrm{Na}^{+}$extraction occurred in the $\mathrm{pH} 4+0 \mathrm{mM} \mathrm{Nd}+\mathrm{Lac}$ system over the range of increasing $[\mathrm{HA}]_{\text {org }}$. A comparison of the $\left[\mathrm{Na}^{+}\right]_{\text {org }}$ and $[\mathrm{Lac}]_{\text {org }}$ for this system showed $2.3 \pm 0.1$ times the $\left[\mathrm{Na}^{+}\right]_{\text {org }}$ as $[\mathrm{Lac}]_{\text {org }}$ (see Figure 2.20 for a plot of the data), indicating that $\mathrm{Na}^{+}$is most likely extracted by more than one mechanism and exists as both $\mathrm{HA}$ sodium salt ( $\mathrm{NaA}$ ) and $\mathrm{NaLac}$ salts within the organic phase. In the $\mathrm{HCl}$ system, the $\mathrm{NaA}$ species was shown to exists as $\mathrm{NaA}(\mathrm{HA})$ in the organic phase because no increase in aggregation was observed under $\mathrm{NaA}$ extraction conditions as shown in equation (2.10) (with $\mathrm{n}=1$ ). The NaLac salt is therefore extracted by the reaction shown in equation (2.28). 


$$
\mathrm{NaLac}+\mathrm{n} \overline{(\mathrm{HA})_{2}} \leftrightarrow \overline{\mathrm{NaLac}(\mathrm{HA})_{2 \mathrm{n}}}
$$

As described in the aggregation modeling section, results give the value for $2 n=Z_{\text {NaLacHAz }}=5.1 \pm$ 0.3 (or $\mathrm{n}=2.5 \pm 0.2$ ). $\mathrm{Na}^{+}$extraction was suppressed in $\mathrm{pH} 4+(30,60) \mathrm{mM} \mathrm{Nd}+$ Lac systems at low $[\mathrm{HA}]_{\text {org }}$ but was observed at higher $[\mathrm{HA}]_{\text {org }}$. The threshold where $\mathrm{Na}^{+}$extraction began in these systems was approximately $0.125 \mathrm{M}$ for the $\mathrm{pH} 4+30 \mathrm{mM} \mathrm{Nd}+$ Lac system and approximately $0.25 \mathrm{M}$ for the $\mathrm{pH} 4+60 \mathrm{mM}+$ Lac system. This corresponds with the $4: 1$ ratio of $\mathrm{HA}$ to $\mathrm{Nd}^{3+}$ in the organic phase. This 4:1 ratio is significant for this system because it is consistent with the formation of the $\mathrm{Nd}(\mathrm{A})_{3}(\mathrm{HA})$ species at lower $\mathrm{HA}$ loading. The $\mathrm{Na}^{+}$extraction began at greater than 4:1 ratio because there was excess $\mathrm{HA}$ available to accommodate $\mathrm{Na}^{+}$ extraction.

\subsubsection{Lactate Distribution Results}

Lactate was shown to extract in various quantities and species depending on experimental conditions. In the pH $1+0 \mathrm{mM} \mathrm{Nd}+$ Lac system, weak extraction of lactic acid (HLac) was observed so that organic Lac concentration increased linearly with HA concentration (slope = $\left.0.0086 \pm 0.0002, R^{2}=0.985\right)$. Log-slope analysis of $[\mathrm{Lac}]_{\text {org }}$ vs. $[H A]_{\text {org }}$ shows a slope of approximately $1\left(0.93 \pm 0.03, R^{2}=0.994\right.$; results shown in Figure 2.17$)$. VPO results indicated average aggregation of 2 in this system, so the suggested mechanism of the HLac extraction is via solvation by HA dimers [equation (2.29)]

$$
\mathrm{HLac}+\overline{(\mathrm{HA})_{2}} \leftrightarrow \overline{\mathrm{HLac}(\mathrm{HA})_{2}}
$$

When $\mathrm{Nd}^{3+}$ was present in the $\mathrm{pH} 1+30 \mathrm{mM} \mathrm{Nd}+$ Lac system, the extraction of Lac was approximately doubled with a linear correlation of $[\mathrm{Lac}]_{\text {org }}$ with $[\mathrm{HA}]_{\text {org }}$ (slope $=0.0171 \pm 0.0006$, $\left.R^{2}=0.994\right)$. The log-slope analysis showed a slope near, but statistically greater than 1 (slope $=$ $1.17 \pm 0.03 ; R^{2}=0.994 ;$ see Figure 2.17). The slope difference between the $\mathrm{pH} 1+(0,30) \mathrm{mM} \mathrm{Nd}$ 
+ Lac systems ( 0.93 vs. 1.17 respectively) indicates that the mechanisms operating in these systems are different.

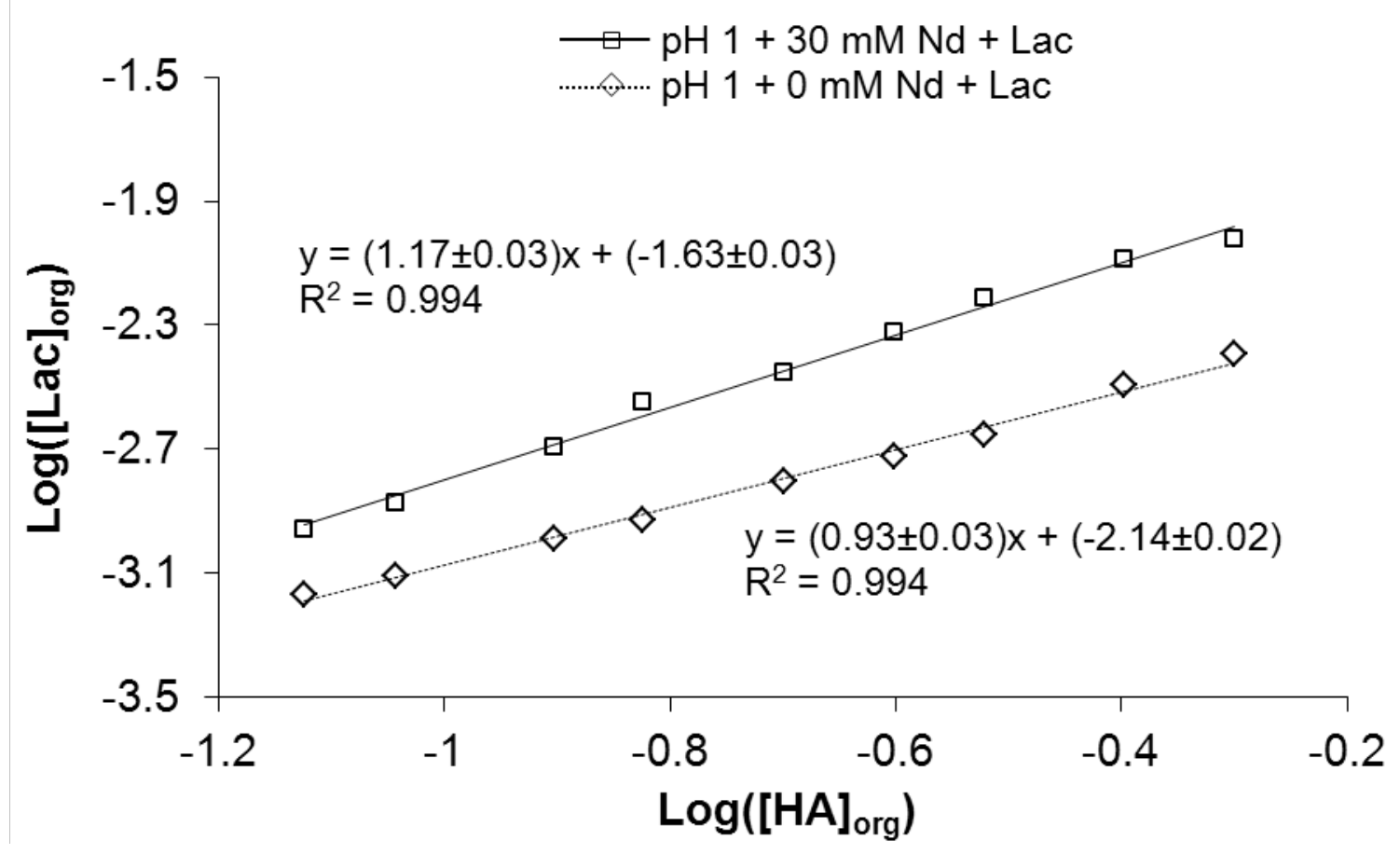

Figure 2.17- Log-slope analysis of Lac extraction vs. [HA] org for the $\mathrm{pH} 1+(0,30) \mathrm{mM} \mathrm{Nd}+\mathrm{Lac}$ systems.

To observe the correlation between $\mathrm{Nd}^{3+}$ and Lac extraction, the [Lac] org values had to be adjusted to remove the $[\mathrm{Lac}]_{\text {org }}$ that is present due to the reaction described by equation (2.29). This was performed by subtracting the $[\mathrm{Lac}]_{\text {org }}$ observed in the $\mathrm{pH} 1+0 \mathrm{mM} \mathrm{Nd}+$ Lac system from the $[\mathrm{Lac}]_{\text {org }}$ in the $\mathrm{pH} 1+30 \mathrm{mM} \mathrm{Nd}+$ Lac system for each $[\mathrm{HA}]_{\text {org }}$. The adjusted Lac concentration is named ' $[\mathrm{Lac}]_{\text {org,excess' }}$ signifying the Lac present in the organic phase 'in excess' of that observed in the corresponding non- $\mathrm{Nd}^{3+}$ system. The log-slope data for the $\mathrm{Nd}^{3+} \mathrm{vs}$. Lac 
extraction is shown in Figure 2.18. The log-slope correlation between $\mathrm{Nd}^{3+}$ and Lac extraction gives a slope of $1.18 \pm 0.04$, but this does yield a unique mechanism operating in this system. More experiments must be run to determine the source of the enhancement of the lactate extraction. It may be due to co-extraction with $\mathrm{Nd}^{3+}$. Another option is that the electronic and steric solvation environment of the organic phase due to highly aggregated $\mathrm{Nd}(\mathrm{A})_{3}(\mathrm{HA})$ complex species provides a more favorable solubility for lactic acid than HA dimers in the nonneodymium system.

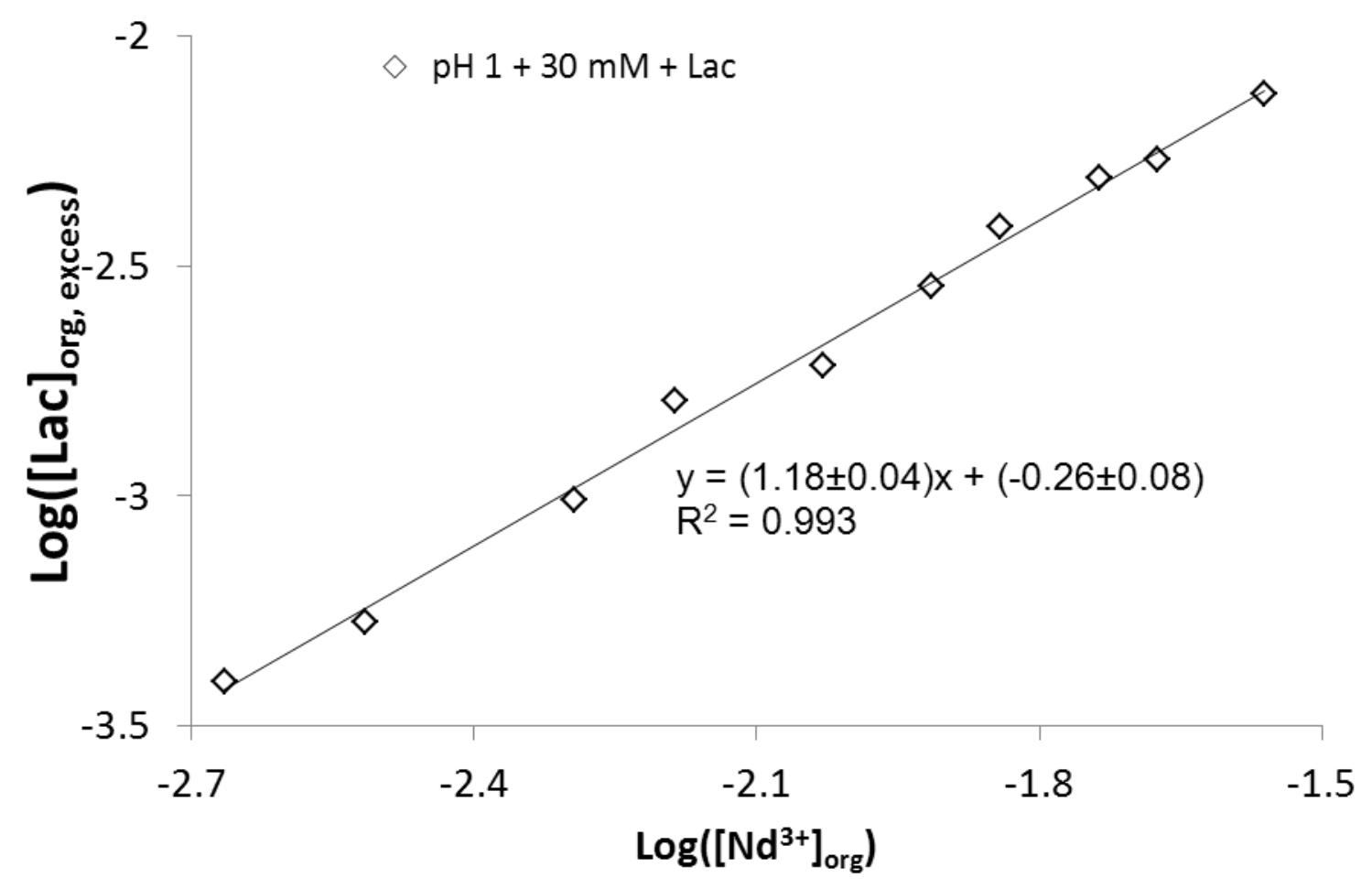

Figure 2.18- Log-slope plot for the $\mathrm{Nd}^{3+}$ vs. Lac dependence in the $\mathrm{pH} 1+30 \mathrm{mM} \mathrm{Nd}+\mathrm{Lac}$ system. 
In the $\mathrm{pH} 4+0 \mathrm{mM} \mathrm{Nd}+$ Lac system, a significantly higher concentration of Lac was extracted compared with $\mathrm{pH} 1+(0,30) \mathrm{mM} \mathrm{Nd}+$ Lac systems, presumably as sodium salt in accord with equation (2.28). The composition of the product species ( $\mathrm{NaLac})(\mathrm{HA})_{2 n}(\mathrm{org})$ was deduced based upon the assumption that in this system HA exists as two type of aggregates, namely a free or $\mathrm{Na}^{+}$salt dimer [as discussed above, our data demonstrated that conversion of $\mathrm{HA}$ into $\mathrm{Na}^{+}$salt, $\mathrm{NaA}(\mathrm{HA})$ does not change its aggregation of 2] and NaLac HA solvate. The analysis of the VPO data, total HA and Lac concentrations in the equilibrium organic phases indicated that the latter contained $5(5.1 \pm 0.3)$ extractant molecules so that ' $n$ ' in the equation (2.28) is equal to approximately $2.5 \pm 0.2$.

The distribution of $\mathrm{Lac}$ in the $\mathrm{pH} 4+(30,60) \mathrm{mM} \mathrm{Nd}+$ Lac systems is a complicated function with respect to the HA increase in HA concentrations. For [Lac] org, there is an initial increase in [Lac] org from 0.025 through $0.075 \mathrm{M} \mathrm{HA}$ for the $\mathrm{pH} 4+30 \mathrm{mM} \mathrm{Nd}+$ Lac system, and from 0.025 through 0.125 M HA for the pH $4+60 \mathrm{mM} \mathrm{Nd}+$ Lac system (see Figure 2.19-A, B, respectively). Following this initial increase there was a decrease in [Lac] org from 0.09 to $0.25 \mathrm{M} \mathrm{HA}$ for the $\mathrm{pH}$ $4+30 \mathrm{mM} \mathrm{Nd}+$ Lac system and from 0.15 to $0.5 \mathrm{M} \mathrm{HA}$ for pH $4+60 \mathrm{mM} \mathrm{Nd}+$ Lac system. Finally there was an increase in $[\mathrm{Lac}]_{\text {org }}$ with $[\mathrm{HA}]_{\mathrm{org}}$ from 0.3 to $1.0 \mathrm{M} \mathrm{HA}$ in the $\mathrm{pH} 4+30 \mathrm{mM}$ $\mathrm{Nd}+$ Lac system and for $1 \mathrm{M} \mathrm{HA}$ in the $\mathrm{pH} 4+60 \mathrm{mM} \mathrm{Nd}+$ Lac system.

In systems with $\mathrm{Na}^{+}$extraction, the trend between $\mathrm{Na}^{+}$and lactate in the organic phase indicates the stoichiometry between these constituents. Some data near the transition between $\mathrm{NdLac}^{2+}$ and NaLac extraction was disregarded because the contribution by the $\mathrm{NdLac}^{2+}$ species to the overall lactate concentration moved the data points off the trend line. The data showing the correlation between $\mathrm{Na}^{+}$and lactate in the organic phase is shown in Figure 2.20. The slope obtained from this correlation $(2.3 \pm 0.1)$ is not indicative of a complex involving two or more $\mathrm{Na}^{+}$with one lactate in the organic phase. The stoichiometry between $\mathrm{Na}^{+}$and lactate cannot be 
more than 1:1 due to the charge of these constituents. This slope merely indicates that since sodium is $2.3 \pm 0.1$ more concentrated in the organic phase, and that there must be two mechanisms and two complexes involved in $\mathrm{Na}^{+}$extraction: $\mathrm{NaLac}$ and $\mathrm{NaA}$.
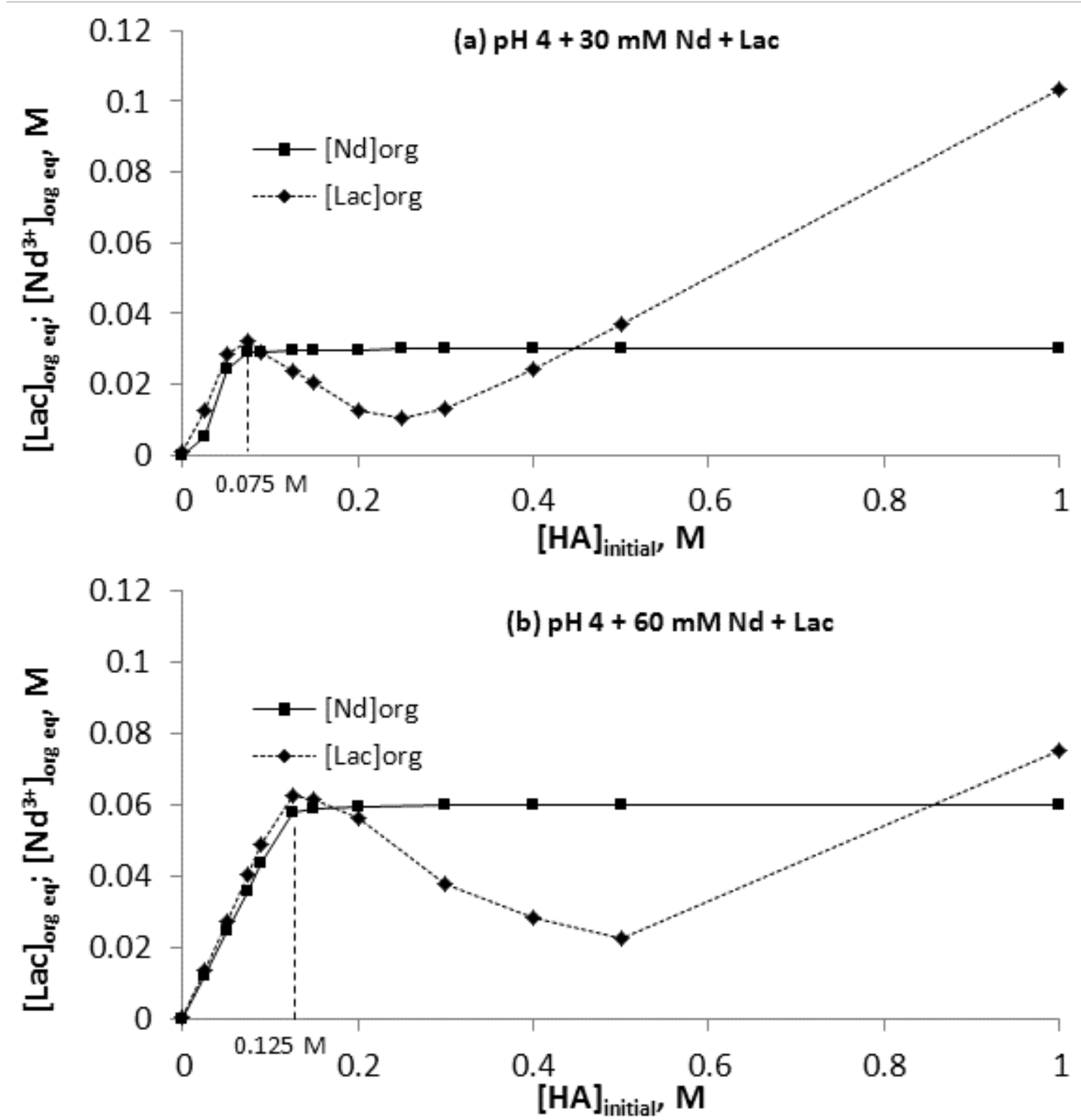

Figure 2.19- Dependence of organic $\mathrm{Nd}^{3+}$ and Lac concentrations on HA molarity in the $\mathrm{pH} 4+30$ $\mathrm{mM} \mathrm{Nd}+$ Lac systems. 
The maximum in Lac extraction at low HA concentration was attributed to the high extractant loading with $\mathrm{Nd}^{3+}$ promoting extraction of the $\mathrm{NdLac}_{\mathrm{b}}{ }^{(3-\mathrm{b})+}(\mathrm{b}=1)$ complex and formation of the mixed Nd/Lac/HA complex species in the organic phase. The initial increase in organic Lac concentration correlated with organic $\mathrm{Nd}^{3+}$ concentration (see Figure 2.19-A,B). The observed linear dependence of the organic Lac and $\mathrm{Nd}^{3+}$ concentrations had a slope of $1.09 \pm$ $0.05\left(R^{2}=0.998\right)$ and $1.08 \pm 0.01\left(R^{2}=0.9993\right)$ for $\mathrm{pH} 4+(30,60) \mathrm{mM} \mathrm{Nd}+$ Lac systems, respectively (see Figure 2.21). Because $\mathrm{Na}^{+}$extraction was below detection, it was not necessary to adjust the Lac concentration. The slope of 1 in both systems is consistent with extraction of the aqueous $\mathrm{NdLac}^{2+}(b=1)$ species in these systems under high extractant loading conditions as described by equation (2.9). Moreover, Figure 2.19 ( $A$ and $B$ ) demonstrates that at high organic loading, maximum organic Lac concentrations of 32 and $62 \mathrm{mM}$ (ratio 1:2 with $\mathrm{HA}$ ) are nearly equal to the organic $\mathrm{Nd}^{3+}$ concentrations (30 and $60 \mathrm{mM}$ ) when $\mathrm{Nd}^{3+}$ extraction becomes quantitative. As HA loading decreased, $\mathrm{Nd}^{3+}$ extraction described by equation (2.9) using a $\mathrm{Nd}(\mathrm{Lac})(\mathrm{HA})_{2}$ extraction complex, was gradually displaced by the extraction mechanism in accord with equation (2.7) where $\mathrm{NdA}_{3} \mathrm{HA}$ predominates as the $\mathrm{Nd}^{3+}$ extraction complex, resulting in the decrease of the organic Lac concentration. Finally under the low organic loading conditions (i.e. high HA concentration), organic Lac concentration increased due to the coextraction of Lac with $\mathrm{Na}^{+}$described by equation (2.28). 


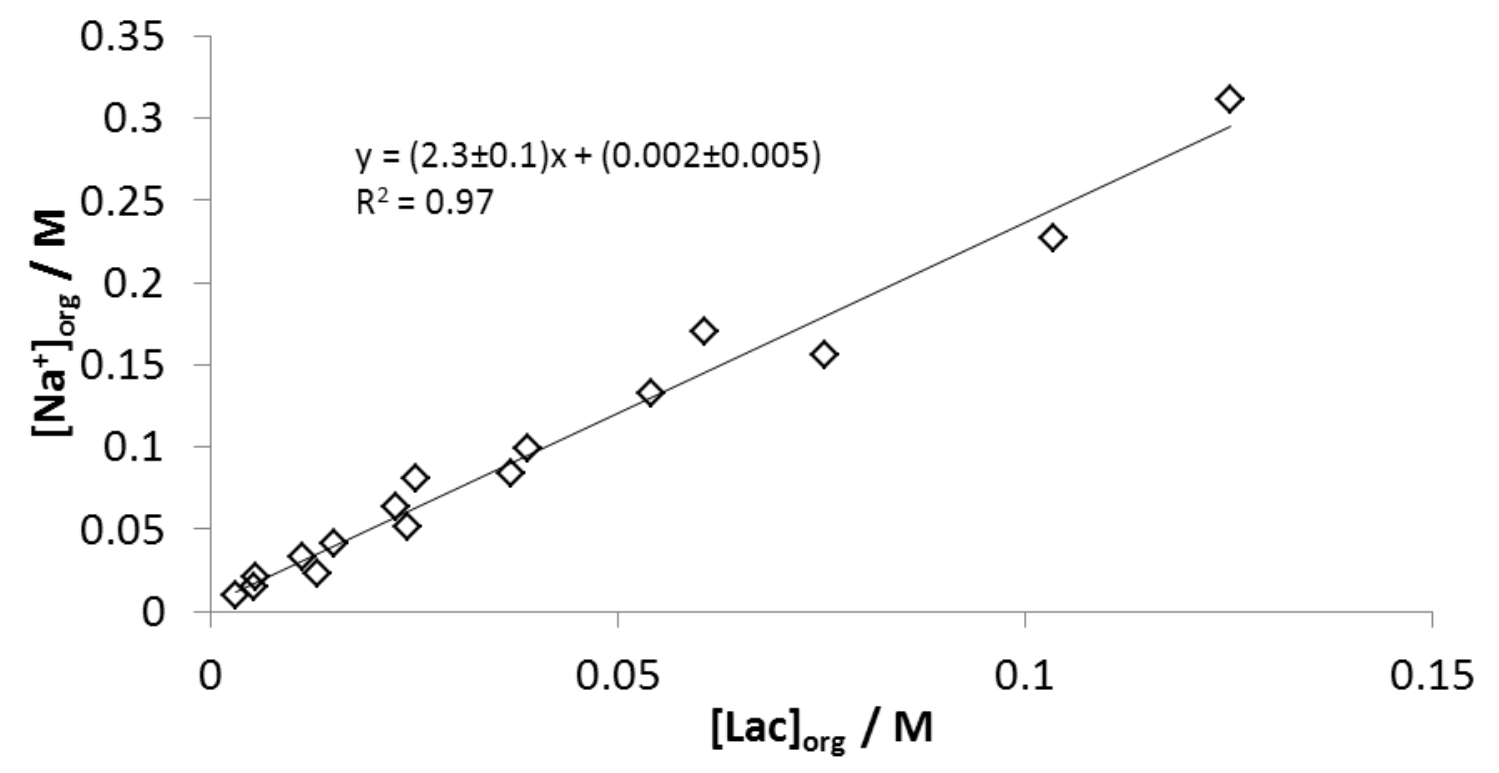

Figure 2.20- Correlation between organic phase $\mathrm{Na}^{+}$and lactate. This slope is not indicative of the stoichiometry between $\mathrm{Na}^{+}$and lactate in the organic phase, but indicates that $\mathrm{Na}+$ must exist as both a lactate and HA salt.

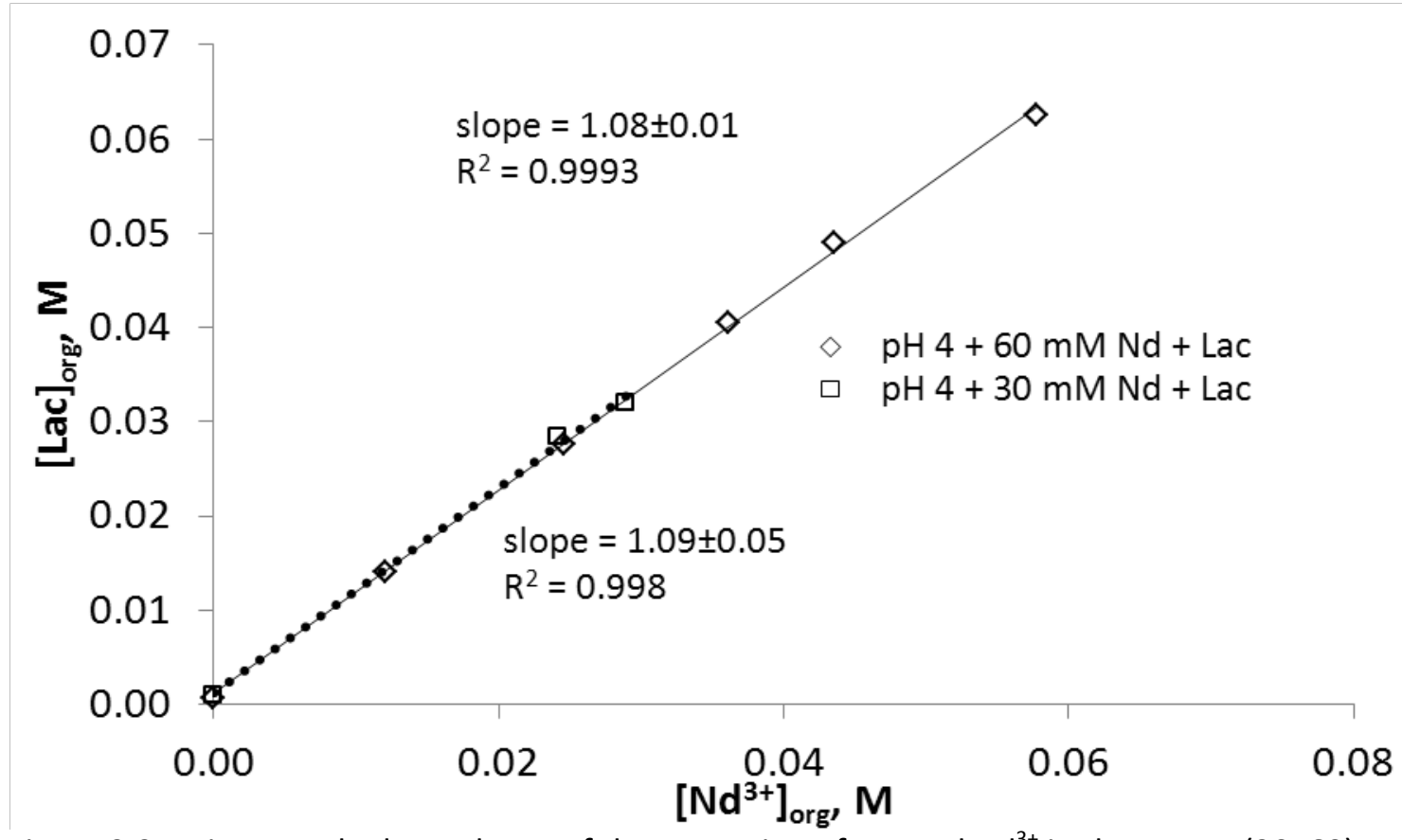

Figure 2.21- Linear scale dependence of the extraction of $\operatorname{Lac}$ and $\mathrm{Nd}^{3+}$ in the $\mathrm{pH} 4+(30,60) \mathrm{mM}$ $\mathrm{Nd}+$ Lac systems. This data is limited to the data points where the $\mathrm{Nd}(\mathrm{Lac})(\mathrm{HA})_{2}$ species was shown to be present. 
Under the conditions of the high organic loading (i.e. low HA concentration) with $\mathrm{NdLac}^{2+}$, the formation of the high-order aggregates was observed as suggested by VPO data (see section 2.5.1 and Figure 2.13 for details). The average aggregation number decreased from $19 \pm 4$ and $94 \pm 42$ at the highest loading to $2.75 \pm 0.04$ and $2.82 \pm 0.06$ at the intermediate loading for the $\mathrm{pH} 4+(30,60) \mathrm{mM} \mathrm{Nd}+$ Lac systems, respectively.

\subsubsection{Water Distribution Results}

To explore the hydration of organic phase species in the studied extraction systems, the water content of the post-contact organic extraction phases was measured by Karl Fischer titration. These results were compared with the water content of the corresponding pre-contact water-washed organic solutions. It was discovered that in all extraction systems except the $\mathrm{pH} 4$ $+(0,30,60) \mathrm{mM} \mathrm{Nd}+$ Lac systems, post-contact organic water concentration was within experimental error equal to the pre-contact organic water concentration in the water-washed $\mathrm{HA}$ /isooctane solutions due to the hydration of HA (see Table 2.17-18). The finding that organic water content did not increase upon $\mathrm{Nd}^{3+}$ extraction is consistent with the results in the $\mathrm{HCl}$ systems (see section 2.4.2.3 for details).

The result that no increase of the organic water was observed for the equilibrated organic phases in the $\mathrm{pH} 4+0 \mathrm{mM} \mathrm{Nd}+\mathrm{HCl}$ system that exhibited $\mathrm{Na}^{+}$extraction suggests very little water co-extraction with the $\mathrm{Na}^{+}$ion. This is most likely due to the small $\mathrm{Na}^{+}$extraction and the limits of detection of water for that experiment. In the extraction systems $\mathrm{pH} 4+(0,30,60) \mathrm{mM}$ $\mathrm{Nd}+$ Lac that exhibited pronounced Lac transport into the solvent phase, equilibrium organic water concentration was significantly greater than in the corresponding washed solvent solutions (see Figure 2.22) where $\left[\mathrm{H}_{2} \mathrm{O}\right]_{\text {org }}$ is plotted as a function of HA concentration. The excess water in the organic phase is not associated with $\mathrm{Nd}^{3+}$ or $\mathrm{Na}^{+}$extraction. Due to the 
similarity of the magnitude of excess water with the trends in lactate extraction, the correlation between water and lactate extraction was analysed. The trends showed a good correlation for the $\mathrm{pH} 4+(0,30) \mathrm{mM} \mathrm{Nd}+$ Lac systems when the conditions where $\mathrm{Na}^{+}$extraction was observed. The linear scale slope (see Figure 2.23 ) is indicative of the ratio of water-to-Lac in the organic phase and was observed at $7.1 \pm 0.2, R^{2}=0.993$ and $5.6 \pm 0.1, R^{2}=0.998$ for $p H 4+(0$, 30) $\mathrm{mM} \mathrm{Nd}+$ Lac systems, respectively. These slope results indicate a stoichiometry between water and lactate in the organic phase of between 5 and 7, which is dependent on the system; the $0 \mathrm{mM}$ Nd system giving a slope of 7 and the $30 \mathrm{mM} \mathrm{Nd}$ system giving a slope of 5.

Table 2.17- Organic phase water concentration for the $\mathrm{pH} 1+(0,30) \mathrm{mM} \mathrm{Nd}+$ Lac systems.

\begin{tabular}{|c|c|c|c|}
\hline & & \multicolumn{2}{|c|}{ pH 1 + Lac } \\
\cline { 3 - 4 } & Washed Solvent & $\mathbf{0 ~ m M ~ N d}$ & $30 \mathrm{mM} \mathrm{Nd}$ \\
\hline$[\mathrm{HA}]_{\text {org }} / \mathrm{M}$ & {$\left[\mathrm{H}_{2} \mathrm{O}\right]_{\text {org }} / \mathrm{M}$} & {$\left[\mathrm{H}_{2} \mathrm{O}\right]_{\text {org }} / \mathrm{M}$} & {$\left[\mathrm{H}_{2} \mathrm{O}\right]_{\text {org }} / \mathrm{M}$} \\
\hline 0 & $0.000 \pm 0.003$ & $0.004 \pm 0.003$ & $0.003 \pm 0.003$ \\
\hline 0.025 & $0.001 \pm 0.003$ & $0.004 \pm 0.003$ & $0.003 \pm 0.003$ \\
\hline 0.05 & $0.003 \pm 0.003$ & $0.005 \pm 0.003$ & $0.005 \pm 0.003$ \\
\hline 0.075 & $0.004 \pm 0.003$ & $0.007 \pm 0.003$ & $0.007 \pm 0.003$ \\
\hline 0.09 & $0.007 \pm 0.003$ & $0.007 \pm 0.003$ & $0.008 \pm 0.003$ \\
\hline 0.125 & $0.011 \pm 0.003$ & $0.010 \pm 0.003$ & $0.011 \pm 0.000$ \\
\hline 0.15 & $0.014 \pm 0.003$ & $0.012 \pm 0.003$ & $0.016 \pm 0.003$ \\
\hline 0.2 & $0.021 \pm 0.003$ & $0.019 \pm 0.003$ & $0.023 \pm 0.003$ \\
\hline 0.25 & $0.023 \pm 0.003$ & $0.026 \pm 0.003$ & $0.030 \pm 0.003$ \\
\hline 0.3 & $0.029 \pm 0.005$ & $0.042 \pm 0.005$ & $0.039 \pm 0.006$ \\
\hline 0.4 & $0.053 \pm 0.007$ & $0.052 \pm 0.007$ & $0.062 \pm 0.008$ \\
\hline 0.5 & $0.07 \pm 0.01$ & $0.08 \pm 0.01$ & $0.08 \pm 0.01$ \\
\hline 1 & $0.21 \pm 0.03$ & $0.21 \pm 0.03$ & $0.23 \pm 0.04$ \\
\hline
\end{tabular}


Table 2.18- Organic phase water concentration for the $\mathrm{pH} 4+(0,30,60) \mathrm{mM} \mathrm{Nd}+$ Lac systems.

\begin{tabular}{|c|c|c|c|}
\hline & \multicolumn{3}{|c|}{ pH 4 + Lac } \\
\hline$[\mathrm{HA}]_{\text {org }} / \mathrm{M}$ & $\mathbf{0 ~} \mathrm{mM} \mathrm{Nd}$ & $\mathbf{3 0} \mathrm{mM}$ Nd & $60 \mathrm{mM} \mathrm{Nd}$ \\
\hline 0 & $0.002 \pm 0.003$ & $0.002 \pm 0.003$ & $0.003 \pm 0.003$ \\
\hline 0.025 & $0.002 \pm 0.003$ & $0.007 \pm 0.003$ & $0.015 \pm 0.003$ \\
\hline 0.05 & $0.009 \pm 0.003$ & $0.027 \pm 0.003$ & $0.028 \pm 0.003$ \\
\hline 0.075 & $0.024 \pm 0.003$ & $0.031 \pm 0.003$ & $0.039 \pm 0.003$ \\
\hline 0.09 & $0.034 \pm 0.003$ & $0.029 \pm 0.003$ & $0.047 \pm 0.003$ \\
\hline 0.125 & $0.064 \pm 0.003$ & $0.027 \pm 0.003$ & $0.064 \pm 0.003$ \\
\hline 0.15 & $0.095 \pm 0.003$ & $0.027 \pm 0.003$ & $0.062 \pm 0.003$ \\
\hline 0.2 & $0.109 \pm 0.003$ & $0.027 \pm 0.003$ & $0.059 \pm 0.003$ \\
\hline 0.25 & $0.172 \pm 0.003$ & $0.032 \pm 0.003$ & $0.059 \pm 0.003$ \\
\hline 0.3 & $0.258 \pm 0.006$ & $0.055 \pm 0.006$ & $0.060 \pm 0.004$ \\
\hline 0.4 & $0.373 \pm 0.006$ & $0.13 \pm 0.01$ & $0.070 \pm 0.006$ \\
\hline 0.5 & $0.49 \pm 0.01$ & $0.21 \pm 0.01$ & $0.101 \pm 0.008$ \\
\hline 1 & $1.07 \pm 0.02$ & $0.73 \pm 0.01$ & $0.48 \pm 0.03$ \\
\hline
\end{tabular}

Trends between the water and $\mathrm{Nd}^{3+}$ and $\mathrm{Na}^{+}$are also of interest in understanding the hydration of metal ions in solution. The $\mathrm{HCl}$ systems showed no water extraction above the washed solvent hydration values as described in section 2.4.2.3. The presence of excess water in the organic phase in the low $[\mathrm{HA}]_{\text {org }}$ values (see Figure 2.22 ) indicates that the $\mathrm{Nd}^{3+}$ organic phase species is hydrated. Due to the high loading of the HA by $\mathrm{Nd}^{3+}$ it was assumed that any hydration water associated with the HA dimers would be lost by complexation with $\mathrm{Nd}^{3+}$, so the total organic phase water concentrations were used for this correlation. Linear scale correlations (Figure 2.24) between the organic $\mathrm{Nd}^{3+}$ and water concentrations showed that there was 1 water of hydration present in this species (slope $=1.01 \pm 0.01, R^{2}=0.9999 ; 1.04 \pm$ $0.02, \mathrm{R}^{2}=0.998$; for $\mathrm{pH} 4+(30,60) \mathrm{mM} \mathrm{Nd}+$ Lac systems, respectively; see Figure 2.24). 

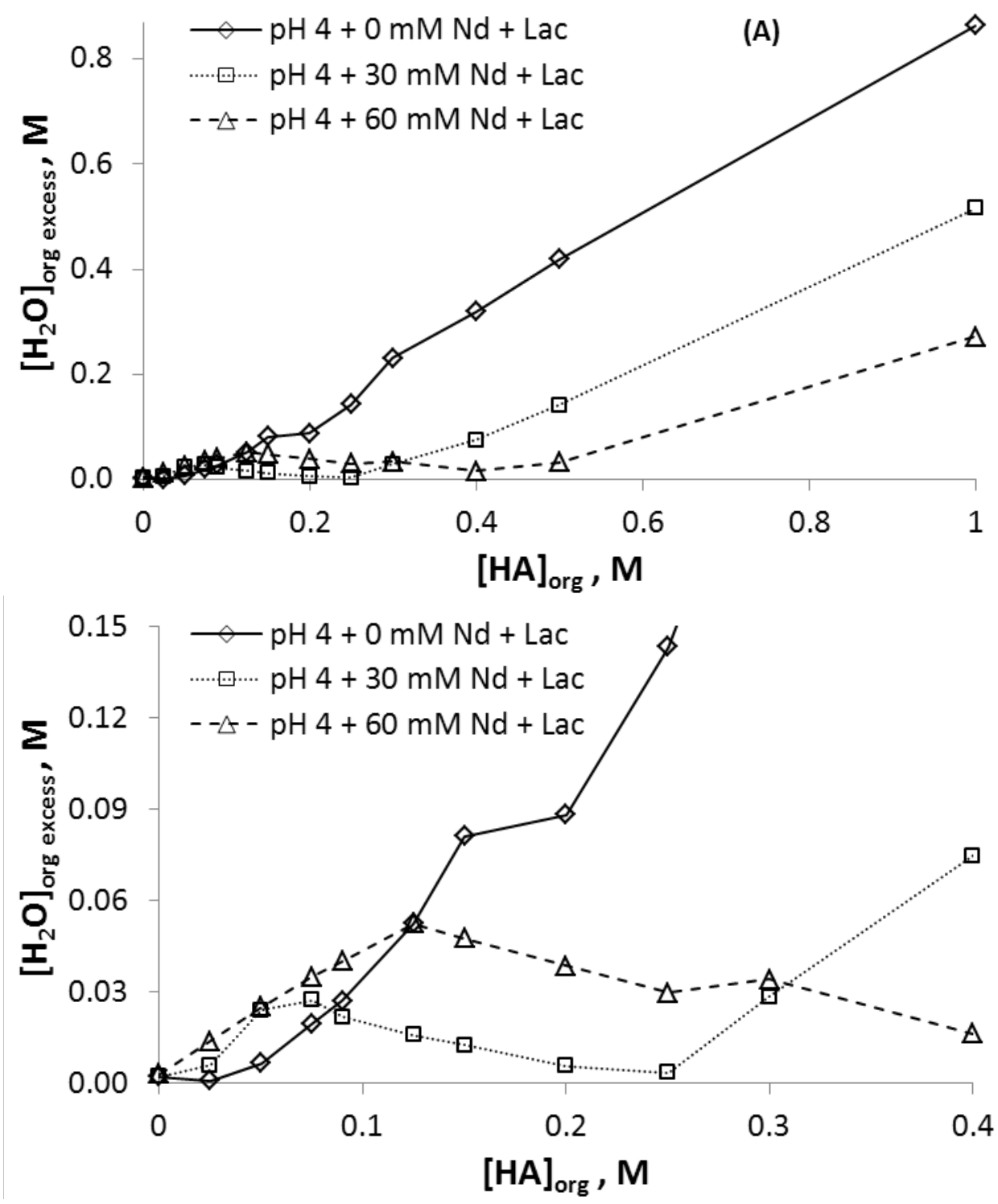

Figure 2.22- The extraction of water in excess of the HA hydration of the washed solvent for the $\mathrm{pH} 4+(0,30,60) \mathrm{mM} \mathrm{Nd}+$ Lac systems. 


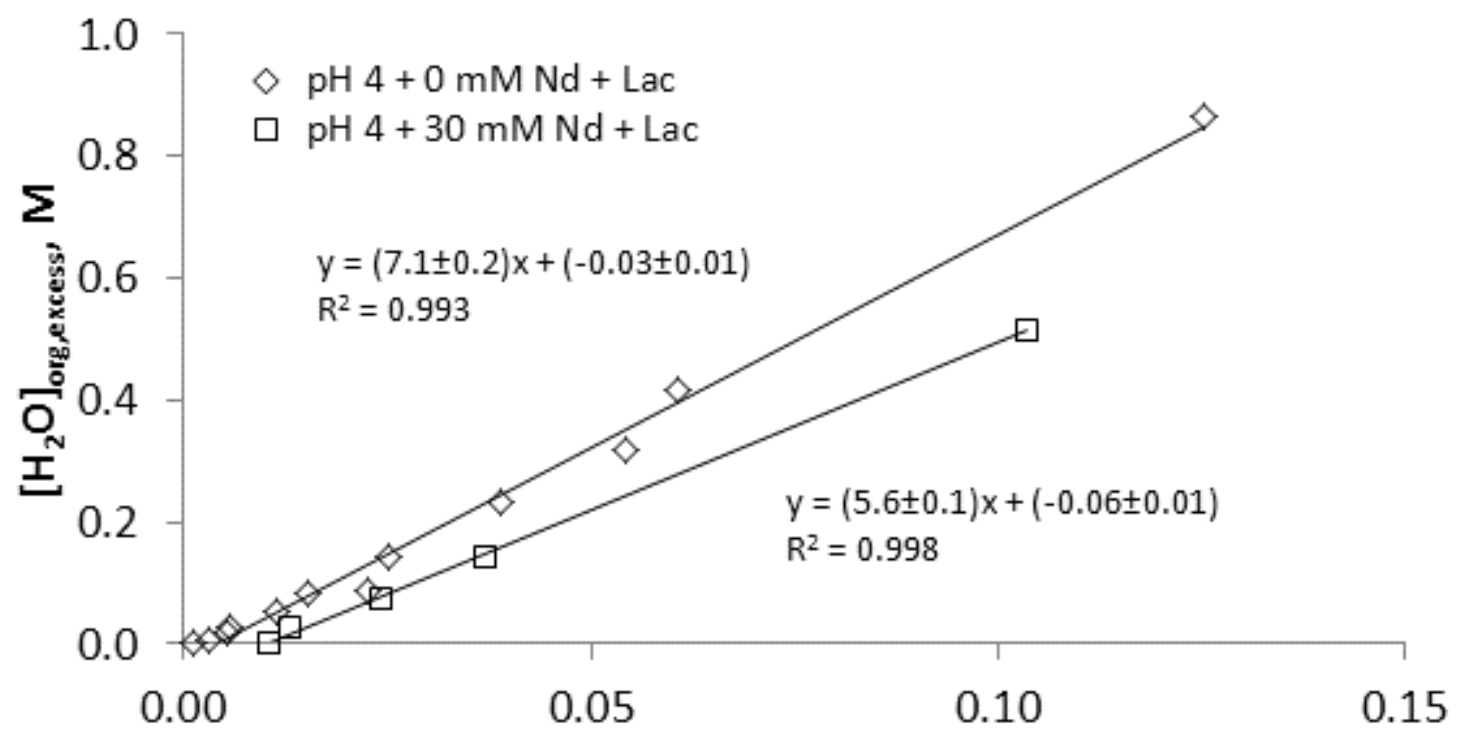

[Lac] $]_{\text {org eq }}, \mathbf{M}$

Figure 2.23- Dependence of organic $\mathrm{H}_{2} \mathrm{O}$ concentration on the organic Lactate molarity. These data were selected from the entire $\mathrm{pH} 4+(0,30) \mathrm{mM} \mathrm{Nd}+$ Lac systems under conditions of NaLac extraction.

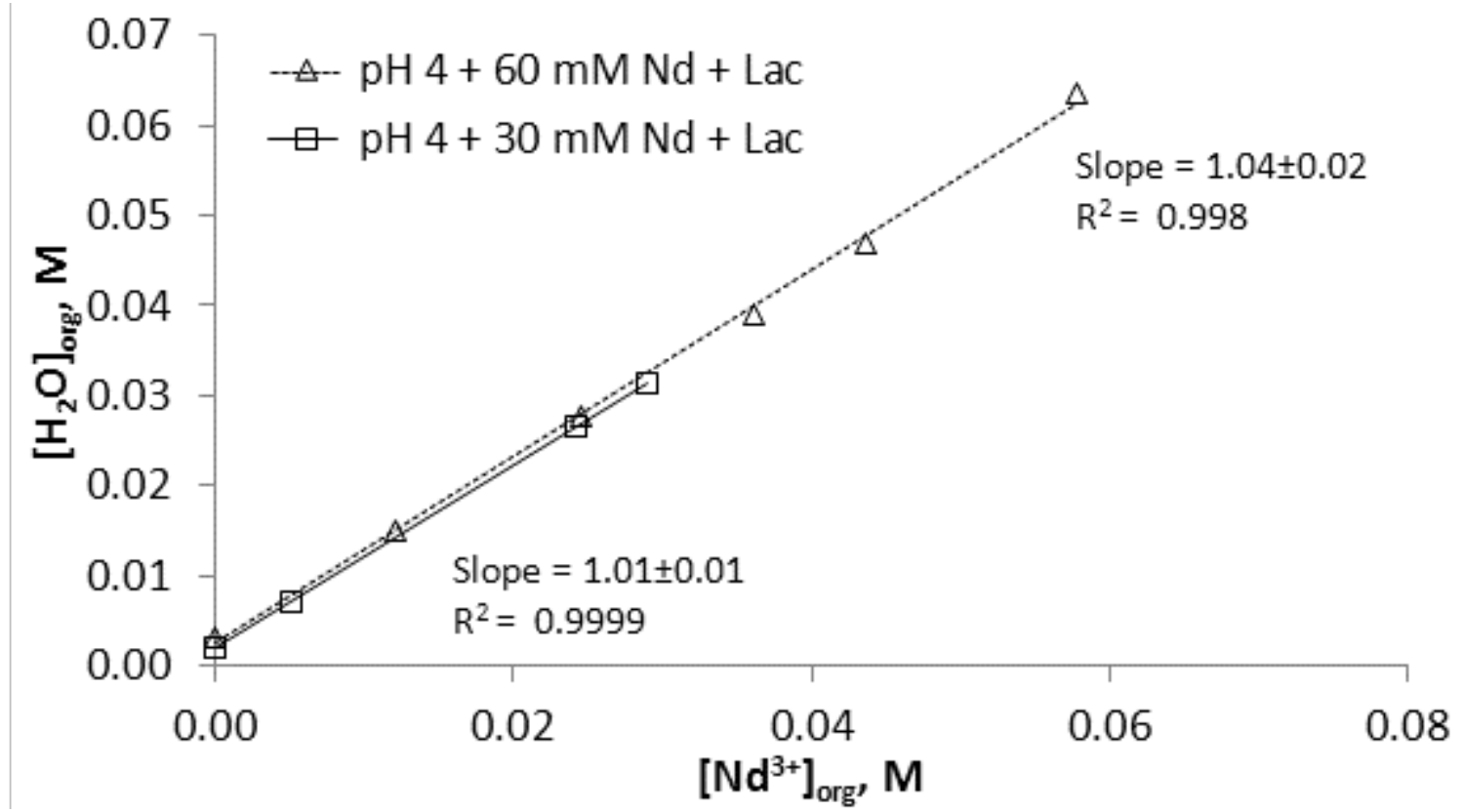

Figure 2.24- Linear scale correlation between organic phase $\mathrm{Nd}^{3+}$ and water concentration in the $\mathrm{pH} 4+(30,60) \mathrm{mM} \mathrm{Nd}+$ Lac systems. This data was selected from the $\mathrm{pH} 4+(30,60) \mathrm{mM} \mathrm{Nd}+$ Lac systems where $\mathrm{Nd}(\mathrm{Lac})(\mathrm{HA})_{2}$ was shown to exist in the organic phase. Data points used were restricted to the $[\mathrm{HA}]_{\text {org }}$ of $0-0.075$ and $0-0.125 \mathrm{M} \mathrm{HA}$ respectively. 
A correlation between the water and $\mathrm{Na}^{+}$extraction was prepared using the same assumption: high loading eliminates the HA hydration water eliminating the need to correct the extracted water concentration. The correlation showed that $\mathrm{Na}^{+}$extracted in the Lac systems has a hydration number of 3 , with the slope being the ratio of water-to- $\mathrm{Na}^{+}$in the organic phase (slope $=3.3 \pm 0.1, \mathrm{R}^{2}=0.98$; for $\mathrm{pH} 4+(0,30,60) \mathrm{mM} \mathrm{Nd}+$ Lac systems, when fit as 1 data set; see Figure 2.25).

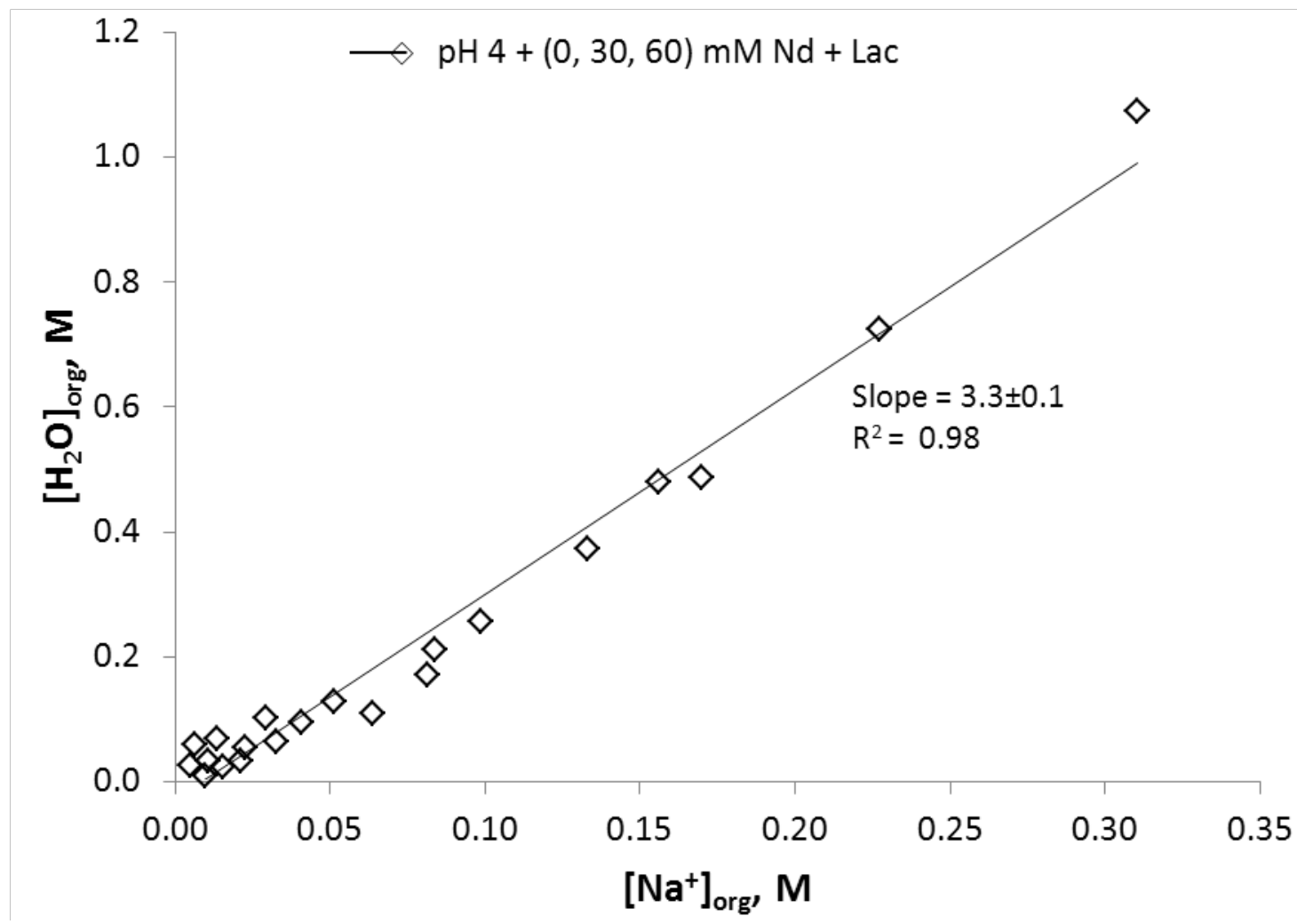

Figure 2.25- Linear scale correlation of organic phase water and $\mathrm{Na}^{+}$concentrations from the $\mathrm{pH}$ $4+(0,30,60) \mathrm{mM} \mathrm{Nd}+$ Lac systems using only data where $\mathrm{Na}^{+}$extraction was observed. $[\mathrm{HA}]_{\text {org }}$ utilized in this plot are: $0.025-1,0.2-1$ and $0.3-1 \mathrm{M}$ HA respectively. 


\subsubsection{Visible Spectroscopy}

\subsubsection{Aqueous Phase}

The absorbance bands observed for the aqueous phase $\mathrm{Nd}^{3+}$ ions in the $\mathrm{pH} 1+30 \mathrm{mM} \mathrm{Nd}+\mathrm{Lac}$ system (see Table 2.19) were the same as those observed for the $\mathrm{pH}(1,4)+30 \mathrm{mM} \mathrm{Nd}+\mathrm{HCl}$ system (see Table 2.6 and Figure 2.9). In the $\mathrm{pH} 4+(30,60) \mathrm{mM} \mathrm{Nd}+$ Lac systems, where $\mathrm{Nd}^{3+}$ complexation occurs in the aqueous phase, certain bands appear and others disappear in the aqueous phase absorbance spectrum. All bands are unchanged from those observed for the $\mathrm{pH}$ $1+30 \mathrm{mM} \mathrm{Nd}+(\mathrm{Lac}, \mathrm{HCl})$ systems except the following noted bands which shift in location when free lactate in solution complexes the $\mathrm{Nd}^{3+}$ in the $\mathrm{pH} 4+$ Lac systems: $575.28 \mathrm{~nm} \rightarrow$ $580.42 \mathrm{~nm}$ (with a shoulder at $575 \mathrm{~nm}$ ), $797.94 \mathrm{~nm}$ (with a shoulder at $794 \mathrm{~nm}$ ) $\rightarrow 794.50 \mathrm{~nm}$, and $870.18 \mathrm{~nm} \rightarrow 865.98 \mathrm{~nm}$ (with shoulder at $870 \mathrm{~nm}$ ) (compare the two solid trace spectra in Figure 2.26).

Table 2.19- Molar absorptivities observed for $\mathrm{Nd}^{3+}$ in the aqueous phase. $\mathrm{R}^{2}$ ranged from 0.9970.9999 for the listed molar absorptivities shown in this table.

\begin{tabular}{|c|c|c|c|c|}
\hline \multirow{4}{*}{$\begin{array}{l}\text { Wavelength } \\
(\mathrm{nm})\end{array}$} & \multirow{4}{*}{$\begin{array}{c}\mathrm{pH} 1+30 \mathrm{mM} \mathrm{Nd}+\mathrm{Lac} \\
\varepsilon \\
\left(\mathrm{L} \mathrm{mol}^{-1} \mathrm{~cm}^{-1}\right)\end{array}$} & \multirow{4}{*}{$\begin{array}{l}\text { Wavelength } \\
(\mathrm{nm})\end{array}$} & \multicolumn{2}{|c|}{ pH $4+$ Lac } \\
\hline & & & \multirow{3}{*}{$\begin{array}{c}30 \mathrm{mM} \mathrm{Nd} \\
\varepsilon \\
\left(\mathrm{L} \mathrm{mol}^{-1} \mathrm{~cm}^{-1}\right)\end{array}$} & \multirow{3}{*}{$\begin{array}{c}60 \mathrm{mM} \mathrm{Nd}^{-1} \\
\left(\mathrm{~L} \mathrm{~mol}^{-1} \mathrm{~cm}^{-1}\right) \\
\end{array}$} \\
\hline & & & & \\
\hline & & & & \\
\hline 511.83 & $2.05 \pm 0.03$ & 511.83 & $2.28 \pm 0.01$ & $2.207 \pm 0.005$ \\
\hline 521.67 & $4.24 \pm 0.02$ & 523.37 & $5.39 \pm 0.02$ & $5.15 \pm 0.01$ \\
\hline 575.28 & $7.65 \pm 0.03$ & 580.42 & $14.65 \pm 0.02$ & $14.04 \pm 0.02$ \\
\hline 680.15 & $0.494 \pm 0.007$ & 678.54 & $0.53 \pm 0.01$ & $0.525 \pm 0.005$ \\
\hline 732.31 & $3.96 \pm 0.02$ & 733.92 & $4.95 \pm 0.01$ & $4.88 \pm 0.01$ \\
\hline 740.66 & $6.99 \pm 0.02$ & 741.63 & $7.64 \pm 0.01$ & $7.68 \pm 0.02$ \\
\hline 794.5 & $9.94 \pm 0.03$ & 797.94 & $10.64 \pm 0.01$ & $10.407 \pm 0.005$ \\
\hline 801.37 & $6.54 \pm 0.02$ & & & \\
\hline 865.98 & $3.50 \pm 0.03$ & 870.18 & $3.02 \pm 0.01$ & $2.90 \pm 0.01$ \\
\hline
\end{tabular}




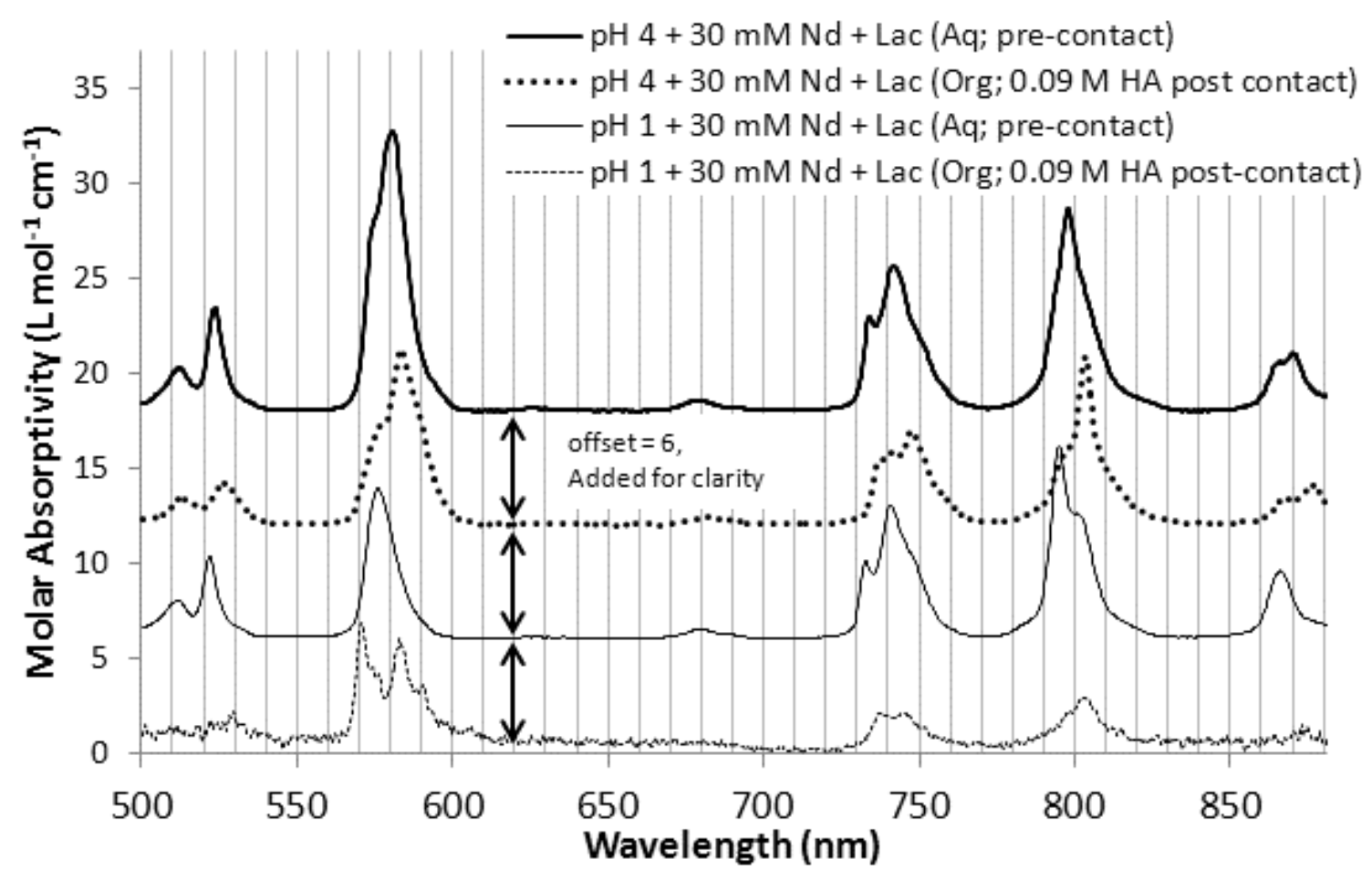

Figure 2.26- Comparison of the molar absorptivities that are representative of those observed for $\mathrm{pH}(1,4)+(30,60) \mathrm{mM} \mathrm{Nd}+$ Lac systems.

\subsubsection{Organic Phase}

The spectra obtained from the organic phase visible absorbance spectroscopy were converted to molar absorptivity scale using the $\left[\mathrm{Nd}^{3+}\right]_{\text {org }}$ measured for each sample. In the $\mathrm{pH} 1$ + $30 \mathrm{mM} \mathrm{Nd}+$ Lac system we observed the same spectral profile and shifts were observed as for the $\mathrm{pH}(1,4) 30 \mathrm{mM} \mathrm{Nd}+\mathrm{HCl}$ systems, including the decrease of the absorptivity in the $570 \mathrm{~nm}$ band with the increase of ligand concentration that is discussed in section 0 . In the $\mathrm{pH} 4+(30,60) \mathrm{mM} \mathrm{Nd}+$ Lac systems at low $[\mathrm{HA}]_{\text {org }}$ the spectral profile was significantly different than previously observed systems. Instead of 5 bands in the 560-615 nm window, there were two, the strongest at 585 , and a shoulder at $577 \mathrm{~nm}$ (see Figure 2.27). This spectral profile is consistent with a different coordination environment surrounding the $\mathrm{Nd}^{3+}$ metal 
center. Based on the other analyses in this work (see sections 2.5.2.1 and 2.5.2.3), the species present at $0.075 \mathrm{M} \mathrm{HA}$ portrayed in Figure 2.27 is $\operatorname{NdLac}(\mathrm{A})_{2}$. This species contains three (one Lactate plus two A) bidentate ligands surrounding the metal center. In papers that show complexes with this coordination chemistry, a similar spectral profile is observed $\left[\right.$ for $\operatorname{Nd}(A)_{3}{ }^{[69]}$, $\mathrm{Nd}(\text { Cyanex301) })_{3}{ }^{[64]}$ and $\mathrm{Nd}\left(\right.$ Cyanex302) $\left.{ }_{3}{ }^{[64]}\right]$.

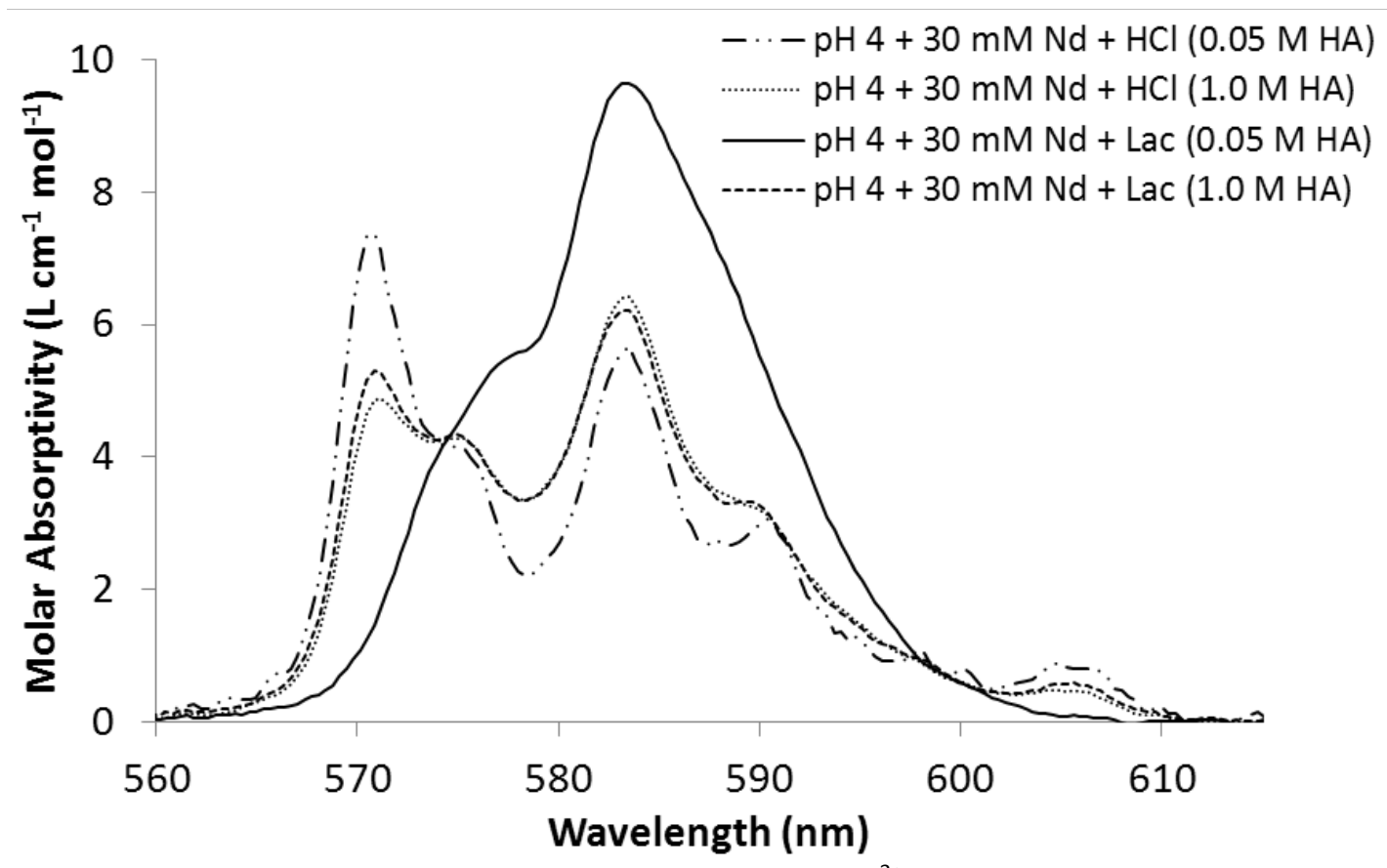

Figure 2.27- A comparison of the molar absorptivities for $\mathrm{Nd}^{3+}$ in the loaded organic phase solutions in the $\mathrm{HCl}$ and Lac systems at $\mathrm{pH} 4+30 \mathrm{mM} \mathrm{Nd}$ systems.

\subsubsection{Fourier Transform Infrared Spectrscopy of the Organic Phase}

The FTIR of the organic phase contained previously observed bands in the $\mathrm{pH} 1+$ Lac system as the $\mathrm{pH} 1+\mathrm{HCl}$ systems, but showed new bands that appeared in the high loading regions of the $\mathrm{pH} 4+$ Lac system, just as in the visible absorbance of the organic phase. In order to focus on 
the extracted complexes in the organic phase, the iso-octane solvent bands were subtracted from the loaded organic phase spectra. For comparison purposes, the spectral intensities were normalized (divided) by $[\mathrm{HA}]_{\text {org }}$ resulting in the HA molar absorbance spectra. In all systems, the two peaks of highest interest for our study are the 1230 and $1035 \mathrm{~cm}^{-1}$ associated with $\mathrm{P}=\mathrm{O}$ and P-O stretching, respectively (see Figure 2.28 for spectra).

The $\mathrm{Nd}^{3+}$ complexation by $\mathrm{HA}$ leads to a withdrawal of electron density in the $\mathrm{P}=\mathrm{O}$ bond resulting in the decrease of the stretching mode frequency. This is supported by the shift in the 1230 band to $1200 \mathrm{~cm}^{-1}$ in all systems with $\mathrm{Nd}^{3+}$ extraction. A smaller shift is observed in systems that show $\mathrm{Na}^{+}$extraction with the maximum at $1222 \mathrm{~cm}^{-1}$. This was supported by a shift from 1230 to broader $1222 \mathrm{~cm}^{-1}$ band in $\mathrm{pH} 4+0 \mathrm{mM} \mathrm{Nd}+\mathrm{Lac}$ and the $\mathrm{pH} 4+30 \mathrm{mM} \mathrm{Nd}+\mathrm{Lac}$ systems at high $[\mathrm{HA}]_{\text {org }}$. Lactate co-extraction with $\mathrm{Nd}^{+3}$ as $\mathrm{NdLac}^{2+}$ complex species in the $\mathrm{pH} 4+$ $30 \mathrm{mM} \mathrm{Nd}+$ Lac systems at low $[\mathrm{HA}]_{\text {org }}$ was confirmed by the appearance of FTIR band at 1565 $\mathrm{cm}^{-1}$ that is indicative of bi-dentate Lac complexation with $\mathrm{Nd}^{3+} \cdot{ }^{[106]} \mathrm{The} \mathrm{pH} 4+30 \mathrm{mM} \mathrm{Nd}+\mathrm{Lac}$ systems with high HA concentration exhibited a band at $1120 \mathrm{~cm}^{-1}$ indicative of the lactate secondary alcohol group. ${ }^{[106]}$ This band appeared at $1116 \mathrm{~cm}^{-1}$ in the $\mathrm{pH} 4+0 \mathrm{mM} \mathrm{Nd}+\mathrm{Lac}$ system associated with lactate co-extracted as NaLac. Formation of highly aggregated $\left(\mathrm{NdLacA}_{2}\right)_{\times}$species leads to bridging -O-P-O- groups as evident from the appearance of the $1100 \mathrm{~cm}^{-1}$ band $^{[71]}$ in the $\mathrm{pH} 4+30 \mathrm{mM} \mathrm{Nd}+$ Lac systems and a $1097 \mathrm{~cm}^{-1}$ band for $\mathrm{pH} 4+30$ $\mathrm{mM} \mathrm{Nd}+\mathrm{HCl}$ systems at low $[\mathrm{HA}]_{\mathrm{org}}$. Although the $\mathrm{pH} 4+60 \mathrm{mM} \mathrm{Nd}+\mathrm{Lac}$ system was not featured in Figure 2.28, the same spectral shifts that were described in $\mathrm{pH} 4+30 \mathrm{mM} \mathrm{Nd}+\mathrm{Lac}$ system were also observed in the $\mathrm{pH} 4+60 \mathrm{mM} \mathrm{Nd}+$ Lac system. 


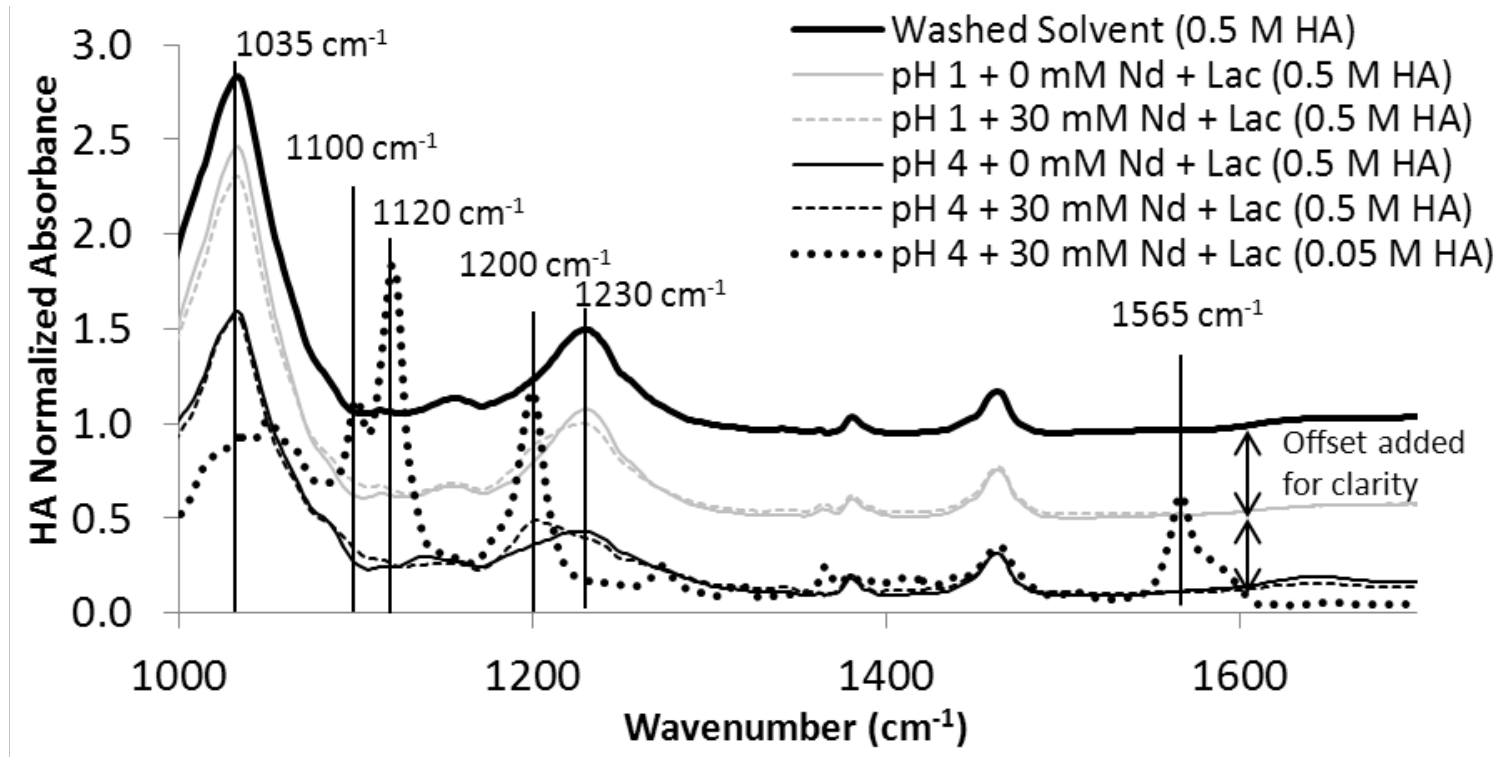

Figure 2.28- A comparison of the FTIR spectra for the $\mathrm{pH}(1,4)+(0,30) \mathrm{mM} \mathrm{Nd}+$ Lac systems and the washed solvent.

\subsubsection{Nuclear Magnetic Resonance Spectroscopy of the Organic Phase}

Organic phase samples prepared as described in section 2.2 .15 were probed using ${ }^{23} \mathrm{Na},{ }^{13} \mathrm{C}$ and ${ }^{31} \mathrm{P}$ NMR. The initial results from the two samples were analysed to assess the feasibility of utilizing this technique to better understand the speciation of the organic phase. Most spectroscopic features are difficult to identify without running a sample set probing the variable loading of the system.

A background experiment was performed to verify that $\mathrm{La}^{3+}$ extraction causes the formation of the large aggregate species that $\mathrm{Nd}^{3+}$ did at high loading. Batch contact experiments were performed that were identical to the $\mathrm{pH} 4+(30,60) \mathrm{mM} \mathrm{Nd}+$ Lac systems, except $\mathrm{La}^{3+}$ was substituted for $\mathrm{Nd}^{3+}$. The average aggregation of the organic phase solutions was determined by vapour pressure osmometry as described in section 2.2.13. The results obtained are shown in Figure 2.29. The aggregation results show that $\mathrm{La}^{3+}$ does form high-order aggregates just as $\mathrm{Nd}^{3+}$ 
under high loading conditions (compare Figure 2.13 and Figure 2.29). It was assumed that all the other distribution trends with $\mathrm{Na}^{+}$, $\mathrm{Lac}, \mathrm{H}_{2} \mathrm{O}$, and $\mathrm{La}^{3+}$ were also the same between the two systems. Based on the aggregation results, two representative samples for the high loading/high aggregate condition, and the lower loading condition were selected: $\mathrm{pH} 4+60 \mathrm{mM} \mathrm{La}+\mathrm{Lac}$ at $0.125 \mathrm{M}[\mathrm{HA}]_{\text {org }}$ and $\mathrm{pH} 4+30 \mathrm{mM} \mathrm{La}+$ Lac at $1 \mathrm{M}[\mathrm{HA}]_{\text {org }}$, respectively.

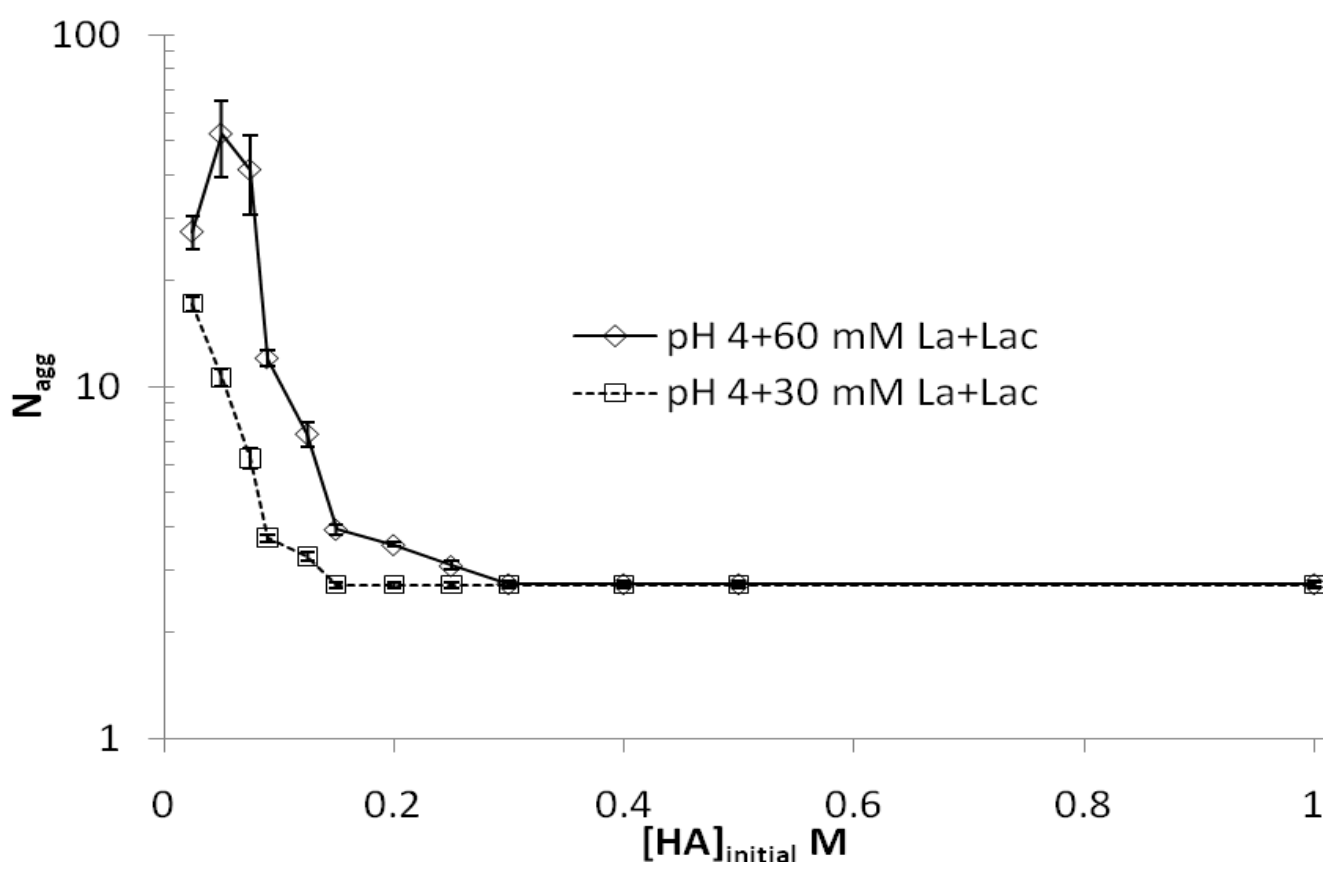

Figure 2.29- Aggregation results for the $\mathrm{pH} 4+(30,60) \mathrm{mM}$ La + Lac systems.

The ${ }^{23} \mathrm{Na}$ scan of the $\mathrm{pH} 4+30 \mathrm{mM}$ La + Lac (1 M $[\mathrm{HA}]_{\text {org }}$ ) low-loading sample showed a single peak centered at $1.864 \mathrm{ppm}$. It was noted in the ${ }^{22} \mathrm{Na}$ distribution section (2.4.2.2) that $\mathrm{Na}^{+}$was most likely extracted by two mechanisms due to the presence of Lac in the same organic phases in a ratio of $2.7 \pm 0.4 \mathrm{Na}$ :Lac (see section 2.4.2.2 for details). One peak in the NMR spectrum may be indicative of one species, or there may be two present, provided that the 
exchange rate between the species is more rapid than the NMR relaxation time, making it impossible to observe two peaks. With more effort in adjusting the relaxation time, two peaks may be resolved, but in the current experiment they were not. No ${ }^{23} \mathrm{Na} N M R$ was performed for the $\mathrm{pH} 4+60 \mathrm{mM} \mathrm{La}+\operatorname{Lac}\left(0.125 \mathrm{M}[\mathrm{HA}]_{\text {org }}\right)$ sample because in the analogous $\mathrm{Nd}^{3+}$ system there was no ${ }^{23} \mathrm{Na}$ present.

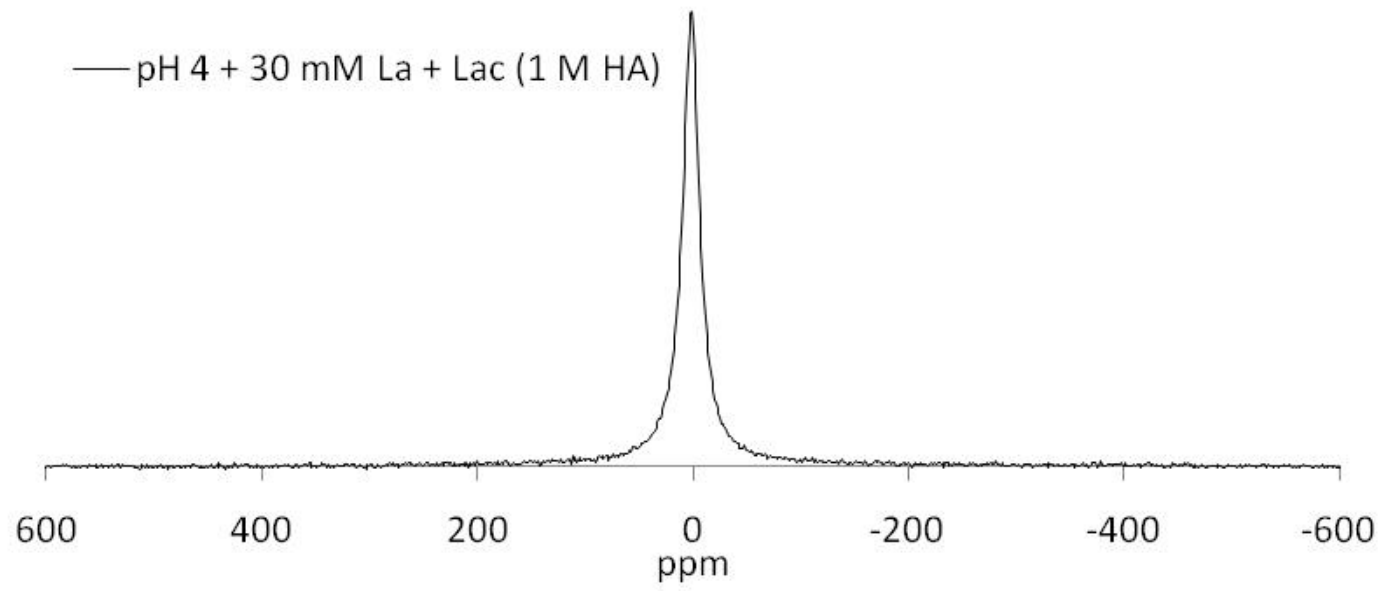

Figure $2.30-{ }^{23} \mathrm{Na}$ NMR scan for pH $4+30 \mathrm{mM}$ La + Lac (1.0 M HA) sample.

The ${ }^{31} \mathrm{P}$ NMR probed the environment of the ${ }^{31} \mathrm{P}$ in the HA molecule, and would thus offer understanding of what species the HA was involved in the organic phase. Both samples were analysed by this method and produced the spectra shown in Figure 2.31. The spectra obtained show peaks with different chemical shifts $[0.477 \mathrm{ppm}$ in the pH $4+30 \mathrm{mM} \mathrm{La}+\operatorname{Lac}(1.0 \mathrm{M} \mathrm{HA})$ sample and 1.456, -0.528 , and $-6.305 \mathrm{ppm}$ in the pH $4+60 \mathrm{mM} \mathrm{La}+\operatorname{Lac}(0.125 \mathrm{M} \mathrm{HA})$ sample], which would be indicative of different speciation in each sample. This is the expected result, with the $\mathrm{pH} 4+60 \mathrm{mM}$ La $+\operatorname{Lac}(0.125 \mathrm{M} \mathrm{HA})$ sample showing the high aggregation speciation, and the $\mathrm{pH} 4+30 \mathrm{mM} \mathrm{La}+\operatorname{Lac}(1.0 \mathrm{M} \mathrm{HA})$ low aggregation speciation. It is difficult to analyze 
these spectra without performing an array of NMR measurements over a dynamic range of loading, but it can be stated that these results show that the ${ }^{31} \mathrm{P}$ NMR spectra are sensitive to the speciation changes in the organic phase. These results definitely warrant further research with a larger sample array.

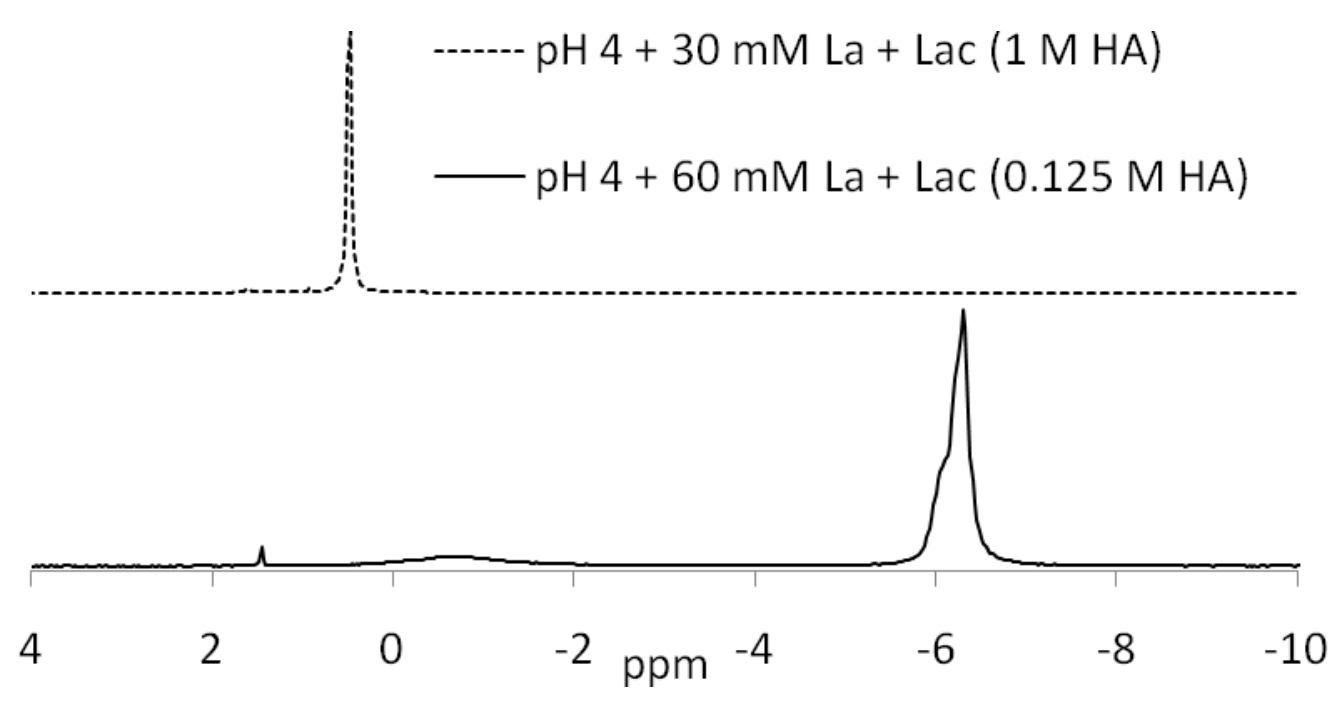

Figure $2.31-{ }^{31} \mathrm{P}$ NMR spectra obtained from the $\mathrm{pH} 4+60 \mathrm{mM} \mathrm{La}+\operatorname{Lac}(0.125 \mathrm{M} \mathrm{HA})$ and $\mathrm{pH} 4+$ $30 \mathrm{mM} \mathrm{La}+$ Lac (1.0 M HA) samples.

In the ${ }^{13} \mathrm{C} N M R$, changes in the environment of carbon containing molecules offers some insight into the speciation of this system. Because of the natural abundance of ${ }^{13} \mathrm{C}(1.07 \%),{ }^{[16]}$ more peaks than those attributed to lactate appear in the spectrum that are associated with the isooctane diluent, and HA. In the pH $4+60 \mathrm{mM} \mathrm{La}+\operatorname{Lac}(0.125 \mathrm{M} \mathrm{HA})$ sample shown in Figure 2.32 most carbon species are too low in concentration for detection except the acid carbonyl at 186 ppm, and the peaks associated with isooctane (see Table 2.20 for isooctane peak assignments). The weak multiplets observed in this spectrum are due to ${ }^{13} \mathrm{C}^{2} \mathrm{H}$ coupling in the deuterated isooctane. Peaks associated with HA were visible in $\mathrm{pH} 4+30 \mathrm{mM} \mathrm{La}+\operatorname{Lac}(1 \mathrm{M} \mathrm{HA})$ 
due to its higher concentration in this sample. Doublets appearing at $\sim 68, \sim 40$, and $\sim 25 \mathrm{ppm}$ in the $\mathrm{pH} 4+30 \mathrm{mM} \mathrm{La}+\operatorname{Lac}(1 \mathrm{M} \mathrm{HA})$ spectrum are not due to coupling between nuclei, but rather due to a small change in the chemical shift of a fraction of HA. This change is likely due to lanthanum bonding through the phosphate group which would lead to a slight increase in the peak's chemical shift. A larger change in chemical shift observed for the acid carbonyl peak between these spectra reflects the variation in the chemical environment that lactate experiences when it complexes lanthanum vs. sodium. Because of the chemical shift data obtained in these two scoping samples, more work using this technique is planned for other experimental conditions.

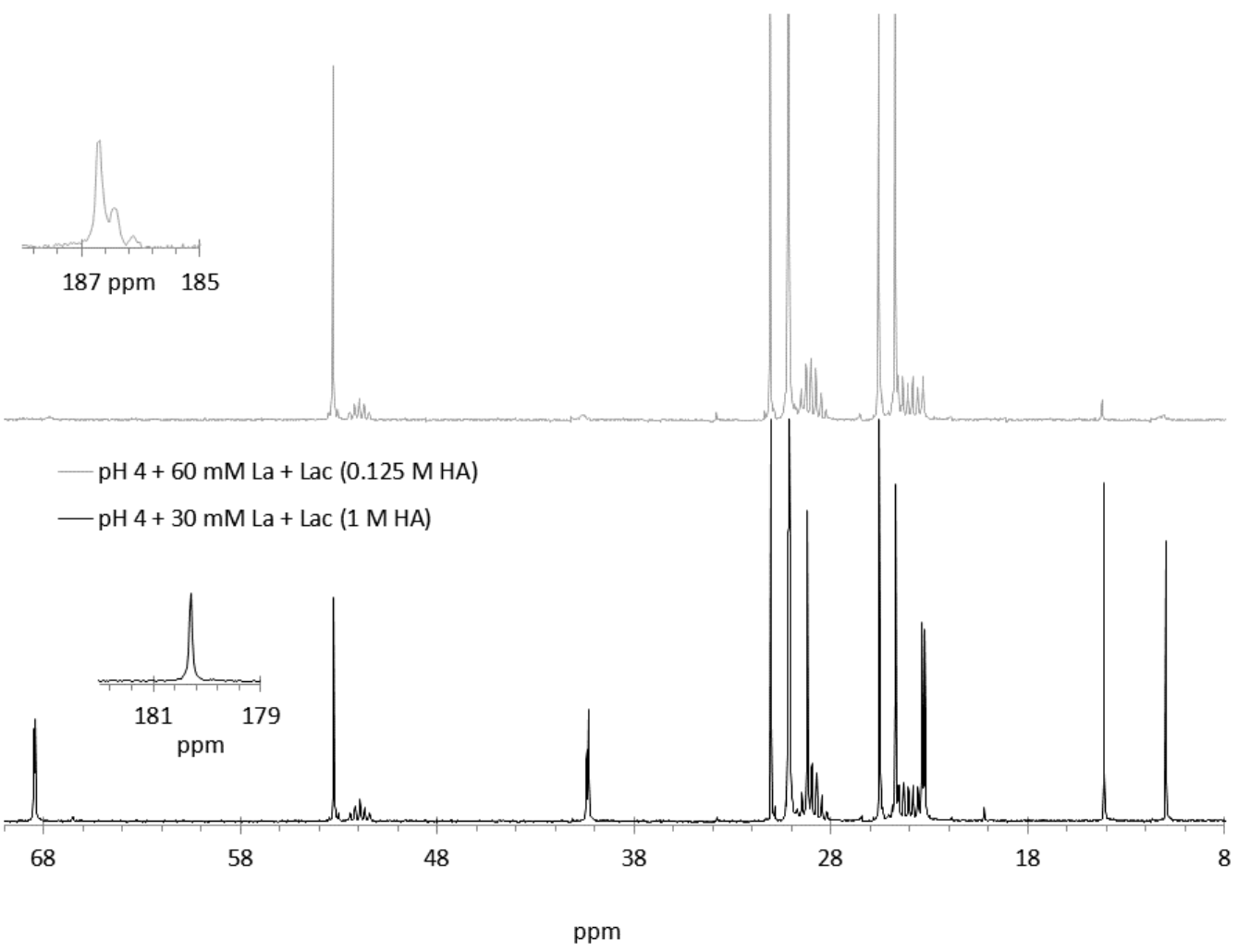

Figure 2.32- ${ }^{13} \mathrm{C}$ NMR scan spectra obtained from the $\mathrm{pH} 4+60 \mathrm{mM}$ La + Lac $(0.125 \mathrm{M} \mathrm{HA})$ and pH $4+30$ mM La + Lac (1.0 M HA) samples. 


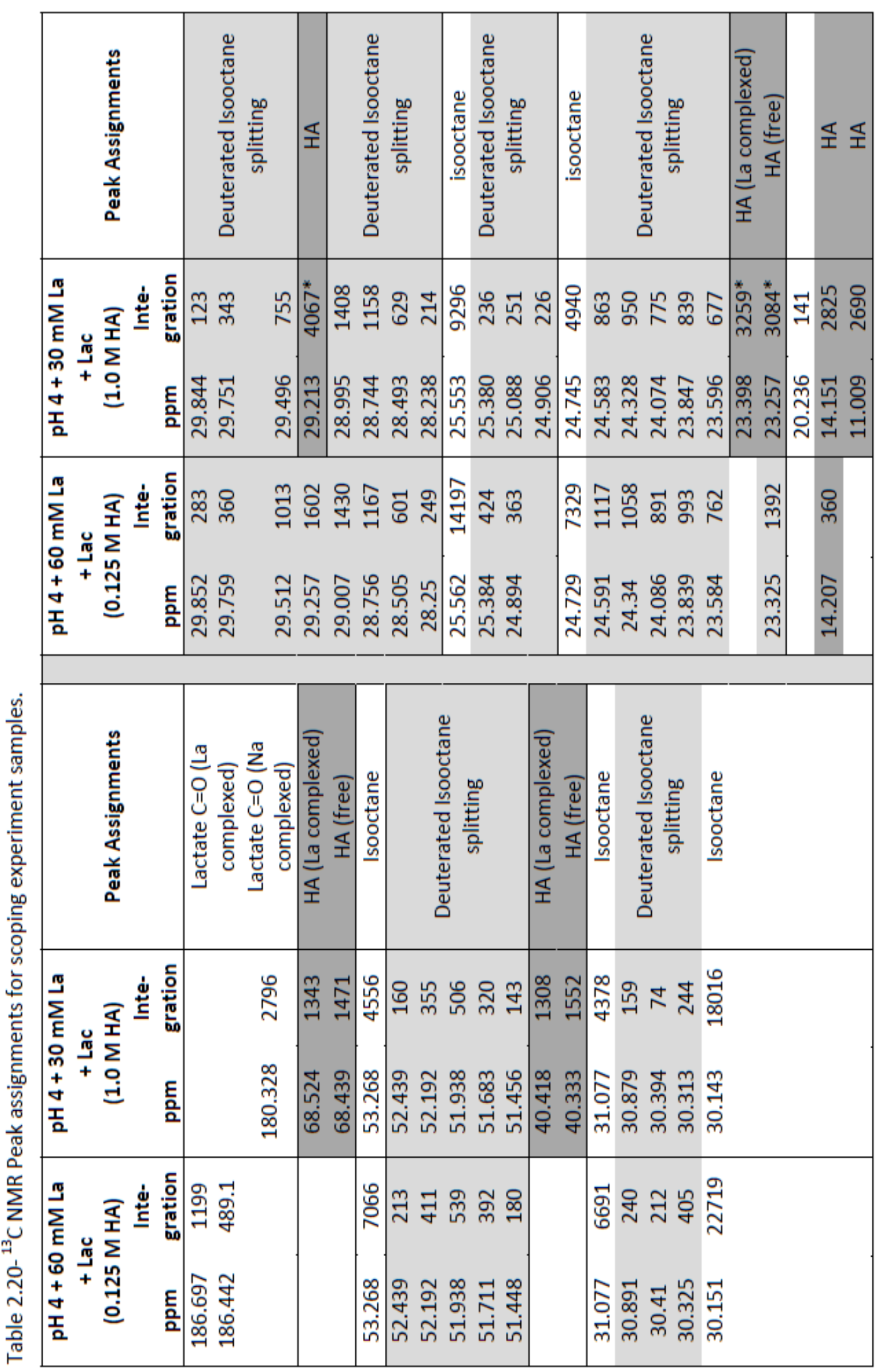




\subsection{Conclusions}

The conditional ionization constant $\left(\mathrm{pK}_{\mathrm{a}}\right)$ of lactic acid at I $=2.0 \mathrm{M} \mathrm{NaCl}$ was quantified over the temperature range of $25-65^{\circ} \mathrm{C}$. The results show very little change with temperature and a relatively small standard enthalpy of reaction. This work is of importance to the development of the TALSPEAK extraction model, specifically providing information that is useful for modeling the aqueous phase activity coefficients for lactate media solutions. Expanding this work to other ionic strengths will provide a more complete data set that will enable modeling of aqueous activity coefficients for lactate solutions in the TALSPEAK system.

The studies described in this chapter explored speciation of $\mathrm{Nd}^{3+}$ in the organic phase, including aggregation of $\mathrm{HA}$ under a range of condition (see Table 2.21). Extractions of $\mathrm{Nd}^{3+}$ by varied concentration $\mathrm{HA}(0-1 \mathrm{M})$ from $\mathrm{HCl}$ media at both $\mathrm{pH} 1$ and 4 were compared with control samples without $\mathrm{Nd}^{3+}$ that were also at $\mathrm{pH} 1$ and 4 . A second set of samples measured the extraction of $\mathrm{Nd}^{3+}$ over the same $\mathrm{HA}$ concentrations from $1 \mathrm{M}$ lactate media at $\mathrm{pH} 1$ and 4 . The initial concentration of $\mathrm{Nd}^{3+}$ was $30 \mathrm{mM}$ for both $\mathrm{HCl}$ and lactate experiments; one additional experiment in lactate medium at $60 \mathrm{mM}$ was also performed. The lactate series of extractions was also compared with non- $\mathrm{Nd}^{3+}$ control experiments at $\mathrm{pH} 1$ and 4 .

In the non- $\mathrm{Nd}^{3+}$ control samples for the $\mathrm{HCl}$ system [i.e. $\mathrm{pH}(1,4)+0 \mathrm{mM} \mathrm{Nd}+\mathrm{HCl}$, and the pH 1 lactate system [pH $1+0 \mathrm{mM} \mathrm{Nd}+$ Lac] an average aggregation number of 2 was observed, indicating that $\mathrm{HA}$ existed predominantly as a dimer. In the corresponding $\mathrm{Nd}^{3+}$ containing systems [i.e. $\mathrm{pH}(1,4)+30 \mathrm{mM} \mathrm{Nd}+\mathrm{HCl}$; pH $1+30 \mathrm{mM} \mathrm{Nd}+$ Lac] there was an increase in aggregation that was consistent with the mechanism for $\mathrm{Nd}^{3+}$ extraction by $\mathrm{HA}$. Buffering in the $\mathrm{pH} 4$ lactate systems led to high loading and the formation of large HA aggregates. In the $\mathrm{pH} 4+$ $0 \mathrm{mM} \mathrm{Nd}+$ Lac system the average aggregation was $3.82 \pm 0.05$ which when modeled, showed the formation of $\mathrm{NaA}(\mathrm{HA})$ and $\mathrm{NaLac}(\mathrm{HA})_{z}$ with $\mathrm{Z}=5.1 \pm 0.3$. The average aggregation in the $\mathrm{pH}$ 
$4+(30,60) \mathrm{mM} \mathrm{Nd}+$ Lac systems showed formation of high order aggregates at low $[\mathrm{HA}]_{\text {org }}$ with an average aggregation as high as 100 observed. These high order aggregates diminished with increasing $[\mathrm{HA}]_{\text {org }}$ until a constant aggregation was reached that corresponded with the lowloading extraction mechanism of $\mathrm{Nd}^{3+}$ by $\mathrm{HA}$.

The $\mathrm{Nd}^{3+}$ distribution results were consistent with the extraction mechanism for $\mathrm{Nd}^{3+}$ by $\mathrm{HA}$ with the HA dimer stoichiometric coefficient $(n)=4$ when $\mathrm{Nd}^{3+}$ loading of $\mathrm{HA}$ was moderate to low. Modeling of these systems by SXLSQI gave an equilibrium constant or Log $\mathrm{K}_{\mathrm{Nd}}=-0.14 \pm$ 0.06. In the $\mathrm{pH} 4+(30,60) \mathrm{mM} \mathrm{Nd}+$ Lac systems where $\mathrm{Nd}^{3+}$ loading of $\mathrm{HA}$ was high, $\mathrm{Nd}^{3+}$ saturated the organic phase at low $[\mathrm{HA}]_{\text {org }}$, and was quantitatively extracted at higher $[\mathrm{HA}]_{\text {org }}$.

The $\mathrm{Na}^{+}$extraction was suppressed in systems with $\mathrm{pH}$ of 1 , with partial $\mathrm{Nd}^{3+}$ loading [pH (1, 4) + $30 \mathrm{mM} \mathrm{Nd}+\mathrm{HCl} ; \mathrm{pH} 1+30 \mathrm{mM} \mathrm{Nd}+$ Lac systems], or where the $\mathrm{HA}$ was saturated with $\mathrm{Nd}^{3+}$ $\left[\mathrm{pH} 4+(30,60) \mathrm{mM} \mathrm{Nd}+\right.$ Lac systems at $\mathrm{HA}$ concentrations $<4 x$ total $\left.\mathrm{Nd}^{3+}\right]$. The $\mathrm{Na}^{+}$extraction occurred in systems where the $\mathrm{pH}$ was 4 and excess $\mathrm{HA}$ existed $[\mathrm{pH} 4+0 \mathrm{mM} \mathrm{Nd}+(\mathrm{HCl}, \mathrm{Lac})$ system; $\mathrm{pH} 4+(30,60) \mathrm{mM} \mathrm{Nd}+$ Lac systems at HA concentrations $>4 x$ total $\left.\mathrm{Nd}^{3+}\right]$, in the Lac systems, $\mathrm{Na}^{+}$was extracted both as the HA sodium salt, and co-extracted with Lac. SXLSQI modeling of the $\mathrm{pH} 4+0 \mathrm{mM} \mathrm{Nd}+\mathrm{HCl}$ system gave an equilibrium constant or $\log \mathrm{K}_{\mathrm{Na}}=-0.989$ \pm 0.009 .

Lactate was found to extract in all the Lac systems in various species. At $\mathrm{pH} 1$, lactate was extracted as lactic acid, and possibly as $\mathrm{NdLac}^{2+}$ salt in the presence of $\mathrm{Nd}^{3+}$. In the $\mathrm{pH} 4+0 \mathrm{mM}$ $\mathrm{Nd}+$ Lac system, and $\mathrm{pH} 4+(30,60) \mathrm{mM} \mathrm{Nd}+$ Lac systems at $\mathrm{HA}$ concentrations $>4 x$ total $\mathrm{Nd}^{3+}$, Lac was co-extracted with $\mathrm{Na}^{+}$. In the $\mathrm{pH} 4+(30,60) \mathrm{mM} \mathrm{Nd}+$ Lac systems at HA concentrations $<4 x$ total $\mathrm{Nd}^{3+}$ Lac was co-extracted with $\mathrm{Nd}^{3+}$ as $\mathrm{NdLac}^{2+}$.

Water was found in the organic phase in all systems. In all systems but the $\mathrm{pH} 4+(0,30,60)$ $\mathrm{mM} \mathrm{Nd}+$ Lac systems the organic phase under low loading retained the levels of water found in 
the washed solvent within experimental error. In the $\mathrm{pH} 4+0 \mathrm{mM} \mathrm{Nd}+\mathrm{HCl}$ system where low $\mathrm{Na}^{+}$loading was observed, a slight increase in the water concentration was observed. In the $\mathrm{pH}$ $4+(0,30,60) \mathrm{mM} \mathrm{Nd}+$ Lac systems, water extraction was observed to increase with $\mathrm{NdLac}^{2+}$ extraction, and increased even more with $\mathrm{Na}^{+}$extraction at higher $\mathrm{HA}$ organic phase concentrations. Correlations of organic phase $\mathrm{H}_{2} \mathrm{O}$ concentration with $\mathrm{Nd}^{3+}$ in the $\mathrm{NdLac}^{2+}$ extraction regime indicates a 1:1 stoichiometry of water with $\mathrm{Nd}^{3+}$. Correlations of organic phase $\mathrm{H}_{2} \mathrm{O}$ concentration with $\mathrm{Na}^{+}$when present in the $\mathrm{pH} 4+(0,30,60) \mathrm{mM} \mathrm{Nd}+$ Lac systems indicates a 3:1 stoichiometry of water with $\mathrm{Na}^{+}$or a hydration number of 3 for $\mathrm{Na}^{+}$.

Organic phase visible spectroscopy in the $\mathrm{pH}(1,4)+30 \mathrm{mM} \mathrm{Nd}+\mathrm{HCl}$ and $\mathrm{pH} 1+30 \mathrm{mM} \mathrm{Nd}$ + Lac systems indicate that the molar absorptivity of $\mathrm{Nd}^{3+}$ does not depend on $\mathrm{pH}$ or $\mathrm{Nd}^{3+}$ loading of the organic phase, but rather on the organic phase HA concentration. The similar spectra in the $\mathrm{pH}(1,4)+30 \mathrm{mM} \mathrm{Nd}+\mathrm{HCl}$ systems indicate that the extraction mechanism is unchanged from $\mathrm{pH} 1$ to $\mathrm{pH} 4$ in $\mathrm{HCl}$ media at $[\mathrm{HA}]_{\text {org }}>0.05 \mathrm{M}$. Changes in the organic phase visible spectra under the high loading conditions support the formation of $\mathrm{Nd}(\mathrm{Lac})(\mathrm{HA})_{2}$. FTIR results support the ion exchange mechanism at low to moderate $\mathrm{Nd}^{3+}$ loading, and the extraction of $\mathrm{NdLac}^{2+}$ and high HA loading, including the formation of high order aggregates with HA participating in phosphate bridging in the organic phase.

The overall speciation results (see Table 2.21 for summary of species and conditions where they were identified) indicate that at high $\mathrm{Nd}^{3+}$ loading in the $\mathrm{pH} 4+$ Lac systems, $\mathrm{Nd}^{3+}$ exists as a highly aggregated, $\left(\mathrm{NdLacA}_{2}\right)_{\times}$species in the organic phase. As loading is decreased (i.e. HA increased), the fraction of $\mathrm{Nd}^{3+}$ in the $\left(\mathrm{NdLacA}_{2}\right)_{\times}$species decreases, while the fraction in the mono-nuclear $\mathrm{NdA}_{3}(\mathrm{HA})$ species increases until a complete conversion to $\mathrm{NdA}_{3}(\mathrm{HA})$ is achieved. Other species identified with the conditions where they were observed is shown in Table 2.21). 
Table 2.21- Summary of species identified in the systems studied with the conditions where they were observed.

\begin{tabular}{|c|c|c|}
\hline Species & System & $\begin{array}{c}\text { Organic Phase Condition } \\
\text { (M HA) }\end{array}$ \\
\hline $\mathrm{H}(\mathrm{Lac})$ & $\mathrm{pH} 1+0 \mathrm{mM} \mathrm{Nd}+\mathrm{Lac}$ & $0.025-1.0$ \\
\hline $\mathrm{Na}(\mathrm{A})$ & $\mathrm{pH} 4+0 \mathrm{mM} \mathrm{Nd}+\mathrm{HCl}$ & $0.05-1.0$ \\
\hline $\mathrm{Nd}(\mathrm{A})_{3}(\mathrm{HA})$ & $\begin{array}{l}\mathrm{pH} 1+30 \mathrm{mM} \mathrm{Nd}+\mathrm{HCl} \\
\mathrm{pH} 4+30 \mathrm{mM} \mathrm{Nd}+\mathrm{HCl} \\
\mathrm{pH} 4+30 \mathrm{mM} \mathrm{Nd}+\mathrm{Lac} \\
\mathrm{pH} 4+60 \mathrm{mM} \mathrm{Nd}+\mathrm{Lac}\end{array}$ & $\begin{array}{c}0.05-1.0 \\
0.05-1.0 \\
0.15-1.0 \\
0.3-1.0\end{array}$ \\
\hline $\begin{array}{l}{\left[\mathrm{Nd}(\operatorname{Lac})(\mathrm{A})_{2}\right]_{\mathrm{x}}} \\
\quad(x=2-50)\end{array}$ & $\begin{array}{l}\mathrm{pH} 4+30 \mathrm{mM} \mathrm{Nd}+\mathrm{Lac} \\
\mathrm{pH} 4+60 \mathrm{mM} \mathrm{Nd}+\mathrm{Lac}\end{array}$ & $\begin{array}{c}0.025-0.15 \\
0.025-0.3\end{array}$ \\
\hline $\mathrm{Na}(\mathrm{Lac})+\mathrm{Na}(\mathrm{A})$ & $\begin{array}{l}\mathrm{pH} 4+0 \mathrm{mM} \mathrm{Nd}+\mathrm{Lac} \\
\mathrm{pH} 4+30 \mathrm{mM} \mathrm{Nd}+\mathrm{Lac} \\
\mathrm{pH} 4+60 \mathrm{mM} \mathrm{Nd}+\mathrm{Lac}\end{array}$ & $\begin{array}{c}0.05-1.0 \\
0.15-1.0 \\
0.3-1.0\end{array}$ \\
\hline
\end{tabular}

The initial NMR results obtained through the scoping experiment indicate that the ${ }^{13} \mathrm{C}$ and

${ }^{31} \mathrm{P}$ spectra obtained from the analogous $\mathrm{pH} 4+(30,60) \mathrm{mM} \mathrm{La}^{3+}+$ Lac systems are sensitive to the speciation changes in the system and more work with these methods is warranted. A complete interpretation of this system using NMR was not undertaken. However identified trends were consistent with the observed results from other methods. 


\section{CHAPTER 3}

\section{THE DETERMINATION OF BINDING CONSTANTS OF 8-HYDROXYQUINOLINE IN THE}

\section{TRITON X-114/WATER MIXED SOLVENT SYSTEM}

\subsection{Introduction}

Cloud point extraction has been shown to separate lanthanum from gadolinium using the surfactant Triton X-114 (see Figure 3.1) and the ligand 8-hydroxyquinoline (HQ) (see Figure 3.2). ${ }^{[5-7]}$ The success of any extraction system to perform such a difficult separation led to interest in applying this method to the separation of the trivalent actinides from the trivalent lanthanides. Preliminary extraction experiments ${ }^{[107]}$ using europium and curium have indicated that there is some selectivity for curium over europium, but the data showed that the optimal separation conditions occur over a very small $\mathrm{pH}$ range (Separation factor of $\sim 10^{3}$ at $\mathrm{pH}=5.5$ ). The desire to model the system and the need for complexation constants under the conditions of the cloud point extraction has motivated this research. The aim of this work is to design a better separation technique through understanding the protonation and $\mathrm{Eu}^{3+}$ complexation behavior of 8-hydroxyquinoline and the $\mathrm{Eu}^{3+}$ hydrolysis behavior in the Triton X-114/water mixed solvent used in cloud point extraction.

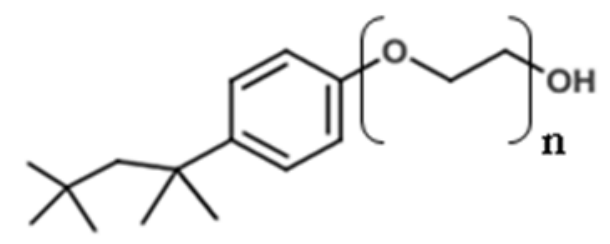

Figure 3.1- Chemical Structure of Triton $\mathrm{X}-114$. This chemical is typically sold as a mixture with $\mathrm{n}$ $=7-8$ with the total mixture having an average molecular weight of $537 \mathrm{gm} / \mathrm{mol}^{\left[{ }^{[108]}\right.}$

Past research with 8-hydroxyquinoline extends back to 1881 when it was first synthesized by Bedall and Fischer. ${ }^{[109-111]}$ It was later discovered to form insoluble chelates with many metals ${ }^{[112-}$ 
${ }^{114]}$ that proved useful for quantitative determination by gravimetric means. Solvent extraction with 8-hydroxyquinoline in chloroform has been explored, ${ }^{[115-123]}$ but this is not a highly favored option for industrial application due to the toxicity and flammability of the solvent. Watanabe and Tanaka ${ }^{[9]}$ introduced a new extraction technique in 1978 using cloud point extraction that relies on non-ionic surfactants and chelators that form water-insoluble complexes to extract metal ions from aqueous solutions. Cloud point extraction has experienced a large growth in research as witnessed by these reviews, ${ }^{[35-37,40]}$ but has generally been employed in laboratory analysis rather than industrial scale separations. Recent work on Continuous Rotating Disk Contactors $^{[11-42,124]}$ is, perhaps, opening the door for industrial scale deployment of cloud point extraction, however this cited work deals with extraction of phenol from waste water rather than metal chelates which would seem to increase the complexity of the process. Industrial scale deployment for recycling of irradiated nuclear fuel is the ultimate goal of the continued research in trivalent actinide-trivalent lanthanide separation research.

The solubility of 8-hydroxyquinoline is the major issue that has driven the selection of experimental media for complexation constant determination. The solubility of 8hydroxyquinoline is $\mathrm{pH}$ dependent due to its protonation states (see Figure 3.2). At pH extremes 8-hydroxyquinoline carries a charge but at intermediate $\mathrm{pH}$ values 8-hydroxyquinoline is uncharged, leading to increased solubility at $\mathrm{pH}$ extremes and low solubility at moderate $\mathrm{pH}$ values. Aqueous solubility of the neutral form of 8-hydroxyquinoline (as the species labeled 'B' in Figure 3.2) is approximately $3 \mathrm{mM}$ (see Figure 3.3). ${ }^{[125-126]}$ The speciation diagram included in Figure 3.3 was calculated from data by Ashton et al. $\left(\mathrm{pk}_{\mathrm{a} 1}=4.88, \mathrm{pK}_{\mathrm{a} 2}=10.05\right) .{ }^{[127]}$ Conditions that increase 8-hydroxyquinoline solubility are elevated temperature, and solvent modification such as mixed solvent conditions. ${ }^{[126]}$ For this reason many of the reported values of the 8hydroxyquinoline metal complexation constant data are given for mixed solvent systems such as 
water/dioxane or water/ethanol. There was a reasonably complete determination of lanthanides complexation constants with 8-hydroxyquinoline in dioxane/water ${ }^{[128]}$ which were used to model the $\mathrm{Gd}^{3+} / \mathrm{La}^{3+}$ cloud point extraction results. ${ }^{[5]}$ It was desired to use parameters for modeling the complexation behavior of $\mathrm{Eu}^{3+}$ and $\mathrm{Cm}^{3+}$ with 8-hydroxyquinoline, but $\mathrm{Eu}^{3+}$ was one of four lanthanides that were omitted from the dioxane/water study. There is very little data published for $\mathrm{Eu}^{3+}$ or $\mathrm{Cm}^{3+}$ complexation with 8-hydroxyquinoline. Constants from the literature ${ }^{[129]}$ only showed 2 values for complexation between 8-hydroxyquinoline and $\mathrm{Eu}^{3+}$ : one ${ }^{[130]}$ was a solubility study on $\left[\mathrm{Eu}(\mathrm{Q})_{3}\right]$ and the other ${ }^{[131]}$ was a study at $0.1 \mathrm{M} \mathrm{NaClO}_{4} / 20^{\circ} \mathrm{C}$ that lists only the $1^{\text {st }}$ complexation constant. No complexation constants were listed in the IUPAC database for $\mathrm{Cm}^{3+}$ 8-hydroxyquinoline complexation.

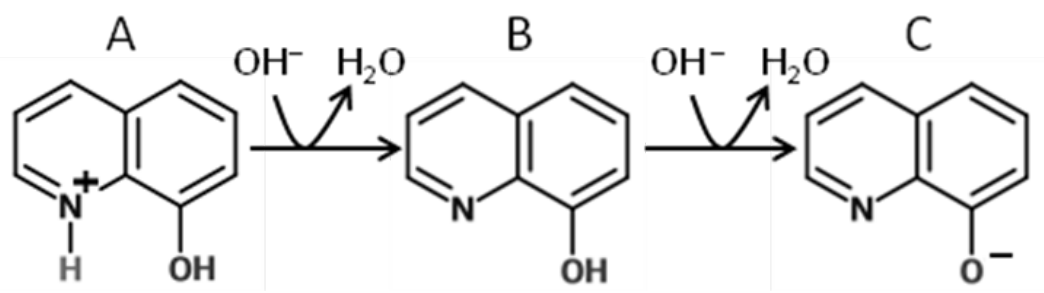

Figure 3.2- The protonation states of 8-hydroxyquinoline.

When modeling behavior under cloud point extraction conditions, the thermodynamic constants should ideally be determined in the experimental medium, which in this case is the Triton/water mixed solvent. One study determined the $\mathrm{pK}_{\mathrm{a} 1}$ and $\mathrm{pK}_{\mathrm{a} 2}$ of 8-hydroxyquinoline in Triton $\mathrm{X}-100^{[132][133]} /$ water as a function of Triton X-100 concentration (0-10 wt\%) at $0.1 \mathrm{M}$ $\mathrm{NaCl} / 20^{\circ} \mathrm{C}^{[134]}$ which in one paper represents the entire literature record on the stability constant modeling of 8-hydroxyquinoline in the water/triton mixed solvent system. This work aims to measure the protonation and $\mathrm{Eu}^{3+}$ complexation behavior of 8-hydroxyquinoline and the 
$\mathrm{Eu}^{3+}$ hydrolysis behavior in the Triton X-114/water mixed solvent used in cloud point extraction to enable the design of a better separation technique.

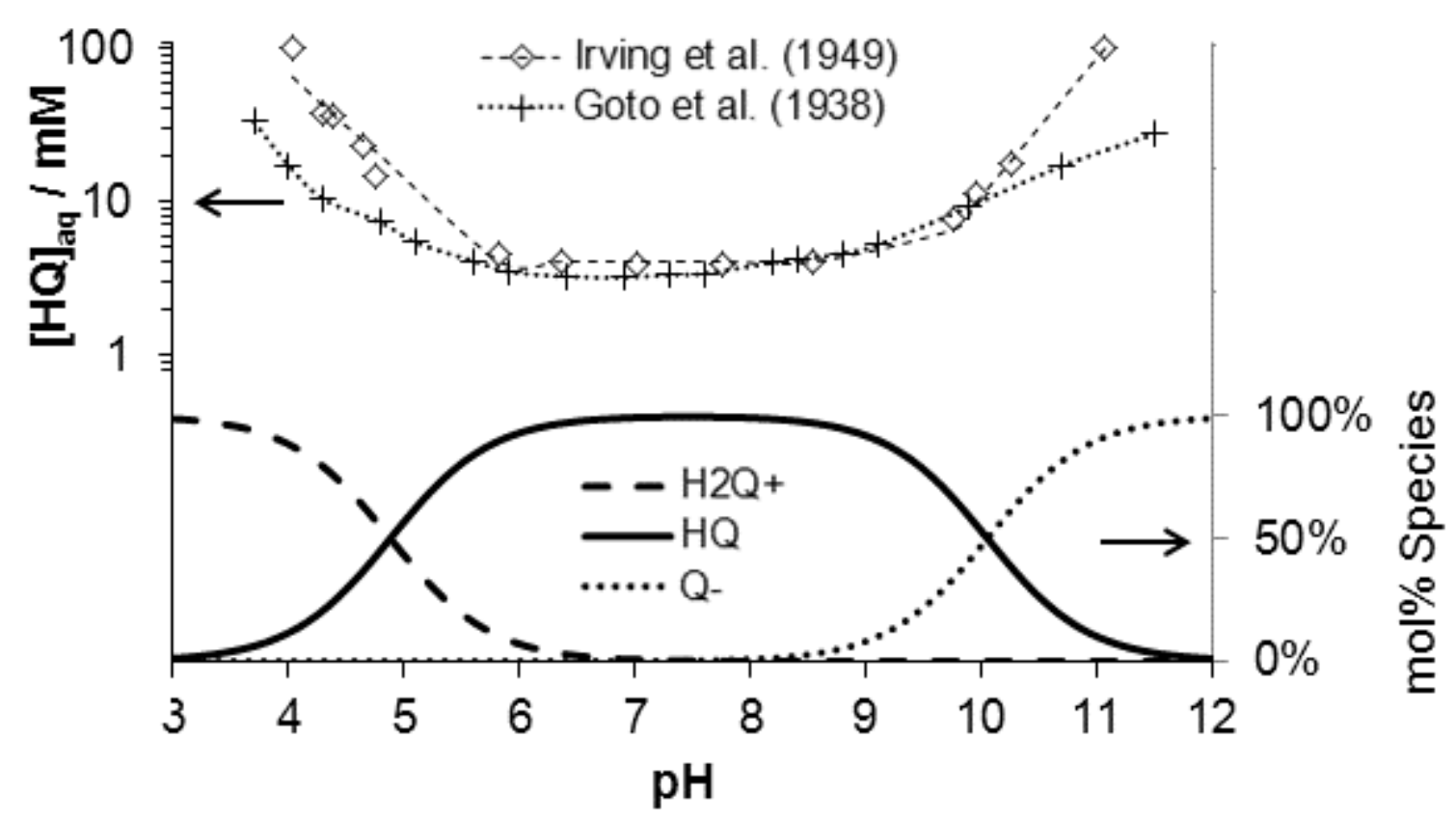

Figure 3.3- The aqueous solubility and speciation of 8-hydroxyquinoline as a function of $\mathrm{pH}$.

The methods selected for the characterization of the protonation, hydrolysis and complexation behavior of 8-hydroxyquinoline and $\mathrm{Eu}^{3+}$ used in this study, are potentiometric and spectrophotometric titration techniques. Potentiometry will measure the $\mathrm{pH}$ buffering effects caused by the formation of different species in the solutions undergoing titration by a strong acid or base. The potentiometric titration curve can be analyzed by modeling software program such as Hyperquad, ${ }^{[135]}$ to yield the conditional protonation, hydrolysis, and/or complexation constants. Hyperquad is a highly regarded program that has been utilized or cited within $\sim 900^{[136]}$ publications since its release in 1996. Similarly, recording the changing $\mathrm{pH}$ and 
absorbance spectra of 8-hydroxyquinoline in a solution undergoing titration by a strong acid or base and analyzing the sequence of spectra using a spectrophotometric modeling program such as SQUAD, ${ }^{[137]}$ will likewise provide the conditional protonation, hydrolysis, and/or complexation

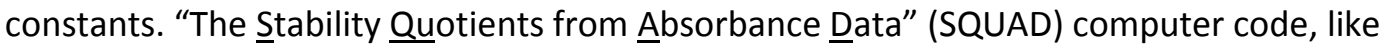
hyperquad, has been widely used and was cited in $230^{[138]}$ publications since its publication in 1975. Hyperquad can find the best fit for speciation parameters under investigation, and the overall system speciation from user-supplied pH titration data, chemical constituent concentrations, chemical equilibria expressions, and the known chemical equilibria constants. SQUAD utilizes the inputs listed above, but also requires the spectrophotometric titration data that correspond to the potentiometric titration data points. With this information, SQUAD can calculate the overall system speciation, the speciation parameters under investigation, and the spectrum of each absorbing species in solution.

Typically, cloud point extraction solutions are mixed below the surfactant cloud point temperature, which for Triton $\mathrm{X}-114$ is $22^{\circ} \mathrm{C} .^{[108]}$ The phase separation in cloud point extraction is performed by heating the solution above the cloud point temperature and centrifuging and separating/disengagement of the phases. The thermal fluctuation of the system over the course of the separation makes it challenging to select a temperature that is representative of the conditions of the system at equilibrium. Further complications are introduced by the formation of two phases during separation, and the turbidity of the solution at temperatures above the cloud point. The use of spectrophotometry necessitates a non-turbid solution so that the solution absorbance spectrum is not obscured. Potentiometry is not affected by turbidity, but does require experiments to be conducted at a constant temperature. Therefore, the current study is carried out at a constant temperature maintained by ice slurry $\left(T=\sim 1{ }^{\circ} \mathrm{C}\right)$ that will prevent turbidity due to cloud point separation. 
The long-term goal to be achieved following the completion of this project is to develop a better trivalent actinide / trivalent lanthanide separation method using cloud point extraction. The modeling results described herein are an initial effort to understand preliminary cloud point extraction data involving $\mathrm{Eu}^{3+}$ and $\mathrm{Cm}^{3+}$ by 8 -hydroxyquinoline using Triton $\mathrm{X}-114$ that show at $\mathrm{pH} 5.5, \mathrm{a} \mathrm{Cm}^{3+} / \mathrm{Eu}^{3+}$ separation factor of $10^{3} \cdot{ }^{[139]}$ It is believed that when the $\mathrm{Eu}^{3+}$ system is fully characterized by the methods described above, that a comparative study of the relative complexation strength of $\mathrm{Eu}^{3+}$ and $\mathrm{Cm}^{3+}$ with 8-hydroxyquinoline by Time Resolved Laser Fluorescence Spectroscopy will provide clarity into the unpublished separation results while conserving $\mathrm{Cm}^{3+}$. For the present time however, this work addresses the characterization of the protonation of 8-hydroxyquinoline, the hydrolysis of $\mathrm{Eu}^{3+}$, and the complexation of 8hydroxyquinoline and $\mathrm{Eu}^{3+}$ in the water/Triton X-114 mixed solvent system at about $1^{\circ} \mathrm{C}$.

\subsection{Experimental}

\subsubsection{Chemicals}

Europium chloride hexahydrate $\left(\mathrm{EuCl}_{3} \cdot 6 \mathrm{H}_{2} \mathrm{O}, 99.99 \%\right)$ was obtained from Alfa Aesar and used as received. Sodium Hydroxide (50 wt\%) and $\mathrm{NaCl}$ were obtained from Aldrich and used as received. Triton X-114 was obtained from Fluka and used as received. 8-hydroxyquinoline was obtained from JT Baker and purified by sublimation prior to use.

\subsubsection{Methods}

Potentiometric data was recorded using a laptop controlled system running the Metrohm Tiamo 1-2 software. Potentiometric titrations were performed using the dynamic $\mathrm{pH}$ titration setting in the Tiamo software. The dosing unit (Metrohm Dosino 800), stir plate (Metrohm 801 Stirrer), and combination temperature/pH electrode (Metrohm 6.0258.010) were all connected to the laptop through a Metrohm Titrando 809 . Temperature control at $1.7 \pm 0.3^{\circ} \mathrm{C}$ was performed using a beaker (as the titration vessel) packed in ice chunks in a crystallizing dish and 
a zippered plastic bag filled with ice chunks that was firmly wrapped around the combination temperature/pH electrode. Carbonate free $\mathrm{NaOH}$ was mixed from $50 \mathrm{wt} \% \mathrm{NaOH}$ and boiled DI water, standardized, and stored in an Ascarite vented bottle.

Spectrophotometric titrations were performed by manual titration (using pipette) and $\mathrm{pH}$ was measured using Tiamo system in $\mathrm{pH} /$ Temperature measurement mode, the spectral data was saved electronically and the $\mathrm{pH}$ data was recorded by hand. Spectra were collected using an OceanOptics HR4000 spectrophotometer (190 - $1140 \mathrm{~nm})$. Light was introduced to the sample using a fiber-optic dip probe from both deuterium and tungsten light sources. The tungsten light source's intensity was controlled using a 2.9 Optical Density glass filter. The spectra recorded were averages of 50 scans of 15 millisecond each with the boxcar averaging feature disabled. Resolution for the scans was $0.26 \mathrm{~nm}$.

Calibration of $\mathrm{pH}$ probes was performed using standard $\mathrm{pH} \mathrm{4,} 7$ and 10 buffers that were chilled to the temperature of the titration solutions. The system was recalibrated for each titration. lonic strength was maintained at $0.1 \mathrm{~mol} / \mathrm{L}$ using $\mathrm{NaCl}$. The solution constituent concentrations for the potentiometric titrations are shown in Table 3.1 and Table 3.2. The solution constituent concentrations for the spectrophotometric titrations are shown in Table 3.3. Standardized $\mathrm{NaOH}$ was used for the potentiometric titrations with $[\mathrm{NaOH}]=$ $0.01024 \pm 0.00002 \mathrm{M}$, and $[\mathrm{NaCl}]=0.09 \mathrm{M}$. Spectrophotometric titrations were performed with manual additions from solutions of $\mathrm{NaOH}$ of $0.1,0.01$, and $0.001 \mathrm{M}$ depending on the needed $\mathrm{pH}$ change and solution buffering conditions on a point-by-point basis. These $\mathrm{NaOH}$ solutions were supplemented with $\mathrm{NaCl}$ to maintain a total ionic strength of $0.1 \mathrm{M}$. 
Table 3.1- Solution constituent concentrations for the $\mathrm{H}_{2} \mathrm{O}$ system potentiometric titrations.

\begin{tabular}{|c|c|c|c|c|c|c|c|c|}
\hline \multirow{2}{*}{$\begin{array}{c}\mathrm{Eu}^{3+}: \mathrm{HQ} \\
\text { Ratio }\end{array}$} & \multirow{2}{*}{$\mathbf{p H}_{\text {initial }}$} & \multirow{2}{*}{$\begin{array}{c}{[\mathrm{HCl}]} \\
\mathbf{M}\end{array}$} & \multirow{2}{*}{$\begin{array}{c}{\left[\mathrm{Eu}^{3+}\right]} \\
\mathrm{M}\end{array}$} & \multirow{2}{*}{$\begin{array}{c}\text { [HQ] } \\
\mathbf{M}\end{array}$} & \multirow{2}{*}{$\begin{array}{c}{[\mathrm{NaCl}]} \\
\mathrm{M}\end{array}$} & \multirow{2}{*}{$\begin{array}{c}\text { [Triton] } \\
w t \% \\
\end{array}$} & \multicolumn{2}{|c|}{$T$} \\
\hline & & & & & & & & C \\
\hline $0: 1$ & 3.20 & 0.001 & 0 & $3 \times 10^{-4}$ & 0.1 & 0 & 1.5 & \pm 0.2 \\
\hline $1: 0$ & 3.30 & 0.001 & $3 \times 10^{-4}$ & 0 & 0.1 & 0 & 1.7 & \pm 0.2 \\
\hline $1: 1$ & 3.22 & 0.001 & $3 \times 10^{-4}$ & $3 \times 10^{-4}$ & 0.1 & 0 & 1.7 & \pm 0.2 \\
\hline $1: 2$ & 3.21 & 0.001 & $1.5 \times 10^{-4}$ & $3 \times 10^{-4}$ & 0.1 & 0 & 1.9 & \pm 0.2 \\
\hline $1: 3$ & 3.26 & 0.001 & $1 \times 10^{-4}$ & $3 \times 10^{-4}$ & 0.1 & 0 & 2.0 & \pm 0.2 \\
\hline
\end{tabular}

Table 3.2- Solution constituent concentrations for the $\mathrm{H}_{2} \mathrm{O} /$ Triton $\mathrm{X}-114$ system potentiometric titrations.

\begin{tabular}{|c|c|c|c|c|c|c|c|c|}
\hline \multirow{2}{*}{$\begin{array}{c}\mathrm{Eu}^{3+}: \mathrm{HQ} \\
\text { Ratio }\end{array}$} & \multirow{2}{*}{$\mathrm{pH}_{\text {initial }}$} & \multirow{2}{*}{$\begin{array}{c}{[\mathrm{HCl}]} \\
\mathbf{M}\end{array}$} & \multirow{2}{*}{$\begin{array}{c}{\left[\mathrm{Eu}^{3+}\right]} \\
\mathrm{M}\end{array}$} & \multirow{2}{*}{$\begin{array}{c}\text { [HQ] } \\
M\end{array}$} & \multirow{2}{*}{$\begin{array}{c}{[\mathrm{NaCl}]} \\
\mathrm{M}\end{array}$} & \multirow{2}{*}{$\begin{array}{c}\text { [Triton] } \\
\text { wt\% } \\
\end{array}$} & \multicolumn{2}{|c|}{$\mathbf{T}$} \\
\hline & & & & & & & \multicolumn{2}{|c|}{${ }^{\circ} \mathrm{C}$} \\
\hline $0: 0$ & 3.13 & 0.001 & 0 & 0 & 0.1 & 1 & 1.9 & \pm 0.2 \\
\hline $0: 1$ & 3.25 & 0.001 & 0 & $3 \times 10^{-4}$ & 0.1 & 1 & 1.8 & \pm 0.2 \\
\hline $1: 0$ & 3.05 & 0.001 & $3 \times 10^{-4}$ & 0 & 0.1 & 1 & 1.8 & \pm 0.2 \\
\hline $1: 1$ & 3.21 & 0.001 & $3 \times 10^{-4}$ & $3 \times 10^{-4}$ & 0.1 & 1 & 1.8 & \pm 0.2 \\
\hline $1: 2$ & 3.24 & 0.001 & $1.5 \times 10^{-4}$ & $3 \times 10^{-4}$ & 0.1 & 1 & 1.9 & \pm 0.1 \\
\hline $1: 3$ & 3.22 & 0.001 & $1 \times 10^{-4}$ & $3 \times 10^{-4}$ & 0.1 & 1 & 1.9 & \pm 0.2 \\
\hline
\end{tabular}

Table 3.3- Solution constituent concentrations for the $\mathrm{H}_{2} \mathrm{O}$ and $\mathrm{H}_{2} \mathrm{O}$ /Triton X-114 system spectrophotometric titrations.

\begin{tabular}{|c|c|c|c|c|c|c|c|c|}
\hline \multirow{2}{*}{$\begin{array}{c}\mathrm{Eu}^{3+}: \mathrm{HQ} \\
\text { Ratio }\end{array}$} & \multirow{2}{*}{$\mathrm{pH}_{\text {initial }}$} & \multirow{2}{*}{$\begin{array}{c}{[\mathrm{HCl}]} \\
\mathrm{M}\end{array}$} & \multirow{2}{*}{$\begin{array}{c}{\left[\mathrm{Eu}^{3+}\right]} \\
\mathrm{M}\end{array}$} & \multirow{2}{*}{$\begin{array}{c}{[\mathrm{HQ}]} \\
\mathrm{M}\end{array}$} & \multirow{2}{*}{$\begin{array}{c}{[\mathrm{NaCl}]} \\
\mathrm{M}\end{array}$} & \multirow{2}{*}{$\begin{array}{c}\text { [Triton] } \\
\text { wt\% }\end{array}$} & \multicolumn{2}{|c|}{$\mathbf{T}$} \\
\hline & & & & & & & & ${ }^{\circ} \mathrm{C}$ \\
\hline $0: 1$ & 3.18 & 0.001 & 0 & $10^{-5}$ & 0.1 & 0 & 1.9 & \pm 0.2 \\
\hline 1:5 & 3.06 & 0.001 & $2 \times 10^{-6}$ & $10^{-5}$ & 0.1 & 0 & 1.9 & \pm 0.2 \\
\hline $0: 1$ & 3.19 & 0.001 & 0 & $10^{-5}$ & 0.1 & 1 & 1.9 & \pm 0.2 \\
\hline
\end{tabular}

To obtain the conditional stability constants, the potentiometric data was modeled using Hyperquad software ${ }^{[135]}$ and the spectrophotometric data were modeled using the SQUAD 
computer code. ${ }^{[137]}$ The $\mathrm{pK}_{\mathrm{w}}$ for the water system was obtained using the Specific Interaction Theorem ${ }^{[140]}$ and Van't Hoff ${ }^{[141]}$ equations to calculate the $\mathrm{pK}_{\mathrm{w}}$ at $\mathrm{T}=1.8^{\circ} \mathrm{C}$ and $\mathrm{I}_{\mu}=0.1 \mathrm{M} \mathrm{NaCl}$ from the $\mathrm{pK}_{\mathrm{w}}$ for pure water at $25^{\circ} \mathrm{C}$ of 14.0 . Enthalpy data used in the Van't Hoff equation was from Harned et al. ${ }^{[142]}$ and calculations were performed using programs obtained from IUPAC/Academic software. ${ }^{[143-144]}$ The value calculated for the $\mathrm{pKW}$ at $\mathrm{T}=1.8^{\circ} \mathrm{C}$ and $\mathrm{I}_{\mu}=0.1 \mathrm{M}$ $\mathrm{NaCl}$ is 14.7.

The notation used in this work for the dissociation of acids is shown in the equations (3.1) and (3.2)

$$
H A \stackrel{\kappa_{a}}{\longleftrightarrow} H^{+}+A^{-}
$$

which is the same as writing

$$
\mathrm{HA}+\mathrm{OH}^{-} \stackrel{\mathrm{K}_{a}}{\longleftrightarrow} \mathrm{H}_{2} \mathrm{O}+\mathrm{A}^{-}
$$

The equilibrium expression that describes both of these reactions is shown in equation (3.3).

$$
K_{a}=\frac{\left[H^{+}\right]\left[A^{-}\right]}{[H A]}=\frac{\left[A^{-}\right]}{[H A]\left[O H^{-}\right]}
$$

The shorthand for the negative logarithm of a value is shown in equation (3.4).

$$
p K_{a}=-\log _{10}\left(K_{a}\right)
$$

The notation used for the step-wise stability constants can be derived from the equilibrium formation reactions outlined using the example of europium in equations (3.5), (3.6), and (3.7).

$$
\begin{aligned}
& \mathrm{Eu}^{3+}+\mathrm{H}_{2} \mathrm{O} \longleftrightarrow \mathrm{K}_{1-10} \longleftrightarrow[\mathrm{Eu}(\mathrm{OH})]^{2+}+\mathrm{H}^{+} \\
& {[\mathrm{Eu}(\mathrm{OH})]^{2+}+\mathrm{H}_{2} \mathrm{O} \longleftrightarrow \mathrm{k}_{1-20} \longrightarrow\left[\mathrm{Eu}(\mathrm{OH})_{2}\right]^{+}+\mathrm{H}^{+}} \\
& {\left[\mathrm{Eu}(\mathrm{OH})_{2}\right]^{+}+\mathrm{H}_{2} \mathrm{O} \longleftrightarrow \mathrm{K}_{1-30} \longleftrightarrow\left[\mathrm{Eu}(\mathrm{OH})_{3}\right]+\mathrm{H}^{+}}
\end{aligned}
$$


The first subscript of any $\mathrm{K}$ or $\beta$ value will indicate the stoichiometry of europium. The second subscript refers to hydroxide $\left(\mathrm{OH}^{-}\right)$when negative or a proton $\left(\mathrm{H}^{+}\right)$when positive. Stepwise stability constants describing reactions adding $Q^{-}$utilize the third subscript to indicate which stepwise addition is taking place. The first addition of $\mathrm{Q}^{-}$to europium is shown in equation (3.8).

$$
\mathrm{Eu}^{3+}+Q^{-} \longleftrightarrow \mathrm{K}_{101} \longrightarrow[\mathrm{Eu}(Q)]^{2+}
$$

Overall stability constants will be used to describe equilibrium reactions as shown in equation (3.9)

$$
\mathrm{Eu}^{3+}+3 \mathrm{H}_{2} \mathrm{O} \longleftarrow \beta_{1-30} \longleftrightarrow\left[\mathrm{Eu}(\mathrm{OH})_{3}\right]+3 \mathrm{H}^{+}
$$

Overall stability constants are a product of the succession of stepwise stability constants that, when used in succession, result in the same reaction. An example is the overall stability constant shown for the formation of $\left[\mathrm{Eu}(\mathrm{OH})_{3}\right]$ shown in equation (3.10)

$$
\beta_{1-30}=K_{1-10} K_{1-20} K_{1-30}
$$

Mixed ligand reactions are a special case of the overall stability constant notation where europium hydrolyzes both waters and complexes $Q^{-}$in one step as shown in equation (3.11)

$$
\mathrm{Eu}^{3+}+2 \mathrm{H}_{2} \mathrm{O}+\mathrm{Q}^{-} \stackrel{\beta_{1-21}}{\longleftrightarrow}\left[\mathrm{Eu}(\mathrm{OH})_{2}(\mathrm{Q})\right]+2 \mathrm{H}^{+}
$$

where the overall stability constant is a product of the successive stepwise reactions as shown in equation (3.12).

$$
\beta_{1-21}=K_{101} K_{1-11} K_{1-21}=K_{1-10} K_{1-20} K_{1-21}
$$

The $\mathrm{K}$ values presented in this text for mixed hydrolysis species are calculated by assuming that the order of addition of the mixed ligands is: first all $Q^{-}$, then the successive hydroxides as is shown by the middle equivalence of equation (3.12). All results displayed in this text will 
indicate parameters that are held constant by displaying no error (i.e. X.X), and parameters that were fit in the model by displaying error (i.e. X.X \pm X.X) in the tables.

\subsection{Results: The $\mathrm{H}_{2}$ O System}

The values of the $\mathrm{pK}_{\mathrm{a}}$ 's of 8-hydroxyquinoline and europium hydrolysis constants were obtained prior to determining the europium 8-hydroxyquinoline stability constants. The europium 8-hydroxyquinoline systems of increasing complexity were created using solutions with $\mathrm{Eu}^{3+}: \mathrm{HQ}$ ratio of $1: 1,1: 2$, and 1:3. This sequential progression provided the opportunity to fit (1) the $[\mathrm{Eu}(\mathrm{Q})]^{2+},(2)\left[\mathrm{Eu}(\mathrm{Q})_{2}\right]^{+}$, with a fixed $[\mathrm{Eu}(\mathrm{Q})]^{2+}$ formation parameter, and (3) $\left[\mathrm{Eu}(\mathrm{Q})_{3}\right]$, with fixed parameters for $[\mathrm{Eu}(\mathrm{Q})]^{2+},\left[\mathrm{Eu}(\mathrm{Q})_{2}\right]^{+}$.

\subsubsection{Determination of the $\mathrm{pK}_{\mathrm{a}}{ }^{\text {'s }} \mathrm{s}$ of 8-Hydroxyquinoline by Potentiometric Titration}

To obtain the protonation constants of 8-hydroxyquinoline, a solution of composition [HQ] = $3 \times 10^{-4} \mathrm{M},[\mathrm{NaCl}]=0.1 \mathrm{M},[\mathrm{HCl}]=0.001 \mathrm{M}$ was titrated with standardized $\mathrm{NaOH}([\mathrm{NaOH}=$ $0.01024 \pm 0.00002 \mathrm{M}$, and $[\mathrm{NaCl}]=0.09 \mathrm{M})$. The temperature for this titration was $1.5 \pm 0.2{ }^{\circ} \mathrm{C}$. The $1^{\text {st }}$ derivative shown here and throughout this work was calculated by the TIAMO software using a modification of Fortuin's method for End Point Recognition. ${ }^{[145-146]}$ Maxima in the $1^{\text {st }}$ derivative curve show how many equivalence points were obtained during the titration and may provide an indication of the number of acid constants that may be fit to the titration curve. In this titration there are two maxima, indicating transitions between three species. This is expected given the protonation states of 8-hydroxyquinoline (see Figure 3.2). The equilibrium expressions shown in equations (3.13) and (3.14) were used to model the titration shown in Figure 3.4 using Hyperquad.

$$
\begin{aligned}
& \mathrm{H}_{2} \mathrm{Q}^{+}+\mathrm{OH}^{-} \longleftrightarrow \kappa_{a 1} \longrightarrow \mathrm{HQ}+\mathrm{H}_{2} \mathrm{O} \\
& \mathrm{HQ}+\mathrm{OH}^{-} \stackrel{\kappa_{a 2}}{\longleftrightarrow Q^{-}}+\mathrm{H}_{2} \mathrm{O}
\end{aligned}
$$


The water dissociation constant or $\mathrm{pk}_{\mathrm{w}}$ was 14.7 for all modeling simulations in the $\mathrm{H}_{2} \mathrm{O}$ system whose reaction is shown in equation (3.15)

$$
\mathrm{H}_{2} \mathrm{O} \stackrel{\mathrm{K}_{w}}{\longleftrightarrow} \mathrm{H}^{+}+\mathrm{OH}^{-}
$$

The parameters that best fit the titration curve are shown in Table 3.4. The modeling results are shown in Figure 3.5.

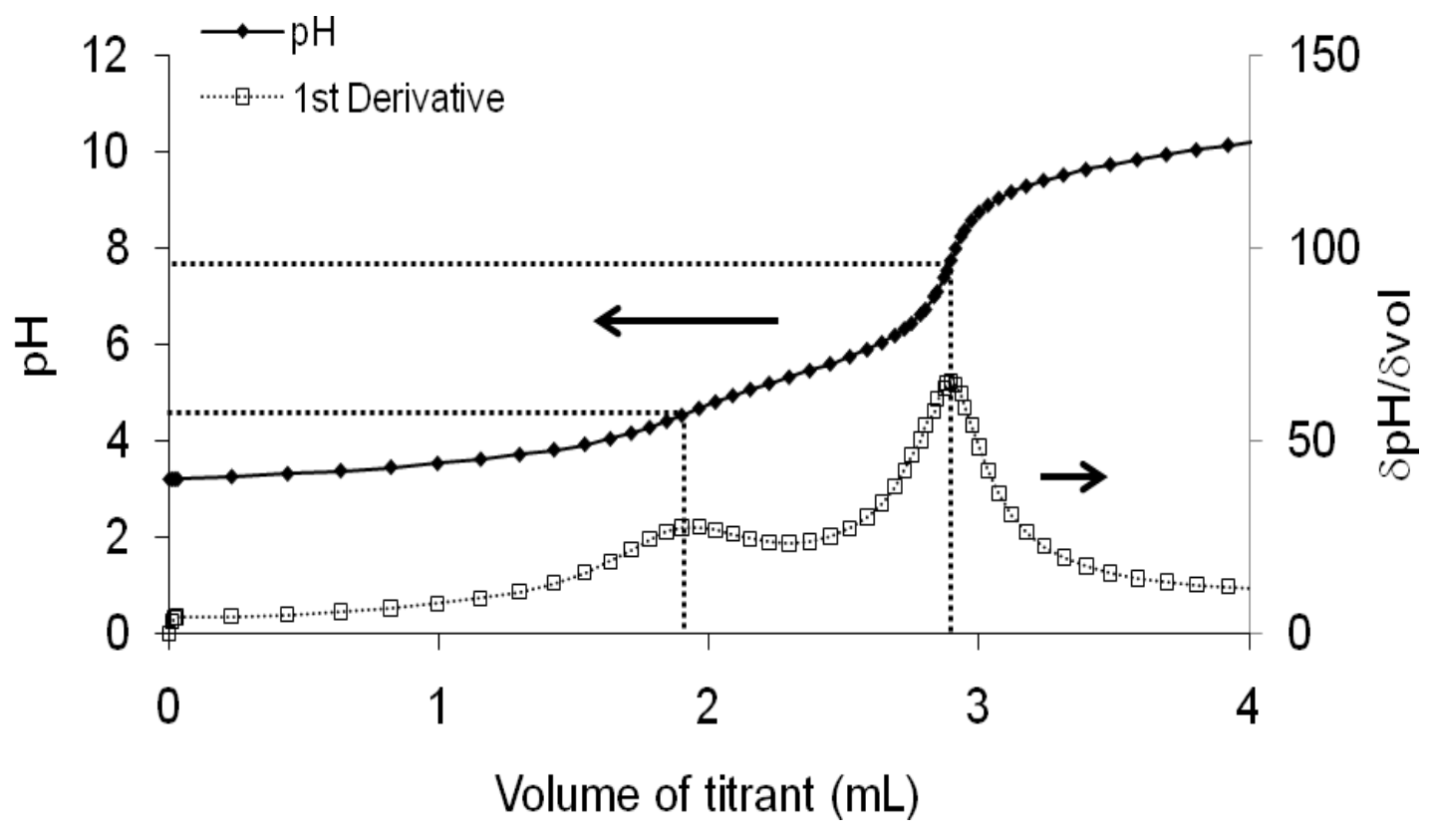

Figure 3.4- Titration of $[\mathrm{HQ}]=3 \times 10^{-4} \mathrm{M},[\mathrm{NaCl}]=0.1 \mathrm{M},[\mathrm{HCl}]=0.001 \mathrm{M}$ by $[\mathrm{NaOH}]$ $0.01024 \pm 0.00002 \mathrm{M}$ and $[\mathrm{NaCl}]=0.09 \mathrm{M} . \mathrm{T}=1.5 \pm 0.2{ }^{\circ} \mathrm{C}$.

Table 3.4- Hyperquad fit parameters for 8-hydroxyquinoline deprotonation constants.

\begin{tabular}{|c|c|c|c|c|c|}
\hline Equation & $\begin{array}{c}\text { Formed } \\
\text { Species }\end{array}$ & Parameter & $\begin{array}{c}\text { Parameter } \\
\text { Refined? }\end{array}$ & $\mathbf{p} \boldsymbol{\beta}$ & $\mathbf{p K}$ \\
\hline$(3.15)$ & $\mathrm{OH}^{-}$ & $\mathrm{K}_{\mathrm{w}}$ & No & 14.7 & 14.7 \\
$(3.13)$ & $\mathrm{H}(\mathrm{Q})$ & $\mathrm{K}_{\mathrm{a} 1}$ & Yes & $5.6 \pm 0.1$ & $5.6 \pm 0.1$ \\
\hline$(3.14)$ & $\mathrm{Q}^{-}$ & $\mathrm{K}_{\mathrm{a} 2}$ & Yes & $15.2 \pm 0.1$ & $9.5 \pm 0.1$ \\
\hline
\end{tabular}




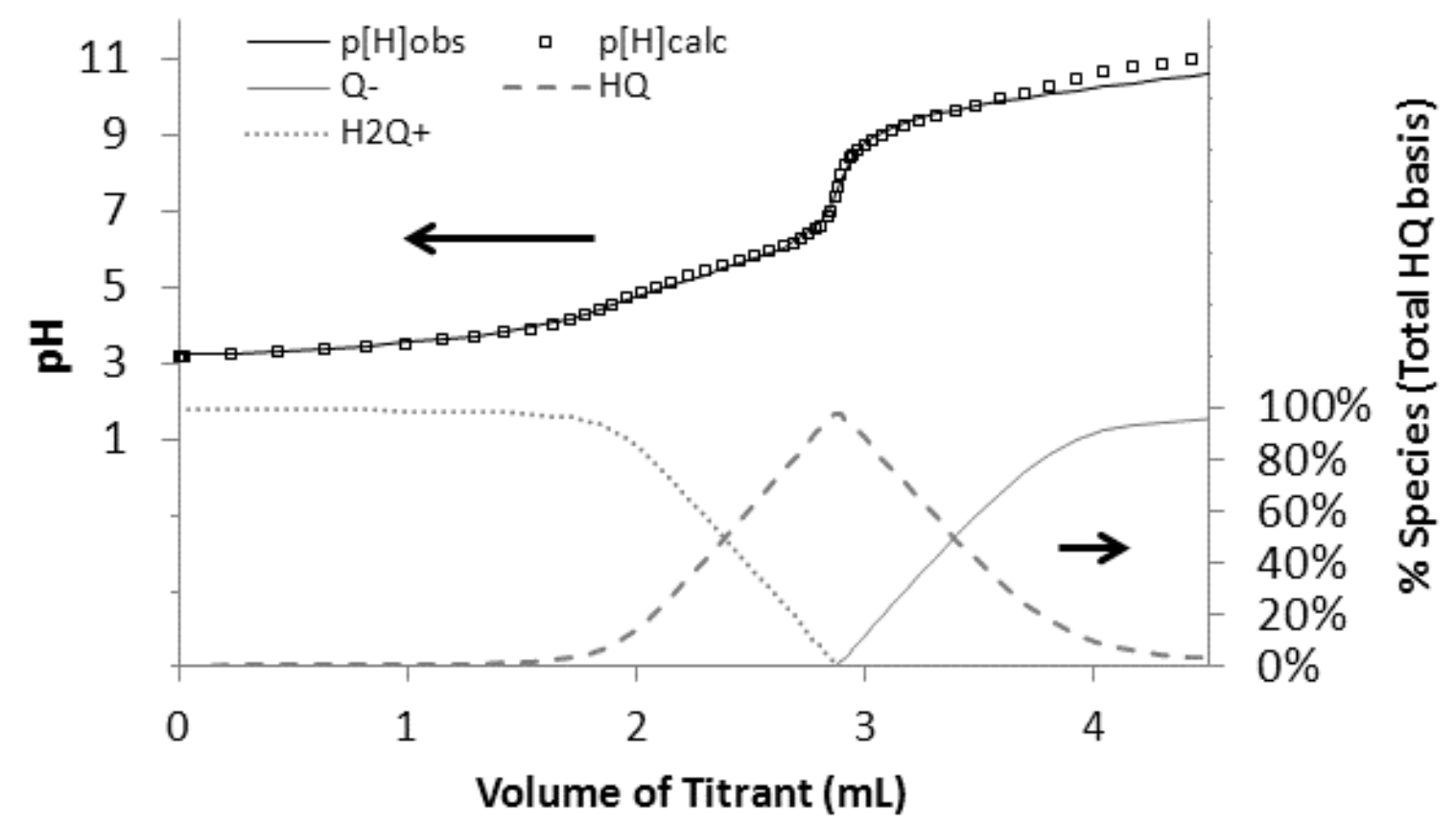

Figure 3.5-Hyperquad modeling results for the potentiometric titration of 8-hydroxyquinoline at $\mathrm{T}=1.5 \pm 0.2^{\circ} \mathrm{C}$.

\subsubsection{Determination of the $\mathrm{pK}_{\mathrm{a}}{ }^{\prime} \mathrm{s}$ of 8-Hydroxyquinoline by Spectrophotometric}

\section{Titration}

The absorbance spectrum of 8-hydroxyquinoline shows bands in the visible and UV regions.

This was immediately evident by the yellow color of the aqueous solution of 8-hydroxyquinoline.

The absorbance changes during a $\mathrm{pH}$ titration are shown in Figure 3.6 and Figure 3.7. The $\mathrm{pH}$ for each spectrum is used as the label for each trace. The spectra in Figure 3.6 and Figure 3.7 show an initial absorbance bands for the $\mathrm{pH}=3.18$ solution at $250.9,306.9,315.5$, and $358.5 \mathrm{~nm}$ (see Table 3.5). This is the absorbance spectrum attributed to $\mathrm{H}_{2} \mathrm{Q}^{+}$, the chemical structure of which is shown in Figure 3.2-structure ' $\mathrm{A}$ '. As the $\mathrm{pH}$ increases the absorbance spectrum shifts to show bands at 241.1, 270, and $306.5 \mathrm{~nm}$ (see Table 3.5). This absorbance spectrum shown in both Figure 3.6 and Figure 3.7 at $\mathrm{pH}=6.87$ and is attributed to $\mathrm{HQ}$, (Figure 3.2-structure ' $\mathrm{B}$ '). At the 
highest $\mathrm{pH}$ shown in Figure 3.6 and Figure 3.7 there is a third spectrum shown for $\mathrm{pH}=10.23$

that is attributed to $Q^{-}$(Figure 3.2-structure ' $C$ ') and shows absorbance bands at 249.3, 331, and $352 \mathrm{~nm}$ (see Table 3.5). Isosbestic points for the transition between $\mathrm{H}_{2} \mathrm{Q}^{+}$and $\mathrm{HQ}$ were observed at 262 and $335 \mathrm{~nm}$. Likewise, Isosbestic points for the transition between $\mathrm{HQ}$ and $\mathrm{Q}$ were observed at 270 and $324 \mathrm{~nm}$. It is notable that the spectrum associated with the species ' $A$ ' and ' $C$ ' show very similar absorbance bands whereas the spectrum of the species ' $B$ ' is much different. This is due to the influence that the proton complexation has on the conjugated aromatic bonds in the double ring structure. For instance, species ' $A$ ' and ' $C$ ' both share the 250 and $360 \mathrm{~nm}$ band and with similar bands at 319 in ' $A$ ' and 330 in ' $C$ '. The ' $B$ ' species shows the 250 band shifted to 243 , and the 360 band shifted to $307 \mathrm{~nm}$.

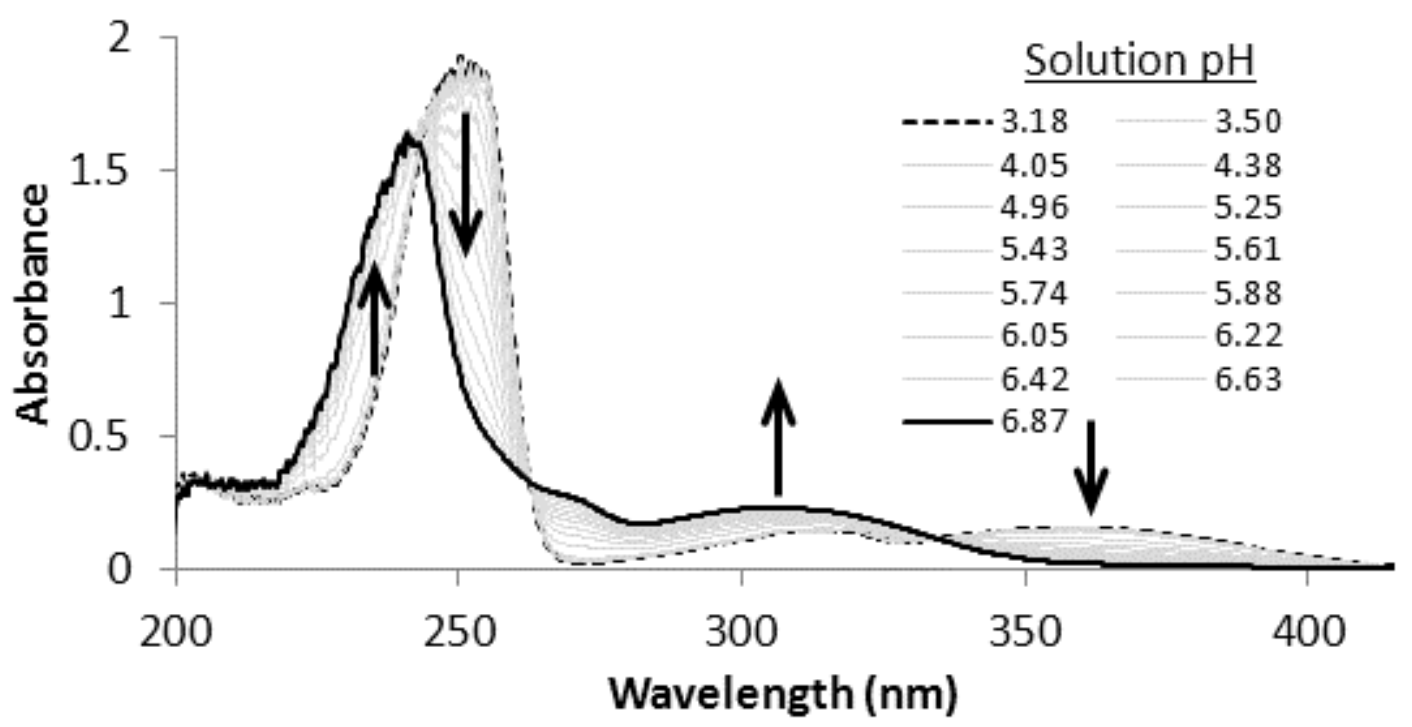

Figure 3.6-Spectral changes for $[\mathrm{HQ}]=10^{-5} \mathrm{M},[\mathrm{NaCl}]=0.1 \mathrm{M},[\mathrm{HCl}]=0.001 \mathrm{M}$ titrated by $\mathrm{NaOH}$ of varying concentration and volume at $1.8 \pm 0.2{ }^{\circ} \mathrm{C}$. The solution $\mathrm{pH}$ for each spectrum is listed in the figure legend. Arrows indicate the direction of the spectral change as $\mathrm{pH}$ increases. These spectral changes represent the transition from 8-hydroxyquinoline protonation state $A$ to $B$ in Figure 3.2. 


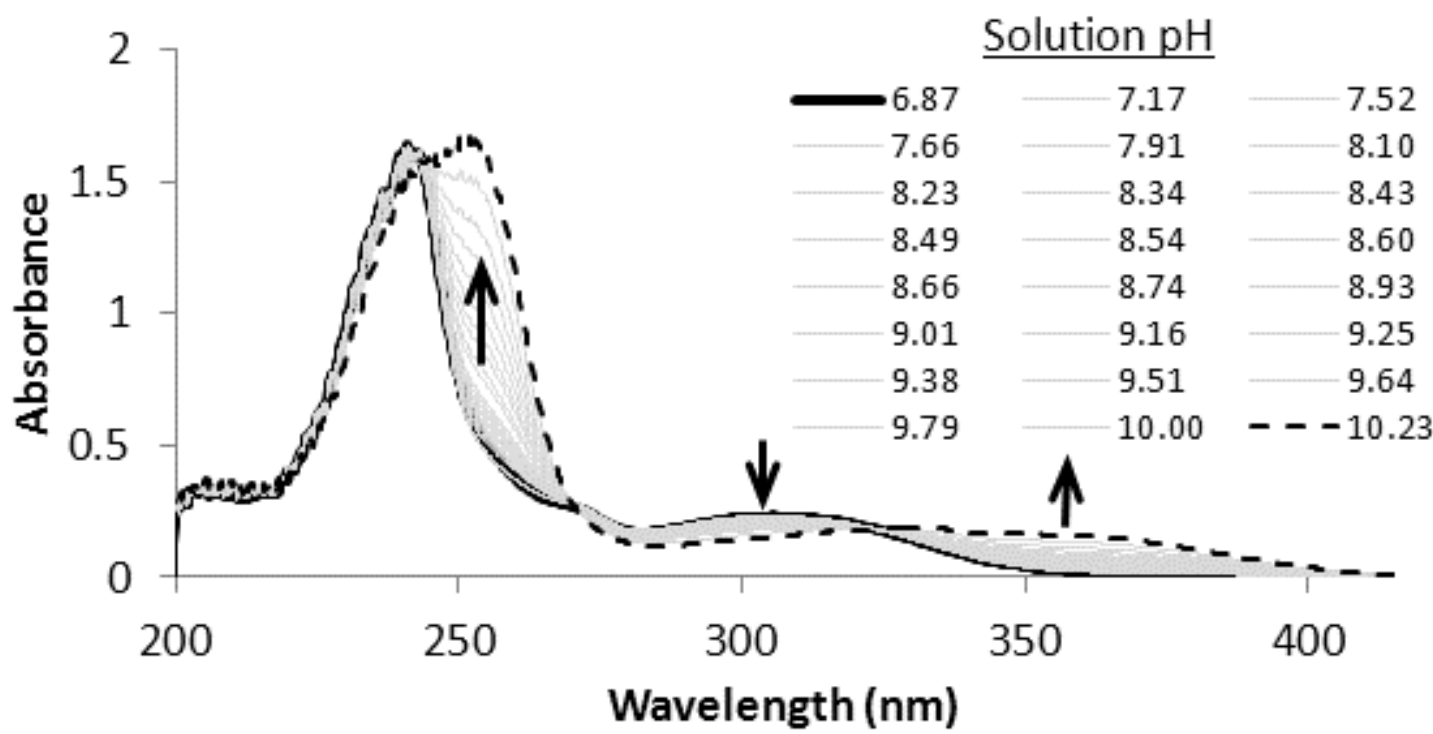

Figure 3.7- Spectral changes for $[\mathrm{HQ}]=10^{-5} \mathrm{M},[\mathrm{NaCl}]=0.1 \mathrm{M},[\mathrm{HCl}]=0.001 \mathrm{M}$ titrated by $\mathrm{NaOH}$ of varying concentration and volume at $1.8 \pm 0.2^{\circ} \mathrm{C}$. The solution $\mathrm{pH}$ for each spectrum is listed in the figure legend. Arrows indicate the direction of the spectral change as $\mathrm{pH}$ increases. These spectral changes represent the transition from 8-hydroxyquinoline protonation state $B$ to $C$ in Figure 3.2.

Table 3.5- The observed spectral maxima in the titration spectrum of 8-hydroxyquinoline. The wavelength of the peak in parenthesis is estimated, but does not have a clearly quantifiable maximum.

\begin{tabular}{|c|c|c|}
\hline $\mathbf{H}_{2} \mathbf{Q}^{+}$ & $\mathbf{H Q}$ & $\mathbf{Q}^{-}$ \\
\hline $\begin{array}{c}\text { Spectrum } \\
\text { Maxima } \\
(\mathbf{n m})\end{array}$ & $\begin{array}{c}\text { Spectrum } \\
\text { Maxima } \\
\text { (nm) }\end{array}$ & $\begin{array}{c}\text { Spectrum } \\
\text { Maxima } \\
(\mathbf{n m})\end{array}$ \\
\hline 250.9 & 241.1 & 249.3 \\
\hline 306.9 & 306.5 & \\
\hline 315.5 & & \\
\hline & & 331 \\
\hline 358.5 & & 352 \\
\hline
\end{tabular}




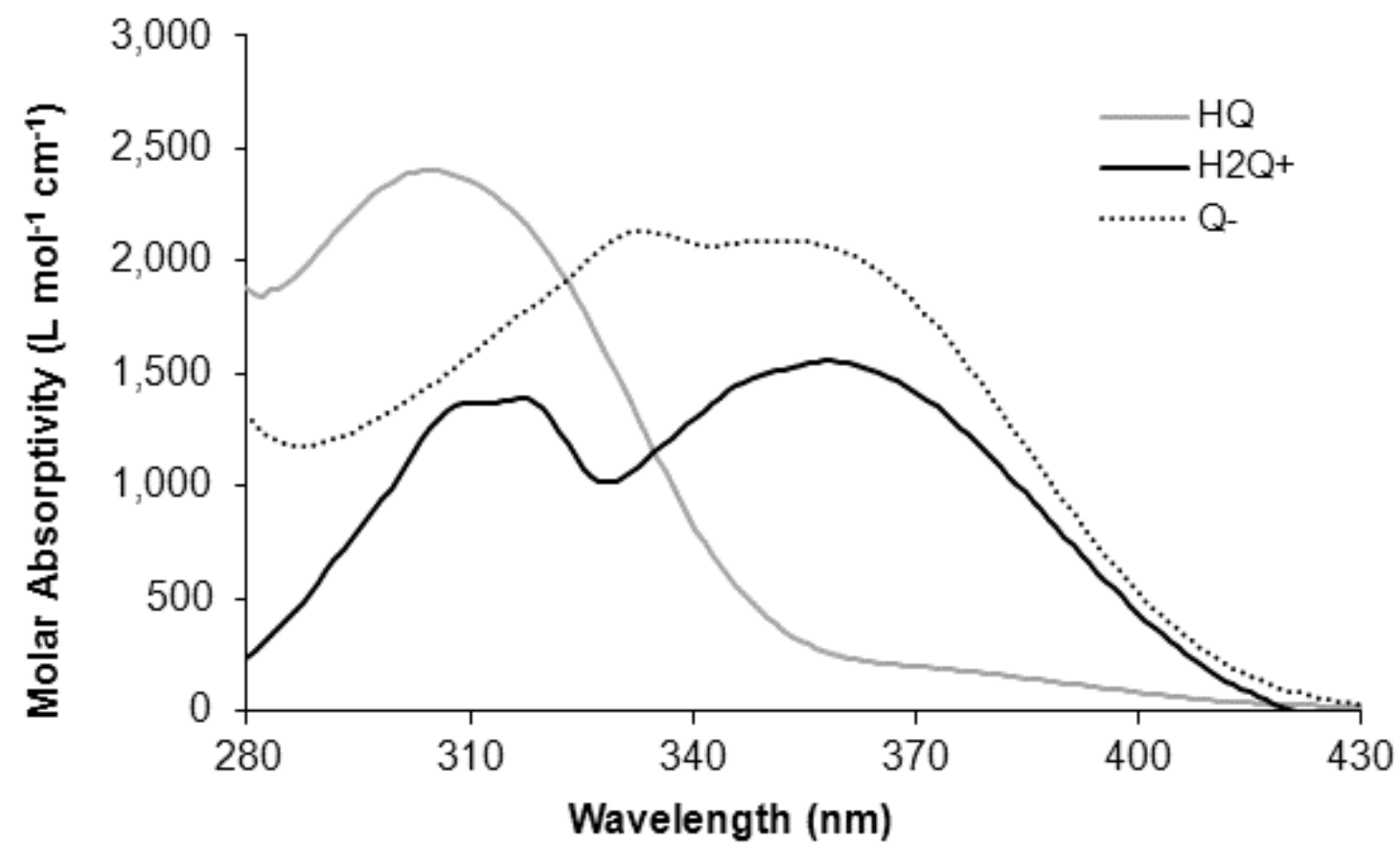

Figure 3.8-Molar absorptivity Spectra obtained from SQUAD modeling using the $280-430 \mathrm{~nm}$ spectral window called window $\mathrm{B}$.

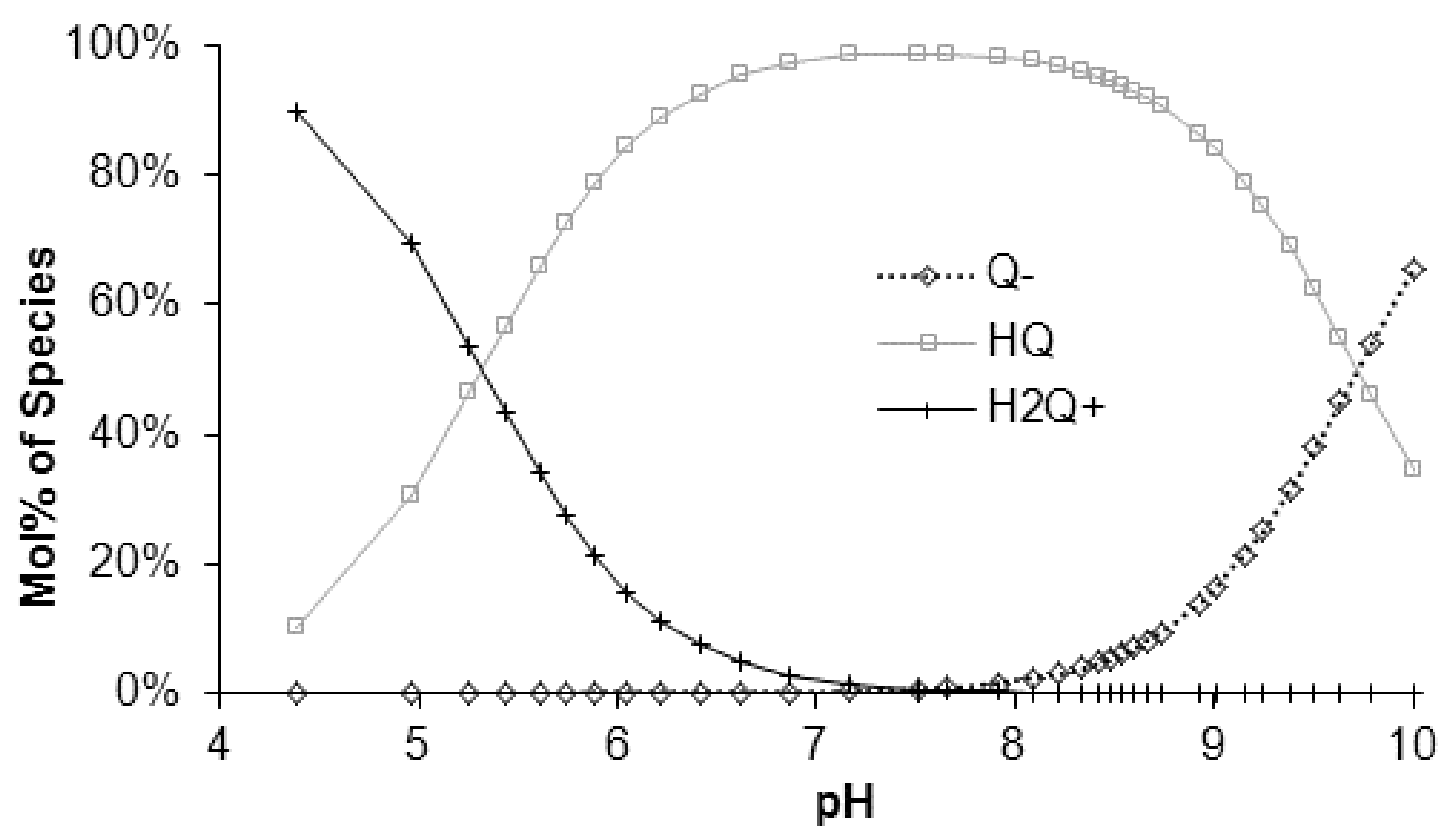

Figure 3.9- Calculated speciation curve obtained from SQUAD modeling using the $280-430 \mathrm{~nm}$ spectral window also called window $B$. 
Equilibrium constants were calculated by SQUAD using different windows of the spectrophotometric data. The modeled windows are designated ' $A$ ' for the entire spectrum (220-445 nm), and two subsets: ' $\mathrm{B}$ ' for the one that contained only the strongest absorbance bands in the UV (220-295 nm), and ' $\mathrm{C}$ ' for the other that contained the weaker absorbance bands in the UV + Visible spectrum (280-430 nm) (see Table 3.6). Spectrophotometric data must be interpreted with caution when the absorbance is greater than $\sim 1$ because spectrophotometers can incorrectly record the absorbance of such samples. ${ }^{[54]}$ It appears that this has happened with the spectra observed in window ' $C$ ' in Figure 3.6 and Figure 3.7 that shows absorbances in the range of 1.5-2.0. The only result that is unaffected by the extreme absorbance in the $220-270 \mathrm{~nm}$ region is window ' $\mathrm{B}$ ' that excludes the high absorbing bands in the spectrum (i.e. $280-439 \mathrm{~nm}$ ) (see Table 3.6). The values of $\mathrm{pK}_{\mathrm{a} 1}=5.32$ and $\mathrm{pK}_{\mathrm{a} 2}=9.72$ are those accepted in this work as most accurate from this technique and the others will be ignored in the future discussion. The molar absorbance spectra obtained for the $\mathrm{H}_{2} \mathrm{Q}^{+}, \mathrm{HQ}$ and $\mathrm{Q}^{-}$species using window ' $\mathrm{B}$ ' is shown in Figure 3.8. The speciation calculated using window ' $\mathrm{B}$ ' obtained by SQUAD modeling is shown in Figure 3.9.

Table 3.6- SQUAD modeled parameters using varied spectral windows.

\begin{tabular}{|c|c|c|c|c|}
\hline \multirow{3}{*}{$\begin{array}{c}\text { Modeling } \\
\text { Window } \\
\text { Designation }\end{array}$} & \multicolumn{2}{|c|}{ Window Limits } & \multirow{2}{*}{$\begin{array}{l}\text { SQUAD } \\
\text { Result }\end{array}$} & \multirow{2}{*}{$\begin{array}{l}\text { SQUAD } \\
\text { Result }\end{array}$} \\
\hline & Lower & Upper & & \\
\hline & $(\mathrm{nm})$ & $(n m)$ & $\mathrm{pK}_{\mathrm{a} 1}$ & $\mathbf{p K}_{\mathrm{a} 2}$ \\
\hline $\bar{A}$ & 220 & 446 & $5.71 \pm 0.01$ & $9.58 \pm 0.01$ \\
\hline B & 280 & 430 & $5.32 \pm 0.01$ & $9.72 \pm 0.01$ \\
\hline C & 220 & 296 & $5.72 \pm 0.01$ & $9.58 \pm 0.01$ \\
\hline
\end{tabular}




\subsubsection{Determination of the Europium Hydrolysis Constants by Potentiometric Titration}

Lanthanides are known to hydrolyze, ${ }^{[147]}$ just as other cations in solution, ${ }^{[148]}$ under conditions of increasing $\mathrm{pH}$. In the systems studied in this work europium hydrolysis is potentially a competitive process with the 8-hydroxyquinoline complexation reaction.

Therefore, both processes must be quantitatively characterized so that the optimum separation can be designed for these systems.

A solution of $\left[\mathrm{Eu}^{3+}\right]=3 \times 10^{-4} \mathrm{M},[\mathrm{NaCl}]=0.1 \mathrm{M}$, and $[\mathrm{HCl}]=0.001 \mathrm{M}$ was titrated with standardized $0.01 \mathrm{M} \mathrm{NaOH}(\mathrm{I}=0.1 \mathrm{M})$. The temperature for this titration was $1.7 \pm 0.2^{\circ} \mathrm{C}$. The titration curve is shown in Figure 3.10. Only two inflection points are indicated by the two maxima of the $1^{\text {st }}$ derivative in Figure 3.10. The first endpoint is attributed to the neutralization of $\mathrm{HCl}$ in solution. The volume of added titrant between the first and second endpoints accounts for 3 equivalents of $\mathrm{NaOH}$ compared to 1 equivalent of europium. Literature data for the lanthanides ${ }^{[147]}$ indicate that the first, second, and third stepwise hydrolysis formation constants are very similar in magnitude, within $0.04-0.66 \log \mathrm{K}$ units of each other. Because the second and third hydrolysis species form in such close succession to the first, it appears that the individual inflection points of the three hydrolysis species are impossible to observe in the titration curve and thus, the second endpoint is attributed to the complete hydrolysis of all $\mathrm{Eu}^{3+}$ in solution. 


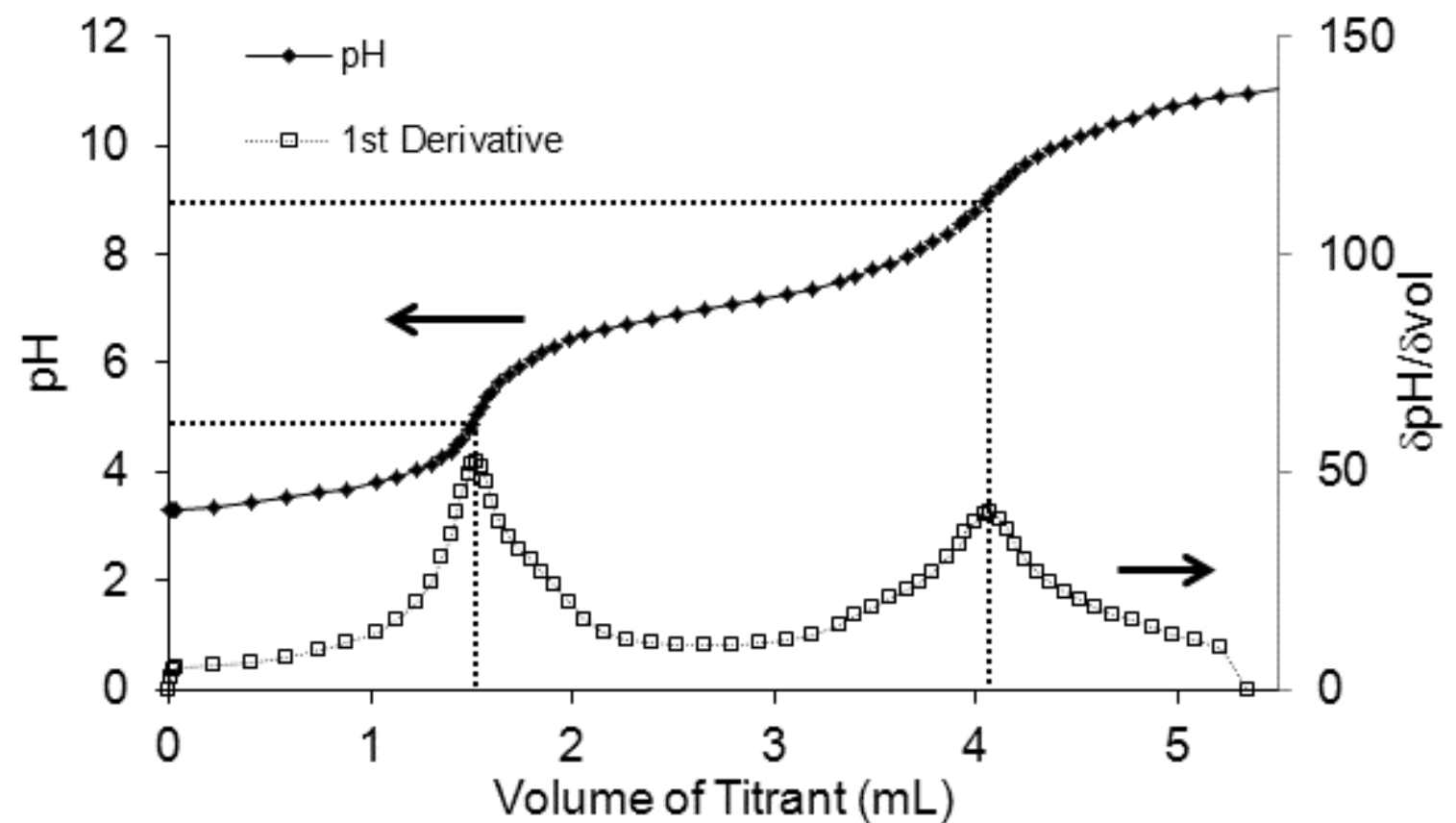

Figure 3.10- Titration of $\left[\mathrm{Eu}^{3+}\right]=3 \times 10^{-4} \mathrm{M},[\mathrm{NaCl}]=0.1 \mathrm{M},[\mathrm{HCl}]=0.001 \mathrm{M}$ by $[\mathrm{NaOH}]$ $0.01024 \pm 0.00002 \mathrm{M}$ and $[\mathrm{NaCl}]=0.09 \mathrm{M} . \mathrm{T}=1.7 \pm 0.2^{\circ} \mathrm{C}$.

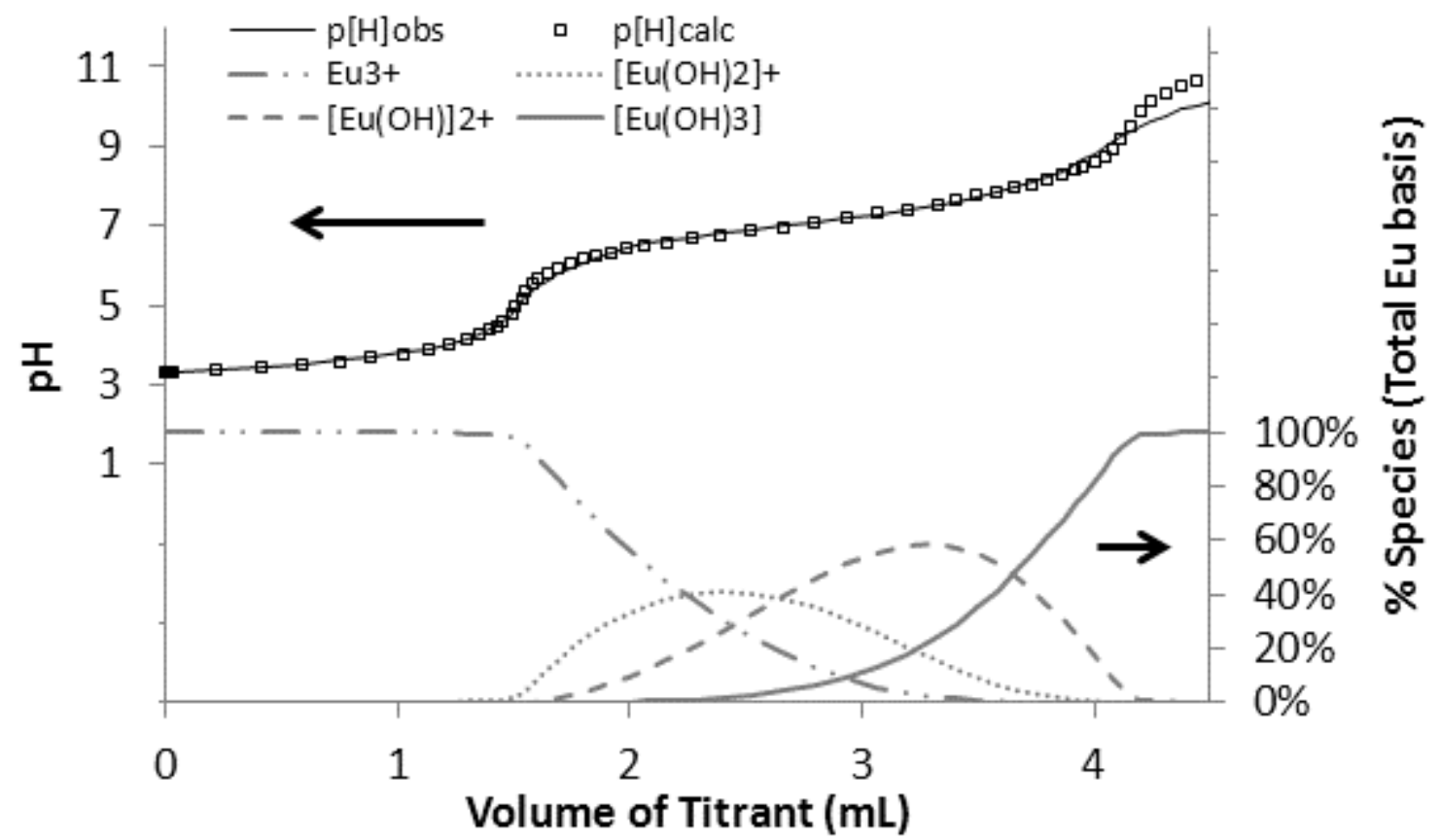

Figure 3.11- Hyperquad modeling results for the titration of $\left[\mathrm{Eu}^{3+}\right]=3 \times 10^{-4} \mathrm{M},[\mathrm{NaCl}]=0.1 \mathrm{M}$, $[\mathrm{HCl}]=0.001 \mathrm{M}$ by $[\mathrm{NaOH}] 0.01024 \pm 0.00002 \mathrm{M}$ and $[\mathrm{NaCl}]=0.09 \mathrm{M} . \mathrm{T}=1.7 \pm 0.2^{\circ} \mathrm{C}$. 
The titration data was modeled in Hyperquad using 14.7 as the $\mathrm{pK}_{\mathrm{w}}$ for the titration conditions. The three hydrolysis reactions used in the model are shown in equations (3.16) (3.18).

$$
\begin{aligned}
& \mathrm{Eu}^{3+}+\mathrm{H}_{2} \mathrm{O} \longleftrightarrow \beta_{1-10} \longleftrightarrow[\mathrm{Eu}(\mathrm{OH})]^{2+}+\mathrm{H}^{+} \\
& \mathrm{Eu}^{3+}+2 \mathrm{H}_{2} \mathrm{O} \stackrel{\beta_{1-20}}{\longleftrightarrow}\left[\mathrm{Eu}(\mathrm{OH})_{2}\right]^{+}+2 \mathrm{H}^{+} \\
& \mathrm{Eu}^{3+}+3 \mathrm{H}_{2} \mathrm{O} \stackrel{\beta_{1-30}}{\longleftrightarrow}\left[\mathrm{Eu}(\mathrm{OH})_{3}\right]+3 \mathrm{H}^{+}
\end{aligned}
$$

The fit parameters obtained from this model are shown in Table 3.7. These data are as expected with the three hydrolysis constants in very close proximity on the $\mathrm{pH}$ scale. The data fitting results are shown in Figure 3.11.

Table 3.7- Hyperquad fit parameters for the europium hydrolysis constants.

\begin{tabular}{|c|c|c|c|c|c|}
\hline Equation & $\begin{array}{c}\text { Formed } \\
\text { Species }\end{array}$ & Parameter & $\begin{array}{c}\text { Parameter } \\
\text { Refined? }\end{array}$ & $\mathbf{p} \boldsymbol{\beta}$ & $\mathbf{p K}$ \\
\hline$(3.15)$ & $\mathrm{OH}^{-}$ & $\mathrm{K}_{\mathrm{w}}$ & No & 14.7 & \\
\hline$(3.16)$ & {$[\mathrm{Eu}(\mathrm{OH})]^{2+}$} & $\beta_{1-10}$ & Yes & $-8.0 \pm 0.2$ & $-8.0 \pm 0.2$ \\
\hline$(3.17)$ & {$\left[\mathrm{Eu}(\mathrm{OH})_{2}\right]^{+}$} & $\beta_{1-20}$ & Yes & $-15.8 \pm 0.1$ & $-7.7 \pm 0.1$ \\
$(3.18)$ & {$\left[\mathrm{Eu}(\mathrm{OH})_{3}\right]$} & $\beta_{1-30}$ & Yes & $-22.5 \pm 0.2$ & $-6.8 \pm 0.2$ \\
\hline
\end{tabular}

\subsubsection{Determination of Europium 8-Hydroxyquinoline Stability Constants by Potentiometric Titration}

The complexation constants from the modeling of $\mathrm{Eu}^{3+}$ hydrolysis reactions presented in Section 3.3.3 were used in the fitting of the stability constants for complexes between europium and 8-hydroxyquinoline. As described earlier, each of the subsequent titrations in the $\mathrm{H}_{2} \mathrm{O}$ system were designed to allow only the formation of certain species and preventing the 
formation of others. In the first titration of a mixed $\mathrm{Eu}^{3+} / \mathrm{HQ}$ solution, an equal concentration of $\mathrm{Eu}^{3+}$ and $\mathrm{HQ}$ were used so that only the $1: 1$ complex $[\mathrm{Eu}(\mathrm{Q})]^{2+}$ would be present. At higher $\mathrm{pH}$ values it was anticipated that the mixed ligand/hydrolysis species will form, requiring the fit of 2 new species for this titration: $[\mathrm{Eu}(\mathrm{OH})(\mathrm{Q})]^{+}$, and $\left[\mathrm{Eu}(\mathrm{OH})_{2}(\mathrm{Q})\right]$. The titration curve obtained for the 1:1 ligand to metal ratio is shown in Figure 3.12. The titration curve showed some very minor maxima in the $1^{\text {st }}$ derivative function shown in Figure 3.12. These results were fit using previously obtained $\mathrm{pK}_{\mathrm{a}}$ 's of 8-hydroxyquinoline for the reactions in equations (3.13), and (3.14). Stability constants for the $\mathrm{Eu}^{3+}-\mathrm{OH}-\mathrm{HQ}$ system were fit for the model according to equations (3.19) - (3.21). The Hyperquad fit results show a gradual transition from one species to the next as the $\mathrm{pH}$ increases with $[\mathrm{Eu}(\mathrm{Q})]^{2+}$ forming first, $[\mathrm{Eu}(\mathrm{OH})(\mathrm{Q})]^{+}$forming next and $\left[\mathrm{Eu}(\mathrm{OH})_{2}(\mathrm{Q})\right]$ forming last. The parameters that resulted from the Hyperquad modeling Fit are shown in Table 3.8.

$$
\begin{gathered}
\mathrm{Eu}^{3+}+\mathrm{Q}^{-} \stackrel{\beta_{101}}{\longleftrightarrow}[\mathrm{Eu}(\mathrm{Q})]^{2+} \\
\mathrm{Eu}^{3+}+\mathrm{H}_{2} \mathrm{O}+\mathrm{Q}^{-} \stackrel{\beta_{1-11}}{\longleftrightarrow}[\mathrm{Eu}(\mathrm{OH})(\mathrm{Q})]^{+}+\mathrm{H}^{+} \\
\mathrm{Eu}^{3+}+2 \mathrm{H}_{2} \mathrm{O}+\mathrm{Q}^{-} \stackrel{\beta_{1-21}}{\longleftrightarrow}\left[\mathrm{Eu}(\mathrm{OH})_{2}(\mathrm{Q})\right]+2 \mathrm{H}^{+}
\end{gathered}
$$

Table 3.8- Hyperquad fit parameters for the 1:1 ligand to metal ratio solutions.

\begin{tabular}{|c|c|c|c|c|c|}
\hline Equation & $\begin{array}{c}\text { Formed } \\
\text { Species }\end{array}$ & Parameter & $\begin{array}{c}\text { Parameter } \\
\text { Refined? }\end{array}$ & $\mathbf{p} \boldsymbol{\beta}$ & pK \\
\hline$(3.15)$ & $\mathrm{OH}^{-}$ & $\mathrm{K}_{\mathrm{w}}$ & No & 14.7 & \\
\hline$(3.13)$ & $\mathrm{HQ}$ & $\mathrm{K}_{\mathrm{a} 1}$ & No & 5.6 & 5.6 \\
\hline$(3.14)$ & $\mathrm{Q}$ & $\mathrm{K}_{\mathrm{a} 2}$ & No & 15.2 & 9.5 \\
\hline$(3.19)$ & {$[\mathrm{Eu}(\mathrm{Q})]^{2+}$} & $\beta_{101}$ & Yes & $-6.9 \pm 0.1$ & $-6.9 \pm 0.1$ \\
\hline$(3.20)$ & {$[\mathrm{Eu}(\mathrm{OH})(\mathrm{Q})]^{+}$} & $\beta_{1-11}$ & Yes & $-13.3 \pm 0.1$ & $-6.4 \pm 0.1$ \\
\hline$(3.21)$ & {$\left[\mathrm{Eu}(\mathrm{OH})(\mathrm{Q})_{2}\right]$} & $\beta_{1-21}$ & Yes & $-18.8 \pm 0.1$ & $-5.6 \pm 0.1$ \\
\hline
\end{tabular}




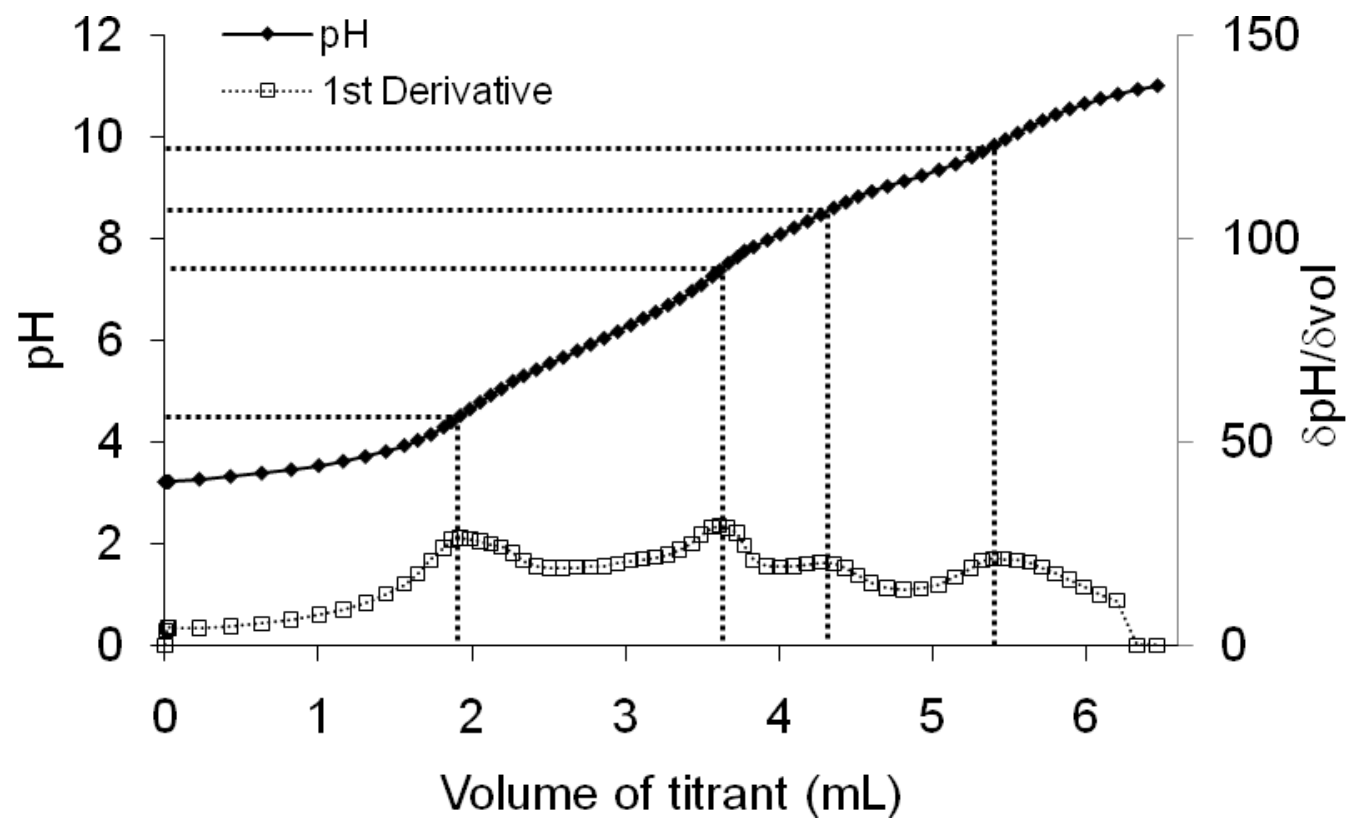

Figure 3.12- Titration of $[\mathrm{HQ}]=3 \times 10^{-4} \mathrm{M},\left[\mathrm{Eu}^{3+}\right]=3 \times 10^{-4} \mathrm{M},[\mathrm{NaCl}]=0.1 \mathrm{M},[\mathrm{HCl}]=0.001 \mathrm{M}$ by $[\mathrm{NaOH}]=0.01024 \pm 0.00002 \mathrm{M}$ and $[\mathrm{NaCl}]=0.09 \mathrm{M} . \mathrm{T}=1.7 \pm 0.2^{\circ} \mathrm{C}$.

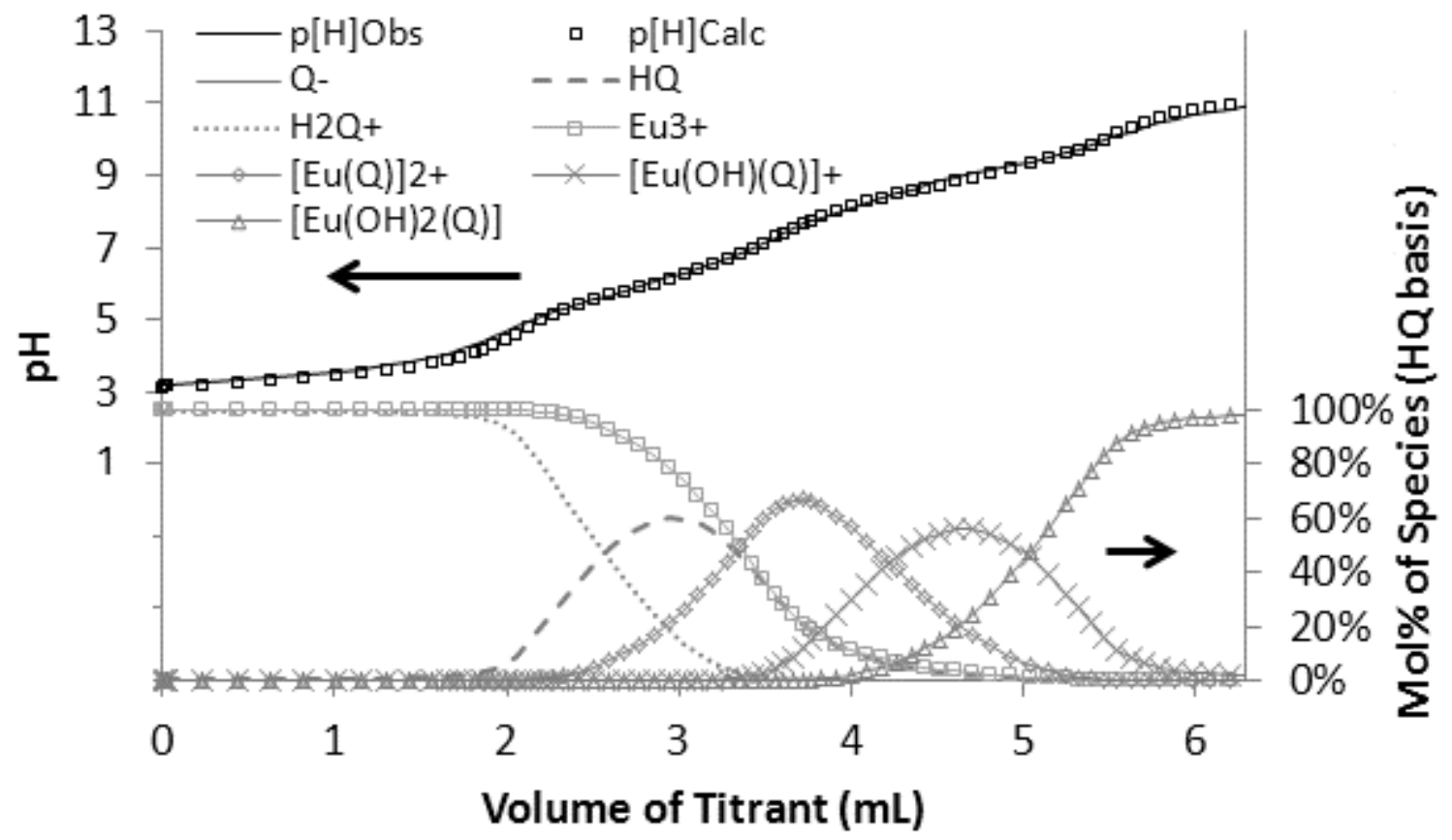

Figure 3.13- Hyperquad fit results for titration of $[\mathrm{HQ}]=3 \times 10^{-4} \mathrm{M},\left[\mathrm{Eu}^{3+}\right]=3 \times 10^{-4} \mathrm{M},[\mathrm{NaCl}]=0.1$ $\mathrm{M},[\mathrm{HCl}]=0.001 \mathrm{M}$ by $[\mathrm{NaOH}]=0.01024 \pm 0.00002 \mathrm{M}$ and $[\mathrm{NaCl}]=0.09 \mathrm{M} . \mathrm{T}=1.7 \pm 0.2^{\circ} \mathrm{C}$. 
The next titration was specifically designed to follow the formation of both $[\mathrm{Eu}(\mathrm{Q})]^{2+}$ and $\left[\mathrm{Eu}(\mathrm{Q})_{2}\right]^{+}$but preventing the formation of $\left[\mathrm{Eu}(\mathrm{Q})_{3}\right]$. The solution designed to achieve this was one with a 2:1 ligand to metal ratio. The titration curve obtained using this solution is shown in Figure 3.14. This titration curve appears very similar to that obtained for the $1: 1$ ligand to metal ratio solution. It was anticipated that this curve when fit would also yield a formation of 3 species just as in the 1:1 curve, but two previously unobserved species would be formed: $\left[\mathrm{Eu}(\mathrm{Q})_{2}\right]^{+}$, and the mixed ligand/hydrolysis species $\left[\mathrm{Eu}(\mathrm{OH})(\mathrm{Q})_{2}\right]$. This curve was fit using the previously obtained 8-hydroxyquinoline $\mathrm{pK}_{\mathrm{a}}$ values [equations (3.13), (3.14)], the formation constant for $[\mathrm{Eu}(\mathrm{Q})]^{2+}$ from the 1:1 ligand to metal solution modeling [equation (3.19)], and two new reactions that are shown in equations $(3.22)-(3.23)$.

$$
\begin{gathered}
\mathrm{Eu}^{3+}+2 \mathrm{Q}^{-} \stackrel{\beta_{102}}{\longleftrightarrow}\left[\mathrm{Eu}(\mathrm{Q})_{2}\right]^{+} \\
\mathrm{Eu}^{3+}+\mathrm{H}_{2} \mathrm{O}+2 \mathrm{Q}^{-} \stackrel{\beta_{1-12}}{\longleftrightarrow}\left[\mathrm{Eu}(\mathrm{OH})(\mathrm{Q})_{2}\right]+\mathrm{H}^{+}
\end{gathered}
$$

The Hyperquad fit results using the above described model are shown in Figure 3.15. The Hyperquad results show a smooth transition between three species as the $\mathrm{pH}$ increases. The order of the species formation is $\mathrm{Eu}^{3+} \rightarrow[\mathrm{Eu}(\mathrm{Q})]^{2+} \rightarrow\left[\mathrm{Eu}(\mathrm{Q})_{2}\right]^{+} \rightarrow\left[\mathrm{Eu}(\mathrm{OH})(\mathrm{Q})_{2}\right]$. The parameters that define this system according to the model are shown in Table 3.9.

Table 3.9- Hyperquad fit Parameters for the 2:1 ligand to metal solution.

\begin{tabular}{|c|c|c|c|c|c|}
\hline Equations & $\begin{array}{c}\text { Formed } \\
\text { Species }\end{array}$ & Parameter & $\begin{array}{c}\text { Parameter } \\
\text { Refined? }\end{array}$ & $\mathbf{p} \boldsymbol{\beta}$ & $\mathbf{p K}$ \\
\hline$(3.15)$ & $\mathrm{OH}^{-}$ & $\mathrm{K}_{\mathrm{w}}$ & No & 14.7 & \\
\hline$(3.13)$ & $\mathrm{HQ}$ & $\mathrm{K}_{\mathrm{a} 1}$ & No & 5.6 & 5.6 \\
\hline$(3.14)$ & $\mathrm{Q}^{-}$ & $\mathrm{K}_{\mathrm{a} 2}$ & No & 15.2 & 9.5 \\
\hline$(3.19)$ & {$[\mathrm{Eu}(\mathrm{Q})]^{2+}$} & $\beta_{101}$ & No & -6.9 & -6.9 \\
\hline$(3.22)$ & {$\left[\mathrm{Eu}(\mathrm{Q})_{2}\right]^{+}$} & $\beta_{102}$ & Yes & $-12.5 \pm 0.7$ & $-5.6 \pm 0.7$ \\
\hline$(3.23)$ & {$\left[\mathrm{Eu}(\mathrm{OH})(\mathrm{Q})_{2}\right]$} & $\beta_{1-12}$ & Yes & $-19.3 \pm 0.3$ & $-6.7 \pm 0.3$ \\
\hline
\end{tabular}




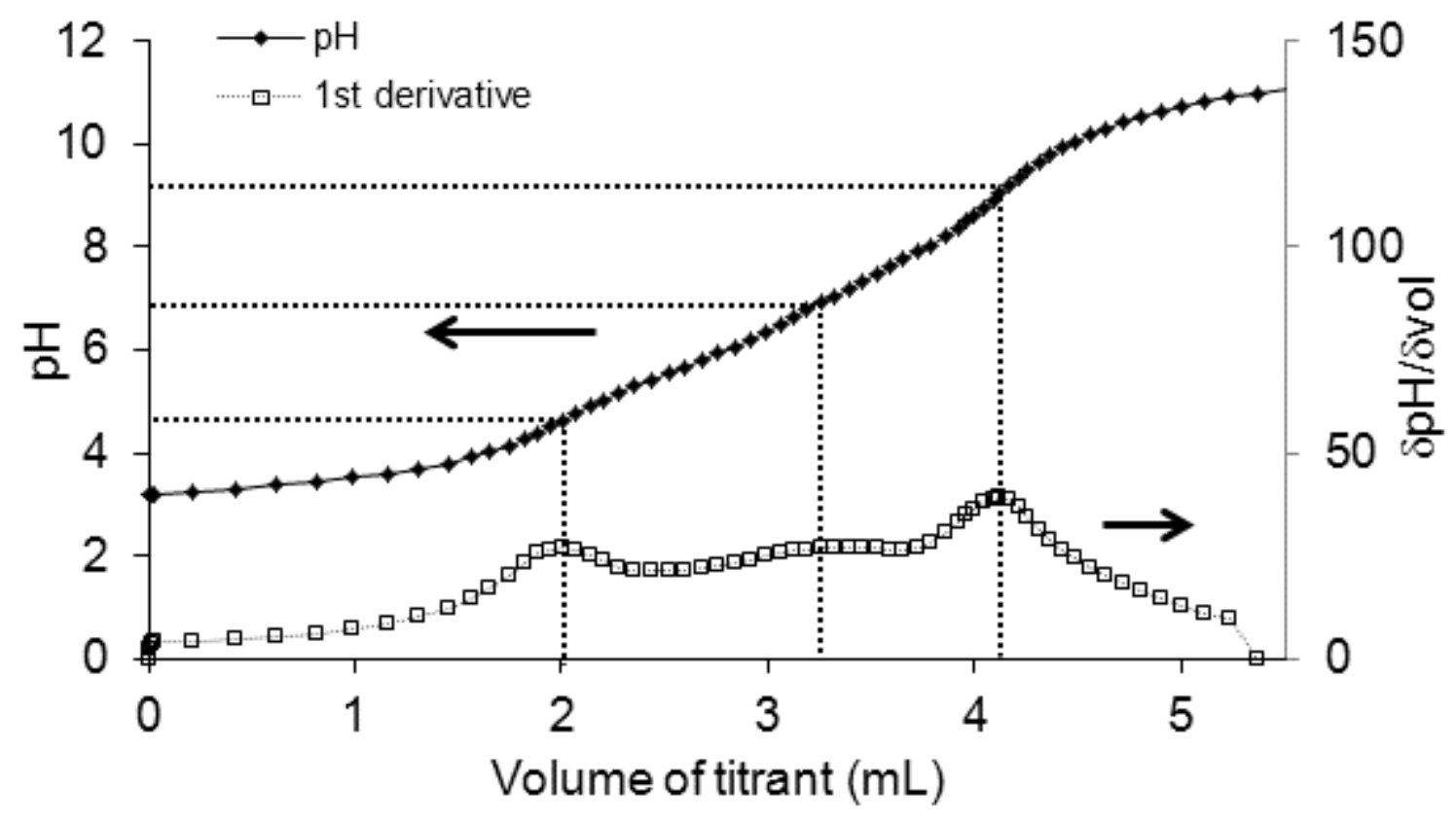

Figure 3.14- Titration of $[\mathrm{HQ}]=3 \times 10^{-4} \mathrm{M},\left[\mathrm{Eu}^{3+}\right]=1.5 \times 10^{-4} \mathrm{M},[\mathrm{NaCl}]=0.1 \mathrm{M},[\mathrm{HCl}]=0.001 \mathrm{M}$ by $[\mathrm{NaOH}]=0.01024 \pm 0.00002 \mathrm{M}$ and $[\mathrm{NaCl}]=0.09 \mathrm{M} . \mathrm{T}=1.9 \pm 0.2^{\circ} \mathrm{C}$.

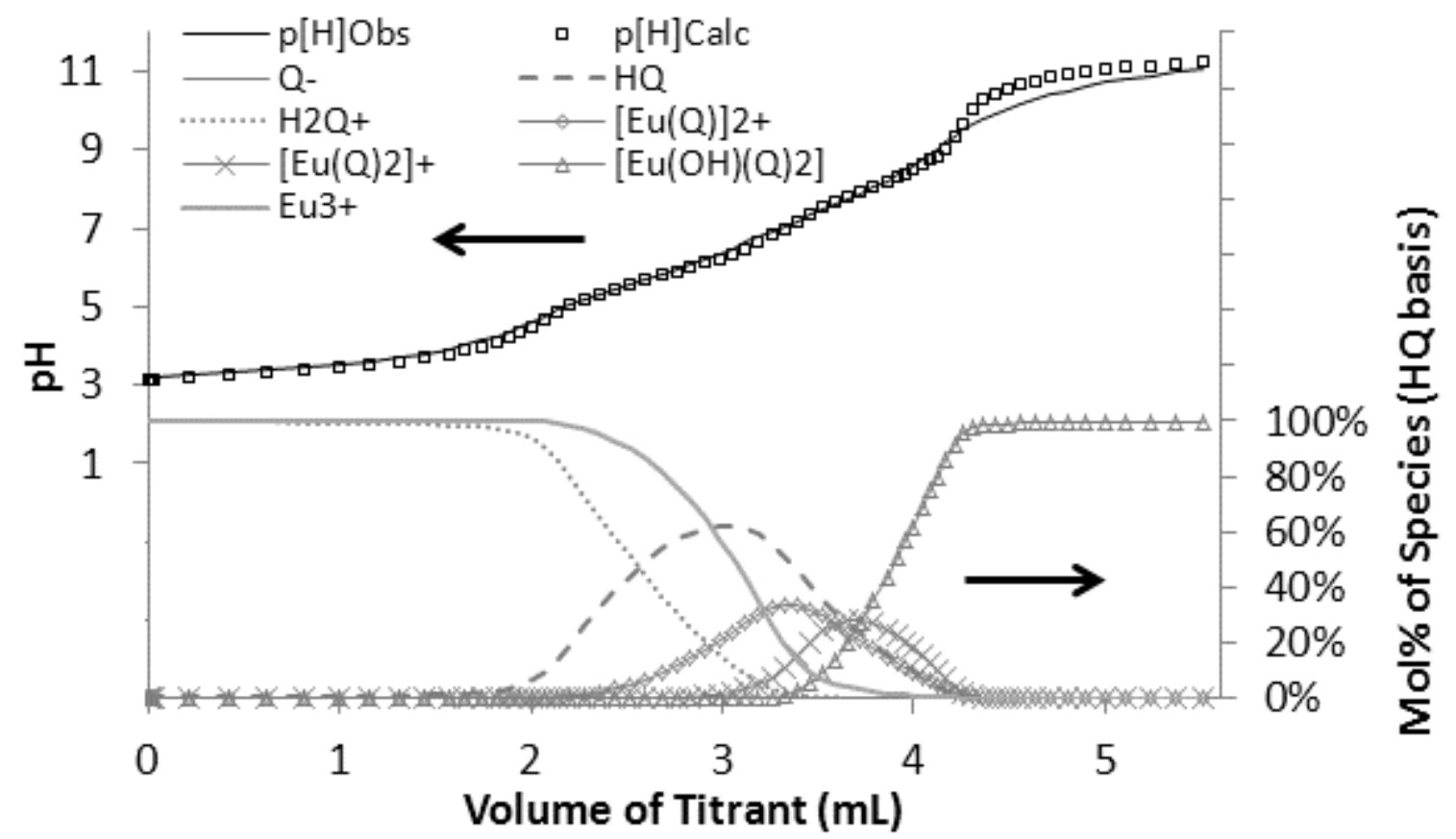

Figure 3.15- Hyperquad fit results for titration of $[\mathrm{HQ}]=3 \times 10^{-4} \mathrm{M},\left[\mathrm{Eu}^{3+}\right]=1.5 \times 10^{-4} \mathrm{M},[\mathrm{NaCl}]=$ $0.1 \mathrm{M},[\mathrm{HCl}]=0.001 \mathrm{M}$ by $[\mathrm{NaOH}]=0.01024 \pm 0.00002 \mathrm{M}$ and $[\mathrm{NaCl}]=0.09 \mathrm{M} . \mathrm{T}=1.7 \pm 0.2^{\circ} \mathrm{C}$. 
The last titration was designed so that all three europium 8-hydroxyquinoline species could form: $[\mathrm{Eu}(\mathrm{Q})]^{2+},\left[\mathrm{Eu}(\mathrm{Q})_{2}\right]^{+},\left[\mathrm{Eu}(\mathrm{Q})_{3}\right]$. This titration contained a 3:1 ligand to metal ratio, however a higher ratio would have also worked. The titration curve is shown in Figure 3.16. Using the previously obtained 8-hydroxyquinoline $\mathrm{pK}_{\mathrm{a}}$ values [equations (3.13), and (3.14)], the complexation constants for the formation of $[\mathrm{Eu}(\mathrm{Q})]^{2+}$, and $\left[\mathrm{Eu}(\mathrm{Q})_{2}\right]^{+}$[equation (3.19), ], (3.22), the parameter for the formation of $\left[\mathrm{Eu}(\mathrm{Q})_{3}\right]$ shown in reaction (3.24) was fit using Hyperquad.

$$
\mathrm{Eu}^{3+}+3 \mathrm{Q}^{-} \stackrel{\beta_{103}}{\longleftrightarrow}\left[\mathrm{Eu}(\mathrm{Q})_{3}\right]
$$

The only parameter that was allowed to vary in this fit was the $\left[\mathrm{Eu}(\mathrm{Q})_{3}\right]$ formation parameter. Attempts to fit more parameters simultaneously led to larger standard deviations in the parameter error, which was interpreted as a poorer result and was thus rejected. The modeling speciation results are shown in Figure 3.17 with the stability constant results shown in Table 3.10 .

Table 3.10- Parameters obtained from the Hyperquad fit of the 3:1 Ligand to metal solution titration.

\begin{tabular}{|c|c|c|c|c|c|}
\hline Equation & $\begin{array}{c}\text { Formed } \\
\text { Species }\end{array}$ & Parameter & $\begin{array}{c}\text { Parameter } \\
\text { Refined? }\end{array}$ & $\mathbf{p} \boldsymbol{\beta}$ & pK \\
\hline$(3.15)$ & $\mathrm{OH}^{-}$ & $\mathrm{K}_{\mathrm{w}}$ & No & 14.7 & \\
\hline$(3.13)$ & $\mathrm{HQ}$ & $\mathrm{K}_{\mathrm{a} 1}$ & No & 5.6 & 5.6 \\
\hline$(3.14)$ & $\mathrm{Q}^{-}$ & $\mathrm{K}_{\mathrm{a} 2}$ & No & 15.2 & 9.5 \\
\hline$(3.19)$ & {$[\mathrm{Eu}(\mathrm{Q})]^{2+}$} & $\beta_{101}$ & No & -6.9 & -6.9 \\
\hline$(3.22)$ & {$\left[\mathrm{Eu}(\mathrm{Q})_{2}\right]^{+}$} & $\beta_{102}$ & No & -12.5 & -5.5 \\
\hline$(3.24)$ & {$\left[\mathrm{Eu}(\mathrm{Q})_{3}\right]$} & $\beta_{103}$ & Yes & $-18.7 \pm 0.5$ & $-6.2 \pm 0.9$ \\
\hline
\end{tabular}




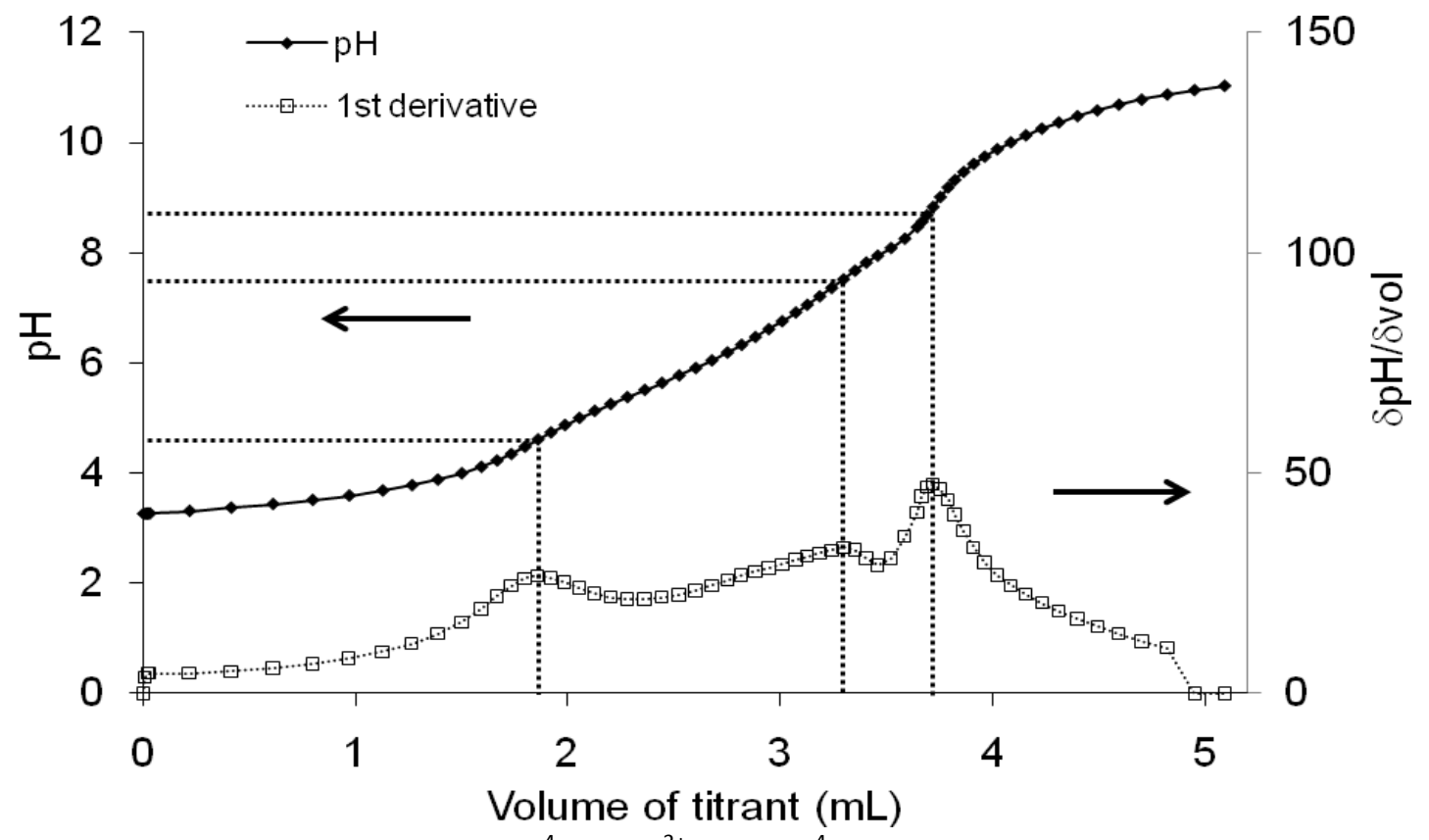

Figure 3.16- Titration of $[\mathrm{HQ}]=3 \times 10^{-4} \mathrm{M},\left[\mathrm{Eu}^{3+}\right]=1 \times 10^{-4} \mathrm{M},[\mathrm{NaCl}]=0.1 \mathrm{M},[\mathrm{HCl}]=0.001 \mathrm{M}$ by $[\mathrm{NaOH}]=0.01024 \pm 0.00002 \mathrm{M}$ and $[\mathrm{NaCl}]=0.09 \mathrm{M} . \mathrm{T}=2.0 \pm 0.2{ }^{\circ} \mathrm{C}$.

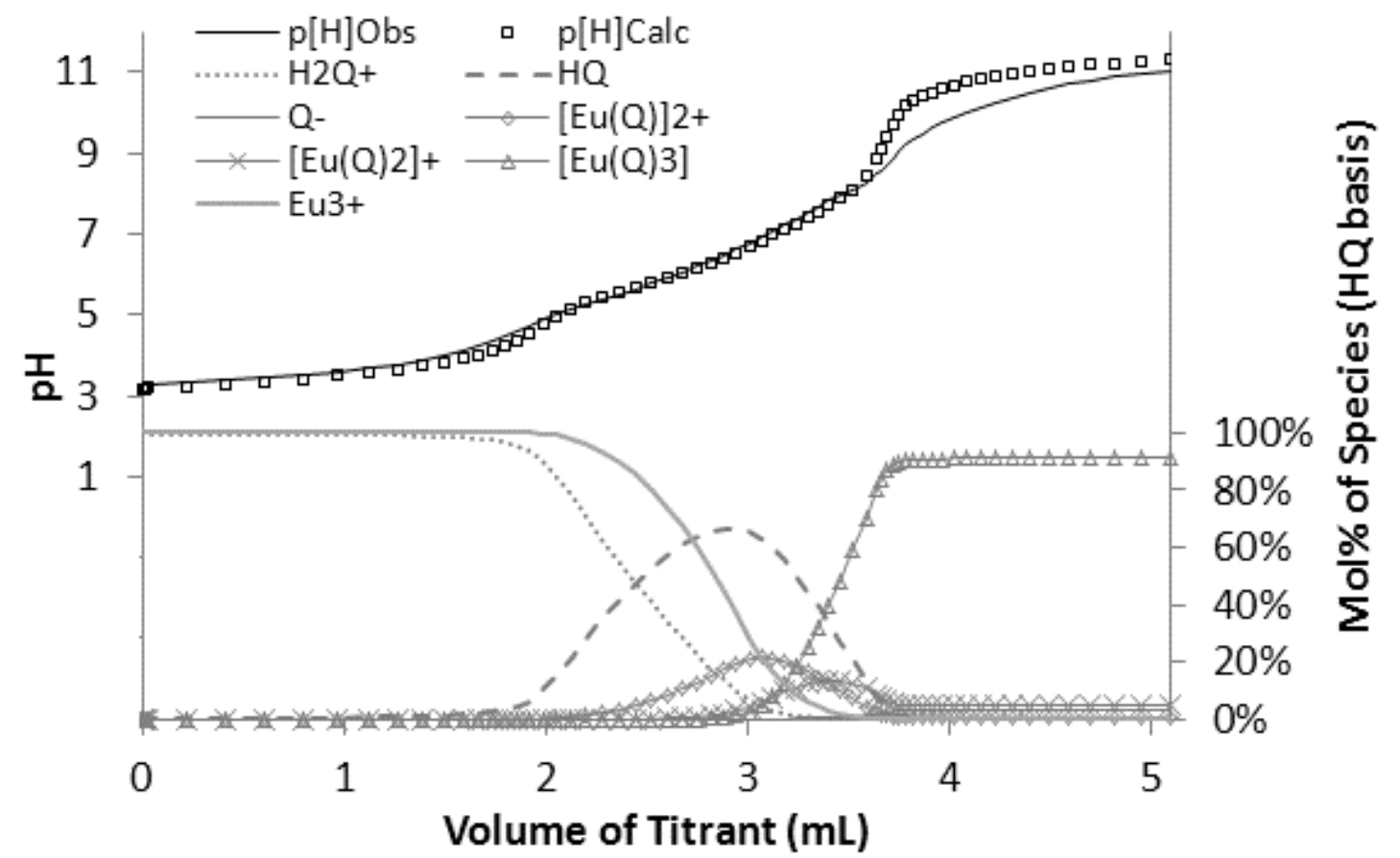

Figure 3.17- Hyperquad fit results for titration of $[\mathrm{HQ}]=3 \times 10^{-4} \mathrm{M},\left[\mathrm{Eu}^{3+}\right]=1 \times 10^{-4} \mathrm{M},[\mathrm{NaCl}]=0.1$ $\mathrm{M},[\mathrm{HCl}]=0.001 \mathrm{M}$ by $[\mathrm{NaOH}]=0.01024 \pm 0.00002 \mathrm{M}$ and $[\mathrm{NaCl}]=0.09 \mathrm{M} . \mathrm{T}=2.0 \pm 0.2^{\circ} \mathrm{C}$. 


\subsubsection{Determination of Europium 8-Hydroxyquinoline Stability Constants by Spectrophotometric Titration}

The use of spectrophotometric titration to obtain the stability constants for europium 8hydroxyquinoline complexation was tested. At the outset this technique poses some challenges due to the difference in molar absorptivity of the constituents involved. Europium absorbs very weakly, with multiple spectral bands in the range of $272-401 \mathrm{~nm} \cdot{ }^{[149]}$ The largest absorbing band in this range is $394 \mathrm{~nm}$ with a molar absorptivity of $3.06 \mathrm{~L} \mathrm{~mol}^{-1} \mathrm{~cm}^{-1}{ }^{[149]}$ By comparison, 8hydroxyquinoline shows a molar absorptivity at $394 \mathrm{~nm}$ of 615,105 , and $738 \mathrm{~L} \mathrm{~mol}^{-1} \mathrm{~cm}^{-1}$ for $\mathrm{H}_{2} \mathrm{Q}^{+}, \mathrm{HQ}$ and $\mathrm{Q}$, respectively (see section 3.3.2). The large absorbance of 8-hydroxyquinoline relative to europium makes it impossible to observe the spectral changes of europium directly.

A spectrophotometric titration of a solution containing both europium and 8hydroxyquinoline was performed in an effort to obtain the stability constants for the europium 8-hydroxyquinoline complexes. The obtained spectra are shown below in Figure 3.18 - Figure 3.20. Attempts at modeling this spectral data using SQUAD were not successful. The spectral changes in the ligand absorbance upon complexation of europium were not sufficient for SQUAD to identify the separate metal:ligand species spectrum. Using a different metal ion with an absorbance band that is not obscured by the absorbance of 8-hydroxyquinoline would increase the likelihood of SQUAD being able to fit stability constant from spectrum. The absorbance of 8-hydroxyquinoline is zero above $430 \mathrm{~nm}$, therefore, other lanthanides that are possible candidates are praseodymium $(440 \mathrm{~nm})$, neodymium $(521,720$, or $794 \mathrm{~nm})$, dysprosium (908 nm), holmium (537 nm), erbium (523 nm), thulim (683), or ytterbium (974 $\mathrm{nm}) \cdot{ }^{[149]}$ 


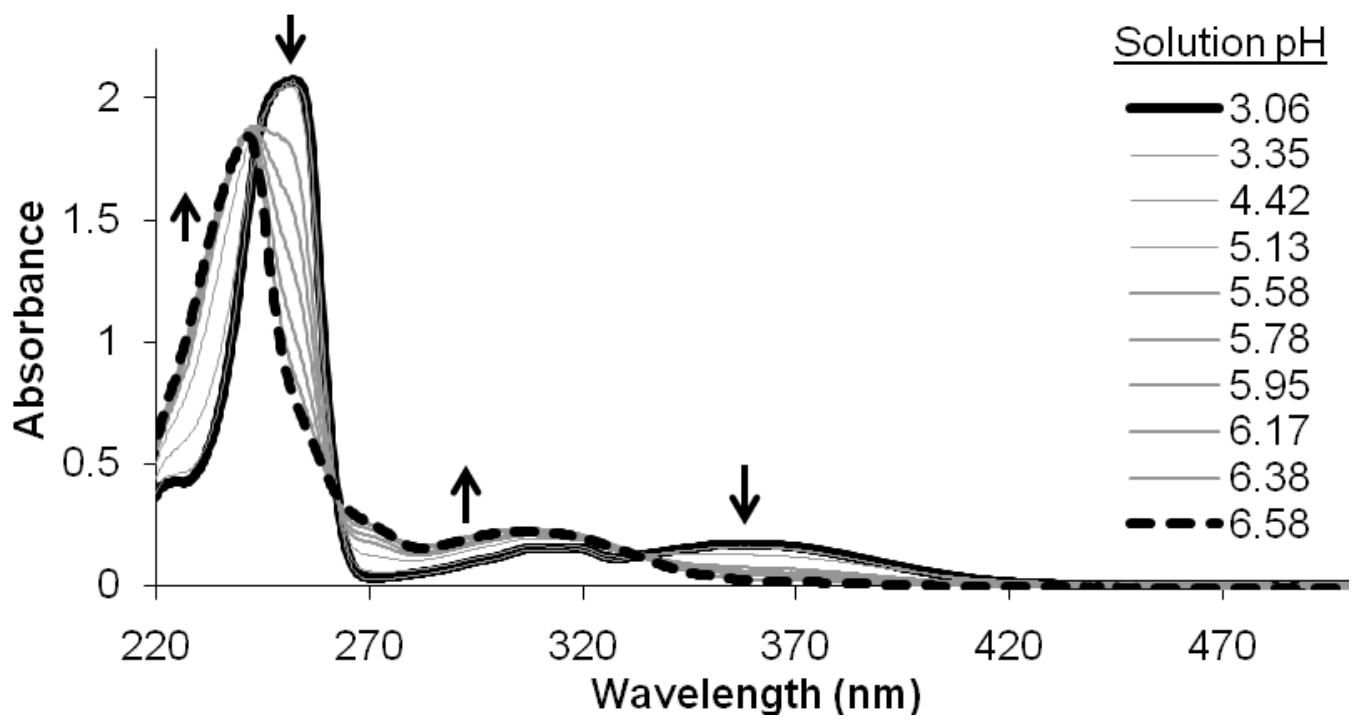

Figure 3.18- Spectral changes for $[\mathrm{HQ}]=10^{-5} \mathrm{M},\left[\mathrm{Eu}^{+3}\right]=2 \times 10^{-6} \mathrm{M},[\mathrm{NaCl}]=0.1 \mathrm{M},[\mathrm{HCl}]=0.001$ $\mathrm{M}$ titrated by $\mathrm{NaOH}$ of varying concentration and volume at $\mathrm{T}=1.8 \pm 0.2^{\circ} \mathrm{C}$. The solution $\mathrm{pH}$ for each spectrum is listed in the figure legend. Arrows indicate the direction of the spectral change as $\mathrm{pH}$ increases.

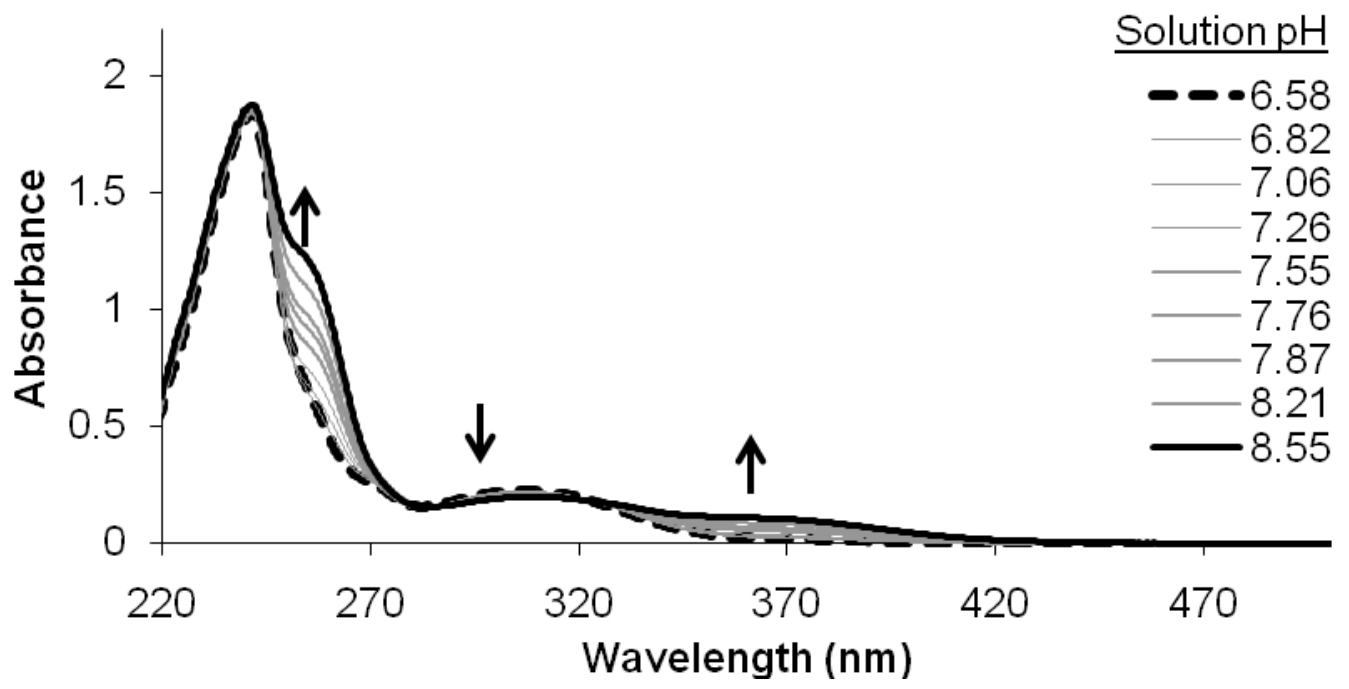

Figure 3.19- Spectral changes for $[\mathrm{HQ}]=10^{-5} \mathrm{M},\left[\mathrm{Eu}^{+3}\right]=2 \times 10^{-6} \mathrm{M},[\mathrm{NaCl}]=0.1 \mathrm{M},[\mathrm{HCl}]=0.001$ $\mathrm{M}$ titrated by $\mathrm{NaOH}$ of varying concentration and volume at $\mathrm{T}=1.8 \pm 0.2^{\circ} \mathrm{C}$. The solution $\mathrm{pH}$ for each spectrum is listed in the figure legend. Arrows indicate the direction of the spectral change as $\mathrm{pH}$ increases. 


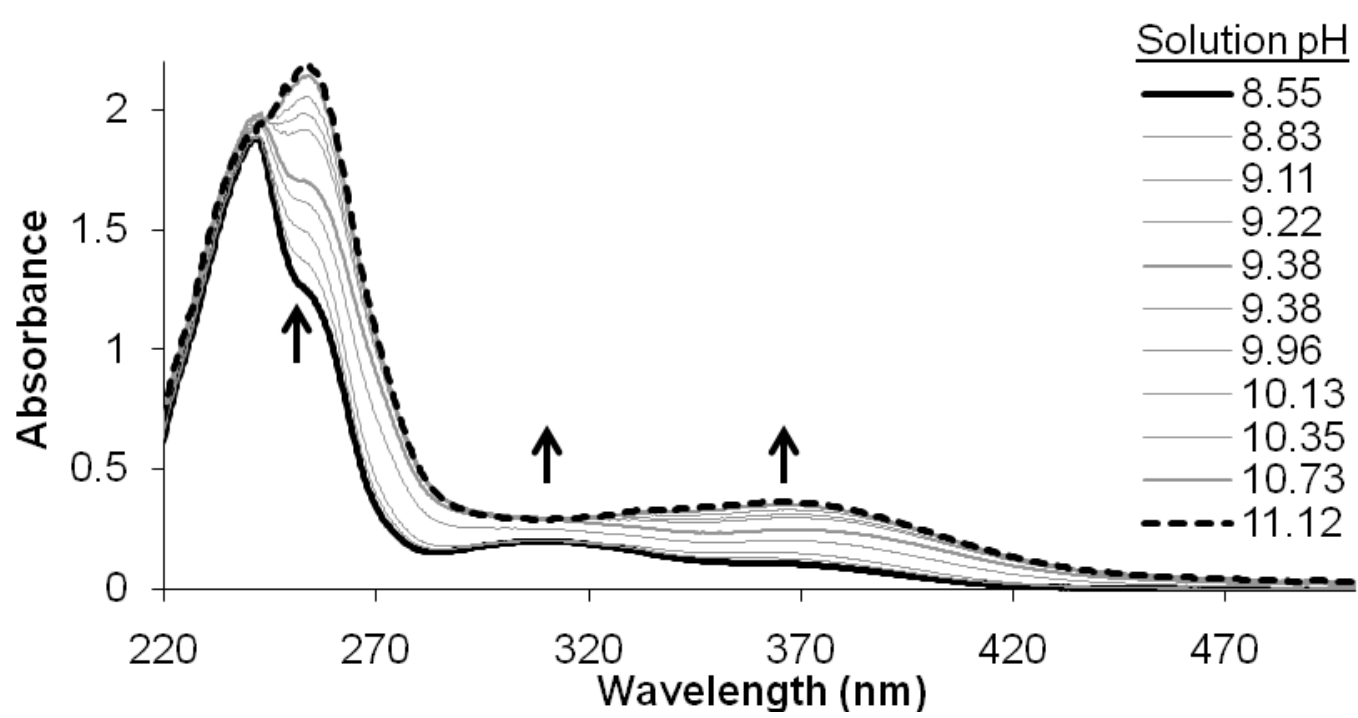

Figure 3.20- Spectral changes for $[\mathrm{HQ}]=10^{-5} \mathrm{M},\left[\mathrm{Eu}^{+3}\right]=2 \times 10^{-6} \mathrm{M},[\mathrm{NaCl}]=0.1 \mathrm{M},[\mathrm{HCl}]=0.001$ $\mathrm{M}$ titrated by $\mathrm{NaOH}$ of varying concentration and volume at $\mathrm{T}=1.8 \pm 0.2^{\circ} \mathrm{C}$. The solution $\mathrm{pH}$ for each spectrum is listed in the figure legend. Arrows indicate the direction of the spectral change as $\mathrm{pH}$ increases.

\subsection{Results: The Triton $\mathrm{X}-114 / \mathrm{H}_{2}$ O System}

A similar approach was used in the Triton $\mathrm{X}-114 / \mathrm{H}_{2} \mathrm{O}$ mixed solvent system as in the $\mathrm{H}_{2} \mathrm{O}$ system for finding the complex stability constants for the europium 8-hydroxyquinoline complexes. No previous literature has reported the water dissociation constant $\left(\mathrm{K}_{\mathrm{w}}\right)$ in the presence of Triton $\mathrm{X}-114$. One paper measured the change in $\mathrm{pK}_{\mathrm{w}}$ as a function of various organic/water mixed solvents with the change in wt\% of the non-water constituent. The mixed solvent solutions included propane and butane diols, methyl/ethyl ethers, butanone, sulfolane, and triethylene glycol. ${ }^{[150]}$ The data showed that the $\mathrm{pK}_{\mathrm{w}}$ of water changed as the co-solvent concentration increased up to $65 \mathrm{wt} \%$, but changed very little at $1 \mathrm{wt} \%$ of any of the tested cosolvents. The co-solvent triethylene glycol, which is similar to Triton X-114, showed a change of 0.02 in the $\mathrm{pK}_{\mathrm{w}}$ at a concentration of $3 \mathrm{wt} \% .{ }^{[150]}$ Because Triton X-114 is present at such a low concentration in this study (i.e. 1 wt\%), it is assumed that Triton's effect on the $\mathrm{pK}_{\mathrm{w}}$ is negligible. 
Therefore, the $\mathrm{pK}_{\mathrm{w}}$ of 14.7 that was used in the water system can also be used in the water/Trion X-114 system.

\subsubsection{Characterization of Protolytic Behavior of Triton X-114 by Potentiometric} Titration

Due to difficulties in modeling data in the Triton/water system and anomalies in the potentiometric titration data, a study of the protonation behavior of Triton X-114 was performed. The difficulties encountered while fitting the 8-hydroxyquinoline $\mathrm{pK}_{\mathrm{a}}$ data are discussed in section 3.4.2. The anomaly noted in the potentiometric titration data is observed in the shape of the first inflection point of all titration data. In the water system data, the first derivative plots of the titrations curves for solutions containing 8-hydroxyquinoline have a peak (see Figure 3.4, Figure 3.12, Figure 3.14, and Figure 3.16), whereas in the Triton/water system data there is a rise to a plateau, but no peak (see Figure 3.23, Figure 3.34, Figure 3.36, and Figure 3.38). The combination of the modeling difficulties and the titration curve shape anomalies shared by the Triton/water data but not the water data led to the decision to explore potential protolytic impurities in the Triton X-114 stock. A titration of Triton X-114 that had been acidified by $\mathrm{HCl}$ is shown in Figure 3.21. There are two inflection points observed for this titration. The distance between the two inflection points is small and correlates to $0.24 \mathrm{~mL}$ of titrant or $2.4 \times 10^{-6}$ moles of $\mathrm{NaOH}$. With only $\mathrm{HCl}, \mathrm{NaCl}$ and Triton X-114 present, it seemed obvious that this small species was some protolytic impurity in the system. The data was modeled by Hyperquad fitting the deprotonation of an unknown mono-protic acid $H(X X)$ shown in equation (3.25).

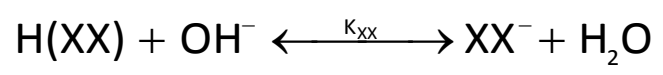




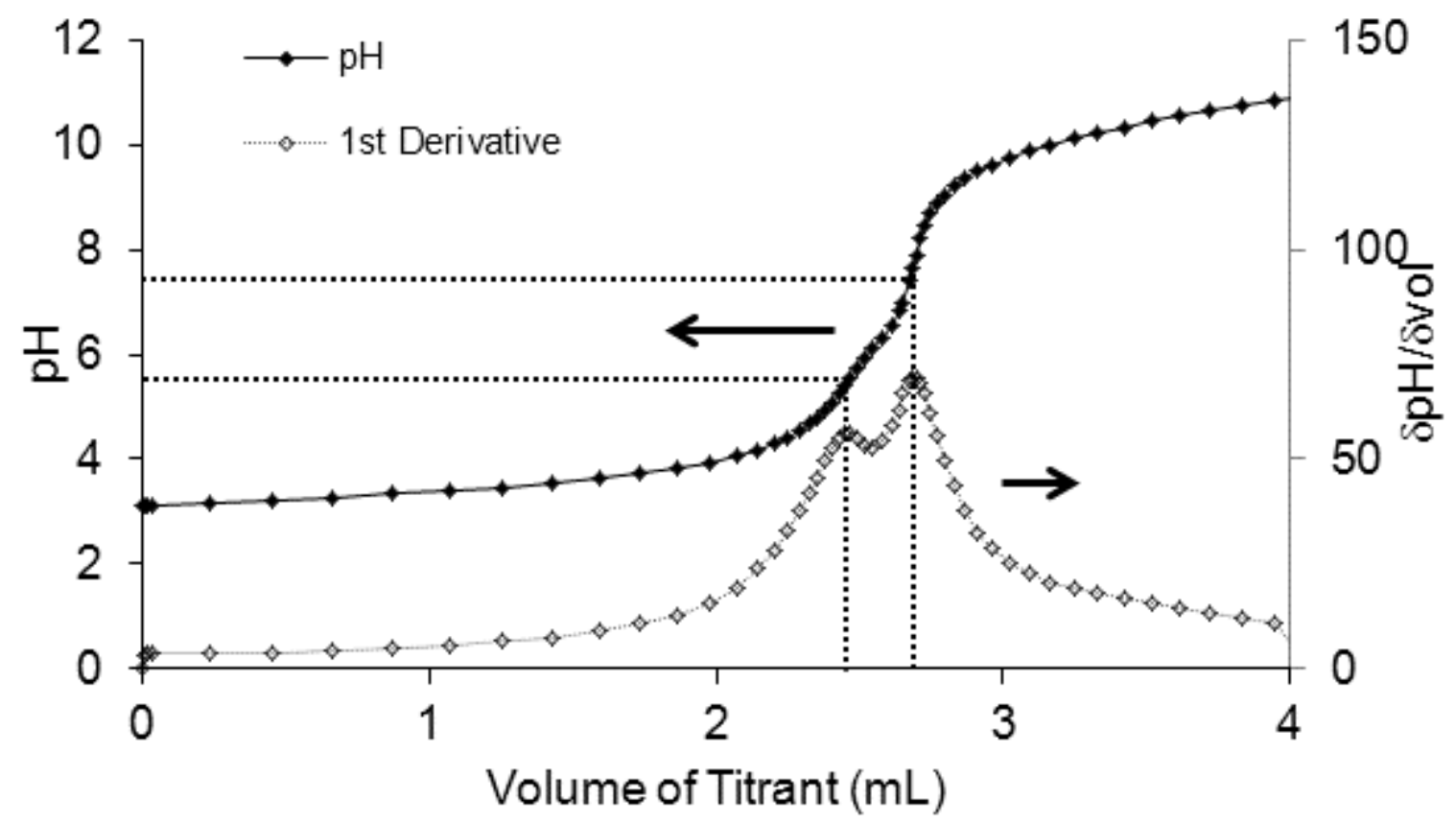

Figure 3.21-Titration of $[\mathrm{HCl}]=0.001 \mathrm{M}$, $[$ Triton X-114] $=1 \mathrm{wt} \%(\sim 0.02 \mathrm{~mol} / \mathrm{L})$ by $[\mathrm{NaOH}]=0.01024 \pm 0.00002 \mathrm{M}$ and $[\mathrm{NaCl}]=0.09 \mathrm{M} . \mathrm{T}=1.9 \pm 0.2^{\circ} \mathrm{C}$.

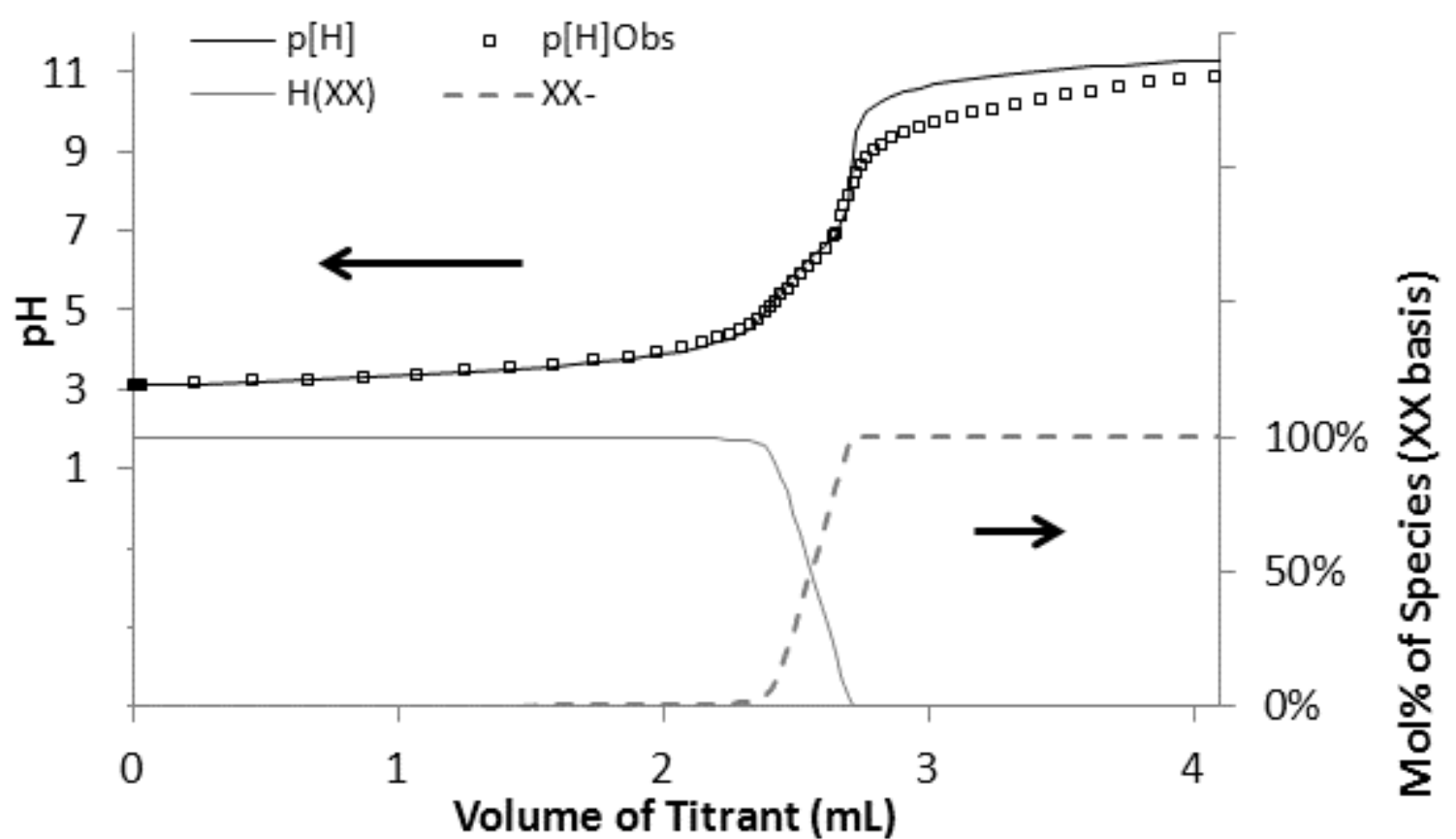

Figure 3.22- Hyperquad fit for the titration of $[\mathrm{HCl}]=0.001 \mathrm{M}$, [Triton X-114] $=1 \mathrm{wt} \%(\sim 0.02$ $\mathrm{mol} / \mathrm{L})$ by $[\mathrm{NaOH}]=0.01024 \pm 0.00002 \mathrm{M}$ and $[\mathrm{NaCl}]=0.09 \mathrm{M} . \mathrm{T}=1.9 \pm 0.2{ }^{\circ} \mathrm{C}$. 
Because the quantity of the impurity present is unknown, the concentration was fit together with the protonation constant and the $\mathrm{pK}_{\mathrm{w}}$. The Hyperquad modeling results are shown in Figure 3.22. The estimated concentration of $X X$ is $0.0001 \pm 0.0015$, indicating that the concentration of impurity is statistically insignificant (see Table 3.12). The percent impurity on a total Triton $\mathrm{X}-114$ basis is $0.5 \pm 8.1 \%$. Although statistically insignificant, the inclusion of this impurity in the Hyperquad models of the Triton/water system leads to more reliable data when compared to literature sources (see section 3.4.2). The source of this impurity is assumed for now to be the Triton X-114, but more experiments are needed to identify the source and eliminate its presence from these solutions.

Table 3.11- Fit parameters for the deprotonation of the protolytic impurity.

\begin{tabular}{|c|c|c|c|c|}
\hline Equation & $\begin{array}{c}\text { Formed } \\
\text { Species }\end{array}$ & Parameter & $\begin{array}{c}\text { Parameter } \\
\text { Refined? }\end{array}$ & $\mathbf{p} \boldsymbol{\beta}, \mathbf{p K}$ \\
\hline$(3.15)$ & $\mathrm{OH}^{-}$ & $\mathrm{K}_{\mathrm{w}}$ & No & 14.7 \\
\hline$(3.25)$ & $\mathrm{XX}^{-}$ & $\mathrm{K}_{\mathrm{xx}}$ & Yes & $6.3 \pm 0.1$ \\
\hline
\end{tabular}

Table 3.12- Concentration of unknown protolytic species obtained from Hyperquad simulation for the $1 \mathrm{wt} \%$ Triton solution titration.

\begin{tabular}{|c|c|}
\hline & $\begin{array}{c}\text { Concentration } \\
\text { (mol/L) }\end{array}$ \\
\hline$[\mathrm{XX}]$ & $0.0001 \pm 0.0015$ \\
\hline
\end{tabular}

\subsubsection{Determination of the $\mathrm{pK}_{\mathrm{a}}{ }^{\text {'s }}$ of 8-Hydroxyquinoline by Potentiometric Titration}

The $\mathrm{pK}_{\mathrm{a}}{ }_{\mathrm{a}}$ 's of 8-hydroxyquinline were obtained for the Triton X-114/water system by titrating a solution that was identical to that used in the $\mathrm{H}_{2} \mathrm{O}$ system except there was 1 wt\% 
(approximately $0.02 \mathrm{~mol} / \mathrm{L}$ ) of Triton X-114 present (see Figure 3.23 ). Inspection of the $1^{\text {st }}$ derivative in Figure 3.23 will reveal that there was only one discernible maxima. This result was completely unexpected and is attributed to the presence of the surfactant, or protolytic impurity (see section 3.4.1). When the titration curve in Figure 3.23 was fit using the same reactions (3.13), and (3.14) as were used for the $\mathrm{H}_{2} \mathrm{O}$ system the resulting fit was not satisfactory (see Figure 3.24). The model used to fit this data imposed an inflection point where there is not one in the observed titration curve leading to a poor fit. The parameters from this model are shown in Table 3.13.

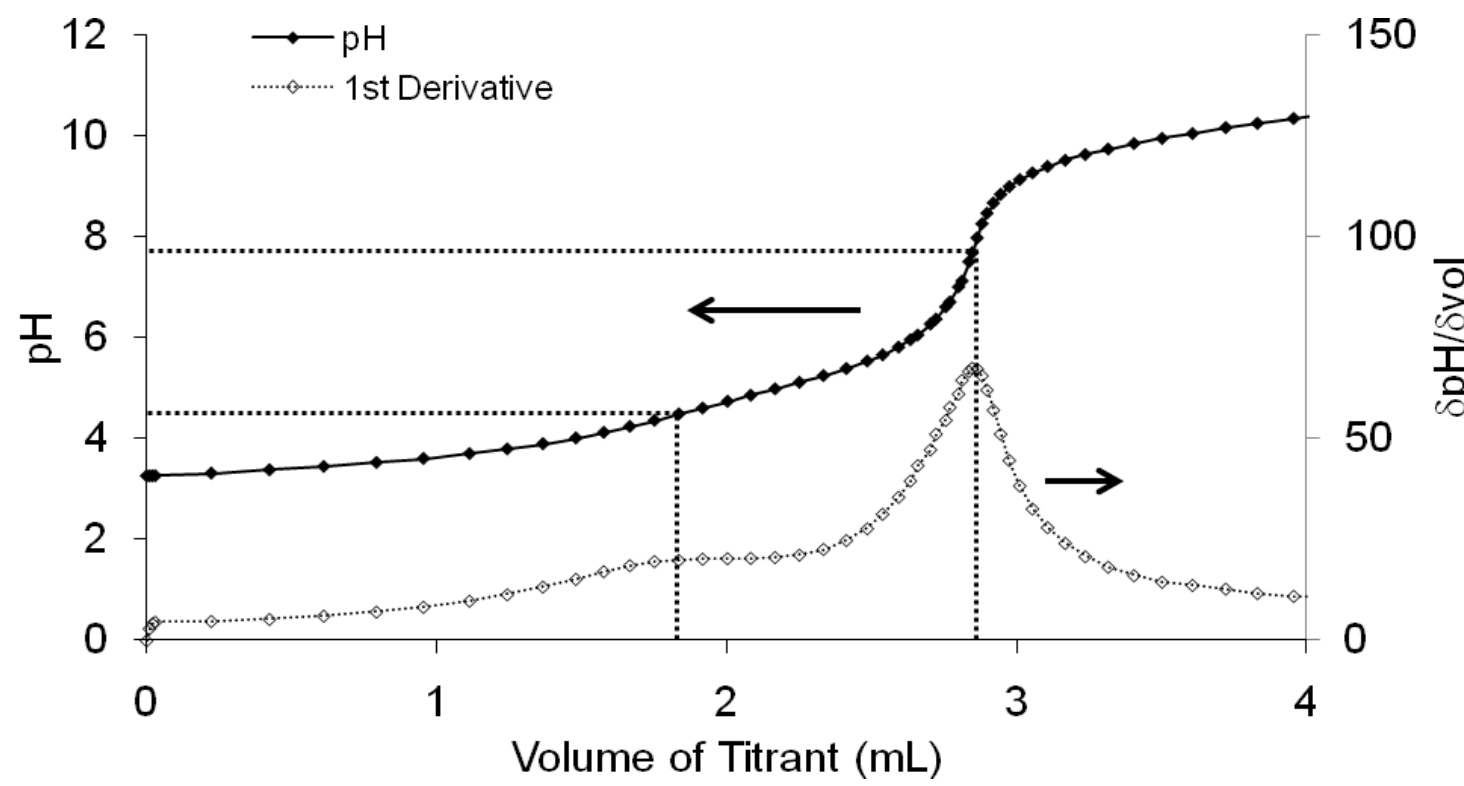

Figure 3.23- Titration of $[\mathrm{HQ}]=3 \times 10^{-4} \mathrm{M},[\mathrm{NaCl}]=0.1 \mathrm{M},[\mathrm{HCl}]=0.001 \mathrm{M}$, [Triton X-114] $=1 \mathrm{wt} \%$ $(\sim 0.02 \mathrm{M})$ by $[\mathrm{NaOH}]=0.01024 \pm 0.00002 \mathrm{M}$ and $[\mathrm{NaCl}]=0.09 \mathrm{M} . \mathrm{T}=1.8 \pm 0.2^{\circ} \mathrm{C}$. 


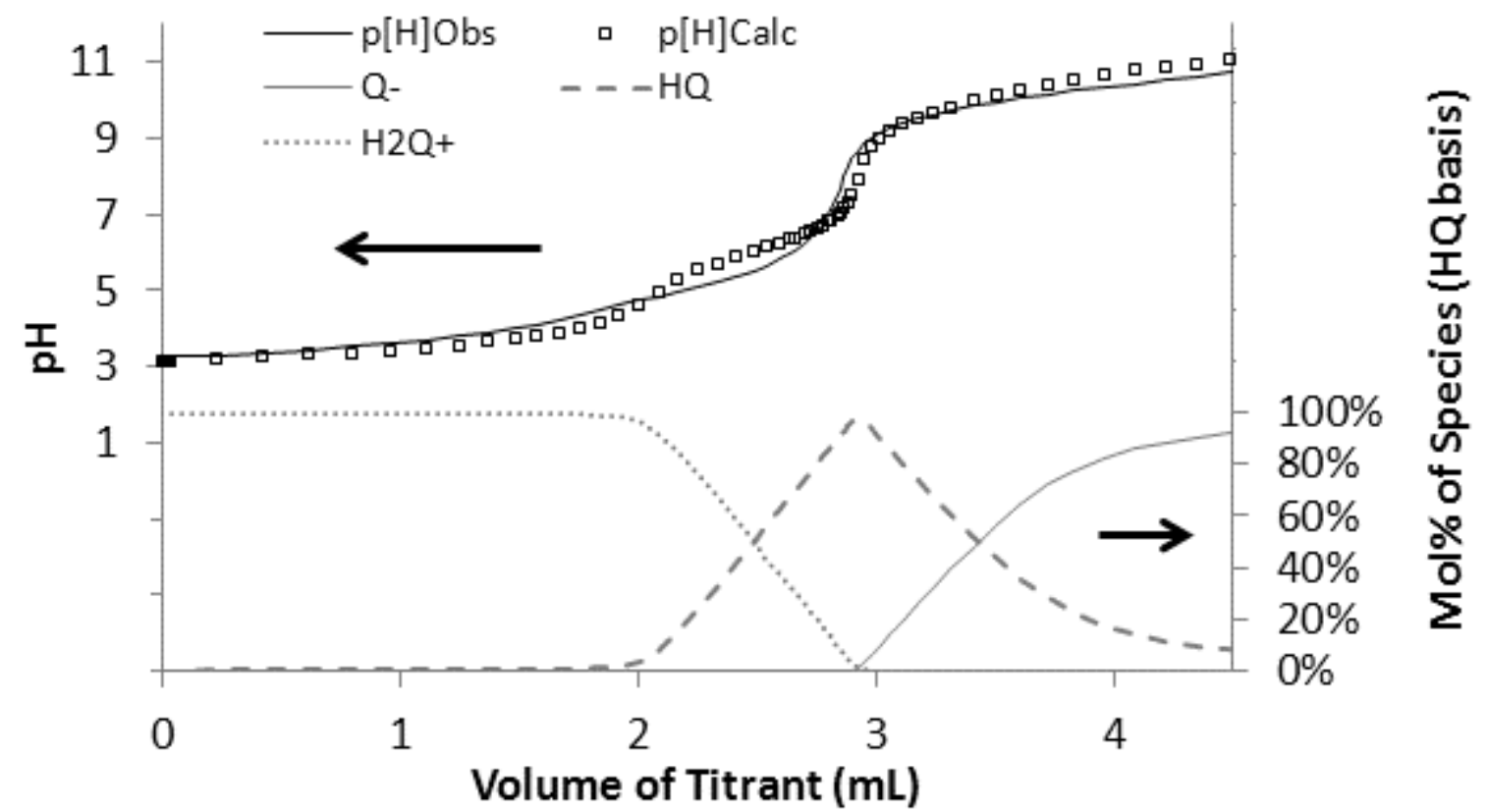

Figure 3.24- Hyperquad fit for the titration of $[\mathrm{HQ}]=3 \times 10^{-4} \mathrm{M},[\mathrm{NaCl}]=0.1 \mathrm{M},[\mathrm{HCl}]=0.001 \mathrm{M}$, $[$ Triton X-114] $=1 \mathrm{wt} \%(\sim 0.02 \mathrm{M})$ by $[\mathrm{NaOH}]=0.01024 \pm 0.00002 \mathrm{M}$ and $[\mathrm{NaCl}]=0.09 \mathrm{M}$. $\mathrm{T}=1.8 \pm 0.2^{\circ} \mathrm{C}$.

Table 3.13- Hyperquad parameters obtained for the protonation of 8-hydroxyquinoline in the $\mathrm{H}_{2} \mathrm{O} /$ Triton $\mathrm{X}-114$ system when the $\mathrm{pK}_{\mathrm{xx}}$ parameter is excluded from the model.

\begin{tabular}{|c|c|c|c|c|c|}
\hline Equation & $\begin{array}{c}\text { Formed } \\
\text { Species }\end{array}$ & Parameter & $\begin{array}{c}\text { Parameter } \\
\text { Refined? }\end{array}$ & $\mathbf{p} \boldsymbol{\beta}$ & $\mathbf{p K}$ \\
\hline$(3.15)$ & $\mathrm{OH}^{-}$ & $\mathrm{K}_{\mathrm{w}}$ & No & 14.7 & \\
\hline$(3.13)$ & $\mathrm{HQ}$ & $\mathrm{K}_{\mathrm{a} 1}$ & Yes & $6.0 \pm 0.1$ & $6.0 \pm 0.1$ \\
\hline$(3.14)$ & $\mathrm{Q}$ & $\mathrm{K}_{\mathrm{a} 2}$ & Yes & $16.0 \pm 0.1$ & $10.0 \pm 0.1$ \\
\hline
\end{tabular}

The results from this model led to speculation that the Triton X-114 was playing a larger role in the system than merely mix-solvent or solvent modifier. With the protolytic impurity characterized, this species was included in the fit of all the subsequent titration data, including the fit of the 8-hydroxyquinoline titration shown in Figure 3.23. Reactions (3.13) and (3.14), (3.25) were fit simultaneously. Results from this modeling experiment are shown in Figure 3.25. The modeling program still introduces a slight inflection point where there is none, but it more 
closely matches the actual titration data than the model without the inclusion of the protolytic impurity reaction. Fit parameters for this fit are shown in Figure 3.14.

Table 3.14- Hyperquad parameters obtained for the protonation of 8-hydroxyquinoline in the $\mathrm{H}_{2} \mathrm{O} /$ Triton X-114 system when the $\mathrm{pK}_{\mathrm{xx}}$ parameter is included in the model.

\begin{tabular}{|c|c|c|c|c|c|}
\hline Equation & $\begin{array}{c}\text { Formed } \\
\text { Species }\end{array}$ & Parameter & $\begin{array}{c}\text { Parameter } \\
\text { Refined? }\end{array}$ & $\mathbf{p} \boldsymbol{\beta}$ & pK \\
\hline$(3.15)$ & $\mathrm{OH}^{-}$ & $\mathrm{K}_{\mathrm{w}}$ & No & 14.7 & \\
\hline$(3.25)$ & $\mathrm{XX}$ & $\mathrm{K}_{\mathrm{xx}}$ & $\mathrm{No}$ & 6.3 & \\
\hline$(3.13)$ & $\mathrm{HQ}$ & $\mathrm{K}_{\mathrm{a} 1}$ & Yes & $5.2 \pm 0.2$ & $5.2 \pm 0.2$ \\
\hline$(3.14)$ & $\mathrm{Q}^{-}$ & $\mathrm{K}_{\mathrm{a} 2}$ & Yes & $15.2 \pm 0.1$ & $10.0 \pm 0.1$ \\
\hline
\end{tabular}

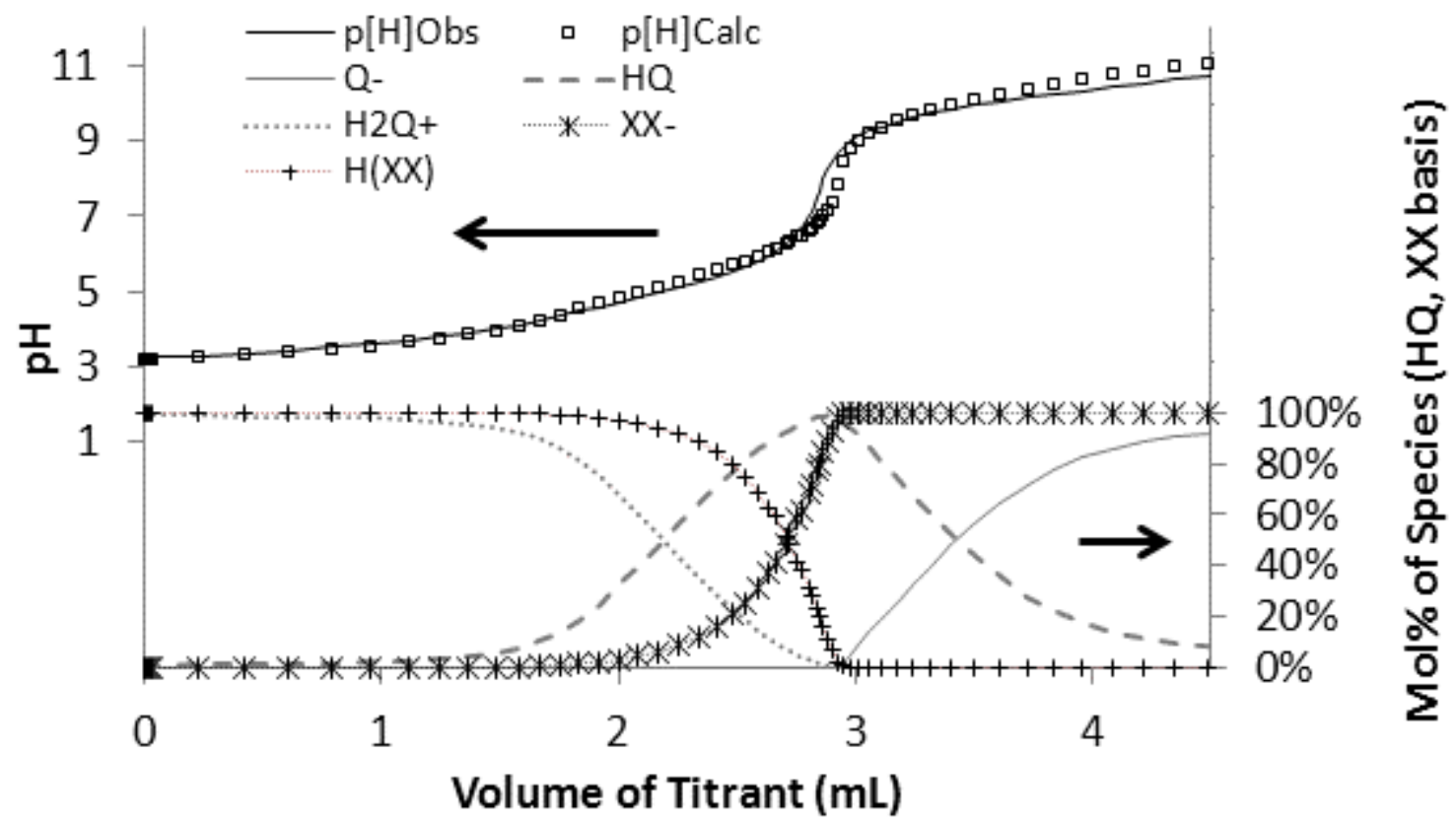

Figure 3.25- Hyperquad fit for the Hyperquad fit for the titration of $[\mathrm{HQ}]=3 \times 10^{-4} \mathrm{M},[\mathrm{NaCl}]=0.1$ $\mathrm{M},[\mathrm{HCl}]=0.001 \mathrm{M},[$ Triton X-114] $=1 \mathrm{wt} \%(\sim 0.02 \mathrm{M})$, by $[\mathrm{NaOH}]=0.01024 \pm 0.00002 \mathrm{M}$ and $[\mathrm{NaCl}]=0.09 \mathrm{M} . \mathrm{T}=1.8 \pm 0.2^{\circ} \mathrm{C}$. 
Although the use of the $\mathrm{pK}_{\mathrm{xx}}$ parameter in the model would appear to act as an extraneous term for obtaining a better fit of the titration curve, the use of the $\mathrm{pK}_{\mathrm{xx}}$ term in the model was supported by several independent sources as will now be explained. First, the $\mathrm{pK}_{\mathrm{a}}$ 's of 8hydroxyquinoline in the Triton X-114/water system obtained by spectrophotometry returned a similar value (4.94 \pm 0.01 ; see Section 3.4.3) as is obtained when including the $\mathrm{pK}_{\mathrm{xx}}$ parameter in the Hyperquad model of the potentiometric data ( $5.2 \pm 0.2$; see Table 3.14$)$. The value obtained by Hyperquad modeling when excluding the $\mathrm{pK}_{\mathrm{xx}}$ parameter is significantly higher $(6.0 \pm 0.1$; see Table 3.13). Second, a comparison of the literature data trend in the $\mathrm{pK}_{\mathrm{a}}$ values of 8hydroxyquinoline show a decrease in $\mathrm{pK}_{\mathrm{a} 1}$ with an increase in surfactant concentration ${ }^{[134]}$ (see Figure 3.42). The $\mathrm{pK}_{\mathrm{a} 1}$ obtained when the $\mathrm{pK}_{\mathrm{xx}}$ is excluded from the model gives a $\mathrm{pK}_{\mathrm{a} 1}$ that creates a trend opposite as the one shown in previous literature, whereas the result from the potentiometric method when the $\mathrm{pK}_{\mathrm{xx}}$ is used gives a trend that is consistent with previously published literature. Thus, inclusion of the $\mathrm{pK}_{\mathrm{xx}}$ parameter in the Hyperquad model gives data that was confirmed by an independent technique, and supported by literature data, and will therefore be included in all models in the Triton X-114/water system.

\subsubsection{Determination of the $\mathrm{pK}_{\mathrm{a}}$ 's of 8-Hydroxyquinoline by Spectrophotometric}

\section{Titration}

An effort to quantify the deprotonation behavior of 8-hydroxyquinoline in the Triton/water mixed solvent by spectrophotometric titration was performed. The series of spectra obtained are shown in Figure 3.26 through Figure 3.29 with Figure 3.27, and Figure 3.29 showing a smaller, higher detail view of the spectral changes. As is observed in Figure 3.26 and Figure 3.28, the solution absorbance in the $220-300 \mathrm{~nm}$ window is large and constant, unlike the changes observed in the $\mathrm{H}_{2} \mathrm{O}$ system spectrophotometric titration. The 8-hydroxyquinoline absorbance bands in this region appear to be obscured by the UV absorption by Triton X-114. The only 
usable portion of the spectra is limited to the spectral window above $300 \mathrm{~nm}$. The spectral data using the 300-412 $\mathrm{nm}$ spectral window were modeled using SQUAD. The modeling parameters obtained are shown in Table 3.15.

Table 3.15- SQUAD modeling parameters for the $\mathrm{pK}_{\mathrm{a}}$ 's of 8-hydroxyquinoline in $1 \mathrm{wt} \%$ Triton, $\mathrm{T}=0{ }^{\circ} \mathrm{C}$.

\begin{tabular}{|c|c|c|c|}
\hline \multicolumn{3}{|c|}{ Window Limits } & \multicolumn{2}{l|}{} \\
\cline { 1 - 2 } Lower & Upper & & \\
\hline (nm) & $(\mathbf{n m})$ & $\mathbf{p K}_{\mathrm{a} 1}$ & $\mathbf{p K}_{\mathrm{a} 2}$ \\
\hline 300 & 412 & $4.94 \pm 0.01$ & $9.69 \pm 0.01$ \\
\hline
\end{tabular}

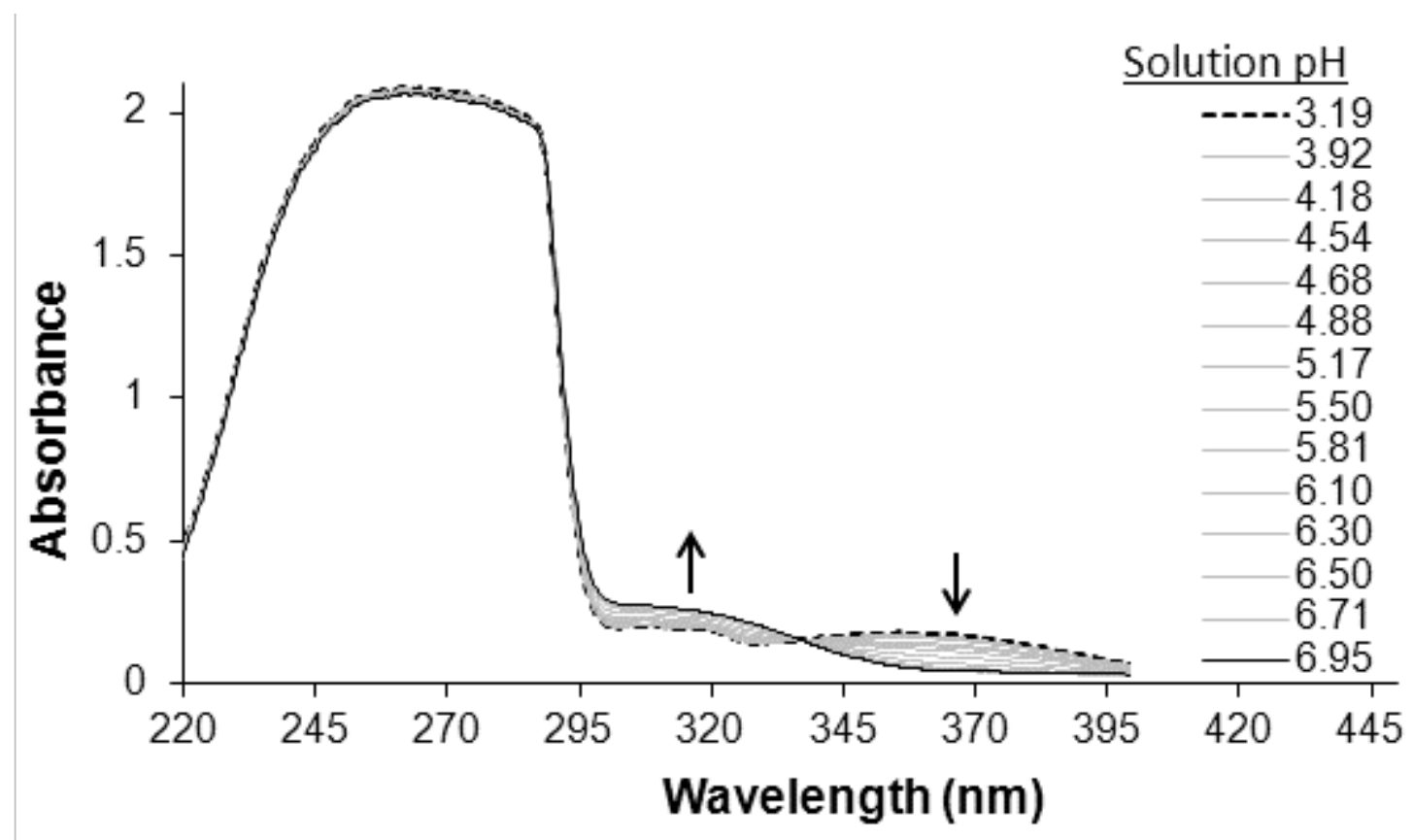

Figure 3.26-Spectra obtained while titrating a solution containing: $[\mathrm{HQ}]=0.0001 \mathrm{M},[\mathrm{NaCl}]=0.1$ $\mathrm{M}$, [Triton X-114] = $1 \mathrm{wt} \%$. Spectra shown are of $\mathrm{pH}$ 3.19-6.95. The arrows indicate the spectral change as $\mathrm{pH}$ increases. 


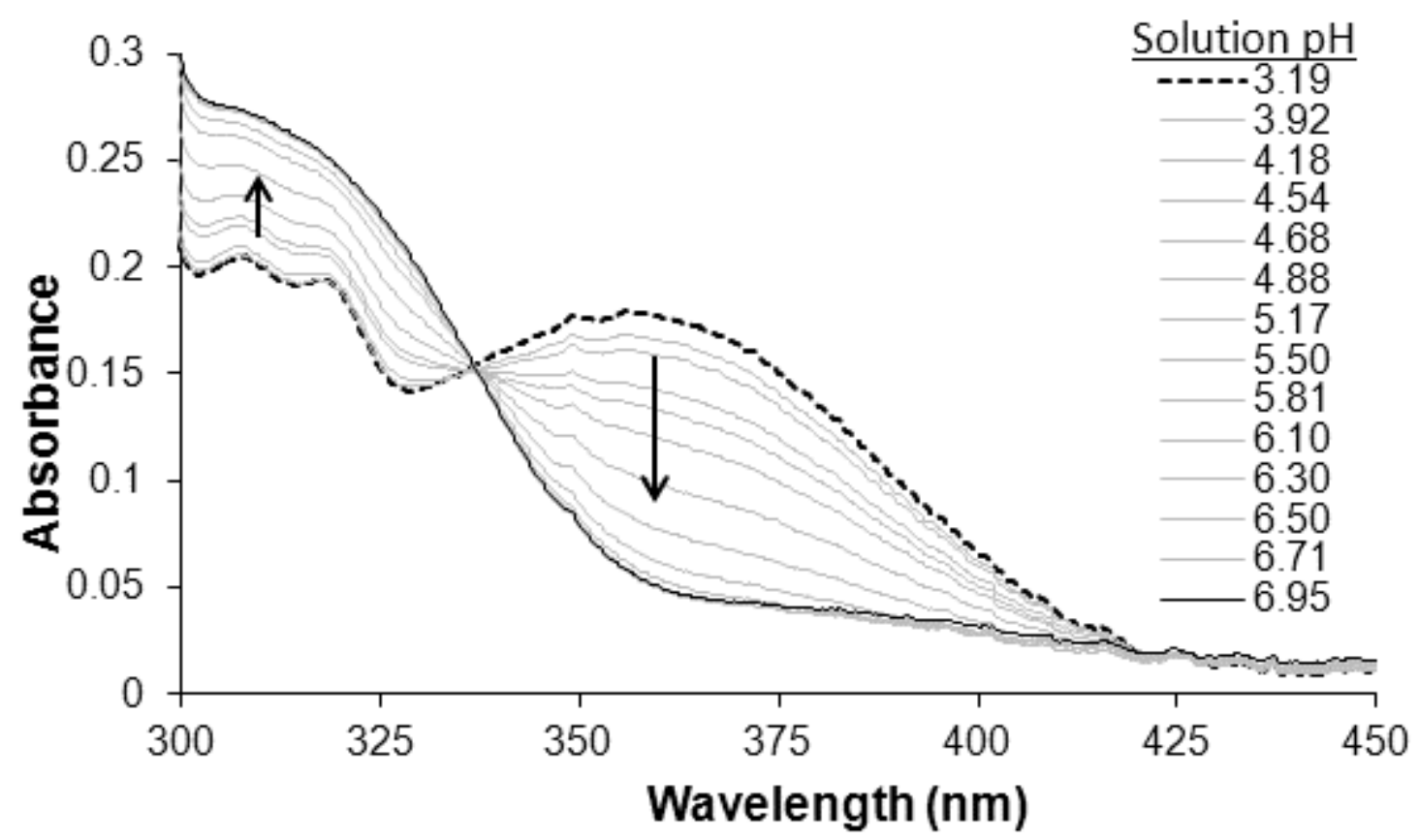

Figure 3.27- $A$ select window of the spectra obtained while titrating a solution containing: $[\mathrm{HQ}]=$ $0.0001 \mathrm{M},[\mathrm{NaCl}]=0.1 \mathrm{M},[$ Triton X-114] $=1 \mathrm{wt} \%$. Spectra shown are of $\mathrm{pH} 3.19-6.95$. The arrows indicate the spectral change as $\mathrm{pH}$ increases.

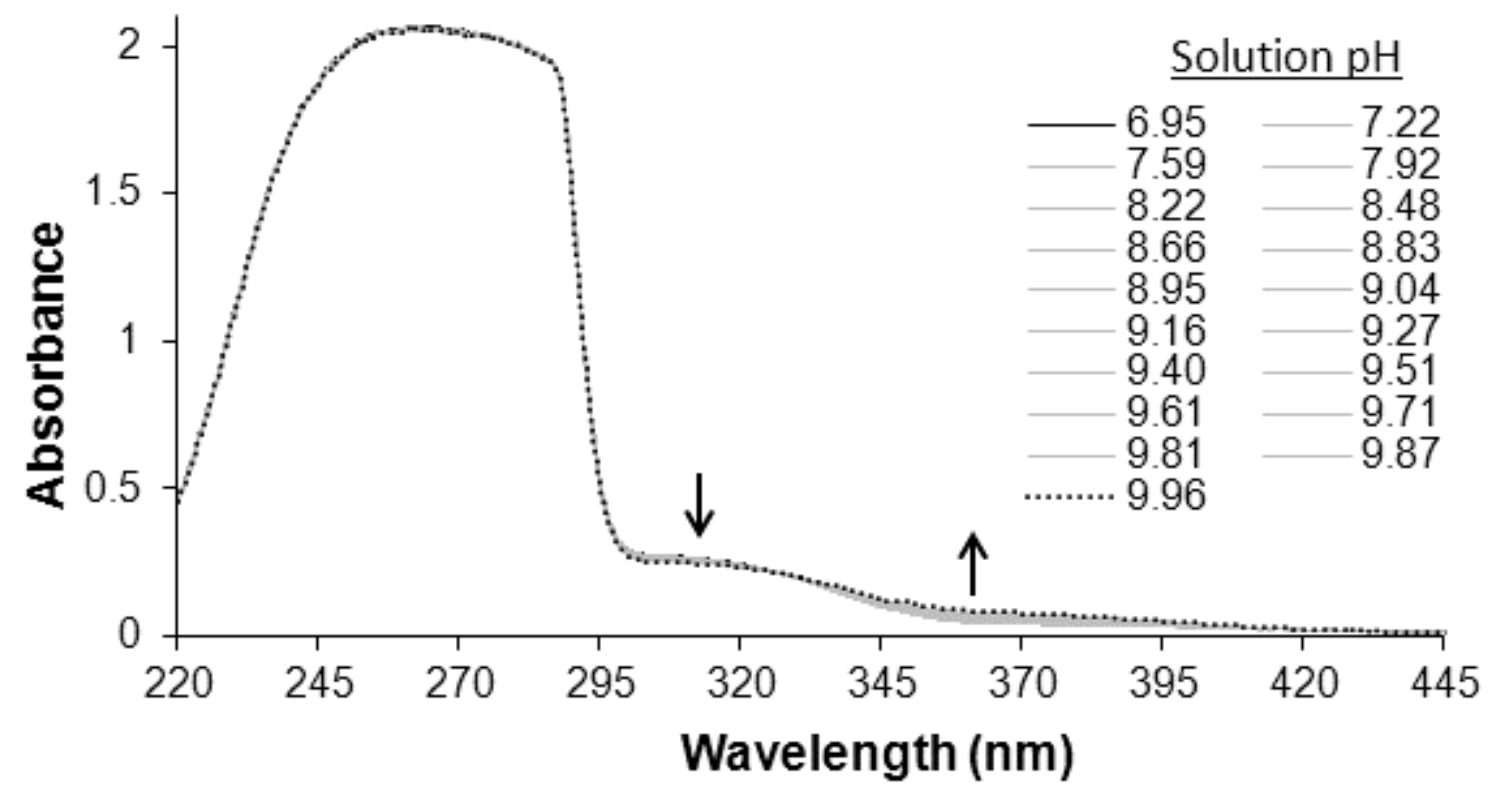

Figure 3.28- Spectra obtained while titrating a solution containing: $[\mathrm{HQ}]=0.0001 \mathrm{M},[\mathrm{NaCl}]=0.1$ $\mathrm{M}$, [Triton X-114] = $1 \mathrm{wt} \%$. Spectra shown are of $\mathrm{pH}$ 6.95-9.96. The arrows indicate the spectral change as $\mathrm{pH}$ increases. 


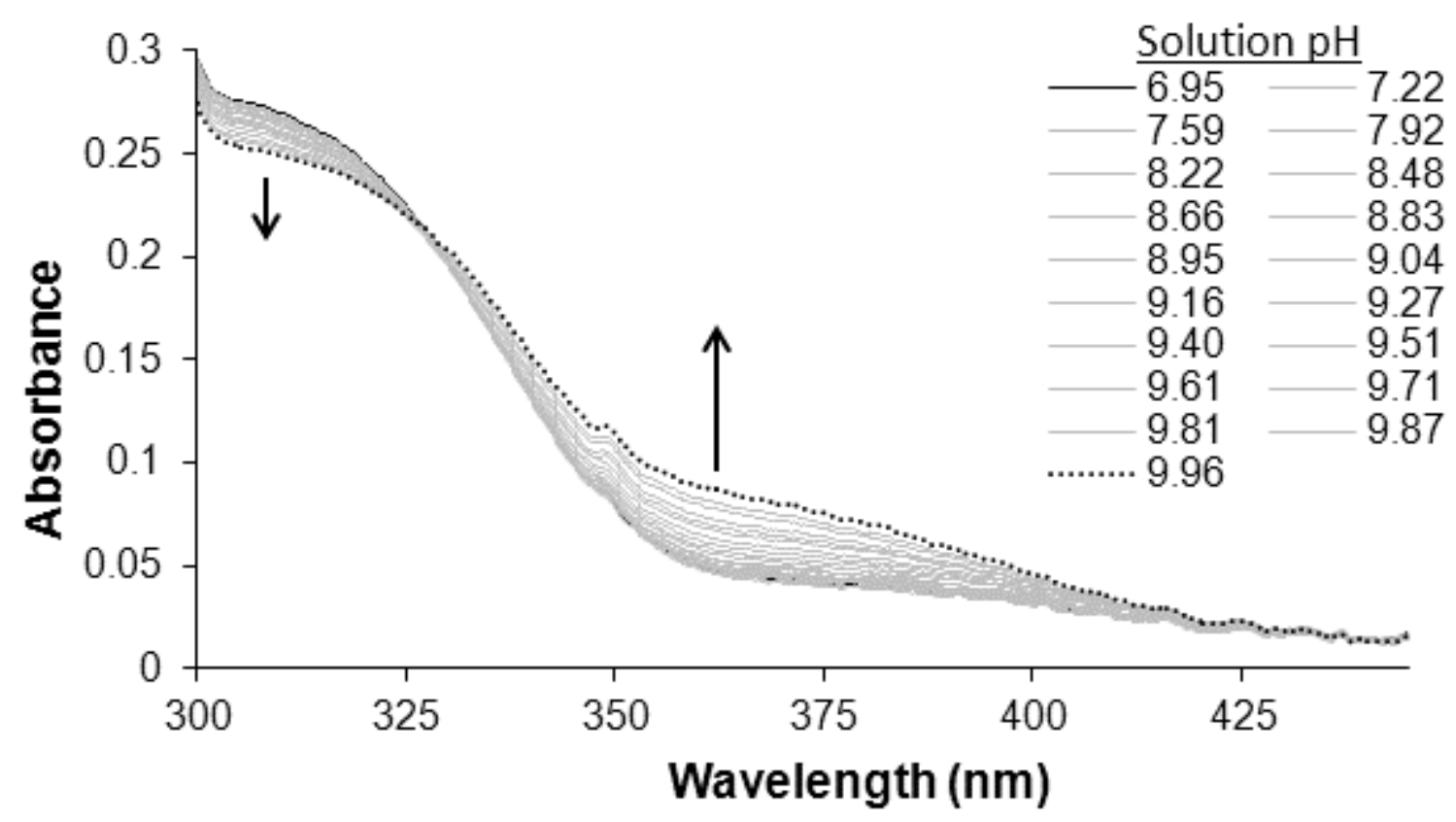

Figure 3.29- $A$ select window of the spectra obtained while titrating a solution containing: $[\mathrm{HQ}]=$ $0.0001 \mathrm{M},[\mathrm{NaCl}]=0.1 \mathrm{M},[$ Triton X-114] $=1 \mathrm{wt} \%$. Spectra shown are of $\mathrm{pH} 6.95-9.96$. The arrows indicate the spectral change as $\mathrm{pH}$ increases.

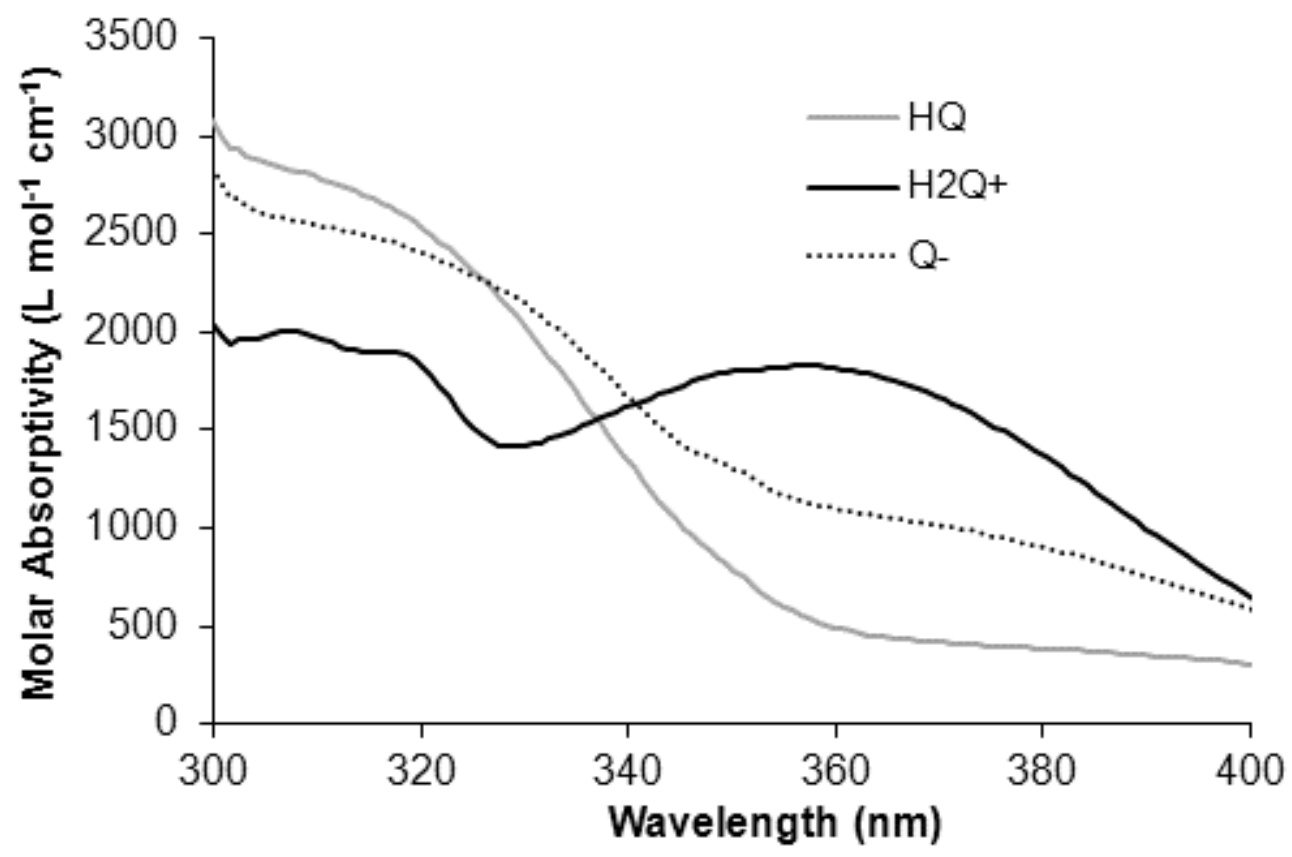

Figure 3.30- Molar absorptivity Spectra obtained from SQUAD modeling using the $300-412 \mathrm{~nm}$ spectral window. 


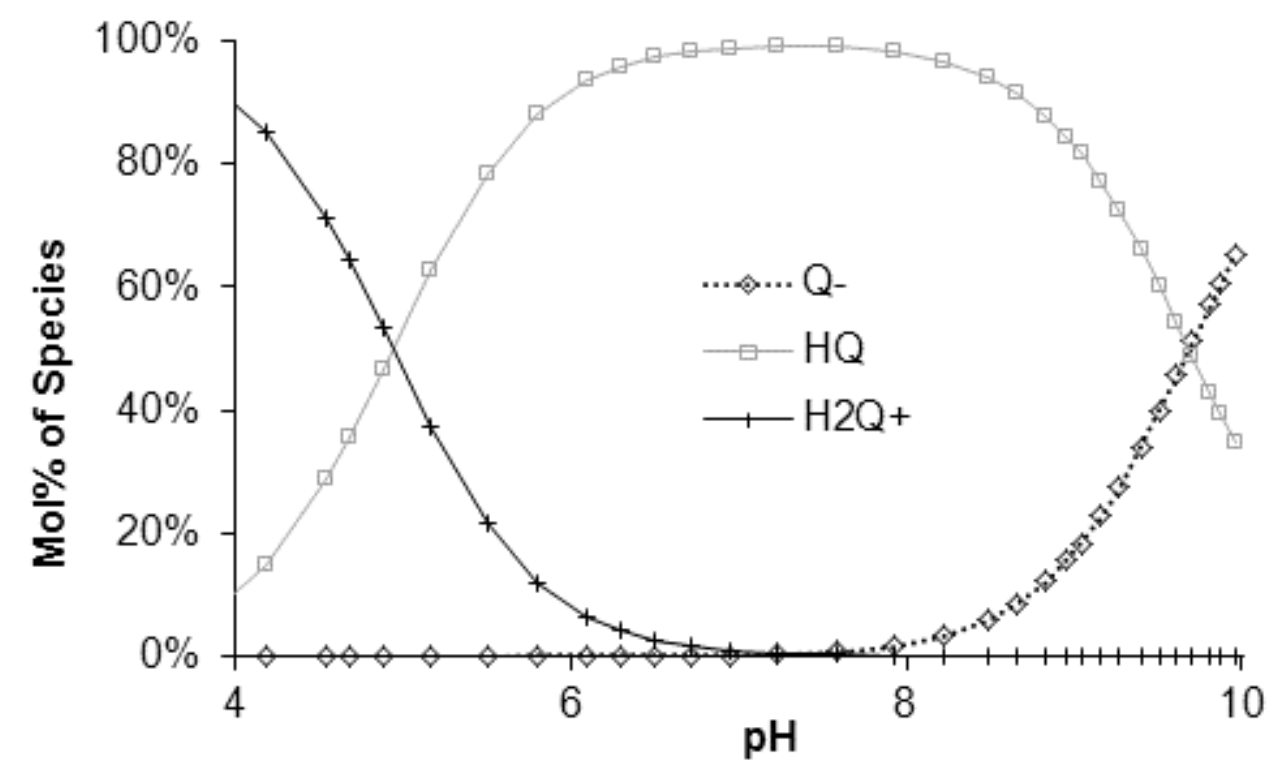

Figure 3.31- Calculated speciation curves obtained from SQUAD modeling using the $300-412 \mathrm{~nm}$ window.

\subsubsection{Determination of the Europium Hydrolysis Constants by Potentiometric Titration}

Modeling using the protolytic impurity was used in the fitting of the europium hydrolysis constants for $\mathrm{Eu}^{3+}$ in $1 \mathrm{wt} \%$ Triton. The titration data obtained for $\mathrm{Eu}^{3+}$ in $1 \mathrm{wt} \%$ Triton is shown in Figure 3.32. The reactions used to fit this system were the europium hydrolysis reactions [equations (3.16) - (3.18)], along with the parameter for the protolytic impurity $\left(\mathrm{pK}_{\mathrm{xx}}\right)$ [equation (3.25)]. The fit results are shown in Figure 3.33. The fit parameters obtained are shown in Table 3.16. 


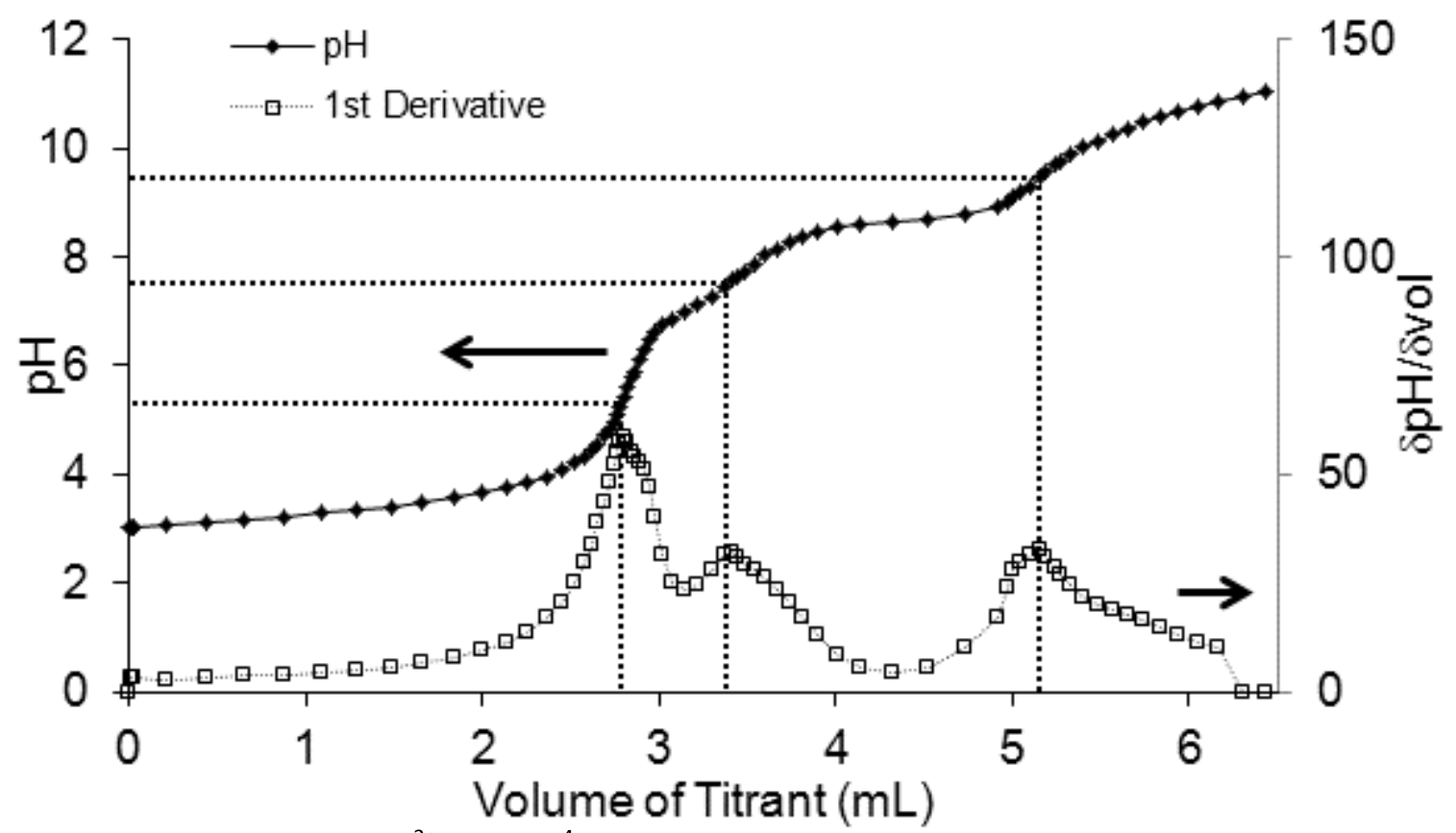

Figure 3.32- Titration of $\left[\mathrm{Eu}^{3+}\right]=3 \times 10^{-4} \mathrm{M},[\mathrm{NaCl}]=0.1 \mathrm{M},[\mathrm{HCl}]=0.001 \mathrm{M},[$ Triton X-114] $=1 \mathrm{wt} \%$ $(\sim 0.02 \mathrm{M})$, by $[\mathrm{NaOH}]=0.01024 \pm 0.00002 \mathrm{M}$ and $[\mathrm{NaCl}]=0.09 \mathrm{M} . \mathrm{T}=1.8 \pm 0.2^{\circ} \mathrm{C}$.

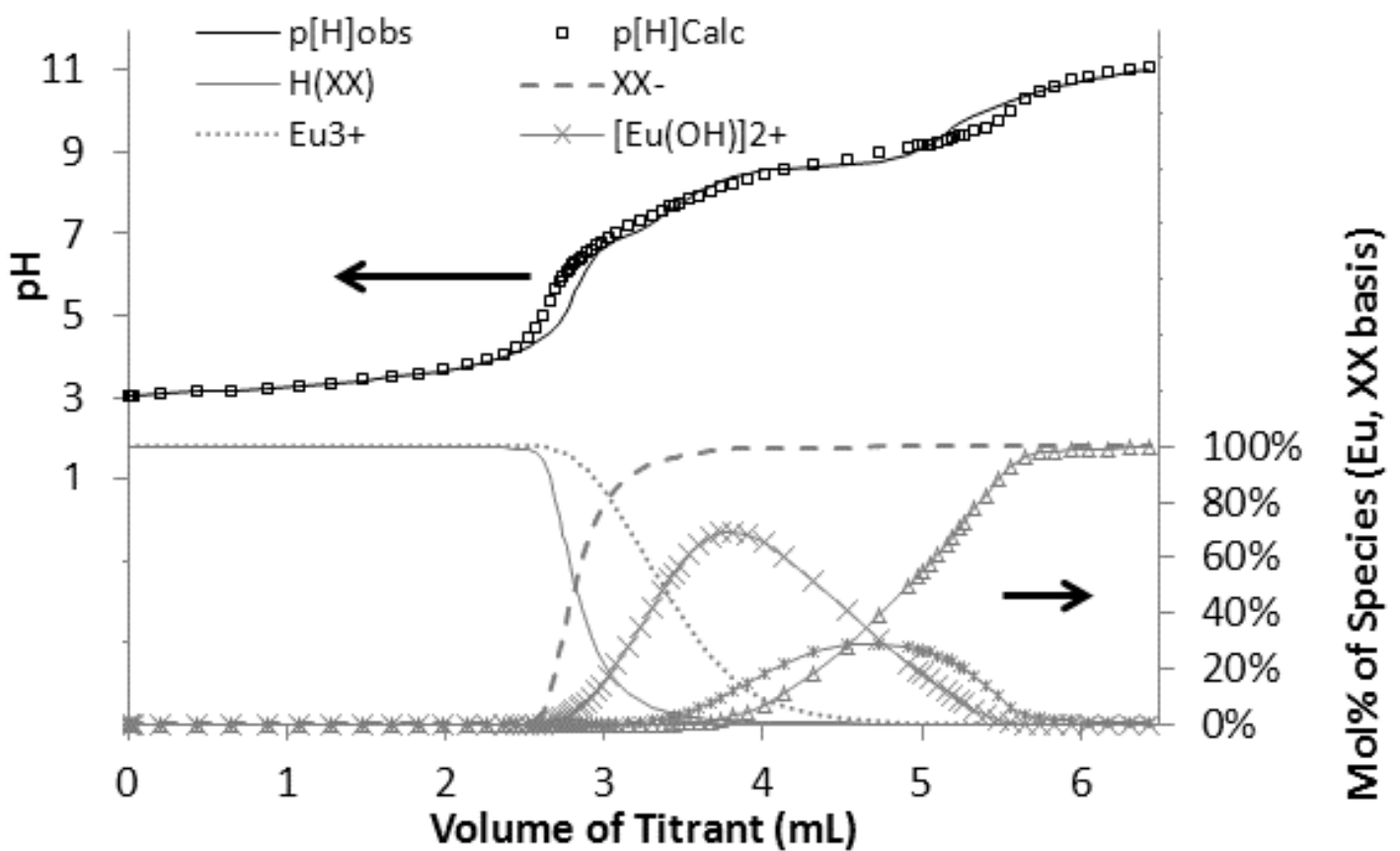

Figure 3.33- Hyperquad fit for the titration of $\left[\mathrm{Eu}^{3+}\right]=3 \times 10^{-4} \mathrm{M},[\mathrm{NaCl}]=0.1 \mathrm{M},[\mathrm{HCl}]=0.001 \mathrm{M}$, $[$ Triton X-114] $=1$ wt\% $(\sim 0.02 \mathrm{M})$, by $[\mathrm{NaOH}]=0.01024 \pm 0.00002 \mathrm{M}$ and $[\mathrm{NaCl}]=0.09 \mathrm{M}$. $\mathrm{T}=1.8 \pm 0.2^{\circ} \mathrm{C}$. 
Table 3.16- Hyperquad fit parameters for the titration data from the $\mathrm{Eu}^{3+}$ in 1 wt\% Triton.

\begin{tabular}{|c|c|c|c|c|c|}
\hline Equation & $\begin{array}{c}\text { Formed } \\
\text { Species }\end{array}$ & Parameter & $\begin{array}{c}\text { Parameter } \\
\text { Refined? }\end{array}$ & $\mathbf{p} \boldsymbol{\beta}$ & $\mathbf{p K}$ \\
\hline$(3.15)$ & $\mathrm{OH}^{-}$ & $\mathrm{K}_{\mathrm{w}}$ & No & 14.7 & \\
\hline$(3.25)$ & $\mathrm{XX}$ & $\mathrm{K}_{\mathrm{xx}}$ & No & 6.3 & \\
\hline$(3.16)$ & {$[\mathrm{Eu}(\mathrm{OH})]^{2+}$} & $\beta_{1-10}$ & Yes & $-7.1 \pm 0.1$ & $-7.1 \pm 0.1$ \\
$(3.17)$ & {$\left[\mathrm{Eu}(\mathrm{OH})_{2}\right]^{+}$} & $\beta_{1-20}$ & Yes & $-12.8 \pm 0.1$ & $-5.7 \pm 0.1$ \\
\hline$(3.18)$ & {$\left[\mathrm{Eu}(\mathrm{OH})_{3}\right]$} & $\beta_{1-30}$ & Yes & $-18.7 \pm 0.1$ & $-5.9 \pm 0.2$ \\
\hline
\end{tabular}

\subsubsection{Determination of Europium 8-Hydroxyquinoline Complex Stability Constants by}

Potentiometric Titration

The stability constants were obtained using the same progression of 1:1, 2:1 and 3:1 ligand to metal ratio solutions used in the $\mathrm{H}_{2} \mathrm{O}$ system. The titration of the 1:1 ligand to metal ratio solution is shown in Figure 3.34. The reactions used to fit this titration curve are shown in Table 3.17, including the reaction for the formation of $[\mathrm{Eu}(\mathrm{HQ})]^{3+}$ shown in equation (3.26) that was added to improve the fit shown in Figure 3.35. The 2:1 ligand to metal solution titration was the performed next (see Figure 3.36). The fit obtained using the reactions shown in Table 3.18 is shown in Figure 3.37.

$$
\mathrm{Eu}^{+3}+\mathrm{H}^{+}+\mathrm{Q}^{-} \stackrel{\beta_{111}}{\longleftrightarrow}[\mathrm{Eu}(\mathrm{HQ})]^{3+}
$$

Table 3.17- Hyperquad fit parameters for the 1:1 ligand to metal solution titration in $1 \mathrm{wt} \%$ Triton

\begin{tabular}{|c|c|c|c|c|c|}
\hline Equation & $\begin{array}{c}\text { Formed } \\
\text { Species }\end{array}$ & Parameter & $\begin{array}{c}\text { Parameter } \\
\text { Refined? }\end{array}$ & $\mathbf{p} \boldsymbol{\beta}$ & $\mathbf{p K}$ \\
\hline$(3.15)$ & $\mathrm{OH}^{-}$ & $\mathrm{K}_{\mathrm{w}}$ & No & 14.7 & \\
\hline$(3.25)$ & $\mathrm{XX}$ & $\mathrm{K}_{\mathrm{xx}}$ & No & 6.3 & \\
\hline$(3.13)$ & $\mathrm{HQ}$ & $\mathrm{K}_{\mathrm{a} 1}$ & No & 5.2 & \\
$(3.14)$ & $\mathrm{Q}^{-}$ & $\mathrm{K}_{\mathrm{a} 2}$ & No & 15.2 & \\
\hline$(3.19)$ & {$[\mathrm{Eu}(\mathrm{Q})]^{2+}$} & $\beta_{101}$ & Yes & $-7.78 \pm 0.04$ & $-7.78 \pm 0.04$ \\
\hline$(3.20)$ & {$[\mathrm{Eu}(\mathrm{OH})(\mathrm{Q})]^{+}$} & $\beta_{1-11}$ & Yes & $-14.4 \pm 0.1$ & $-6.6 \pm 0.1$ \\
\hline$(3.21)$ & {$\left[\mathrm{Eu}(\mathrm{OH})(\mathrm{Q})_{2}\right]$} & $\beta_{1-21}$ & Yes & $-19.8 \pm 0.1$ & $-5.4 \pm 0.1$ \\
\hline$(3.26)$ & {$[\mathrm{Eu}(\mathrm{HQ})]^{3+}$} & $\beta_{111}$ & Yes & $-14.07 \pm 0.06$ & $-6.28 \pm 0.06$ \\
\hline
\end{tabular}




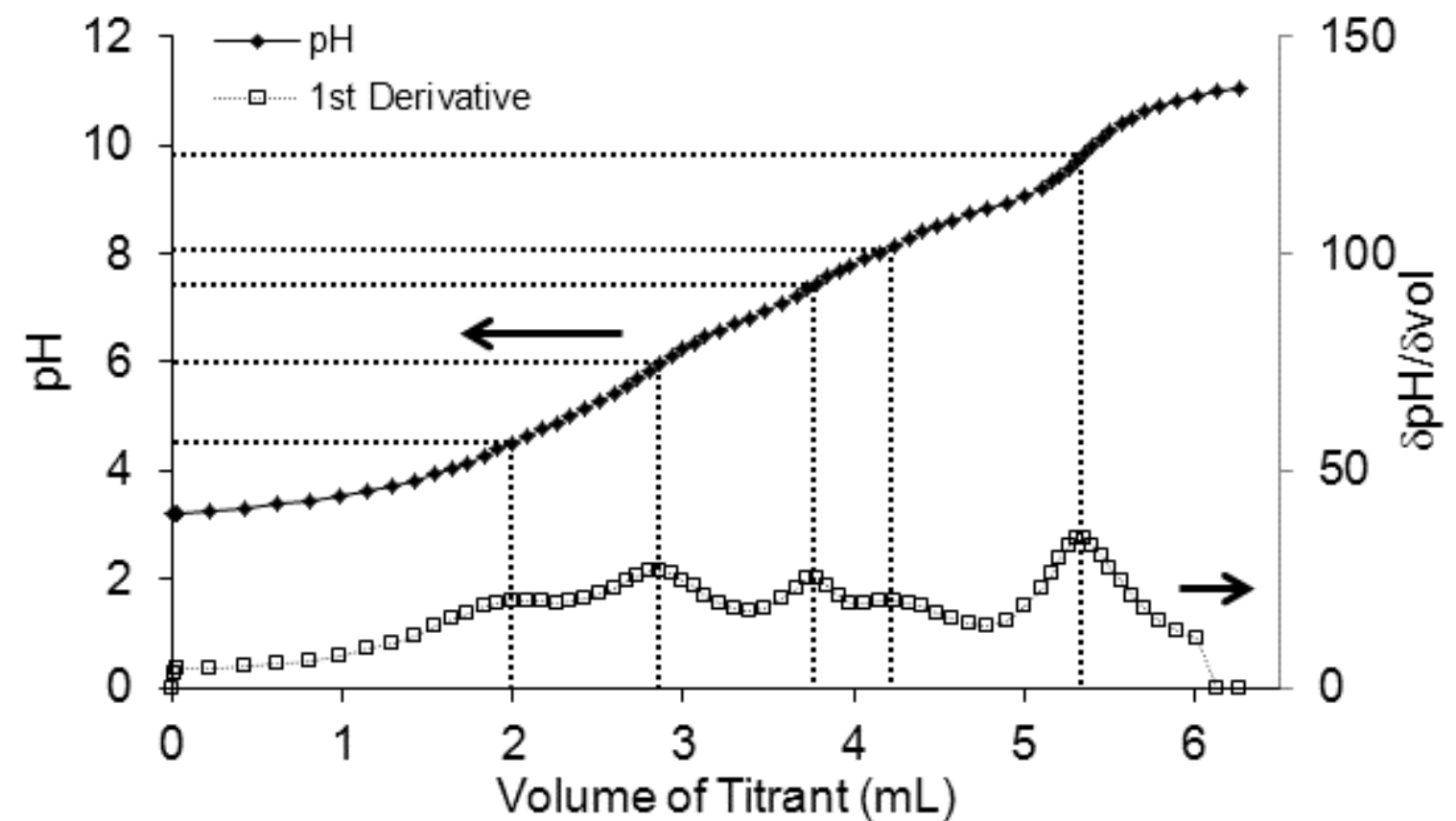

Figure 3.34- Titration of $[\mathrm{HQ}]=3 \times 10^{-4} \mathrm{M},\left[\mathrm{Eu}^{3+}\right]=3 \times 10^{-4} \mathrm{M},[\mathrm{NaCl}]=0.1 \mathrm{M},[\mathrm{HCl}]=0.001 \mathrm{M}$, $[$ Triton X-114] $=1$ wt\% $(\sim 0.02 \mathrm{M})$, by $[\mathrm{NaOH}]=0.01024 \pm 0.00002 \mathrm{M}$ and $[\mathrm{NaCl}]=0.09 \mathrm{M}$. $\mathrm{T}=1.8 \pm 0.2^{\circ} \mathrm{C}$.

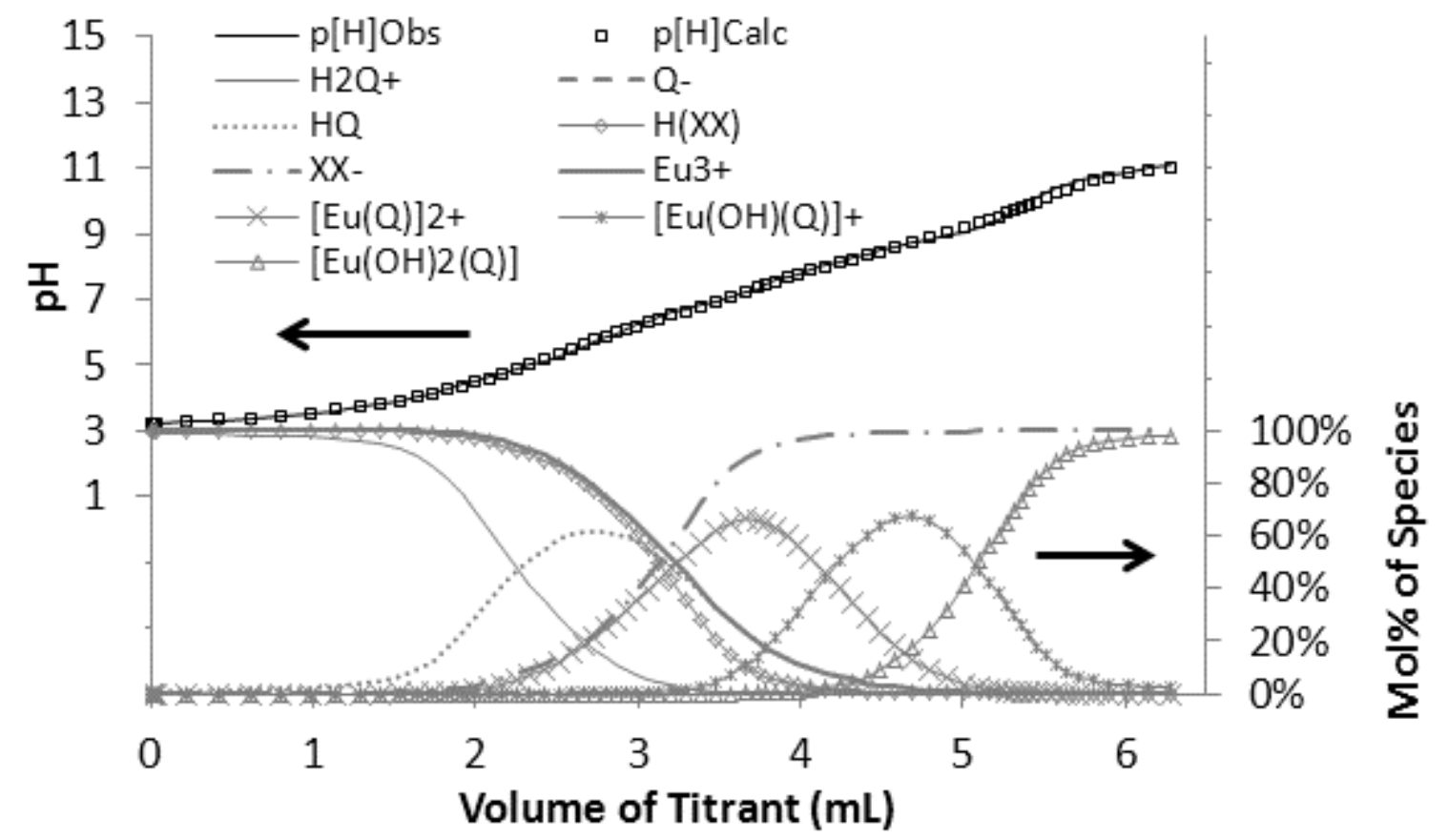

Figure 3.35- Hyperquad fit for the titration of $[\mathrm{HQ}]=3 \times 10^{-4} \mathrm{M},\left[\mathrm{Eu}^{3+}\right]=3 \times 10^{-4} \mathrm{M},[\mathrm{NaCl}]=0.1 \mathrm{M}$, $[\mathrm{HCl}]=0.001 \mathrm{M},[$ Triton X-114] $=1 \mathrm{wt} \%(\sim 0.02 \mathrm{M})$, by $[\mathrm{NaOH}]=0.01024 \pm 0.00002 \mathrm{M}$ and $[\mathrm{NaCl}]=0.09 \mathrm{M} \cdot \mathrm{T}=1.8 \pm 0.2^{\circ} \mathrm{C}$. 


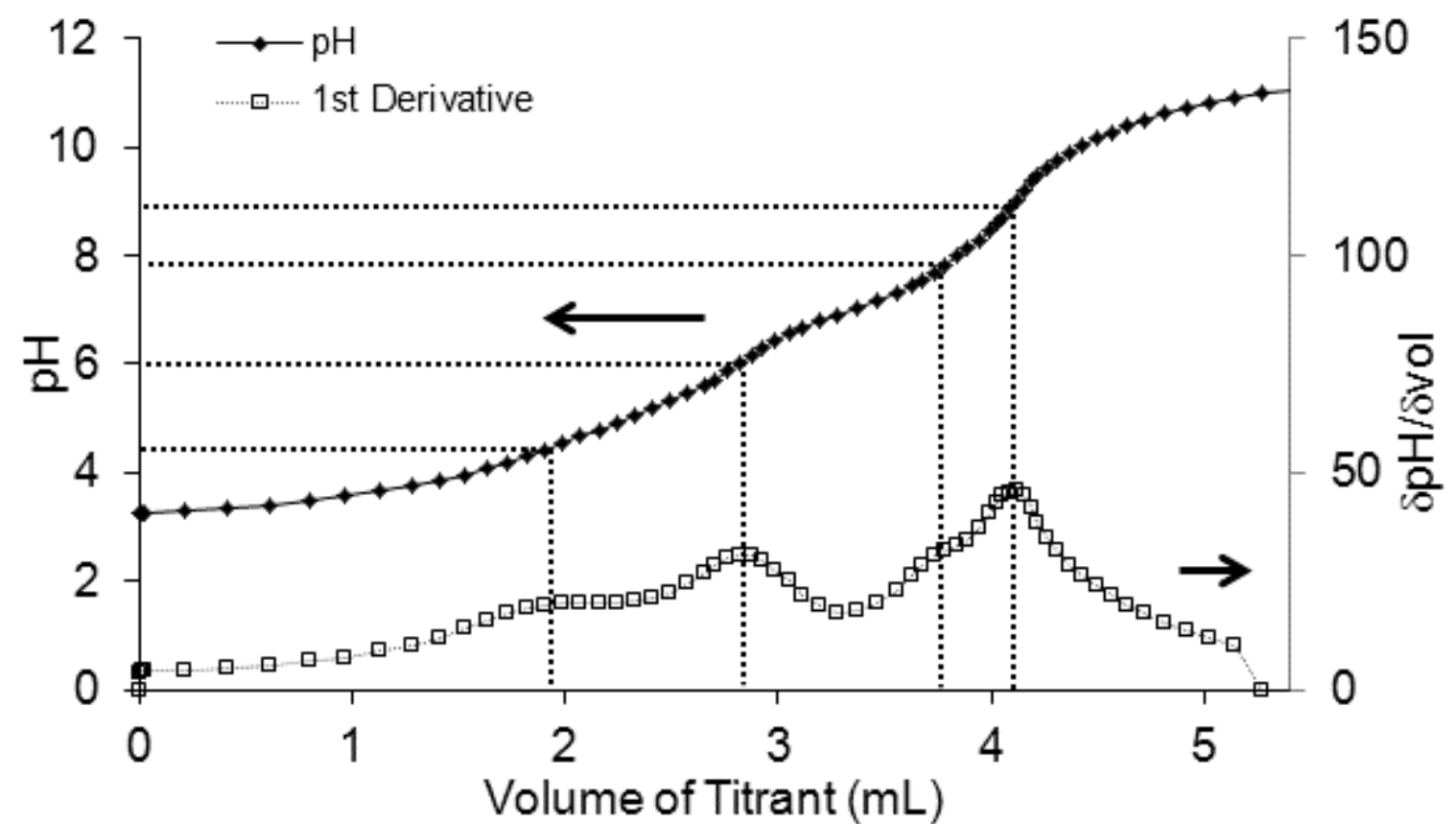

Figure 3.36- Titration of $[\mathrm{HQ}]=3 \times 10^{-4} \mathrm{M},\left[\mathrm{Eu}^{3+}\right]=1.5 \times 10^{-4} \mathrm{M},[\mathrm{NaCl}]=0.1 \mathrm{M},[\mathrm{HCl}]=0.001 \mathrm{M}$, $[$ Triton X-114] $=1$ wt\% $(\sim 0.02 \mathrm{M})$, by $[\mathrm{NaOH}]=0.01024 \pm 0.00002 \mathrm{M}$ and $[\mathrm{NaCl}]=0.09 \mathrm{M}$. $\mathrm{T}=1.9 \pm 0.1^{\circ} \mathrm{C}$.

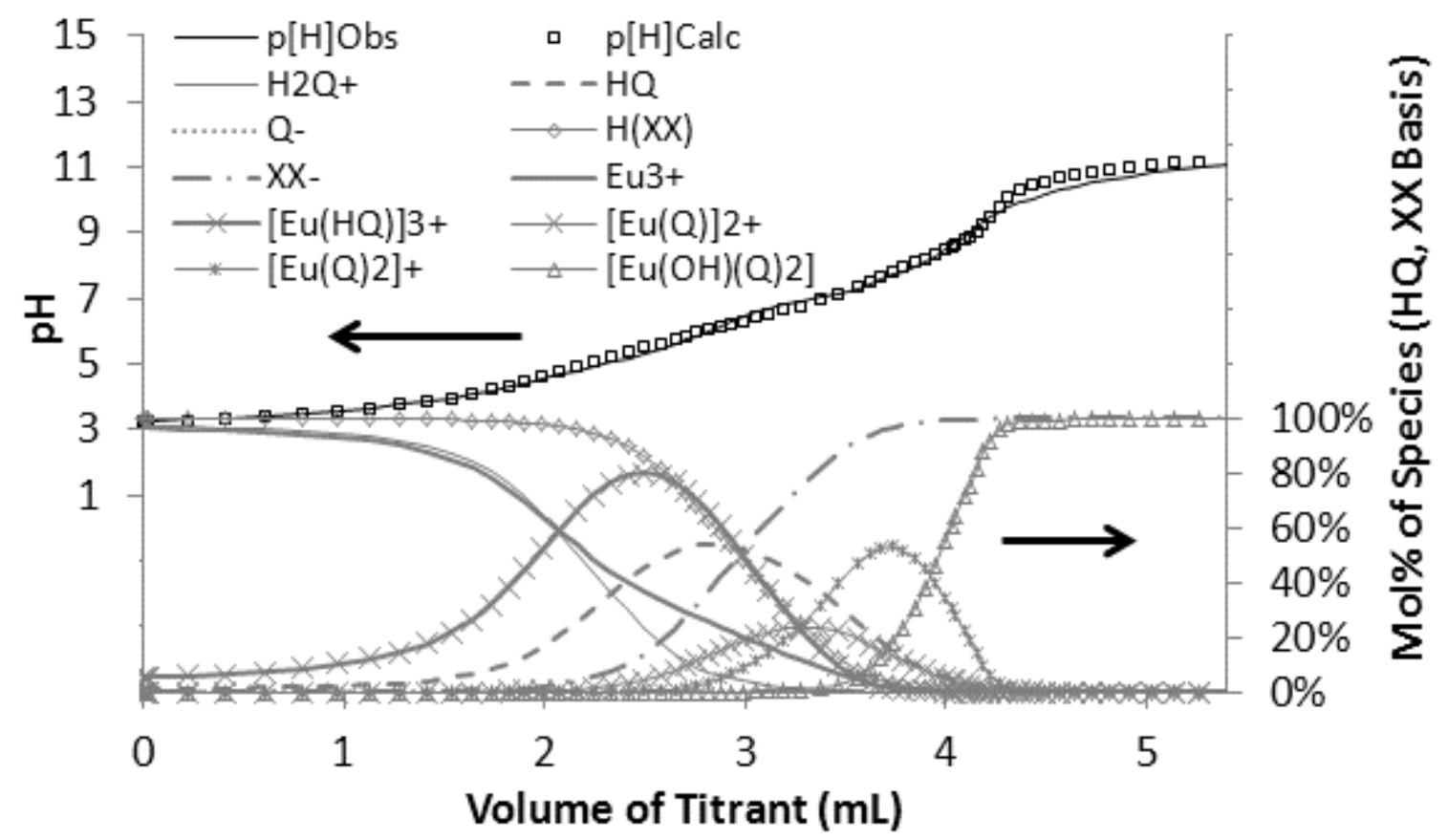

Figure 3.37- Hyperquad fit for the titration of $[\mathrm{HQ}]=3 \times 10^{-4} \mathrm{M},\left[\mathrm{Eu}^{3+}\right]=1.5 \times 10^{-4} \mathrm{M},[\mathrm{NaCl}]=0.1$ $\mathrm{M},[\mathrm{HCl}]=0.001 \mathrm{M},[$ Triton X-114] $=1 \mathrm{wt} \%(\sim 0.02 \mathrm{M})$, by $[\mathrm{NaOH}]=0.01024 \pm 0.00002 \mathrm{M}$ and $[\mathrm{NaCl}]=0.09 \mathrm{M} . \mathrm{T}=1.9 \pm 0.1^{\circ} \mathrm{C}$. 
Table 3.18- Hyperquad fit parameters for the 2:1 ligand to metal solution titration in $1 \mathrm{wt} \%$ Triton.

\begin{tabular}{|c|c|c|c|c|c|}
\hline Equation & $\begin{array}{c}\text { Formed } \\
\text { Species }\end{array}$ & Parameter & $\begin{array}{c}\text { Parameter } \\
\text { Refined? }\end{array}$ & $\mathbf{p} \boldsymbol{\beta}$ & pK \\
\hline$(3.15)$ & $\mathrm{OH}^{-}$ & $\mathrm{K}_{\mathrm{w}}$ & No & 14.7 & \\
\hline$(3.25)$ & $\mathrm{XX}$ & $\mathrm{K}_{\mathrm{xx}}$ & No & 6.3 & \\
\hline$(3.13)$ & $\mathrm{HQ}$ & $\mathrm{K}_{\mathrm{a} 1}$ & No & 5.2 & \\
\hline$(3.14)$ & $\mathrm{Q}^{-}$ & $\mathrm{K}_{\mathrm{a} 2}$ & No & 15.2 & \\
\hline$(3.19)$ & {$[\mathrm{Eu}(\mathrm{Q})]^{2+}$} & $\beta_{101}$ & No & -7.78 & \\
\hline$(3.22)$ & {$\left[\mathrm{Eu}(\mathrm{Q})_{2}\right]^{+}$} & $\beta_{102}$ & Yes & $-14.7 \pm 0.2$ & $-6.9 \pm 0.2$ \\
\hline$(3.23)$ & {$\left[\mathrm{Eu}(\mathrm{OH})(\mathrm{Q})_{2}\right]$} & $\beta_{1-12}$ & Yes & $-21.1 \pm 0.2$ & $-6.4 \pm 0.2$ \\
\hline$(3.26)$ & {$[\mathrm{Eu}(\mathrm{HQ})]^{3+}$} & $\beta_{111}$ & No & -14.1 & -6.3 \\
\hline
\end{tabular}

Four different models were used in an effort to find the best fit for the 3:1 ligand to metal ratio titration data with differing results. The models all relied on equations (3.13), (3.14), (3.19), (3.22), (3.24), and (3.25), which were all used for fitting the 3:1 titration in the $\mathrm{H}_{2} \mathrm{O}$ system. Equation (4.20) associated with the $H(X X)$ species was added to all models to be consistent with the Triton/water system evaluation. The equation (3.26) that relates to the formation of $[\mathrm{Eu}(\mathrm{HQ})]^{3+}$ was used in Models $\mathrm{C}$ and $\mathrm{D}$ to test its effect on curve fit and parameter values. A new reaction between europium and the protolytic impurity shown in equation (3.27) was included in Model A and D to see if it would improve the modeling results.

$$
\mathrm{Eu}^{+3}+\mathrm{XX}^{-} \stackrel{\beta_{1001}}{\longleftrightarrow}[\mathrm{Eu}(\mathrm{XX})]^{2+}
$$

The fits from the parameters obtained in Table 3.19 are shown in Figure 3.39 and show that all modeling attempts give a reasonable fit for the data with the best fit shown for the model with the most parameters (Model D). Despite a slightly superior fit, the inclusion of the formation of the $[\mathrm{Eu}(\mathrm{XX})]^{2+}$ species leads to larger error in the $\left[\mathrm{Eu}(\mathrm{Q})_{3}\right]$ formation constant. Given that the parameter for the formation of $\left[\mathrm{Eu}(\mathrm{Q})_{3}\right]$ from all modeling attempts are all statistically the same, the model using the most parameters that give a largest errors (Model D) must be rejected for 
the simpler model providing less error (Model C). Model C was chosen over the simplest (A) because $[\mathrm{Eu}(\mathrm{HQ})]^{3+}$ included in the other Triton/water system parameter calculations.

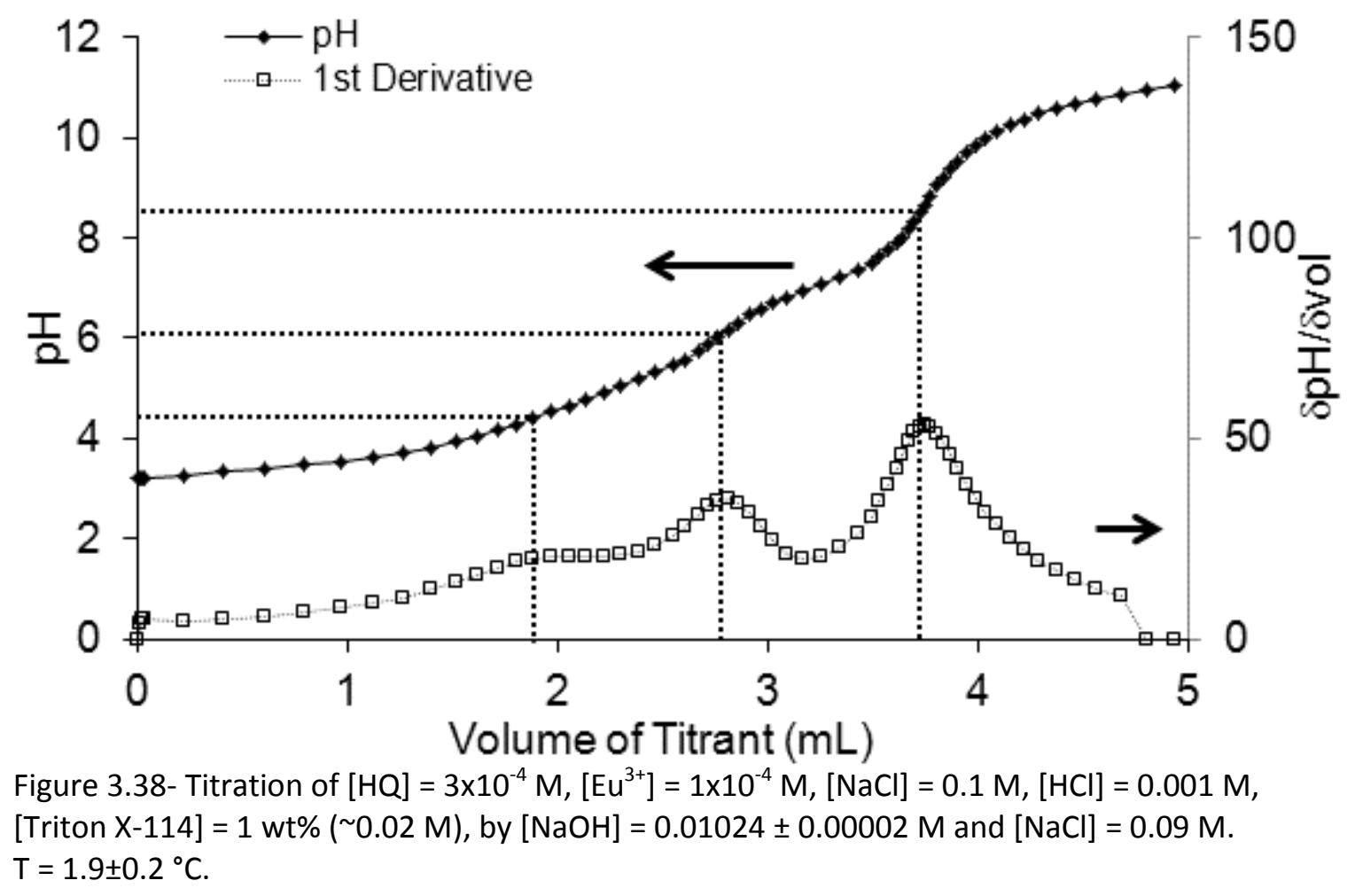

Table 3.19-Parameters from the four models used to fit the 3:1 ligand to metal ratio solution titration.

\begin{tabular}{|c|c|c|c|c|c|c|c|}
\hline Equation & $\begin{array}{c}\text { Formed } \\
\text { Species }\end{array}$ & Parameter & & Model A & Model B & Model C & Model D \\
\hline$(3.15)$ & $\mathrm{OH}^{-}$ & $\mathrm{K}_{\mathrm{w}}$ & & 14.7 & 14.7 & 14.7 & 14.7 \\
\hline$(3.25)$ & $\mathrm{XX}$ & $\mathrm{K}_{\mathrm{xX}}$ & & 6.3 & 6.3 & 6.3 & 6.3 \\
\hline$(3.13)$ & $\mathrm{HQ}$ & $\mathrm{K}_{\mathrm{a} 1}$ & & 5.2 & 5.2 & 5.2 & 5.2 \\
$(3.14)$ & $\mathrm{Q}$ & $\mathrm{K}_{\mathrm{a} 2}$ & $\mathrm{p} \beta$ & 15.2 & 15.2 & 15.2 & 15.2 \\
& & $\mathrm{pK}$ & 10.0 & 10.0 & 10.0 & 10.0 \\
\hline$(3.27)$ & {$[\mathrm{Eu}(\mathrm{XX})]^{2+}$} & $\beta_{1001}$ & & (not used) & $-6.4 \pm 0.5$ & (not used) & $-6.2 \pm 0.5$ \\
$(3.19)$ & {$[\mathrm{Eu}(\mathrm{Q})]^{2+}$} & $\beta_{101}$ & & -7.78 & -7.78 & -7.78 & -7.78 \\
$(3.22)$ & {$\left[\mathrm{Eu}(\mathrm{Q})_{2}\right]^{+}$} & $\beta_{102}$ & $\mathrm{p} \beta$ & -14.7 & -14.7 & -14.7 & -14.7 \\
& & $\mathrm{pK}$ & -6.9 & -6.9 & -6.9 & -6.9 \\
\hline$(3.24)$ & {$\left[\mathrm{Eu}(\mathrm{Q})_{3}\right]$} & $\beta_{103}$ & $\mathrm{p} \beta$ & $-21.3 \pm 0.2$ & $-22.1 \pm 0.5$ & $-21.4 \pm 0.2$ & $-22.0 \pm 0.4$ \\
& & $\mathrm{pK}$ & $-6.6 \pm 0.2$ & $-7.4 \pm 0.5$ & $-6.7 \pm 0.2$ & $-7.3 \pm 0.4$ \\
\hline$(3.26)$ & {$[\mathrm{Eu}(\mathrm{HQ})]^{3+}$} & $\beta_{111}$ & $\mathrm{p} \beta$ & (not used) & (not used) & -14.1 & -14.3 \\
& & & & & & -6.5 & -6.5 \\
\hline
\end{tabular}




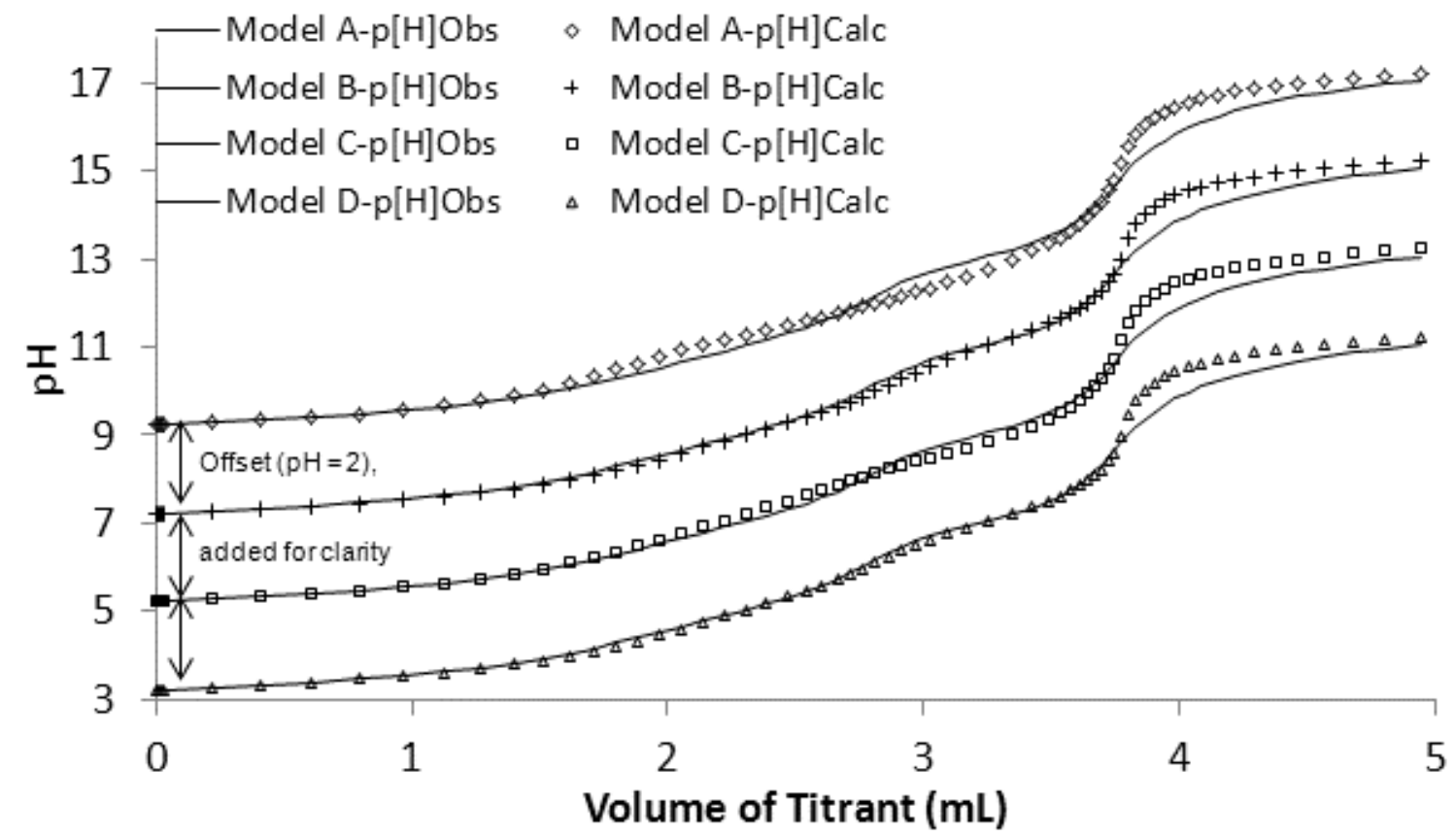

Figure 3.39- Results from four attempts at fitting the 3:1 ligand to metal titration curve.

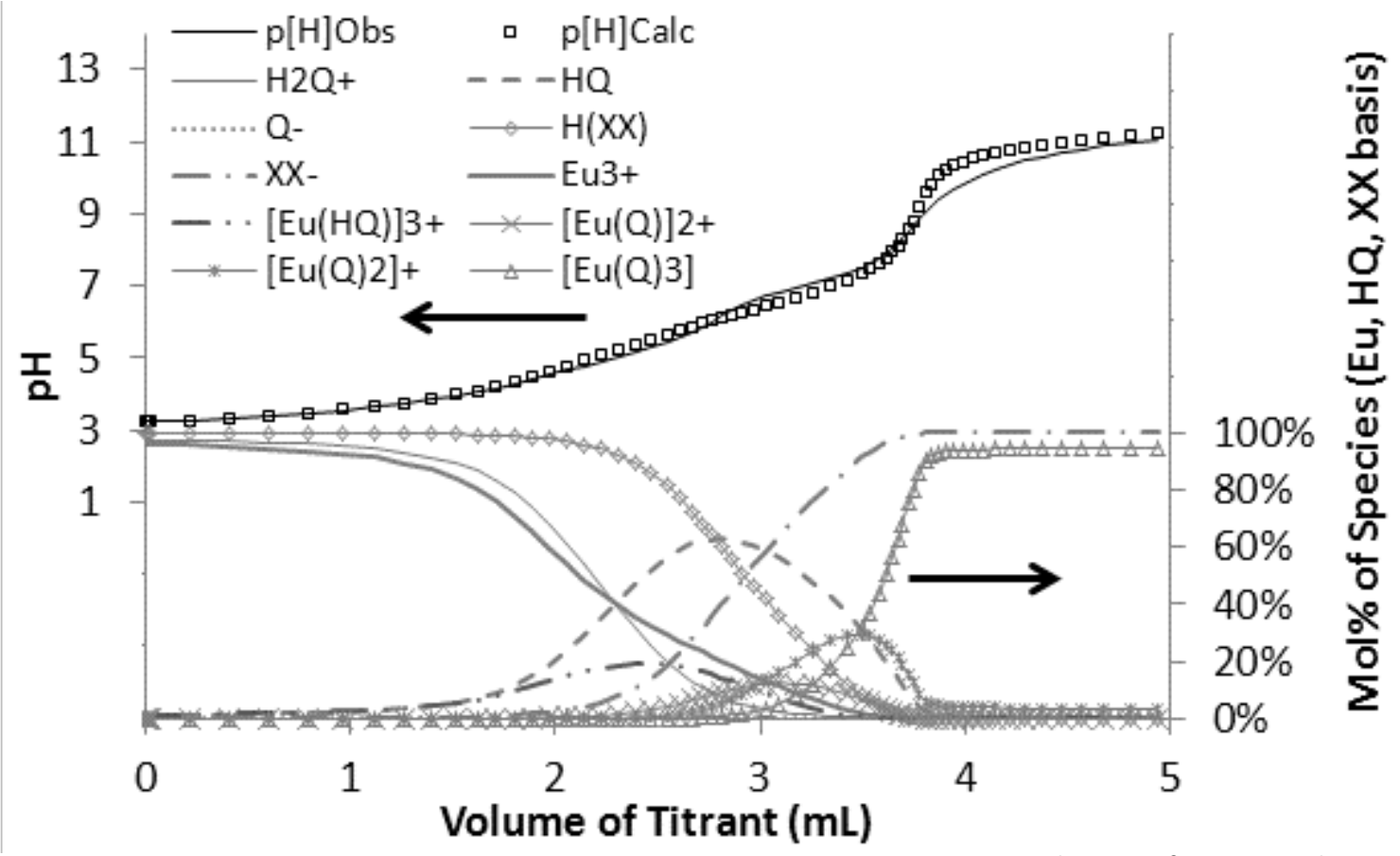

Figure 3.40- Hyperquad fit using Model $\mathrm{C}$ for the titration of $[\mathrm{HQ}]=3 \times 10^{-4} \mathrm{M},\left[\mathrm{Eu}^{3+}\right]=1 \times 10^{-4} \mathrm{M}$, $[\mathrm{NaCl}]=0.1 \mathrm{M},[\mathrm{HCl}]=0.001 \mathrm{M},[$ Triton X-114] $=1 \mathrm{wt} \%(\sim 0.02 \mathrm{M})$, by $\mathrm{NaOH}]=0.01024 \pm 0.00002$ $\mathrm{M}$ and $[\mathrm{NaCl}]=0.09 \mathrm{M} . \mathrm{T}=1.9 \pm 0.1^{\circ} \mathrm{C}$. 
Table 3.20- Hyperquad fit parameters for the 3:1 ligand to metal solution titration in $1 \mathrm{wt} \%$ Triton.

\begin{tabular}{|c|c|c|c|c|c|}
\hline Equation & $\begin{array}{c}\text { Formed } \\
\text { Species }\end{array}$ & Parameter & $\begin{array}{c}\text { Parameter } \\
\text { Refined? }\end{array}$ & $\mathbf{p} \boldsymbol{\beta}$ & pK \\
\hline$(3.15)$ & $\mathrm{OH}^{-}$ & $\mathrm{K}_{\mathrm{w}}$ & No & 14.7 & \\
\hline$(3.25)$ & $\mathrm{XX}$ & $\mathrm{K}_{\mathrm{xx}}$ & No & 6.3 & \\
\hline$(3.13)$ & $\mathrm{HQ}$ & $\mathrm{K}_{\mathrm{a} 1}$ & No & 5.2 & \\
\hline$(3.14)$ & $\mathrm{Q}^{-}$ & $\mathrm{K}_{\mathrm{a} 2}$ & No & 15.2 & \\
$(3.19)$ & {$[\mathrm{Eu}(\mathrm{Q})]^{2+}$} & $\beta_{101}$ & No & -7.78 & \\
\hline$(3.22)$ & {$\left[\mathrm{Eu}(\mathrm{Q})_{2}\right]^{+}$} & $\beta_{102}$ & No & -14.7 & -6.9 \\
\hline$(3.24)$ & {$\left[\mathrm{Eu}(\mathrm{Q})_{3}\right]$} & $\beta_{103}$ & Yes & $-21.4 \pm 0.2$ & $-6.7 \pm 0.2$ \\
\hline$(3.26)$ & {$[\mathrm{Eu}(\mathrm{HQ})]^{3+}$} & $\beta_{111}$ & No & -14.1 & -6.3 \\
\hline
\end{tabular}

\subsection{Discussion}

\subsubsection{The $\mathrm{pK}_{\mathrm{a}}{ }^{\prime} \mathrm{s}$ of 8-Hydroxyquinoline}

Literature data for the 8-hydroxyquinoline protonation constants measured at $1^{\circ} \mathrm{C}$ in $0.1 \mathrm{M}$ $\mathrm{NaCl}$ are not available for comparison. However, using the Van't Hoff equation, these results can be compared with data measured under the same ionic strength, but at different temperatures. Previously published $\mathrm{pK}_{\mathrm{a}}$ data for 8-hydroxyquinoline measured in $0.1 \mathrm{M} \mathrm{NaCl}$ at $\mathrm{T}=25$ and 60 ${ }^{\circ} \mathrm{C}$ are available. ${ }^{[151-152]}$ These data as presented are shown in a modified Van't Hoff plot, which shows the data in $\log _{10}$ instead of In (see Figure 3.41 ). The linearity of data with $1 / T$ is still preserved as presented. The comparison of $\mathrm{pK}_{\mathrm{a}}$ values measured in this work with the literature shows very good consistency with previously published data. Using the Van't Hoff equation, the standard enthalpy of reaction was calculated using the data from the literature and results of this work (see Table 3.21).

Comparing the $\mathrm{pK}_{\mathrm{a}}$ results of the water system with the Triton $\mathrm{X}-114 /$ water system gives insight into the effect of the Triton on the deprotonation of 8-hydroxyquinoline. Literature values measured in Triton X-100 at $20^{\circ} \mathrm{C}^{[134]}$ show the same trend (see Figure 3.42 ) as the 
observed results measured in this work (see Table 3.23, the $\mathrm{pK}_{\mathrm{a}}$ average of both techniques). When comparing the average values it is notable that the $\mathrm{pK}_{\mathrm{a}}{ }^{\prime}$ 's were changed in opposite directions with the $\mathrm{pK}_{\mathrm{a} 1}$ lowered by $0.4 \pm 0.2$ and the $\mathrm{pK}_{\mathrm{a} 2}$ increased by $0.3 \pm 0.1$ (see Table 3.23). In this work, the $\mathrm{pK}_{\mathrm{a} 1}$ refers to the deprotonation of the aromatic nitrogen, and the $\mathrm{pK}_{\mathrm{a} 2}$ refers to the deprotonation of the phenolic oxygen. The divergent trend in the $\mathrm{pK}_{\mathrm{a}}$ values of 8 hydroxyquinoline is consistent with data presented by Irving et al. ${ }^{[153]}$ which indicate that ligands with $-\mathrm{OH}$ functional groups experience an increase in $\mathrm{pK}_{\mathrm{a}}$ with increasing organic content of a mixed solvent, whereas ligands with $-\mathrm{NH}$ functional groups show a decrease. Irving et al. ${ }^{[153]}$ interprets the data to mean that increasing the organic content of the mixed solvent will decrease the dielectric constant of the medium. This leads to increased ionic interaction between the proton and negatively charged oxygen in $-\mathrm{OH}$ functional groups [see equation (3.28)]. ${ }^{[153]}$ In the $-\mathrm{NH}$ functional groups there is a smaller change in the ion-dipole forces between the nitrogen and proton than in the ion-dipole forces between the proton and the more electronegative oxygen donors of the solvent. ${ }^{[153]}$ In the case of $-\mathrm{OH}$ functional groups, electrostatic effects can explain the change in $\mathrm{pK}_{\mathrm{a}}$ with increased organic content of a mixed solvent, but with -NH functional groups the reaction is charge neutral [see equation (3.29)]. Because the change in $\mathrm{pK}_{\mathrm{a}}$ for $-\mathrm{NH}$ functional groups cannot be described by electrostatic effect, it has been linked to such variables as basicity of solvent, ${ }^{[154-155]}$ solvation effects, ${ }^{[154-155]}$ stabilization of the free base, ${ }^{[154]}$ and interaction of ionic solutes with solvent species. ${ }^{[153-154]}$ When dealing with the medium effects caused by mixed solvents, the thermodynamic treatment of the system involves the inclusion of the Gibbs free energy of transfer of the solute species from water to the mixed solvent solution $\left(\Delta G_{t}\right) \cdot{ }^{[153,156]}$ These factors when taken together offer an explanation for the divergent trends observed in this work for the $\mathrm{pK}_{\mathrm{a}}{ }^{\text {'s }}$ of 8hydroxyquinoline as the surfactant concentration increases. 


$$
\begin{gathered}
{[R-O H] \leftrightarrow H^{+}+[R-O]^{-}} \\
{[R-N H]^{+} \leftrightarrow H^{+}+[R-N]}
\end{gathered}
$$

Table 3.21- Calculated Standard Enthalpy of reaction for the deprotonation of 8hydroxyquinoline. Data utilized for this calculation are shown in Table 3.22.

\begin{tabular}{|c|c|c|}
\hline & $\mathrm{K}_{\mathrm{a} 1}$ & $\mathrm{~K}_{\mathrm{a} 2}$ \\
\hline Van't Hoff Slope & $-2700 \pm 300$ & $-760 \pm 620$ \\
$\Delta \mathbf{H}^{\circ}(\mathrm{kJ} / \mathrm{mol})$ & $22 \pm 3$ & $6 \pm 5$ \\
\hline
\end{tabular}

Table 3.22- Data used in the Van't Hoff Analysis of the $\mathrm{pK}_{\mathrm{a}}$ 's of 8-hydroxyquinoline in the water system.

\begin{tabular}{|c|c|c|c|c|}
\hline Medium & $\begin{array}{c}\text { Temp } \\
\left({ }^{\circ} \mathbf{C}\right)\end{array}$ & $\mathbf{p K}_{\mathrm{a} 1}$ & $\mathbf{p K}_{\mathrm{a} 1}$ & Source \\
\hline $0.1 \mathrm{M} \mathrm{NaCl}$ & 1 & $5.6 \pm 0.1$ & $9.52 \pm 0.06$ & $\begin{array}{c}\text { This Work } \\
\text { (Potentiometry) }\end{array}$ \\
\hline $0.1 \mathrm{M} \mathrm{NaCl}$ & 1 & $5.32 \pm 0.01$ & $9.72 \pm 0.01$ & $\begin{array}{c}\text { This work } \\
\text { (Spectrophotometry) }\end{array}$ \\
$0.1 \mathrm{M} \mathrm{KCl}$ & 25 & 5.02 & 9.68 & Matrosovich et al..$^{[151]}$ \\
$0.1 \mathrm{M} \mathrm{KCl}$ & 60 & 4.63 & 9.38 & Lobanov et al. ${ }^{[152]}$ \\
\hline
\end{tabular}

Table 3.23-Summary of $\mathrm{pK}_{\mathrm{a}}$ results from potentiometric (Potent.) and spectrophotometric (Spect.) titration methods. The results from these methods were averaged for a final result from this study.

\begin{tabular}{|c|cc|c|c|c|c|}
\hline \multirow{2}{*}{ Parameter } & \multicolumn{3}{|c|}{$\mathrm{H}_{2} \mathrm{O}$} & \multicolumn{3}{c|}{ Triton X-114/ $\mathrm{H}_{2} \mathbf{O}$} \\
\cline { 7 - 8 } & Potent. & Spect. & Avg. & Potent. & Spect. & Avg. \\
\hline $\mathrm{pK}_{\mathrm{a} 1}$ & $5.6 \pm 0.1$ & $5.32 \pm 0.01$ & $5.5 \pm 0.1$ & $5.2 \pm 0.2$ & $4.94 \pm 0.01$ & $5.1 \pm 0.2$ \\
$\mathrm{pK}_{\mathrm{a} 2}$ & $9.5 \pm 0.1$ & $9.72 \pm 0.01$ & $9.6 \pm 0.1$ & $10.0 \pm 0.1$ & $9.69 \pm 0.01$ & $9.9 \pm 0.1$ \\
\hline
\end{tabular}




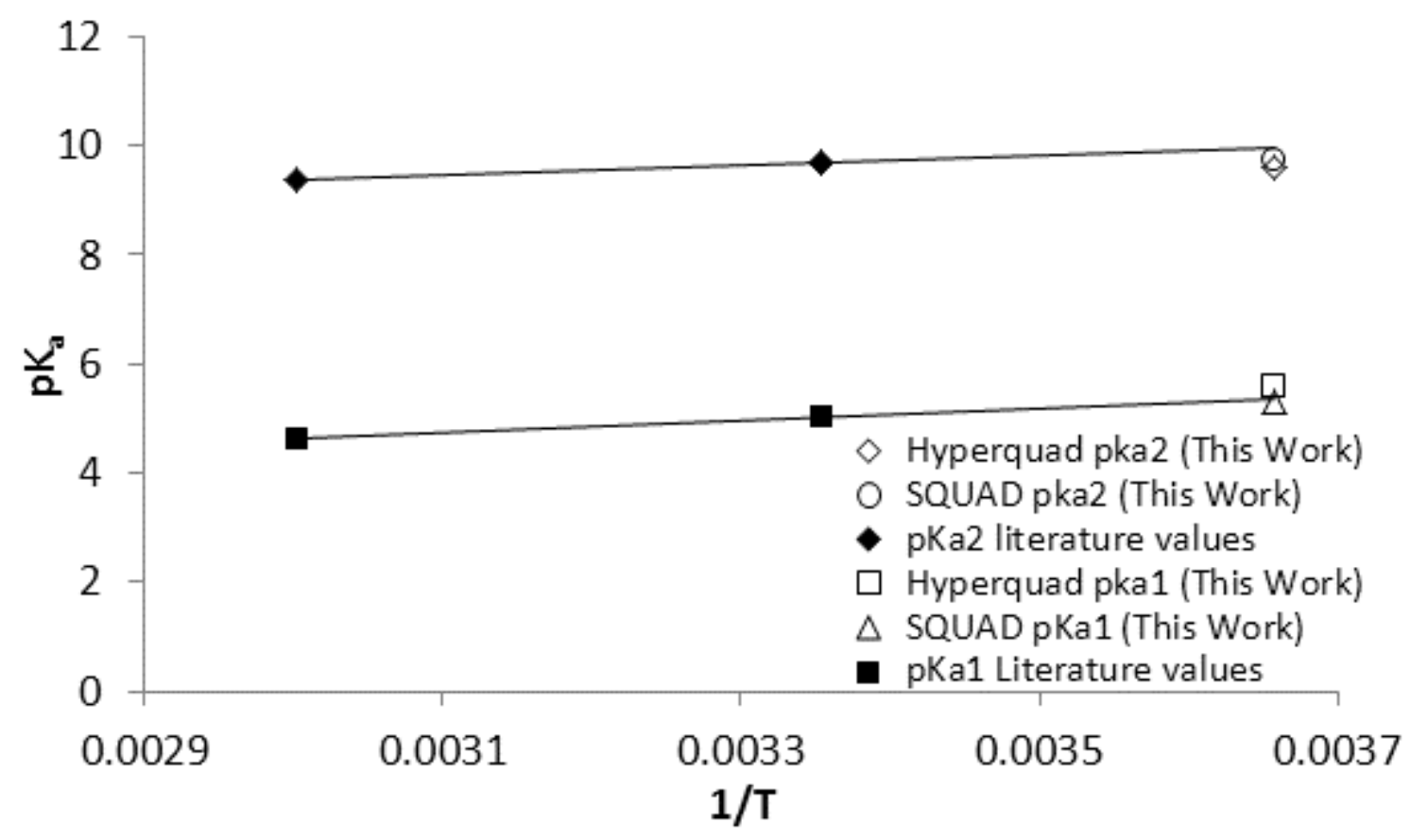

Figure 3.41- Comparison of measured $\mathrm{pK}_{\mathrm{a}}$ values for 8-hydroxyquinoline with literature data from Lobanov et al. ${ }^{[151-152]}$ utilizing the Van't Hoff relation. Data used in this plot are shown in Table 3.22.

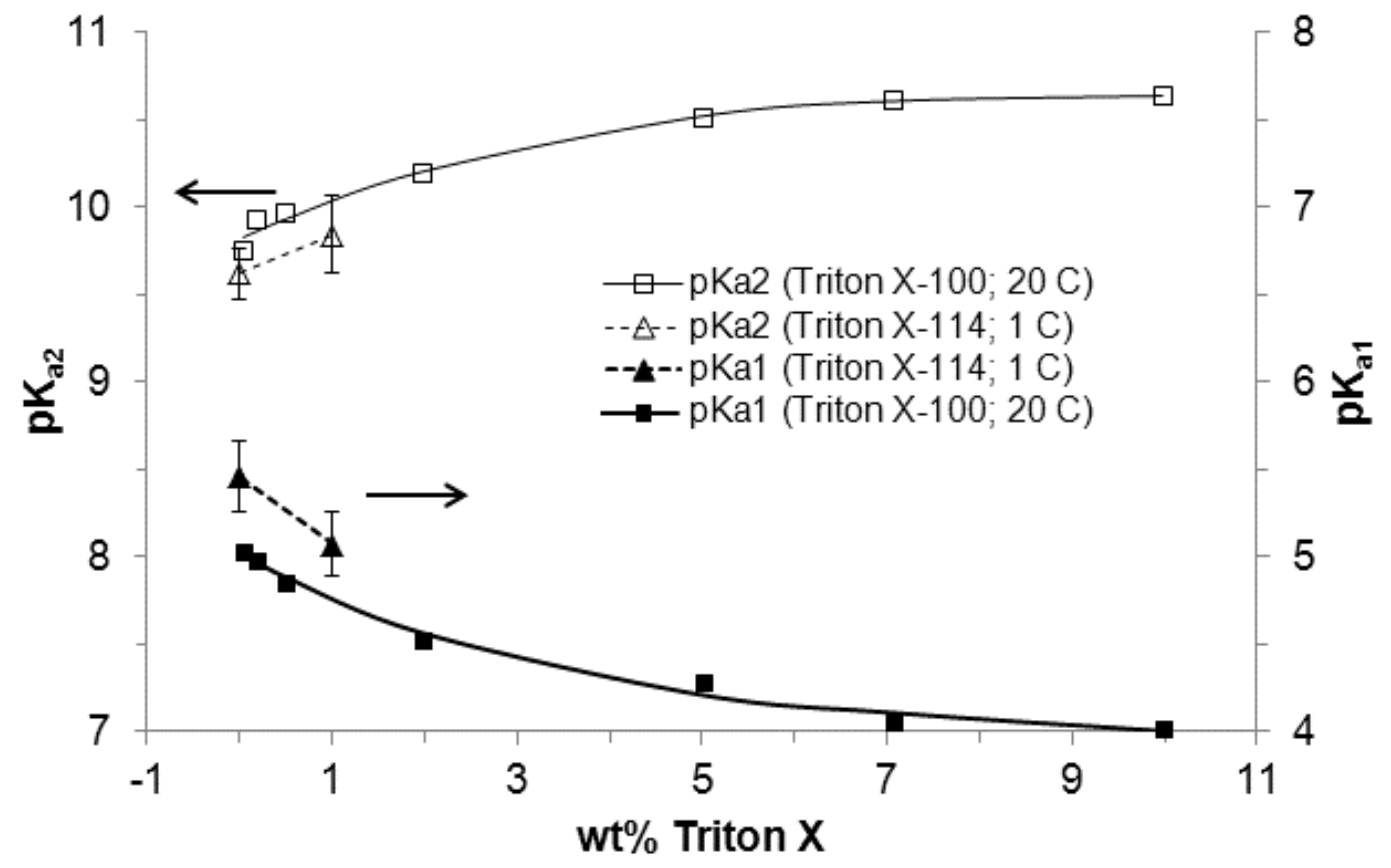

Figure 3.42- Comparison of the $\mathrm{pK}_{\mathrm{a}}$ of 8-hydroxyquinoline in Triton X-100/water with the $\mathrm{pK}_{\mathrm{a}}$ of 8-hydroxyquinoline in Triton X-114/water measured in this work. The literature values are from Hoshino et al. ${ }^{[134]}$ 


\subsubsection{Europium Hydrolysis Behavior}

Europium hydrolysis constants have not been measured previously at $1{ }^{\circ} \mathrm{C}$, however, there exists a rather complete set of data for the first hydrolysis constant at $25^{\circ} \mathrm{C}$ over a range of ionic strength presented in the literature. ${ }^{[157]}$ This data compares reasonably well with the data measured in this work at $1{ }^{\circ} \mathrm{C}$ and $0.1 \mathrm{M} \mathrm{NaCl}$ as shown in Figure 3.43and Table 3.7. Because there is not a great deal of europium hydrolysis data that is comparable with the values measured in this work, it is reasonable to compare with hydrolysis data from the entire lanthanide series. Lanthanides, as trivalent ions, share a similar chemistry that is altered very slightly by the lanthanide contraction, giving fairly predictable data trends. Any holes in series of data are reasonably easy to interpolate. The lanthanide series data presented here will be shown as a function of inverse ionic radius (Table 3.24). Figure 3.44 shows a comparison of observed data with literature is that of the first, second and third hydrolysis constants of select lanthanides with the observed values for europium measured at $1{ }^{\circ} \mathrm{C}$ in $0.1 \mathrm{M} \mathrm{NaCl}$. Literature data in Figure 3.44 were calculated from the data in Baes ${ }^{[147]}$ at $25^{\circ} \mathrm{C}$ and infinite dilution using an accompanying equation to adjust the data to $0.1 \mathrm{M} \mathrm{NaClO}_{4}$ conditions. The data observed in this work agrees with the general trend of the literature data, but as the values progress from $1^{\text {st }}$ to $2^{\text {nd }}$ to $3^{\text {rd }}$, the agreement is poorer. 


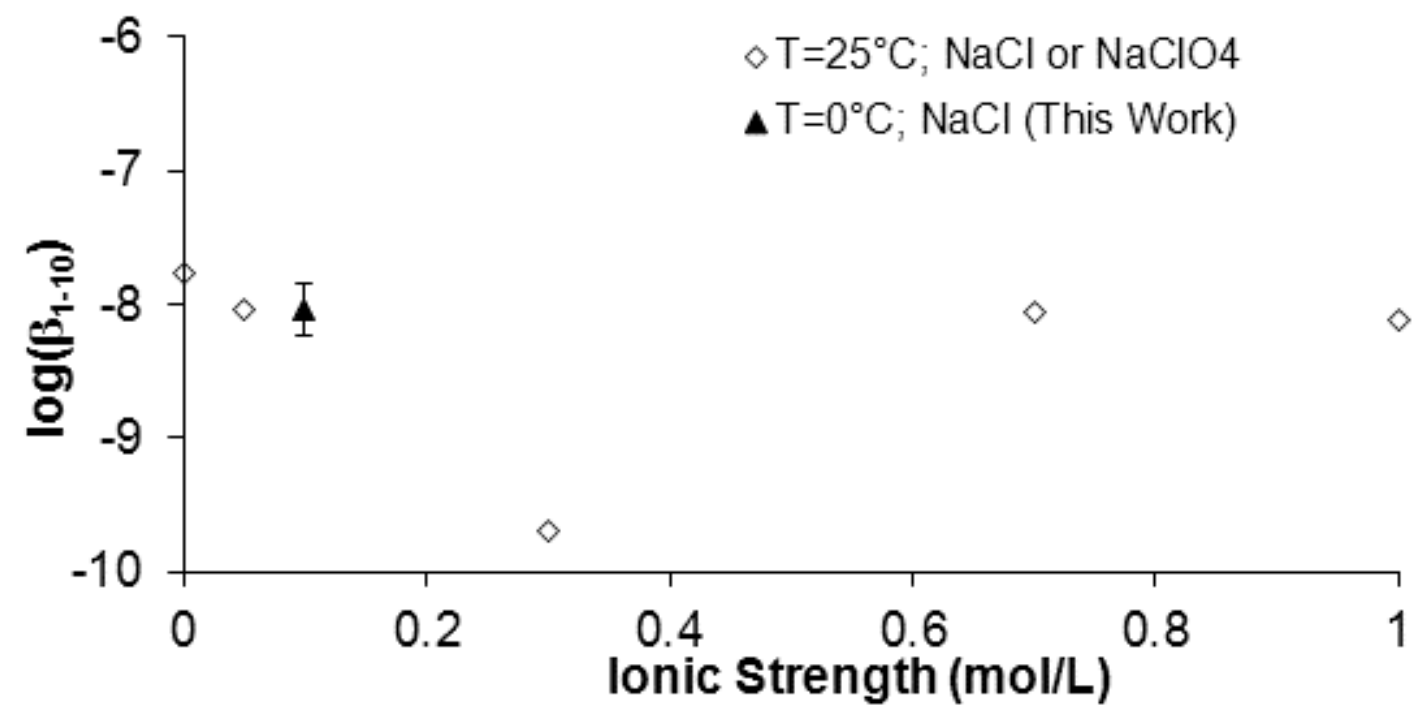

Figure 3.43-Comparison of the first hydrolysis constant $\left(\log \beta_{1-10}\right)$ obtained in this work at $1{ }^{\circ} \mathrm{C}$ with literature data at $25^{\circ} \mathrm{C}$.

Table 3.24- Values used for the inverse ionic radius of the trivalent lanthanides. ${ }^{[14]}$

\begin{tabular}{|c|c|}
\hline Ion & $1 / r\left(\AA^{-1}\right)$ \\
\hline $\mathrm{La}^{3+}$ & 0.969 \\
\hline $\mathrm{Ce}^{3+}$ & 0.990 \\
\hline $\mathrm{Pr}^{3+}$ & 1.010 \\
\hline $\mathrm{Nd}^{3+}$ & 1.017 \\
\hline $\mathrm{Pm}^{3+}$ & 1.031 \\
\hline $\mathrm{Sm}^{3+}$ & 1.044 \\
\hline $\mathrm{Eu}^{3+}$ & 1.056 \\
\hline $\mathrm{Gd}^{3+}$ & 1.066 \\
\hline $\mathrm{Tb}^{3+}$ & 1.083 \\
\hline $\mathrm{Dy}^{3+}$ & 1.096 \\
$\mathrm{Ho}^{3+}$ & 1.110 \\
\hline $\mathrm{Er}^{3+}$ & 1.124 \\
$\mathrm{Tm}^{3+}$ & 1.136 \\
\hline $\mathrm{Yb}^{3+}$ & 1.152 \\
$\mathrm{Lu}^{3+}$ & 1.161 \\
\hline
\end{tabular}




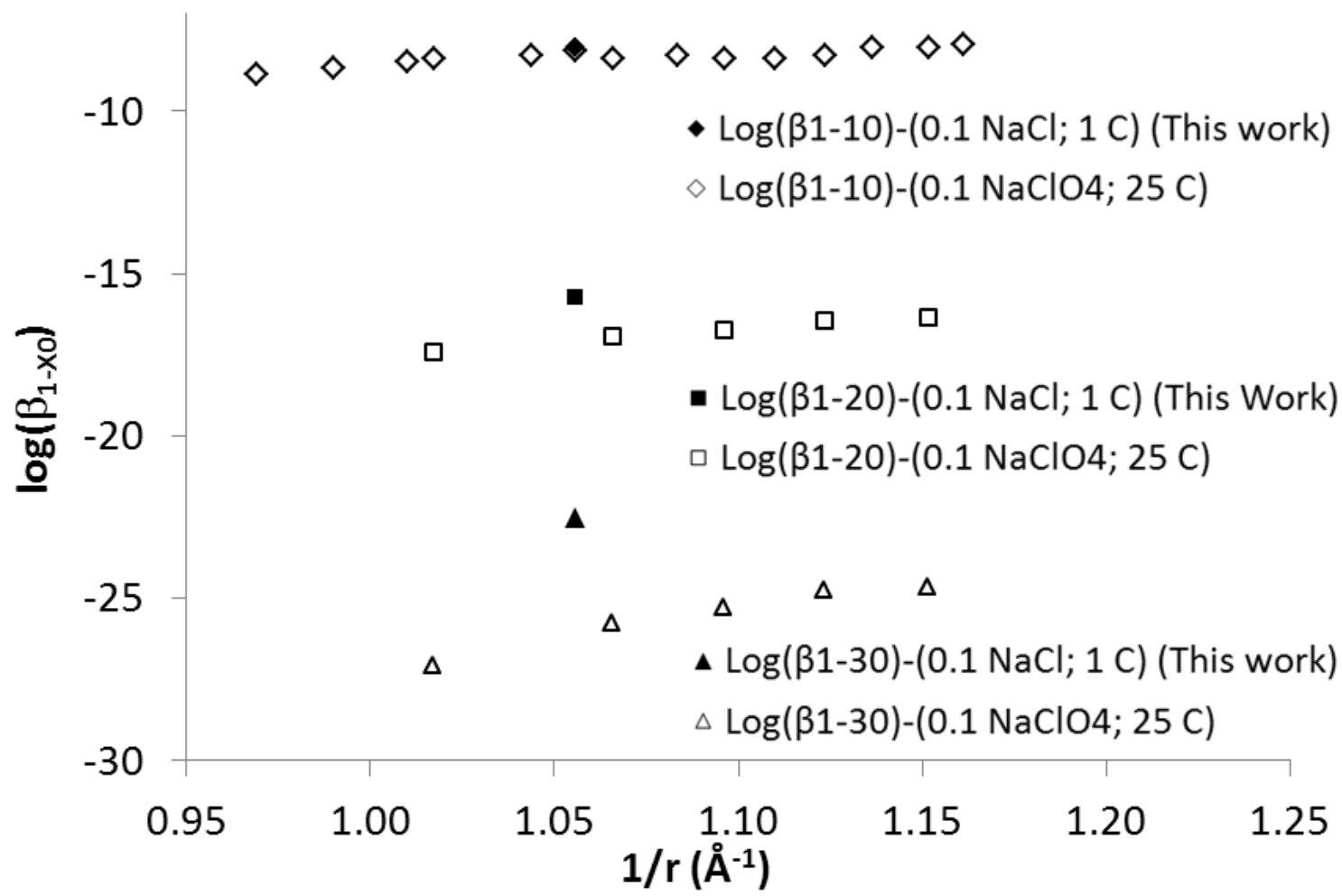

Figure 3.44- Comparison of observed and literature data values for the first hydrolysis constant as a function of inverse ionic radius.

The comparison of hydrolysis constants between the water and the Triton X-114/water system offers insight as to the role that Triton X-114 plays in the hydrolysis of lanthanides in this system. There is a significant change in the hydrolysis constants when Triton X-114 is added to the system with higher stability in the complexes (as indicated by higher $\log \beta$ values) (see Table 3.25). These results are consistent with the description by Irving et al. ${ }^{[153]}$ of ligands, or in the case of europium hydrolysis, hydrated metal centers with -OH functional groups. In the hydrolysis reaction, the ligand with $-\mathrm{OH}$ functional group is the hydroxide, the proton is the $\mathrm{Eu}^{3+}$ ion, and the europium hydrolysis species are analogous to the protonated oxygen group. Adapting the description of the system in Irving et al. ${ }^{[153]}$ to the hydrolysis system, the increase in organic character of the mixed solvent leads to increased ionic interaction between the 
europium and negatively charged oxygen in the hydroxide causing the formation of hydrolysis species.

The system can also be described in terms of $\Delta \mathrm{G}_{\mathrm{t}}$. Based on tabulated $\Delta \mathrm{G}_{\mathrm{t}}$ data in Kalidas et $a l .{ }^{[158]}$ the only data available involving lanthanides is for the methanol/water mixed solvent. These data show an approximately $8-10$ fold increase in $\Delta G_{t}$ as methanol content is increased from $10-30 \%$. An increase in Gibbs free energy indicates lower stability of a species. Therefore, the increase in $\Delta \mathrm{G}_{\mathrm{t}}$ for $\mathrm{Eu}^{3+}$ in the mixed solvent favors its elimination from the system, including the formation of other species, leading to higher stability of $[\mathrm{Eu}(\mathrm{OH})]^{2+},\left[\mathrm{Eu}(\mathrm{OH})_{2}\right]^{+}$, and $\left[\mathrm{Eu}(\mathrm{OH})_{3}\right]$

When considering the case of the mixed hydrolysis species (those involving europium with both $\mathrm{Q}^{-}$and $\mathrm{OH}^{-}$simultaneously) the comparison between the systems shows that no significant change was observed (see Table 3.26). This result indicates that the mixed solvent has no effect on the formation of these species. This is an unexpected result considering the description of the hydrolysis species above, and the europium 8-hydroxyquinoline complexation data discussed in section 3.5.3.

Table 3.25- Comparison of the $\mathrm{H}_{2} \mathrm{O} /$ Triton X-114 vs. $\mathrm{H}_{2} \mathrm{O}$ system europium hydrolysis constants.

\begin{tabular}{|c|c|c|c|}
\hline $\begin{array}{c}\text { Species } \\
\text { Formed }\end{array}$ & \multirow{2}{*}{ Parameter } & $\mathbf{H}_{\mathbf{2}} \mathbf{O}$ & $\mathbf{H}_{\mathbf{2}} \mathbf{O} /$ Triton X-114 \\
\cline { 3 - 4 } & $\mathbf{p K}$ & $\mathbf{p K}$ \\
\hline$[\mathrm{Eu}(\mathrm{OH})]^{2+}$ & $\beta_{1-10}$ & $-8.0 \pm 0.2$ & $-7.1 \pm 0.1$ \\
\hline$\left[\mathrm{Eu}(\mathrm{OH})_{2}\right]^{+}$ & $\beta_{1-20}$ & $-7.7 \pm 0.1$ & $-5.7 \pm 0.1$ \\
\hline$\left[\mathrm{Eu}(\mathrm{OH})_{3}\right]$ & $\beta_{1-30}$ & $-6.8 \pm 0.2$ & $-5.9 \pm 0.2$ \\
\hline
\end{tabular}


Table 3.26- Comparison of the mixed ligand hydrolysis species parameters.

\begin{tabular}{|c|c|c|c|}
\hline $\begin{array}{c}\text { Species } \\
\text { Formed }\end{array}$ & \multirow{2}{*}{ Parameter } & $\mathbf{H}_{\mathbf{2}} \mathbf{O}$ & $\mathbf{H}_{\mathbf{2}} \mathbf{O} /$ Triton X-114 \\
\cline { 3 - 4 } & $\mathbf{p K}$ & $\mathbf{p K}$ \\
\hline$[\mathrm{Eu}(\mathrm{OH})(\mathrm{Q})]^{+}$ & $\beta_{1-11}$ & $-6.4 \pm 0.1$ & $-6.6 \pm 0.1$ \\
{$\left[\mathrm{Eu}(\mathrm{OH})_{2}(\mathrm{Q})\right]$} & $\beta_{1-21}$ & $-5.6 \pm 0.1$ & $-5.4 \pm 0.1$ \\
\hline$\left[\mathrm{Eu}(\mathrm{OH})(\mathrm{Q})_{2}\right]$ & $\beta_{1-12}$ & $-6.6 \pm 0.4$ & $-6.4 \pm 0.2$ \\
\hline
\end{tabular}

\subsubsection{The Europium 8-Hydroxyquinline Stability Constants}

Previous measurements of the formation constant for $[\mathrm{Eu}(\mathrm{Q})]^{2+}\left(\mathrm{i} . \mathrm{e} \cdot \log \beta_{101}\right.$ ) have been performed at $20-25^{\circ} \mathrm{C}$ in $0.1 \mathrm{M}$ of various ionic media. ${ }^{[131,159]}$ These results are shown compared with the value measured in this work at $1{ }^{\circ} \mathrm{C}$ and $0.1 \mathrm{M} \mathrm{NaCl}$ in Figure 3.45. The data are reasonably close given the fact that most the literature data is at $20-25^{\circ} \mathrm{C}$ and the value measured in this work is at $1{ }^{\circ} \mathrm{C}$. The one value in the literature for $[\mathrm{Eu}(\mathrm{Q})]^{2+}$ formation at $20^{\circ} \mathrm{C}$ was 7.2 compared with this work's value of $6.9 \pm 0.1$ at $1{ }^{\circ} \mathrm{C}$.

The effect of the presence of Triton $\mathrm{X}-114$ on the $[\mathrm{Eu}(\mathrm{Q})]^{2+}$ formation constant has not been reported in the literature. However, one work ${ }^{[160]}$ measured this formation constant in $50 \%$ dioxane, which would cause a similar effect as Triton X-114. The $50 \%$ dioxane data are shown with the data from this work using 1 wt\% Triton X-114, and the data measured without any cosolvent present ${ }^{[131]}$ for comparison. These data are shown in Figure 3.46. The data show that increasing the organic component of the mixed-solvent causes an increase in the stability of the formation of $[\mathrm{Eu}(\mathrm{Q})]^{2+}$ that is consistent between the $50 \%$ dioxane data and the $1 \mathrm{wt} \%$ Triton data. A similar comparison can be made for the data concerning the formation of $\left[\mathrm{Eu}(\mathrm{Q})_{2}\right]^{+}$also using data from the previously cited $50 \%$ dioxane paper ${ }^{[160]}$, and one with no dioxin. ${ }^{[159]}$ These data are shown for comparison with the data observed in this work at 0 and 1 wt\% Triton X-114 in Figure 3.47. Just as with the formation of $[\mathrm{Eu}(\mathrm{Q})]^{2+}$, the formation of $\left[\mathrm{Eu}(\mathrm{Q})_{2}\right]^{+}$also appears to be more favored (forming a stronger complex) by increasing the organic component of the 
mixed solvent. This is consistent between the 0 and $50 \%$ dioxane as it is with the 0 and $1 \mathrm{wt} \%$ Triton X-114 data.

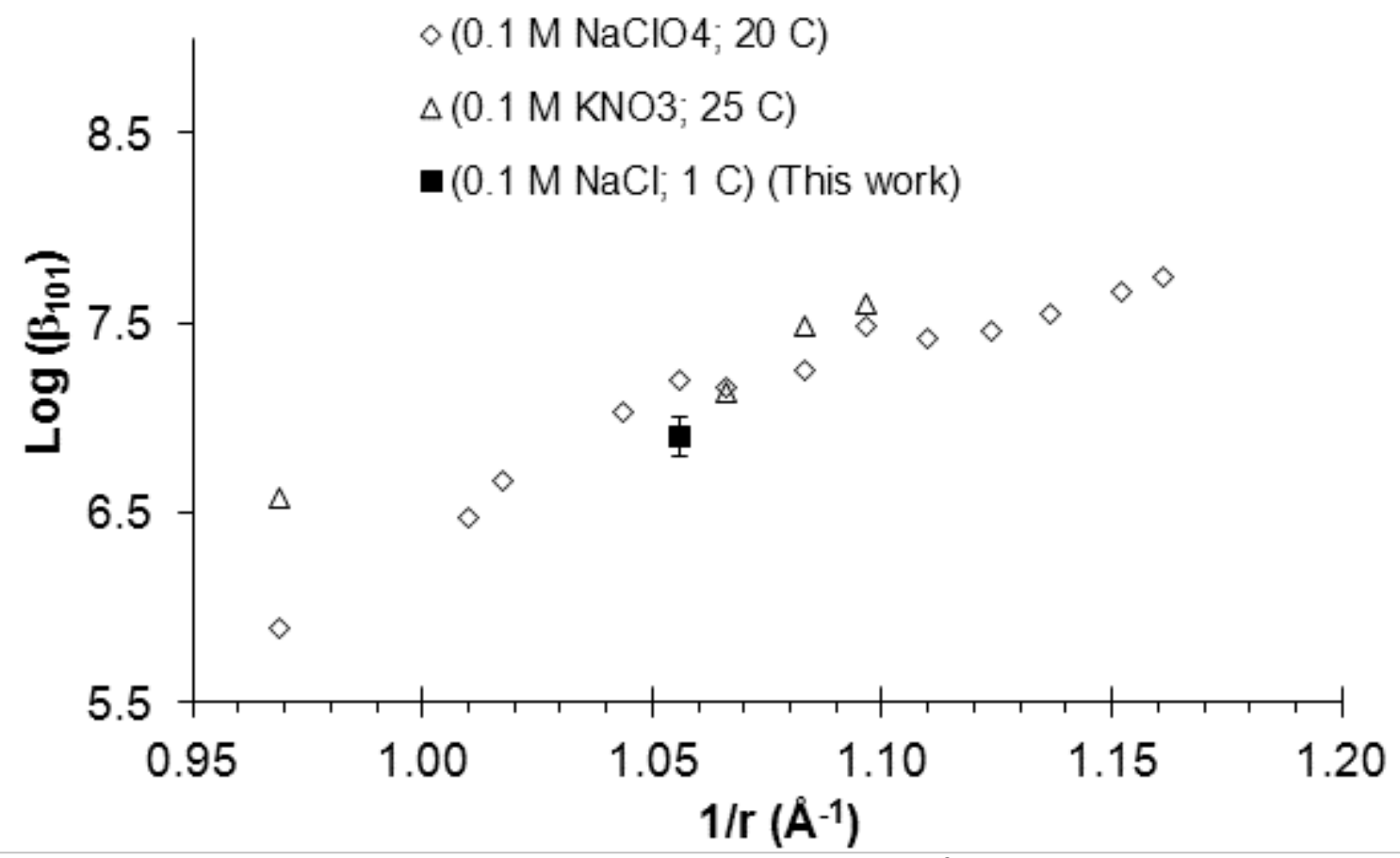

Figure 3.45- A Comparison of the formation constants for $[\mathrm{Eu}(\mathrm{Q})]^{2+}\left(\log \beta_{101}\right)$ in $0.1 \mathrm{M}$ lonic media at various temperatures, to various lanthanides' $[\operatorname{Ln}(Q)]^{2+}$ formation constants represented as a function of ionic radius.

When comparing only the data observed in this work, it is clear that the stability of the complexes between europium and 8-hydroxyquinoline were affected by the presence of Triton $\mathrm{X}-114$. There is a significant increase in the strength of the strength of the $1^{\text {st }}$ and $2^{\text {nd }}$ europium 8-hydroxyquinoline complexation constants in the Triton/water mixed solvent with an increase of $0.9 \pm 0.1$ and $1.3 \pm 0.6$ respectively (see Table 3.27 ). A statistically insignificant increase was also noted for the $3^{\text {rd }}$ complexation constant of $0.5 \pm 0.9$. These results are also consistent with the 
description by Irving et al. ${ }^{[153]}$ of ligands with -OH functional groups. In the europium 8hydroxyquinoline complexation reaction, the ligand with $-\mathrm{OH}$ functional group is the deprotonated 8-hydroxyquinoline, the proton is the $\mathrm{Eu}^{3+}$ ion, and the europium 8hydroxyquinoline complex is analogous to the protonated oxygen group. Adapting the description of the system in Irving et al. ${ }^{[153]}$ to the 8-hydroxyquinoline complexation, the increase in organic character of the mixed solvent leads to increased ionic interaction between the europium and negatively charged oxygen in the deprotonated 8-hydroxyquinoline causing the formation of hydrolysis species. The discussion involving $\Delta G_{t}$ in the hydrolysis reactions also apply to the formation of europium 8-hydroxyquinoline complexes. The increase of $\Delta G_{t}$ for free europium in the system still favors its elimination from the system through 8-hydroxyquinoline complexation.

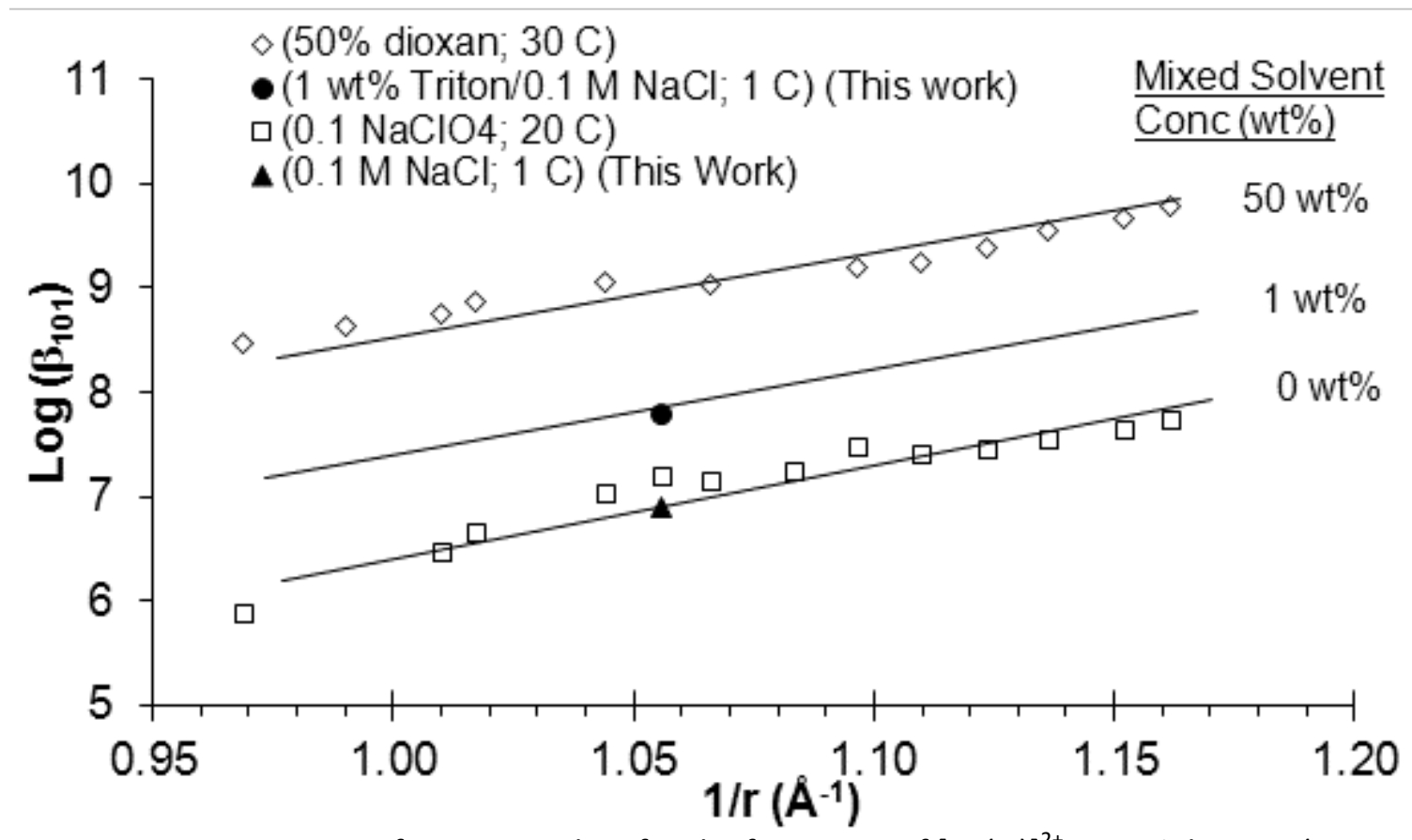

Figure 3.46- Comparison of Literature data for the formation of $[\mathrm{Eu}(\mathrm{Q})]^{2+}$ in $50 \%$ dioxane (at $\mathrm{T}=30{ }^{\circ} \mathrm{C}$ ), and $0 \%$ dioxane $\left(\right.$ at $\mathrm{T}=20^{\circ} \mathrm{C}$ ) with the observed values at $1 \mathrm{wt} \%$ Triton X-114 (at $1{ }^{\circ} \mathrm{C}$ ) and 0 wt\% Triton $\mathrm{X}-114$ (at $1^{\circ} \mathrm{C}$ ). 


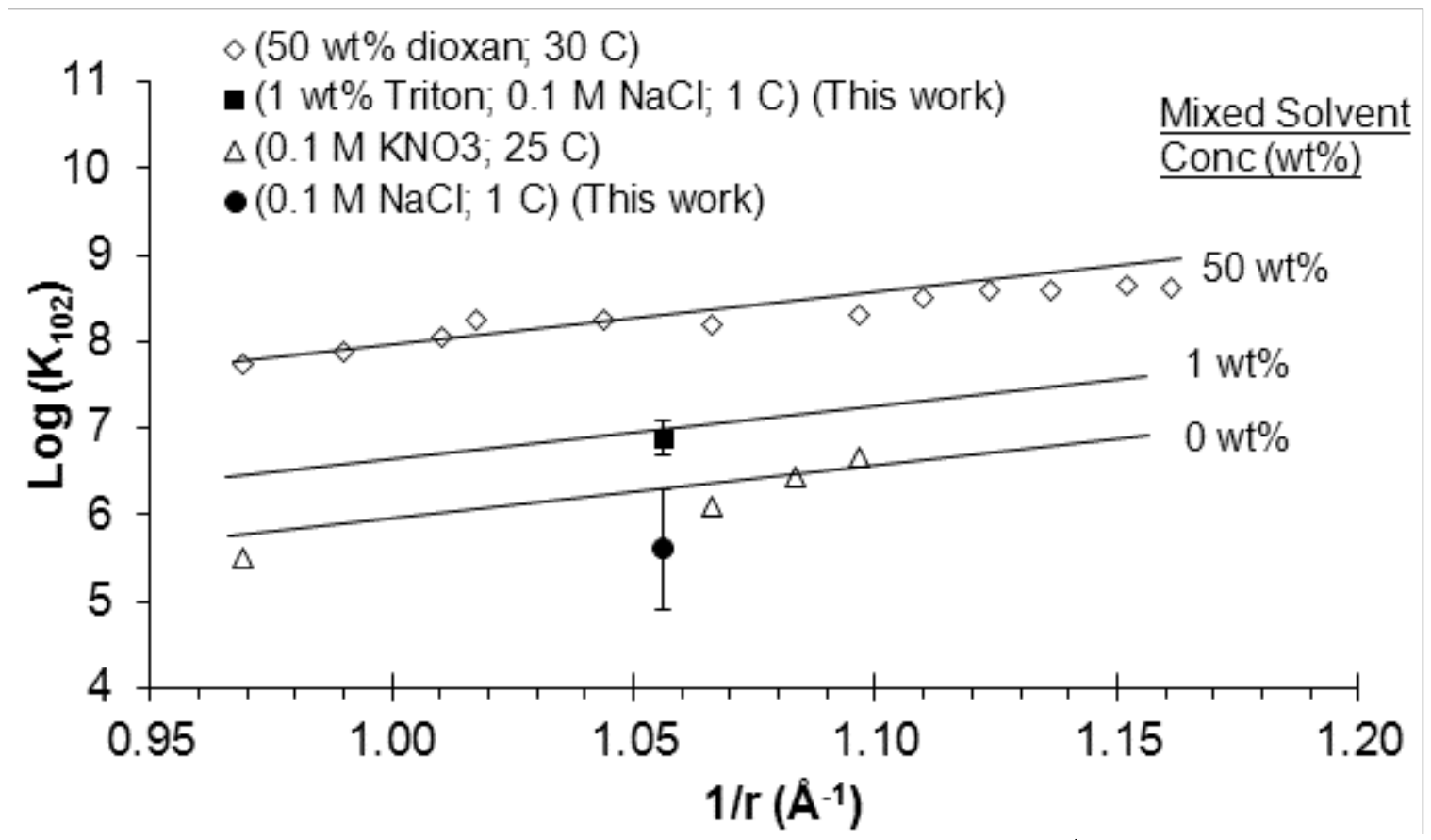

Figure 3.47-- Comparison of Literature data for the formation of $\left[\mathrm{Eu}(\mathrm{Q})_{2}\right]^{+}$in $50 \%$ dioxane (at $\mathrm{T}=30{ }^{\circ} \mathrm{C}$ ), and $0 \%$ dioxane $\left(\right.$ at $\mathrm{T}=25^{\circ} \mathrm{C}$ ) with the observed values at $1 \mathrm{wt} \%$ Triton $\mathrm{X}-114\left(\right.$ at $1{ }^{\circ} \mathrm{C}$ ) and 0 wt\% Triton $\mathrm{X}-114$ (at $1{ }^{\circ} \mathrm{C}$ ).

Table 3.27- A comparison of the stability constants obtained from the $\mathrm{H}_{2} \mathrm{O}$ and the $\mathrm{H}_{2} \mathrm{O}$ /Triton $\mathrm{X}$-114 systems.

\begin{tabular}{|c|c|c|c|}
\hline $\begin{array}{c}\text { Species } \\
\text { Formed }\end{array}$ & \multirow{2}{*}{ Parameter } & $\mathbf{H}_{\mathbf{2}} \mathbf{O}$ & $\mathbf{H}_{\mathbf{2}} \mathbf{O} /$ Triton X-114 \\
\cline { 3 - 4 } & $\mathbf{p K}$ & $\mathbf{p K}$ \\
\hline$[\mathrm{Eu}(\mathrm{Q})]^{2+}$ & $\beta_{101}$ & $-6.9 \pm 0.1$ & $-7.78 \pm 0.04$ \\
\hline$\left[\mathrm{Eu}(\mathrm{Q})_{2}\right]^{+}$ & $\beta_{102}$ & $-5.6 \pm 0.7$ & $-6.9 \pm 0.2$ \\
{$\left[\mathrm{Eu}(\mathrm{Q})_{3}\right]$} & $\beta_{103}$ & $-6.2 \pm 0.9$ & $-6.7 \pm 0.2$ \\
\hline
\end{tabular}

\subsection{Conclusions}

The speciation of 8-hydroxyquinoline and europium in both water and the Triton X-

$114 /$ water mixed solvent at $1^{\circ} \mathrm{C}$ and $0.1 \mathrm{M} \mathrm{NaCl}$ has been quantified. The results are summarized in Table 3.28. The complex stability constants in the Triton X-114/Water system are all affected by the presence of Triton X-114. In the case of europium hydrolysis and europium 8- 
hydroxyquinoline complexation, the formation of these species is favored in the presence of Triton. The formation of mixed 8-hydroxyquinoline/hydrolysis species defied this trend showing no change between the water and the Triton/water mixed solvent. 8-Hydroxyquinoline protonation behavior showed divergent trends in its $\mathrm{pK}_{\mathrm{a}}$ values. The aromatic nitrogen group and the phenolic oxygen group showing a decrease and increase in $\mathrm{pK}_{\mathrm{a}}$ in the Triton/water system, respectively. 


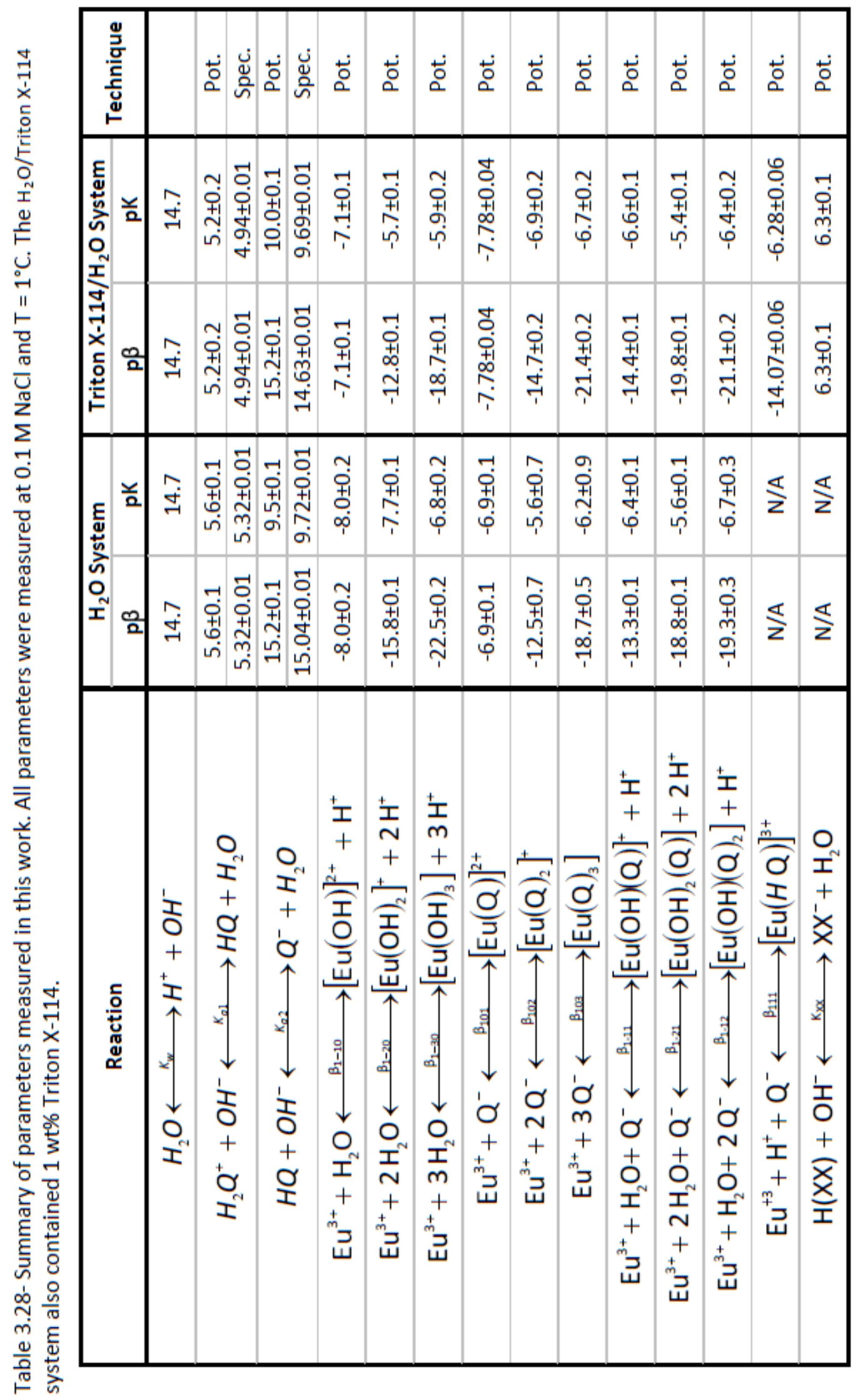




\section{CHAPTER 4}

\section{CONSLUSIONS}

\subsection{Conclusions Concerning the TALSPEAK Study}

The conditional ionization constant $\left(\mathrm{pK}_{\mathrm{a}}\right)$ of lactic acid at I $=2.0 \mathrm{M} \mathrm{NaCl}$ was quantified over the temperature range of $25-65^{\circ} \mathrm{C}$. The results show very little change with temperature and a relatively small standard enthalpy of reaction. This work is of importance to the development of the TALSPEAK extraction model, specifically providing information that is useful for modeling the aqueous phase activity coefficients for lactate media solutions. Expanding this work to other ionic strengths will provide a more complete data set that will enable modeling of aqueous activity coefficients for lactate solutions in the TALSPEAK system.

Additional studies described in this work explored speciation of $\mathrm{Nd}^{3+}$ in the organic phase, including aggregation of $\mathrm{HA}$ under a range of conditions. Extractions of $\mathrm{Nd}^{3+}$ by varied concentration $\mathrm{HA}(0-1 \mathrm{M})$ from $\mathrm{HCl}$ media at both $\mathrm{pH} 1$ and 4 were compared with control samples without $\mathrm{Nd}^{3+}$ that were also at $\mathrm{pH} 1$ and 4 . A second set of samples measured the extraction of $\mathrm{Nd}^{3+}$ over the same $\mathrm{HA}$ concentrations from $1 \mathrm{M}$ lactate media at $\mathrm{pH} 1$ and 4 . The initial concentration of $\mathrm{Nd}^{3+}$ was $30 \mathrm{mM}$ for both $\mathrm{HCl}$ and lactate experiments; one additional experiment in lactate medium at $60 \mathrm{mM}$ was also performed. The lactate series of extractions was also compared with non- $\mathrm{Nd}^{3+}$ control experiments at $\mathrm{pH} 1$ and 4.

In the non- $\mathrm{Nd}^{3+}$ control samples for the $\mathrm{HCl}$ system [i.e. $\mathrm{pH}(1,4)+0 \mathrm{mM} \mathrm{Nd}+\mathrm{HCl}$ ], and the $\mathrm{pH} 1$ lactate system [pH $1+0 \mathrm{mM} \mathrm{Nd}+\mathrm{Lac}]$ an average aggregation number of 2 was observed, indicating that HA existed predominantly as a dimer. In the corresponding $\mathrm{Nd}^{3+}$ containing systems [i.e. $\mathrm{pH}(1,4)+30 \mathrm{mM} \mathrm{Nd}+\mathrm{HCl} ; \mathrm{pH} 1+30 \mathrm{mM} \mathrm{Nd}+$ Lac] there was an increase in aggregation that was consistent with the mechanism for $\mathrm{Nd}^{3+}$ extraction by $\mathrm{HA}$. Buffering in the $\mathrm{pH} 4$ lactate systems led to high loading and the formation of large $\mathrm{HA}$ aggregates. In the $\mathrm{pH} 4+$ 
$0 \mathrm{mM} \mathrm{Nd}+$ Lac system the average aggregation was $3.82 \pm 0.05$ which when modeled, showed the formation of $\mathrm{NaA}(\mathrm{HA})$ and $\mathrm{NaLac}(\mathrm{HA})_{z}$ with $\mathrm{Z}=5.1 \pm 0.3$. The average aggregation in the $\mathrm{pH}$ $4+(30,60) \mathrm{mM} \mathrm{Nd}+$ Lac systems showed formation of high order aggregates at low $[\mathrm{HA}]_{\text {org }}$ with an average aggregation as high as 100 observed. These high order aggregates diminished with increasing $[\mathrm{HA}]_{\text {org }}$ until a constant aggregation was reached that corresponded with the lowloading extraction mechanism of $\mathrm{Nd}^{3+}$ by $\mathrm{HA}$.

The $\mathrm{Nd}^{3+}$ distribution results were consistent with the extraction mechanism for $\mathrm{Nd}^{3+}$ by $\mathrm{HA}$ with the HA dimer stoichiometric coefficient $(n)=4$ when $\mathrm{Nd}^{3+}$ loading of HA was moderate to low. Modeling of these systems by SXLSQI gave an equilibrium constant or Log $\mathrm{K}_{\mathrm{Nd}}=-0.14 \pm$ 0.06. In the $\mathrm{pH} 4+(30,60) \mathrm{mM} \mathrm{Nd}+$ Lac systems where $\mathrm{Nd}^{3+}$ loading of HA was high, $\mathrm{Nd}^{3+}$ saturated the organic phase at low $[\mathrm{HA}]_{\text {org }}$, and was quantitatively extracted at higher $[\mathrm{HA}]_{\text {org }}$.

The $\mathrm{Na}^{+}$extraction was suppressed in systems with $\mathrm{pH}$ of 1 , with partial $\mathrm{Nd}^{3+}$ loading [pH (1, 4) + $30 \mathrm{mM} \mathrm{Nd}+\mathrm{HCl} ; \mathrm{pH} 1+30 \mathrm{mM} \mathrm{Nd}+$ Lac systems], or where the HA was saturated with $\mathrm{Nd}^{3+}$ $\left[\mathrm{pH} 4+(30,60) \mathrm{mM} \mathrm{Nd}+\right.$ Lac systems at HA concentrations $<4 x$ total $\left.\mathrm{Nd}^{3+}\right] . \mathrm{Na}^{+}$extraction occurred in systems where the $\mathrm{pH}$ was 4 and excess $\mathrm{HA}$ existed $[\mathrm{pH} 4+0 \mathrm{mM} \mathrm{Nd}+(\mathrm{HCl}, \mathrm{Lac})$ system; $\mathrm{pH} 4+(30,60) \mathrm{mM} \mathrm{Nd}+$ Lac systems at HA concentrations $>4 x$ total $\left.\mathrm{Nd}^{3+}\right]$, in the Lac systems, $\mathrm{Na}^{+}$was extracted both as the HA sodium salt, and co-extracted with Lac. SXLSQI modeling of the $\mathrm{pH} 4+0 \mathrm{mM} \mathrm{Nd}+\mathrm{HCl}$ system gave an equilibrium constant or Log $\mathrm{K}_{\mathrm{Na}}=-0.989$ \pm 0.009 .

Lactate was found to extract in all the Lac systems in various species. At $\mathrm{pH} 1$, lactate was extracted as lactic acid, and possibly as $\mathrm{NdLac}^{2+}$ salt in the presence of $\mathrm{Nd}^{3+}$. In the $\mathrm{pH} 4+0 \mathrm{mM}$ $\mathrm{Nd}+$ Lac system, and $\mathrm{pH} 4+(30,60) \mathrm{mM} \mathrm{Nd}+$ Lac systems at HA concentrations $>4 x$ total $\mathrm{Nd}^{3+}$, Lac was co-extracted with $\mathrm{Na}^{+}$. In the $\mathrm{pH} 4+(30,60) \mathrm{mM} \mathrm{Nd}+$ Lac systems at HA concentrations $<4 x$ total $\mathrm{Nd}^{3+}$ Lac was co-extracted with $\mathrm{Nd}^{3+}$ as $\mathrm{NdLac}^{2+}$. 
Water was found in the organic phase in all systems. In all systems but the $\mathrm{pH} 4+(0,30,60)$ $\mathrm{mM} \mathrm{Nd}+$ Lac systems the organic phase under low loading retained the levels of water found in the washed solvent within experimental error. In the $\mathrm{pH} 4+0 \mathrm{mM} \mathrm{Nd}+\mathrm{HCl}$ system where low $\mathrm{Na}^{+}$loading was observed, a slight increase in the water concentration was observed. In the $\mathrm{pH}$ $4+(0,30,60) \mathrm{mM} \mathrm{Nd}+$ Lac systems, water extraction was observed to increase with $\mathrm{NdLac}^{2+}$ extraction, and increased even more with $\mathrm{Na}^{+}$extraction at higher $\mathrm{HA}$ organic phase concentrations. Correlations of organic phase $\mathrm{H}_{2} \mathrm{O}$ concentration with $\mathrm{Nd}^{3+}$ in the $\mathrm{NdLac}^{2+}$ extraction regime indicates a 1:1 stoichiometry of water with $\mathrm{Nd}^{3+}$. Correlations of organic phase $\mathrm{H}_{2} \mathrm{O}$ concentration with $\mathrm{Na}^{+}$when present in the $\mathrm{pH} 4+(0,30,60) \mathrm{mM} \mathrm{Nd}+$ Lac systems indicates a 3:1 stoichiometry of water with $\mathrm{Na}^{+}$or a hydration number of 3 for $\mathrm{Na}^{+}$.

Organic phase visible spectroscopy in the $\mathrm{pH}(1,4)+30 \mathrm{mM} \mathrm{Nd}+\mathrm{HCl}$ and $\mathrm{pH} 1+30 \mathrm{mM} \mathrm{Nd}$ + Lac systems indicate that the molar absorptivity of $\mathrm{Nd}^{3+}$ does not depend on $\mathrm{pH}$ or $\mathrm{Nd}^{3+}$ loading of the organic phase, but rather on the organic phase HA concentration. The similar spectra in the $\mathrm{pH}(1,4)+30 \mathrm{mM} \mathrm{Nd}+\mathrm{HCl}$ systems indicate that the extraction mechanism is unchanged from $\mathrm{pH} 1$ to $\mathrm{pH} 4$ in $\mathrm{HCl}$ media at $[\mathrm{HA}]_{\mathrm{org}}>0.05 \mathrm{M}$. Changes in the organic phase visible spectra under the high loading conditions support the formation of $\mathrm{Nd}(\mathrm{Lac})(\mathrm{HA})_{2}$. FTIR results support the ion exchange mechanism at low to moderate $\mathrm{Nd}^{3+}$ loading, and the extraction of $\mathrm{NdLac}^{2+}$ and high HA loading, including the formation of high order aggregates with HA participating in phosphate bridging in the organic phase.

The overall speciation results (see Table 2.21 for summary of species and conditions where they were identified) indicate that at high $\mathrm{Nd}^{3+}$ loading in the $\mathrm{pH} 4+$ Lac systems, $\mathrm{Nd}^{3+}$ exists as a highly aggregated, $\left(\mathrm{NdLacA}_{2}\right)_{\times}$species in the organic phase. As loading is decreased (i.e. HA increased), the fraction of $\mathrm{Nd}^{3+}$ in the $\left(\mathrm{NdLacA}_{2}\right)_{\times}$species decreases, while the fraction in the mono-nuclear $\mathrm{NdA}_{3}(\mathrm{HA})$ species increases until a complete conversion to $\mathrm{NdA}_{3}(\mathrm{HA})$ is achieved. 
Other species identified with the conditions where they were observed is shown in Table 2.21).

Table 4.1-Summary of species identified in the systems studied with the conditions where they were observed.

\begin{tabular}{|c|c|c|}
\hline Species & System & $\begin{array}{c}\text { Organic Phase Condition } \\
\text { (M HA) }\end{array}$ \\
\hline $\mathrm{H}(\mathrm{Lac})$ & $\mathrm{pH} 1+0 \mathrm{mM} \mathrm{Nd}+\mathrm{Lac}$ & $0.025-1.0$ \\
\hline $\mathrm{Na}(\mathrm{A})$ & $\mathrm{pH} 4+0 \mathrm{mM} \mathrm{Nd}+\mathrm{HCl}$ & $0.05-1.0$ \\
\hline $\mathrm{Nd}(\mathrm{A})_{3}(\mathrm{HA})$ & $\begin{array}{l}\mathrm{pH} 1+30 \mathrm{mM} \mathrm{Nd}+\mathrm{HCl} \\
\mathrm{pH} 4+30 \mathrm{mM} \mathrm{Nd}+\mathrm{HCl} \\
\mathrm{pH} 4+30 \mathrm{mM} \mathrm{Nd}+\mathrm{Lac} \\
\mathrm{pH} 4+60 \mathrm{mM} \mathrm{Nd}+\mathrm{Lac}\end{array}$ & $\begin{array}{c}0.05-1.0 \\
0.05-1.0 \\
0.15-1.0 \\
0.3-1.0\end{array}$ \\
\hline $\begin{array}{c}{\left[\mathrm{Nd}(\operatorname{Lac})(\mathrm{A})_{2}\right]} \\
x \\
(x=2-50)\end{array}$ & $\begin{array}{l}\mathrm{pH} 4+30 \mathrm{mM} \mathrm{Nd}+\mathrm{Lac} \\
\mathrm{pH} 4+60 \mathrm{mM} \mathrm{Nd}+\mathrm{Lac}\end{array}$ & $\begin{array}{l}0.025-0.15 \\
0.025-0.3\end{array}$ \\
\hline $\begin{array}{c}\mathrm{Na}(\mathrm{Lac})+ \\
\mathrm{Na}(\mathrm{A})\end{array}$ & $\begin{array}{l}\mathrm{pH} 4+0 \mathrm{mM} \mathrm{Nd}+\text { Lac } \\
\mathrm{pH} 4+30 \mathrm{mM} \mathrm{Nd}+\mathrm{Lac} \\
\mathrm{pH} 4+60 \mathrm{mM} \mathrm{Nd}+\text { Lac }\end{array}$ & $\begin{array}{c}0.05-1.0 \\
0.15-1.0 \\
0.3-1.0\end{array}$ \\
\hline
\end{tabular}

The initial NMR results obtained through the scoping experiment indicate that the ${ }^{13} \mathrm{C}$ and

${ }^{31} \mathrm{P}$ spectra obtained from the analogous $\mathrm{pH} 4+(30,60) \mathrm{mM} \mathrm{La}^{3+}+$ Lac systems are sensitive to the speciation changes in the system and more work with these methods is warranted. A complete interpretation of this system was not undertaken.

\subsection{Recommendations for Future Research: TALSPEAK}

A full characterization of the TALSPEAK extraction behavior was not quite realized in this work. A characterization of the chloride extraction is necessary to resolve whether chloride coextraction is occurring. This can be done using $a^{36} \mathrm{Cl}$ radioactive tracer study using liquid scintillation counting. The full set of NMR data must be collected and interpreted to understand $\mathrm{HA}$, lactate, $\mathrm{Ln}^{3+}$ and $\mathrm{Na}^{+}$speciation at high and low loading as well as through the transition.

The method for this experiment was shown to work in the previous studies for ${ }^{13} \mathrm{C},{ }^{31} \mathrm{P}$, and ${ }^{23} \mathrm{Na}$ 
nuclei but must be extended to ${ }^{139} \mathrm{La}$. The inclusion of DTPA is not anticipated to change the organic phase speciation of the system, but this assumption must be rigorously tested before accepted. This can be done by adding 0.05 M DTPA to the previously studied lactate system contact experiments and characterizing by VPO. If VPO measurements in the presence and absence of DTPA are consistent, then the influence of DTPA can be assumed to be negligible. The formation of DTPA neodymium complexes in the aqueous phase will need to be accounted for in the results, because DTPA is known to complex lanthanides strongly at $\mathrm{pH}$ greater than 2.5.

Characterization of the TALSPEAK system generated some unanswered questions that could be investigated. In the lactate distribution experiments, there was an increase in lactate distributions at $\mathrm{pH} 1$ when neodymium was present than when it was absent. The lactate extraction exactly doubled under the conditions of the experiments in this work. It was not possible to conclude if the extra lactate extracted in the presence of neodymium was due to coextraction with $\mathrm{Nd}$ as $[\mathrm{Nd}(\operatorname{Lac})]^{2+}$ as it was at $\mathrm{pH} 4$, or if the extra lactate was lactic acid that was extracted due to a more favorable solvation environment in the organic phase. This could be tested by performing other extractions at $\mathrm{pH} 1$ in lactate, but with other concentrations of neodymium such as 15 and $45 \mathrm{mM}$. It is anticipated that the pattern in the excess lactate extraction would reveal more about the species present.

Another question in the TALSPEAK characterization experiments was in the water distribution results. In the $\mathrm{HCl}$ media experiments, and the lactate media at $\mathrm{pH} 1$ there was no excess water extracted with neodymium in the organic phase. This was consistent with literature results for europium extraction under similar conditions. However, at high loading, one water per neodymium and per lactate was observed in the organic phase. It was never clear if the water in the organic phase was present in the inner coordination sphere of neodymium or 
if it was associated with the lactate only. This question could be answered by performing Time Resolved Laser Fluorescence Spectroscopy (TRLFS) experiments using europium or terbium in place of neodymium at a single high loading condition. By increasing the percent of deuterated water in the aqueous phase through a series of extractions at the same loading condition, the change in the fluorescence lifetime of the europium would indicate if the water was in the inner coordination sphere of the extracted lanthanide. If there was no change in fluorescence lifetime of europium as a function of percent deuterated water in the aqueous phase, then the extracted under the high loading conditions is associated with lactate and is not in the inner coordination sphere of the metal.

Once the TALSPEAK systems are fully characterized, extraction modeling is needed to understand the equilibrium constants that describe the system. This modeling has already been performed for the systems involving extraction from the $\mathrm{HCl}$ media because pitzer parameters were available to model the activities of the species in the aqueous phase. There are no pitzer parameters available for solutions containing mixtures of lanthanides, lactate and sodium. These parameters must be quantified before the extraction modeling in the lactate media can be performed. The characterization of the $\mathrm{pK}_{\mathrm{a}}$ of lactate at various ionic strengths and temperatures is a good beginning. The technique described in this work is effective for a quantitative analysis.

In addition to the $\mathrm{pK}_{\mathrm{a}}$ of lactate, the activity of water, lactate and neodymium must be quantified. This can be done through the measurement of the water activity of solutions of these constituents alone and mixed together in a systematic series. By modeling the change in water activity as a function of the concentrations of each constituent, the thermodynamic parameters used to predict the activity of the constituents can be obtained. 


\subsection{Conclusions Concerning the Europium 8-Hydroxyquinoline Work}

The speciation of 8-hydroxyquinoline and europium in both water and the Triton X$114 /$ water mixed solvent at $1^{\circ} \mathrm{C}$ and $0.1 \mathrm{M} \mathrm{NaCl}$ has been quantified. The complex stability constants in the Triton X-114/Water system are all affected by the presence of Triton X-114. In the case of europium hydrolysis and europium 8-hydroxyquinoline complexation, the formation of these species is favored in the presence of Triton. The formation of mixed 8hydroxyquinoline/hydrolysis species defied this trend showing no change between the water and the Triton/water mixed solvent. The 8-hydroxyquinoline protonation behavior showed divergent trends in its $\mathrm{pK}_{\mathrm{a}}$ values. The aromatic nitrogen group and the phenolic oxygen group showing a decrease and increase in $\mathrm{pK}_{\mathrm{a}}$ in the Triton/water system, respectively.

\subsection{Recommendations for Future Research: Europium 8-Hydroxyquinoline}

The work involving europium 8-hydroxyquinoline complexation is promising, but needs additional effort to be complete. Because cloud point extraction relies on a separation technique involving a temperature change, the complexation parameters of the system must be measured under different conditions so the system behavior is understood. A similar study of the behavior of europium and 8-hydroxyquinoline as was present in this work should be repeated using the Triton $\mathrm{X}-100$ surfactant that is very similar to Triton X-114 but has a higher cloud point temperature of $60^{\circ} \mathrm{C}$. This will allow potentiometric determination of system behavior at higher temperatures. The issue of the protolytic impurity must also be resolved. Efforts to eliminate carbon dioxide absorption during titration, such as utilizing an argon gas purge, may resolve this issue if the impurity is carbonate absorbed from the atmosphere. If efforts to eliminate carbon dioxide prove unsuccessful, then performing a purification of the Triton, or obtaining a higher purity material may solve the problem. 
Measuring the stability constants for actinides in this system by the potentiometric technique employed in this study is problematic due to the high specific activity of the trivalent actinides. An alternative would be to use TRLFS to measure the stability constants of curium. Curium has been shown to fluoresce, allowing for its study by TRLFS at very low concentrations.

Cloud point extraction data comparing the separation of the lanthanides and the actinides must be collected if the trivalent actinide trivalent lanthanide separation potential is to be understood. This can be done by tracer study using europium, americium or curium and measuring the distribution in cloud point extraction experiments. By keeping the concentration of the metal much less than the ligand (less than $10 \%$ of theoretical loading), log-slope data can be analyzed for mechanistic information. Separation factors can also be calculated from the distribution ratios.

With the complexation constants and the extraction data, the separation of the trivalent lanthanides from the trivalent actinides can be modeled. Modeled data would be compared with extraction data to ensure a good fit. Modeling of the system can show if the optimum separation condition was observed during experimental separations, or if a different condition should be re-tested for optimum separation.

One unanswered question in the europium 8-hydroquinoline complexation work is why the mixed hydrolysis 8-hydroxyquinoline species formation constants were not changed in the presence of Triton. An initial effort to study this question would be to try and crystallize the mixed hydrolysis 8-hydroxyquinoline species from the water system and perform powder or single crystal X-ray diffraction measurements to work out the complex's coordination chemistry. While crystallized compounds are not always the same as the species in solution, they may offer clues as to why the surfactant did not affect their formation in solution. 


\section{APPENDIX 1 \\ Instructions and Description of the Use of SQUAD}

\section{A1.1 General Description}

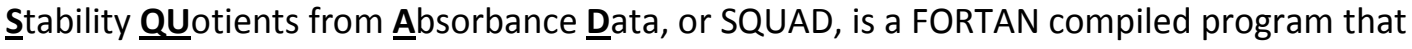
uses user-supplied absorbance spectra, metal and ligand concentrations, and $\mathrm{pH}$ to calculate complexation stability constants. The program limitations depend on the compiled program and differ slightly for different compilations. In the current manual, the compilation used is entitled "sqd5.exe" and has the following limits: spectra are limited to 140 data points, up to 35 spectra per run, 10 modeled complexes, with 6 varied complexation constants allowed. It is expected that much of the information provided herein will apply to other SQUAD compilations that have different limitations. The limitations are described in accompanying "Readme" type files, or can be discovered by the user through trial and error.

\section{A1.2 Data Preparation}

Before SQUAD can model absorbance data an appropriate input file must be created. Most spectrometers today have a resolution of less than $1 \mathrm{~nm}$, which means that, given the 140 data points per spectrum limitation, only a small window of the spectrum can be fit. An alternative is to use a wider window of the spectrum by only using every $2^{\text {nd }}, 3^{\text {rd }}$ or $n^{\text {th }}$ data point. A spreadsheet program such as Excel can be programmed to provide these truncated spectra. To provide an example, the titration of 8-hydroxyquinoline $(\mathrm{HQ})$ by $\mathrm{NaOH}$ will be used to demonstrate the data preparation process. The following spectra were obtained at $0^{\circ} \mathrm{C}$ (see Figure A1.1 and Figure A1.2). 


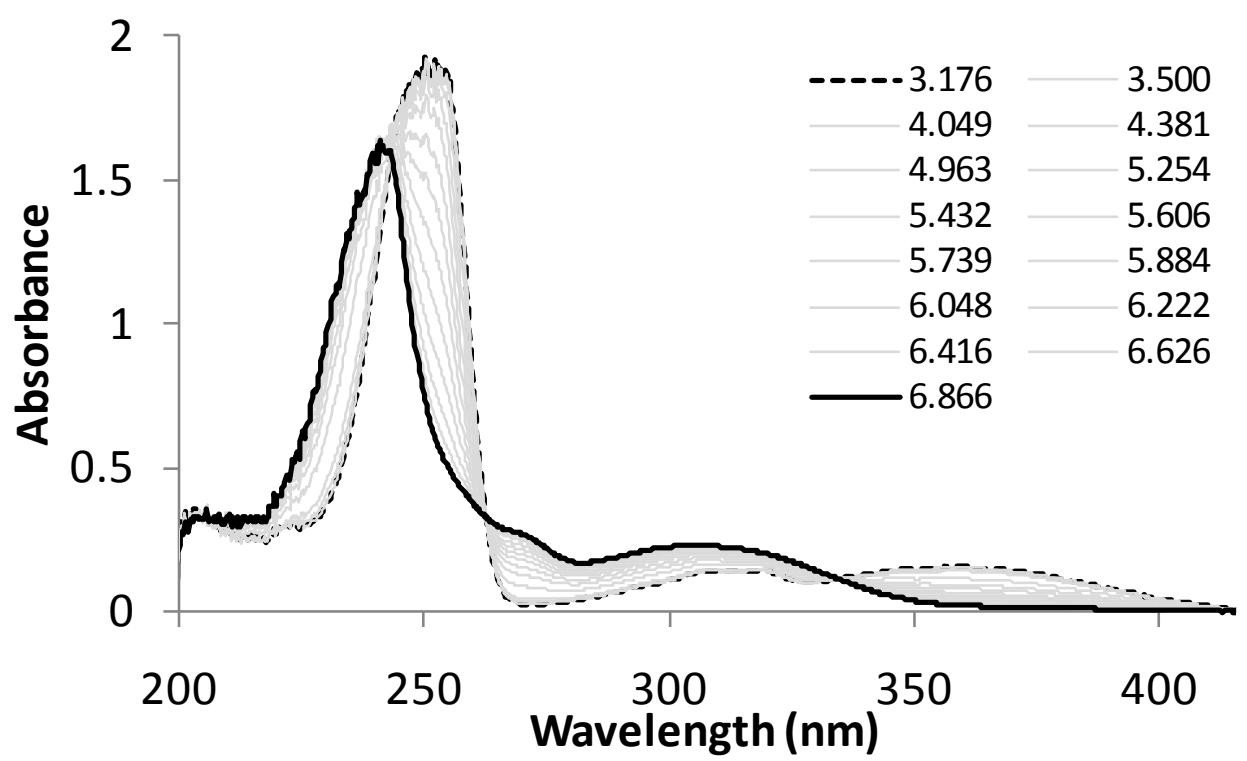

Figure $\mathrm{A} 1.1-\mathrm{pH}$ titration of $\mathrm{HQ}$ from $\mathrm{pH} 3.18$ to 6.87 at $\mathrm{T}=0^{\circ} \mathrm{C}$.

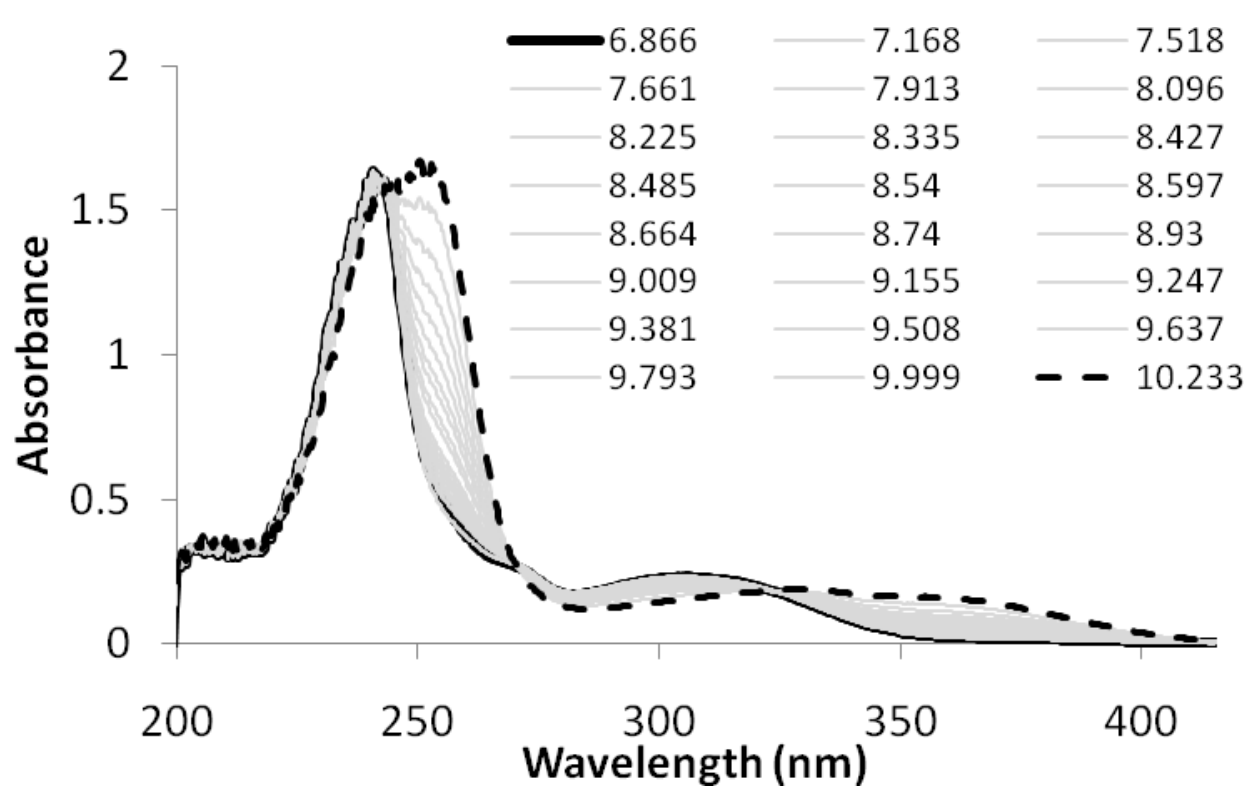

Figure A1.2- $\mathrm{pH}$ titration of $\mathrm{HQ}$ from $\mathrm{pH} 6.87$ to 10.23 at $\mathrm{T}=0^{\circ} \mathrm{C}$.

The HQ titration spectrum is from $220-445 \mathrm{~nm}$. The average resolution for these spectra is $0.27 \mathrm{~nm}$ for a total of 883 data points. Since this exceeds the data limit for SQUAD, a 
spreadsheet was programmed to use only a 1 out of every $n$ spectra for a total of 140 data points. A screen shot shows the spreadsheet parameters used in preparing the data.

\begin{tabular}{|c|c|c|c|c|c|c|c|c|c|}
\hline 4 & A & B & C & D & $E$ & $\mathrm{~F}$ & G & $\mathrm{H}$ & \\
\hline 1 & & Start $\lambda$ & Avg Step & Spec jump & & & 0 & 0 & \\
\hline 2 & & 220 & 1.625 & 6 & & & 3.176 & 3.5 & \\
\hline 3 & & Start $\lambda$ & Final $\lambda$ & total pts & & & 0 & 0.0 & \\
\hline 4 & & 219.77 & 445.69 & \begin{tabular}{r|}
140 \\
\end{tabular} & 0 & 0.0 & 0.00000000 & 0.00000000 & 0.01 \\
\hline 5 & & & & & [8-HQ] & 0.0 & 0.00009942 & 0.00009889 & 0.01 \\
\hline 6 & & 2 & & 3 & 4 & 5 & 6 & 7 & \\
\hline 7 & \# & Spec \# & Sep $(n m)$ & WL (nm) & & \begin{tabular}{|l|}
1.8163 \\
\end{tabular} & 1.8163 & 1.8076 & \\
\hline 8 & & & & & & & 0.0069 & 0.0075 & \\
\hline 9 & & & & & & & & & \\
\hline 10 & 1 & 92 & & 219.77 & 92 & 0 & 0.2652 & 0.2619 & \\
\hline 11 & 2 & 98 & 1.64 & 221.41 & 98 & 0 & 0.2935 & 0.2911 & \\
\hline 12 & 3 & 104 & 1.64 & 223.05 & 104 & 0 & 0.3167 & 0.3143 & \\
\hline 13 & 4 & 110 & 1.65 & 224.7 & 110 & 0 & 0.3077 & 0.3079 & \\
\hline 14 & 5 & 116 & 1.64 & 226.34 & 116 & 0 & 0.3300 & 0.3316 & \\
\hline 15 & 6 & 122 & 1.64 & 227.98 & 122 & 0 & 0.3591 & 0.3622 & \\
\hline 16 & 7 & 128 & 1.64 & 229.62 & 128 & 0 & 0.4007 & 0.4052 & \\
\hline 17 & 8 & 134 & $1 \mathrm{fd}$ & 23126 & 134 & ก & $\cap \Delta € \Delta \Delta$ & $\cap \Delta 7 \cap 3$ & \\
\hline
\end{tabular}

Figure A1.3- Spectrum preparation spreadsheet screenshot.

As is shown in Figure A1.3, the nominal starting wavelength $(\lambda)$ was 220 , with the spreadsheet function used returning the actual value of $219.77 \mathrm{~nm}$. The "spec jump" parameter, which is the ' $n$ ' in the " 1 out of every $n$ spectra" shows that every $6^{\text {th }}$ point was used. The final $\lambda$ is $445.69 \mathrm{~nm}$ if all 140 data points are used. The spreadsheet creates a truncated spectrum where only every $6^{\text {th }}$ data point is used, and from this truncated spectrum calculates the average step between data points which is a necessary parameter to know that will be input into the SQUAD program.

The truncated spectra now need to be saved into individual files that can be accessed by the GWBASIC.EXE program. The GWBASIC.EXE program changes the orientation of a spectral file 
from a vertical column of numbers to a horizontal row with text-wrapping so that it will become a block of numbers as shown in Figure A1.4.

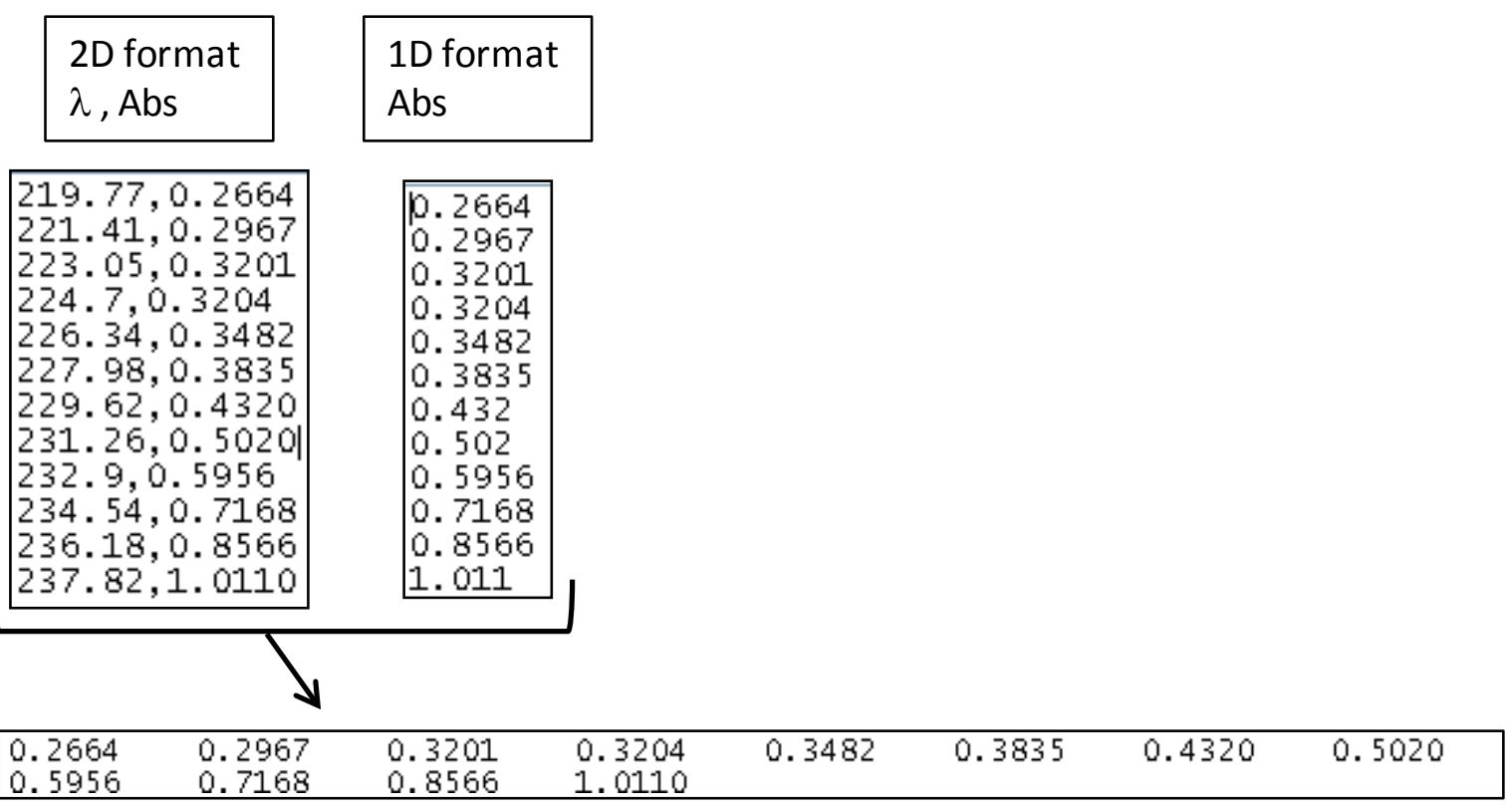

Figure A1.4- Format manipulation by "GWBASIC.EXE" on each Spectum file.

GWBASIC.EXE accepts the "comma separated values" file format or .CSV. This format can be created if the data are copied and pasted one spectrum at a time into a blank Excel spreadsheet and each file is saved as a .CSV file.

The user can create either a 1D or 2D absorbance files (see Figure A1.4). The 1D consists of one column of absorbance values only. The $2 \mathrm{D}$ file format contains the wavelength values in the first column and the absorbance values in the second column. The GWBASIC.EXE program will discard the wavelength column of data in the 2D file format. While the GWBASIC.EXE program 
does not make use of these values, the user may find this format useful if they return to these data files at a later date. After saving the spectral data files in the .CSV file format, the user must remove the .CSV file extension manually prior to manipulating or reading these files into the GWBASIC.EXE program. Once this is done the user can move on to making a header file described in the next section.

\section{A1.3 Creating a SQUAD Header File}

Once individual spectra are in the ".CSV" file format it is time to create the SQUAD input file. GWBASIC.EXE and the SQUAD-IN-BAS must be moved to the same folder as the spectral data files. In addition, a header file must be moved to this folder. Generally a header file is created by the user, or the user can alter a previously existing header file. There is no program that will generate the header file, but the "Notepad" program can be used to create or alter header and input files. ${ }^{1}$ An example header file that will be explained in detail is shown below.

\section{A1.3.1 Example of the SQUAD Header File}

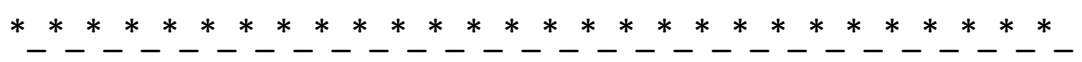

1 8-hydroxyquinoline pka fitting experiment. Wavelength range: $219 \ldots 445.0 \mathrm{~nm}$

2 System 0.1M NaCl; April 2, 2009 experiment, Dip-probe with normal pathlength $(10 \mathrm{~mm})$

3 DICTIONARY:

$4 \quad$ MTL1=Nd;LIG1=Q;PROT=H;HYDX=OH:

5 END:

6 SPECIES:

$7 \quad \mathrm{Nd}(1) \mathrm{Q}(1) ; 5.81 ; \mathrm{FB} ; \mathrm{FE}$ :

$8 \quad \mathrm{H}(1) \mathrm{Q}(1) ; 9.02 ; \mathrm{VB} ; \mathrm{VE}$ :

$9 \quad \mathrm{H}(2) \mathrm{Q}(1) ; 15.02 ; \mathrm{VB} ; \mathrm{VE}$ :

10 END:

11 OTHER:

${ }^{1}$ Column spacing can be verified in "Notepad" by activating the status bar in the "View" dropdown menu. The status bar will display the cursor position in the lower right corner. To check the correct column spacing, place the cursor at the immediate left of the character of interest. 


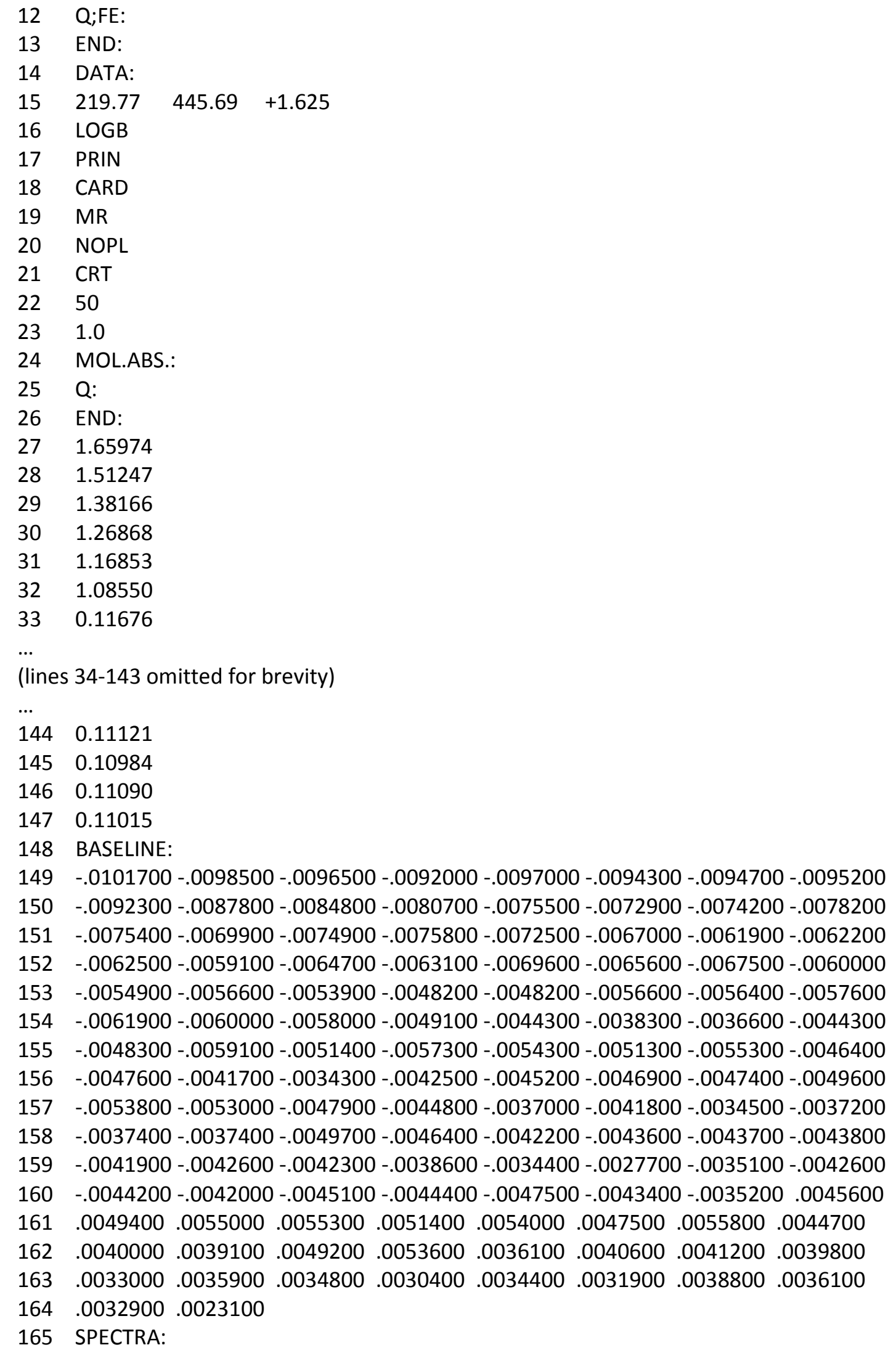




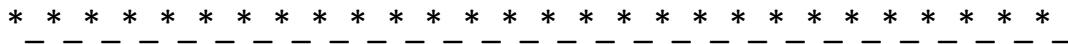

The left hand column of numbers was not a part of the original file but is included to increase the ease of referencing specific lines of the header file while describing their function.

\section{A1.3.2 Description of the SQUAD Header File Line-by-line}

A) Lines 1 and 2: Descriptive text

a) Function: To provide a description of the current experiment. It does not have any effect on the SQUAD program's operation

b) Commands and Syntax: Regular text, numbers and punctuation are used here. This section is always limited to the first two lines. No COLON is required to finish these lines.

B) Line 3: Initiation of the "Dictionary section

a) Function: This begins the dictionary section where all metal and ligands used in the model are defined by user supplied names.

b) Commands and Syntax: "DICTIONARY" followed by a COLON to finish the line.

C) Line 4: Declaration of metal and ligands used in the model

a) Function: This section assigns specific names to the metals (up to 2), ligands (up to 2), and proton and hydroxide ions that will be used in the model.

b) Commands and Syntax: SQUAD programs are CASE SENSITVE, so all commands are in capitol letters. Names supplied by user can be mixtures of upper and lower case, however. SQUAD recognizes up to 2 metals ("MTL1", "MTL2"), 2 ligands ("LIG1", "LIG2"), proton "PROT", and hydroxide "HYDX". Even if a chemical system does not contain a metal, a metal must be included in the program or SQUAD will crash. If there is no metal present in the actual system, simply set the metal concentration to an extremely low number. A concentration of $1 \mathrm{E}-19 \mathrm{M}$ has worked in this author's experience.

D) Line 5: End of "Dictionary" section

a) Function: This ends the dictionary section.

b) Commands and Syntax: "END" followed by a COLON to finish the line.

E) Line 6: Beginning of "Species" declarations

a) Function: This begins the species declaration section where the species are defined by stoichiometry, and formation constants are entered.

b) Commands and Syntax: "SPECIES" followed by a COLON to finish the line. 
F) Line 7: Species declarations

a) Function: All formation constants are mathematically defined by the user.

b) Commands and Syntax:

i) Species Formed: Using the user-defined names from the "Dictionary" section (see line 4) the species stoichiometry is defined. The stoichiometric coefficient is enclosed in parentheses (such as ' $\mathrm{H}(2) \mathrm{Q}(1)^{\prime}$ ' for the species $\left.\left[\mathrm{H}_{2} \mathrm{Q}\right]^{+}\right)$. An example of the type of expression for the species formation by SQUAD is shown in equation (1). Up to 10 species can be included in this section with 1 species per line. The species definition is followed by a semi-colon.

$$
M+2 L \stackrel{\beta_{12}}{\longrightarrow} M(1) L(2)
$$

ii) Stability constant Value or initial guess: This value is the $\log \beta$ for the expression defined in the previous step. This number is followed by a semi-colon.

iii) Fixed or Variable log $\beta$ : The two commands allowed here are "VB" for "variable log $\beta$ " or "FB" for "fixed log $\beta$ ". "Variable" refers to an unknown value that SQUAD will solve for using the user-supplied guess, whereas fixed is a known value supplied by the user. SQUAD will only allow a total of 6 species to have a variable $\log \beta$. If the guessed value is outside the program's test criteria, SQUAD will usually replace it with a better one at the initiation of the calculations. All caps must be used here or SQUAD will return an error. This command is followed by a semi-colon.

iv) Fixed or Variable Molar Absorptivity, $\varepsilon$ : SQUAD can solve for the molar absorptivity of different complexes in the system. The two commands allowed here are "FE" for Fixed Molar Absorptivity or "VE" for Variable Molar Absorptivity. If "FE" is declared here and no molar absorptivity spectrum is supplied to the program later in the header, SQUAD assumes that the species has a constant, zero molar absorptivity. All caps must be used here or SQUAD will return an error. This command is followed by a COLON because it is the end of the line.

G) Line 10: End of Species declaration section

a) Function: Ends the section where species can be declared

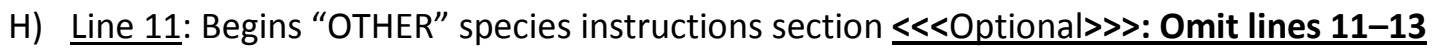
together if the "OTHER" section is not used.

a) Function: Introduces the section that allows the user to specify any free species that contain a molar absorbance.

b) Commands and Syntax: "OTHER" followed by a COLON to finish the line.

I) Line 12: Other species declaration $\leq<<O p t i o n a l \gg \gg$ : Omit lines 11-13 together if the "OTHER" section is not used. 
a) Function: Allows the user to specify which free species have a molar absorbance.

b) Commands and Syntax: As many lines can be used as there are free species that have a molar absorbance; one species per line.

i) Free Species name: Use the free species as it was defined by the user in the dictionary. This is followed by a semi-colon.

ii) Fixed or Variable Molar Absorptivity or $\varepsilon$ : The two commands allowed here are "FE" for Fixed Molar Absorptivity or "VE" for Variable Molar Absorptivity. If "FE" is declared here and no molar absorptivity spectrum is supplied to the program later in the header, SQUAD assumes that the species has a constant, zero molar absorptivity (this declaration would be useless since SQUAD assumes this if no declaration is made in the "OTHER" section. So if using the "FE" command here, make sure the spectrum is supplied later). All caps must be used here or SQUAD will return an error. This command is followed by a COLON because it is the end of the line.

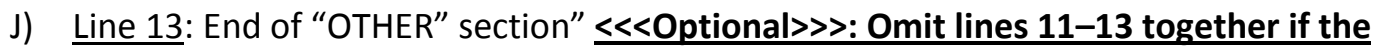
"OTHER" section is not used.

a) Function: Signals to SQUAD that there are no other free species with a molar absorbance to be declared.

b) Commands and Syntax: "END" followed by a COLON to finish the line.

K) Line 14: Beginning of the "DATA" section

a) Function: Gives instruction and clarification for modeling the data.

b) Commands and Syntax: "DATA" followed by a COLON to end the line.

L) Line 15: Wavelength declaration

a) Function: This line defines the wavelength range of the spectrum. It is not mathematically important in modeling, but is necessary for accurately displaying the SQUAD-calculated molar absorptivities. The program will refuse to run if the correct information is not supplied here. No error is displayed in the output file to indicate that the wavelength range or increment is incorrect, so this line must be checked manually.

b) Commands and Syntax:

i) Column 1= Starting wavelength: The lower bound of the spectrum wavelength. This number is in decimal format. There is NO SEMI-COLON after this number. Add spaces until column 13 is reached and then enter the next value (described below) in this line.

ii) Column 13=Ending wavelength: The lower bound of the spectrum wavelength. This number is in decimal format. Because no semi-colon is used after the previous 
number, it is important that this number's first digit be placed in column 13 by adding spaces after the previous number in this line. The column number can be checked by placing the cursor immediately to the left of the character in question and checking the status bar in the lower right-hand corner of window when using the "Notepad" program. It is not recommended to use "Word" or other word processing software for editing SQUAD input files. There is NO SEMI-COLON after this number. Add spaces until column 23 is reached and then enter the next value (described below) in this line.

iii) Column 23=Wavelength Increment Value: Because wavelengths of the absorbance values are not explicitly provided to the program, SQUAD calculates the wavelengths for each absorbance value using an increment value. The increment between each wavelength in the spectrum is written with a ' + ' leading the decimal number. The ' + ' must be positioned in the 23 column by spaces or SQUAD will not acquire the value correctly. If the (starting wavelength) $+[$ (the number of points in a spectrum) $\times$ (Wavelength Increment Value)] is not equal to the final Ending Wavelength, then SQUAD will crash, generating an incomplete output file that has no error message, so it is necessary to manually check this input line.

M) Line 16: Stability constant format options

a) Function: Instructs SQUAD what form the stability constants are supplied in the "SPECIES" section and determines the format for the calculated/refined stability constants.

b) Commands and Syntax: Allowed values here are "LOGB" or "EXPB", in all caps. These signify that the stability constants will be refined as $\log (\beta)$, or $\beta$ (i.e. $10^{\wedge}(\log \beta)$ ).

N) Line 17: Full or abbreviated display of input data in the output file

a) Function: Instructs SQUAD whether to display the full set of input data or an abbreviated version in the output file

b) Commands and Syntax: Allowed values here are "PRIN" for full input data display, or "NOPR" for the abbreviated input data display. These commands must be in all caps.

O) Line 18: Option to check initial guess values prior to calculation

a) Function: Instructs SQUAD whether to test the initial guesses provided by the user prior to calculation. This test is called a "GRID SEARCH" in the output file.

b) Commands and Syntax: Allowed values here are "CARD" if the test of the initial estimate is desired, or "NOCD" for no test of the initial estimated values. These commands must be in all caps. The "NOCD" command would only be used if the user were adamant that the program start calculations with the user supplied values. It is the experience of the author that using the "CARD" option is generally preferred.

P) Line 19: Algorithm used to calculate the molar absorbance of the variable absorbance species. 
a) Function: This instructs SQUAD whether to use a multiple regression or non-negative least squares fitting algorithm.

b) Commands and Syntax: "MR" is used for multiple regression and "NNLS" is used for nonnegative least squares. Based on the author's experiences, the algorithm has little effect on the data outcome. If the spectra, $\mathrm{pH}$ and concentrations are correct, then either algorithm is appropriate.

Q) Line 20: Instructs SQUAD whether to plot or not to plot

a) Function: This command line has no perceived function in the SQUAD program when run on modern computers. It is believed to be an artifact from an older type of system and maybe instructed a printer whether to plot the results or not. The only important thing is that one of the allowed commands must be used or the program will crash.

b) Commands and Syntax: "PLOT" or "NOPL". If omitted there will be an error and SQUAD will not run.

R) Line 21: Another artifact that must be included, but does not appear to affect the function of SQUAD

a) Function: Must be present or the program will prematurely stop and provide an error message.

b) Commands and Syntax: "PAGE" or "CRT"

S) Line 22: Maximum number of iterations

a) Function: Tells SQUAD how many interations are allowed before SQUAD assumes that no solution was reached.

b) Commands and Syntax: Positive integer value. " 50 " is the default.

T) Line 23: Activity coefficient for $\mathrm{H}^{+}$ions.

a) Function: Informs SQUAD of the activity of the $\mathrm{H}^{+}$ions in solution.

b) Commands and Syntax: This value can be any decimal value. "1.0" is the default value. When the unreasonable value of " 50.0 " was used, SQUAD did perform calculations, so this input line must be checked manually.

U) Line 24: Begins the declaration of species with a declared molar absorbance in the header

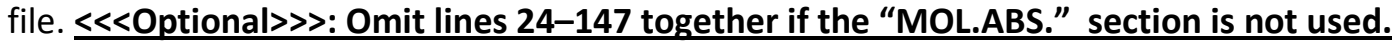

a) Function: Begins the section where species with a fixed and non-zero molar absorbance are declared.

b) Commands and Syntax: The command for this line is "MOL.ABS." and the line ends with a COLON. 
V) Line 25: Declaration of species with a fixed and non-zero molar absorbance.

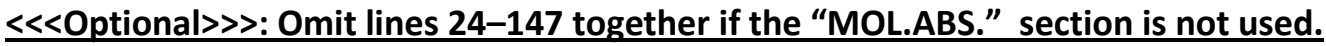

a) Function: This line tells SQUAD the column order in which the species with molar absorbances will be specified by the user.

b) Commands and Syntax: All species with a fixed and non-zero absorbance are listed separated by a semi-colon. A COLON ends the line after the last species. Any type of species, whether a free species, or one that forms a complex can be declared in this section. Species that form a complex are listed in the same syntax as in the "SPECIES" section e.g.: " $\mathrm{Q}$ " (for free hq) or " $\mathrm{H}(1) \mathrm{Q}(1)$ " [for the species $\mathrm{H}(1) \mathrm{hq}(1)$ ]. All species that are listed on this line must be declared either in the "OTHER:" or the "SPECIES" sections as having a fixed molar absorbance or "FE". Any species listed as "FE" in the "OTHER" or the "SPECIES" sections that are not listed in the "MOL.ABS." section will be assumed by SQUAD to have zero molar absorbance.

W) Line 26: End of the "MOL.ABS." section. «<<Optional >>: Omit lines 24-147 together if the "MOL.ABS." section is not used.

a) Function: This tells SQUAD that this section is complete

X) Lines 27-147: This is the fixed molar absorbance for the species declared in line 25 in this

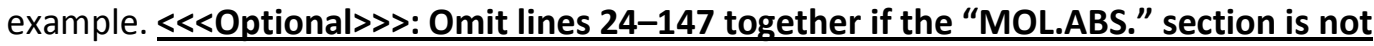
used.

a) Function: These are the actual values read in for the species listed in line 25 .

b) Commands and Syntax: In the event that more than one species is listed in the "MOL.ABS." section the molar absorbance for the listed species should be added as columns in the order that they are declared, left to right. Data columns must be aligned with the following text columns: $1,11,21,31,41,51$, etc. for as many columns are needed. No semi-colon or colons are needed to separate the values. They need only be aligned in the proper text columns to be read in properly.

Y) Line 148: Begins the declared baseline correction. $\lfloor<<$ Optional $\gg>$ : Omit lines 148-164 together if the "BASELINE" section is not used.

a) Function: If all spectra are in need of a baseline correction, this instructs SQUAD that a baseline will be provided.

b) Commands and Syntax: "BASELINE" followed by COLON to end the line.

Z) Lines 149-164: Values provided as the baseline to be applied to all the spectra. $\leq<<$ Optional $\gg>$ : Omit lines 148-164 together if the "BASELINE" section is not used.

a) Function: These are the values that represent the baseline occurring in all the spectra.

b) Commands and Syntax: This block of text is a horizontal row of absorbance data that is wrapped just as the GWBASIC.EXE program will do to the spectra files. It is 
recommended that the baseline be prepared in the same manner as the spectra files are in the GWBASIC.EXE program and pasted into either the header, or the input file. If GWBASIC.EXE is used to prepare this block of numbers, the spacing for proper read-in to the SQUAD program will be already done.

AA) Line 166: Beginning of the "SPECTRA" section

a) Function: Informs SQUAD that all necessary information has been provided, except the solution absorbance data.

b) Commands and Syntax: "SPECTRA:" followed by a COLON to end the line.

This concludes the description of the header file. It is noted that the line numbers cited in this description are not absolute values of the lines where these commands should appear, but are merely references to the header file example presented. The order of the lines is important, but the optional sections, when not needed, will change the actual line numbers where these different commands appear. NOTE: Before the header file can be used by the GWBASIC.EXE program, the file extension. TXT must be manually removed by the user.

\section{A1.4 Creating the SQUAD input file using GWBASIC.EXE while running the}

\section{$\underline{\text { SQUAD-IN.BAS instruction file }}$}

It is important to verify that all files needed by the GWBASIC.EXE program are in the same folder. The files required for the operations described in this section are: 1) all the absorbance data files saved individually in the .CSV file format, but with the .CSV file extension removed, 2) the user created header file described in the previous section saved in the .TXT file format, but with the .TXT file extension removed, 3) the GWBASIC.EXE program, and 4) the SQUAD-IN.BAS data file. Once all of this is done, the following instructions can be followed to prepare the SQUAD input file. 


\section{A1.4.1 Step-by-step instructions for using GWBASIC.EXE and SQUAD-IN.BAS to}

create a SQUAD Input File

1. Run the "GWBASIC.EXE" program by double clicking. The following screen will appear (See Figure A1.5):

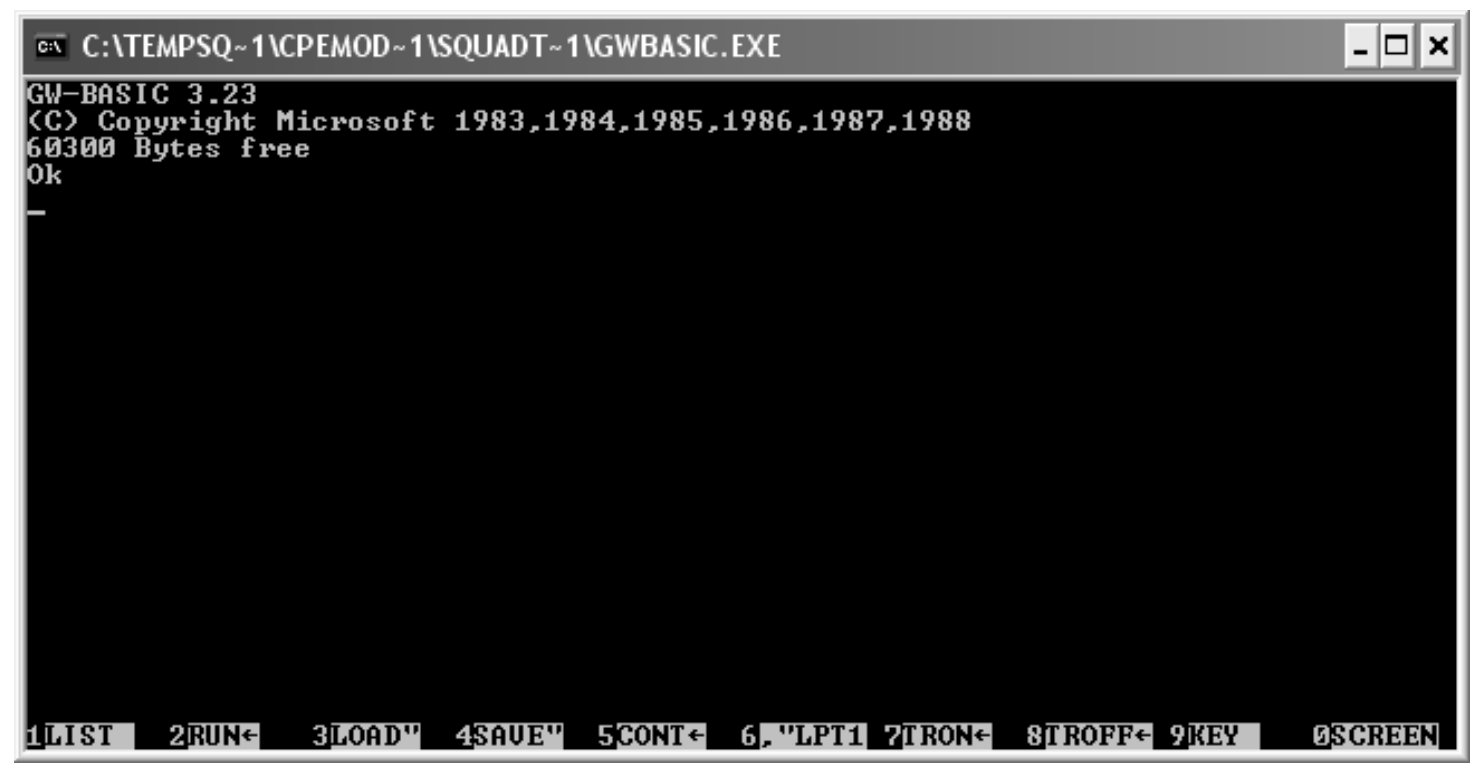

Figure A1.5-Screen shot showing the start-up of the "GWBASIC.EXE" program.

2. Press the "F3" function key which will cause the string "LOAD" to appear.

3. Next type "squad-in.bas" and press enter. "OK" will appear.

4. Press the "F2" function key to run the "squad-in.bas" program.

5. A new window will appear, prompting the user for the following information:

a. "This is going to process LUMINESCENCE data or ABSORPTION data ? (L/A) :" to which the user responds with either " $L$ " or " $A$ " depending on data type.

b. "Your data format is only INTENSITY or WAVELENGTH-VS-INTENSITY? (1D/2D) :" to which the user responds either "1D" or "2D" depending on if the user's spectrum files have 1 or 2 columns. An example and description of the 1D and 2D data formats are shown in Figure A1.4 and the accompanying text. 
c. "Please INPUT the SQUAD-INPUT filename:" to which the user types the name you want the newly generated input file to be named. If you enter the name of a file that currently exists, it will be overwritten so care must be taken with naming the SQUAD-INPUT file.

d. "Please INPUT the HEADING-FILE-NAME:" to which the user types the name of the user created header file. (See screen shot in Figure A1.6)

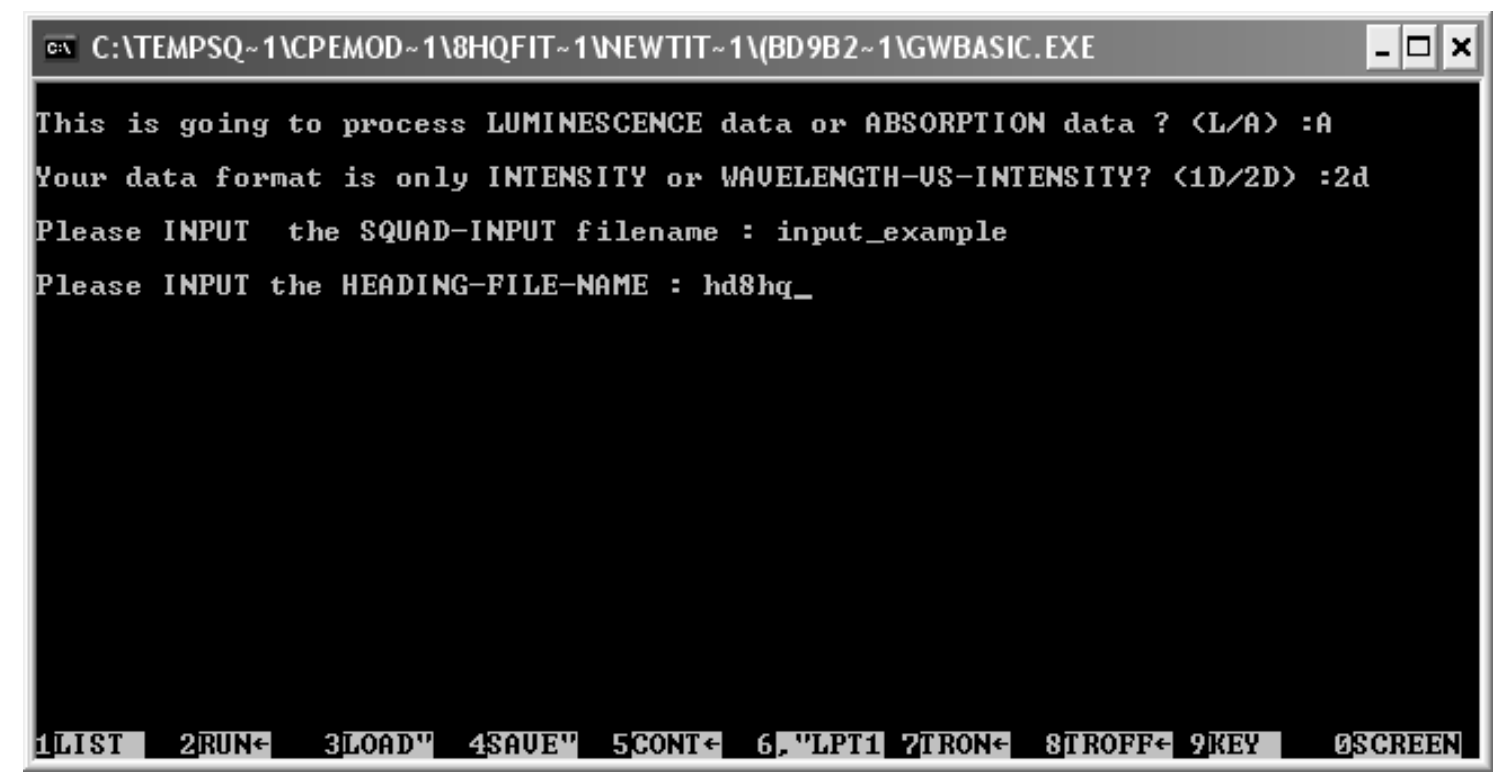

Figure A1.6- Screen shot showing the first user prompts upon starting the "SQUAD-IN.BAS" program.

a. If a HEADING-FILE-NAME is entered that does not exist in the same file folder with the GWBASIC.EXE or SQUAD-IN.BAS programs that are running then the following error will result (see Figure A1.7): 


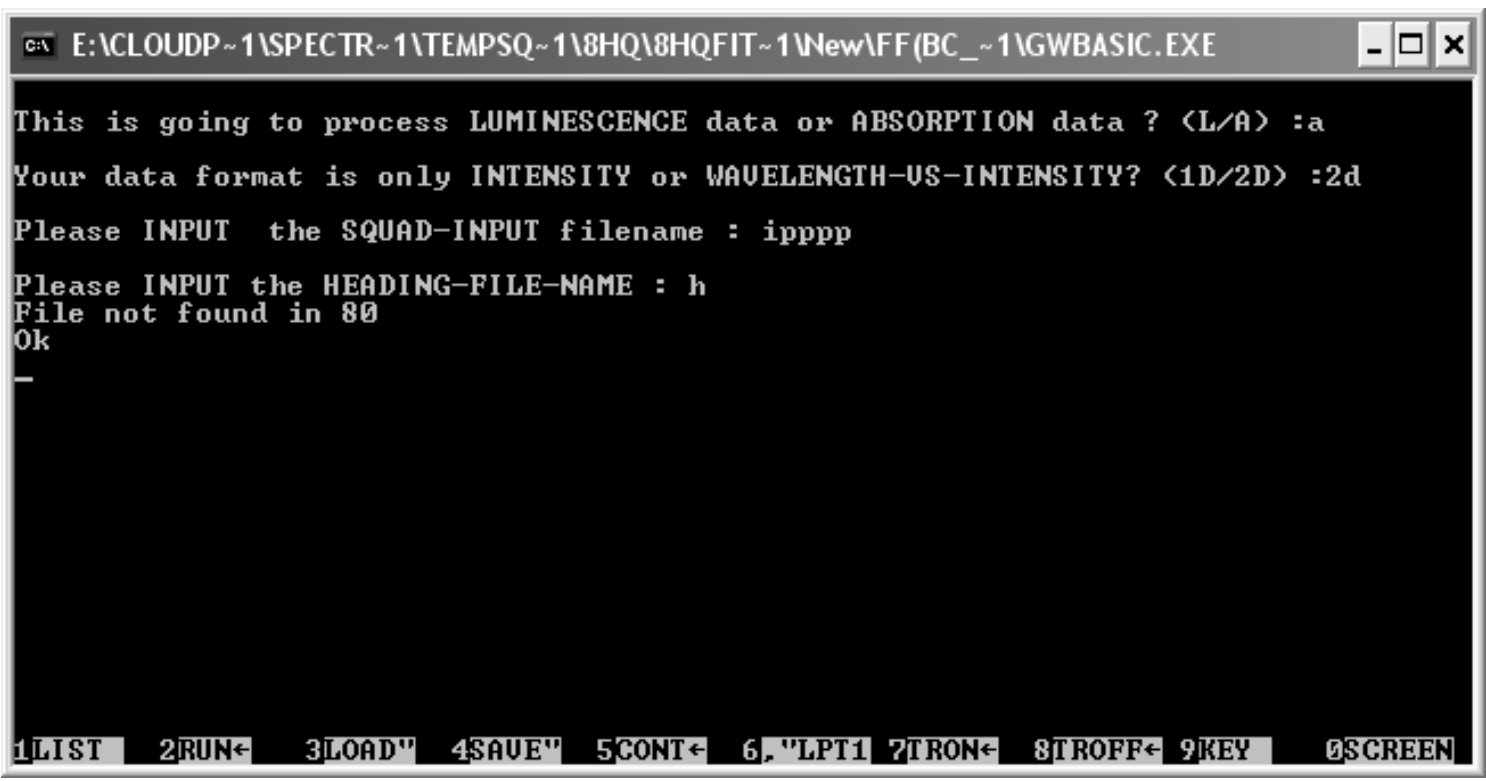

Figure A1.7- Screen shot showing the error result from entering a header file name that does not exist in the folder that contains the "SQUAD-IN.BAS" program that is running.

If this happens, the program can be restarted by pressing the ' $F 2$ ' function key and the user will be returned to step 5 . Otherwise, the program will continue to the next step.

6. Following this, a new window will display: "Now we start to INPUT and REARRANGE the data file:" and it asks the user for the following information:

a. "Please INPUT name of file \#1 :" where the user inputs the $1^{\text {st }}$ spectral data file name.

i. Note: These files must be saved in the comma delimited format or ".csv", but prior to importing these spectrum files, the file name must be altered to remove the ".csv" suffix.

b. "The conditions of solution $1<<$ Filename: "XXXX >> is:" where "XXXX" is the file name that was previously entered, and on the next line is "Concentration of METAL $1=$ " where the user enters it in and pushes enter.

i. Note: In solutions were the METAL concentration is zero, it is important to the program that there is not exactly zero concentration present so input instead a small number such as 1 e19 for zero.

c. "Concentration of LIGAND $1=$ " where the user enters the ligand concentration and pushes enter.

d. "Ph OF THIS SAMPLE =" where the user enters the $\mathrm{pH}$ and pushes enter. 
i. If the user entered the name of a spectral file (in step 6a) that does not appear in the file folder with the GWBASIC.EXE or SQUAD-IN.BAS programs that are running then the following error will result (see Figure A1.8):

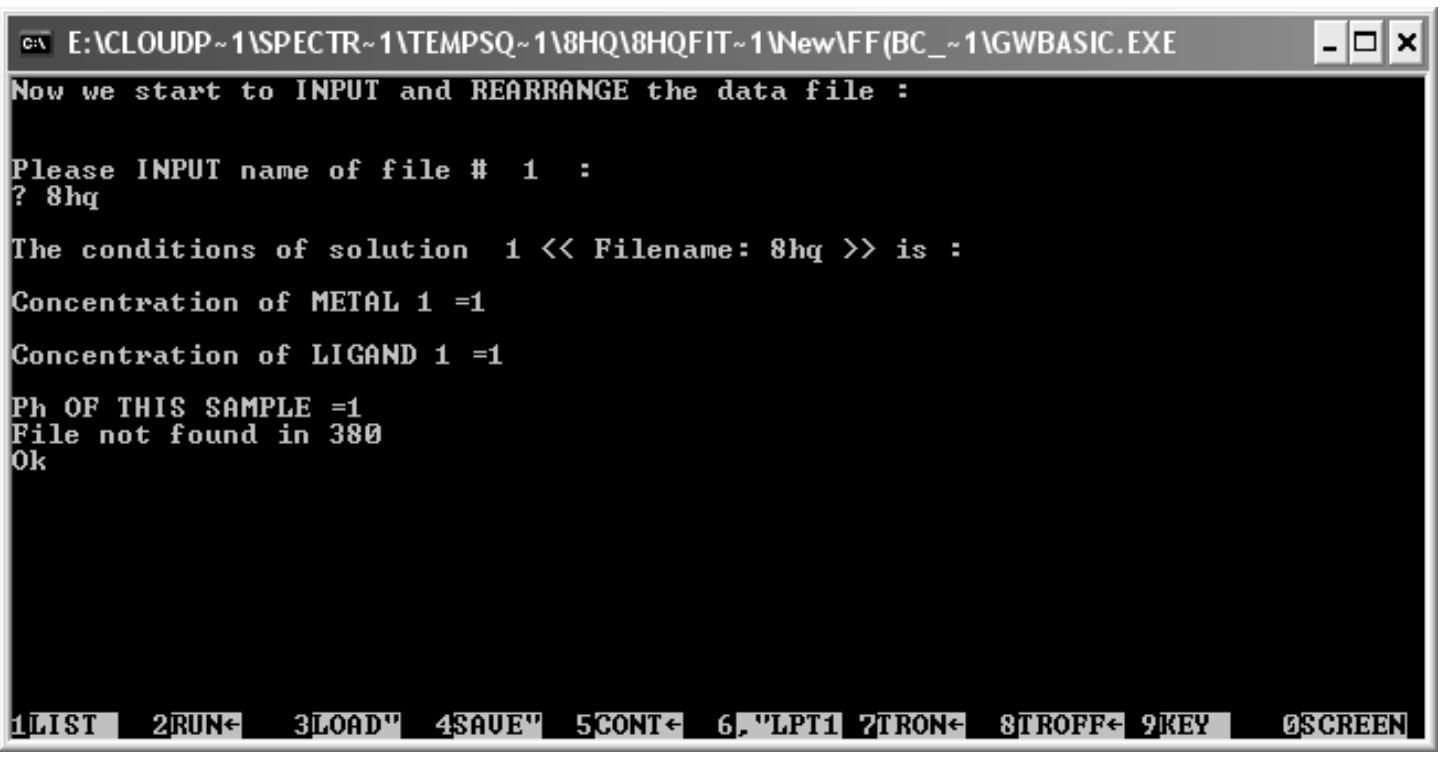

Figure A1.8- Screen shot showing the result of entering an input file name that does not exist in the folder that contains the "SQUAD-IN.BAS" program that is running.

If this happens, the program can be restarted by pressing the ' $F 2$ ' function key and the user will be returned to step 5 . Otherwise, the program will continue to the next step.

e. This is followed by "file XXXX has been input. Do you want to INPUT one more data file?" you answer either " $Y$ " or "N". (Screen shot shown in Figure A1.9): 


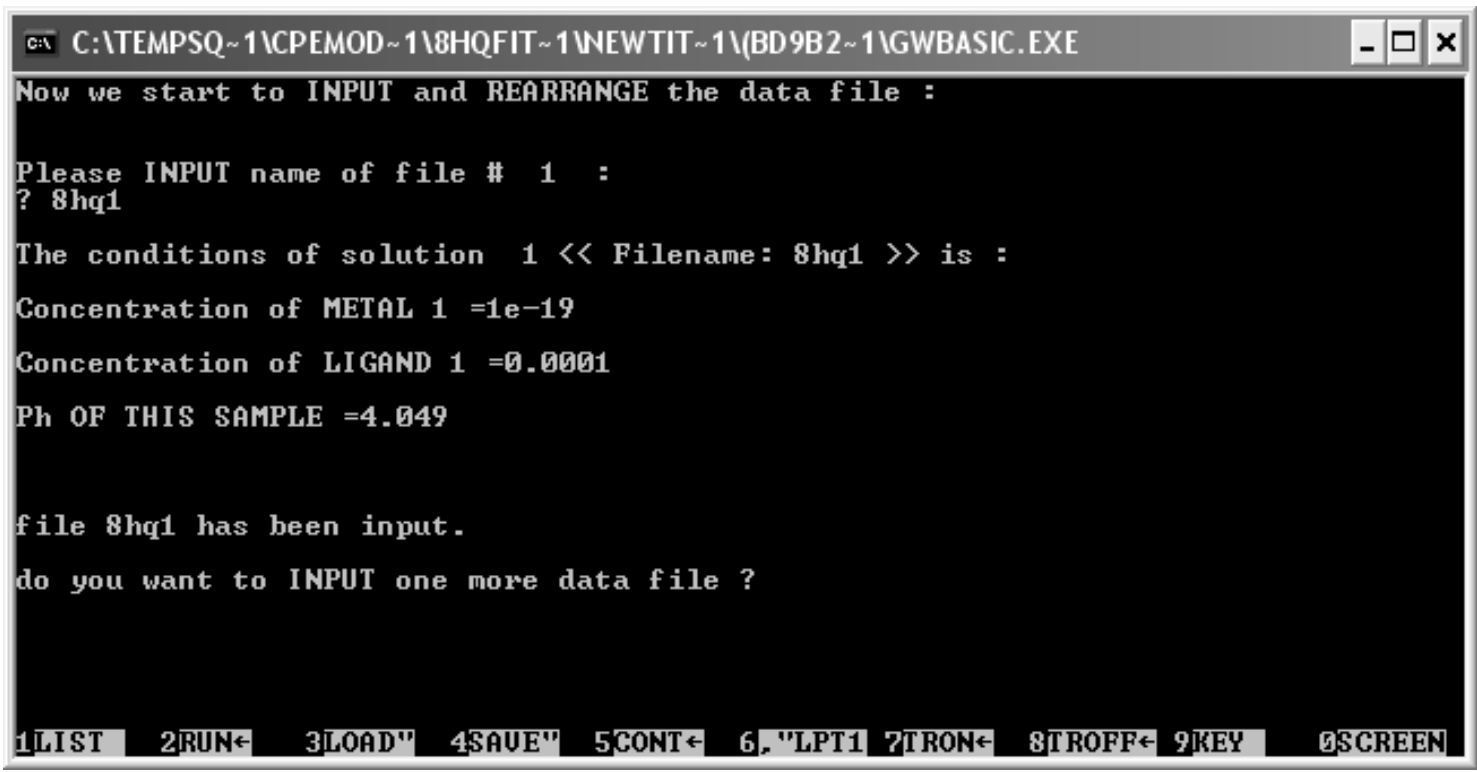

Figure A1.9- Screen shot showing the user prompt displayed upon supplying the "SQUADIN.BAS" program with the correct information for 1 spectrum file.

f. If 'YES', then steps $6(a-e)$ are repeated.

i. It has been observed that the SQUAD-IN.BAS can only be used to compile 25 spectral files. If a $26^{\text {th }}$ file is input in step 6(a) following error appears (see Figure A1.10): 


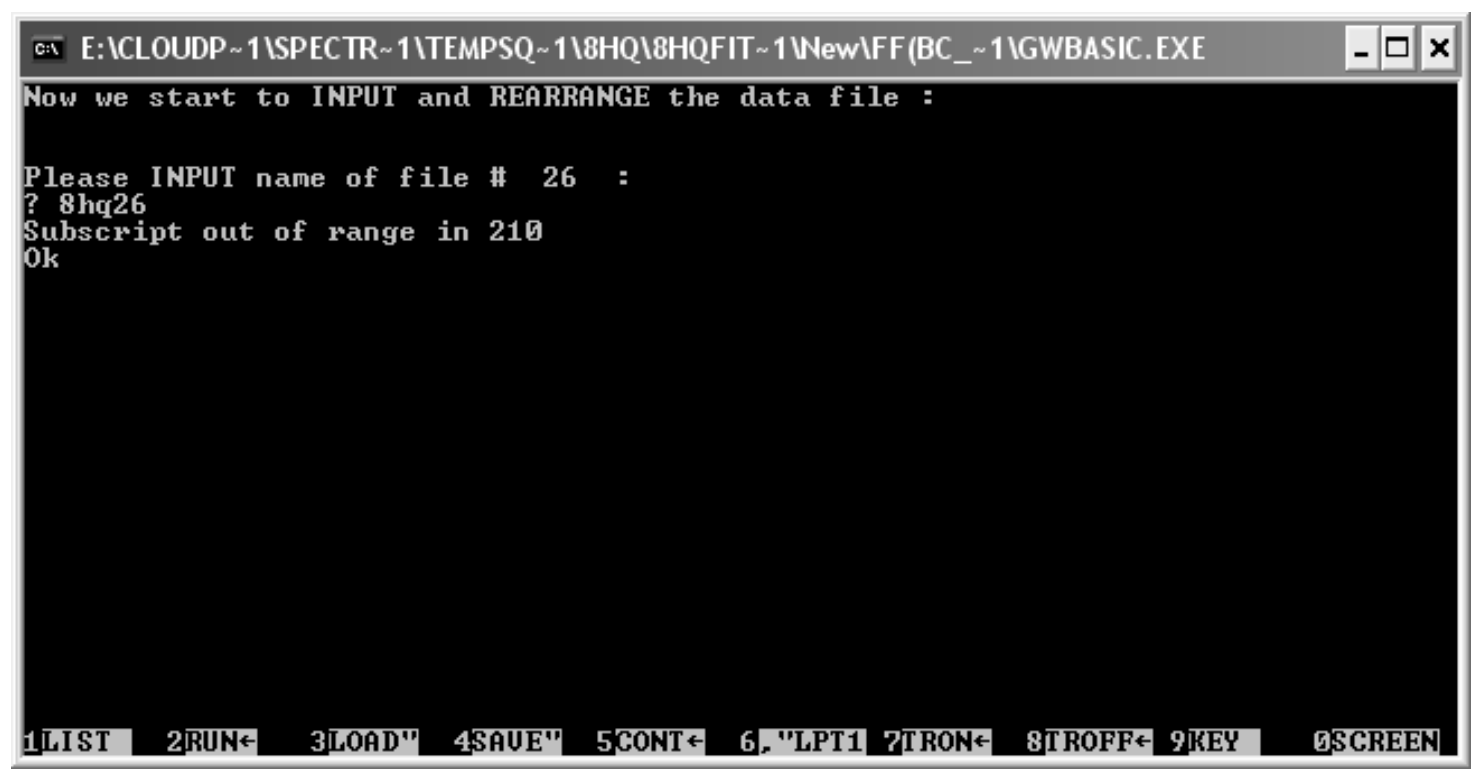

Figure A1.10-Screen shot showing the error that results following the input of the 26th consecutive spectrum file.

The SQUAD-IN.BAS program will have quit, but can be restarted by simply pressing the ' $\mathrm{F} 2$ ' function key. A second input file can be created with spectra 26 and greater by following the steps $5-a$. The two files can then be opened in "Notepad" and the spectral files from the second input file can be pasted at the bottom of the first input file.

g. If 'NO', then the following will appear: "All of the data files have been put into the $<<$ [your input file name here] $\gg$. Do you want to print out :" with the options:

$<1>$ nothing.

$<2>$ The headings only.

$<3>$ The solution conditions only.

$<4>$ The headings and solution conditions only.

$<5>$ The Whole [your input file name here].

Please make your choice $<1 / 2 / 3 / 4 / 5 />$ :" to which the user enters a number.

h. If selecting " $<1>$ Nothing" then the polite program responds with: "WE FINISHED, SIR." And "OK" 
7. This sequence has created an input file, but the SQUAD program is very particular about spacing. Correct spacing must be verified, and corrected if necessary.

The following is an example of a GWBASIC.EXE generated input file with the required spacing. The file was shortened by deleting all of the spectral data except for two spectra. A column of numbers was added to aid in referencing each line clearly in the accompanying description of the correct spacing.

A1.4.2 Example of a SQUAD Input File

1 8-hydroxyquinoline pka fitting experiment. Wavelength range: 226..272.0nm

2 System 0.1M NaCl; April 2, 2009 experiment, Dip-probe with normal pathlength $(10 \mathrm{~mm})$

3 DICTIONARY:

4 MTL1=Nd;LIG1=Q;PROT=H;HYDX=OH:

5 END:

6 SPECIES:

$7 \mathrm{Nd}(1) \mathrm{Q}(1) ; 5.81 ; \mathrm{FB} ; \mathrm{FE}$ :

$8 \quad H(1) Q(1) ; 9.02 ; V B ; V E$ :

$9 \mathrm{H}(2) \mathrm{Q}(1) ; 15.02 ; \mathrm{VB} ; \mathrm{VE}$ :

10 END:

11 OTHER:

12 Q;VE:

13 END:

14 DATA:

$\begin{array}{llll}15 & 219.77 \quad 295.61 & +0.546\end{array}$

16 LOGB

17 PRIN

18 CARD

$19 \mathrm{MR}$

20 NOPL

21 CRT

2250

231.0

24 SPECTRA:

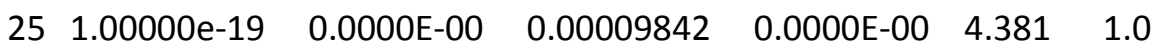

$\begin{array}{lllllllll}26 & 0.2687 & 0.2961 & 0.2896 & 0.3023 & 0.3106 & 0.3146 & 0.3215 & 0.3271\end{array}$

$\begin{array}{llllllllll}27 & 0.3299 & 0.3350 & 0.3387 & 0.3447 & 0.3516 & 0.3591 & 0.3687 & 0.3819\end{array}$

$\begin{array}{llllllllll}28 & 0.3985 & 0.4158 & 0.4366 & 0.4604 & 0.4862 & 0.5140 & 0.5455 & 0.5786\end{array}$

$\begin{array}{llllllllll}29 & 0.6136 & 0.6506 & 0.6912 & 0.7353 & 0.7812 & 0.8279 & 0.8775 & 0.9320\end{array}$

$\begin{array}{lllllllll}30 & 0.9876 & 1.0475 & 1.1050 & 1.1626 & 1.2232 & 1.2842 & 1.3399 & 1.3962\end{array}$ 


$\begin{array}{lllllllll}31 & 1.4520 & 1.5060 & 1.5607 & 1.6068 & 1.6416 & 1.6754 & 1.7082 & 1.7342 \\ 32 & 1.7544 & 1.7698 & 1.7862 & 1.8017 & 1.8164 & 1.8345 & 1.8398 & 1.8458 \\ 33 & 1.8571 & 1.8554 & 1.8610 & 1.8629 & 1.8512 & 1.8492 & 1.8454 & 1.8189 \\ 34 & 1.7900 & 1.7399 & 1.6729 & 1.5872 & 1.4802 & 1.3572 & 1.2310 & 1.1004 \\ 35 & 0.9721 & 0.8475 & 0.7324 & 0.6269 & 0.5314 & 0.4462 & 0.3717 & 0.3071 \\ 36 & 0.2522 & 0.2061 & 0.1681 & 0.1375 & 0.1132 & 0.0943 & 0.0799 & 0.0690 \\ 37 & 0.0610 & 0.0553 & 0.0512 & 0.0483 & 0.0464 & 0.0450 & 0.0441 & 0.0436 \\ 38 & 0.0432 & 0.0431 & 0.0430 & 0.0431 & 0.0432 & 0.0434 & 0.0437 & 0.0440 \\ 39 & 0.0445 & 0.0450 & 0.0456 & 0.0464 & 0.0472 & 0.0481 & 0.0491 & 0.0502 \\ 40 & 0.0514 & 0.0526 & 0.0538 & 0.0552 & 0.0565 & 0.0580 & 0.0595 & 0.0611 \\ 41 & 0.0627 & 0.0644 & 0.0662 & 0.0681 & 0.0699 & 0.0719 & 0.0740 & 0.0761 \\ 42 & 0.0782 & 0.0805 & 0.0828 & 0.0852 & 0.0876 & 0.0898 & 0.0920 & 0.0942 \\ 43 & 0.0963 & 0.0973 & 0.0982 & 0.0991 & & & & \\ 44 & 1.00000 \mathrm{e}-19 & 0.0000 \mathrm{E}-000 & 0.00009837 & 0.0000 \mathrm{E}-00 & 4.963 & 1.0 \\ 45 & 0.3005 & 0.3319 & 0.3250 & 0.3441 & 0.3563 & 0.3643 & 0.3765 & 0.3877 \\ 46 & 0.3963 & 0.4082 & 0.4191 & 0.4335 & 0.4482 & 0.4641 & 0.4826 & 0.5057 \\ 47 & 0.5335 & 0.5612 & 0.5934 & 0.6294 & 0.6669 & 0.7056 & 0.7488 & 0.7923 \\ 48 & 0.8362 & 0.8804 & 0.9274 & 0.9773 & 1.0265 & 1.0728 & 1.1200 & 1.1716 \\ 49 & 1.2209 & 1.2738 & 1.3180 & 1.3602 & 1.4044 & 1.4471 & 1.4817 & 1.5174 \\ 50 & 1.5511 & 1.5853 & 1.6232 & 1.6530 & 1.6737 & 1.6958 & 1.7188 & 1.7369 \\ 51 & 1.7522 & 1.7636 & 1.7781 & 1.7924 & 1.8066 & 1.8253 & 1.8321 & 1.8390 \\ 52 & 1.8501 & 1.8478 & 1.8523 & 1.8520 & 1.8352 & 1.8245 & 1.8094 & 1.7708 \\ 53 & 1.7274 & 1.6630 & 1.5833 & 1.4888 & 1.3776 & 1.2556 & 1.1344 & 1.0128 \\ 54 & 0.8958 & 0.7841 & 0.6816 & 0.5884 & 0.5045 & 0.4300 & 0.3652 & 0.3095 \\ 55 & 0.2624 & 0.2232 & 0.1912 & 0.1656 & 0.1455 & 0.1299 & 0.1181 & 0.1093 \\ 56 & 0.1027 & 0.0979 & 0.0944 & 0.0917 & 0.0897 & 0.0881 & 0.0868 & 0.0856 \\ 57 & 0.0845 & 0.0834 & 0.0822 & 0.0810 & 0.0799 & 0.0787 & 0.0776 & 0.0766 \\ 58 & 0.0757 & 0.0749 & 0.0743 & 0.0740 & 0.0738 & 0.0739 & 0.0742 & 0.0746 \\ 59 & 0.0752 & 0.0760 & 0.0769 & 0.0779 & 0.0790 & 0.0804 & 0.0818 & 0.0834 \\ 60 & 0.0851 & 0.0868 & 0.0886 & 0.0905 & 0.0924 & 0.0944 & 0.0965 & 0.0987 \\ 61 & 0.1009 & 0.1032 & 0.1056 & 0.1080 & 0.1104 & 0.1128 & 0.1151 & 0.1173 \\ 62 & 0.1193 & 0.1204 & 0.1214 & 0.1224 & & & & \\ 63 & -1 & & & & & & & \\ & & & & & & & & \end{array}$

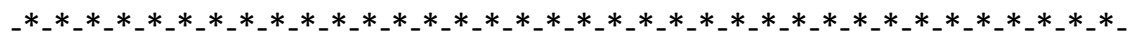

\section{A1.4.3 Line-by-line Description of the SQUAD Input File}

A) Lines 1-24: These lines make up the header and were described in section A1.3.2.

B) Line 25: This line contains the first solution's concentration make-up. The spacing of the numbers on this line is important. If the spacing provided by the GWBASIC.EXE program is not correct, the user must correct the column spacing by inserting or deleting spaces until the values are in the columns specified. Column spacing can be verified in the "notepad" program when the "status bar" is activated in the "View" drop down menu. When the cursor is placed to the immediate left of the character in question, the column number will be shown in the status bar at the lower right corner. 
a) Column 1: This number is the value representing the MTL1 concentration. It is critical that this number be something other than zero, otherwise SQUAD will crash. If there is no metal present in the solution, then a small number such as $1 \mathrm{E}-19$ can be substituted (see section A1.3.2).

b) Column 17: This number is the value representing the MTL2 concentration. It can be left at zero.

c) Column 32: This number is the value representing the LIG1 concentration.

d) Column 47: This number is the value representing the LIG2 concentration. It can be left at zero.

e) Column 61: This number is the value representing the solution $\mathrm{pH}$.

f) Column 72: This number is the value representing the optical pathlength for the solution measured.

C) Lines 26-43: These values represent the solution absorbance values that were read-in by the GWBASIC.EXE program. These values are transposed from the column format that was saved in the .CSV format and are now a horizontal row of absorbance values that are textwrapped to fit in the width of the "notepad" file. Their spacing should be perfectly aligned; however, the user can verify that they are correct. The columns that the first digit of the numbers should be in are: $3,13,23,33, \ldots 73$.

D) Line 44: Same spacing and description as line 25 except this is for second solution in the series.

E) Lines 45-62: These are the same as lines 26-43 above, except this is for the second solution in the series.

F) Line 63: "-1" marks the end of the input file and instructs SQUAD that there is no more data to be read-in. This " -1 " sometimes appears as " -1.0 " but it doesn't matter to the program which is used.

It is necessary to save the spacing changes to the input file before it can be run by SQUAD.

To do this the user can press "Save" and the file will be ready to use. If the user desires to use the "Saved As" option, the input file will be saved as a .TXT file format. If this is done, the .TXT file extension will need to be removed prior to reading the input file into the SQUAD program. If desired, after removing any file extensions from the input file, the user may leave the input file open on their computer while the SQUAD program reads the file in and creates the output file with the modeling results. 


\section{A1.5 Using SQUAD to Read-in Data from the Input File and Create an Output File}

Once the input file is properly formatted with all the correct commands included, the SQUAD program can be run. Running the SQUAD program is probably the simplest part of the entire SQUAD modeling process

\section{A1.5.1 Step-by-step Instructions for Using SQUAD to create an Output File}

1) Before running the program it must be copied into the file folder that contains the input file.

2) Once the SQUAD program of choice has been moved to the file folder containing your input file, run the SQUAD program by double clicking on the program

3) A new command prompt window will appear with the following instruction:

"INPUT file name for SQUAD run ="

4) The user then types the input file name and presses 'ENTER'. If a file name is entered that is not in the file folder where the SQUAD program is located then a brief error message is flashed in the window concerning a run-time error and the window automatically closes. If this happens, simply restart the program by returning to Step 2 ) in this section.

5) If the input file name is correct then the following instruction will appear next:

"OUTPUT file name for SQUAD run ="

6) The user then types the desired output file name and presses 'ENTER'. If a file name is entered that already exists in the file folder where the SQUAD program is located then a brief error message is flashed in the window concerning a run-time error and the window automatically closes. If this happens, simply restart the program by returning to Step 2 ) in this section.

7) If the input and output file names are correct then SQUAD begins to calculate and brief messages concerning iterations will be flashed up in the window. SQUAD will automatically close the window that is running the program and the user will notice a newly created output file in the file folder where the SQUAD program is located.

The data obtained from the SQUAD calculations for the data shown in Figure A1.1 and

Figure A1.2 will be used as the example to explain the output file.

\section{A1.5.2 Example of a SQUAD Output File}

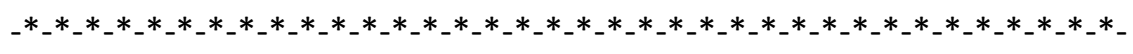

Lines 1-9 are the following ASCII art picture: 


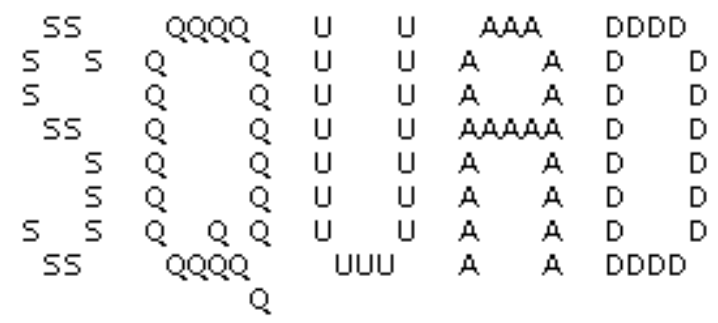

10 8-hydroxyquinoline pka fitting experiment. Wavelength range: $226 . .272 .0 \mathrm{~nm}$

11 System 0.1M NaCl; April 2, 2009 experiment, Dip-probe with normal pathlength (10

12 DICTIONARY...

13 METAL1 METAL2 LIGAND1 LIGAND2 PROTON HYDROXO

$14 * * * * * * * * * * * * * * * * * * * * * * * * * * * * * * * * * * * * * * *$

$15 \mathrm{Nd}$ ABSENT Q ABSENT $\mathrm{H}$ OH

16 SPECIES FORMULA LOG BETA FOR V MOL.ABS.

$171 \mathrm{Nd}(1) \mathrm{Q}(1) \ldots \ldots \ldots \ldots \ldots \ldots . . . . . . . .5100 \quad$ FIXED FIXED

$182 \mathrm{H}(1) \mathrm{Q}(1) \ldots \ldots \ldots \ldots \ldots \ldots . . . . . . .11 .0200$ VARY VARY

$193 \mathrm{H}(2) \mathrm{Q}(1) \ldots \ldots \ldots \ldots \ldots . . . . . . . .16 .0200$ VARY VARY

20 NUMBER OF METALS $=1$ NUMBER OF LIGANDS = 1 NUMBER OF COMPLEXES $=3$

21 NUMBER OF CONSTANTS TO BE VARIED $=2$

22 NUMBER OF SETS OF MOLAR ABSORPTIVITIES TO BE FOUND $=3$

23 THE FOLLOWING MOL. ABS. OF THE COMPONENT(S) WILL BE VARIED.

24 I.E... Q

25 THE MOL. ABS. OF ALL OTHER COMPONENTS WILL BE FIXED

26 THE FOLLOWING SPECIES AND/OR COMPLEXES HAVE FIXED (ZERO OR READ IN) MOL. ABS.

$27 \mathrm{Nd}$

$28 \mathrm{Nd}(1) \mathrm{Q}(1)$

29 THE FOLLOWING PRINT-OUT AND CALCULATION OPTIONS ARE IN EFFECT:-

30 LOGARITHMIC STABILITY CONSTANTS WILL BE REFINED

31 FULL INPUT DATA WILL BE PRINTED OUT

32 GRID SEARCH WILL BE EXECUTED 
33 MOLAR ABSORPTIVITIES WILL BE CALCULATED USING A MULTIPLE REGRESSION ALGORITHM

34 NO PRINTER PLOTS WILL BE PRODUCED

35 GAMMA FOR $\mathrm{H}+=1.0000$

36 NUMBER OF CYCLES DESIRED $=50$

37 TEMPERATURE IS 25.0 DEGREE C

38 SPECTRAL REGION COVERED IS 219.7700 TO 445.6900 AT 1.6250 INTERVALS

39 KNOWN AND FIXED MOLAR ABSORPTIVITIES

40 HEADING 1 REFERS TO THE SPECIES Nd

41 HEADING 2 REFERS TO THE SPECIES MTL2 IS ABSENT

42 HEADING 4 REFERS TO THE SPECIES LIG2 IS ABSENT

43 HEADING 5 REFERS TO THE SPECIES Nd(1)Q(1)

$\begin{array}{lllll}44 & 1 & 2 & 4 & 5\end{array}$

$\begin{array}{lllllll}45 & 219.77 & 0.0000 D+00 & 0.0000 D+00 & 0.0000 D+00 & 0.0000 D+00\end{array}$

$\begin{array}{lllllll}46 & 221.40 & 0.0000 D+00 & 0.0000 D+00 & 0.0000 D+00 & 0.0000 D+00\end{array}$

$\begin{array}{llllllll}47 & 223.02 & 0.0000 \mathrm{D}+00 & 0.0000 \mathrm{D}+00 & 0.0000 \mathrm{D}+00 & 0.0000 \mathrm{D}+00\end{array}$

$\begin{array}{lllllll}48 & 224.65 & 0.0000 D+00 & 0.0000 D+00 & 0.0000 D+00 & 0.0000 D+00\end{array}$

$\begin{array}{llllllllll}49 & 226.27 & 0.0000 \mathrm{D}+00 & 0.0000 \mathrm{D}+00 & 0.0000 \mathrm{D}+00 & 0.0000 \mathrm{D}+00\end{array}$

$\begin{array}{lllllll}50 & 227.90 & 0.0000 \mathrm{D}+00 & 0.0000 \mathrm{D}+00 & 0.0000 \mathrm{D}+00 & 0.0000 \mathrm{D}+00\end{array}$

...

Lines 51-181 were omitted for brevity

...

$\begin{array}{lllll}182442.39 & 0.0000 D+00 & 0.0000 D+00 & 0.0000 D+00 & 0.0000 D+00\end{array}$

$183444.02 \quad 0.0000 \mathrm{D}+00 \quad 0.0000 \mathrm{D}+00 \quad 0.0000 \mathrm{D}+00 \quad 0.0000 \mathrm{D}+00$

$184445.64 \quad 0.0000 \mathrm{D}+00 \quad 0.0000 \mathrm{D}+00 \quad 0.0000 \mathrm{D}+00 \quad 0.0000 \mathrm{D}+00$

185 COMPOSITION OF SOLUTIONS USED TO OBTAIN SPECTRA

186SPECTRUM METAL 1 METAL 2 LIGAND 1 LIGAND 2 PH PATHLENGTH 187 NUMBER MOLES PER LITER

(CMS.)

1881 1.0000D-19 0.0000D+00 9.8420D-05 0.0000D+00 4.38101 .000

$\begin{array}{lllllll}1892 & 1.0000 \mathrm{D}-19 & 0.0000 \mathrm{D}+00 & 9.8370 \mathrm{D}-05 & 0.0000 \mathrm{D}+00 & 4.9630 & 1.000\end{array}$

1903 1.0000D-19 0.0000D+00 9.8170D-05 $0.0000 \mathrm{D}+00 \quad 5.25401 .000$

1914 1.0000D-19 0.0000D+00 9.8040D-05 $0.0000 \mathrm{D}+005.43201 .000$

1925 1.0000D-19 0.0000D+00 9.7920D-05 $0.0000 \mathrm{D}+00 \begin{array}{llll}5.6060 & 1.000\end{array}$

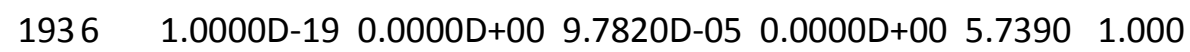

1947 1.0000D-19 0.0000D+00 9.7720D-05 0.0000D+00 5.88401 .000

1958 1.0000D-19 0.0000D+00 9.7630D-05 $0.0000 \mathrm{D}+00 \quad 6.04801 .000$

1969 1.0000D-19 0.0000D+00 9.7530D-05 $0.0000 \mathrm{D}+00 \quad 6.22201 .000$ 
19710 1.0000D-19 0.0000D+00 9.7440D-05 0.0000D+00 6.41601 .000 19811 1.0000D-19 0.0000D+00 9.7340D-05 0.0000D+00 6.62601 .000 $\begin{array}{lllllll}19912 & 1.0000 \mathrm{D}-19 & 0.0000 \mathrm{D}+00 & 9.7250 \mathrm{D}-05 & 0.0000 \mathrm{D}+00 & 6.8660 & 1.000\end{array}$ 20013 1.0000D-19 0.0000D+00 9.7150D-05 $0.0000 D+00 \quad 7.16801 .000$ 20114 1.0000D-19 $0.0000 \mathrm{D}+00 \quad 9.7060 \mathrm{D}-05 \quad 0.0000 \mathrm{D}+00 \quad 7.51801 .000$ $20215 \quad 1.0000 D-19 \quad 0.0000 D+00 \quad 9.6960 D-05 \quad 0.0000 D+007.66101 .000$ $\begin{array}{lllllll}20316 & 1.0000 D-19 & 0.0000 D+00 & 9.6870 D-05 & 0.0000 D+00 & 7.9130 & 1.000\end{array}$ 20417 1.0000D-19 $0.0000 \mathrm{D}+00 \quad 9.6770 \mathrm{D}-05 \quad 0.0000 \mathrm{D}+00 \quad 8.09601 .000$ 20518 1.0000D-19 0.0000D+00 9.6680D-05 0.0000D+00 8.22501 .000 20619 1.0000D-19 0.0000D+00 9.6580D-05 $0.0000 \mathrm{D}+00 \quad 8.3350 \quad 1.000$ $\begin{array}{lllllll}20720 & 1.0000 D-19 & 0.0000 D+00 & 9.6490 \mathrm{D}-05 & 0.0000 \mathrm{D}+00 & 8.4270 & 1.000\end{array}$ 20821 1.0000D-19 0.0000D+00 9.6400D-05 0.0000D+00 8.48501 .000 $\begin{array}{lllllll}20922 & 1.0000 \mathrm{D}-19 & 0.0000 \mathrm{D}+00 & 9.6300 \mathrm{D}-05 & 0.0000 \mathrm{D}+00 & 8.5400 & 1.000\end{array}$ 21023 1.0000D-19 0.0000D+00 9.6210D-05 0.0000D+00 8.59701 .000 21124 1.0000D-19 0.0000D+00 9.6090D-05 0.0000D+00 8.66401 .000 $\begin{array}{lllllll}21225 & 1.0000 D-19 & 0.0000 D+00 & 9.5930 D-05 & 0.0000 D+00 & 8.7400 & 1.000\end{array}$ $\begin{array}{lllllll}21326 & 1.0000 D-19 & 0.0000 D+00 & 9.5440 \mathrm{D}-05 & 0.0000 \mathrm{D}+00 & 8.9300 & 1.000\end{array}$ $\begin{array}{llllllll}21427 & 1.0000 \mathrm{D}-19 & 0.0000 \mathrm{D}+00 & 9.5170 \mathrm{D}-05 & 0.0000 \mathrm{D}+00 & 9.0090 & 1.000\end{array}$ $\begin{array}{lllllll}21528 & 1.0000 \mathrm{D}-19 & 0.0000 \mathrm{D}+00 & 9.5110 \mathrm{D}-05 & 0.0000 \mathrm{D}+00 & 9.1550 & 1.000\end{array}$ $21629 \quad 1.0000 \mathrm{D}-19 \quad 0.0000 \mathrm{D}+00 \quad 9.5060 \mathrm{D}-05 \quad 0.0000 \mathrm{D}+00 \quad 9.2470 \quad 1.000$ $21730 \quad 1.0000 \mathrm{D}-19 \quad 0.0000 \mathrm{D}+00 \quad 9.4980 \mathrm{D}-05 \quad 0.0000 \mathrm{D}+00 \quad 9.38101 .000$ $\begin{array}{llllllll}21831 & 1.0000 \mathrm{D}-19 & 0.0000 \mathrm{D}+00 & 9.4890 \mathrm{D}-05 & 0.0000 \mathrm{D}+00 & 9.5080 & 1.000\end{array}$ $\begin{array}{lllllll}21932 & 1.0000 D-19 & 0.0000 D+00 & 9.4770 D-05 & 0.0000 D+00 & 9.6370 & 1.000\end{array}$ $\begin{array}{lllllll}22033 & 1.0000 D-19 & 0.0000 D+00 & 9.4620 D-05 & 0.0000 D+00 & 9.7930 & 1.000\end{array}$ $22134 \quad 1.0000 D-19 \quad 0.0000 D+00 \quad 9.4380 D-05 \quad 0.0000 D+00 \quad 9.99901 .000$ 22235 1.0000D-19 0.0000D+00 9.4090D-05 0.0000D+00 ****** 1.000

223 BASELINE CORRECTION NOT APPLIED

$224 * * * *$ INTERMEDIATE CALCULATIONS $* * * * *$

225 USER-SUPPLIED INITIAL ESTIMATES HAVE BEEN REPLACED

\author{
226 HESSIAN MATRIX APPROXIMATION \\ 2276.868194D+00 -6.847144D+00 \\ $228-6.847144 \mathrm{D}+00 \quad 6.950316 \mathrm{D}+00$ \\ 229 ELEMENTS OF CONSTANT VECTOR \\ 230-4.197037D+00 \\ 2313.828173D+00 \\ 232 HESSIAN (INVERTED) \\ 2338.150616D+00 8.029627D+00 \\ 2348.029627D+00 8.054312D+00
}

235 EXTREME OVERSHIFT, VARIABLE CONSTANT NO. $1 \mathrm{X}(\mathrm{I})=-3.470 \mathrm{E}+00$ 
236 EXTREME OVERSHIFT, VARIABLE CONSTANT NO. 2 X(I)=-2.867E +00

237 CORRELATION MATRIX

238.100000D+01

$239.991028 D+00 \quad .100000 D+01$

240 THE STANDARD DEVIATION IN THE ABSORBANCE DATA WITH THE INPUT CONSTANTS IS 3.7108D-02

241 THE STANDARD DEVIATION IN THE ABSORBANCE DATA IS 3.3470D-02

24210.040000 .0956 SHIFT $=-.9800$ FOR H(1)Q(1)

$24315.040000 \quad .0950 \quad$ SHIFT $=-.9800$ FOR H(2)Q(1)

2441 CYCLE(S) CALCULATED

245 HESSIAN MATRIX APPROXIMATION

$2468.405028 \mathrm{D}+00-6.926368 \mathrm{D}+00$

247-6.926368D+00 7.539305D+00

248 ELEMENTS OF CONSTANT VECTOR

249-4.795847D+00

2504.391715D+00

251 HESSIAN (INVERTED)

2524.897721D-01 4.499541D-01

2534.499541D-01 5.460116D-01

254 CORRELATION MATRIX

$255.100000 D+01$

$256.870102 D+00 \quad .100000 D+01$

257THE STANDARD DEVIATION IN THE ABSORBANCE DATA IS 1.7179D-02

$2589.667199 \quad .0120 \quad$ SHIFT $=-.3728$ FOR H(1)Q(1)

25915.280017 .0127 SHIFT $=.2400 \mathrm{FOR} \mathrm{H(2)Q(1)}$

260 CYCLE(S) CALCULATED

261 HESSIAN MATRIX APPROXIMATION

$2629.368666 \mathrm{D}+00-6.811129 \mathrm{D}+00$

263-6.811129D+00 8.065930D+00

264 ELEMENTS OF CONSTANT VECTOR

265-8.699003D-01

2666.998994D-01

267 HESSIAN (INVERTED)

2682.764622D-01 2.334535D-01

2692.334535D-01 3.211139D-01 
270 CORRELATION MATRIX

$271.100000 \mathrm{D}+01$

$272.783525 \mathrm{D}+00 \quad .100000 \mathrm{D}+01$

273 THE STANDARD DEVIATION IN THE ABSORBANCE DATA IS 1.6639D-02

$2749.590098 \quad .0087 \quad$ SHIFT $=-.0771 \mathrm{FOR} \mathrm{H}(1) \mathrm{Q}(1)$

27515.301683 .0094 SHIFT $=.0217$ FOR H(2)Q(1)

276 CYCLE(S) CALCULATED

277 HESSIAN MATRIX APPROXIMATION

2789.223845D+00 -6.498355D+00

279-6.498355D+00 7.879332D+00

280 ELEMENTS OF CONSTANT VECTOR

281-6.300704D-03

282-3.888409D-02

283 HESSIAN (INVERTED)

2842.587704D-01 2.134168D-01

2852.134168D-01 3.029264D-01

286 CORRELATION MATRIX

287.100000D+01

$288.762259 D+00 \quad .100000 D+01$

289THE STANDARD DEVIATION IN THE ABSORBANCE DATA IS 1.6635D-02

2909.580169 .0085 SHIFT $=-.0099$ FOR H(1)Q(1)

29115.288559 .0092 SHIFT $=-.0131 \mathrm{FOR} \mathrm{H(2)Q(1)}$

292 CYCLE(S) CALCULATED

293 HESSIAN MATRIX APPROXIMATION

2949.245685D+00 -6.512480D+00

295-6.512480D+00 7.907041D+00

296 ELEMENTS OF CONSTANT VECTOR

297-8.051318D-03

2982.563333D-03

299 HESSIAN (INVERTED)

3002.576119D-01 2.121770D-01

3012.121770D-01 3.012250D-01

302 CORRELATION MATRIX

303.100000D+01

$304.761675 D+00 \quad .100000 D+01$

305 THE STANDARD DEVIATION IN THE ABSORBANCE DATA IS 1.6635D-02 


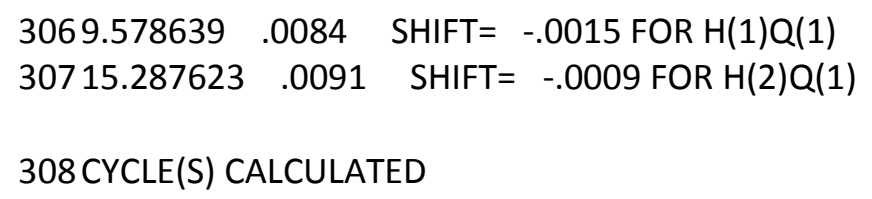

321 THE STANDARD DEVIATION IN THE ABSORBANCE DATA IS 1.6635D-02 3229.578416 .0084 SHIFT $=-.0002$ FOR H(1)Q(1)

326STAND. DEV. OF FIT FOR SPECTRUM NUMBER $1=5.9624 \mathrm{E}-02$ 327STAND. DEV. OF FIT FOR SPECTRUM NUMBER $2=1.3362 \mathrm{E}-02$ 328STAND. DEV. OF FIT FOR SPECTRUM NUMBER $3=1.5027 \mathrm{E}-02$ 329STAND. DEV. OF FIT FOR SPECTRUM NUMBER $4=2.5973 E-02$ 330STAND. DEV. OF FIT FOR SPECTRUM NUMBER $5=2.8568 \mathrm{E}-02$ 331STAND. DEV. OF FIT FOR SPECTRUM NUMBER $6=2.6721 \mathrm{E}-02$ 332 STAND. DEV. OF FIT FOR SPECTRUM NUMBER $7=2.2282 \mathrm{E}-02$ 333 STAND. DEV. OF FIT FOR SPECTRUM NUMBER $8=1.6887 \mathrm{E}-02$ 334STAND. DEV. OF FIT FOR SPECTRUM NUMBER $9=1.2959 \mathrm{E}-02$ 335STAND. DEV. OF FIT FOR SPECTRUM NUMBER $10=1.1980 \mathrm{E}-02$ 336STAND. DEV. OF FIT FOR SPECTRUM NUMBER $11=1.1874 \mathrm{E}-02$ 337STAND. DEV. OF FIT FOR SPECTRUM NUMBER $12=1.3943 \mathrm{E}-02$ 338STAND. DEV. OF FIT FOR SPECTRUM NUMBER $13=1.4091 \mathrm{E}-02$ 339STAND. DEV. OF FIT FOR SPECTRUM NUMBER $14=1.2175 \mathrm{E}-02$ 340STAND. DEV. OF FIT FOR SPECTRUM NUMBER $15=8.3334 \mathrm{E}-03$ 341STAND. DEV. OF FIT FOR SPECTRUM NUMBER $16=8.0491 \mathrm{E}-03$ 342 STAND. DEV. OF FIT FOR SPECTRUM NUMBER $17=7.4988 E-03$ 
343STAND. DEV. OF FIT FOR SPECTRUM NUMBER $18=7.0119 \mathrm{E}-03$

344STAND. DEV. OF FIT FOR SPECTRUM NUMBER $19=6.5921 \mathrm{E}-03$

345STAND. DEV. OF FIT FOR SPECTRUM NUMBER $20=6.7444 \mathrm{E}-03$

346STAND. DEV. OF FIT FOR SPECTRUM NUMBER $21=7.2449 \mathrm{E}-03$

347STAND. DEV. OF FIT FOR SPECTRUM NUMBER $22=7.1423 \mathrm{E}-03$

348STAND. DEV. OF FIT FOR SPECTRUM NUMBER $23=6.6514 \mathrm{E}-03$

349STAND. DEV. OF FIT FOR SPECTRUM NUMBER $24=6.6122 \mathrm{E}-03$

350STAND. DEV. OF FIT FOR SPECTRUM NUMBER $25=5.9381 \mathrm{E}-03$

351STAND. DEV. OF FIT FOR SPECTRUM NUMBER $26=4.9635 \mathrm{E}-03$

352 STAND. DEV. OF FIT FOR SPECTRUM NUMBER $27=5.4146 \mathrm{E}-03$

353STAND. DEV. OF FIT FOR SPECTRUM NUMBER $28=4.1101 \mathrm{E}-03$

354STAND. DEV. OF FIT FOR SPECTRUM NUMBER $29=3.9495 \mathrm{E}-03$

355STAND. DEV. OF FIT FOR SPECTRUM NUMBER $30=6.9922 \mathrm{E}-03$

356STAND. DEV. OF FIT FOR SPECTRUM NUMBER $31=8.8144 \mathrm{E}-03$

357STAND. DEV. OF FIT FOR SPECTRUM NUMBER $32=8.1250 E-03$

358STAND. DEV. OF FIT FOR SPECTRUM NUMBER $33=6.6455 \mathrm{E}-03$

359STAND. DEV. OF FIT FOR SPECTRUM NUMBER $34=1.7384 \mathrm{E}-03$

360STAND. DEV. OF FIT FOR SPECTRUM NUMBER $35=1.2596 \mathrm{E}-02$

361 SUM OF SQUARES OF (OBSVD. - CALC.) $=1.2392 \mathrm{D}+00$

362 CONCENTRATION MATRIX Q(NUMPH,NSPECIES)

363 FREE Nd FREE MTL2 FREE Q FREE LIG2

364SOLN. 1 9.9998E-20 0.0000E+00 2.8037E-11 0.0000E+00

365SOLN. 2 9.9977E-20 0.0000E+00 3.6288E-10 0.0000E+00

366SOLN. 3 9.9922E-20 0.0000E+00 1.2078E-09 0.0000E+00

367SOLN. 4 9.9844E-20 0.0000E+00 2.4195E-09 0.0000E+00

368SOLN. 5 9.9704E-20 0.0000E+00 4.6011E-09 0.0000E+00

369SOLN. 6 9.9529E-20 0.0000E+00 7.3231E-09 0.0000E+00

370SOLN. 7 9.9242E-20 0.0000E+00 1.1837E-08 0.0000E+00

371SOLN. 8 9.8742E-20 0.0000E+00 1.9737E-08 0.0000E+00

372SOLN. 9 9.7924E-20 $0.0000 \mathrm{E}+00 \quad 3.2835 \mathrm{E}-08 \quad 0.0000 \mathrm{E}+00$

373SOLN. 10 9.6510E-20 0.0000E+00 5.6004E-08 0.0000E+00

374SOLN. 11 9.4118E-20 0.0000E+00 9.6787E-08 0.0000E+00

375 SOLN. 12 8.9798E-20 0.0000E+00 1.7597E-07 0.0000E+00

376SOLN. 13 8.0990E-20 0.0000E+00 3.6355E-07 0.0000E+00

377 SOLN. 14 6.5258E-20 0.0000E+00 8.2457E-07 0.0000E+00

378SOLN. 15 5.7474E-20 0.0000E+00 1.1460E-06 0.0000E+00

379SOLN. 16 4.3201E-20 $0.0000 \mathrm{E}+00 \quad 2.0363 \mathrm{E}-06 \quad 0.0000 \mathrm{E}+00$

380SOLN. 17 3.3513E-20 0.0000E+00 3.0728E-06 0.0000E+00

381 SOLN. 18 2.7463E-20 0.0000E+00 4.0909E-06 0.0000E+00

382 SOLN. 19 2.2934E-20 0.0000E+00 5.2046E-06 0.0000E+00

383 SOLN. 20 1.9612E-20 0.0000E+00 6.3486E-06 0.0000E+00

384SOLN. 21 1.7738E-20 0.0000E+00 7.1830E-06 0.0000E+00

385SOLN. 22 1.6111E-20 0.0000E+00 8.0647E-06 0.0000E+00

386SOLN. 23 1.4569E-20 0.0000E+00 9.0820E-06 0.0000E+00

387 SOLN. 24 1.2939E-20 0.0000E+00 1.0421E-05 0.0000E+00 
388SOLN. 25 1.1311E-20 0.0000E+00 1.2144E-05 0.0000E+00 389SOLN. 26 8.1301E-21 0.0000E+00 1.7501E-05 0.0000E+00 390SOLN. 27 7.1226E-21 0.0000E+00 2.0196E-05 0.0000E+00 391SOLN. 28 5.6132E-21 0.0000E+00 2.6043E-05 0.0000E+00 392 SOLN. 29 4.8752E-21 0.0000E+00 3.0220E-05 0.0000E+00 393 SOLN. 30 4.0310E-21 0.0000E+00 3.6874E-05 0.0000E+00 394 SOLN. 31 3.4302E-21 0.0000E+00 4.3603E-05 0.0000E+00 395SOLN. 32 2.9715E-21 0.0000E+00 5.0573E-05 0.0000E+00 396SOLN. 33 2.5680E-21 0.0000E+00 5.8764E-05 0.0000E+00 397SOLN. 34 2.2140E-21 0.0000E+00 6.8406E-05 0.0000E+00 398SOLN. 35 1.9711E-21 0.0000E+00 7.7026E-05 0.0000E+00

399 NSPECIES 12123

400SOLN. 1 1.8102E-24 4.4172E-06 9.4003E-05

401SOLN. 2 2.3424E-23 1.4969E-05 8.3401E-05

402SOLN. 3 7.7920E-23 2.5492E-05 7.2677E-05

403SOLN. 4 1.5598E-22 3.3896E-05 6.4141E-05

404SOLN. 5 2.9619E-22 4.3180E-05 5.4736E-05

405SOLN. 6 4.7059E-22 5.0595E-05 4.7217E-05

406SOLN. 7 7.5845E-22 5.8567E-05 3.9142E-05

407SOLN. 8 1.2583E-21 6.6942E-05 3.0668E-05

408SOLN. 9 2.0760E-21 7.4602E-05 2.2895E-05

409SOLN. 10 3.4897E-21 8.1402E-05 1.5982E-05

410SOLN. 11 5.8815E-21 8.6742E-05 1.0501E-05

411SOLN. 12 1.0202E-20 9.0752E-05 6.3219E-06

412SOLN. 13 1.9010E-20 9.3536E-05 3.2506E-06

413SOLN. 14 3.4742E-20 9.4764E-05 1.4711E-06

414SOLN. 15 4.2526E-20 9.4756E-05 1.0583E-06

415SOLN. 16 5.6799E-20 9.4245E-05 5.8918E-07

416SOLN. 17 6.6487E-20 9.3314E-05 3.8277E-07

417SOLN. 18 7.2537E-20 9.2308E-05 2.8134E-07

418SOLN. 19 7.7066E-20 9.1160E-05 2.1567E-07

419SOLN. 20 8.0388E-20 8.9969E-05 1.7222E-07

420SOLN. 21 8.2262E-20 8.9068E-05 1.4918E-07

421SOLN. 22 8.3889E-20 8.8105E-05 1.3001E-07

422 SOLN. 23 8.5431E-20 8.7015E-05 1.1261E-07

423SOLN. 24 8.7061E-20 8.5574E-05 9.4914E-08

424SOLN. 25 8.8689E-20 8.3708E-05 7.7939E-08

425SOLN. 26 9.1870E-20 7.7892E-05 4.6825E-08

426SOLN. 27 9.2877E-20 7.4936E-05 3.7556E-08

427SOLN. 28 9.4387E-20 6.9042E-05 2.4723E-08

428SOLN. 29 9.5125E-20 6.4821E-05 1.8780E-08

429SOLN. 30 9.5969E-20 5.8094E-05 1.2363E-08

430SOLN. 31 9.6570E-20 5.1279E-05 8.1456E-09

431SOLN. 32 9.7028E-20 4.4191E-05 5.2159E-09

432 SOLN. 33 9.7432E-20 3.5853E-05 2.9547E-09

433SOLN. 34 9.7786E-20 2.5972E-05 1.3320E-09

434SOLN. 35 9.8029E-20 1.7063E-05 5.1056E-10 


\begin{abstract}
435 MOLAR ABSORPTIVITIES OF INDIVIDUAL SPECIES CALCULATED BY PROGRAM 436 USING MULTIPLE REGRESSION ALGORITHM.
\end{abstract}

437 HEADING 1 REFERS TO THE SPECIES $H(1) Q(1)$ 438 HEADING 2 REFERS TO THE SPECIES H(2)Q(1) 439HEADING 3 REFERS TO THE SPECIES $Q$

$\begin{array}{lccc}\text { 440 WAVELENGTH } & 1 & 2 & 3 \\ 441219.77 & 3.8477 \mathrm{E}+03 & 2.9827 \mathrm{E}+03 & 3.9572 \mathrm{E}+03 \\ 442221.40 & 4.6363 \mathrm{E}+03 & 3.4218 \mathrm{E}+03 & 4.6347 \mathrm{E}+03 \\ 443223.02 & 5.6608 \mathrm{E}+03 & 3.8614 \mathrm{E}+03 & 5.4501 \mathrm{E}+03 \\ 444224.65 & 6.4788 \mathrm{E}+03 & 4.1640 \mathrm{E}+03 & 5.9961 \mathrm{E}+03 \\ 445226.27 & 7.5971 \mathrm{E}+03 & 4.7461 \mathrm{E}+03 & 6.8618 \mathrm{E}+03 \\ 446227.90 & 8.7195 \mathrm{E}+03 & 5.4318 \mathrm{E}+03 & 7.7667 \mathrm{E}+03 \\ 447229.52 & 9.8757 \mathrm{E}+03 & 6.2679 \mathrm{E}+03 & 8.7584 \mathrm{E}+03 \\ \ldots & & & \\ \text { Lines 448-577 were omitted for brevity } & \\ \ldots & & & \\ 578442.39 & 1.0416 \mathrm{E}+01 & -7.4789 \mathrm{E}+01 & -2.1898 \mathrm{E}+00 \\ 579444.02 & 1.0359 \mathrm{E}+01 & -7.5821 \mathrm{E}+01 & -3.5862 \mathrm{E}+00 \\ 580445.64 & 1.0393 \mathrm{E}+01 & -7.5956 \mathrm{E}+01 & -3.3932 \mathrm{E}+00\end{array}$

581STANDARD DEVN. OF CALCULATED MOLAR ABSORPTIVITIES

\begin{tabular}{|c|c|c|c|}
\hline \multicolumn{2}{|c|}{582 WAVELENGTH } & \multicolumn{2}{|l|}{2} \\
\hline 583219.77 & $2.1639 E+01$ & $4.6063 E+01$ & $5.8835 E+0$ \\
\hline 584221.40 & $2.9588 \mathrm{E}+01$ & $6.2983 E+01$ & $8.0447 \mathrm{E}+\mathrm{C}$ \\
\hline 585223.02 & $3.9691 \mathrm{E}+01$ & $8.4489 E+01$ & $1.0792 \mathrm{E}+02$ \\
\hline 86224.65 & $5.6437 E+01$ & $1.2014 \mathrm{E}+02$ & $1.5345 \mathrm{E}+0$ \\
\hline
\end{tabular}

Lines 587-716 were omitted for brevity ...

$\begin{array}{llll}717437.52 & 2.5558 \mathrm{E}+00 & 5.4405 \mathrm{E}+00 & 6.9490 \mathrm{E}+00 \\ 718439.14 & 2.5343 \mathrm{E}+00 & 5.3947 \mathrm{E}+00 & 6.8905 \mathrm{E}+00 \\ 719440.77 & 2.5691 \mathrm{E}+00 & 5.4687 \mathrm{E}+00 & 6.9850 \mathrm{E}+00 \\ 720442.39 & 2.5545 \mathrm{E}+00 & 5.4377 \mathrm{E}+00 & 6.9454 \mathrm{E}+00 \\ 721444.02 & 2.5481 \mathrm{E}+00 & 5.4240 \mathrm{E}+00 & 6.9279 \mathrm{E}+00 \\ 722445.64 & 2.5283 \mathrm{E}+00 & 5.3820 \mathrm{E}+00 & 6.8742 \mathrm{E}+00\end{array}$

A1.5.3 Description of the SQUAD Output File Line-by-line

A) Lines 1-9: ASCll art spelling out the word SQUAD.

B) Lines 10-11: This is a reproduction of the text description of this experiment taken from the first two lines of the header/input (H/I) file. 
C) Lines 12-15: These lines display the user-defined components as they were provided in the header file "DICTIONARY" section. Note: In a text file the columns align much better than in MS Word.

D) Lines 16-19: These lines display the formed species, log beta values, and the user-provided instructions on whether the log beta or molar absorptivity should be varied or not.

E) Line 20: This line summarizes the number of metals, ligands and complexes.

F) Line 21: This line displays the number of constants to be varied.

G) Line 22: This line displays the number of molar absorptivities to be found.

H) Line 23-24: These lines display the components whose molar absorptivities will be calculated. The species listed are those identified in the "OTHER" section with instructions to SQUAD to "VE" or "vary the molar absorptivity".

I) Line 25-28: These lines display the components or complexes that possess fixed molar absorptivities that will be assumed to be zero, or explicitly read-in.

J) Line 29: Beginning of the explanation of the user requested calculation instructions.

K) Line 30: Messages correspond to the instructions entered in $(\mathrm{H} / \mathrm{I})$ file Line 16 that is described in Section A1.3 of this manual. When the instruction "LOGB" is used on line 16 of the $(H / I)$ file, the message: "LOGARITHMIC STABILITY CONSTANTS WILL BE REFINED" appears on this line. When the instruction "EXPB" is used on line 16 of the $(H / I)$ file, the message: “ANTILOGARITHM OF LOG(BETA) WILL BE REFINED” appears instead.

L) Line 31: Messages correspond to the instructions entered in Line 17 of the $(\mathrm{H} / \mathrm{I})$ file that is described in Section A1.3 of this manual. When the instruction "PRIN" is used on line 17 of the $(\mathrm{H} / \mathrm{I})$ file, the message: "FULL INPUT DATA WILL BE PRINTED OUT" appears on this line. When the instruction "NOPR" is used on line 16 of the $(\mathrm{H} / \mathrm{I})$ file, the message: "ABBREVIATED INPUT DATA WILL PRINTED OUT" appears instead.

a) The abbreviated version of the input data omits lines 39-222 that are displayed in the example output file shown.

M) Line 32: Messages correspond to the instructions entered in Line 18 of the header file that is described in Section A1.3 of this manual. When the instruction "CARD" is used on line 18 of the header, the message: "GRID SEARCH WILL BE EXECUTED" appears on this line. When the instruction "NOCD" is used on line 16 of the $(H / I)$ file, the message: "GRID SEARCH WILL NOT BE EXECUTED" appears instead.

a) When the Grid search is performed, the SQUAD program judges the user supplied values to see if they are good. If so, then the message "USER-SUPPLIED INITIAL ESTIMATES ARE GOOD" appears on line 225. If they user-supplied values are not good, then the message: "USER-SUPPLIED INITIAL ESTIMATES HAVE BEEN REPLACED" appears on line 225. SQUAD does not explicitly indicate what values are used in the place of the usersupplied are replaced. 
b) If no grid search is performed, then the message on line 225 is omitted.

N) Line 33: Messages correspond to the instructions entered in Line 19 of the header file that is described in section A1.3 of this manual. When the instruction "MR" is used on line 19 of the header, the message: "MOLAR ABSORPTIVITIES WILL BE CALCULATED USING A MULTIPLE REGRESSION ALGORITHM" appears on this line. When the instruction "NNLS" is used on line 16 of the (H/I) file, the message: "MOLAR ABSORPTIVITIES WILL BE CALCULATED USING A NON-NEGATIVE LLSQ ALGORITHM" appears instead.

O) Line 34: Messages correspond to the instructions entered in Line 20 of the header file that is described in section A1.3 of this manual. When the instruction "PLOT" is used on line 20 of the header, the message: "NO PRINTER PLOTS WILL BE PRODUCED" appears on this line. When the instruction "NNLS" is used on line 16 of the $(\mathrm{H} / \mathrm{I})$ file, the message: "NO PRINTER PLOTS WILL BE PRODUCED" appears instead.

a) It would seem that this feature was disabled by the programmer prior to the compilation of this version of SQUAD. It is not known what this feature does in a version of SQUAD where this option is activated.

P) Line 35: This indicates what the user-supplied value for the $\mathrm{H}^{+}$activity coefficients is for this modeling calculation. This value is specified in line 23 of the $(H / I)$ file.

Q) Line 36: This indicates the user-supplied maximum number of iterations allowed by SQUAD. This value is defined in line 22 of the $(\mathrm{H} / \mathrm{I})$ file.

R) Line 37: This indicates the assumed temperature for the experiment is 25.0 degrees. The author has tried, but failed to find a way to change the assumed temperature for the experiment. It is assumed that temperature is not used in the computations performed by SQUAD.

S) Line 38: This indicates what the lower and upper bounds of the wavelengths and the interval between wavelengths for the absorbance data that were supplied by the user in line 15 of the $(\mathrm{H} / \mathrm{I})$ file.

T) Lines 39-44: These lines offer clarification of the Fixed Molar Absorptivities being displayed in lines 45-184, specifying which column pertains to which component or species.

U) Lines 45-184: These lines show the "Fixed Molar Absorptivities" for the components that were identified as having fixed molar absorptivities.

V) Lines 185-187: These lines act as column headings for the data table showing the "Composition of Solutions used to Obtain Spectra" indicating units for concentration and optical pathlength.

W) Lines 188-222: These lines contain the data values that SQUAD imported from the $(\mathrm{H} / \mathrm{I})$ file concerning the solution concentrations. The lines in the $(H / I)$ file these data come from are lines 25 and 44 shown in the example $(\mathrm{H} / \mathrm{I})$ file in Section A1.3. 
X) Line 223: This line indicates whether there was a baseline spectrum read in to the SQUAD program. If a baseline spectrum was read in then "BASELINE CORRECTION APPLIED" will appear in this line. If no baseline spectrum was read in then "BASELINE CORRECTION NOT APPLIED" will appear in this line.

Y) Line 224: This shows the beginning of the actual iterations that SQUAD performed while fitting the data.

Z) Line 225: This indicates whether the "GRID SEARCH" or check of the "goodness" of the guess values was performed based on the user-supplied information. If no "GRID SEARCH" was performed then no notification will appear here. If the "GRID SEARCH" determines that estimates are good then the message appears: "USER-SUPPLIED INITIAL ESTIMATES ARE GOOD". If the "GRID SEARCH" determines that estimates are not good then the message, "USER-SUPPLIED INITIAL ESTIMATES HAVE BEEN REPLACED" appears on line 225. SQUAD never indicates what numbers are used if the user estimates are replaced.

AA) Lines 226-244: These lines contain the data obtained from the first iteration of calculations.

a) Lines 226-234: These lines contain the Hessian matrix, the constant vector and the inverted Hessian matrix.

b) Lines 235-236: These lines contain information concerning the "EXTREME OVERSHIFT, VARIABLE CONSTANT". This message and reported values disappear as SQUAD approaches a solution.

c) Lines 237-239: These lines contain information concerning the "CORRELATION MATRIX". The documentation supplied with SQUAD did not provide the significance of these values.

d) Line 240: This line shows "THE STANDARD DEVIATION IN THE ABSORBANCE DATA WITH THE INPUT CONSTANTS". The documentation supplied with SQUAD did not provide the significance of this value.

e) Line 241: This line shows "THE STANDARD DEVIATION IN THE ABSORBANCE DATA"; The documentation supplied with SQUAD did not provide the significance of this value, nor the difference between this value and that shown on line 240 .

f) Line 242-243: These lines show the $\log \beta$ values calculated by the program:

i) First number: $\log \beta$ resulting from the first iteration.

ii) Second number: Standard Deviation of the $\log \beta$ resulting from the first iteration.

iii) "SHIFT="

iv) Third number: The shift that SQUAD made in the $\log \beta$ value from the previous iteration value. 
v) Species identification: "FOR XXXXX" the XXX identifies the species to which the $\log \beta$ corresponds.

g) Line 244: This line indicates the end of the presentation of data relating to the $1^{\text {st }}$ iteration with the message: "1 CYCLE(S) CALCULATED"

BB) Lines 245-324: These lines show the other 5 iterations and are identical in nature to those explained above in lines 226-244.

CC) Line 325: This line indicates that a solution has been achieved and the number of iterations that were performed.

DD) Lines 326-360: These lines show the "Standard Deviation of the Fit" for each spectrum. These data would help a user to identify particular spectra that have a poor fit that might be excluded for a better solution.

EE) Line 361: This shows the sum of squares for the calculations performed.

FF) Lines 362-363: These lines introduce and clarify the columns of data as they relate to the final free concentrations of the components that were obtained from the calculations.

GG)Lines 364-398: The data for the final free concentrations of the components that were obtained from the calculations.

$\mathrm{HH}$ ) Lines 299-434: These are the concentrations of the species that were obtained from the calculations. The column numbers shown here correspond to the numbers shown in lines 17-19 of the output file. These column numbers should not be equated or confused with the upcoming column numbers shown in lines 437-439 for the "Molar Absorptivity" calculations that appear almost directly under this data table. Note: SQUAD renumbers the species in the "Molar Absorptivities" section which could lead to some confusion.

II) Lines 435-440: These lines clarify the "Molar Absorptivity" data presented in lines 441-722.

a) Lines 435-436: These lines restate the algorithm used to fit the molar absorptivities: Multiple Regression or Non-Negative Least Squares methods.

b) Lines 437-439: These lines establish a new numbering for the presentation of molar absorptivities of the species and components with varied molar absorptivities. These heading numbers are different, and should not be confused with the heading numbers used in line 399 of this example output file.

c) Line 440: Displays the column headings for the molar absorptivities.

JJ) Lines 441-580: Displays the molar absorptivities of the species and components with varied molar absorptivities.

KK) Line 581: This line divides the molar absorptivities from the standard deviations of the molar absorptivities. 
LL) Line 582: Displays the column headings for the table of standard deviations for the calculated molar absorptivities of the species and components with varied molar absorptivities.

MM) Lines 583-722: This table gives the standard deviations for the calculated molar absorptivities of the species and components with varied molar absorptivities. 


\section{BIBLIOGRAPHY}

1. Weaver, B.; Kappelmann, F. A., TALSPEAK: A New Method of Separating Americium and Curium from the Lanthanides by Extraction from an Aqueous Solution of an Aminopolyacetic Acid Complex with a Monoacidic Organophosphate or Phosphonate; ORNL-3559; Oak Ridge National Laboratory: Oak Ridge, TN, 1964.

2. Gelis, A. V.; Vandegrift, G. F.; Bakel, A.; Bowers, D. L.; Hebden, A. S.; Pereira, C.; Regalbuto, M., Radiochim. Acta 2009, 97, 231-232.

3. Nilsson, M.; Nash, K., Solvent Extr. Ion Exch. 2007, 25, 665-701.

4. Leggett, C. J.; Liu, G.; Jensen, M. P., Solvent Extr. Ion Exch. 2010, 28, 313-334.

5. De Jong, N.; Draye, M.; Favre-Reguillon, A.; LeBuzit, G.; Cote, G.; Foos, J., J. Colloid Interface Sci. 2005, 291, 303-306.

6. Draye, M.; Thomas, S.; Cote, G.; Favre-Reguillon, A.; LeBuzit, G.; Guy, A.; Foos, J., Sep. Sci. Technol. 2005, 40, 611-622.

7. Favre-Reguillon, A.; Draye, M.; Lebuzit, G.; Thomas, S.; Foos, J.; Cote, G.; Guy, A., Talanta 2004, 63, 803-806.

8. Miura, J.; Ishii, H.; Watanabe, H., Bunseki Kagaku 1976, 25, 808-809.

9. Watanabe, H.; Tanaka, H., Talanta 1978, 25, 585-589.

10. Madic, C.; Boullis, B.; Baron, P.; Testard, F.; Hudson, M. J.; Liljenzin, J. O.; Christiansen, B.; Ferrando, M.; Facchini, A.; Geist, A.; Modolo, G.; Espartero, A. G.; De Mendoza, J., J. Alloys Compd. 2007, 444-445, 23-27.

11. Seaborg, G. T., Extraction Method for Separating Uranium, Plutonium, and Fission Products from Compositions Containing Same. U.S. Patent 2,811,415,1957.

12. Mathur, J. N.; Murali, M. S.; Krishna, M. V. B.; Iyer, R. H.; Chitnis, R. R.; Wattal, P. K.; Theyyunni, T. K.; Ramanujam, A.; Dhami, P. S.; Gopalakrishnan, V., Sep. Sci. Technol. 1996, 31, 2045-2063.

13. Mathur, J.; Murali, M.; Balarama Krishna, M.; Iyer, R.; Chitnis, R.; Wattal, P.; Bauri, A.; Banerji, A., J. Radioanal. Nucl. Chem. 1996, 213, 419-429.

14. Shannon, R. D., Acta Crystallogr. Sect. A 1976, 32, 751-767.

15. Nilsson, M.; Nash, K. L., Solvent Extraction and Ion Exchange 2007, 25, 665 - 701.

16. Baum, E. M.; Knox, H. D.; Miller, T. R. Nuclides and Isotopes, 16th ed., Knolls Atomic Power Laboratory, Niskayuna, NY, 2002.

17. Croff, A. G., ORIGEN2 - A Revised and Updated Version of the Oak Ridge Isotope Generation and Depletion Code; ORNL-5621; Oak Ridge National Laboratory: 1980.

18. Guenther, R. J.; Blahnik, D. E.; Campbell, T. K.; Jenquin, U. P.; Mendel, J. E.; Thomas, L. E.; Thornhill, C. K., Characterization of Spent Fuel Approved Testing Material - ATM-105; PNL-5109-105; Pacific Northwest Laboratory: Richland, WA, 1989.

19. Irish, E. R.; Reas, W. H., The PUREX Process- A Solvent Extraction Reprocessing Method for Irradiated Uranium; HW-49483A; Hanford Atomic Products Operation: Richland, WA, 1957.

20. Musikas, C., Miner. Process. Extr. Metall. Rev. 1997, 17, 109-142.

21. Law, J. D.; Herbst, R. S.; Peterman, D. R.; Tillotson, R. D.; Todd, T. A., J. Am. Chem. Soc. 2004, 147, 284-290.

22. Stoyanov, E. S.; Smirnov, I. V.; Babain, V. A.; Antonov, N. G.; Peterman, D.; Herbst, R. S.; Todd, T. A.; Luther, T. A., Radiochem. 2004, 46, 540-545.

23. Leonard, R. A.; Aase, S. B.; Arafat, H. A.; Conner, C.; Chamberlain, D. B.; Falkenberg, J. R.; Regalbuto, M. C.; Vandegrift, G. F., Solvent Extr. Ion Exch. 2003, 21, 505-526. 
24. Delmau, L. H.; Bonnesen, P. V.; Engle, N. L.; Haverlock, T. J.; Sloop, F. V.; Moyer, B. A., Solvent Extr. Ion Exch. 2006, 24, 197-217.

25. Law, J. D.; Garn, T. G.; Meikrantz, D. H.; Warburton, J., Sep. Sci. Technol. 2010, 45, 17691775.

26. Street, K.; Seaborg, G. T., J. Am. Chem. Soc. 1950, 72, 2790-2792.

27. Madic, C.; Hudson, M. J.; Liljenzin, J.-O.; GLatz, J.-P.; Nannicini, R.; Facchini, A.; Kolarik, Z.; Odoj, R., Prog. Nucl. Energy 2002, 40, 523-526.

28. Mehdoui, T.; Berthet, J.-C.; Thuéry, P.; Ephritikhine, M., J. Chem. Soc., Dalton Trans. 2004, 579-590.

29. Vandegrift, G. F.; Regalbuto, M. C.; Aase, S. B.; Arafat, H. A.; Bakel, A. J.; Bowers, D. L.; Byrnes, J. P.; Clark, M. A.; Emergy, J. W.; Falkenberg, J. R.; Gelis, A. V.; Hafenrichter, L. D.; Leonard, R. A.; Pereira, C.; Quigley, K. J.; Tsai, Y.; Vander Pol, M. H.; Laidler, J. J., LabScale Demonstration of the UREX+ Process. Proceedings of Waste Management 2004 International Symposium, Tucson, AZ, February 29-March 4, 2004.

30. Vandegrift, G. F.; Regalbuto, M. C.; Aase, S.; Bakel, A.; Battisti, T. J.; Bowers, D. L.; Byrnes, J. P.; Clark, M. A.; Cummings, D. G.; Emery, J. W.; Falkenberg, J. R.; Gelis, A. V.; Pereira, C.; Hafenrichter, L.; Tsai, Y.; Quigley, K. J.; Vander Pol, M. H., Designing and Demonstration of the UREX+ Process Using Spent Nuclear Fuel. Proceedings of ATALANTE 2004 - Advances for Future Nuclear Fuel Cycles, Nimes, France, June 21-24, 2004.

31. Romanovskiy, V. N.; Smirnov, I. V.; Babain, V. A.; Todd, T. A.; Herbst, R. S.; Law, J. D.; Brewer, K. N., Solvent Extr. Ion Exch. 2001, 19, 1-21.

32. Law, J. D.; Herbst, R. S.; Todd, T. A.; Romanovskiy, V. N.; Babain, V. A.; Esimantovskiy, V. M.; Smirnov, I. V.; Zaitsev, B. N., Solvent Extr. Ion Exch. 2001, 19, 23-36.

33. Lumetta, G. L.; Sinkov, S.; Neiner, D.; Levitskaia, T.; Braley, J. C.; Carter, J. C.; Warner, M. G.; Pittman, J. W.; Rapko, B. M., Sigma Team for Minor Actinide Separation: PNNL FY 2010 Status Report; Pacific Northwest National Laboratory: 2010.

34. Lumetta, G. J.; Carter, J. C.; Gelis, A. V.; Vandegrift, G. F., Nucl. Energy Environ., Vol. 1046, American Chemical Society, 2010, pp. 107-118.

35. Stalikas, C. D., Trends Anal. Chem. 2002, 21, 343-355.

36. Paleologos, E. K.; Giokas, D. L.; Karayannis, M. I., Trends Anal. Chem. 2005, 24, 426-436.

37. Bezerra, M. d. A.; Arruda, M. A. Z.; Ferreira, S. L. C., Appl. Spectrosc. Rev. 2005, 40, 269299.

38. Tani, H.; Kamidate, T.; Watanabe, H., J. Chromatogr., A 1997, 780, 229-241.

39. Pires, M. J.; Aires-Barros, M. R.; Cabral, J. M. S., Biotechnol. Prog. 1996, 12, 290-301.

40. Hinze, W. L.; Pramauro, E., Crit. Rev. Anal. Chem. 1993, 24, 133-177.

41. Trakultamupatam, P.; Scamehorn, J. F.; Osuwan, S., Sep. Sci. Technol. 2002, 37, 12911305.

42. Trakultamupatam, P.; Scamehorn, J. F.; Osuwan, S., Sep. Sci. Technol. 2005, 39, 479-499.

43. Choppin, G. R.; Silva, R. J., J. Inorg. Nucl. Chem. 1956, 3, 153-154.

44. Smith, H. L.; Hoffman, D. C., J. Inorg. Nucl. Chem. 1956, 3, 243-247.

45. Wheelwright, E. J.; Spedding, F. H.; Schwarzenbach, G., J. Am. Chem. Soc. 1953, 75, 4196-4201.

46. Wheelwright, E. J.; Spedding, F. H., J. Am. Chem. Soc. 1953, 75, 2529-2530.

47. Pearson, R. G., J. Am. Chem. Soc. 1963, 85, 3533-3539.

48. Nash, K. L., Solvent Extr. Ion Exch. 1993, 11, 729-768.

49. Choppin, G. R., J. Alloys Compd. 2002, 344, 55-59.

50. Kolarik, Z., Miner. Process. Extr. Metall. Rev. 2000, 21, 89-141. 
51. Cotton, F. A.; Wilkinson, G., Advanced Inorganic Chemistry, 5th ed., Wiley Interscience: New York, 1988, pp. 1-1455.

52. Nilsson, M.; Nash, K. L., Solvent Extr. Ion Exch. 2007, 25, 665-701.

53. Weaver, B.; Kappelmann, F. A., J. Inorg. Nucl. Chem. 1968, 30, 263-272.

54. Harris, D. C., Quantitative Chemical Analysis, 4th ed., W. H. Freeman and Company: New York, 1995, p. 186.

55. Portanova, R.; Lajunen, L. H. J.; Piispanen, J., Pure Appl. Chem. 2003, 75, 495-540.

56. Peppard, D. F.; Mason, G. W.; Maier, J. L.; Driscoll, W. J., J. Inorg. Nucl. Chem. 1957, 4, 334-343.

57. Lundqvist, R.; Lu, J.; Svantesson, I., Acta Chem. Scand. 1983, A37, 743-753.

58. Dubuquoy, C.; Guillaumont, R.; Bouissières, G., Radiochimica Acta 1967, 8, 49-57.

59. Thomas, N. E.; Burkhart, L. E., J. Inorg. Nucl. Chem. 1974, 36, 1369-1376.

60. Mel'nik, M. I.; Filimonov, V. T.; Karelin, E. A., Radiochem. 1999, 41, 70-73.

61. Sato, T.; Ueda, M., J. Inorg. Nucl. Chem. 1973, 35, 1003-1010.

62. Mikhailichenko, A. I.; Pimenova, R. M., Sov. Radiochem. 1969, 11, 6-10.

63. Svantesson, I.; Persson, G.; Hagström, I.; Liljenzin, J. O., J. Inorg. Nucl. Chem. 1980, 42, 1037-1043.

64. Jensen, M. P.; Chiarizia, R.; Urban, V., Solvent Extr. Ion Exch. 2001, 19, 865-884.

65. Yurtov, E.; Murashova, N.; Datsenko, A., Russ. J. Inorg. Chem. 2006, 51, 670-675.

66. Anticó, E.; Masana, A.; Hidalgo, M.; Salvadó, V.; Iglesias, M.; Valiente, M., Anal. Chim. Acta 1996, 327, 267-276.

67. Kizim, N. F.; Lar'kov, A. P., Sov. Radiochem. 1988, 30, 177-181.

68. Trifonov, Y. I.; Legin, E. K.; Suglobov, D. N., Sov. Radiochem. 1992, 34, 373-377.

69. Trifonov, Y. I.; Legin, E. K.; Suglobov, D. N., Sov. Radiochem. 1985, 27, 387-393.

70. Spiryakov, V. I.; Krutikov, P. G.; Barinov, V. M.; Solovkin, A. S.; Yakovlev, G. N., Sov. Radiochem. 1972, 14, 589-591.

71. Mel'nik, M. I.; Spiryakov, V. I.; Filimonov, V. T.; Karelin, E. A., Radiochem. 1997, 39, 432437.

72. Trifonov, Y. I.; Legin, E. K.; Suglobov, D. N., Sov. Radiochem. 1992, 34, 369-372.

73. Myers, A. L.; McDowell, W. J.; Coleman, C. F., J. Inorg. Nucl. Chem. 1964, 26, 2005-2011.

74. Wang, D.; Li, Y.; Wu, J.; Xu, G., Solvent Extr. Ion Exch. 1996, 14, 585-601.

75. Blake, C. A.; Crouse, D. J.; Coleman, C. F.; Brown, K. B.; Kelmers, A. D., Progress Report: Further Studies of the Dialkylphosphoric Acid Extraction (DAPEX) Process for Uranium; ORNL-2172; Oak Ridge National Laboratory: Oak Ridge, TN, 1956.

76. Blake, C. A.; Brown, K. B.; Coleman, C. F., The Extraction and Recovery of Uranium (and Vanadium) From Acidic Liquors with Di(2-ethylhexyl) Phosphoric Acid and some other Organophosphorus Acids; ORNL-1903; Oak Ridge Naional Laboratory: Oak Ridge, TN, 1955.

77. Jensen, M. P.; Bond, A. H., Radiochimica Acta 2002, 90, 205-209.

78. Sokolov, E. I.; Melkaya, R. F.; Tevelev, L. G.; Nikolaev, V. M., Russ. J. Inorg. Chem. 1975, 20, 1399-1402.

79. Peppard, D. F.; Ferraro, J. R., J. Inorg. Nucl. Chem. 1959, 10, 275-288.

80. Nakamoto, K.; Ferraro, J. R.; Mason, G. W., Appl. Spectrosc. 1969, 23, 521-527.

81. Li, Q.; Weng, S. F.; Wu, J. G.; Zhou, N. F., J. Phys. Chem. B 1998, 102, 3168-3174.

82. Beitz, J. V.; Sullivan, J. C., J. Less-Common Met. 1989, 148, 159-166.

83. Lis, S.; Mathur, J. N.; Choppin, G. R., Solvent Extr. Ion Exch. 1991, 9, 637-647.

84. McDowell, W. J.; Coleman, C. F., J. Inorg. Nucl. Chem. 1965, 27, 1117-1139. 
85. Unknown, VAPRO Vapor Pressure Osmometer Model 5520 User's Manual, M2468-4 rev B ed., Wescor, Inc., 2004, pp. 29-42.

86. Boiani, J. A., J. Chem. Educ. 1986, 63, 724-726.

87. Gran, G., Analyst (Cambridge, U.K.) 1952, 77, 661-671.

88. Rossotti, F. J.; Rossotti, H., J. Chem. Educ. 1965, 42, 375-378.

89. Partanen, J. I.; Juusola, P. M.; Minkkinen, P. O., Fluid Phase Equilib. 2003, 204, 245-266.

90. Bukietynska, K.; Mondry, A.; Osmeda, E., J. Inorg. Nucl. Chem. 1981, 43, 1321-1330.

91. Nilsson, M. University of California, Irvine, Irvine, CA. Personal communication, 2010.

92. Ryabchikov, D. I.; Ryabukhin, V. A., Analytical Chemistry of Yttrium and the Lanthanide

Elements (Ed.: A. P. Vinogradov), Ann Arbor-Humphrey Science Publisher, Ann Arbor, MI, 1970, pp. 159-177.

93. Moeller, T.; Brantley, J. C., Anal. Chem. 1950, 22, 433-441.

94. Baes, J. C. F., SXLSQI, A Program for Modeling Solvent Extraction System; ORNL-TM13604; Oak Ridge, TN, 1998.

95. Kolarik, Z., Solvent Extr. Ion Exch. 2010, 28, 707-763.

96. Pitzer, K. S., Activity Coefficients in Electrolyte Solutions (Ed.: K. S. Pitzer), CRC Press, Boca Raton, Florida, 1991, pp. 75-153.

97. Barton, A. F. M., CRC Handbook of Solubility Parameters and Other Cohesion Parameters, CRC Press: Boca Raton, Florida, 1991.

98. Baes, C. F.; Moyer, B. A., J. Phys. Chem. B 1997, 101, 6566-6574.

99. Harris, R. K.; Becker, E. D.; De Menezes, S. M. C.; Goodfellow, R.; Granger, P., Pure Appl. Chem. 2001, 73, 1795-1818.

100. Adamson, A. W., A Textbook of Physical Chemistry, 3rd ed., Academic Press, Inc.: Orlando, FL, 1986, pp. 1-972.

101. De Stefano, C.; Foti, C.; Giuffrè, O.; Sammartano, S., J. Chem. Eng. Data 2001, 46, 14171424.

102. The reported reaction in De Stefano et al. (protonation) is the reverse of what is reported in this work (deprotonation). When comparing enthalpy of reaction, reversing the direction of the reaction merely changes the sign of the enthalpy. De Stefano et al. show a decrease in the enthalpy for the reverse reaction, which equates to an increase in the enthalpy for the reaction presented in this paper.

103. Miralles, N.; Sastre, A.; Martinez, M.; Aguilar, M., Anal. Sci. 1992, 8, 773-777.

104. Jensen, M.; Chiarizia, R.; Urban, V., Solvent Extr. Ion Exch. 2001, 19, 865 - 884.

105. Colthrup, N. B.; Daly, L. H.; Wiberly, S. E., Introduction to Infrared and Raman Spectroscopy, 3rd ed., Academic Press, Inc., 1990, pp. 355-386.

106. Socrates, G., Infrared and Raman Characteristic Group Frequencies, 3rd ed., Wiley and Sons, Inc., New York, 2001, pp. 96, 128.

107. Czerwinski, K.; Draye, M.; Favre-Reguillon, A., in 232nd ACS National Meeting, San Francisco, CA, 2006.

108. Sigma-Aldrich, "Triton X-114 Product Description", http://www.sigmaaldrich.com/catalog/ProductDetail.do?lang=en\&N4=X114|SIAL\&N5=S EARCH_CONCAT_PNO|BRAND_KEY\&F=SPEC, accessed April 22, 2011.

109. Bedall, K.; Fischer, O., Ber. Dtsch. Chem. Ges. 1881, 14, 442.

110. Bedall, K.; Fischer, O., Ber. Dtsch. Chem. Ges. 1881, 14, 1366.

111. Bedall, K.; Fischer, O., Ber. Dtsch. Chem. Ges. 1882, 15, 683.

112. Berg, R., Fresenius J. Anal. Chem. 1927, 71, 23-36.

113. Hahn, F., Angew. Chem. 1926, 39, 1198. 
114. Hollingshead, R. G. W., Oxine and its Derivatives, Vol. 1, Butterworths Scientific Publications: London, 1954.

115. Czakissulikowska, D.; Kuznik, B.; Malinowska, A.; Kijanowicz, U., Chem. Anal. (Warsaw) 1993, 38, 53-62.

116. Czakis-Sulikowska, D.; Pustelnik, N.; Kuznik, B.; Malinowska, A., J. Alloys Compd. 2000, 300-301, 234-237.

117. Starý, J., Anal. Chim. Acta 1963, 28, 132-149.

118. Dyrssen, D.; Dahlberg, V., Acta Chem. Scand. 1953, 7, 1186-1196.

119. Schweitzer, G. K.; Bramlitt, E. T., Anal. Chim. Acta 1960, 23, 419-422.

120. Schweitzer, G. K.; Coe, G. R., Anal. Chim. Acta 1961, 24, 311-315.

121. Oosting, M., Anal. Chim. Acta 1959, 21, 301-308.

122. Oosting, M., Anal. Chim. Acta 1959, 21, 397-406.

123. Oosting, M., Anal. Chim. Acta 1959, 21, 505-510.

124. Taechangam, P.; Scamehorn, J. F.; Osuwan, S.; Rirksomboon, T., Sep. Sci. Technol. 2008, 43, 3601-3623.

125. Irving, H.; Ewart, J. A. D.; Wilson, J. T., J. Chem. Soc. 1949, 2672-2674.

126. Goto, H., Scientific Reports of the Tohoku Imperial University, First Series 1938, 418-428.

127. Ashton, L. A.; Bullock, J. I.; Simpson, P. W. G., J. Chem. Soc., Faraday Trans. 1 1982, 78, 1961-1970.

128. Gupta, R. D.; Manku, G. S.; Bhat, A. N.; Jain, B. D., J. Less-Common Met. 1970, 20, 345352.

129. Powell, K. J.; Solov'ev, V., The IUPAC Stability Constants Database, ScQuery Vn. 5.7 ed., Academic Software: Timble, Otley, UK, 2001.

130. Firsching, F. H.; Cuca, R. C., J. Chem. Eng. Data 1981, 26, 116-118.

131. Skorik, N. A., Zh. Neorg. Khim. 1977, 22, 1425-1427.

132. Triton $\mathrm{X}-100$ is identical to Triton $\mathrm{X}-114$ except the $\mathrm{n}=9-10$ with an average molecular weight of 625.

133. Sigma-Aldrich, "Triton X-100 Product Description", http://www.sigmaaldrich.com/catalog/ProductDetail.do?lang=en\&N4=T9284|SIAL\&N5= SEARCH_CONCAT_PNO|BRAND_KEY\&F=SPECi, accessed May 2, 2011.

134. Hoshino, H.; Saitoh, T.; Taketomi, H.; Yotsuyanagi, T.; Watanabe, H.; Tachikawa, K., Anal. Chim. Acta 1983, 147, 339-345.

135. Gans, P.; Sabatini, A.; Vacca, A., Talanta 1996, 43, 1739-1753.

136. 899 papers citing the Hyperquad publication on Web of Science on the access date $5 / 3 / 2011$.

137. Leggett, D. J.; McBryde, W. A. E., Anal. Chem. 1975, 47, 1065-1070.

138. 230 papers citing the SQUAD publication on Web of Science on the access date $5 / 3 / 2011$.

139. Robinson, T. A. University of Nevada, Las Vegas, Las Vegas, NV, Unpublished work, 2007.

140. Harned, H. S.; Owen, B. B., The Physical Chemistry of Electrolytic Solutions, 2nd ed., Reinhold Publishing Corp: New York, 1950, pp. 1-645.

141. Levine, I. N., Physical Chemistry, 2nd ed., McGraw-Hill Book Company, New York, 1983, pp. 170-173.

142. Harned, H. S.; Hamer, W. J., J. Am. Chem. Soc. 1933, 55, 2194-2206.

143. Pettit, L. D.; Puigdomenech, I.; Wanner, H.; Sukhno, I.; Buzko, V., lonic Strength Corrections for Stability Constants, 2.0 ed., IUPAC, 2004.

144. Buzko, V.; Polushin, A.; Sukhno, I.; Pettit, L. D., Temp Effects, 1.2 ed., IUPAC, 2004.

145. Unknown, Metrohm AG, Switzerland, 2006, p. 372. 
146. Fortuin, J. M. H., Anal. Chim. Acta 1961, 24, 175-191.

147. Baes, J. C. F.; Mesmer, R. E., The Hydrolysis of Cations, John Wiley and Sons: New York, NW, 1986, pp. 137.

148. Baes, J. C. F.; Mesmer, R. E., The Hydrolysis of Cations, John Wiley and Sons: New York, NY, 1976, pp. 1-489.

149. Ryabchikov, D. I.; Ryabukhin, V. A., Analytical Chemistry of Yttrium and the Lanthanide Elements, Ann Arbor-Humphrey Science Publisher: Ann Arbor, MI, 1970, pp. 292.

150. Woolley, E. M.; George, R., J. Solution Chem. 1974, 3, 119-126.

151. Matrosovich, T. Y.; Lobanov, F. I.; Makarov, N. V., Zh. Neorg. Khim. 1986, 31, 1441-1446.

152. Lobanov, F. I.; Matrosovich, T. Y., Zh. Neorg. Khim. 1981, 26, 3001-3006.

153. Irving, H.; Rossotti, H., Acta Chem. Scand. 1956, 10, 72-93.

154. Bates, R. G., J. Electroanal. Chem. Interfacial Electrochem. 1971, 29, 1-19.

155. Demirelli, H.; Köseoğlu, F., J. Solution Chem. 2004, 33, 1501-1515.

156. Kılıç, E.; Köseoğlu, F.; Başgut, Ö., Anal. Chim. Acta 1994, 294, 215-220.

157. Ramírez-García, J. J.; Solache-Ríos, M.; Jiménez-Reyes, M.; Rojas-Hernández, A., J. Solution Chem. 2003, 32, 879-896.

158. Kalidas, C.; Hefter, G.; Yizhak, M., Chem. Rev. 2000, 100, 819-852.

159. Narayana, V. G.; Swamy, S. J.; Lingaiah, P., Indian J. Chem., Sect A 1986, 25, 491-493.

160. Gupta, R. D.; Manku, G. S.; Bhat, A. N.; Jain, B. D., Aust. J. Chem. 1970, 23, 1387-1395. 


$$
\begin{gathered}
\text { VITA } \\
\text { Graudate College } \\
\text { University of Nevada, Las Vegas }
\end{gathered}
$$

Troy A Robinson

Degrees:

Bachelor of Science, Chemical Engineering, 2005

Brigham Young University

Special Honors and Awards:

US DOE Office of Civilian Radioactive Waste Management Graduate Fellow, 2006-2010

Publications:

Herbst, R.S.; Peterman, D.R.; Robinson, T.A. "PEG-400 Partitioning in the HCCD/PEG Process for Cs and Sr Recovery" Separation Science and Technology (2008), 43(9), 2557-2571.

Dissertation Title: Equilibrium Speciation of Select Lanthanides in the Presence of Acidic Ligands in Homo- and Heterogeneous Solutions

Dissertation Examination Committee:

Chairperson, Kenneth R. Czerwinski, Ph.D.

Committee Member, Tatiana G. Levitskaia, Ph.D.

Committee Member, Sam A. Bryan, Ph.D.

Committee Member, Patricia Paviet-Hartmann, Ph.D.

Graduate Faculty Representative, Ralf Sudowe, Ph.D. 